\title{
Der Einsatz von Nachhaltigkeitslabeln im Marketing
}

am Beispiel von Gütesiegeln mit Tierwohlkriterien (Tierwohl-Labeln) für artgerechter und nachhaltiger produzierte Fleisch- und Wurstwaren

\author{
Dissertation \\ zur Erlangung des Doktorgrades \\ der Fakultät für Agrarwissenschaften \\ der Georg-August-Universität Göttingen
}

\author{
vorgelegt von \\ Marko Freckmann \\ geboren in Duderstadt
}

Göttingen, im Oktober 2020 
D7

1. Gutachter: Prof. Dr. Ulrich Enneking

2. Gutachter: Prof. Dr. Achim Spiller

3. Gutachter: Prof. Dr. Ulrich Hamm

Tag der mündlichen Prüfung: 17. Dezember 2020 
Die Größe und den moralischen Fortschritt einer Nation kann man daran messen, wie sie ihre Tiere behandelt.

Mahatma Gandhi 


\section{Danksagung}

Zum erfolgreichen Gelingen dieser Arbeit haben zahlreiche Personen einen wertvollen Beitrag geleistet. Ein großer Dank geht zunächst an die Initiatoren des Promotionsprogramms, Animal Welfare in Intensive Livestock Production Systems Tierhaltung im Spannungsfeld von Tierwohl, Ökonomie und Gesellschaft ${ }^{\star}$ und das Niedersächsische Ministerium für Wissenschaft und Kultur (MWK) für die Finanzierung. Im Rahmen des Promotionsprogramms konnten spannende Fragestellungen zu den Herausforderungen der Nutztierhaltung in Deutschland diskutiert werden. Die Module des Promotionsprogramms an den Standorten Göttingen, Hannover, Vechta und Osnabrück gewährten interessante Einblicke in die Praxis der intensiven und extensiven Nutztierhaltung. In guter Erinnerung bleiben z. B. die Exkursionen zu landwirtschaftlichen Betrieben und die Gespräche mit Landwirten, Lebensmitteleinzelhändlern und Marketingexperten. Für die Organisation der Module gebührt ein großer Dank Dr. Gesa Busch, Dr. Christina Ikinger und Dr. Sarah Kühl. Herrn Prof. Dr. Recke und Frau Dr. Niens danke ich herzlich für die Übernahme von Korreferaten in den Doktorandenseminaren und Frau Dr. Christoph-Schulz vom ThünenInstitut danke ich für wertvolles Feedback zu meiner Arbeit.

Ein besonderer Dank gilt Herrn Prof. Dr. Enneking von der Hochschule Osnabrück für die Betreuung der Doktorarbeit. Im Rahmen der Dissertation kamen regelmäßig Fragen auf, für die kaum eine Antwort zu finden war. Herr Prof. Dr. Enneking stand jederzeit als engagierter Doktorvater zur Verfügung und hatte stets pragmatische Lösungsvorschläge parat. Besonders dankbar bin ich für die große Geduld und das Verständnis dafür, dass einzelne Arbeitsschritte länger gedauert haben als geplant sowie die Unterstützung bei den komplexen Auswertungen in SPSS und die stets sinnvollen Verbesserungsvorschläge für meine Arbeit. Besonders gefreut hat mich, dass ich an verschiedenen Beratungsprojekten des Lehrstuhls für Agrarmarketing mitarbeiten durfte, so etwa bei der Konzeption und Durchführung einer Zukunftsstudie für die APETITO AG oder einer Marketingstudie zum Apfelkonsum in Deutschland für EDEKA. 
Darüber hinaus gilt ein großer Dank dem Sprecher des Promotionsprogramms Herrn Prof. Dr. Spiller für das große Engagement im Rahmen des Promotionsprogramms und die Übernahme des Zweitgutachtens für meine Arbeit. Herrn Prof. Dr. Hamm von der Universität Kassel danke ich sehr herzlich für die Zusage zur Mitwirkung in der Prüfungskommission.

Besonders hervorzuheben ist des Weiteren die sehr gute Zusammenarbeit mit dem Marktforschungsinstitut RESPONDI AG in Köln. Trotz zahlreicher besonderer Wünsche bei der Programmierung des Fragebogens blieben alle Mitarbeiter stets freundlich und führten die Online-Befragung zügig und professionell durch.

Der größte Dank geht an meine Familie, die immer an meiner Seite steht und auf die ich mich in allen Lebenslagen verlassen kann. Daneben danke ich allen Freunden, die dazu beigetragen haben, dass ich meine Motivation für das Schreiben der Doktorarbeit stets aufrechterhalten konnte.

Göttingen, im Oktober 2020 


\section{Inhaltsverzeichnis}

Inhaltsverzeichnis ..............................................................................................................

Abbildungsverzeichnis..................................................................................................

Tabellenverzeichnis ..............................................................................................................VI

Abkürzungsverzeichnis ..........................................................................................................VIII

1. Einleitung..................................................................................................................... 1

1.1 Ausgangssituation .................................................................................................. 1

1.2 Zielsetzung der Arbeit ............................................................................................ 6

1.3 Aufbau der Arbeit.................................................................................... 11

2. Veränderung gesellschaftlicher Anforderungen .................................................. 14

2.1 Ethische Grundsätze und gesellschaftlicher Wertewandel ...................... 15

2.2 Abnahme der gesellschaftlichen Akzeptanz der Nutztierhaltung ......... 24

2.3 Reaktionen der Fleischbranche und der Agrarpolitik ............................. 27

2.4 Fazit zu den gesellschaftlichen Anforderungen ......................................... 29

3. Tierwohl-Labels als Einkaufshilfe...............................................................35

3.1 Einkaufs- und Ernährungsverhalten ........................................................... 37

3.2 Beachtung von Tierwohl-Labels....................................................................... 47

3.3 Hinderungsgründe für den Kauf................................................................... 64

3.4 Informationsbedarf bei Tierwohlprodukten ................................................ 67

3.5 Politische Präferenzen und politische Partizipation.................................. 74

4. Methodisches Vorgehen ....................................................................................... 79

4.1 Stichprobenwahl ...................................................................................................... 79

4.2 Konstruktion des Fragebogens ................................................................... 81

4.3 Pretest ……...............................................................................................................99

4.4 Durchführung der Befragung und Datenaufbereitung ........................... 94 
5. Ergebnisse der empirischen Studie ........................................................99

5.1 Beschreibung der Stichprobe....................................................................... 99

5.2 Einkaufs- und Ernährungsverhalten .................................................. 100

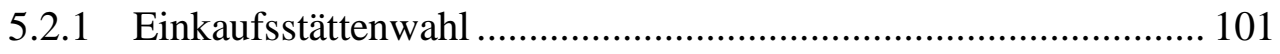

5.2.2 Angebotsformen Fleischtheke oder Selbstbedienung ................. 104

5.2.3 Verzehrmenge und Reduktionsbereitschaft .............................. 106

5.2.4 Klassische und ethische Kaufkriterien ........................................ 107

5.2.5 Präferenzen für Fleischarten und -sorten ................................... 116

5.3 Tierwohl-Labels als Einkaufshilfe ........................................................ 118

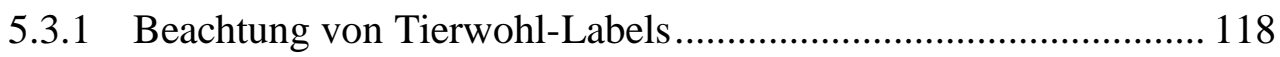

5.3.2 Hinderungsgründe für den Kauf .............................................. 139

5.3.3 Informationsbedarf bei Tierwohlprodukten ................................ 153

5.3.4 Politische Präferenzen und politische Partizipation..................... 161

5.3.5 Zusammenfassung der deskriptiven und bivariaten Ergebnisse ... 169

5.4 Ergebnisse der explorativen Faktorenanalysen ................................. 178

5.4.1 Faktorenextraktion aus Tierwohl-Label-Präferenzen .................. 179

5.4.2 Soziodemografische Unterschiede der Motivgruppen.................. 183

5.4.3 Vertriebsrelevante Unterschiede der Motivgruppen ..................... 185

5.4.4 Marketingrelevante Unterschiede der Motivgruppen .................. 192

5.4.5 Faktorenextraktion aus Kaufkriterien .......................................... 199

5.4.6 Zusammenfassung der Ergebnisse der Faktorenanalyse ............. 202 
5.5 Ergebnisse der hierarchischen Clusteranalyse ....................................205

5.5.1 Clusterbildung auf Basis der Tierwohl-Label-Präferenzen .......... 206

5.5.2 Soziodemografische Unterschiede zwischen den Clustern........... 210

5.5.3 Vertriebsrelevante Unterschiede zwischen den Clustern............. 216

5.5.4 Marketingrelevante Unterschiede zwischen den Clustern ............ 221

5.5.5 Zusammenfassung der Ergebnisse der Clusteranalyse ................ 232

6. Diskussion und Implikationen der Ergebnisse......................................... 235

6.1 Einkaufs- und Ernährungsverhalten .................................................... 236

6.2 Tierwohl-Labels als Einkaufshilfe.................................................... 247

6.3 Informationsbedarf bei Tierwohlprodukten ....................................... 262

6.4 Politische Präferenzen und politische Partizipation .............................. 270

6.5 Motiv- und segmentspezifische Unterschiede.................................... 273

6.5.1 Interpretation und Diskussion der Faktorenanalyse................... 274

6.5.2 Interpretation und Diskussion der Clusteranalyse ..................... 288

6.5.3 Handlungsempfehlungen für die identifizierten Marktsegmente . 308

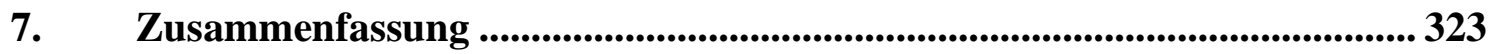

7.1 Limitationen der Forschung ................................................................... 325

7.2 Weiterer Forschungsbedarf ................................................................3328

Literaturverzeichnis ............................................................................................... 335

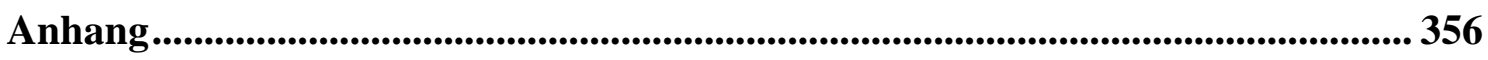

Anhang A: Fragebogen ........................................................................356

Anhang B: Soziodemografie ................................................................................... 373

Anhang C: Quotierung der Stichprobe ............................................................... 379

Anhang D: Eidesstattliche Erklärungen ....................................................... 381

Anhang E: Vermerk Sprachgebrauch .......................................................... 382 


\section{Abbildungsverzeichnis}

Abbildung 1: Schematische Darstellung der Arbeit ..................................................... 13

Abbildung 2: Assoziationen zum Begriff der Massentierhaltung ................................26

Abbildung 3: Die vier zentralen Ziele einer nachhaltigeren Ernährung ......................... 34

Abbildung 4: Informationsbedarf bei unterschiedlichen Lebensmitteln ........................69

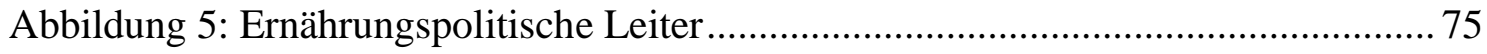

Abbildung 6: Einkaufsstätten für Fleisch- und Wurstwaren .................................... 103

Abbildung 7: Haupteinkaufsstätten für Fleisch- und Wurstwaren .............................. 104

Abbildung 8: Reduktion des Konsums von Fleisch- und Wurstwaren........................ 107

Abbildung 9: Kaufkriterien beim Einkauf von Fleisch- und Wurstwaren..................... 108

Abbildung 10: Zusammenhang Kaufkriterien und Haupteinkaufsstätte ..................... 110

Abbildung 11: Kaufhäufigkeit von Fleischarten und -sorten ................................... 117

Abbildung 12: Kaufinteresse an Tierwohlprodukten für Haustiere............................... 120

Abbildung 13: Bekanntheitsgrad ausgewählter Tierwohl-Labels................................ 121

Abbildung 14: Zusammenhang Bekanntheitsgrad und Haupteinkaufsstätte ................ 122

Abbildung 15: Kauf von Produkten mit Tierwohl-Labels ........................................ 126

Abbildung 16: Ranking der Tierwohl-Labels beim Fleischkauf ............................... 127

Abbildung 17: Zusammenhang Label-Präferenzen und Haupteinkaufsstätte ............... 129

Abbildung 18: Zusammenhang Label-Präferenzen und Reduktionsbereitschaft ......... 132

Abbildung 19: Kaufhäufigkeit von Produkten mit Tierwohl-Labels.......................... 137

Abbildung 20: Präferenzen zum Multi-Labeling von Tierwohlprodukten ................... 138

Abbildung 21: Hinderungsgründe beim Kauf von Produkten mit Tierwohl-Labels .... 140

Abbildung 22: Zusammenhang Kaufbarrieren und Haupteinkaufsstätte ..................... 141

Abbildung 23: Einschätzung der Strenge von Gesundheit und Tierwohl .................... 142 
Abbildung 24: Einschätzung der Strenge von Umwelt und Gesundheit 143

Abbildung 25: Einschätzung der Strenge von Tierwohl und Umwelt 144

Abbildung 26: Einschätzung negativer Auswirkungen des Fleischkonsums 145

Abbildung 27: Kundenzufriedenheit mit dem Angebot an Tierwohlprodukten 147

Abbildung 28: Zusammenhang Kundenzufriedenheit und Haupteinkaufsstätte 148

Abbildung 29: Sortierung von Tierwohlprodukten im LEH 150

Abbildung 30: Zusammenhang Sortierung und Haupteinkaufsstätte 151

Abbildung 31: Einkaufsstättenwechsel aufgrund von Tierwohlprodukten 152

Abbildung 32: Zusammenhang Wechselbereitschaft und Haupteinkaufsstätte. 153

Abbildung 33: Informationsbedarf beim Kauf von Fleisch- und Wurstwaren 154

Abbildung 34: Bereitstellung von Tierwohlinformationen 155

Abbildung 35: Zusammenhang Tierwohlinformationen und Haupteinkaufsstätte 156

Abbildung 36: Detaillierung von Herkunftsangaben als Kaufargument 159

Abbildung 37: Bereitsteller von Tierwohlinformationen 160

Abbildung 38: Politikansätze für mehr Tierwohl aus Verbrauchersicht 162

Abbildung 39: Bewertung politischer Ansätze nach gewählter Partei 163

Abbildung 40: Verbraucherpräferenzen für Tierwohlkennzeichnungsformen 165 Abbildung 41: Zusammenhang Kennzeichnungsformen und Partei 166 Abbildung 42: Möglichkeiten der politischen Partizipation für mehr Tierwohl 167

Abbildung 43: Einschätzung politischer Partizipation nach Parteien 169

Abbildung 44: Tierwohl-Label-Typologie nach Freckmann und Enneking 182 


\section{Tabellenverzeichnis}

Tabelle 1: Marketingbezogene Ziele der durchgeführten Verbraucherstudie .8

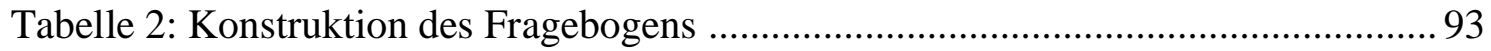

Tabelle 3: Durchführung der Befragung im Überblick .............................................. 96

Tabelle 4: Soziodemografische Merkmale der Stichprobe im Überblick...................... 99

Tabelle 5: Präferierte Angebotsform für Wurstwaren oder Aufschnitt ....................... 105

Tabelle 6: Präferierte Angebotsform für Fleisch zum Braten und Grillen .................... 105

Tabelle 7: Korrelation Kaufkriterien und Angebotsform - Wurst und Aufschnitt....... 111

Tabelle 8: Korrelation Kaufkriterien und Angebotsform - Fleisch zum Braten .......... 111

Tabelle 9: Zusammenhang Kaufkriterien und Soziodemografie ............................... 113

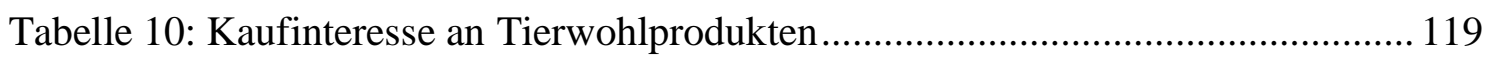

Tabelle 11: Zusammenhang Bekanntheitsgrad Tierwohl-Label und Soziodemografie 124

Tabelle 12: Korrelation Tierwohl-Label-Präferenzen und Angebotsform ................... 131

Tabelle 13: Label-Präferenzen nach soziodemografischen Kriterien .......................... 135

Tabelle 14: Korrelation Tierwohlinformationen und Angebotsform (Wurst) .............. 157

Tabelle 15: Korrelation Tierwohlinformationen und Angebotsform (Fleisch) ............ 158

Tabelle 16: Übersicht der Forschungsergebnisse ....................................................... 170

Tabelle 17: Überblick der bivariaten Analysen zu einzelnen Tierwohl-Labels ........... 175

Tabelle 18: Ergebnisse der Faktorenanalyse zu den Label-Präferenzen ...................... 180

Tabelle 19: Unterschiede Soziodemografie bei Tierwohl-Label-Präferenzgruppen .... 184

Tabelle 20: Präferierte Einkaufsstätten unterschiedlicher Tierwohl-Label-Fans .......... 186

Tabelle 21: Präferierte Angebotsformen unterschiedlicher Tierwohl-Label-Fans ....... 191

Tabelle 22: Motivbasierte Unterschiede bei Präferenzen zur Produktplatzierung ....... 192

Tabelle 23: Reduktionsbereitschaft unterschiedlicher Tierwohl-Label-Fans ................ 193 
Tabelle 24: Multi-Labeling-Präferenzen unterschiedlicher Tierwohl-Label-Fans 194

Tabelle 25: Gewichtung von Kaufkriterien unterschiedlicher Tierwohl-Label-Fans... 198

Tabelle 26: Ergebnisse der Faktorenanalyse zu Kaufkriterien beim Fleischkauf ........202

Tabelle 27: Kompakte Charakterisierung der Tierwohl-Label-Präferenzgruppen ....... 204

Tabelle 28: Stärke der Einflüsse der Motivfaktoren auf die gefundenen Cluster.........207

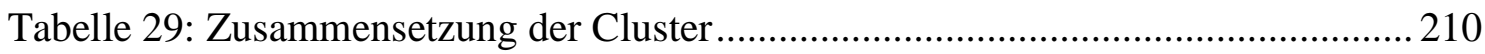

Tabelle 30: Soziodemografische Charakterisierung der Cluster .................................213

Tabelle 31: Segmentspezifische Unterschiede nach genutzten Einkaufsstätten I ........217

Tabelle 32: Segmentspezifische Unterschiede nach genutzten Einkaufsstätten II ....... 219

Tabelle 33: Ausgewählte segmentspezifische Unterschiede mit Tierwohlbezug .........222

Tabelle 34: Tierwohl-Label-Bekanntheit in den verschiedenen Segmenten ................ 227

Tabelle 35: Multi-Label-Präferenzen in den verschiedenen Segmenten ...................... 231

Tabelle 36: Überblick über die Ergebnisse der hierarchischen Clusteranalyse ............ 234

Tabelle 37: Übersicht der heterogenen Items beim Vergleich der Marktsegmente...... 289 


\section{Abkürzungsverzeichnis}

AG

BDI Bundesverband der Deutschen Industrie

BfR Bundesamt für Risikobewertung

BMEL Bundesministerium für Ernährung und Landwirtschaft

BÖLW Bund Ökologische Lebensmittelwirtschaft

BUND Bund für Umwelt- und Naturschutz Deutschland

CMA Centrale Marketing-Gesellschaft der deutschen Agrarwirtschaft

$\mathrm{CO}_{2} \quad$ Kohlendioxid

DBV Deutscher Bauernverband

DFB Deutscher Fußballbund

DGE Deutsche Gesellschaft für Ernährung

DGHM Deutsche Gesellschaft für Hygiene und Mikrobiologie

DLG Deutsche Landwirtschafts-Gesellschaft

DOSB Deutscher Olympischer Sportbund

EC European Commission

ESBL Extended-Spectrum-Betalaktamasen

ELER Europäischer Landwirtschaftsfonds für die Entwicklung des ländlichen Raums

et al. et alii

EU Europäische Union

FAO Food and Agriculture Organization of the United Nations

FMCGs $\quad$ Fast Moving Consumer Goods

GEPA Gesellschaft zur Förderung der Partnerschaft mit der Dritten Welt

GFK Gesellschaft für Konsumforschung 
IPCC Intergovernmental Panel on Climate Change

LEH Lebensmitteleinzelhandel

lit. littera

LOHAS Lifestyles of Health and Sustainability

MRSA Multi-resistenter Staphylococcus aureus

NABU Naturschutzbund Deutschland

NGO Non-Governmental Organisation

OECD Organisation for Economic Co-operation and Development

PR Public Relations

QR Quick Response

QS Qualität und Sicherheit

SB Selbstbedienung

SDGs $\quad$ Sustainable Development Goals

TSchG Tierschutzgesetz

TTIP Transatlantic Trade and Investment Partnership

UBA Umweltbundesamt

UN United Nations (Vereinte Nationen)

UNEP United Nations Environment Programme

USA United States of America

WBAE Wissenschaftlicher Beirat für Agrarpolitik beim Bundesministerium für Ernährung und Landwirtschaft

WHO World Health Organization

WTO World Trade Organization

WWF World Wide Fund For Nature 


\section{Einleitung}

\subsection{Ausgangssituation}

Die globale Fleischproduktion und der Fleischkonsum steigen von Jahr zu Jahr. Insbesondere aufgrund der steigenden Nachfrage in Asien wird auch für das nächste Jahrzehnt erwartet, dass sich der Wachstumstrend fortsetzt. $77 \%$ der landwirtschaftlichen Flächen der Erde werden für die industrielle Tierhaltung genutzt (BLUE HORIZON, 2020). Die negativen Auswirkungen des übermäßigen Fleischkonsums auf Tiere, Umwelt, Gesundheit und Soziales sind dabei massiv. Um den globalen Herausforderungen in den Bereichen Klimaschutz, Erhalt der Artenvielfalt, Welternährung, Wasserverbrauch oder Gesundheit besser begegnen zu können, wurden verschiedene Nachhaltigkeitsziele formuliert, so z. B. die Sustainable Development Goals (LEE et al., 2020; NAIDOO \& FISHER, 2020; UN, 2020). Die Reduktion des Fleischkonsums bzw. eine vorwiegend pflanzliche Ernährung, die weniger Ressourcen verbraucht und Treibhausgase verursacht, wird regelmäßig empfohlen (THE LANCET, 2019; WBAE, 2020; WWF, 2020).

In Deutschland empfiehlt der Wissenschaftliche Beirat des BMEL im aktuellen Gutachten „Politik für eine nachhaltigere Ernährung“, dass deutsche Verbraucher ihren Fleischkonsum reduzieren sollten, wobei gleichzeitig mehr Wert auf ethische Produktqualitäten gelegt werden sollte, um die ,Big-Four'-Ziele einer nachhaltigeren Ernährung (Tierwohl, Umwelt, Gesundheit und Soziales) erreichen zu können (WBAE, 2020). In den letzten Jahren ist bzgl. des Verbraucherverhaltens ein deutlicher Wandel festzustellen. Immer mehr Verbraucher wollen sich gesünder und umweltverträglicher ernähren und hinterfragen verstärkt die Produktionsbedingungen insbesondere bei Lebensmitteln. Eine der zentralen Fragen für die Verbraucher ist dabei, wie die Tiere gehalten wurden, von denen Fleisch, Milch oder Eier stammen. Daneben werden auch die sozialen Kontexte kritisch hinterfragt, in denen Lebensmittel produziert werden (CHRISTOPH-SCHULZ et al., 2018; ZÜHLSDORF et al., 2016). 
Das Thema Tierwohl wird in diesem Zusammenhang seit der Jahrtausendwende in der gesellschaftlichen, politischen und wissenschaftlichen Diskussion vermehrt thematisiert. Aufgrund zahlreicher Berichterstattungen in den Medien mit Bildern von Tierquälerei von Nutztieren in Deutschland hinterfragen immer mehr Verbraucher die Produktionsbedingungen für tierische Erzeugnisse und fordern von der Politik und der Fleischbranche eine größere Berücksichtigung des Tierschutzes in der intensiven Nutztierhaltung (KANIS et al., 2003; KAYSER et al., 2012b; VANHONACKER et al., 2010).

Die Fleischbranche, als ökonomisch bedeutendster Teil der deutschen Agrar- und Ernährungswirtschaft, steht dabei im Fokus der Diskussion. Im Vergleich zu anderen Branchen besitzt die Fleischbranche ein eher schlechtes Image (ALBERSMEIER \& SPILLER, 2010; SPILLER, 2013a). Zum einen bezieht sich die Kritik auf Skandale wie etwa Kontaminierungen von Fleisch- und Wurstwaren mit gesundheitsschädlichen Keimen (ALBERSMEIER \& SPILLER, 2009; KAYSER et al., 2012a).

Davon zu unterscheiden ist die Kritik an den vielfältigen Problemfeldern der intensiven Nutztierhaltung, die sich vor allem auf die Haltungsbedingungen konzentriert (BMEL, 2015). Dahingehend werden die seit Jahrzehnten zunehmenden Konzentrations- und Intensivierungsprozesse über die gesamte Wertschöpfungskette der Fleischproduktion kritisch betrachtet (BÄURLE \& TAMÁSY, 2012; KIRSCHKE et al., 2007; MCKENDREE, 2016; POUTA et al., 2010; PRÄTTÄLÄ et al., 2007; RÖDIGER \& HAMM, 2020; SCHIPMANN-SCHWARZE \& HAMM, 2020; SPILLER et al., 2015; STAMPA et al., 2020; THEUVSEN \& RECKE, 2008).

In Deutschland sinkt die Anzahl der landwirtschaftlichen Betriebe. Die Bestandszahlen in den Tierställen haben jedoch beträchtliche Größenordnungen angenommen (SPILLER et al., 2015). Für die zunehmende gesellschaftliche Kritik werden unterschiedliche Gründe diskutiert. Häufig wird in diesem Kontext auf die Entfremdung der Bevölkerung gegenüber der Landwirtschaft hingewiesen (ALBERSMEIER \& SPILLER, 2010; FEINDT et al., 2004; SPILLER et al., 2015; VON ALVENSLEBEN, 1995). 
Diese Entfremdung wurde womöglich durch die Tatsache weiter verstärkt, dass immer größere Bevölkerungsteile in den Städten fernab der Landwirtschaft wohnen und keinen persönlichen Bezug mehr zu Landwirten haben. SCHULZE et al. (2008) nennen als weiteren Grund für die Kritik der Bevölkerung die „Haustierperspektive‘, wobei die Nutztiere von den Menschen wie Haustiere betrachtet werden.

Daneben wird auf die realitätsferne Werbung verwiesen, die jahrzehntelang ein zu romantisches Bild von der Nutztierhaltung vermittelt hat (ALBERSMEIER \& SPILLER, 2010; ALBERSMEIER \& SPILLER, 2009, SCHULZE et al., 2008; SPILLER, 2006). Die Fleischbranche hat viele Jahre über die Centrale Marketing-Gesellschaft der deutschen Agrarwirtschaft (CMA) für den Kauf von Lebensmitteln geworben. Dabei wurden oft kleine idyllische landwirtschaftliche Betriebe gezeigt, die indes wenig mit den modernen Betrieben von heute $\mathrm{zu}$ tun haben. Umso irritierter waren die Verbraucher womöglich, als in den Medien über die realen Produktionsbedingungen berichtet wurde. Dieser Effekt wurde dadurch verstärkt, dass häufig Berichte über grausame Tierquälerei gezeigt wurden.

Des Weiteren hat sich durch die stärkere Berücksichtigung des Eigenwerts von Tieren in der Bevölkerung ein höheres Tierschutzbewusstsein entwickelt. Dies ist ein Grund für die zunehmende Kritik (EDWARDS \& SCHNEIDER, 2005; VERBEKE \& VIANE, 2000; VON ALVENSLEBEN, 2002). Der Eigenwert von Tieren spielt eine zentrale Rolle hinsichtlich der Unterscheidung ethischer Ansätze, bei denen der Umfang der Objekte, denen ein Eigenwert zugesprochen wird und die somit schützenswert sind, variiert. Während bei anthropozentrischen Ansätzen nur der Mensch einen Eigenwert besitzt, wird aus pathozentrischer Sicht auch Tieren ein Eigenwert zugesprochen (DRZE, 2019).

Die Kritik der Gesellschaft an der intensiven Nutztierhaltung wurde in den letzten Jahren immer mehr von den Medien aufgenommen. Auf diesem Weg wurden große Teile der Bevölkerung über die Probleme in der Nutztierhaltung aufmerksam gemacht und die Relevanz des Themas ist stetig größer geworden. Heute scheint das Thema Tierwohl in der Nutztierhaltung zu den Themen zu gehören, die die Menschen am meisten bewegen (FRASER \& DUNCAN, 1998; KEELING et al., 2013; SPILLER et al., 2015). 
Diese Tendenz ist auch in vielen anderen Ländern der Welt zu beobachten. SPILLER et al. (2015) erkennen diesen Trend zu mehr Tierwohl besonders stark in Nordwesteuropa. Neben einem immer stärkeren Ausbau der ökologischen Landwirtschaft mit Bio-Labels, die Tierwohl- und ökologische Kriterien beinhalten, wurden in verschiedenen Ländern spezielle Tierwohl-Labels eingeführt, durch die schwerpunktmäßig die artgerechtere Tierhaltung in den Vordergrund gestellt wird. So gibt es beispielsweise in Dänemark das Siegel BEDRE DYREVELFÆRD (Besserer Tierschutz) und in den Niederlanden das Siegel BETER LEVEN (BUNDESTAG, 2016).

In Schweden gibt es seit 1985 das besondere Tierwohlsiegel KRAV (,Kontrollföreningen för Alternativ Odling'), dem die Kriterien des DE-BIO-Labels zugrunde liegen, das jedoch in verschiedenen Bereichen des Tierwohls über die Anforderungen des DE-BIOLabels hinausgeht. Daneben existieren vereinzelte private Labels, die ebenfalls Tierwohlkriterien beinhalten. In Großbritannien gibt es spezielle Zertifizierungssysteme für die Industrie, die Tierwohlkriterien beinhalten, wie z. B. den RED TRACTOR oder LION EGGS (BUNDESTAG, 2016).

In der Politik hat das Thema Tierwohl ebenfalls eine hohe Relevanz erreicht, und es wird über unterschiedlichste politische Lösungsansätze diskutiert (FRASER, 2008; KEELING et al., 2013; SPILLER et al., 2015). Die Bundesregierung könnte z. B. die gesetzlichen Mindeststandards in den verschiedenen Bereichen der Nutztierhaltung anheben, was jedoch mit zahlreichen Implementierungsproblemen einhergehen würde und ökonomische Nachteile mit sich brächte, wie beispielsweise eine Schwächung der internationalen Marktposition der deutschen Wirtschaft (DEIMEL et al., 2010). Daher werden strikte Gesetzesänderungen von der Fleischbranche und der Landwirtschaft mehrheitlich abgelehnt.

Durch eine stärkere Umverteilung der EU-Agrarsubventionen von der 1. Säule (Direktzahlungen nach Fläche) in die 2. Säule (Förderprogramme für eine nachhaltigere und umweltschonendere Bewirtschaftung) könnte die Politik noch mehr Landwirte in ihrem Einsatz für mehr Tierwohl finanziell unterstützen (BMEL, 2015). Eine weitere Möglichkeit bestünde in der Einführung einer gesetzlich verpflichtenden Haltungskennzeichnung (SPILLER \& ZÜHLSDORF, 2018). 
Daneben könnte die Politik private Initiativen, wie die Brancheninitiative Tierwohl oder verschiedene andere Tierwohl-Labels, durch begleitende Werbung oder finanzielle Unterstützung fördern. Nicht außer Acht gelassen werden sollten auch die Effekte lenkender bzw. unterstützender politischer Maßnahmen, die auf die Verteuerung tierischer Erzeugnisse, Preissenkungen für alternative Proteinquellen sowie Gemüse und Obst, indirekte Anreize für eine gesündere Ernährung, eine bessere Bereitstellung von Informationen oder ein besseres Monitoring abzielen (SPILLER \& ZÜHLSDORF, 2018; WBAE, 2020). Durch diese Maßnahmen könnte indirekt eine Senkung des Fleischkonsums gefördert werden, was sich evtl. ebenfalls positiv auf das Tierwohl auswirken könnte. Eine politische Förderung von mehr Tierwohl könnte auch durch die Einführung eines staatlichen bzw. europäischen Tierwohl-Labels erfolgen (BMEL, 2015).

Vor allem in freiwillige Tierwohl-Labels werden in Deutschland große Hoffnungen gesetzt, damit Verbraucher Fleisch- und Wurstwaren aus einer artgerechteren und nachhaltigeren Nutztierhaltung im Lebensmitteleinzelhandel besser erkennen können. Diese Tierwohl-Labels beinhalten oft ökologische Kriterien und Tierwohlkriterien. Beispielhaft genannt seien in diesem Zusammenhang die ökologischen Tierwohl-Labels DEMETER, BIOLAND, NATURLAND, DE-BIO und EU-BIO. Des Weiteren gibt es reine Tierwohl-Labels, die keine Bio-Labels sind, wie z. B. NEULAND (BMEL, 2019b). Der Erfolg dieser Tierwohl-Labels ist jedoch überschaubar, was auf zahlreiche Probleme zurückzuführen ist (SPILLER et al., 2010; WBAE, 2020; WEINRICH et al., 2015).

$\mathrm{Zu}$ den häufig aufgeführten Problemen in der Wissenschaft zählt die Diskrepanz zwischen den Einstellungen der Verbraucher (mehr Tierwohl erwünscht) sowie dem Einkaufs- und Ernährungsverhalten (übermäßiger Konsum preisgünstiger Fleisch- und Wurstwaren). Die zu hohen Preisdifferenzen zwischen Tierwohlprodukten und konventionellen Fleisch- und Wurstwaren, die zu geringe Zahlungsbereitschaft der Verbraucher sowie die Verwirrung stiftende hohe Anzahl unterschiedlicher Labels im LEH sind weitere Gründe (FRANZ et al., 2012; LAGERKVIST \& HESS, 2011; NOCELLA et al., 2010; PADEL \& FOSTER, 2005; SCHRÖDER \& MCEACHERN, 2004; VAN KLEEF et al., 2007; VERMEIR \& VERBEKE, 2006). 
Aufgrund der hohen gesellschaftlichen Relevanz des Tierwohls setzt sich auch die Wissenschaft intensiv mit diesem Thema auseinander. Dies spiegelt sich in der Entstehung eines eigenen globalen Forschungsgebietes, der Tierwohlforschung, wider. In einer Vielzahl internationaler Verbraucherstudien wurde das Thema aus verschiedensten Perspektiven untersucht. So existieren zahlreiche Verbraucherstudien zur allgemeinen Wahrnehmung des Themas Tierwohl in der Gesellschaft und zum Tierwohl bei allgemeinen oder auch besonderen Lebensmitteln sowie Zahlungsbereitschaftsanalysen bei einzelnen Vertriebsformen oder für spezifische Produkte.

Daneben liegen Segmentierungsstudien vor, die sich auf den allgemeinen respektive den ökologischen Lebensmittelkonsum oder bestimmte Kaufkriterien der Verbraucher (ALONSO et al., 2020; BOAITEY \& MINEGISHI, 2020; CLARK et al., 2017; DE JONGE et al., 2015; DE JONGE et al., 2015; HAMM et al., 2011; HEMMERLING et al., 2016; LAI \& YUE, 2020; SCHULZE et al., 2008; SPILLER et al., 2015; TOMASEVIC et al., 2020; VANHONACKER et al., 2010; VAN LOO et al., 2014).

\subsection{Zielsetzung der Arbeit}

Fleisch- und Wurstwaren wurden in der Vergangenheit zumeist als standardisierte Massenware vermarktet. Bei der Vermarktung von Fleisch- und Wurstwaren aus einer artgerechteren und nachhaltigeren Nutztierhaltung werden jedoch eine stärkere Marktdifferenzierung und ein Wechsel von der Strategie der Kostenführerschaft hin zu einer Qualitätsführerschaft empfohlen (AMINE \& CADENAT, 2003; WBAE, 2020; WEINRICH et al., 2015).

Daraus resultieren zahlreiche vermarktungsrelevante Fragen, die sich Anbietern von Fleisch- und Wurstwaren stellen, die differenzierte Vermarktungsstrategien entwickeln möchten. Vor diesem Hintergrund ist das Hauptziel der vorliegenden Arbeit, einen Beitrag für die bessere Vermarktung von Fleisch- und Wurstwaren aus einer artgerechteren und nachhaltigeren Nutztierhaltung zu leisten. 
$\mathrm{Zu}$ diesem Zweck sollen die Tierwohl-Label-Präferenzen der Verbraucher, die für die erfolgreiche Umsetzung von Premiumstrategien für Anbieter von Fleisch- und Wurstwaren von großer Bedeutung sind, umfassend analysiert werden. Auch wenn, wie in der Ausgangssituation der Arbeit erwähnt, zahlreiche Studien zum allgemeinen Verbraucherverhalten beim Fleisch- und Wurstkauf vorliegen, konnte eine Forschungslücke im Hinblick auf den gezielten Einsatz von Marketinginstrumenten je nach Tierwohl-Label-Präferenzen der Verbraucher identifiziert werden, die mit der vorliegenden Studie etwas mehr geschlossen werden soll. Hierfür sollen erstmals ausgewählte ökologische und nichtökologische Tierwohl-Labels genauer untersucht werden, es soll eine Tierwohl-Label-Typologie erstellt werden und eine Marktsegmentierung auf Basis der Tierwohl-Label-Präferenzen deutscher Verbraucher erfolgen. Dabei gilt es, auch das allgemeine Einkaufs- und Ernährungsverhalten der Verbraucher zu berücksichtigen. Einzelne Segmente werden umfassend charakterisiert und es werden Handlungsempfehlungen im Hinblick auf die zentralen Marketinginstrumente für Anbieter von Fleisch- und Wurstwaren ausgesprochen.

In Tabelle 1 sind im Hinblick auf den strategischen Einsatz der Marketinginstrumente die Ziele der vorliegenden Arbeit zusammenfassend dargestellt, um den Forschungsbeitrag für die Anbieter von Fleisch- und Wurstwaren zu verdeutlichen. Für Anbieter von Fleisch- und Wurstwaren sind dies im Wesentlichen Entscheidungen, die die Produkt-, Kommunikations-, Vertriebs- und Preispolitik betreffen. Da sich Entscheidungen zur Preispolitik auf andere Methoden zur Ermittlung von Zahlungsbereitschaften stützen (wie z. B. Discrete-Choice-Analysen), wurde der Fokus in dieser Arbeit auf die Produkt-, Vertriebs- und Kommunikationspolitik gesetzt. Diese drei Instrumente bieten das höchste Optimierungspotenzial für die Angebotsseite hinsichtlich der Vermarktung von Tierwohlprodukten. Daneben werden auch einige Empfehlungen zur Gestaltung der Preispolitik für Tierwohlprodukte auf Basis der Soziodemografie (z. B. Einkommen) und der Präferenzen (z. B. Preisorientierung) ausgesprochen, die dabei helfen können, die Preise je Segment besser zu positionieren und den Einsatz weiterer preispolitischer Instrumente zu begründen. 
Tabelle 1: Marketingbezogene Ziele der durchgeführten Verbraucherstudie

\begin{tabular}{|l|l|l|l|}
\hline $\begin{array}{l}\text { Ziel 1: Produkt- } \\
\text { entscheidungen }\end{array}$ & $\begin{array}{l}\text { Ziel 2: Vertriebs- } \\
\text { entscheidungen }\end{array}$ & $\begin{array}{l}\text { Ziel 3: Kommunikations- } \\
\text { entscheidungen }\end{array}$ & $\begin{array}{l}\text { Ziel 4: Preis- } \\
\text { entscheidungen }\end{array}$ \\
\hline $\begin{array}{l}\text { Auswahl von Tierwohl- } \\
\text { Labels für die Produkte }\end{array}$ & $\begin{array}{l}\text { Auswahl von Absatz- } \\
\text { wegen und Regionen }\end{array}$ & $\begin{array}{l}\text { Bereitstellung von } \\
\text { Tierwohlinformationen }\end{array}$ & $\begin{array}{l}\text { Allgemeine } \\
\text { Preispositionierung }\end{array}$ \\
\hline $\begin{array}{l}\text { Gestaltung des } \\
\text { Produktangebots nach } \\
\text { wichtigen Kaufkriterien }\end{array}$ & $\begin{array}{l}\text { Auswahl Angebotsform } \\
\text { (Theke/SB) }\end{array}$ & $\begin{array}{l}\text { Gestaltung des } \\
\text { Informationsangebots }\end{array}$ & $\begin{array}{l}\text { Einsatz weiterer } \\
\text { preispolitischer } \\
\text { Instrumente }\end{array}$ \\
\hline $\begin{array}{l}\text { Auswahl zusätzlicher } \\
\text { Gütesiegel zu den }\end{array}$ & $\begin{array}{l}\text { Vertriebsstrategie am } \\
\text { Point of Sale bzgl. der } \\
\text { Pierwohl-Labels }\end{array}$ & $\begin{array}{l}\text { Detaillierungsgrad von } \\
\text { Herkunftsangaben }\end{array}$ & \\
\hline
\end{tabular}

\section{Quelle: Eigene Darstellung}

Um möglichst zahlreiche Erkenntnisse für produktpolitische Entscheidungen von Anbietern zu erhalten, sollen (aufbauend auf den Fragen zum Einkaufs- und Ernährungsverhalten) umfangreiche Erkenntnisse zur Beachtung von Tierwohl-Labels aus der Perspektive der Verbraucher gewonnen werden.

Von besonderem Interesse für die Vermarktung sind dabei der Bekanntheitsgrad der Tierwohl-Labels, die Berücksichtigung der Tierwohl-Labels beim Kauf von Fleisch- und Wurstwaren (Kauferfahrung, Kaufpräferenzen, Kaufhäufigkeit und Hinderungsgründe für den Kauf) sowie das Image der Tierwohl-Labels in Bezug auf die drei Kriterien artgerechtere Tierhaltung, Umweltfreundlichkeit und Gesundheitsaspekte.

Für distributionspolitische Entscheidungen bei der Vermarktung von Tierwohlprodukten soll in der vorliegenden Arbeit neues Wissen generiert werden, damit Anbieter von Fleisch- und Wurstwaren strategischere vertriebspolitische Entscheidungen treffen können. Von zentraler Bedeutung in diesem Zusammenhang ist die Auswahl der Vertriebskanäle (Einkaufsstätten) für unterschiedliche Zielgruppen. 
Diesbezüglich soll in der vorliegenden Arbeit erstmals untersucht werden, welche konkreten Einkaufsstätten verschiedene Tierwohl-Label-Fans für den Einkauf von Fleisch- und Wurstwaren nutzen. Dabei sollen detaillierte Erkenntnisse hinsichtlich spezifischer Einkaufsstätten wie z. B. EDEKA oder ALDI wie auch gruppenspezifische Erkenntnisse bzgl. der unterschiedlichen Betriebsformen Supermarkt, Discounter, Verbrauchermarkt und Metzger gewonnen werden. Daneben wird der Versuch unternommen, geographische Unterschiede bei den Verbraucherpräferenzen bzgl. verschiedener Tierwohl-Labels in verschiedenen Regionen Deutschlands (Bundesländer, Stadt-Land-Vergleich) zu ermitteln, die als Basis für die Entwicklung gezielterer Vertriebsstrategien dienen können. Weitere Aspekte, die im Rahmen der Distributionspolitik untersucht werden sollen, umfassen die Auswahl der Angebotsform (Fleischtheke oder SB-Bereich) für verschiedene Verbrauchergruppen und TierwohlLabels sowie die Platzierung von Tierwohlprodukten im Handel.

Eine besondere Rolle bei der Vermarktung von Tierwohlprodukten spielt das Angebot an Informationen, das die Anbieter von Fleisch- und Wurstwaren im Rahmen unterschiedlicher Kommunikationsentscheidungen gestalten müssen. Für die Glaubwürdigkeit von Tierwohl-Labels sind Zusatzinformationen von zentraler Bedeutung.

Die Preisaufschläge für mehr Tierwohl oder mehr Umweltfreundlichkeit sollten in transparenter Form begründet werden, z. B. durch die besonderen ethischen Produktvorteile. Im Rahmen der vorliegenden Studie soll ermittelt werden, wie viele Verbraucher sich bessere Informationen $\mathrm{zu}$ den Haltungsbedingungen der Tiere wünschen, wo und in welcher Form die Informationen aus Verbrauchersicht bereitgestellt werden sollen, welche Organisationen als Informationsquellen das Vertrauen der Verbraucher genießen und wie detailliert Herkunftsangaben bei Tierwohlprodukten sein sollten.

Da das Tierwohl-Labeling bei Fleisch- und Wurstwaren nicht im politikfreien Raum erfolgt, sollen ergänzend verschiedene Politikoptionen für mehr Tierwohl von den Verbrauchern bewertet werden, um daraus Handlungsempfehlungen für die Politik zur Verbesserung des Tierwohls ableiten zu können. 
Tierschutzorganisationen fordern eine deutlich stärkere Regulation der intensiven Nutztierhaltung. Die Bundesregierung setzt jedoch schwerpunktmäßig auf freiwillige Tierwohl-Labels. Vor diesem Hintergrund ist die Sicht der Verbraucher auf verschiedene politische Optionen für mehr Tierwohl interessant. Daher soll in der vorliegenden Studie eine vergleichende Bewertung politischer Instrumente für mehr Tierwohl durch die befragten Verbraucher vorgenommen werden. Neben gesetzlichen Änderungen des Tierschutzgesetzes werden Subventionen für mehr Tierwohl, eine staatliche Haltungskennzeichnung, Tierwohl-Labels und Tierwohlinitiativen zur Auswahl gestellt. Des Weiteren sollen verschiedene Kennzeichnungsformen (Sterne, Ampel, Zahlen etc.) bewertet werden, die für alle Tierwohlkennzeichnungen mit mehreren Abstufungen eingesetzt werden können. Somit sind diese Ergebnisse im Hinblick auf die Gestaltung staatlicher Haltungskennzeichnungen oder auch für die diskutierte Einführung eines europäischen Tierwohl-Labels interessant. Um die Perspektive der Verbraucher auf ihren eigenen Einfluss für eine verbesserte Nutztierhaltung in Deutschland zu verstehen, sollen die Befragten unterschiedliche Möglichkeiten der politischen Partizipation beim Thema Tierwohl bewerten. So kann festgestellt werden, welche Bedeutung die Verbraucher ihrem persönlichen Einkaufs- und Konsumverhalten für die Verbesserung der Nutztierhaltung in Deutschland beimessen und wie sie andere Möglichkeiten der politischen Partizipation (Bundestagswahl, Petitionen, Demonstrationen etc.) bewerten.

Das Meta-Ziel der Arbeit liegt in einer besseren Kenntnis der unterschiedlichen Verbraucherpräferenzen bei Tierwohlprodukten, um die Vermarktung von Fleisch- und Wurstwaren aus einer artgerechteren und nachhaltigeren Nutztierhaltung besser an den Verbraucherpräferenzen auszurichten, damit der Einsatz von Nachhaltigkeitssiegeln bzw. Tierwohl-Labels im Lebensmittelmarketing mit Hilfe wissenschaftlich abgesicherter Erkenntnisse noch strategischer und zielorientierter erfolgen kann. Die Erstellung einer Tierwohl-Label-Typologie sowie die Segmentierung deutscher Verbraucher $(n=930)$ auf Basis der unterschiedlichen Tierwohl-Label-Präferenzen stellen dabei den Schwerpunkt der vorliegenden Arbeit dar. 


\subsection{Aufbau der Arbeit}

Der Aufbau der Arbeit, die einen theoretischen Teil und einen empirischen Teil beinhaltet, ist in Abbildung 1 schematisch dargestellt. Kapitel 2 befasst sich zunächst, aufbauend auf dem Gutachten „Wege $\mathrm{zu}$ einer gesellschaftlich akzeptierten Nutztierhaltung“ des Wissenschaftlichen Beirats für Agrarpolitik beim Bundesministerium für Ernährung und Landwirtschaft (BMEL), mit der Veränderung gesellschaftlicher Anforderungen an die Nutztierhaltung in Deutschland.

Die durch den globalen Megatrend der Neo-Ökologie deutlich gestiegenen Anforderungen deutscher Verbraucher an ethische Aspekte der Fleischproduktion (z. B. im Hinblick auf das Tierwohl oder Umweltfreundlichkeit) sind der zentrale Grund dafür, dass Produzenten und Händler von Fleisch- und Wurstwaren einem immer stärkeren Handlungsdruck ausgesetzt sind. Um deutschen Verbrauchern ein aus ethischer Perspektive besseres Angebot an Produkten unterbreiten und auf die veränderten Rahmenbedingungen optimal reagieren zu können, müssen Hersteller und Händler von Fleisch- und Wurstwaren den gesellschaftlichen Wertewandel sowie die stark abnehmende Akzeptanz der intensiven Nutztierhaltung in Deutschland zunächst einmal nachvollziehen und richtig einordnen.

In Kapitel 3 werden Studien vorgestellt, in denen eine Auseinandersetzung mit dem Thema Tierwohl aus Verbraucherperspektive erfolgt. In Unterkapitel 3.1 wird das Einkaufs- und Ernährungsverhalten bei Fleisch- und Wurstwaren charakterisiert. Dabei wird auf die Einkaufsstättenwahl, die Angebotsformen (Fleischtheke und Selbstbedienungsbereich), das Ernährungsverhalten, die Kaufkriterien und Sortenpräferenzen eingegangen. Anschließend werden die Beachtung von TierwohlLabels, die Hinderungsgründe für den Kauf von Tierwohlprodukten, der Informationsbedarf bei Tierwohlprodukten und die Politikoptionen beschrieben (Unterkapitel 3.2 bis 3.5). Bereits existierende wissenschaftliche Forschungserkenntnisse sowie Praxisstudien sollen in diesem Kapitel dargestellt werden, um Forschungslücken aufzuzeigen und eine theoretische Grundlage für die empirischen Untersuchungen der vorliegenden Arbeit zu schaffen. 
Im ersten empirischen Teil der Arbeit (Methodenteil) werden anschließend die Stichprobenwahl, die Konstruktion des Fragebogens, der Pretest und die Durchführung der Befragung beschrieben (Kapitel 4). Die umfangreichen Ergebnisse der empirischen Studie werden anschließend im Ergebnisteil (Kapitel 5) beschrieben.

Zunächst wird im Ergebnisteil die Stichprobe beschrieben, bevor anschließend einzelne Erkenntnisse zum Einkaufs- und Ernährungsverhalten sowie zum Tierwohl-Labeling vorgestellt werden (Unterkapitel 5.2 bis 5.3). Diese Erkenntnisse basieren auf den Forschungshypothesen, die aus dem Theorieteil abgeleitet werden konnten. In den Unterkapiteln 5.4 und 5.5 des Ergebnisteils werden die Ergebnisse weiterführender Analysen vorgestellt. Dabei handelt es sich um die gruppenspezifischen Untersuchungsergebnisse der durchgeführten Faktorenanalyse (Tierwohl-LabelTypologie; Unterkapitel 5.4) und der durchgeführten Clusteranalyse (Verbrauchertypologie; Unterkapitel 5.5). Für diesen Teil wurden aufgrund der bis dato unbekannten Verbrauchergruppen keine Forschungshypothesen erstellt, sondern es wurde ein exploratives Vorgehen gewählt.

Im Rahmen der Diskussion (Kapitel 6) werden zunächst die zentralen empirischen Forschungsergebnisse der Unterkapitel 5.2 und 5.3 (bivariate Analysen) interpretiert und diskutiert. Dabei werden die Ergebnisse kritisch betrachtet und an geeigneten Stellen praxisnahe Handlungsempfehlungen für Anbieter von Fleisch- und Wurstwaren, die Politik und die Wissenschaft abgeleitet. Daran schließen sich in Unterkapitel 6.5 die Interpretation und eine Diskussion der Ergebnisse der Faktorenanalyse und der Clusteranalyse (multivariate Analysen) an. Abschließend werden konkrete Handlungsempfehlungen im Hinblick auf den strategischen Einsatz der zentralen Marketinginstrumente für die identifizierten Marktsegmente ausgesprochen. Die Zusammenfassung der Arbeit erfolgt in Kapitel 7. Nach einer kompakten Darstellung der gewonnenen Erkenntnisse wird auf einige Limitationen der Arbeit hingewiesen und weiterer Forschungsbedarf beschrieben, der aus der vorliegenden Studie abgeleitet werden konnte und die Basis für weitere interessante Forschungsprojekte bilden kann. 


Ausgangssituation
Zielsetzung
Aufbau der Arbeit

Theorie

Ethischer Wertewandel in der Gesellschaft

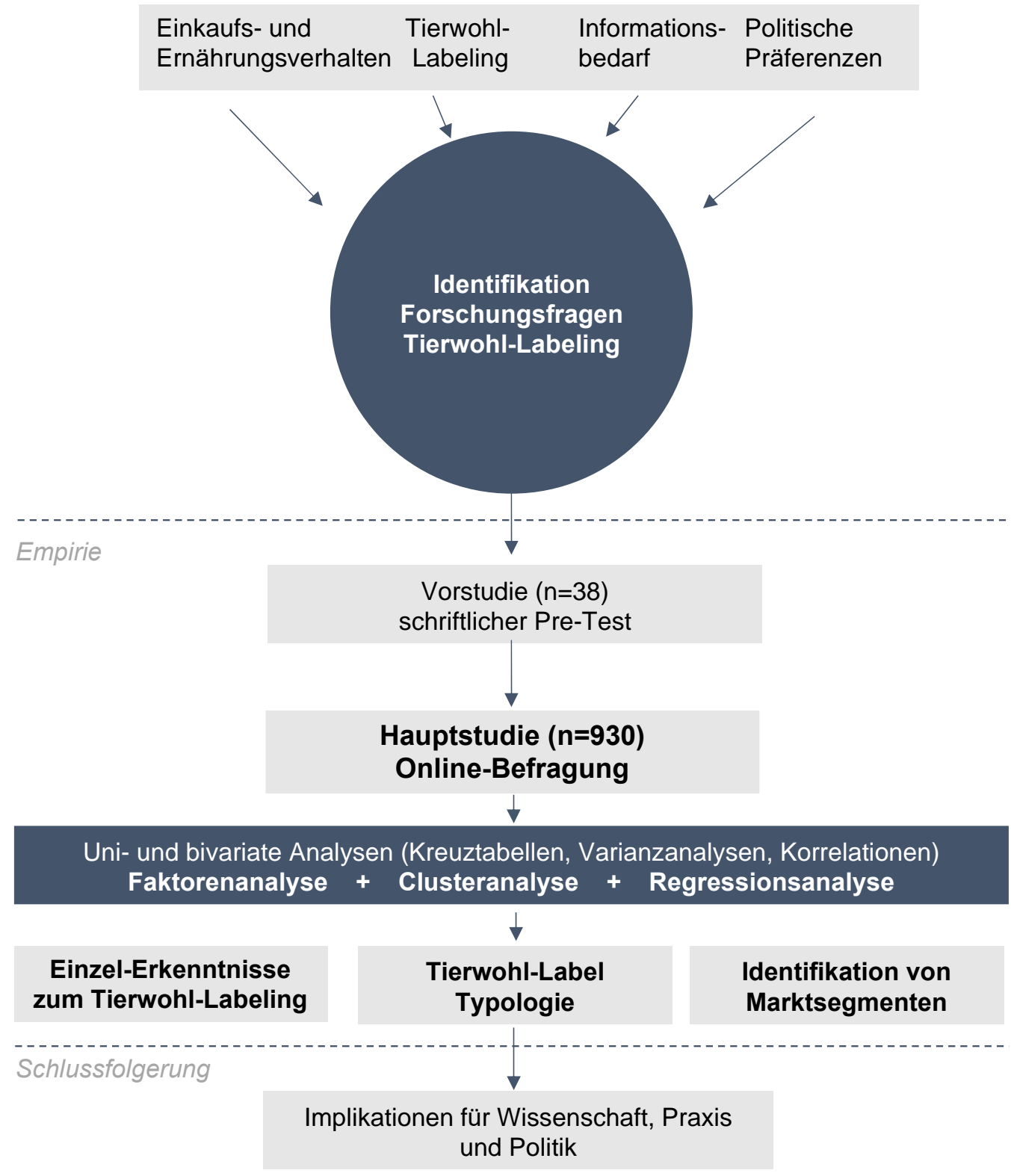

Abbildung 1: Schematische Darstellung der Arbeit

(Quelle: Eigene Darstellung) 


\section{Veränderung gesellschaftlicher Anforderungen}

Die gesellschaftlichen Anforderungen an das Tierwohl haben sich in den letzten Jahren stark verändert. Basierend auf ethischen Grundsätzen wird aktuell eine breite gesellschaftliche Diskussion zum Umgang mit Nutztieren und zum Konsum von Fleischprodukten geführt. Dabei ist in der Bevölkerung ein Wertewandel zu erkennen, der dazu geführt hat, dass die gesellschaftliche Akzeptanz der intensiven Nutztierhaltung in den letzten Jahren deutlich abgenommen hat (WBAE, 2020).

Dazu beigetragen hat der globale Megatrend der Neo-Ökologie, durch den Anforderungen an die Politik und die Wirtschaft auf der ganzen Welt neu definiert wurden. In diesem Zusammenhang wird u. a. ein besserer Umgang mit der Natur, der Gesundheit oder Tieren eingefordert. Die intensive Nutztierhaltung wird von dem neuen Zeitgeist daher besonders stark getroffen. Megatrends sind durch einen grundlegenden und langfristigen Wandel charakterisiert, der normalerweise länger als 50 Jahre dauert und tiefgreifende Konsequenzen mit sich bringt (ZUKUNFTSINSTITUT, 2019).

Die Kenntnis der in der Bevölkerung unterschiedlich ausgeprägten Moralvorstellungen hilft dabei, das unterschiedliche Einkaufs- und Ernährungsverhalten der Verbraucher beim Fleischkonsum sowie die Präferenzen von Verbrauchern für mehr Tierwohl besser $\mathrm{zu}$ verstehen. In Unterkapitel 2.1 sollen zunächst die drei wesentlichen ethischen Grundpositionen hinsichtlich des Umgangs mit Tieren vorgestellt werden, die für das Verständnis der veränderten Anforderungen der Verbraucher an das Tierwohl notwendig sind. Hierzu zählen der Anthropozentrismus, der Pathozentrismus und der Biozentrismus. Dabei wird ein deutlicher gesellschaftlicher Wertewandel erkennbar. In Unterkapitel 2.2 wird die abnehmende Akzeptanz der intensiven Nutztierhaltung beschrieben, deren Ursprung im zuvor beschriebenen Wertewandel liegt. Die abnehmende Akzeptanz der intensiven Nutztierhaltung in der Gesellschaft veranlasst die Fleischbranche und die Politik dazu, auf die veränderten gesellschaftlichen Ansprüche zu reagieren. Wie die Reaktionen in der Vergangenheit ausfielen, wird in Unterkapitel 2.3 kritisch betrachtet. Die wesentlichen Erkenntnisse werden im Fazit zusammengefasst. 


\subsection{Ethische Grundsätze und gesellschaftlicher Wertewandel}

Fleisch- und Wurstwaren stammen von Lebewesen, die dem Menschen genetisch ähnlich sind. Dadurch werden besondere ethische Fragen aufgeworfen, die sich in dieser Form nur bei Produkten tierischen Ursprungs stellen. In diesem Punkt unterscheiden sich Produkte tierischen Ursprungs deutlich von Lebensmitteln pflanzlichen Ursprungs wie z. B. Obst oder Gemüse. An Landwirte, Verarbeiter bzw. Hersteller und Händler von Fleisch- und Wurstwaren werden daher andere ethische Anforderungen gestellt als an Unternehmen anderer Branchen; angefangen von der Produktion bis hin zur Vermarktung der Produkte.

Das Thema Tierwohl wird vor allem im Hinblick auf die intensive Nutztierhaltung und den Verzehr von Fleisch- und Wurstwaren intensiv diskutiert. Daneben ist es auch in anderen Branchen immer häufiger Gegenstand von Debatten. Beispielhaft genannt seien an dieser Stelle Unternehmen der Bekleidungs- und Lederwarenindustrie, die neben Lederwaren (vor allem von Rindern, Schweinen, Schafen, Ziegen; jedoch nach wie vor auch von diversen anderen Tieren wie Reptilien, Känguru oder Strauß) verschiedene Produkte aus Merinowolle vom Schaf, Angorawolle vom Kaninchen oder Edelhaaren von Kaschmir-Ziegen vermarkten.

Weitere Branchen, die sich mit den neuen gesellschaftlichen Anforderungen an das Tierwohl befassen müssen, sind u. a. die Tierfutterindustrie (z. B. Hunde- oder Katzenfutter für Haustiere, das aus Nutztieren hergestellt wird), Kosmetik- und Drogerieartikelanbieter (z. B. tierische Fette in Cremes) sowie Medikamenten- und Nahrungsergänzungsmittelhersteller (z. B. bzgl. in Kapseln enthaltener Gelatine oder im Hinblick auf ethisch bedenkliche Tierversuche).

Daneben müssen sich Unternehmen der Freizeitindustrie wie Zoologische Gärten oder Universitäten mit Tierversuchsabteilungen immer stärker mit dem Thema Tierwohl beschäftigen, da die Haltung der Tiere in diesem Bereich immer kritischer betrachtet wird. Mehr Berücksichtigung des Tierwohls wird z. B. auch von Reiseanbietern (z. B. bei Jeep- oder Boot-Safaris) gefordert. 
Hersteller von Verbrauchs- (wie z. B. Benzin), Gebrauchs- (wie z. B. Smartphones) oder Industriegütern (wie z. B. Maschinen) und Anbieter von Dienstleistungen (wie z. B. Finanz- oder Versicherungsdienstleistungen) werden dagegen mit tierethischen Fragen eher seltener konfrontiert. In der vorliegenden Studie steht das Wohl von Tieren, aus denen in Deutschland Fleisch- und Wurstwaren hergestellt werden, im Fokus; zumindest für die ohnehin stark verkürzte Lebensdauer. Daneben wird im Rahmen der vorliegenden Arbeit ergänzend die Bedeutung des Tierwohls für Haustierbesitzer bzw. die Tierfutterindustrie untersucht.

Fragen zum Umgang mit Tieren stellen unter ethischen Gesichtspunkten bedeutsame Charakteristika einer Zivilisation dar und werden im religiösen und philosophischen Kontext bereits seit langer Zeit im Rahmen der Tierethik kontrovers diskutiert. Diese sind dem Bereich der angewandten (Bio-)Ethik zuzuordnen (BOSSERT, 2014). Heutige tierethische Diskussionen basieren häufig auf Henry Stephens Salts Manifest „Animal Rights Considered in Relation to Social Progress“, das 1892 erschien, Benthams Utilitarismus und Darwins Evolutionstheorie. Die deutsche Tierethik wurde im 19. Jahrhundert maßgeblich von Kant beeinflusst und später innerhalb der Schopenhauerschen Mitleidsethik diskutiert.

In den siebziger und achtziger Jahren des 20. Jahrhunderts wurde vor allem durch das Buch „Animal Liberation“ von PETER SINGER die Debatte darüber eröffnet, ob und aufgrund welcher Eigenschaften Tiere eine moralische Berücksichtigung verdienen. Ein wesentlicher Beitrag, den die Tierethik leistet, besteht in der Analyse existierender Moralvorstellungen in Bezug auf den Umgang von Menschen mit Tieren sowie der Explikation der zugrundeliegenden Voraussetzungen und der kritischen Überprüfung ihrer Geltungsansprüche (BMEL, 2015). Die Tierethik umfasst dabei den generellen Umgang mit allen Tieren und folglich nicht nur den Umgang mit landwirtschaftlichen Nutztieren, sondern auch mit Haus-, Zoo- und Wildtieren.

$\mathrm{Ob}$ und in welchem Maße Tiere moralisch Berücksichtigung finden sollten, wird in der Tierethik kontrovers diskutiert. Im folgenden Abschnitt werden die drei meistdiskutierten Perspektiven bzw. Moralvorstellungen vorgestellt. 
Die drei zentralen Perspektiven sind die anthropozentrische, die pathozentrische und die biozentrische Perspektive (BOSSERT, 2014; VERHOOG et al., 2004). Bei der anthropozentrischen Perspektive entstehen moralische Pflichten gegenüber Tieren nicht um der Tiere willen, sondern werden nur indirekt abgeleitet als moralische Pflicht der Menschen gegenüber sich selbst, da Tierquälerei durch den Menschen die moralische Verfassung des Menschen negativ beeinflusst. Kant diskutiert dieses Phänomen im Rahmen seiner Verrohungsthese und betont dabei ausdrücklich die moralische Verpflichtung zum achtsamen Umgang mit Tieren als Schutz des Menschen selbst (BMEL, 2015).

Heute dominieren in der Ethik pathozentrische und biozentrische Konzepte, die moralische Pflichten gegenüber leidensfähigen Tieren um ihrer selbst willen ableiten. Pathozentrische Moralvorstellungen werden in der philosophischen Literatur als sentientistische Moralvorstellungen bezeichnet. In der vorliegenden Arbeit wird ausschließlich der Begriff, pathozentrisch“ verwendet. Wenn bei pathozentrischen Ansätzen diskutiert wird, ob und in welchem Ausmaß Tieren Rechte zuzusprechen sind, wird die Frage aufgeworfen, ob Tiere den gleichen moralischen Status wie Menschen haben sollten, und wodurch eine übergeordnete Position des Menschen begründet werden kann (GRUEN, 2012). Eine Vielzahl an Philosophen konstatiert seit vielen Jahren hohe Anforderungen an den Schutz von Tieren. Diese Anforderungen sind in jüngster Zeit stetig gestiegen, u. a. aufgrund neuerer Erkenntnisse aus der biologischen Forschung, durch die belegt wird, dass sich die kognitiven, emotionalen und sozialen Fähigkeiten vieler Tierarten prinzipiell nur unwesentlich vom Menschen unterscheiden und die genetische Übereinstimmung von Mensch und Tier höher ist als bisher angenommen (SINGER, 1995).

Der pathozentrische Ansatz zur Bewertung des moralischen Status von Tieren wird in den gradualistischen Pathozentrismus und den egalitären (radikalen) Pathozentrismus unterteilt, was weitreichende Auswirkungen auf die moralische Bewertung der Nutztierhaltung hat (BOSSERT, 2014). 
Eine Sichtweise in der Philosophie ist die grundsätzliche Ablehnung der Nutztierhaltung, die in der Regel dem egalitären Pathozentrismus folgt. Eng verwandt mit dem egalitären Pathozentrismus sind Tierrechts- und Tierbefreiungsethiken.

Die Tierrechts- und Tierbefreiungsethiken lehnen die Nutzung von Tieren zu menschlichen Zwecken $\mathrm{ab}$ und sprechen Tieren eigenständige Rechte $\mathrm{zu}$, so beispielsweise das Recht auf körperliche Unversehrtheit. Das Recht auf körperliche Unversehrtheit gilt sowohl für die Züchtung und Haltung der Tiere als auch für die Tötung der Tiere und schließt damit eine Tötung generell aus (BMEL, 2015).

Da die Nutztierhaltung aus Sicht des egalitären Pathozentrismus grundsätzlich abgelehnt wird, erfährt eine ,weiche` Reform der intensiven Nutztierhaltung, bei der graduelle Verbesserungen der intensiven Nutztierhaltung im Fokus stehen, ebenfalls häufig Ablehnung. Aufgrund der Tatsache, dass eine generelle Abschaffung der Nutztierhaltung von der Mehrheit der Menschen nicht gewünscht ist, sind allerdings für die aktuelle Tierschutzdiskussion zu Reformen in der intensiven Nutztierhaltung die verschiedenen Formen des gradualistischen Pathozentrismus von größerer Bedeutung. Ein wesentlicher ethischer Gesichtspunkt für gradualistische Pathozentriker ist die Leidens- und Glücksempfindungsfähigkeit von Lebewesen, die Grund genug dafür ist, dass Tieren um ihrer selbst willen möglichst wenig Leid zugefügt wird. Diese mitleids- oder fürsorgeethische Position hat in der Philosophie eine lange Tradition und wurde vor allem von Schopenhauer und Bentham thematisiert (BMEL, 2015).

Die gesellschaftliche Diskussion zum Tierschutz in der Nutztierhaltung stützt sich häufig auf utilitaristische Tierschutzansätze (lat. utilitas: Nutzen, Vorteil), die dem gradualistischen Pathozentrismus zuzuordnen sind. Dabei wird versucht, zwischen dem Leiden der Nutztiere und dem Nutzen für den Menschen abzuwägen (SINGER, 1995). Abwägungen dieser Art werden in verschiedenen Bereichen der Nutztierhaltung getroffen. Im deutschen Tierschutzgesetz heißt es beispielsweise in $\S 1$ Satz 2: „Niemand darf einem Tier ohne vernünftigen Grund Schmerzen, Leiden oder Schaden zufügen“. Als vernünftige Gründe werden heute z. B. der Verzehr der Tiere zum Genuss, aber auch ökonomische Aspekte wie Wertschöpfung oder Arbeitsplatzsicherung angesehen. 
Ein weiteres Beispiel für die Abwägung von Tierleid und Nutzen für den Menschen ist die EU-Verordnung 63/2010, durch die Harm-Benefit-Analysen für Tierversuche vorgeschrieben werden (BMEL, 2015). Hierbei werden Vorteile für den Menschen wie etwa die (Weiter-)Entwicklung von Medikamenten für Menschen mit dem Leid der Versuchstiere abgewogen (MASTERTON et al., 2014; VON ROTEN, 2012). Der Nutzen der Tierversuche, die für verschiedene Medikamente gesetzlich vorgeschrieben sind, wird von Teilen der Gesellschaft immer kritischer hinterfragt, und entsprechende Abwägungsansätze werden aus ethischer Perspektive kontrovers diskutiert.

Dass sich diese gesellschaftlichen Anforderungen konkret auf die Daseinsberechtigung, die sogenannte licence to operate, für ganze Industriezweige auswirken kann, wird anhand des Beispiels der Pelzindustrie offensichtlich. Angesichts des Leids vieler Tiere in der Pelztierhaltung und des aus Sicht des Gesetzgebers relativ geringen Nutzens wurden beispielsweise 2006 in Deutschland erstmalig Detailregelungen mit teilweise erhöhten Anforderungen an die Pelztierhalter erlassen, die keiner verpflichtenden EURichtlinie folgten. Die Branche hat im Lauf der Zeit ihre gesellschaftliche Akzeptanz verloren, weil der Zweck ,Pelz als Statussymbol zu tragen“ nicht mehr als ,vernünftiger Grund‘, wie vom Tierschutzgesetz gefordert, anerkannt wurde (BMEL, 2015).

In anderen europäischen Ländern, die in Bezug auf den Tierschutz in verschiedenen Fällen gesetzlich deutlich höhere Standards durchsetzen als Deutschland (z. B. Schweiz, das Vereinigte Königreich, Dänemark, Niederlande etc.), wurde inzwischen die Haltung oder der Handel bestimmter Pelztierarten zur Pelzgewinnung gesetzlich verboten (BMEL, 2015). Die Haltung und Schlachtung von Nutztieren für die Ernährung der Menschen wird im Gegensatz zur Haltung von Tieren auf Pelzfarmen noch von großen Teilen der Gesellschaft weitestgehend unkritisch akzeptiert.

Die gesellschaftliche Akzeptanz der intensiven Nutztierhaltung hat jedoch seit der Jahrtausendwende in Deutschland und in vielen anderen EU-Staaten stetig abgenommen (EUROPEAN COMMISSON, 2005; EUROPEAN COMMISSON 2016). Mithilfe zahlreicher politischer und wirtschaftlicher Initiativen soll diesen Entwicklungen entgegengewirkt werden. 
In der Vergangenheit stellte das auf dem Anthropozentrismus basierende deutsche Tierschutzgesetz die Vermeidung von Tierleid in den Vordergrund. Ergänzt wird diese Perspektive mittlerweile durch die Berücksichtigung des Wohlbefindens von Tieren während ihres Lebens in der Nutztierhaltung.

Durch verschiedene Studien ist belegt, dass das Ausleben angeborener Verhaltensmuster eine positive Erfahrung für die Tiere darstellt und einen wesentlichen Beitrag leisten kann, das Wohlbefinden und die Lebensqualität zu erhöhen (FRASER \& DUNCAN, 1998; KNIERIM, 2001; SPINKA, 2006). Dieses Konzept passt in verschiedener Hinsicht besser zum geänderten Mensch-Tier-Verhältnis einer aufgeklärteren Gesellschaft, die die soziale Ebene der Mensch-Tier-Beziehung in den Vordergrund rückt (GRUEN, 2012).

In der tierethischen Diskussion wird die Berücksichtigung von Wohlbefinden bzw. Glück von Nutztieren (Animal Welfare) als bedeutsame Ergänzung zur reinen Absenz von Leid gesehen. Weitestgehend unbeantwortet bleiben in der ethischen Diskussion bisher Fragen bzgl. der Art und des Ausmaßes von Gelegenheiten zum Erleben positiver Emotionen. Im Hinblick auf die praktische Integration in die Nutztierhaltung müssen zudem Fragen zur tiergerechteren Gestaltung von Ställen (z. B. mehr Platz, Beschäftigungsmaterialien etc.) unter Berücksichtigung ökonomischer Aspekte z. B. durch die Integration der Kosten für zusätzliche Tierschutzmaßnahmen in die einzelbetriebliche Kosten- und Leistungsrechnung beantwortet werden (BMEL, 2015).

Eine dritte Sichtweise, die oftmals mit dem Anthropozentrismus und dem Pathozentrismus vergleichend diskutiert wird, ist in der Ethik unter dem Modell des Biozentrismus zusammengefasst. Dabei wird allen Lebewesen ein intrinsischer Wert (Eigenwert) zugesprochen, der eine moralische Relevanz begründet.

Von radikalem bzw. egalitärem Biozentrismus wird gesprochen, wenn für alle Lebewesen (Mensch und Tier) derselbe Eigenwert ohne Abstufung angenommen wird. Von einem hierarchischen beziehungsweise schwachen Biozentrismus ist dagegen die Rede, wenn Menschen ein höherer Eigenwert zugesprochen wird als Tieren, oder der Eigenwert verschiedener Tiere variiert (BMEL, 2015). 
Der Integrität von Tieren im Rahmen der Eigenwert-Theorie kommt in der Diskussion zu aktuellen Tierschutzthemen eine große Bedeutung zu. Die Integrität von Tieren kann beispielsweise durch Amputationen, Medikamente oder auch züchterische Einwirkungen verletzt werden. So wurden beispielsweise blinde Hühner gezüchtet, um das Auftreten von Federpicken zu reduzieren (ALI \& CHENG, 1985).

Auf der einen Seite konnte so Tierleid reduziert werden, auf der anderen Seite wurden aber die Möglichkeiten für artgemäßes Verhalten und positive Empfindungen eingeschränkt, wodurch die Integrität der Tiere auf diesem Weg stark verletzt und dadurch wiederum Tierleid erzeugt wurde (BOVENKERK et al., 2002; THOMPSON, 2008).

Ein dem biozentrischen Konzept der Integrität verwandter Ansatz ist das Konzept bzw. der Begriff der Würde, der beispielsweise im Schweizer Tierschutzgesetz in Art. 3 lit. a TSchG in Bezug auf Tiere wie folgt definiert wird: „Würde: Eigenwert des Tieres, der im Umgang mit ihm geachtet werden muss. Die Würde des Tieres wird missachtet, wenn eine Belastung des Tieres nicht durch überwiegende Interessen gerechtfertigt werden kann. Eine Belastung liegt vor, wenn dem Tier insbesondere Schmerzen, Leiden oder Schäden zugefügt werden, es in Angst versetzt oder erniedrigt wird, wenn tiefgreifend in sein Erscheinungsbild oder seine Fähigkeiten eingegriffen oder es übermäßig instrumentalisiert wird.“ (BMEL, 2015).

In Art. 3 lit. b TSchG wird ergänzend das Wohlergehen von Tieren definiert: „Wohlergehen: Wohlergehen der Tiere ist namentlich gegeben, wenn: 1. die Haltung und Ernährung so sind, dass ihre Körperfunktionen und ihr Verhalten nicht gestört sind und sie in ihrer Anpassungsfähigkeit nicht überfordert sind, 2. das artgemäße Verhalten innerhalb der biologischen Anpassungsfähigkeit gewährleistet ist, 3. sie klinisch gesund sind, 4. Schmerzen, Leiden, Schäden und Angst vermieden werden." (SCHWEIZER TIERSCHUTZVERORDNUNG, 2008)

An diesen beiden juristischen Beispielen wird deutlich, dass sich die Formulierungen in Tierschutzgesetzen aktuell noch schwerpunktmäßig auf die Abwesenheit von Leid konzentrieren (BMEL, 2015). 
Die gesellschaftlichen Anforderungen an das Tierwohl sind vor dem Hintergrund der skizzierten ethischen Positionen besonders in Nordwesteuropa in den letzten Jahrzehnten deutlich angestiegen (BMEL, 2015). Als Reaktion wurde im Bundestag 2002 eine Gesetzesänderung beschlossen, durch die dem Tierschutz in Deutschland erstmalig Verfassungsrang eingeräumt wurde. In Artikel 20a heißt es, der Staat solle „die natürlichen Lebensgrundlagen und die Tiere im Rahmen der verfassungsmäßigen Ordnung"s schützen.

Der Umgang mit Tieren ist daneben auch im Tierschutzgesetz geregelt, wobei manche Verstöße gegen die Regelungen als Ordnungswidrigkeit ( $\$ 18)$ gelten und andere Verstöße bestraft werden. Wer laut Tierschutzgesetz ein Wirbeltier „ohne vernünftigen Grund“ tötet oder ihm ,aus Rohheit erhebliche Schmerzen“ zufügt, muss in Deutschland mit einer Freiheitsstrafe von bis zu drei Jahren oder einer Geldstrafe rechnen (§ 17), je nach Art und Schwere des Verstoßes.

Das deutsche Tierschutzgesetz ist allerdings durch unkonkrete Definitionen, die einen großen Interpretationsspielraum bieten, und zahlreiche Ausnahmeregelungen gekennzeichnet. Mangelhafte Kontrollen und Sanktionen sind weitere wesentliche Gründe, warum das Tierschutzgesetz in Deutschland praktisch kaum Anwendung findet (BMEL, 2015).

In zahlreichen Studien wurden in den letzten Jahren die kognitiven, emotionalen und sozialen Kompetenzen der Tiere herausgearbeitet, wobei der Eigenwert der Tiere in den Vordergrund gerückt wurde (ARMSTRONG \& BOTZLER, 2008; KÄMPER, 2003). Daraus haben sich zwei unterschiedliche soziale Bewegungen entwickelt.

Die Tierrechtsbewegung (Animal Rights Movement) setzt sich dafür ein, dass höher entwickelten Tierarten aufgrund ihrer Leidensfähigkeit eigene Rechte zugesprochen werden. Untergruppen der Tierrechtsbewegung kritisieren daneben die Unterscheidung zwischen verschiedenen Tierarten und die Unterscheidung zwischen Tier und Mensch. Geprägt wurde die Tierrechtsbewegung vom australischen Philosophen PETER SINGER, der in den siebziger Jahren durch die Veröffentlichung des Buchs „Animal Liberation“" weltweit Befürworter seiner Thesen fand (SINGER, 1995). 
Bekannte Tierrechtsorganisationen sind beispielsweise die internationale Organisation People for the Ethical Treatment of Animals (PETA), Stop Huntingdon Animal Cruelty (SHAC in Großbritannien und Irland), der österreichische Verein gegen Tierfabriken (VGT), Animal Rights Watch (ARIWA), Soko Tierschutz, die Tierbefreier oder die Albert Schweitzer Stiftung. Daneben existieren sowohl verschiedene globale Tierrechtsbewegungen wie die Animal Liberation Front als auch zahlreiche kleine lokale Initiativen wie z. B. die verschiedenen Tierrechtsinitiativen an Universitäten und Hochschulen. Die vorgestellten Organisationen sind dem egalitären Pathozentrismus zuzuordnen (BMEL, 2015).

Im Rahmen der Tierschutzbewegung (Animal Welfare Movement) steht dagegen eher die Reform von Institutionen und Praktiken der Nutztierhaltung im Vordergrund, um das Leid von Nutztieren zu reduzieren. Als wesentliche Verbände der Tierschützer in Deutschland sind beispielsweise der DEUTSCHE TIERSCHUTZBUND, VIER PFOTEN, PRO VIEH oder der BUND GEGEN MISSBRAUCH DER TIERE (BMT) zu nennen. Eine Sonderstellung nimmt in Deutschland der DEUTSCHE TIERSCHUTZBUND ein, der im Verbund mit Partnern aus Wirtschaft, Forschung und Beratung die Initiative zur Entwicklung eines zweistufigen Tierschutzlabels (Einstiegsstufe, Premiumstufe) für Fleisch- und Wurstwaren ergriffen hat (BMEL, 2015).

Tierrechts- und Tierschutzbewegung stehen aufgrund der unterschiedlichen Reichweite der Forderungen und der differierenden ethischen Legitimationsbasis in einem Spannungsverhältnis zueinander, wobei die Tierschutzbewegung in der Bevölkerung stärker akzeptiert ist (BMEL, 2015). Die Fleischwirtschaft und die Agrarpolitik stehen einer Diskussion mit Tierschützern wie z. B. dem DEUTSCHEN TIERSCHUTZBUND aufgeschlossen gegenüber, da diese Teile der Tierschutzbewegung den Fleischverzehr als ,vernünftigen Grund‘ für die Haltung und das Töten von Nutztieren anerkennen. Aufgrund der weitergehenden Forderungen der Tierrechtsbewegung kommt es allerdings häufig zu nicht überwindbaren Zielkonflikten. Tierrechtsorganisationen versuchen häufig, möglichst viele Menschen über Kampagnen auf die Missstände in der Nutztierhaltung aufmerksam zu machen, und sprechen sich für eine vegane Ernährung aus. 
In den letzten Jahren wurden aus der Perspektive der Tierrechtsorganisationen große Erfolge mit diesen Kampagnen erzielt. Da sich das Image der Fleischwirtschaft und der Agrarpolitik jedoch durch diese Kampagnen verschlechtert, ist die Dialogbereitschaft der Fleischwirtschaft und der Agrarpolitik mit den Tierrechtsorganisationen vice versa eher gering (BMEL, 2015).

\subsection{Abnahme der gesellschaftlichen Akzeptanz der Nutztierhaltung}

Die kritisch geführte Diskussion in der Tierschutzethik sowie der veränderte Wertewandel haben zu deutlich gestiegenen Anforderungen an die Fleischwirtschaft geführt, und die Akzeptanz der intensiven Nutztierhaltung ist stark gesunken (WBAE, 2020). Innerhalb der Fleischindustrie haben vor allem die Schlacht- und Verarbeitungsindustrie sowie die Landwirtschaftsverbände ein schlechtes Image in der Bevölkerung, wobei das Ansehen einzelner Tierhalter geringfügig besser ist (ALBERSMEIER \& SPILLER, 2010).

Das Ringen um neues Vertrauen und die gesellschaftliche Akzeptanz findet mittlerweile vor allem in einem national wie international stark politisch-medial geprägten Diskurs statt. Unterschieden werden können davon die gesellschaftlichen Konflikte auf regionaler und lokaler Ebene. Als Beispiele für öffentlichen Widerstand auf lokaler Ebene können die Auseinandersetzungen um den Bau von großen Haltungssystemen oder Schlachtbetrieben aufgeführt werden, die zur Gründung verschiedener Bürgerinitiativen beigetragen haben (BMEL, 2015). Das Netzwerk ,Bauernhöfe statt Agrarfabriken` setzt sich z. B. aktiv gegen die intensive Nutztierhaltung ein und wird von Vertretern aus der Kirche, Umwelt- und Tierschutzverbänden und der Arbeitsgemeinschaft bäuerliche Landwirtschaft unterstuitzt (DEUTSCHER TIERSCHUTZBUND, 2019). Regional kommt es in landwirtschaftlich intensiv bewirtschafteten Regionen oft zu Konflikten aufgrund der Verunreinigung des Trinkwassers durch übermäßiges Ausbringen von Tierexkrementen auf Felder sowie die großflächige Verwendung stickstoffhaltiger Düngemittel, Pflanzenschutzmittel (Herbizide, Fungizide und Insektizide) oder Tierarzneimittel (UMWELTBUNDESAMT, 2019). 
Auf nationaler Branchenebene sieht sich die Fleischindustrie mit verschiedenen anderen gesellschaftlichen Anforderungen konfrontiert. Dabei wird die Branche häufig grundsätzlich im Hinblick auf die Produktivitätsentwicklung kritisiert. Dass immer mehr Tiere in immer kürzerer Zeit gezüchtet, gemästet und getötet werden, ist ein zentraler Bestandteil der öffentlichen Kritik.

Daneben werden vor allem Missstände im Bereich des Tierschutzes, des Umweltschutzes und des Verbraucherschutzes kritisiert. SPILLER et al. (2012) belegten in einer kombinierten Verbraucher- und Medienanalyse, dass die Einstellung der Gesellschaft zur Produktivitätssteigerung in der Land- und Ernährungswirtschaft sehr kritisch ist. Dies zeigte sich vor allem bei der Analyse von Internetberichten und Diskussionen über die Agrar- und Ernährungswirtschaft in sozialen Medien und der Presse.

Demnach bewerteten 70 bis $85 \%$ der Bevölkerung die intensive Landwirtschaft negativ. Bei der Verbraucherbefragung gab ein großer Teil der Befragten an, dass eine stärkere Berücksichtigung des Naturschutzes, des Tierschutzes und von Regionalität sowie eher kleinere handwerkliche bzw. bäuerliche Strukturen erwünscht seien. Die Kritik an der intensiven Landwirtschaft ist beim Thema Tierschutz besonders groß (BMEL, 2015).

Positive Entwicklungen in der intensiven Tierhaltung werden von der Öffentlichkeit dagegen kaum wahrgenommen (KAYSER et al., 2012b; ZANDER et al., 2013). Von Landwirten werden oftmals die Verbesserungen von Hygienestandards bei sterileren abgeschlossenen Stallsystemen, ein professionelleres Management (z. B. ausgebildetes Personal oder effizientere Gestaltung der Produktionsprozesse) sowie die technische Ausstattung der Ställe (z. B. Melkroboter) in der intensiven Tierhaltung als Verbesserungen aufgeführt (BMEL, 2015).

Während im gesellschaftlichen Diskurs häufig von ,Massentierhaltung ‘ oder ,industrieller Tierhaltung' gesprochen wird, werden in der wissenschaftlichen Literatur überwiegend die Begriffe, intensive Nutztierhaltung ‘ oder ,moderne Nutztierhaltung ‘ verwendet. KAYSER et al. (2012a) stellten im Rahmen einer Verbraucherstudie eine offene Assoziationsfrage zum Begriff ,Massentierhaltung‘. 
Die befragten Verbraucher beschrieben dabei die ,Massentierhaltung' mit verschiedenen Begriffen, die insgesamt eine Ablehnung der ,Massentierhaltung' ausdrücken, wie Abbildung 2 zeigt (KAYSER et al., 2012a; ZANDER et al., 2013). Dabei wurde die ,Massentierhaltung' von vielen Befragten als Tierquälerei bezeichnet. Häufig genannt wurden daneben u. a. die Begriffe ,Enge', ,Krankheiten', ,Antibiotika' und ,Gestank'.

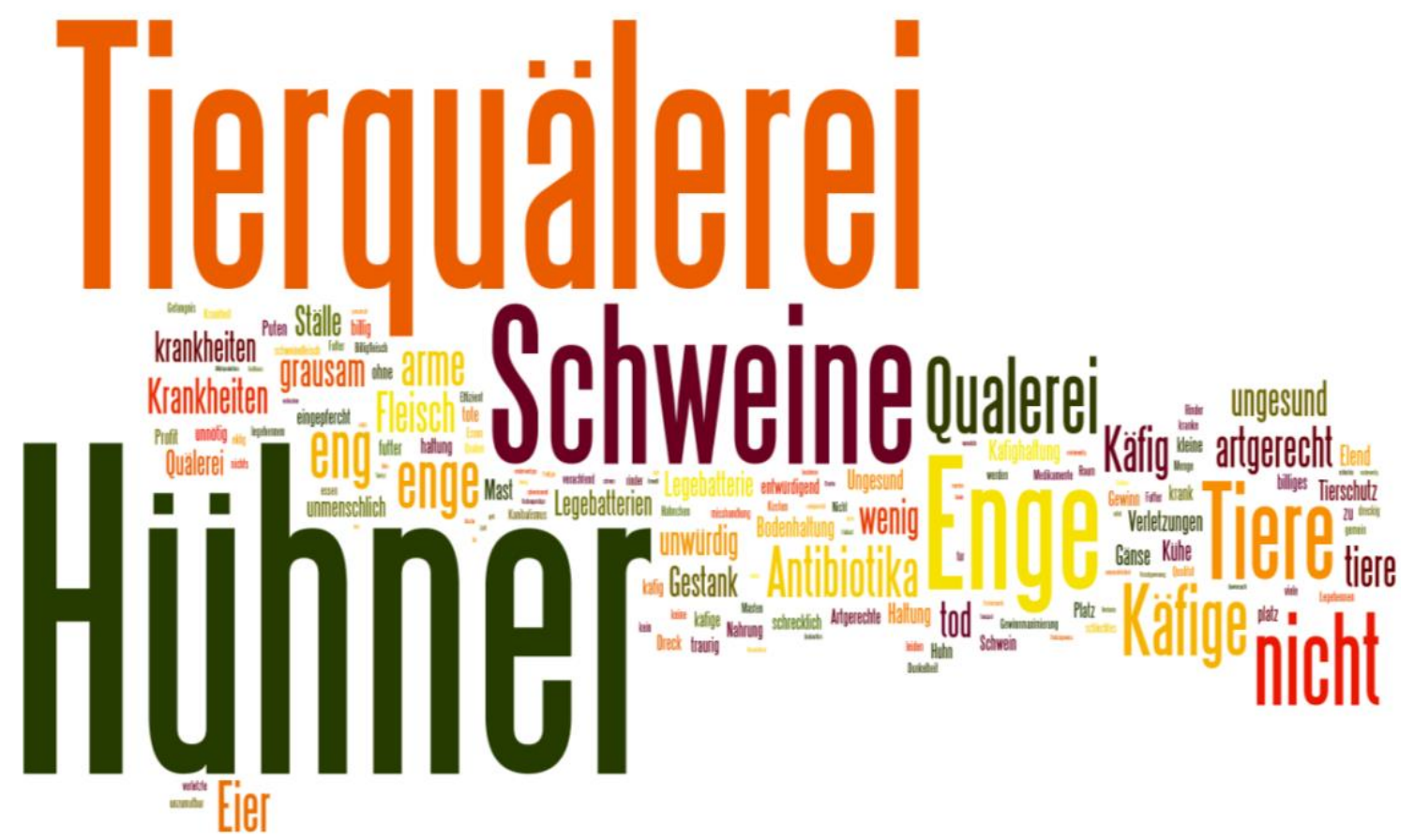

\section{Abbildung 2: Assoziationen zum Begriff der Massentierhaltung (Quelle: KAYSER et al., 2012a)}

In einer Studie von ZANDER et al. (2013) wurden die Haltungsbedingungen in Ställen von Verbrauchern bewertet. Im Rahmen einer Gruppendiskussion beschrieben die Verbraucher Schweineställe als große Hallen, in denen die Tiere in großer Enge gehalten würden. Hinsichtlich des Hygienezustands wurden die Ställe unterschiedlich beurteilt. Eine Gruppe schätzte die Ställe als zu steril und sauber ein, während eine andere Gruppe von eher unhygienischen Zuständen, vor allem durch die Exkremente der Tiere, ausging. Als weiterer Kritikpunkt wurde die strikte Abschirmung der geschlossenen Schweineställe von der Bevölkerung erwähnt. 
Die Landwirte wurden im Rahmen der Diskussionsrunde so charakterisiert, dass ökonomische Interessen bei der Nutztierhaltung im Vordergrund stünden, und es wurde moniert, dass Landwirten die emotionale Beziehung $\mathrm{zu}$ den Tieren fehle. Verbesserungsmöglichkeiten für die Gestaltung von Ställen sahen die Verbraucher im Platzangebot und der Bewegungsfreiheit, in Zugängen zu verschiedenen Klimazonen (Außenbereich) sowie in der Reduktion des Medikamenteneinsatzes (ZANDER et al., 2013).

Zusammenfassend lässt sich sagen, dass die Akzeptanz der intensiven Nutztierhaltung in den letzten Jahren stetig abgenommen hat und die Landwirtschaft von weiten Teilen der Bevölkerung immer kritischer betrachtet wird. Sowohl seitens der Fleischbranche als auch der Agrarpolitik wurde die abnehmende Akzeptanz in der Bevölkerung in den letzten Jahrzehnten überwiegend ignoriert. Im Folgenden werden die Reaktionen der Fleischbranche kritisch betrachtet.

\subsection{Reaktionen der Fleischbranche und der Agrarpolitik}

Die Fleischbranche und die Agrarpolitik haben den gesellschaftlichen Veränderungsprozessen in den letzten Jahrzehnten bisher nur wenig Beachtung geschenkt. Sie reagierten viele Jahre zurückhaltend auf die gesellschaftliche Kritik. Statt adäquate Lösungsmöglichkeiten und Maßnahmen zu entwickeln, um den geforderten Transformationsprozess zu unterstützen, wurde häufig die wenig erfolgreiche ,Augenzu-und-durch-Strategie' verfolgt.

Skandale wurden in der Regel ,ausgesessen“, statt Änderungen durchzusetzen. Die Tierhaltungssysteme wurden zudem immer mehr von der Gesellschaft abgeschottet. Diese geringe Öffentlichkeitsorientierung (,Low-Profile-Strategie“) ist den gesellschaftlichen Anforderungen jedoch nicht gerecht geworden (ALBERSMEIER \& SPILLER, 2009). Die Öffentlichkeitsarbeit wurde traditionell in Form einer monologischen Informationspolitik betrieben. 
Es wurde zudem versucht, die Verbraucher über das aus Sicht der Branche hohe Niveau der deutschen Nutztierhaltung aufzuklären, wobei häufig auf ein niedrigeres Tierwohlniveau in verschiedenen Entwicklungs- und Schwellenländern hingewiesen wurde (KAYSER \& SPILLER, 2012). Häufig wurde Kritik auch begegnet, indem die Haltung der Nutztiere in Deutschland als unproblematisch dargestellt wurde. Der Deutsche Bauernverband griff beispielsweise im Rahmen der Internationalen Grünen Woche 2014 das Thema Tierwohl in der Milchviehhaltung auf und lud zu einer Fachtagung nach Berlin ein, die den Titel ,Unseren Kühen geht es gut!‘ trug (DBV, 2019).

Akzeptanz-PR ohne das Signal der Dialogbereitschaft, ohne das ehrliche Anerkennen von Missständen in der Nutztierhaltung, ohne ernsthafte Bemühungen um Nachbesserung im Bereich Tierschutz und ohne die Umsetzung deutlicher Verbesserungen für das Tierwohl in der Praxis, trägt nicht zum Vertrauensaufbau bei (BMEL, 2015). Besonders kontraproduktiv kann Öffentlichkeitsarbeit in dieser Form werden, wenn für Verbraucher der Eindruck entsteht, dass die Kommunikation lediglich auf die Beschönigung von Missständen in der Nutztierhaltung abzielt, und versucht wird, die Verbraucher davon zu überzeugen, dass es in der Nutztierhaltung keine nennenswerten Probleme gebe (BMEL, 2015).

Vor allem von naturwissenschaftlichen, technischen oder medizinischen Experten der Nutztierhaltung wird häufig eine ,Defizit-Hypothese‘ aufgestellt, die den Verbrauchern eine adäquate Beurteilung der tatsächlichen Haltungsbedingungen der Tiere und des Tierwohls in der deutschen Nutztierhaltung abspricht. Nach der ,Defizit-Hypothese“ beruht die geringe Akzeptanz der Nutztierhaltung lediglich auf dem Unwissen der Verbraucher über die realen Zustände in der Fleischproduktion. Um die gesellschaftliche Akzeptanz zu erhöhen, müsse lediglich der Wissensstand der Verbraucher erhöht werden. Verschiedene Experten plädierten daher dafür, das Wissen der Verbraucher über die Tierhaltung zu erhöhen, damit die tatsächlichen Haltungsbedingungen von Verbrauchern besser eingeschätzt werden können. Presseberichte, TV-Reportagen und Dokumentationen von NGOs über Tierquälerei in der Nutztierhaltung sollten auf diesem Weg entkräftet bzw. widerlegt werden (BMEL, 2015). 
Es wurden verschiedene Initiativen und Programme ins Leben gerufen, wie z. B. die Bereitstellung von Informationsmaterialien für Schulkinder, Besuchertage auf dem Bauernhof, Besichtigungen von Schlachthöfen, Demonstrationsställe auf Anhängern von Lastkraftwagen oder Webcams in vorzeigbaren Ställen (BMEL, 2015).

Kritiker der ,Defizit-Hypothese“ gehen allerdings davon aus, dass der Versuch, auf diesem Weg für mehr Akzeptanz zu werben, nicht erfolgreich ist. Durch verschiedene Studien ist belegt, dass die kritische Haltung mit zunehmendem Wissen über die Nutztierhaltung stark zunimmt (BAUER, 2009). Wissenschaftliche Auswertungen zum Erfolg derartiger Maßnahmen liegen nur in sehr begrenztem Umfang vor. Vor allem die Reichweite von Aktionen wie einem ,Tag der offenen Tür' wird aufgrund der begrenzten Personenanzahl kritisiert. Als positiv kann der grundsätzlich dialogische Ansatz bei Stallbesichtigungen gesehen werden, da Verbraucher hierdurch die Möglichkeit erhalten, dem Landwirt direkt Feedback zu persönlichen Eindrücken zu geben. Diese diskursive Form der Auseinandersetzung zwischen Verbrauchern und Landwirten ist womöglich zielführender als eine einseitige Akzeptanz-PR. Akzeptanz stellt sich verschiedenen Studien zufolge eher entlang von Werten, Einstellungen und Vertrauen statt entlang von Wissen ein (BMEL, 2015).

\subsection{Fazit zu den gesellschaftlichen Anforderungen}

Die gesellschaftliche Diskussion zum Thema Tierwohl ist in erster Linie ethisch motiviert. Dabei sind drei zentrale ethische Positionen in der Gesellschaft zu differenzieren: Anthropozentrismus, Pathozentrismus und Biozentrismus. Die gesellschaftlichen Anforderungen an eine artgerechtere Nutztierhaltung basieren auf der Fähigkeit von Tieren, Schmerz, Leid und Emotionen zu empfinden. Diese Fähigkeit bildet die Grundlage für das deutsche und europäische Tierschutzgesetz, mit dem Tierleid vermieden werden soll. Die gesellschaftlichen Anforderungen an eine artgerechtere Nutztierhaltung basieren außerdem auf dem Bedürfnis der Tiere, das jeweils artspezifische Verhalten ausüben zu können. 
Beispielsweise gehören hierzu das Schwimmen bzw. Baden von Enten im Wasser, das Picken und Scharren von Hühnern oder das Suhlen von Schweinen (BMEL, 2015). Zu bemerken ist in diesem Zusammenhang, dass sich die ethischen Standpunkte hinsichtlich des Umgangs mit Nutztieren im Zeitverlauf teilweise stark verändert haben.

Neue Erkenntnisse aus ethologischen, verhaltensbasierten und tiermedizinischen Forschungsarbeiten mit Nutztieren haben maßgeblich zu dieser Entwicklung beigetragen. Daneben wurden die ethischen Standpunkte auch durch die Veränderung der gesellschaftlichen Werte beeinflusst. Zukünftig werden die Berücksichtigung positiver Emotionen und die Integrität der Tiere deutlich an Relevanz gewinnen, was sich in einer grundlegenden Reform des Tierschutzgesetzes ausdrücken könnte (BMEL, 2015).

Die gesellschaftliche Akzeptanz der Branche hat vor dem Hintergrund der skizzierten ethischen Positionen, vor allem im nordwestlichen Teil der Europäischen Union, stark abgenommen. Deutlich wird der Wertewandel an der zunehmenden Zahl an Vegetariern und Veganern, der Neugründung von Tierschutzorganisationen und -initiativen, häufigeren Kampagnen und Protesten gegen die intensive Nutztierhaltung sowie an der zunehmenden Anzahl an investigativen Dokumentationen und Berichten über Skandale in der Nutztierhaltung in den Medien (BMEL, 2015).

Die gesellschaftliche Diskussion gestaltet sich durch die unterschiedlichen Standpunkte der verschiedenen sozialen Bewegungen (Tierschutzbewegung, Tierrechtler, konventionelle Landwirtschaft, Bio-Bewegung etc.) als äußerst schwierig. Die oftmals emotional aufgeladenen Diskussionen und die unterschiedlichen Sichtweisen erschweren häufig Konsenslösungen bzw. Kompromisse, die sowohl die Bedürfnisse der Tiere als auch die Bedürfnisse der Fleischbranche umfassen (BMEL, 2015).

Tierschutzorganisationen wie der DEUTSCHE TIERSCHUTZBUND oder VIER PFOTEN stehen Reformbemühungen der Branche offen gegenüber, während Tierrechtsorganisationen oftmals die Abschaffung der Nutztierhaltung in ihrer jetzigen Form fordern (BMEL, 2015). 
In anderen Branchen haben derartige gesellschaftliche Veränderungen gezeigt, dass ganze Industriezweige ihre gesellschaftliche Akzeptanz verlieren können, so z. B. bei der Haltung von Nerzen für die Pelzproduktion oder der Durchführung von Tierversuchen für Kosmetika. Die Gesellschaft hat bei diesen Beispielen den Zweck der Tierhaltung (z. B. das Tragen von Pelzen) nicht mehr als , vernünftigen Grund' für die Tötung der Tiere angesehen (Anm.: Formulierung des Tierschutzgesetzes). Inwiefern die ,vernünftigen Gründe' für die intensive Nutztierhaltung ausreichend sind, wird in Zukunft weiterhin Bestandteil der ethischen gesellschaftlichen Diskussion sein (BMEL, 2015).

Die Branche, die es eher weniger gewohnt ist, mit kritischen Anspruchsgruppen umzugehen, hat sich in der Vergangenheit eher von der Öffentlichkeit abgeschottet und die Anschuldigungen von Verbrauchern, Medien und Tierschutzorganisationen mit Argwohn betrachtet. Die Werbung verschiedener Hersteller und Händler von Fleischund Wurstwaren hat jahrzehntelang ein falsches Bild von der Landwirtschaft erzeugt. Gleichzeitig wurden zahlreiche Missstände in der Nutztierhaltung verschwiegen. Die Diskrepanz zwischen der gesellschaftlichen Erwartungshaltung und der Realität ist dabei groß geworden und in der Bevölkerung kam es zu einem massiven Vertrauensverlust (BMEL, 2015).

Die öffentliche Kritik wurde von der Branche lange Zeit ignoriert oder technokratisch beantwortet und das Niveau der Öffentlichkeitsarbeit war im Vergleich zu anderen Branchen gering. Die Veröffentlichung einzelner Pressemitteilungen, wie z. B. Stellungnahmen zu Skandalen durch den Deutschen Bauernverband, entsprachen lediglich einem einfachen ,Aufklärungskonzept ${ }^{\star}$, das den kritischen Gruppen in der Bevölkerung in dieser Form nicht ausreicht. Die Branche sollte sich Prozessen des gegenseitigen Lernens und des Dialoges daher mehr öffnen (BMEL, 2015).

Erst in den letzten Jahren wurden aufgrund des zunehmenden öffentlichen Drucks neben der Kampagne ,No Farmer, No Food, No Future“ neue dialogische Initiativen ins Leben gerufen, mit denen der immer stärker sinkenden Akzeptanz gegenüber der Nutztierhaltung entgegengewirkt werden soll. Beispielhaft genannt seien an dieser Stelle die Kampagnen ,Bauer Willi‘, ,Frag den Landwirt` oder ,Massentierhaltung aufgedeckt‘. 
Daneben hat die Initiative, Wir machen Euch satt' durch zahlreiche Demonstrationen auf sich aufmerksam gemacht. Die Agrarpolitik hat unter dem ehemaligen Bundesminister Christian Schmidt (CSU) beispielsweise auf die Kritik aus der Bevölkerung mit einem Internetportal ,tierwohl-staerken` reagiert und zahlreiche weitere Aktivitäten initiiert.

Um der Forderung der Gesellschaft nach mehr Tierwohl nachzukommen, stehen der Politik verschiedene Möglichkeiten zur Verfügung. Unterscheiden lassen sich diesbezüglich grundsätzlich verpflichtende gesetzliche Vorschriften und auf freiwilliger Basis nutzbare Marktlösungen. Die Bundesregierung könnte beispielsweise bestehende Tierschutzgesetze verschärfen und die Einhaltung der Gesetze in der Praxis stärker kontrollieren. Von der Fleischbranche werden jedoch überwiegend freiwillige Marktlösungen wie Tierwohl-Labels oder private Initiativen für mehr Tierwohl (z. B. die Brancheninitiative Tierwohl) präferiert. Daher setzt die Politik bei der Suche nach Lösungsmöglichkeiten für mehr Tierwohl neben verschiedenen anderen Maßnahmen wie etwa Forschungsförderung oder Öffentlichkeitsarbeit vor allem auf den Einsatz freiwilliger Tierwohl-Labels. Diese Tierwohl-Labels stellen neben dem Screening (Aufklärungsarbeit, z. B. durch NGOs) ein Signaling-Instrument dar, das von Unternehmen für die Vermarktung von Fleisch- und Wurstwaren aus einer artgerechteren und umweltfreundlicheren Haltung eingesetzt werden kann (BMEL, 2015).

Der theoretische Rahmen, in den derartige Gütesiegel eingebettet sind, stammt aus der Ökonomie. Laut LANCASTER besteht ein Produkt für den Verbraucher aus einem Bündel an unterschiedlichen Produkteigenschaften. Diese Produkteigenschaften lassen sich in Such-, Erfahrungs- und Vertrauenseigenschaften unterteilen. Sucheigenschaften, wie z. B. Preis oder Farbe des Produkts, können vom Verbraucher bereits vor dem Kauf erkannt werden (CASWELL \& Mojduszka, 1996; LANCASTER, 1971).

Erfahrungseigenschaften wie z. B. Geschmack können hingegen erst nach dem Kauf identifiziert werden. Vertrauenseigenschaften wie eine artgerechtere und umweltfreundlichere Produktionsweise sind nach NELSON (1970) sowie DARBY und KARNI (1973) weder vor noch nach dem Kauf überprüfbar. Das Wissen über diese Produkteigenschaften ist asymmetrisch zwischen Käufer und Verkäufer verteilt. 
Der Verbraucher ist daher auf vertrauenswürdige Informationen angewiesen. Diese Art des Marktversagens aufgrund asymmetrischer Information zwischen Produzenten und Konsumenten kann durch den Einsatz von Gütesiegeln behoben werden, indem die Vertrauens- in Sucheigenschaften umgewandelt und somit dem Verbraucher direkt zugänglich gemacht werden (CRESPI \& MARETTE, 2005; LOUREIRO et al., 2001; TEISL \& ROE, 1998; THOGERSEN \& ÖLANDER, 2003; THOMPSON, 2008). Mittlerweile wird eine Vielzahl unterschiedlicher Tierwohl-Labels im Lebensmitteleinzelhandel eingesetzt, und das Bundesministerium für Ernährung und Landwirtschaft (BMEL) diskutiert die Einführung eines staatlichen Tierwohl-Labels in Deutschland oder auf europäischer Ebene. Dabei spielt jedoch die Glaubwürdigkeit der Gütesiegel eine zentrale Rolle, die als Instrument der Qualitätskommunikation dienen, um potenziellen Käufern die Vorteile eines Produkts (z. B. mehr Tierwohl oder Umweltfreundlichkeit) zu kommunizieren. Da Verbraucher die vermeintlich besseren Produkt- oder Prozesseigenschaften häufig nicht überprüfen können, besteht die Gefahr, dass die Anbieter (z. B. von Fleisch- und Wurstwaren) den Verbraucher durch unlauteres Anbieterverhalten hinsichtlich der tatsächlichen Produkteigenschaften täuschen (z. B. durch Greenwashing). In diesem Fall stimmt die Produktaufmachung oder Werbung nicht mit der Produktrealität überein. Aus unternehmerischer Perspektive wird dadurch die Funktionsfähigkeit des Wettbewerbs gestört (ZÜHLSDORF \& SPILLER, 2012).

Der Nobelpreisträger AKERLOF kommt in seinem Artikel „The Market for Lemons“ zu dem Ergebnis, dass bei unzutreffenden Marktinformationen für die Nachfrager die ,guten` Qualitäten allmählich aus dem Markt verdrängt werden. Dabei gibt es Anreize für unbeobachtete Qualitätsverschlechterungen bzw. opportunistisches Verhalten von Anbietern, wenn die Nachfrager bestimmte Eigenschaften eines Gutes vor dem Kauf nicht überprüfen können (AKERLOF, 1970). Käufer, die keinen Unterschied zwischen ,guten“ und ,schlechten' Produkten erkennen können, zahlen keinen Aufpreis. In der Folge passt sich der Marktpreis immer mehr dem Preis eines geringerwertigen Gutes an. Dadurch verkleinert sich das Premium-Segment insgesamt, da Qualitätsproduzenten zu einer Absenkung ihrer Produktanforderungen gezwungen werden (ZÜHLSDORF \& SPILLER, 2012). 
Durch den in Kapitel 2 beschriebenen starken gesellschaftlichen Wandel und den Megatrend der Neo-Ökologie können Hersteller und Händler von Fleisch- und Wurstwaren sowie die Politik besser verstehen, warum Verbraucher heute insgesamt mehr auf ethische Kaufkriterien achten als noch vor einigen Jahrzehnten (CORDTS et al., 2013). Im aktuellen Gutachten „Politik für eine nachhaltigere Ernährung“ des Wissenschaftlichen Beirats des Agrarministeriums werden die sogenannten Big Four Tierwohl, Umweltschutz, Gesundheit und Soziales - als die vier zentralen Aspekte einer nachhaltigeren Ernährung genannt (Abbildung 3).

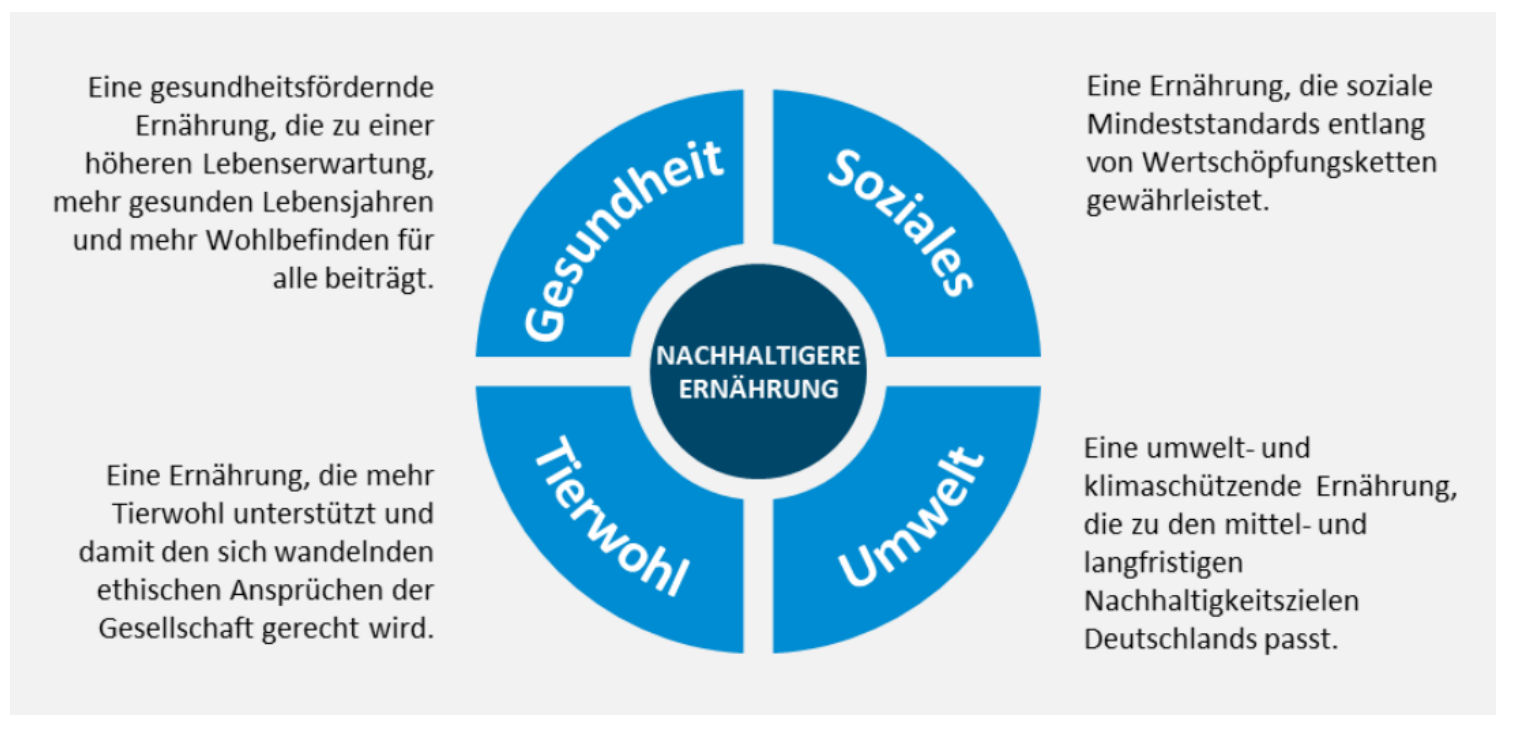

\section{Abbildung 3: Die vier zentralen Ziele einer nachhaltigeren Ernährung}

(Quelle: WBAE, 2020)

Die in Abbildung 3 präsentierten Aspekte Tierwohl, Umwelt und Gesundheit stellen heute für viele Verbraucher bedeutsame Kaufkriterien dar und stehen bei zahlreichen Nachhaltigkeitslabeln im Fokus. Diese drei Kaufkriterien entsprechen laut BMEL (2015) den drei wesentlichen Problemfeldern der Nutztierhaltung mit dem höchsten Optimierungspotenzial und bilden daher einen zentralen Schwerpunkt des folgenden Kapitels, in dessen Rahmen verschiedene wissenschaftliche Studien und Praxisstudien über Tierwohl-Labels vorgestellt werden, die die Basis für den empirischen Teil der vorliegenden Arbeit bilden. 


\section{Tierwohl-Labels als Einkaufshilfe}

Wie in Kapitel 2 erläutert möchte die Politik Tierschutz in Deutschland vor allem auf freiwilliger Basis voranbringen. Flächendeckende Gesetzesänderungen würden Landwirte in ihrem Handeln einschränken und sind vor dem Hintergrund ökonomischer Ziele schwer $\mathrm{zu}$ rechtfertigen. Daher werden schwerpunktmäßig freiwillige marktorientierte Ansätze diskutiert, die die Verbesserung des Tierwohls in der Nutztierhaltung durch die Einführung bzw. Nutzung staatlicher und privater Tierwohlzertifizierungssysteme erreichen sollen. Landwirte, die die höheren Tierwohlstandards umsetzen möchten, können sich dabei freiwillig zertifizieren lassen und versuchen, die Produkte mit den besonderen Produktqualitäten zu höheren Preisen abzusetzen. Die erzeugten Produkte werden mit einem Tierwohl-Label gekennzeichnet und können interessierten Verbrauchern mit entsprechender Mehrzahlungsbereitschaft eine Alternative zu konventionell erzeugten Produkten bieten.

Der Begriff ,Tierwohl' ist bis heute nicht einheitlich definiert und wurde womöglich vom englischen Begriff ,Animal Welfare‘ abgeleitet bzw. übersetzt. Allgemein wird unter einem Tierwohlprodukt ein Produkt verstanden, das aus einer Produktion stammt, in der höhere Tierwohlstandards gelten als beim gesetzlichen Standard. Ein zentraler Unterschied im Vergleich zu ökologischen Produkten besteht darin, dass bei der Produktion nicht zwingend auf Umweltfreundlichkeit geachtet werden muss.

So können Produzenten von Tierwohlfleisch auf den Einsatz biologisch erzeugter Futtermittel verzichten oder unterliegen auch in zahlreichen anderen Bereichen, z. B. beim Medikamenteneinsatz, keinen strengen Vorschriften (HEISE et al., 2016; NOCELLA et al., 2010; PIRSICH et al., 2015). Während die Begriffe ,Bio' und ,Öko'an gesetzliche Vorschriften gebunden sind, kann der Begriff, Tierwohl' auch für die Bewerbung konventioneller Fleisch- und Wurstwaren aus der intensiven Nutztierhaltung verwendet werden, auch wenn Nutztieren z. B. nur $5 \%$ mehr Platz zur Verfügung gestellt wird, was in manchen Fällen nur etwa einem DIN-A4-Blatt entspricht (HEISE et al., 2016). 
Dabei sind klassische ,Third-Party-Zertifizierungssysteme', die sich durch externe unabhängige Kontrollen von den Gütesiegelherausgebern (z. B. BIOLAND) als Qualitätskriterium auszeichnen, von Selbstzertifizierungen und Markenkonzepten von Unternehmen zu unterscheiden (PIRSICH, 2017).

Bei Tierwohlmarkenkonzepten wird eine Hersteller- oder Handelsmarke für Produkte aus einer artgerechteren Tierhaltung entwickelt. Die Definition der Tierwohlstandards, die Umsetzung und die Kontrolle obliegen dabei in der Regel dem Unternehmen bzw. Markeninhaber selbst. Bei Tierwohlmarkenkonzepten und Selbstzertifizierungen fehlt jedoch das bedeutsame Kriterium einer unabhängigen Kontrolle (DEIMEL et al., 2010).

Auch in anderen Branchen finden sich Beispiele für Selbstzertifizierungen, wie etwa die ,Caring-Dairy-Initiative“ des Eiscremeherstellers BEN\&JERRYS (UNILEVER), bei der den Milchlieferanten Lieferbedingungen gestellt werden, die artgerechtere Haltungsbedingungen für die Milchkühe zur Folge haben sollen.

In diesem Kapitel zum Tierwohl-Labeling soll zunächst das allgemeine Einkaufs- und Ernährungsverhalten im Hinblick auf Fleisch- und Wurstwaren charakterisiert werden. Im Mittelpunkt des darauffolgenden Abschnitts steht die Beachtung von TierwohlLabels. Dieser Teil befasst sich zunächst mit dem Interesse der Verbraucher an Tierwohlprodukten, um eine Vorstellung vom Marktpotenzial von Tierwohlprodukten zu erhalten.

Des Weiteren wird in diesem Abschnitt u. a. auf den Bekanntheitsgrad und die Einschätzung von Tierwohl-Labels eingegangen. Anschließend werden verschiedene Hinderungsgründe für den Kauf von Tierwohlprodukten vorgestellt und es wird auf den Informationsbedarf und politische Präferenzen eingegangen, um eine theoretische Basis für die empirische Studie der vorliegenden Arbeit zu schaffen. 


\subsection{Einkaufs- und Ernährungsverhalten}

Es besteht eine Vielzahl unterschiedlicher Studien zum Einkaufs- und Ernährungsverhalten. Die meisten Studien, wie etwa der ERNÄHRUNGSREPORT, beziehen sich dabei auf den Einkauf allgemeiner Lebensmittel (BMEL, 2019a). In weiteren Studien, beispielsweise im ÖKOBAROMETER, werden häufig nur Bio-Käufer untersucht (BMEL, 2016). Die Anzahl an wissenschaftlichen Studien, die sich konkret auf Fleisch- und Wurstwaren konzentrieren, ist hingegen deutlich geringer. In diesem Abschnitt werden die relevantesten Studien beschrieben.

So untersuchten WEINRICH et al. (2015) verschiedene Aspekte beim Einkauf von Tierwohlprodukten und fanden heraus, dass bei Verbrauchern, denen das Tierwohl wichtig war, ein höheres Wissen über die Tierhaltung bestand und diese Personen die Fleischtheke präferierten. STAUS (2010) stellte fest, dass die Einkaufsstättenwahl von den persönlichen Einstellungen hinsichtlich verschiedener Kriterien abhängt, wie beispielsweise Qualität, Frische, Umwelt, Bio und Preis.

Soziodemografische Unterschiede bei der Einkaufsstättenwahl stellten NILSSON et al. (2015) fest. HUDDLESTON et al. (2009) versuchten einen Zusammenhang zwischen der Kundenzufriedenheit und der Einkaufsstätte nachzuweisen, mit dem Ergebnis, dass vor allem die Preise, die Produktauswahl, der Service und die Qualität die Kundenzufriedenheit beeinflussen. Fachgeschäftskunden waren zufriedener als Supermarktkunden.

INDERHEES et al. (2004) stellten fest, dass Metzgereien einen hohen Anteil an Stammkunden haben, die fortgeschrittenen Alters sind. Sie unterstreichen auch die besondere Bedeutung der Qualität und der Warenpräsentation für Metzgerei-Käufer.

ZENNER et al. (2004) sowie SPILLER und SCHULZE (2008) identifizierten zudem Einstellungsfaktoren wie Vertrauen, Preisbewusstsein, Gesundheitsbewusstsein, Umwelt- und Sozialbewusstsein, Markenbewusstsein, politische Motivation sowie besondere regionale Präferenzen und Einkaufsstättenpräferenzen. 
Seit einigen Jahren besteht beim Angebot von Fleisch- und Wurstwaren ein starker Trend zum SB-Bereich. SCHULZE und SPILLER (2008) sehen hier die Möglichkeit, Fleisch und Wurstwaren besser über die Produktverpackungen kennzeichnen zu können. Die Verpackung ist bei der Vermarktung von Produkten, die mit Tierwohl-Labels gekennzeichnet werden, letztlich von zentraler Bedeutung, da sie gleichzeitig als Informationsmedium für das Label dient.

Die Fleischtheke steht nach wie vor für mehr Frische und eine bessere Qualität als der SB-Bereich. Beim SB-Bereich stehen der geringere Preis und die Zeitersparnis im Vordergrund (SCHULZE \& SPILLER, 2008). Vor allem das Verkaufspersonal an der Fleischtheke schafft Vertrauen und sorgt für ein höheres Qualitätsempfinden bei den Verbrauchern. Außerdem wird Thekenfleisch stärker als regional wahrgenommen (69\%) und viele Verbraucher gehen davon aus, dass die Tiere aus einer artgerechteren Haltung stammen $(42 \%)$.

Rund ein Drittel gab in der Studie von SCHULZE und SPILLER (2008) an, keinen Unterschied zwischen Fleisch vom Metzger und Fleisch vom Discounter zu sehen. Der Vertrauensvorsprung geht dabei womöglich vor allem in der jungen Zielgruppe verloren. Unter den Thekenkäufern gibt es jedoch eine einkommensstarke Zielgruppe für Premiumprodukte.

Auch verschiedene soziodemografische Faktoren beeinflussen das Verhalten von Konsumenten beim Fleischkauf. Wissenschaftliche Studien, in denen das Konsumverhalten bei Fleischwaren untersucht wurde, konzentrierten sich in der Vergangenheit vor allem auf Unterschiede hinsichtlich der soziodemografischen Kriterien Einkommen, Geschlecht und Alter.

Dem Faktor Einkommen wurde in zahlreichen Studien ein hoher Einfluss auf den Konsum von Fleisch zugesprochen. Da Fleisch nach dem Zweiten Weltkrieg im Vergleich zu Getreideprodukten, Kartoffeln oder Gemüse teuer war, musste man über entsprechende finanzielle Mittel verfügen, um häufig Fleisch essen zu können. 
Insbesondere Menschen mit hohem Einkommen oder Vermögen konnten sich folglich einen höheren Fleischkonsum leisten. Eine Ausnahme bildeten teilweise die Landwirte, die aufgrund der eigenen Haltung von Nutztieren Zugang zu kostengünstigem Fleisch besaßen. Durch den Industrialisierungsprozess, den technologischen Fortschritt, effizienteres Management, ökonomische Skalen- und Verbundeffekte sowie Konzentrationsprozesse in der Fleischbranche konnte Fleisch immer kostengünstiger produziert und Konsumenten zu immer günstigeren Preisen zum Kauf angeboten werden. In der Folge konnten sich im Zeitverlauf mehr einkommensschwächere gesellschaftliche Gruppen einen höheren Fleischkonsum leisten. Die nationale Verzehrstudie aus dem Jahr 2008 hat ergeben, dass Menschen mit niedrigem Einkommen mittlerweile sogar signifikant mehr Fleisch konsumieren als Menschen mit höherem Einkommen (MAXRUBNER-INSTITUT, 2008). Dies entspricht einer gegensätzlichen Situation im Vergleich zur Situation in den Nachkriegsjahren in Deutschland.

Das Einkommen wird in westeuropäischen Ländern nur als begrenzter Einflussfaktor für den Fleischkonsum gesehen und als relativ unbedeutend im Vergleich zu geschmacklichen Präferenzen oder psychographischen Faktoren eingestuft (GOSSARD \& YORK, 2003; THIELE, 2008). In vielen Entwicklungsländern hat das Einkommen dagegen immer noch einen großen Einfluss auf den Fleischkonsum (BMEL, 2015). Die Einkommenselastizität beim Gesamtfleischverzehr ist in Deutschland jedoch vergleichsweise gering. Interessanterweise scheint es aber Unterschiede hinsichtlich der Präferenzen für bestimmte Tier- bzw. Fleischarten in verschiedenen sozialen Milieus zu geben. Gemäß der nationalen Verzehrstudie der DGE variiert die Einkommenselastizität hinsichtlich der Fleisch- bzw. Tierart teilweise stark (MAX-RUBNER-INSTITUT, 2008). Die Einkommenselastizität beim Konsum von Rindfleisch ist z. B. deutlich höher als bei Schweinefleisch. Dies bedeutet, dass Menschen mit höherem Einkommen signifikant häufiger Rindfleisch essen als Menschen mit niedrigerem Einkommen. Dass Rindfleisch eine Ausnahme darstellt, zeigt sich auch in der geringeren Preissensibilität im Vergleich zu anderen Fleisch- und Wurstwaren. Menschen mit höherem Einkommen reagieren beim Fleischkauf häufig weniger stark auf Preisunterschiede als Menschen mit niedrigerem Einkommen (SCHRÖCK, 2013; THIELE, 2008). 
Ein weiteres häufig untersuchtes soziodemografisches Kriterium von Studien zum Fleischkonsum ist das Geschlecht von Konsumenten, das oftmals einen starken Einfluss auf die Menge des Fleischkonsums und die Präferenzen für bestimmtes Fleisch hat. In zahlreichen Studien wurde herausgefunden, dass Männer mehr Fleisch essen als Frauen (BEARDSWORTH \& BRYMAN, 1999; BEARDSWORTH \& BRYMAN, 2004; GOSSARD \& YORK, 2003; LEA \& WORSLEY, 2001; PRÄTTÄLÄ et al., 2007).

CORDTS et al. (2013) identifizierten im Rahmen einer Studie eine Gruppe von ,Fleischfans‘ unter den Männern (rd. $6 \%$ der Bevölkerung), die ungefähr die dreifache Menge des durchschnittlichen Verbrauchs an Fleisch verzehren. Gleichwohl konnte eine Gruppe an Männern bestimmt werden, die rd. $40 \%$ weniger Fleisch konsumieren als der durchschnittliche Fleischverbrauch. Weitere signifikante Unterschiede hinsichtlich des Fleischkonsums bestehen bei unterschiedlichen Altersgruppen. Gemäß Studien von DE BOER et al. (2007) sowie GOSSARD und YORK (2003) essen ältere Menschen unabhängig von ihrem generell reduzierten Konsum von Lebensmitteln durchschnittlich weniger Fleisch als jüngere Menschen. Daher ist davon auszugehen, dass der Fleischkonsum aufgrund des demographischen Wandels in Deutschland zukünftig abnehmen wird.

Bezüglich der Reduktion des Fleischverzehrs empfiehlt die Deutsche Ernährungsgesellschaft (DGE) seit vielen Jahren den wöchentlichen Verzehr von 300 bis $600 \mathrm{~g}$ fettarmem Fleisch und fettarmer Wurst. Die von der DGE angegebene Menge, die insgesamt nicht mehr als $31 \mathrm{~kg}$ pro Jahr betragen soll, entspricht damit etwa der Hälfte der in Deutschland tatsächlich konsumierten Fleischmenge von rd. 60 kg (DGE, 2019).

Weitere Organisationen wie beispielsweise die Ernährungs- und Landwirtschaftsorganisation der Vereinten Nationen (FAO), die Weltgesundheitsorganisation (WHO), Umweltschutzorganisationen, die sich für den Klima- und Artenschutz einsetzen wie z. B. der WWF oder GREENPEACE, Verbraucherschutzorganisationen, kirchliche Organisationen wie Misereor und natürlich Tierschutzorganisationen fordern eine deutliche Reduktion des Fleischkonsums. 
Anfang 2019 haben Experten der Lancet-Kommission EAT in der Studie „Food in the Anthropocene: the EAT-Lancet Commission on healthy diets from sustainable food systems“ in den Empfehlungen für eine nachhaltigere Ernährung neben anderen Maßnahmen wie etwa der Reduktion der Lebensmittelverschwendung noch einmal betont, dass der zu hohe Konsum an Fleisch- und Wurstwaren dringend reduziert werden müsse, um globalen Herausforderungen wie dem Umwelt-/Klimaschutz, Gesundheitsthemen, der Welternährung, dem Erhalt der Artenvielfalt oder dem Wasserverbrauch besser begegnen zu können (THE LANCET, 2019).

Im Rahmen verschiedener Verbraucherbefragungen wurden die Gründe für die Reduktion des Fleischkonsums untersucht. Abgesehen von weiteren ethischen Einflussfaktoren auf den Fleischkonsum (die sich häufig auf externe Effekte der Nutztierhaltung beziehen) gaben Verbraucher häufig an, dass sie neben gesundheitlichen Aspekten am ehesten aufgrund von Tierwohlaspekten ihren Fleischkonsum reduzieren würden (BEARDSWORTH \& KEIL, 1991; DE BOER et al., 2007; GRUNERT, 2006; RICHARDSON et al., 1993; VERBEKE \& VIANE, 2000; WOODWARD, 1988).

LEA und WORSLEY (2001) sehen den Einfluss von Bezugsgruppen als einen weiteren bedeutsamen Faktor an. Daneben beeinflussen auch das Vertrauen in Hersteller und Händler sowie bereitgestellte Informationen und angebotene Produkte das Konsumverhalten (LEA \& WORSLEY, 2001; VERBEKE \& VIANE, 2000). Das ambivalente Image von Fleisch (ANDERSEN et al., 2005) sowie die schlechte Reputation der Fleischwirtschaft (ALBERSMEIER \& SPILLER, 2010; TONSOR \& OLYNK, 2010) sind ebenfalls Faktoren, die zu einer Reduktion des Fleischkonsums führen können.

Manche Konsumenten haben außerdem eine Abneigung gegen den Geschmack, Geruch oder die Konsistenz von Fleisch entwickelt. Einige Personen reduzieren daher ihren Fleischkonsum bzw. verzichten gänzlich auf den Verzehr von Fleisch (BEARDSWORTH \& KEIL, 1991; RICHARDSON et al., 1993; WOODWARD, 1988). Auch durch das zunehmende Tierschutzbewusstsein nimmt der Fleischkonsum ab. Die geringere Nachfrage tierschutzbewusster Konsumenten hat allerdings auch einen geringeren Einfluss auf die Angebotsseite (NORWOOD \& LUSK, 2009). 
In verschiedenen Studien wurde bereits die Reduktionsbereitschaft verschiedener Verbrauchergruppen mit unterschiedlichen Schwerpunkten analysiert. Dabei wurde im Rahmen repräsentativer Bevölkerungsbefragungen der Verzehr von Fleisch- und Wurstwaren anhand der Verzehrhäufigkeit quantifiziert und den Verbrauchern wurden unterschiedliche Ernährungsstile zugeordnet.

CORDTS et al. (2013) zeigten die Besonderheiten im Fleischkonsum bei Flexitariern auf und fanden heraus, dass sich die verschiedenen Typen von Fleischessern deutlich hinsichtlich ihrer Reduktionsbereitschaft unterscheiden. So gaben fast $60 \%$ der Befragten an, nur ganz bestimmtes Fleisch bzw. Wurst zu essen, wie z. B. Bio-Fleisch oder Geflügel. $40 \%$ gaben an, Fleisch nur zu besonderen Anlässen zu essen. Die vorliegende Studie, die sich im Gegensatz zu den bereits durchgeführten Studien auf nachhaltigkeitsinteressierte Fleischkäufer fokussiert (Screening-Fragen), orientiert sich bei der Frage zur Reduktionsbereitschaft an der Studie von CORDTS et al. (2013). Es soll untersucht werden, ob sich spezielle Tierwohl-Labels bei unterschiedlichen Fleischessern signifikant unterscheiden, um die Forschungsergebnisse von CORDTS et al. (2013) mit neuen Erkenntnissen zu ergänzen.

Neben der im vorherigen Abschnitt beschriebenen Reduktionsbereitschaft deutscher Verbraucher ist die Kenntnis der Verbraucherpräferenzen hinsichtlich der verschiedenen Kaufkriterien von Fleisch- und Wurstwaren aus einer artgerechteren und nachhaltigeren Nutztierhaltung für Hersteller und Händler im Hinblick auf eine differenziertere Gestaltung des Angebots an Fleisch- und Wurstwaren von großer Bedeutung und stellt eine zentrale Voraussetzung für strategische unternehmerische Entscheidungen dar (BMEL, 2015). Im Mittelpunkt der vorliegenden Studie stehen die Präferenzen der Verbraucher für Tierwohl-Labels. Die Tierwohl-Labels beinhalten häufig Verbesserungen hinsichtlich der artgerechten Tierhaltung, der Umweltfreundlichkeit und verschiedener Gesundheitsaspekte. Diese drei Kaufkriterien entsprechen laut des Wissenschaftlichen Beirats des BMEL den zentralen Problemfeldern der intensiven Nutztierhaltung (BMEL, 2015). 
Im ÖKOBAROMETER wurden Kaufkriterien für Bio-Fleisch oder -Wurstwaren erfragt. Dabei waren die bedeutsamsten Kaufkriterien die ,bessere Behandlung der Tiere', Vorteile für die Gesundheit (weniger Antibiotika), besserer Geschmack, Umwelt- und Klimaschutz sowie moralische bzw. ethische Gründe (BMEL, 2016). Es wurden jedoch keine klassischen Kaufkriterien erfragt und mit dem ÖKOBAROMETER wurden nur Bio-Käufer untersucht. In der vorliegenden Studie soll dagegen eine vergleichende Bewertung der bedeutsamsten klassischen und ethischen Kaufkriterien durch nachhaltigkeitsinteressierte Fleischkäufer vorgenommen werden (F8).

Hinsichtlich der Kaufkriterien ergab eine Studie von CORDTS et al. (2013), dass ethische Einflussfaktoren, wie Tierwohl oder Umweltfreundlichkeit, die Einstellung und das Verhalten beim Fleischkauf womöglich nicht so stark beeinflussen wie andere Faktoren (z. B. Gesundheits- und Figurbewusstsein) oder klassische Kaufkriterien (z. B. Preis, Geschmack etc.). Tierwohlaspekte haben jedoch bei vielen Verbrauchern einen stärkeren Effekt auf die Kaufentscheidung als negative externe Umwelteffekte der Nutztierhaltung (CORDTS et al., 2013). Durch die bessere Beachtung der ethischen Kaufkriterien Tierwohl, Umwelt und Gesundheit können landwirtschaftliche Betriebe, die hinsichtlich dieser drei Problemfelder Verbesserungen nachweisen, von Verbrauchern beim Fleischkauf honoriert werden. Es ist bisher jedoch noch nicht untersucht worden, ob Kunden der unterschiedlichen Lebensmitteleinzelhändler beim Einkauf von Fleisch- und Wurstwaren unterschiedlich stark auf ethische Kaufkriterien achten. Dahingehend kann davon ausgegangen werden, dass es Unterschiede zwischen den verschiedenen Kunden gibt. Aus diesem Grund sollten weiterführende Analysen durchgeführt werden, um herauszufinden, wie verschiedene Einkaufstypen beim Einkauf von Fleisch- und Wurstwaren auf die ethischen Kaufkriterien achten. Hierdurch können detaillierte Erkenntnisse hinsichtlich einzelner Einkaufsstätten (wie z. B. EDEKA oder ALDI) sowie weiterführende Erkenntnisse durch die Analyse der unterschiedlichen Betriebsformen Supermarkt, Discounter, Verbrauchermarkt und Metzger gewonnen werden (H1). Solche Erkenntnisse können zu fundierteren strategischen Vertriebsentscheidungen führen, und zudem lassen sich konkrete Handlungsempfehlungen für die verschiedenen Lebensmitteleinzelhändler bzw. Betriebsformen ableiten. 
Darüber hinaus wird vermutet, dass sich die Beachtung der ethischen Kaufkriterien hinsichtlich der Angebotsform (Theke/SB) für Wurstwaren oder Aufschnitt sowie Fleisch zum Braten und Grillen unterscheidet, weshalb auch hierzu Untersuchungen angestellt werden sollen (H2 und $\mathrm{H} 3)$. Die Erkenntnisse können $\mathrm{zu}$ deutlich fundierteren strategischen Entscheidungen von Anbietern beitragen, z. B. dahingehend, ob die Fleischund Wurstwaren für Menschen, die besonders auf Tierwohl achten, eher an der Fleischtheke oder im SB-Bereich angeboten werden sollten, oder ob z. B. an der Fleischtheke eher als im SB-Bereich die Umweltfreundlichkeit in Werbematerialien betont werden sollte.

Durch diese Aspekte lassen sich wesentliche Fragen für die Angebotsseite beantworten, da Unterschiede der Beachtung dieser drei Kaufkriterien sowohl bei der Auswahl von Labels als auch bei der Produktkommunikation eine große Rolle spielen. Bisher wurden derart detaillierte praxisnahe Fragestellungen im wissenschaftlichen Kontext noch nicht ausreichend für alle drei Kriterien beantwortet.

Des Weiteren wird vermutet, dass sich die Beachtung dieser drei ethischen Kaufkriterien hinsichtlich verschiedener soziodemographischer Kriterien der Verbraucher unterscheidet (H4). Dass der Fleischkonsum im Allgemeinen bei verschiedenen soziodemografischen Kriterien divergiert, wurde bereits zu Beginn dieses Kapitels beschrieben. Im Hinblick auf die soziodemografischen Unterschiede bei den Kaufkriterien wurden jedoch verschiedene Forschungslücken identifiziert. In der vorliegenden Arbeit werden daher Unterschiede hinsichtlich des Geschlechts, des Alters, der Region in Deutschland, der Bildung und des Einkommens der Befragten in Bezug auf diese Kaufkriterien analysiert und interpretiert, da diese Kriterien von besonderem Interesse für das Marketing und die richtige Zielgruppenansprache sind.

Die bessere Kenntnis dieser Unterschiede hat verschiedene Vorteile. Beispielsweise können Anbieter ihre Marketing-Mix-Entscheidungen besser an den Zielgruppen ausrichten, indem sie z. B. geographische Unterschiede bei der Beachtung der Kaufkriterien besser berücksichtigen. So können bestimmte Tierwohlprodukte in Großstädten womöglich erfolgreich vermarktet werden, während sie in bestimmten anderen Regionen eher einen ,Ladenhüter' darstellen. 
Ebenso könnte aus den Ergebnissen z. B. die Sinnhaftigkeit abgeleitet werden, bei Produkten für jüngere oder einkommensstärkere Zielgruppen besonders umweltfreundliche Produktalternativen anzubieten und die Vorteile für die Umwelt ausdrücklich zu betonen, z. B. durch die Kombination eines Tierwohl-Labels mit einem Klimaneutral-Label oder einem Logo einer Naturschutzorganisation, um sich bei diesen Zielgruppen positiv von Wettbewerbern zu differenzieren.

Als weitere Besonderheit der vorliegenden Studie, speziell hinsichtlich des Kaufkriteriums der artgerechteren Tierhaltung, soll ebenfalls der Einfluss eines Haustiers, der Religion und der gewählten politischen Partei untersucht werden. Diese Fragen stellen in der tierwohlbezogenen Verbraucherforschung bei Fleisch- und Wurstwaren teilweise noch ein Novum dar. Es wird vermutet, dass Haustierbesitzer, religiöse Menschen und Wähler der Grünen und Tierschutzparteien beim Einkauf von Fleisch- und Wurstwaren besonders auf eine artgerechtere Tierhaltung achten (H5 bis H7).

Die Religion der Verbraucher wird erfragt, da vermutet wird, dass innovative Erkenntnisse für die Verbraucherforschung sowie die philosophische und theologische Diskussion gewonnen werden können. So lässt sich dadurch beispielsweise eruieren, ob z. B. religiöse Menschen beim Konsum von Fleisch- und Wurstwaren stärker auf eine artgerechtere Tierhaltung achten und sich somit ein christlicher Ethos auch im alltäglichen Einkaufsverhalten zeigt. Hinweise darauf finden sich in der Tierwohlforschung bei Fleisch- und Wurstwaren bisher noch nicht.

Dabei wird Tierschutz teilweise als christlicher Auftrag gesehen. Sollten die Kirchen zukünftig zu einer stärkeren Berücksichtigung ethischer Aspekte des Fleischkonsums aufrufen, könnte dadurch die Nachfrage nach Tierwohlprodukten zusätzlich gesteigert werden. Des Weiteren ist es vor dem Hintergrund der starken Immigrationsbewegungen nach Deutschland bzw. in die Europäische Union von Interesse, ob signifikante Unterschiede bei verschiedenen Weltreligionen bei der Beachtung des Kaufkriteriums artgerechtere Tierhaltung, bei Sortenpräferenzen oder auch bei bestimmten TierwohlLabels existieren. 
Diese besonderen interkulturellen Unterschiede können bei einem zielgruppenorientierteren Produkt- und Beratungsangebot von Tierwohlprodukten in Deutschland bzw. der Europäischen Union berücksichtigt werden. In vielen weiteren Branchen finden sich konkrete Angebote an bestimmte ethnische Gruppen. So hat die Telekom bereits vor ca. 15 Jahren gezielt Werbematerialien für in Deutschland lebende Türken gestaltet. Eine derartige, im Hinblick auf die unterschiedlichen Religionen differenzierte, Zielgruppenansprache ist auch für Fleisch- und Wurstwaren denkbar.

Auch vor dem Hintergrund der großen Bedeutung des Exports von Fleisch- und Wurstwaren kann religiösen Unterschieden bei der Beachtung ethischer Kaufkriterien eine hohe Relevanz beigemessen werden. In Debatten zur Erhöhung von Tierwohlstandards in Deutschland wird seitens der Fleischindustrie seit Jahrzehnten das Argument aufgeführt, dass in den Exportpartnerländern Deutschlands nur ein sehr geringes Interesse an Tierwohlprodukten mit besonders hohen Standards bestehe und sich höherpreisige Fleisch- und Wurstwaren mit besonderen Tierwohlstandards nicht im Ausland absetzen lassen würden.

Vor dem Hintergrund des globalen langfristigen Trends der Neo-Ökologie sind jedoch deutliche Tendenzen zu beobachten, dass die Kritik an der Nutztierhaltung auch in zahlreichen anderen Ländern (z. B. in Indien oder Brasilien) deutlich steigt und auch dort seit Jahren höhere ethische Standards für Tierwohlprodukte gefordert werden (ZUKUNFTSINSTITUT, 2019).

Eine weitere für die Verbraucherforschung interessante Frage ist zudem, ob der Besitz eines Haustiers auch die Empathie für Nutztiere erhöht. Diesbezüglich wird vermutet, dass Haustierbesitzer auch beim Einkauf von Fleisch- und Wurstwaren stärker auf eine artgerechtere Tierhaltung achten als Menschen ohne Haustier (H5). Diese Erkenntnisse sind in der Praxis nicht nur für Anbieter von Fleisch- und Wurstwaren für Menschen, sondern auch für Anbieter von zertifiziertem Bio-Tierfutter von Relevanz. So könnten beispielsweise Möglichkeiten evaluiert werden, um mit besonderen Tierwohlprodukten oder auch Werbeslogans Haustierbesitzer gezielter anzusprechen. 
Das Kaufverhalten unterschiedlicher Wählergruppen beim Einkauf von Fleisch- und Wurstwaren wurde ebenfalls noch nicht in der Verbraucherforschung untersucht und kann eine neue Diskussion anstoßen, ob sich das Wahlverhalten bei politischen Wahlen auch im alltäglichen (Einkaufs-)Verhalten widerspiegelt. Dabei wird davon ausgegangen, dass sich das Konsumverhalten unterschiedlicher Wählergruppen (nach gewählter Partei) bei Fleisch- und Wurstwaren signifikant unterscheidet (H7).

\subsection{Beachtung von Tierwohl-Labels}

Im Mittelpunkt dieses Abschnitts steht die Beachtung von Tierwohl-Labels beim Kauf von Fleisch- und Wurstwaren. Dieser Teil befasst sich zunächst mit dem allgemeinen Interesse der Verbraucher an Tierwohlprodukten, um eine Vorstellung des Marktpotenzials von Tierwohlprodukten zu erhalten. Des Weiteren wird in diesem Abschnitt auf den Bekanntheitsgrad und die Einschätzung bzw. das Image von TierwohlLabels eingegangen.

In den letzten Jahren wurde in verschiedenen allgemeinen Verbraucherstudien auf hohe Marktpotenziale für Tierwohlprodukte aufmerksam gemacht (DE JONGE et al., 2015; DE JONGE \& VAN TRIJP, 2013; LAGERKVIST \& HESS, 2011; NOCELLA et al., 2010; SCHULZE et al., 2008; VAN LOO et al., 2014; ZANDER et al., 2013). Laut ERNÄHRUNGSREPORT 2018 des Bundesministeriums für Ernährung und Landwirtschaft ist eine große Mehrheit (90\%) der Befragten bereit, mehr Geld für Lebensmittel zu bezahlen, wenn die Tiere besser gehalten würden, als es die Gesetze in Deutschland vorschreiben.

Des Weiteren wünschen sich dem Ernährungsreport des BMEL zufolge vier von fünf Verbrauchern (79\%) ein staatliches Tierwohl-Label (BMEL, 2018). Werte, Einstellungen und Zahlungsbereitschaften für mehr Tierwohl wurden bisher jedoch nur wenig erforscht. LAGERKVIST und HESS (2010) konnten im Rahmen einer Metaanalyse nur 24 Zahlungsbereitschaftsanalysen für mehr Tierwohl nutzen (BMEL, 2015). 
DE JONGE und VAN TRIJP (2013) versuchten 2013 im Rahmen einer weit beachteten Studie die Präferenzen der Verbraucher für Tierwohlprodukte am Beispiel des niederländischen Tierwohl-Labels ,Beter Leven`zu untersuchen. ,Beter Leven` ist ein Beispiel für ein Tierwohl-Label, das Verbesserungen für das Tierwohl in verschiedenen Stufen beinhaltet, jedoch keinen ökologischen Standards entspricht (PIRSICH et al., 2017). Das Beter-Leven-Tierwohl-Label zielt auf ein Kundensegment ab, in dem nach DE JONGE und VAN TRIJP (2013) zwar großes Interesse an Tierwohlprodukten besteht, aber eine relativ geringe Zahlungsbereitschaft für höhere Preise für Bio-Produkte vorhanden ist. SCHULZE et al. (2008) schätzten in ihrer Studie den Anteil eines ähnlichen Segments in Deutschland auf etwa $20 \%$ der Bevölkerung. Dabei wurde eine Mehrzahlungsbereitschaft bei den interessierten Verbrauchern zwischen 10 und $35 \%$ ermittelt. Ähnliche Zahlen gingen auch aus verschiedenen weiteren Studien hervor. Beispielhaft genannt sei hier die Studie von ENNEKING et al. (2019), die im Praxistest bei $16 \%$ der Verbraucher eine Mehrzahlungsbereitschaft für ein neues Tierwohlprodukt ermittelten.

Segmentierungsstudien basierten in der Verbraucherforschung bislang überwiegend auf allgemein gehaltenen Charakterisierungen von Verbrauchern bzgl. psychographischer oder soziodemografischer Kriterien, so beispielsweise die Studie „Verbraucherverständnis von Transparenz“ von SPILLER und NITZKO (2014), in deren Rahmen verschiedene Verbrauchergruppen auf Basis ihrer allgemeinen Informationsbedürfnisse bei Lebensmitteln typologisiert wurden. Differenziertere Einstellungen und Gruppenbildungen von Verbrauchern zum Thema Tierwohl wurden bisher nur selten erforscht, insbesondere speziell für Fleisch- und Wurstwaren. Als eine Ausnahme ist die Studie von SCHULZE et al. (2008) zu erwähnen, bei der mit Hilfe multivariater Analysen verschiedene Verbrauchergruppen identifiziert wurden, zumindest im Hinblick auf allgemeine Produkte tierischer Herkunft ohne eine nähere Differenzierung hinsichtlich der Produktart (Fleisch, Milch, Eier etc.) oder gar einzelnen Sorten an Produkten (Aufschnitt, Grillfleisch, Tierart etc.). Anhand der Einstellungen zum Thema Tierschutz und der Bewertung von Tierschutzstandards identifizierten SCHULZE et al. (2008) eine Gruppe von Menschen, die sie als ,Besorgte Tierschützer` bezeichnen. 
Diese Gruppe, die zu $75 \%$ aus Frauen besteht und rd. $20 \%$ der Bevölkerung ausmacht, eignet sich nach SCHULZE et al. (2008) am ehesten für die Ansprache mit TierwohlLabels und weist die höchste Zahlungsbereitschaft für entsprechende Produkte auf. Die intensive Tierhaltung wird von dieser Gruppe überaus negativ bewertet. Personen aus dieser Gruppe haben ihren Fleischkonsum häufig reduziert und verzehren insgesamt wenig Fleisch- und Wurstwaren. Eine andere Verbrauchergruppe in ähnlicher Größenordnung wird von SCHULZE et al. (2008) als ,Tierschutzbewusste Fleischesser“ bezeichnet und ähnelt in vielen Punkten der Gruppe der ,Besorgten Tierschützer ${ }^{6}$, jedoch mit dem Unterschied, dass diesem Cluster eher ältere Personen angehören und die Haltungsbedingungen positiver bewertet werden (BMEL, 2015). Diese Gruppe kann als erweiterte Zielgruppe für Fleisch- und Wurstwaren, die mit Tierwohl-Labels gekennzeichnet sind, gesehen werden.

ZANDER et al. (2013) stellten im Rahmen einer Studie fest, dass es in der Bevölkerung keine klare Zustimmung bzw. Unterstützung der heutigen Landwirtschaft mehr gibt. Der Großteil der Bevölkerung schätzt die Haltungsbedingungen in der intensiven Nutztierhaltung demnach als schlecht bzw. nicht artgerecht ein.

Im Rahmen der durchgeführten Clusteranalyse von ZANDER et al. zur Schweinehaltung konnten drei ähnlich große Gruppen (Cluster) in der deutschen Bevölkerung identifiziert werden: rd. $30 \%$ Gegner der modernen Schweinehaltung, rd. $30 \%$ moderate Kritiker der Schweinehaltung und rd. $30 \%$ Tolerierende, die eine deutlich höhere Akzeptanz gegenüber der Schweinehaltung haben, diese aber dennoch weder positiv einschätzen noch Verbesserungen fordern (ZANDER et al., 2013). Die Ergebnisse dieser Studie lassen vermuten, dass der Anteil der potenziellen Käufer von Fleisch- und Wurstwaren mit Tierwohl-Labels rd. 60 \% der Bevölkerung entspricht.

MEUWISSEN et al. (2007) untersuchten mittels einer Conjoint-Analyse 24 Eigenschaften von Schweinefleisch, wobei rd. 1200 niederländische Verbraucher in die folgenden sechs Segmente eingeteilt wurden: unpronounced consumers (20\%), economists $(12 \%)$, health concerned consumers (18\%), animal friends (16\%), traditional minded consumers $(17 \%)$ und ecologists $(17 \%)$. 
Eine Haupterkenntnis der Studie von MEUWISSEN et al. (2007) besteht darin, dass sich einzelne Segmente wie beispielsweise animal friends und ecologists (zusammen rd. $30 \%$ ) für die Ansprache mit Tierwohl-Labels im Marketing eignen. Des Weiteren weisen MEUWISSEN et al. (2007) darauf hin, dass Fleisch generell nicht länger als standardisiertes Massenprodukt (Bulk Product) vermarktet werden sollte. Dieser Meinung schließt sich der WBAE an und plädiert für eine stärkere Qualitätsorientierung und Marktdifferenzierung bei Fleisch- und Wurstwaren (BMEL, 2015). Die Mehrzahl der Verbraucher steht der intensiven Nutztierhaltung (Massentierhaltung) kritisch gegenüber. Die Gruppengröße der Konsumenten, denen Tierwohl ein ernsthaftes und verhaltensrelevantes Anliegen ist und die damit Ziel entsprechender Marketingmaßnahmen werden können, wird vom Wissenschaftlichen Beirat des Bundesministeriums für Ernährung und Landwirtschaft (WBAE) auf ca. 30 bis $40 \%$ der Bevölkerung geschätzt (BMEL, 2015).

Diese wenigen Segmentierungsstudien mit konkretem Tierwohlbezug sind jedoch nicht mehr aktuell. Trotz intensiver gesellschaftlicher Diskussion zum Thema Tierwohl und des stark veränderten Konsumentenverhaltens in den letzten zehn Jahren wurden zudem keine weiteren wissenschaftlichen Studien durchgeführt, in denen eine Segmentierung von Verbrauchern nach Tierwohlaspekten erfolgte. Insbesondere fehlt bislang eine Segmentierungsstudie, in der Tierwohl-Labels bei der Bildung verschiedener Verbrauchergruppen berücksichtigt werden. In Studien wie beispielsweise dem ÖKOBAROMETER (BMEL, 2016) oder der Studie ,Verbraucherverständnis von Transparenz' von SPILLER und NITZKO (2014) wurde der Nachweis erbracht, dass verschiedene Verbrauchergruppen im Hinblick auf Tierwohlpräferenzen bei Fleisch- und Wurstwaren zu differenzieren sind. Da das Angebot an Fleisch- und Wurstwaren einen der bedeutendsten Faktoren bei der Einkaufsstättenwahl darstellt, sind die Erkenntnisse eines derartigen Segmentierungsansatzes von hoher Relevanz für die Anbieter von Fleisch- und Wurstwaren und können dabei helfen, den verschiedenen Verbrauchergruppen ein passenderes Angebot an Fleisch- und Wurstwaren nach ethischen Gesichtspunkten bzw. unterschiedlichen Tierwohlstandards im Lebensmitteleinzelhandel zu präsentieren. 
Die Beantwortung dieser Frage soll einen Beitrag zum Metaziel der vorliegenden Arbeit leisten und die Vermarktung von Fleisch- und Wurstwaren aus einer artgerechteren und nachhaltigeren Nutztierhaltung verbessern. Aktuell wird davon ausgegangen, dass das Angebot an Tierwohlprodukten nicht optimal auf die verschiedenen Kundengruppen abgestimmt ist. Ebenfalls wird die These vertreten, dass dies ein wesentlicher Grund für den bisher trotz stetig steigender Umsatzzahlen geringen Markterfolg von Tierwohlprodukten ist.

Die Bereitschaft der Verbraucher, Tierwohlprodukte zu kaufen, wurde in den hier vorgestellten Studien in verschiedenen Formen erfragt. Zum einen wurde das allgemeine Interesse an Tierwohlprodukten mit Hilfe allgemeiner Fragen evaluiert und zum anderen wurden Zahlungsbereitschaftsanalysen für Tierwohlprodukte durchgeführt. Die allgemeinen Studien konzentrierten sich dabei häufig auf den generellen Kauf von Lebensmitteln. In der vorliegenden Arbeit soll jedoch das Kaufinteresse bei Tierwohlprodukten speziell für Fleisch- und Wurstwaren mit Hilfe verschiedener Fragen analysiert werden. Zudem soll sich die Analyse im Gegensatz zu den oben vorgestellten Studien sowohl auf das konkrete Kaufverhalten bei Tierwohlprodukten als auch auf konkrete Tierwohl-Labels beziehen. Dadurch kann besser eingeschätzt werden, ob das Interesse an Tierwohlprodukten tatsächlich so hoch ist, wie im ERNÄHRUNGSREPORT (BMEL, 2019a) angegeben wurde, oder ob das Interesse an Fleisch- und Wurstwaren aus einer artgerechteren und nachhaltigeren Nutztierhaltung im Zeitverlauf angestiegen ist.

Die Fragen zum Verbraucherinteresse werden in der vorliegenden Arbeit nicht in Form von Zahlungsbereitschaftsanalysen gestellt, sondern in Form psychographischer Fragen. Dieses Vorgehen ist darin begründet, dass verschiedenste Aspekte einen maßgeblichen Einfluss auf die Zahlungsbereitschaft der Verbraucher haben und die psychographischen Aspekte vorerst umfassend evaluiert werden sollten, um sie anschließend bei differenzierteren Zahlungsbereitschaftsanalysen zu berücksichtigen. Die Ergebnisse und die Interpretation von Zahlungsbereitschaftsanalysen bei Tierwohlprodukten sollen dadurch verbessert werden. Durch differenzierte Antwortmöglichkeiten bei dieser Frage kann zudem eine genauere Unterteilung der Verbrauchergruppen erfolgen. 
Insgesamt lässt sich bezüglich der in diesem Abschnitt vorgestellten Studien feststellen, dass der Fokus häufig auf einen konkreten Bereich wie beispielsweise eine bestimmte Sorte bzw. Tierart, einen Tierwohlstandard (z. B. DE-BIO) oder eine Vertriebsschiene (z. B. Fleischerfachgeschäfte) gelegt wird. Je nach untersuchtem Teilaspekt kann dies jedoch zu heterogenen Ergebnissen führen. Zudem können in derartigen Studien keine Unterschiede zwischen den verschiedenen Tierwohlstandards, Vertriebsschienen, Fleischarten oder auch speziellen Verbrauchergruppen ermittelt werden. Insbesondere bei den Verbrauchern bestehen heterogene Gruppen, die sich deutlich in ihrem Einkaufs- und Konsumverhalten unterscheiden, weshalb pauschalisierende Aussagen auch in diesem Beispiel als undifferenzierte Verallgemeinerung abzulehnen sind. An dieser Stelle unterscheidet sich der breitere differenzierte Ansatz der vorliegenden Studie.

Daneben existieren nur vereinzelt Studien, in denen der Bekanntheitsgrad von Gütesiegeln für allgemeine Lebensmittel untersucht wurde, obwohl dieser, wie bei anderen Marken ebenfalls, einen wesentlichen Erfolgsfaktor darstellt. Verschiedene Autoren (FRANZ et al., 2012; KROEBER-RIEL \& ESCH, 2004; MALHOTRA, 1984; VAN KLEEF et al., 2007) sehen in der Vielzahl neuer Gütesiegel im Lebensmittelbereich jedoch einen negativen Einfluss auf den Bekanntheitsgrad und damit den Erfolg von Tierwohl-Labels. In den Studien wird darauf hingewiesen, dass die Einführung unterschiedlichster neuer Gütesiegel zu einem ,Label-Dschungel` geführt habe und viele Verbraucher überfordert seien. Dieser ,Informationsoverload` sei ein bedeutender Grund dafür, dass vor allem die neueren reinen Tierwohl-Labels keine hohe Bekanntheit erreichen und deswegen am Markt scheitern (PIRSICH, 2017).

ZÜHLSDORF et al. (2016) untersuchten im Jahr 2016 im Rahmen einer bundesweiten Verbraucherstudie die gestützten Bekanntheitsgrade ausgewählter Tierwohlkennzeichen. Den höchsten Bekanntheitsgrad erzielte in der Umfrage das deutsche Bio-Label. Nahezu jeder Befragte hat das deutsche Bio-Label schon einmal gesehen. Bekannt ist auch das Label des deutschen ökologischen Anbauverbands BIOLAND, das drei Viertel der Befragten schon einmal gesehen haben. Das europäische Bio-Label und das DEMETERLabel erreichen ebenfalls relativ hohe Bekanntheitsgrade (über $40 \%$ ). 
Immerhin jeder fünfte deutsche Verbraucher kennt das Tierwohl-Label von NEULAND. Der gestützte Bekanntheitsgrad der anderen Tierwohl-Labels wie z. B. des Siegels des DEUTSCHEN TIERSCHUTZBUNDES oder VIER PFOTEN ist jedoch sehr gering. Den geringsten Bekanntheitsgrad besitzt in der Studie das Label der Initiative Tierwohl $(3,7 \%)$.

BUXEL und SCHULZ (2010) konnten in einer Online-Untersuchung zu Güte- und Qualitätssiegeln bei Lebensmitteln nachweisen, dass knapp 80 \% der Befragten Prüf- und Gütesiegel auf Lebensmitteln nützlich finden. Dabei wurde der gestützte Bekanntheitsgrad verschiedener Siegel und Gütezeichen abgefragt. Besonders hohe Bekanntheitsgrade erzielten beispielsweise das DLG-Zeichen mit $73 \%$ und das deutsche BIO-Siegel mit einem gestützten Bekanntheitsgrad von $87 \%$. Dagegen erreichte z. B. das QS-Siegel in der Münsteraner Studie lediglich einen Bekanntheitsgrad von $28 \%$. BIOund FAIRTRADE-Siegel wurden von den Probanden in dieser Studie bei verschiedenen Einstellungsfragen am häufigsten als vertrauenswürdig und sympathisch bewertet und bei gleichem Preis gegenüber einem ansonsten gleichen Alternativprodukt ohne Siegel bevorzugt gekauft. Des Weiteren wurde diesen Produkten am häufigsten eine sehr hohe Qualität zugesprochen (BUXEL \& SCHULZ, 2010).

Eine offene Forschungsfrage ist jedoch, wie hoch der Bekanntheitsgrad der verschiedensten Tierwohl-Labels aktuell in Deutschland ist (F10). Im deutschen Lebensmitteleinzelhandel finden sich mittlerweile sehr unterschiedliche Tierwohl-Labels in verschiedensten Einkaufsstätten. Daher wird in der vorliegenden Studie vermutet, dass die Bekanntheitsgrade der Tierwohl-Labels mittlerweile insgesamt höher sind. Des Weiteren wird davon ausgegangen, dass sich der Bekanntheitsgrad der verschiedenen Tierwohl-Labels je nach für den Fleisch- und Wursteinkauf genutzter Einkaufsstätte signifikant unterscheidet (H8). Der Bekanntheitsgrad einzelner Tierwohl-Labels wurde bisher in nur wenigen wissenschaftlichen Studien untersucht.

Derartige Studien wie die hier vorgestellten Befragungen von BUXEL (2018) oder ZÜHLSDORF et al. (2016) beziehen sich größtenteils allgemein auf Lebensmittel oder es wurde teilweise nur die Bekanntheit von Bio-Labels erfragt. 
Als einzige Ausnahme ist die Studie von ZÜHLSDORF et al. (2016) zu nennen, in der der gestützte Bekanntheitsgrad mehrerer Tierwohl-Labels analysiert wurde. Daneben wurde in Praxisstudien, z. B. im ÖKOBAROMETER 2013 (BLE, 2013), lediglich untersucht, welchen Bekanntheitsgrad Bio-Siegel bei Lebensmitteln haben, während zahlreiche weitere Tierwohl-Labels außer Acht gelassen wurden. Bei all diesen Studien zum Tierwohl-Labeling erfolgten zudem keine weiterführenden Analysen zur Größe des Bekanntheitsgrads, es wurde kein Bezug zum zielgruppenorientieren Marketing hergestellt und es wurden auch keine Handlungsempfehlungen für Anbieter von Fleischund Wurstwaren abgeleitet. Dabei stellt der Bekanntheitsgrad von Markenzeichen eine der Grundvoraussetzungen für die erfolgreiche Produktvermarktung dar und gilt als eines der bedeutendsten psychologischen bzw. qualitativen Marketingziele, das allen anderen psychologischen Marketingzielen (wie z. B. Image, Kundenzufriedenheit oder Kauf) und auch allen ökonomischen Zielgrößen (wie z. B. Umsatz, Marktanteil oder Renditen) vorgelagert ist (BRUHN et al., 2018).

Der gestützte Bekanntheitsgrad bei eindeutig definierten Zielgruppen ist von grundlegender Bedeutung für die Definition von Marketingzielen auf der Instrumentalebene. In der Marketingpraxis werden in den meisten Unternehmen entgegen der wissenschaftlichen Empfehlung häufig keine verbindlichen Ziele definiert, wie z. B. die Erhöhung des Bekanntheitsgrads oder die Verbesserung des Images in festgelegten Kategorien um eine gewisse Prozentzahl. Fehlt eine derart konkrete Marketingplanung, ist kein Marketingcontrolling möglich und somit auch kein konkretisierter Erfolgsbeitrag von Marketinginstrumenten nachweisbar (BRUHN et al., 2018). Die genaue Kenntnis des Bekanntheitsgrads in definierten Zielgruppen ist womöglich bei Tierwohl-Labels, die Vertrauen in eine artgerechtere und nachhaltigere Nutztierhaltung schaffen sollen, ebenso notwendig wie bei klassischen Marken. Nur wenn Gütesiegel bekannt sind und ein gutes Image haben bzw. glaubwürdig für strenge Kriterien stehen, sind Verbraucher bereit, höhere Preise für die besondere Produktqualität zu bezahlen (BUXEL, 2018). Beim Angebot von Tierwohlprodukten ist demzufolge im Wesentlichen der Einsatz möglichst bekannter und vertrauenswürdiger Tierwohl-Labels für die entsprechenden Zielgruppen entscheidend. 
Anhand der bereits vorgestellten Studien wurde deutlich, dass sich die zahlreichen Tierwohl-Labels im Hinblick auf den Bekanntheitsgrad mehr oder weniger stark unterscheiden, ohne dass jedoch weitere Untersuchungen durchgeführt wurden. Diese Lücke soll mit der vorliegenden Arbeit geschlossen werden, wobei das Vorgehen im Methodenteil näher erläutert wird.

Von besonderer Relevanz sind diese Erkenntnisse für die Anbieter von Fleisch- und Wurstwaren, die sich aus der Vielzahl an bestehenden Gütesiegeln für bestimmte Tierwohl-Labels entscheiden müssen. Der Bekanntheitsgrad eines Tierwohl-Labels sollte dabei neben dem Image der Gütesiegel eines der wesentlichen Auswahlkriterien für die Anbieter von Fleisch- und Wurstwaren (Hersteller und Händler) sein. Anbietern stellt sich schließlich bei der Auswahl eines geeigneten Tierwohl-Labels die Frage, welche Tierwohl-Labels bei welchen Zielgruppen besonders bekannt sind (F10) und ein gute Reputation besitzen bzw. hohes Vertrauen (F14) genießen.

Hier sollen die Ergebnisse der vorliegenden Arbeit eine Hilfestellung bei der Auswahl geeigneter Tierwohl-Labels bieten und es sollen differenzierte Empfehlungen ausgesprochen werden. Daher wird der Bekanntheitsgrad einer umfassenden Auswahl an Tierwohl-Labels untersucht, um zunächst einen aktuellen Forschungsstand zum Bekanntheitsgrad aller relevanter Tierwohl-Labels in Deutschland zu erfassen (F10).

Im deutschen Lebensmitteleinzelhandel finden sich mittlerweile sehr unterschiedliche Tierwohl-Labels in verschiedensten Einkaufsstätten. Noch nicht untersucht wurde indes, ob der Bekanntheitsgrad aller relevanter Tierwohl-Labels bei den für den Fleisch- und Wursteinkauf genutzten Einkaufsstätten variiert.

Sollte dies der Fall sein, hätte dies einen maßgeblichen Einfluss auf die Auswahl der Tierwohl-Labels nach Bekanntheitsgrad durch die Anbieter. Hersteller sollten im Idealfall Tierwohl-Labels für ihre Produkte auswählen, die bei den Kunden eines Lebensmitteleinzelhändlers eine möglichst hohe Bekanntheit erreichen. Es wird erwartet, dass sich der Bekanntheitsgrad unterschiedlicher Tierwohl-Labels je nach Einkaufsstätte signifikant unterscheidet (H8). 
Zudem werden hinsichtlich des Bekanntheitsgrads der Tierwohl-Labels auch Unterschiede hinsichtlich der Soziodemografie der Fleisch- und Wurstwarenkäufer erwartet (H9). Dies ist von besonderem Interesse für Anbieter von Fleisch- und Wurstwaren, um beispielsweise das Produkt- und Beratungsangebot an Tierwohlprodukten besser an den unterschiedlichen Zielgruppen auszurichten. Eine besondere Bedeutung kommt in diesem Zusammenhang den soziodemografischen Kriterien Geschlecht, Einkommen, Region, Ortsgröße und Bildung zu, da so festgestellt werden kann, welchen Kernzielgruppen welche Tierwohl-Labels am bekanntesten sind. So können beispielsweise zentrale Fragen wie ,Welche Tierwohl-Labels sind einkommensstarken Personen am bekanntesten?` beantwortet oder Annahmen wie ,In Großstädten sind den Menschen andere Tierwohl-Label bekannt als auf dem Land. überprüft werden.

Aus diesen verschiedenen Erkenntnissen lassen sich unmittelbare Handlungsempfehlungen für Anbieter von Fleisch- und Wurstwaren ableiten, wie z. B. in bestimmten Regionen keine bestimmten oder am ehesten bestimmte Tierwohl-Labels anzubieten oder beispielsweise besonders einkommensstarken Käufern am ehesten bestimmte Tierwohl-Labels anzubieten. Zudem können diese Ergebnisse darauf hindeuten, dass in bestimmten Regionen mehr Informationskampagnen für bestimmte Tierwohl-Labels durchgeführt werden sollten, wenn deren Bekanntheitsgrad zu niedrig ist. Dieses neue Wissen ist nicht nur für die Angebotsseite interessant, sondern liefert auch den Label-Herausgebern wertvolle Hinweise auf nicht genutzte Marktpotenziale. Diese Erkenntnisse werden ausführlich im Ergebnisteil der vorliegenden Arbeit und in der Diskussion beschrieben.

Eine genauere Kenntnis der Bekanntheitsgrade von Nachhaltigkeitssiegeln ist auch für die Politik von Relevanz. Beispielsweise ist für eine Neueinführung eines staatlichen Tierwohl-Labels in Deutschland oder auf EU-Ebene aufgrund des nicht vorhandenen Bekanntheitsgrads zunächst kein positives Image zu erzielen und daher das Risiko sehr groß, dass die Verbraucher für derartige Produkte nicht mehr bezahlen. 
Der Aufbau eines hohen Bekanntheitsgrads und eines positiven Images ist mit sehr hohen Investitionen verbunden, die der überschuldete Staat im Gegensatz zu zahlungskräftigen Unternehmen nicht für Werbung aufwenden kann. Daher ist er bei der Bewerbung des neuen Labels auf die Hilfe der Hersteller und Händler angewiesen. Bei der Kaufentscheidung kommt dem Bekanntheitsgrad der Gütesiegel eine große Bedeutung $\mathrm{zu}$, jedoch bedeutet dies nicht zwangsläufig, dass ein hoher Bekanntheitsgrad automatisch zu hohem Vertrauen in die Tierwohl-Labels führt. Tierwohlkennzeichnungen mit einem hohen Bekanntheitsgrad in der Bevölkerung können dennoch nur ein geringes Vertrauen besitzen.

Neben dem Bekanntheitsgrad hat schließlich das Image des Gütesiegels einen maßgeblichen Einfluss auf den Kauf der Produkte. Wird beispielsweise ein TierwohlLabel von NGOs aus dem Tierschutz- und Umweltbereich aufgrund zu schwacher Kriterien als eine nicht empfehlenswerte Kaufalternative bewertet bzw. als Greenwashing identifiziert, so kann in breiten Kreisen der Bevölkerung nicht das nötige Vertrauen in dieses Gütesiegel aufgebaut werden, unabhängig davon, wie stark eine derartige Tierwohlkennzeichnung von Staat, Herstellern und Händlern beworben wird. Unbekannteren Gütesiegeln kann dagegen von wenigen Personen, die dieses Gütesiegel gut kennen, ein hohes Vertrauen ausgesprochen werden.

Eine besondere Bedeutung kommt dem Einsatz bekannter Tierwohl-Labels vor dem Hintergrund der relativ unbekannten Marken für Fleisch- und Wurstwaren zu; insbesondere den vielfältigen Handelsmarken. Laut SPILLER und SCHULZE (2008) trägt die Bekanntheit von Marken eine große Bedeutung. Bei Marken für Fleisch- und Wurstwaren ist die Bekanntheit jedoch gering ausgeprägt.

Es gibt nur wenige Marken, die höhere Werte erzielen als die bekannten Tierwohl-Labels. Beispielhaft genannt seien hier WIESENHOF oder bestimmte Wurstmarken wie RÜGENWALDER oder REINERT. In der von SPILLER und SCHULZE (2008) durchgeführten Befragung konnten nur wenige hohe Bekanntheitsgrade für Fleischmarken ermittelt werden. Abgesehen von WIESENHOF weisen die meisten Fleischmarken nur einen geringen Bekanntheitsgrad auf. 
Die Namensähnlichkeit der überwiegenden Markenkonzepte für Handelsmarken von Fleisch- und Wurstwaren trägt ebenfalls zur Verbraucherverwirrung bei. Die ungestützte Markenbekanntheit ist entsprechend deutlich niedriger. WIESENHOF wurde von 27,5\% der Befragten genannt, BAUERNGLÜCK von 16,8 \%, GUTFLEISCH von 14,1\% und W. BRANDENBURG von nur $4,7 \%$.

Insgesamt lässt sich feststellen, dass vor allem Handelsmarken an Bedeutung gewinnen. Nach SPILLER und SCHULZE (2008) stellen die Bekanntheit des Herstellers sowie die Rückverfolgbarkeit der Fleisch- und Wurstwaren wesentliche Anliegen für Verbraucher in Deutschland dar. Der gezielte Einsatz von Tierwohl-Labels, die teilweise als sehr bekannte und glaubwürdige Marken wahrgenommen werden, könnte demzufolge der Substitution der zahlreichen sehr schwachen Fleischmarken dienen bzw. diese Marken ergänzen bzw. aufwerten.

Während in nur wenigen wissenschaftlichen Studien eine nähere Beschäftigung mit dem Bekanntheitsgrad einzelner Tierwohl-Labels erfolgte, wurden Untersuchungen hinsichtlich des Images einzelner Tierwohl-Labels noch seltener durchgeführt. Lediglich ZÜHLSDORF et al. (2016) befragten Verbraucher dazu, bei welchen Gütesiegeln ihrer Ansicht nach die Lebensmittel tiergerechter hergestellt worden sind und welche Labels für mehr Tierwohl stehen. Dabei schnitten DE-BIO, BIOLAND, DEMETER und EUBIO am besten ab. NEULAND und das Label des DEUTSCHEN TIERSCHUTZBUNDES wurden von einigen zumindest ein wenig mit ,mehr Tierwohl` assoziiert. Dies ist jedoch die einzige wissenschaftliche Studie in Deutschland, in der Verbrauchern die Möglichkeit gegeben wurde, verschiedene Labels im Hinblick auf Tierwohl zu bewerten.

Ungeklärt ist jedoch, wie die Verbraucher Unterschiede zwischen Tierwohl-Labels einschätzen. Die bedeutendsten Kriterien, die hinter den Tierwohl-Labels stehen, beziehen sich zudem nicht nur auf eine artgerechtere Tierhaltung, sondern ebenso auf Umweltfreundlichkeit, Gesundheitsaspekte und soziale Gerechtigkeit (BMEL, 2015). Diese Kriterien werden von SPILLER (2019) auch als die ,Big Four‘ einer nachhaltigen Ernährung bezeichnet. 
In der vorliegenden Studie soll eine Vielzahl an unterschiedlichen Tierwohl-Labels hinsichtlich ihrer Strenge in drei Kategorien der ,Big Four ${ }^{6}$ - Tierwohl, Umweltfreundlichkeit und Gesundheit - von Verbrauchern bewertet werden, um noch differenzierte Erkenntnisse hinsichtlich der Einschätzung unterschiedlicher TierwohlLabels zu erlangen (F14). Die soziale Verträglichkeit wurde dabei aus forschungsökonomischen Gründen nicht detaillierter untersucht. Aus älteren Studien wie dem ÖKOBAROMETER 2013 (BLE, 2013) geht hervor, dass die Sozialverträglichkeit den Verbrauchern deutlich weniger wichtig ist, als beispielsweise eine artgerechte oder umweltfreundliche Tierhaltung. Diese Erkenntnisse sind für die Anbieter von Fleischund Wurstwaren bedeutsam, da erstmals eine nach diesen drei Kriterien differenzierte Einschätzung der Verbraucher zu allen relevanten Tierwohl-Labels in Deutschland empirisch untersucht wird und Anbieter aus den Ergebnissen Rückschlüsse auf das Image der einzelnen Tierwohl-Labels ziehen können. Anbieter können diese Erkenntnisse in die Entscheidung einfließen lassen, welche Tierwohl-Labels sie für ihre Fleisch- und Wurstwaren auswählen.

Die Einschätzung der Strenge der Tierwohl-Labels spielt eine bedeutende Rolle für das Image und die Glaubwürdigkeit der Tierwohl-Labels. Nur wenn Tierwohl-Labels für deutliche Verbesserungen in der Nutztierhaltung stehen und eine entsprechende Glaubwürdigkeit haben, sind Verbraucher bereit, höhere Preise zu zahlen. Dennoch wurde bisher noch keine umfassende Studie zur Bewertung der verschiedenen TierwohlLabels durch die Verbraucher durchgeführt. Da Tierwohl-Labels verschiedenartige Kriterien zugrunde liegen, wird davon ausgegangen, dass es deutliche Unterschiede bei der Einschätzung verschiedener Tierwohl-Labels gibt. Unklar ist jedoch, wie die Einschätzung bei welchen Tierwohl-Labels differiert. Die Ergebnisse können Anbieter von Fleisch- und Wurstwaren nutzen, indem sie z. B. Tierwohl-Labels, die im Kriterium Gesundheit als streng eingeschätzt werden, für besonders gesundheitsorientierte Kundengruppen auswählen und diese beispielsweise mit entsprechenden Health Claims kombinieren. Die neuen Erkenntnisse bieten Herstellern und Händlern von Fleisch- und Wurstwaren zahlreiche Möglichkeiten, die Verbraucherwünsche beim Produkt- und Beratungsangebot von Tierwohlprodukten besser zu berücksichtigen. 
Zum Wissen der Verbraucher zu Tierwohlkennzeichnungen liegt ebenfalls nur eine sehr geringe Anzahl an Studien vor. Als Ausnahme kann hier die Studie von VECCHIO und ANNUNZIATA (2012) genannt werden, die das Wissen um gesetzliche Tierschutzstandards, Label-Systeme und landwirtschaftliche Tierhaltung bei Verbrauchern als eher gering einschätzen (VECCHIO \& ANNUNZIATA, 2012). Aufgrund der zahlreichen Medienberichte über die intensive Nutztierhaltung und der Bereitstellung von Informationen zum Thema Tierwohl durch NGOs, Tierwohl-LabelHerausgeber oder den Lebensmitteleinzelhandel könnte jedoch vermutet werden, dass die Verbraucher mittlerweile besser informiert sind, als dies zur Zeit der Veröffentlichung der Studie von VECCHIO und ANNUNZIATA (2012) der Fall war. Die sehr hohe Produktion und der hohe Konsum von Fleisch- und Wurstwaren in Deutschland haben positive Effekte für Hersteller- und Handelsunternehmen oder Landwirte, die mit der Fleischvermarktung ihr Einkommen erzielen. Daneben existieren positive Effekte für den Staat sowie für zahlreiche weitere Akteure wie Stallbauer, Forschungsinstitute, die Futtermittelbranche, Logistikdienstleister, Werbeagenturen, Banken, Versicherungen, öffentliche Verwaltungen oder Pharmaunternehmen. Zahlreiche Menschen erzielen direkt oder indirekt Einkommen in Zusammenhang mit der Fleischproduktion. Daneben ergeben sich durch den übermäßigen Verzehr bzw. die hohe Produktion von Fleisch- und Wurstwaren jedoch auch negative Effekte auf das Klima (globale Erwärmung), Wasser, Böden, Artenvielfalt, Luft, Gesundheit sowie die Verfügbarkeit und Qualität von Rohstoffen (UMWELTBUNDESAMT, 2019). Besonders häufig werden in diesem Zusammenhang die negativen Klimaeffekte betont, so z. B. vom Weltklimarat IPCC, der den hohen Fleischkonsum kritisch betrachtet und eine Umstellung auf eine überwiegend pflanzlich basierte Ernährung empfiehlt, um klimaschädliche Emissionen einzusparen.

Neben den hohen Umwelt- und Gesundheitskosten für den Staat und dem Leid der Tiere in der intensiven Nutztierhaltung existieren auch soziale Probleme wie beispielsweise die Zerstörung der Lebensgrundlage von Kleinbauern in Entwicklungsländern durch Fleischexporte aus Deutschland, die Zerstörung der Lebensräume bedrohter indigener Völker durch Futtermittelanbau oder auch prekäre Arbeitsverhältnisse von Mitarbeitern in Fleischfabriken. 
Der Wissensstand der Verbraucher soll in der vorliegenden Studie hinsichtlich der Auswirkungen eines übermäßigen Fleischkonsums bzw. einer übermäßigen Fleischproduktion näher untersucht werden, um die sich daraus ergebenden zentralen negativen Auswirkungen aus der Perspektive der Verbraucher zu evaluieren (F16). Daraus lassen sich die zentralen Problemfelder der intensiven Nutztierhaltung aus der Perspektive der Verbraucher aus einer anderen Perspektive ableiten, als dies beispielsweise über die Evaluation von Kaufkriterien möglich ist.

Für Anbieter von Fleisch- und Wurstwaren handelt es sich hierbei um eine wertvolle Information, weil sie sich so besser auf die aus Verbrauchersicht bedeutsamsten Problemfelder einstellen können und vorgenommene Verbesserungen in diesen Bereichen bei der Produktvermarktung gezielt über den Einsatz passender TierwohlLabels und einer entsprechenden Produktkommunikation demonstrieren können, um konkrete Kaufanreize für die in verschiedenen Bereichen besseren Produkte zu setzen.

Beispielhaft könnten bei Bio-Labels zusätzlich die gesundheitlichen Vorteile von BioFleisch (z. B. höherer Anteil an gesundheitsfördernden Omega-Fettsäuren) in der Kommunikation hervorgehoben werden.

In quantitativen Verbraucherbefragungen wurden zudem bisher kaum Fragen zum konkreten Kauf spezieller Tierwohlprodukte, die mit bestimmten Tierwohl-Labels ausgezeichnet sind, beantwortet. Es ist beispielsweise noch nicht bekannt, wie häufig zertifizierte Fleisch- und Wurstwaren gekauft werden (F12) und ob beispielsweise die Kaufhäufigkeit einzelner Tierwohl-Labels variiert.

Was die Berücksichtigung von Tierwohl-Labels beim Kauf von Fleisch- und Wurstwaren betrifft, sind verschiedene Forschungsfragen zum Label-Kauf noch nicht hinreichend beantwortet. In der vorliegenden Studie soll untersucht werden, welche Tierwohl-Labels bei Fleisch- und Wurstwaren von deutschen Verbrauchern bereits gekauft wurden (F11) und welche Tierwohl-Labels welche Verbraucher am ehesten kaufen würden (F15). Die Evaluierung der Tierwohl-Label-Präferenzen für die relevantesten Tierwohl-Labels in Deutschland bildet einen zentralen Schwerpunkt der Studie. 
Es wurde bisher noch nicht untersucht, wie sich die Verbraucherpräferenzen bei Tierwohl-Labels hinsichtlich verschiedener Einkaufsstätten (H10), der Angebotsformen Theke/SB (H11; H12) und der Reduktionsbereitschaft (H13) der Befragten beim Fleischkonsum unterscheiden. Dies ist für die Angebotsseite jedoch interessant und dient der Verbesserung der Produktentscheidungen. Existierende Unterschiede zwischen den Präferenzen der Tierwohl-Labels sind bei der Auswahl einer geeigneten Einkaufsstätte für die Hersteller von Fleisch- und Wurstwaren bzw. für die Händler bei der Zusammenstellung ihres Produktsortiments von Bedeutung, um das Produktangebot besser auf die Nachfrage nach speziellen Tierwohl-Labels abstimmen zu können.

Möchte beispielsweise ein EDEKA-Markt besonders anspruchsvolle Kundengruppen mit Tierwohlprodukten erreichen, die ausschließlich Fleisch- und Wurstwaren nach den Standards der deutschen ökologischen Anbauverbände kaufen, so ist ein Produktsortiment, das ausschließlich Fleisch- und Wurstwaren nach DE-BIO-Standard beinhaltet, nicht zielführend und könnte $\mathrm{zu}$ einer Abwanderung der anvisierten Kundengruppe zu anderen Händlern führen. Das gleiche Beispiel lässt sich auch auf die Angebotsformen übertragen. Kauft beispielsweise ein ,BIOLAND-Fan“ Fleisch- und Wurstwaren ausschließlich an der Frischetheke, so wird er nur eine Einkaufsstätte nutzen, die eine Frischetheke mit BIOLAND-Produkten anbietet. Sowohl Thekenkäufern als auch SB-Käufern sollten in der Einkaufsstätte demnach die passenden Standards angeboten werden, um wirklich zufriedene Kunden zu haben und eine Abwanderung zu Wettbewerbern zu vermeiden.

Ebenfalls wurde spezifisch für die ausgewählten Tierwohl-Labels noch nicht untersucht, ob sich die Label-Präferenzen hinsichtlich der Soziodemografie der Befragten unterscheiden (H14). Hier werden jedoch signifikante Unterschiede vermutet und diese Informationen sind von großer Bedeutung für Anbieter von Fleisch- und Wurstwaren, die sich für ihr gesamtes Produktsortiment für unterschiedliche Tierwohl-Labels entscheiden müssen. Basierend auf den in der vorliegenden Studie ermittelten Präferenzen können die Anbieter ihr Produktangebot und die Produktkommunikation für bestimmte TierwohlLabel-Produkte besser auf ausgewählte soziodemografische Zielgruppen ausrichten. 
Beispielsweise soll ermittelt werden, ob einkommensstarke und damit kaufkräftige Kundengruppen andere Tierwohl-Labels bevorzugen als einkommensschwächere Personen. Ebenso werden Unterschiede bei den Tierwohl-Label-Präferenzen bzgl. des Geschlechts und des Alters, der Region, der Ortsgröße und der Bildung erwartet, die im Rahmen der Studie analysiert werden.

Eine weitere neue Forschungsfrage ist vor dem Hintergrund des im Lebensmitteleinzelhandel seit vielen Jahren $\mathrm{zu}$ beobachtenden Trends des ,MultiLabelings ' entstanden, wozu in der wissenschaftlichen Literatur zu Fleisch- und Wurstwaren jedoch bis heute noch keine expliziten Untersuchungen durchgeführt wurden (F17). Theoretisch bestehen Schnittstellen zu den bereits vorgestellten Studien zum Bekanntheitsgrad oder Vertrauen in verschiedene allgemeine Gütesiegel sowie zum Vertrauen in verschiedene Organisationen. In der vorliegenden Studie sollen jedoch nicht nur Labels bzw. Logos von Organisationen abgefragt werden, sondern zahlreiche unterschiedliche Labels, wobei ebenfalls beurteilt werden soll, ob sich diese womöglich als zusätzliche Gütesiegel zu den Tierwohl-Labels eignen könnten.

Weiterhin finden sich theoretische Anknüpfungspunkte bei der wissenschaftlichen Diskussion zum Thema ,Labelflut' und ,Information Overload“ (BMEL, 2012; MALHOTRA, 1984; SPILLER, 2013b), die bisher jedoch nur auf allgemeiner Ebene quantifiziert wurden und keinen Produktbezug herstellen, sondern lediglich erfragen, ob die zahlreichen Gütesiegel für Verbraucher eher verwirrend als hilfreich sind. Ob der Einsatz mehrerer Label jedoch einen positiven oder doch eher einen negativen Effekt auf die Kaufentscheidung der Verbraucher bei Fleisch- und Wurstwaren hat, ist bisher noch nicht detaillierter wissenschaftlich untersucht worden. Für die Angebotsseite kann dieses Ergebnis eine Hilfestellung geben, ob der Einsatz eines Tierwohl-Labels zum einen mit zusätzlichen anderen Gütesiegeln ergänzt werden sollte, und zum anderen zeigen, welche der untersuchten Gütesiegel sich für einen derartigen Einsatz eignen. Des Weiteren sollen gänzlich neue zusätzliche Label zu Tierwohl-Labels getestet werden, über deren Einführung bzw. Entwicklung bei positiven Bewertungen seitens der Verbraucher diskutiert werden kann. 


\subsection{Hinderungsgründe für den Kauf}

Aus der geringen Marktdurchdringung von Tierwohlprodukten in Deutschland im Vergleich zu konventionellen Produkten lässt sich auf verschiedenste Hinderungsgründe bzw. Kaufbarrieren für Tierwohlprodukte schließen. Insbesondere die reinen TierwohlLabels, die keinen ökologischen Standards folgen, werden von den meisten Verbrauchern nicht gekauft (SPILLER et al., 2010; WEINRICH et al., 2015). Für dieses Phänomen werden verschiedene Gründe diskutiert.

Häufig wird in diesem Kontext auf die Einstellung-Verhalten-Lücke (AttitudeBehaviour-Gap) verwiesen. Die weit verbreitete Aufgeschlossenheit gegenüber Tierwohlprodukten ist nicht in gleichem Maße an den Verkaufszahlen abzulesen. Daraus könnte geschlussfolgert werden, dass sich viele Verbraucher trotz hoher Tierwohlpräferenzen beim alltäglichen Einkauf von Fleisch- und Wurstwaren doch häufiger für konventionelle Produkte entscheiden (PIRSICH, 2017). Hierfür kommen verschiedenste Gründe in Frage. Zum einen ist anzuführen, dass es in den Verbraucherstudien einen Effekt der sozialen Erwünschtheit gibt (VERMEIR \& VERBEKE, 2006). Zum anderen könnten sich auch die Auswahlvarianten in Verbraucherstudien vom Angebot der genutzten Einkaufsstätte der Verbraucher unterscheiden. Des Weiteren werden aus forschungsökonomischen Gründen in wissenschaftlichen Studien häufig zahlreiche weitere Einflussfaktoren auf die Kaufentscheidung vernachlässigt, da nicht alle Einflussfaktoren untersucht werden können. Daneben wird auch der höhere Preis von Tierwohlprodukten im Vergleich zu konventionellen Produkten als zentrale Kaufbarriere diskutiert, der die Mehrzahlungsbereitschaft mancher Verbraucher übersteigt (LAGERKVIST \& HESS, 2011; NOCELLA et al., 2010; PADEL \& FOSTER, 2005; SCHRÖDER \& MCEACHERN, 2004). Wie bereits beschrieben hängt jedoch die Mehrzahlungsbereitschaft von verschiedenen Faktoren ab, wie etwa der Bekanntheit und Glaubwürdigkeit der eingesetzten Tierwohl-Labels, dem Haushaltseinkommen oder auch der realen Verfügbarkeit in den genutzten Einkaufsstätten bzw. der Bereitschaft, weitere Wege in Kauf zu nehmen, um die gewünschten Tierwohlprodukte zu kaufen. 
Der sogenannte Information Overload wird oft als ein weiterer Hinderungsgrund für den Kauf von Tierwohlprodukten diskutiert (PIRSICH, 2017). $\mathrm{Zu}$ viele Labels im Lebensmitteleinzelhandel würden Verbraucher verunsichern und überfordern (FRANZ et al., 2012; KROEBER-RIEL \& ESCH, 2004; MALHOTRA, 1984; VAN KLEEF et al., 2007).

Neben diesen eher allgemeinen Vermutungen zu Kaufbarrieren für Tierwohlprodukte stellten WEINRICH et al. (2015) sowie AMINE und CADENAT (2003) fest, dass womöglich auch das Angebot von speziellen Tierwohlprodukten in den richtigen Einkaufsstätten für die richtigen Zielgruppen entscheidend für den Vermarktungserfolg der Tierwohlprodukte sein könnte.

Daneben wird auch die Kundenzufriedenheit mit dem Beratungs- und Produktangebot von Fleisch- und Wurstwaren aus einer artgerechteren und nachhaltigeren Nutztierhaltung evaluiert (F19) und weiterführend analysiert, inwiefern sich die Kundenzufriedenheit in diesem Punkt bei den verschiedenen Lebensmitteleinzelhändlern unterscheidet (H15). Für die Kundenzufriedenheit spielt auch die Auffindbarkeit der Produkte im Lebensmitteleinzelhandel eine große Rolle, da die meisten Kunden die gewünschte Ware schnell finden möchten.

Daher werden den Verbrauchern in der vorliegenden Arbeit zwei verschiedene CategoryManagement-Varianten (F20) zur Auswahl gestellt, wie Tierwohlprodukte in den Regalen des LEH angeboten werden sollten, damit sie möglichst schnell gefunden werden (HÜBNER \& KUHN, 2012): zum einen die separate Platzierung von Tierwohlprodukten gemeinsam in einem Regal und zum anderen die Platzierung nach Sorten (z. B. konventionelle und ökologische Salami nebeneinander).

Zur Validierung der Forschungsergebnisse werden diese Unterschiede bei den Präferenzen zur Produktplatzierung hinsichtlich der verschiedenen Lebensmitteleinzelhändler analysiert, um bei signifikanten Unterschieden entsprechend differenzierte Handlungsempfehlungen aussprechen zu können (H17). 
Um eine bessere Einschätzung der Bedeutung eines aus Kundenperspektive guten Beratungs- und Produktangebots von Fleisch- und Wurstwaren aus einer artgerechteren und nachhaltigeren Nutztierhaltung vornehmen zu können, wird letztlich auch nach der Wechselbereitschaft potenziell unzufriedener Kunden gefragt (F21), um die Wichtigkeit eines guten Beratungs- und Produktangebots an Tierwohlprodukten besser beurteilen zu können und gleichzeitig potenziell unterschiedlich treue bzw. wechselbereite Kundengruppen im Lebensmitteleinzelhandel zu identifizieren. Die Wechselbereitschaft wird je nach Einkaufsstätte analysiert, da daraus geschlossen werden kann, dass neben allen anderen Händlern besonders die Lebensmitteleinzelhändler, deren Kunden eine hohe Wechselbereitschaft aufweisen, auf ein adäquates Tierwohlsortiment achten sollten. Bezüglich der Wechselbereitschaft werden signifikante Unterschiede zwischen den einzelnen Lebensmitteeinzelhändlern vermutet (H18).

Des Weiteren könnte auch die falsch gewählte Angebotsform (Theke/SB) eine Kaufbarriere darstellen (HÜBNER \& KUHN, 2012; WEINRICH et al., 2015). So konnten SCHULZE und SPILLER (2008) belegen, dass z. B. das Preisbewusstsein und das Beratungsbedürfnis der Verbraucher einen signifikanten Einfluss auf die Wahl der Angebotsform haben. SB-Käufer legen laut WEINRICH et al. (2015) weniger Wert auf Qualität und mehr Wert auf niedrige Preise als Thekenkäufer, weshalb die Autoren das Angebot von Tierwohlprodukten an der Fleischtheke empfehlen.

Zahlreiche weitere Gründe können eine Kaufbarriere darstellen. Umfassend erforscht sind diese Gründe noch nicht. Eine große Auswahl verschiedener Kaufbarrieren bei Tierwohlprodukten wie beispielsweise die Erkennbarkeit bzw. die Sichtbarkeit der entsprechenden Tierwohlprodukte im Handel oder in der Werbung wird daher in der vorliegenden Studie untersucht. Diese Kaufbarrieren sind bedeutsame Gründe für die oftmals noch niedrige Marktdurchdringung von Tierwohlprodukten. Jedoch wurden sie bisher nur in einigen Praxisstudien wie etwa dem ÖKOBAROMETER (BLE, 2013) und nur für Bio-Produkte erhoben, ohne jedoch weiterführende Analysen durchzuführen oder aus den Ergebnissen konkrete Lösungsansätze $\mathrm{zu}$ entwickeln oder gar Handlungsempfehlungen auszusprechen. 
Daher sollen in der vorliegenden Studie die Kaufbarrieren je Einkaufsstätte analysiert werden, um hier erstmalig auf der Ebene des Lebensmitteleinzelhandels die wesentlichen Kaufbarrieren darzustellen und die Unterschiede bzgl. der Kaufbarrieren je Einkaufsstätte bzw. Betriebsform sichtbar zu machen und zu analysieren, um gezielte Lösungsansätze bzw. Handlungsempfehlungen für die Angebotsseite je Haupteinkaufsstätte bzw. Betriebsform $\mathrm{zu}$ diskutieren. Bezüglich der verschiedenen Kaufbarrieren werden aufgrund des stark unterschiedlichen Sortiments signifikante Unterschiede zwischen den verschiedenen Lebensmitteleinzelhändlern vermutet (H16). Die zukünftige Beseitigung dieser derzeit noch zahlreich bestehenden Kaufbarrieren kann maßgeblich dazu beitragen, den Anteil an zertifizierten Fleisch- und Wurstwaren aus einer artgerechteren und nachhaltigeren Nutztierhaltung weiter $\mathrm{zu}$ erhöhen, um die Nachfrage nach Tierwohlprodukten weiter zu steigern und noch besser zu decken.

\subsection{Informationsbedarf bei Tierwohlprodukten}

Laut ERNÄHRUNGSREPORT 2018 legen die Verbraucher großen Wert auf umfassende Informationen bei Lebensmitteln. $79 \%$ der Befragten finden Angaben $\mathrm{zu}$ darin enthaltenen Inhalts- und Zusatzstoffen, zur geografischen Herkunft der Waren sowie etwaige Warnhinweise wichtig oder sehr wichtig (BMEL, 2018). Auch das Mindesthaltbarkeitsdatum zählt zu den klassischen Informationen, die für die meisten Menschen (73\%) beim Einkauf relevant sind. Hinweise auf Stoffe, die möglicherweise Allergien auslösen können, haben heute einen noch höheren Stellenwert als vor einigen Jahren. Mittlerweile sind diese Informationen für zwei Drittel der Befragten (67\%) wichtig. Vor einigen Jahren empfand nur rd. die Hälfte der Bevölkerung diese Angaben als bedeutsam (BMEL, 2018). Viele deutsche Verbraucher sind jedoch aus verschiedenen Gründen beim Kauf von Lebensmitteln verunsichert. SPILLER und NITZKO (2014) geben in der Studie „Verbraucherverständnis von Transparenz“ neben den zahlreichen Lebensmittelskandalen auch die große Distanz zwischen realer Lebensmittelproduktion und Konsumenten, komplexe Produktionstechnologien und die hohe Kennzeichnungsvielfalt als Gründe an. 
Die Vielfalt der unterschiedlichen Auswahlmöglichkeiten an Produkten, Gütesiegeln sowie die vielen Werbebotschaften können demnach zur Verwirrung der Verbraucher (Consumer Confusion) führen. Aus der Verunsicherung resultiert bei den meisten Verbrauchern (rd. zwei Drittel) die Forderung nach mehr Informationen, um bessere Kaufentscheidungen treffen zu können. Vor allem interessieren sich die Verbraucher laut SPILLER und NITZKO (2014) für die Herkunft der Produkte, die Zusammensetzung und Inhaltstoffe sowie die Herstellungsprozesse (TNS EMNID, 2014). Zusätzliche weiterführende Informationen zum Beispiel zu den Haltungsbedingungen der Nutztiere fungieren laut SPILLER und NITZKO (2014) als Vertrauenssignal für die Verbraucher und erfüllen somit einen indirekten entscheidungsbezogenen Nutzen (SPILLER \& NITZKO, 2014; TNS EMNID, 2014).

Eine zentrale Rolle spielt dabei die Produktverpackung. $76 \%$ der Befragten gaben bei einer Befragung von TNS EMNID an, dass ihnen Informationen auf der Verpackung wichtig seien, und über $90 \%$ forderten, dass Landwirte Tierwohlaspekte besonders beachten sollten (TNS EMNID, 2014). Zahlreiche Menschen wünschen sich darüber hinaus weitere Informationen. Vor allem bei tierischen Produkten ist das Interesse an zusätzlichen Informationen groß. Besonders erwünscht sind laut TNS EMNID (2014) Informationen zu den Haltungsbedingungen (85 \%) und dazu, ob ein Produkt fair (84\%) sowie umweltverträglich ( $82 \%$ ) erzeugt wurde und ob es gentechnikfrei ist ( $81 \%)$.

Der Informationsbedarf der deutschen Verbraucher variiert jedoch stark zwischen verschiedenen Lebensmitteln. SPILLER und NITZKO (2014) fragten Verbraucher wie wichtig ihnen Informationen über Lebensmittel sind. Aus Abbildung 4 wird ersichtlich, dass Verbrauchern die Transparenz bei Getränken wie Mineralwasser, Limonade oder alkoholischen Getränken weniger wichtig ist als bei Kaffee, Tee oder Kakao. Ebenso ist vielen Verbrauchern die Bereitstellung zusätzlicher Informationen bei Reis, Nudeln oder Müsli weniger wichtig als für Obst oder Gemüse. Am wichtigsten ist den deutschen Verbrauchern die Bereitstellung von Informationen bei tierischen Produkten, wobei neben Milchprodukten, Eiern oder Fisch die meisten Verbraucher Informationen über Fleisch wünschen (SPILLER \& NITZKO, 2014). 


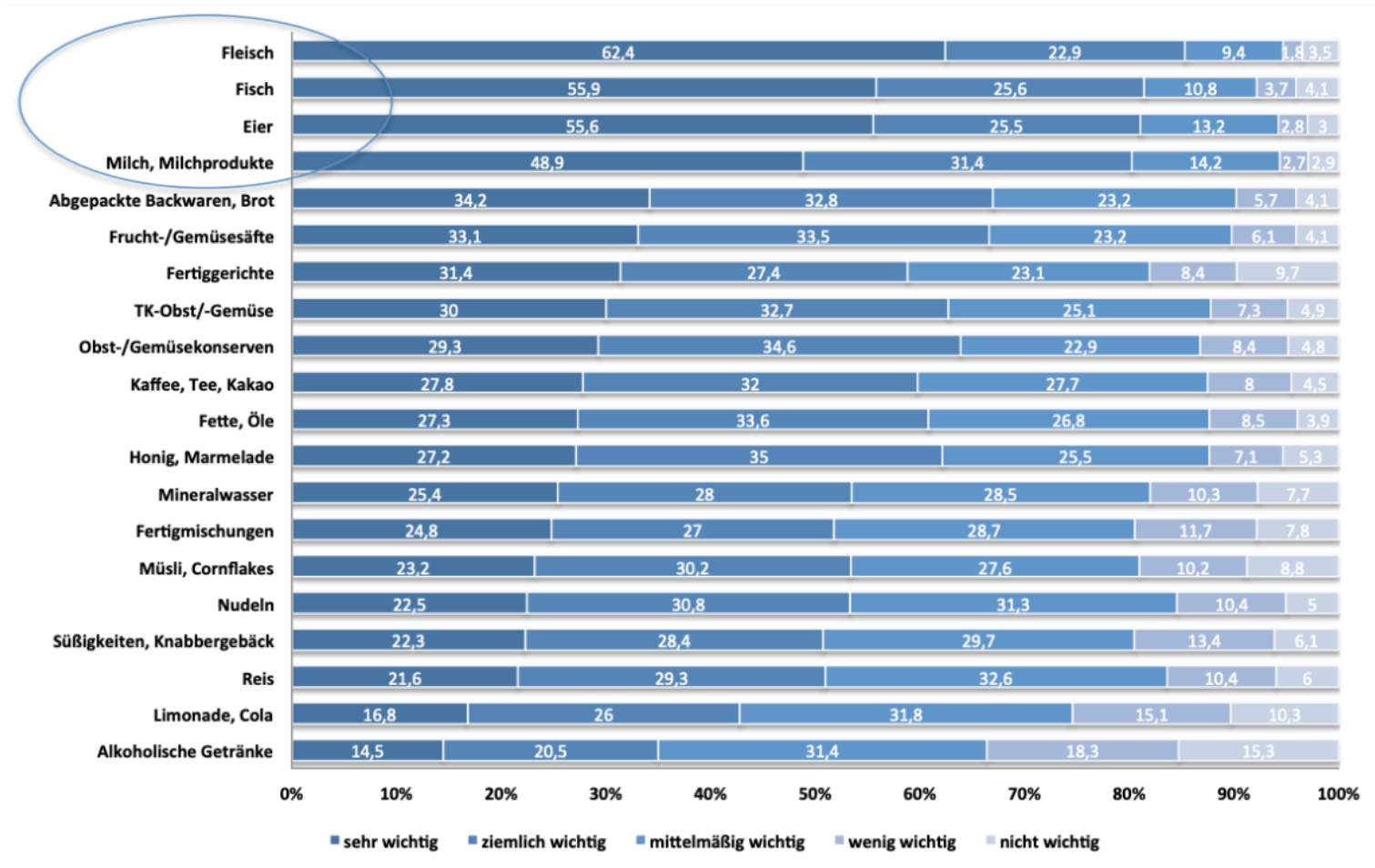

\section{Abbildung 4: Informationsbedarf bei unterschiedlichen Lebensmitteln} (Quelle: SPILLER \& NITZKO, 2014)

Nur rd. $7 \%$ der Verbraucher wünschen sich nicht mehr Informationen über Lebensmittel, während über die Hälfte aller Verbraucher fordert, dass mehr Informationen über Lebensmittel zur Verfügung gestellt werden. Allerdings variieren die Bedürfnisse nach Informationen in verschiedenen Verbrauchergruppen (SPILLER \& NITZKO, 2014). Neben den gesetzlich verpflichtenden Angaben wie dem Mindesthaltbarkeitsdatum oder der Herkunft haben die Befragten des ERNÄHRUNGSREPORTS 2019 ein hohes Interesse an weiteren Informationen, die beispielsweise auf Verpackungen zu finden sind.

$86 \%$ der Verbraucher möchten wissen, wie bei Produkten tierischen Ursprungs die Tiere gehalten wurden. Informationen über die Haltungsbedingungen stehen an erster Stelle der gewünschten freiwilligen Informationen. Neben dem Tierwohl sind den Befragten zwei weitere freiwillige Informationen wichtig: zum einen, ob die Lebensmittel umweltfreundlich produziert wurden, und zum anderen, ob sie unter fairen sozialen Bedingungen produziert wurden (BMEL, 2019a). 
Beim Kauf von Lebensmitteln informieren sich laut ERNÄHRUNGSREPORT 2018 des BMEL mehr als zwei Drittel der Menschen in Deutschland am Ort des Einkaufs über die Lebensmittel. Daneben informieren sich fast ebenso viele Menschen im Internet über Onlinerecherchen, Webforen und soziale Medien. Werbung, Infobroschüren und Verbraucherzentralen sind ebenfalls bedeutsame Informationsquellen. Dabei nutzt heute fast ein Drittel der jungen Menschen (19 bis 29 Jahre alt) die sozialen Medien, um sich über Lebensmittel zu informieren. Bei den älteren Menschen (über 60 Jahre alt) waren es im Jahr 2018 im Vergleich nur 3 \% (BMEL, 2018).

Wie in einer Studie von TNS EMNID (2014) wurde auch im Rahmen der Studie von SPILLER und NITZKO (2014) herausgefunden, dass die Produktverpackung einer der bedeutendsten ,Orte‘ ist, an denen sich Verbraucher über Produkte informieren. Mehr als zwei Drittel der Befragten bestätigten in der Studie „Verbraucherverständnis von Transparenz“, Informationen auf der Verpackung vor dem erstmaligen Kauf eines Produkts in der Einkaufsstätte zu lesen. Ein weiteres Drittel der Befragten gab an, sich die Informationen auf der Verpackung zuhause durchzulesen. Bei Lebensmittelskandalen erhöht sich der Studie zufolge der Informationsbedarf der Verbraucher signifikant. Da in den letzten Jahren in regelmäßigen Abständen Lebensmittelskandale auftraten, ist davon auszugehen, dass der ohnehin schon hohe Informationsbedarf bei tierischen Produkten in diesen Zeiten noch höher ist (SPILLER \& NITZKO, 2014).

Laut ERNÄHRUNGSREPORT 2019 möchten die Menschen immer genauer wissen, was auf ihren Tellern liegt. Das persönliche Gespräch mit Freunden oder Familie stellt diesbezüglich die hauptsächliche Informationsquelle für die meisten Befragten dar. Auf Produktverpackungen oder in der Werbung informieren sich $70 \%$ der Befragten anhand der Herstellerangaben. Knapp die Hälfte der Befragten greift auf Informationsangebote des Staates oder von unabhängigen Organisationen zurück (BMEL, 2019a). Vor allem digitale Angebote gewinnen bei der Information über Lebensmittel an Bedeutung. Mittlerweile informieren sich bundesweit über alle befragten Altersklassen $60 \% \mathrm{im}$ Internet über Lebensmittel. Die Online-Affinität ist in der Generation der 14- bis 44Jährigen (rd. $70 \%$ ) und bei Eltern am höchsten (BMEL, 2019a). 
Ein wesentlicher Faktor für die Glaubwürdigkeit von Informationen ist der Anbieter bzw. Absender der Information. So haben SPILLER und NITZKO (2014) festgestellt, dass die meisten Verbraucher am ehesten Informationen von Verbraucherzentralen, Familie und Freunden sowie ,unabhängigen“ Testinstitutionen vertrauen. Eher mittelmäßiges Vertrauen genießen Ernährungsberater, Wissenschaftler, Umwelt- und Tierschutzorganisationen sowie Landwirte. Am schlechtesten schneiden der Studie zufolge die Bundesregierung, Medien und Lebensmittelindustrie/-hersteller ab, wenn es um das Vertrauen deutscher Verbraucher geht.

Laut der europäischen Studie „Generation What“, die in Deutschland vom Sinus Institut betreut wird, haben insbesondere jüngere Menschen zwischen 16 und 34 ein sehr geringes Vertrauen in Politik oder Medien, das nur noch vom geringen Vertrauen in religiöse Institutionen übertroffen wird (GENERATION WHAT, 2019). Tierwohl ist im ökonomischen Sinne jedoch eine ausgewiesene Vertrauenseigenschaft, d. h. Verbraucher können anhand des Endprodukts nicht beurteilen, wie das Tier gehalten wurde (AKERLOF, 1970). Daher kommt dem Vertrauen in den Absender der Tierwohlinformationen eine zentrale Bedeutung zu.

Des Weiteren verhindert nach ZÜHLSDORF et al. (2016) ein Mangel an entscheidenden Produktinformationen die Markterschließung. Insbesondere bei der Einschätzung von Tierwohlprodukten besteht eine hohe Unsicherheit. Der Großteil der Verbraucher (rd. $65 \%$ ) gab in der Umfrage an, mehr Informationen über die Nutztierhaltung erhalten zu wollen. Von besonderer Bedeutung ist dahingehend die Glaubwürdigkeit.

Nur wenn sich die Verbraucher sicher sind, dass die höheren Preise tatsächlich den Tieren zugutekommen, sind sie auch bereit, mehr Geld für Fleisch auszugeben. Dafür sind jedoch Informationen notwendig, anhand derer die Verbraucher die Verbesserungen in der Haltung nachvollziehen können. Eine Mehrzahlungsbereitschaft ist der Studie zufolge nur gegeben, wenn es sich um bekannte und glaubwürdige Tierwohl-Labels handelt und zusätzliche Informationen bereitgestellt werden. Fast $50 \%$ der Verbraucher gaben jedoch $\mathrm{zu}$, nicht zu wissen, woran Fleisch aus artgerechterer Tierhaltung wirklich erkannt werden könne (ZÜHLSDORF et al., 2016). 
Zusammenfassend lässt sich sagen, dass bereits einige bedeutsame Erkenntnisse zur Informationsbereitstellung in den hier vorgestellten Praxisstudien und vereinzelten wissenschaftlichen Studien gewonnen werden konnten. Nicht detailliert genug untersucht wurde gleichwohl die Bereitstellung von Informationen zum Tierwohl bei Fleisch- und Wurstwaren, die einen unmittelbaren Nutzen für konkrete Herausforderungen bei der Implementierung der Maßnahmen in der Praxis stiften können.

Daher werden in der vorliegenden Arbeit explizit verschiedene Fragen gestellt, die bisher noch nicht differenziert genug untersucht wurden, zum allgemeinen Interesse an Informationen zu den Haltungsbedingungen und der Herkunft der Tiere (F22), zum präferierten Ort der Informationsbereitstellung bzw. zum Informationsmedium (F23) und zum Absender von Tierwohlinformationen bei der Bereitstellung von Informationen im Internet (F24). Diese Fragen werden in der vorliegenden Arbeit nicht nur mit Blick auf soziodemografische Unterschiede analysiert, sondern weiterführend auch hinsichtlich psychographischer Unterschiede verschiedener Zielgruppen.

Ergänzend wird eine Frage zum Detaillierungsgrad der Herkunft der Fleisch- und Wurstwaren gestellt (F25). In den bereits vorgestellten Studien wurde die Bedeutung der Herkunft bisher lediglich im Rahmen sehr allgemeiner Fragen zu den Kaufkriterien in Praxisstudien und häufig nur bei allgemeinen Lebensmitteln abgefragt. Es wird jedoch vermutet, dass die Art und Weise der Herkunftsangaben insbesondere bei Tierwohlprodukten einen großen Unterschied für die Verbraucher machen kann, was das Vertrauen der Verbraucher in die Tierwohlkennzeichnungen auf Produktverpackungen betrifft. So wird davon ausgegangen, dass das Vertrauen der Verbraucher in Tierwohlprodukte steigt, je detaillierter die Herkunftsangaben sind bzw. je höher die Transparenz ist.

Daraus abgeleitet sollen die Verbraucher in der vorliegenden Studie erstmals die Möglichkeit erhalten, ihre persönlichen Präferenzen zum Detaillierungsgrad der Herkunftsangaben anzugeben. Da sich ein Großteil der Verbraucher im Hinblick auf die Herkunftsangaben genauere Angaben wünscht als nur Herkunftsländer (z. B. Irland), sollte in Forschungsstudien oder Kaufexperimenten eruiert werden, ob die Angabe des konkreten Landwirts für Verbraucher ein Kaufargument darstellt. 
Zudem soll diese Frage dabei helfen, das Verbraucherverständnis von Regionalität, neben dem Tierwohl einer der größten Trends bei der Fleischvermarktung, in verschiedenen geographischen Ebenen differenzierter zu untersuchen und somit besser zu verstehen. Sollten sich hier klare Präferenzen seitens der Verbraucher offenbaren, können Konzepte entwickelt werden, die sich besser an den Verbraucherwünschen orientieren.

Denkbar wären beispielsweise das Angebot von Fleisch- und Wurstwaren aus einer artgerechteren und nachhaltigeren Nutztierhaltung mit konkreter Hofangabe für den Direktvertrieb vom Hof respektive über eine Website oder auch die Vermarktung besonderer Produkte (z. B. Bratwürste vom Bentheimer Landschwein in der Grillsaison), die regelmäßig in der Theke eines lokalen EDEKA-Supermarkts unter Angabe des Familiennamens des Landwirts aus der näheren Umgebung des Supermarkts angeboten und entsprechend am Point of Sale beworben werden, z. B. kombiniert mit einem Hoffest.

Die Untersuchung des Informationsverhaltens der Verbraucher (F22 bis F25) dient der Verbesserung kommunikationspolitischer Entscheidungen bei der Vermarktung von Tierwohlprodukten. Zusätzlich soll durch weiterführende Analysen ein Erkenntnisgewinn generiert werden. Zunächst wurde in der vorgestellten Literatur noch nicht untersucht, ob sich die Präferenzen zur Informationsbereitstellung bei verschiedenen Lebensmitteleinzelhändlern nach den vier bedeutsamsten Betriebsformen (Supermarkt, Discounter, Verbrauchermarkt und Metzger) unterscheiden. Es wird davon ausgegangen, dass in diesem Punkt signifikante Unterschiede zwischen den Betriebsformen existieren (H19).

Aus den Ergebnissen dieser Analyse können gezielte Handlungsempfehlungen je Betriebsform abgeleitet werden, wie beispielweise, dass sich Supermarkt-Käufer über die Haltungsbedingungen der Nutztiere eher in den kostenlosen Werbeprospekten informieren möchten, während Discounter-Käufer die Information auf einem Internetportal bevorzugen. Zudem wird vermutet, dass es beim Einkauf von Aufschnitt und Wurst als Brotbelag sowie Fleisch zum Braten und Grillen deutliche Unterschiede im Hinblick auf das Informationsverhalten von Thekenkäufern oder SB-Käufern gibt (H20; H21). 
Daher sollen diesbezüglich konkrete Analysen durchgeführt werden. Anbieter von Fleisch- und Wurstwaren können mit Hilfe dieser Informationen noch besser auf die Kundenwünsche eingehen und ihren Kunden entsprechende Tierwohlinformationen an den geeignetsten Stellen zur Verfügung stellen.

\subsection{Politische Präferenzen und politische Partizipation}

In der politischen Debatte wird intensiv und kontrovers über die sukzessive Umgestaltung hin zu einer artgerechteren und umweltfreundlicheren Nutztierhaltung diskutiert. Dabei werden auch verschiedene soziale Herausforderungen wie beispielsweise sinkende Einkommen bei kleinen landwirtschaftlichen Betrieben oder prekäre Arbeitsverhältnisse in Schlachthöfen berücksichtigt. Der Wissenschaftliche Beirat für Agrarpolitik stellt jedoch vor allem die Defizite im Bereich Tierschutz und im Umweltschutz als die beiden zentralen Herausforderungen in den Mittelpunkt. Spürbare Verbesserungsmaßnahmen würden laut WBAE Mehrkosten für Tierhalter von etwa 13 bis $23 \%$ bedeuten und die Verbraucherpreise für Fleisch- und Wurstwaren um 3 bis $6 \%$ ansteigen lassen (BMEL, 2015).

Zahlreiche internationale Organisationen und Thinktanks haben Ziele und Strategien für eine nachhaltigere Wirtschaftsweise und einen nachhaltigeren Konsum formuliert, die von den einzelnen Nationalstaaten umgesetzt werden sollen. Beispielhaft genannt seien hier die global empfohlenen Sustainable Development Goals (SDGs) der Vereinten Nationen (UN, 2020) oder die Empfehlungen zu einer nachhaltigeren Ernährung der LANCET-Kommission (THE LANCET, 2019).

Das übergreifende Ziel besteht in der Reduktion der Produktion und des Konsums von Fleisch- und Wurstwaren sowie im Wandel hin $\mathrm{zu}$ einer artgerechteren und nachhaltigeren Nutztierhaltung, um negative Auswirkungen auf Tiere und Umwelt (z. B. durch klimaschädliche Emissionen oder den Verlust der Artenvielfalt) zu minimieren. Zur Erreichung dieser Reduktionsziele stehen unterschiedliche wirtschaftspolitische Instrumente zur Verfügung (WBAE, 2020). 
In der GREENPEACE-Studie „Tierwohl fördern, Klima schützen“ werden allgemeine Lösungsansätze sowie verschiedene politische Instrumente für mehr Tierwohl vorgestellt. Im Fokus stehen dabei die drei Problemfelder Umwelt, Gesundheit und Tierwohl. Neben einer Stickstoffüberschussabgabe und einer Mineraldüngemittelsteuer wird auch die Erhöhung der Mehrwertsteuer bei Fleischerzeugnissen von aktuell $7 \%$ auf den Normalsteuersatz von $19 \%$ empfohlen. Daneben sollen eine emissionsabhängige Steuer auf Fleischerzeugnisse sowie eine Tierwohlabgabe eingeführt werden. Auch BUSCH und SPILLER (2020) empfehlen eine Tierwohlabgabe auf Fleisch von ca. 40 Cent pro Kilogramm zur Förderung des Tierwohls, um mit den Mehreinnahmen den mittelfristigen Umbau hin zu einer artgerechteren und klimafreundlicheren Tierhaltung zu finanzieren (BUSCH \& SPILLER, 2020). SPILLER (2018) hat im Fleischatlas 2018 der HeinrichBöll-Stiftung die möglichen staatlichen Eingriffe zur Senkung des zu hohen Fleischverbrauchs schematisch dargestellt (Abbildung 5).

\section{ERNÄHRUNGSPOLITISCHE LEITER}

Stufen möglicher staatlicher Eingriffe zur Senkung des Fleischverbrauchs, Schema

\begin{tabular}{|c|c|c|}
\hline $\mathrm{MaB}$ & Mittel & Beispiele \\
\hline \multirow{3}{*}{$\begin{array}{l}\text { beschränkte } \\
\text { Entscheidung }\end{array}$} & Verbote & Werbung für XXL-Portionen untersagen \\
\hline & & \\
\hline & neue Standards & Fleischanteil in Mensen und Kantinen begrenzen \\
\hline \multirow{3}{*}{$\begin{array}{c}\text { gelenkte } \\
\text { Entscheidung }\end{array}$} & negative Anreize & teureres Fleisch, um Konsum zu senken \\
\hline & positive Anreize & billigeres Obst und Gemüse, um Konsum zu steigern \\
\hline & indirekte Anreize & Lockmittel zur gesünderen Ernährung („Nudging“) \\
\hline \multirow{3}{*}{$\begin{array}{l}\text { unterstützte } \\
\text { Entscheidung }\end{array}$} & erleichterte Auswahl & Informationen beim Einkauf, Apps, Label \\
\hline & informierte Auswahl & bessere Verbraucherbildung und -aufklärung \\
\hline & unregulierte Auswahl & Monitoring des Fleischkonsums \\
\hline
\end{tabular}

\section{Abbildung 5: Ernährungspolitische Leiter \\ (Quelle: SPILLER et al., 2018)}


Da eine Untersuchung aller dargestellten Instrumente den Rahmen der vorliegenden Studie jedoch überschreiten würde, werden Ansätze wie das ,Nudging (das ,Stupsen“ der Verbraucher hin $\mathrm{zu}$ einer nachhaltigeren Ernährung beispielsweise durch Umweltbildungsmaßnahmen) nicht näher untersucht. Ebenso wird eine Tierschutzsteuer, die von BUSCH und SPILLER (2020) vorgeschlagen wurde, in der vorliegenden Arbeit nicht weiter berücksichtigt.

Der Fokus der vorliegenden Studie richtet sich auf fünf Politikoptionen, die aus Verbrauchersicht erstmals vergleichend bewertet werden sollen. Neben Gesetzesänderungen werden gesetzlich verpflichtende Haltungskennzeichnungen, freiwillige Gütesiegel, Subventionen und die Unterstützung privatwirtschaftlicher Initiativen ausgewählt (F26).

All diese Möglichkeiten stellen Beispiele dar, wie die Agrarpolitik einen Beitrag zu mehr Tierwohl leisten könnte. So könnte die Bundesregierung z. B. die gesetzlichen Mindeststandards in den verschiedenen Bereichen der Nutztierhaltung anheben. Eine Änderung des Tierschutzgesetzes zur Verbesserung des Tierwohls wird jedoch von der Fleischbranche und der Landwirtschaft mehrheitlich abgelehnt, wenn die Änderungen ökonomischen Interessen entgegenstehen. Als Grund für die ablehnende Haltung wird in diesem Zusammenhang z. B. aufgeführt, dass Verbraucher durch strengere Tierschutzgesetze höhere Preise zahlen müssten, was dazu führen könnte, dass sich einige Menschen nicht mehr täglich Fleisch- und Wurstwaren leisten könnten.

Des Weiteren könnten in Deutschland keine höheren Tierwohlstandards als in anderen Ländern der EU eingeführt werden, da sich dadurch die Marktposition der Wirtschaft im internationalen Wettbewerb verschlechtern würde (DEIMEL et al., 2010). In diesem Zusammenhang wird oft darauf hingewiesen, dass Unternehmen der Fleischbranche und Landwirte die Tierhaltung bei Gesetzesänderungen in andere EU-Länder verlagern würden. Dem könnte z. B. mit einem einheitlichen gesetzlichen Tierwohlstandard in der Europäischen Union entgegengewirkt werden, der seit vielen Jahren ähnlich wie ein einheitliches Steuerrecht diskutiert wird (EUROPEAN COMMISSION, 2016). 
Subventionen an Landwirte, die besonders artgerechte Haltungsbedingungen nachweisen, sind ein weiteres politisches Instrument, um Tierwohl zu fördern. Durch eine stärkere Umverteilung der EU-Agrarsubventionen von der 1. Säule (Direktzahlungen nach Fläche) in die 2. Säule (Förderprogramme für eine nachhaltigere und umweltschonendere Bewirtschaftung und die ländliche Entwicklung) könnten noch mehr Landwirte beim Einsatz für mehr Tierwohl finanziell unterstützt werden (BMEL, 2015).

Daneben wäre auch eine gesetzlich verpflichtende Haltungskennzeichnung denkbar, die z. B. eine gesetzlich vorgeschriebene Struktur für alle Tierwohlstandards vorgibt. Als weitere Lösungsmöglichkeiten werden die staatliche Unterstützung privater Initiativen wie etwa der Brancheninitiative Tierwohl und die Einführung des staatlichen TierwohlLabels diskutiert (BMEL, 2015). Laut ERNÄHRUNGSREPORT 2019 wünscht sich die Mehrheit der Verbraucher (81\%) ein staatliches Tierwohlkennzeichen (BMEL, 2019a).

Empfehlenswert wäre gegebenenfalls die Kombination unterschiedlicher politischer Instrumente zur Förderung von mehr Tierwohl. Unklar ist dabei jedoch, wie die Verbraucher diese fünf einzelnen politischen Instrumente bzw. Ansätze bewerten. Daher werden die Verbraucher in der vorliegenden Studie zu ihren politischen Präferenzen befragt. Da sich für alle Tierwohl-Labels die Frage stellt, wie eine Tierwohlkennzeichnung, gestalterisch` umgesetzt werden soll, werden ergänzend verschiedene Kennzeichnungsformen wie beispielsweise eine Ampelkennzeichnung oder die Kennzeichnung mit Sternen oder Zahlen abgefragt (F27). Die Erkenntnisse können von der Angebotsseite für die Produkt- und Kommunikationsentscheidungen genutzt werden und auch der Politik bei der Gestaltung eines neuen deutschen oder europäischen Tierwohl-Labels helfen, sich besser an den Wünschen der Verbraucher zu orientieren.

Darüber hinaus wurde noch nicht untersucht, wie Verbraucher denken, die Nutztierhaltung in Deutschland persönlich positiv beeinflussen zu können (F28). In politischen und wissenschaftlichen Diskussionen wird häufig argumentiert, dass letztlich der Verbraucher im Supermarkt bestimme, wie die Tiere in Deutschland produziert werden. Daher sollen die Verbraucher verschiedene Möglichkeiten der politischen Partizipation für mehr Tierwohl auf einer Skala bewerten. 
Unter anderem werden das persönliche Einkaufs- und Konsumverhalten, die Teilnahme an Petitionen und politischen Wahlen oder die Unterstützung verschiedener NGOs zur Auswahl gestellt. Diese Frage kann aufschlussreiche Informationen liefern, um das allgemeine Vertrauen in die Politik oder auch in unterschiedliche NGOs besser einschätzen zu können und festzustellen, ob die Verbraucher davon ausgehen, dass sie selbst einen Beitrag für eine verbesserte Tierhaltung in Deutschland leisten können. Sollte dies nicht der Fall sein, würde u. a. auch die Effektivität des Einsatzes von TierwohlLabels insgesamt abgeschwächt.

Da Tierwohl, Umweltschutz und Gesundheit wichtige Themen bei politischen Wahlen sind, soll außerdem analysiert werden, ob sich die Bewertung der Politikfragen (F26 bis F28) nach den gewählten Parteien der Verbraucher (Bundestagswahl 2017) unterscheidet (H22 bis H24). Diese Vorgehensweise ermöglicht unterschiedliche Interpretationen und Handlungsempfehlungen an verschiedene Parteien. 


\section{Methodisches Vorgehen}

In diesem Kapitel wird das methodische Vorgehen der vorliegenden Forschungsarbeit vorgestellt. Hierzu wird in Unterkapitel 4.1 zunächst auf die Stichprobenwahl eingegangen. Die Konstruktion des Fragebogens für die standardisierte OnlineBefragung wird danach in Unterkapitel 4.2 beschrieben. Abschließend erfolgt eine Darstellung der Durchführung des Pretests und der Befragung (Unterkapitel 4.3 und 4.4).

\subsection{Stichprobenwahl}

Die Forschungsfragen der Studie richten sich ausschließlich an Personen, die Fleischund Wurstwaren kaufen und ein gewisses Grundinteresse an Gütesiegeln wie auch am Thema Nachhaltigkeit haben. Daher wurde die Stichprobe in der vorliegenden Studie bewusst eingeschränkt.

$\mathrm{Zu}$ diesem Zweck wurden allen potenziellen Teilnehmern der Befragung vor Beginn des Fragebogens zwei dichotome Screening-Fragen gestellt. Die erste Screening-Frage lautete: ,Haben Sie in den letzten zwölf Monaten für sich selbst oder für andere Fleischoder Wurstwaren gekauft?`. Die zweite Screening-Frage lautete: ,Für viele Menschen sind besondere Produktqualitäten wie zum Beispiel Bio-Qualität, Gentechnikfreiheit, fairer Handel, Umweltfreundlichkeit oder eine artgerechte Tierhaltung in den letzten Jahren immer wichtiger geworden. Haben Sie beim Einkauf von Lebensmitteln schon einmal auf derartige Aspekte geachtet oder können Sie sich vorstellen, in Zukunft auf so etwas zu achten?‘. Nur Personen, die beide Fragen mit ,Ja‘ beantworteten, konnten an der Befragung teilnehmen.

Die Grundgesamtheit der Befragten in der vorliegenden Studie besteht demnach aus nachhaltigkeitsinteressierten Fleischkäuferinnen und Fleischkäufern in Deutschland im Alter zwischen 18 und 69 Jahren. Im Rahmen der Dissertation werden die Befragten aus diesem Grund des Öfteren als ,nachhaltigkeitsinteressierte Fleischkäufer' charakterisiert, da dieser Begriff die Zielgruppe am ehesten treffend beschreibt. 
Die Eingrenzung der Zielgruppe ist von großer Bedeutung, da die Berücksichtigung von Tierwohl-Labels beim Einkauf von Fleisch- und Wurstwaren den zentralen Schwerpunkt der Studie bildet. So sollten ausdrücklich keine Personen befragt werden, die keine Fleisch- und Wurstwaren kaufen und die kein Interesse am Thema Nachhaltigkeit haben. Zum einen hätte bei diesen Personen mit einer hohen Abbruchquote gerechnet werden müssen und zum anderen hätte bei diesen Personen ein hohes Risiko einer Verzerrung der Befragungsergebnisse bestanden. Die Stichprobenauswahl beschränkte sich daher auf die Personen, deren Antworten als besonders relevant für die Befragung eingeschätzt wurden (HAMMANN \& ERICHSON, 2000).

Die Zielgruppe der Befragung war durch die weit gefassten Formulierungen der oben dargestellten Screening-Fragen deutlich größer als in Studien, in denen beispielsweise das Einkaufs- und Ernährungsverhalten von Bio-Käufern untersucht wurde. Die Zielgruppe war durch die systematische Eingrenzung mit Hilfe der Screening-Fragen gleichwohl deutlich kleiner als in den zahlreichen Studien, in denen keinerlei Einschränkungen bei der Stichprobe vorgenommen wurden. Dies ist bei Vergleichen mit anderen Studien mit anderen Zielgruppen wie z. B. der Gesamtbevölkerung zu beachten.

Da das Auswahlverfahren der Stichprobe durch die Screening-Fragen nicht zufällig war, kann die Stichprobe der vorliegenden Befragung nicht als repräsentativ für die Gesamtbevölkerung bezeichnet werden. Durch die Quotierung der Grundgesamtheit (vgl. Unterkapitel 4.4) anhand der fünf Kriterien Geschlecht, Alter, Bundesland, Einkommen und Bildung wurde in der Studie dennoch eine der Gesamtbevölkerung in Deutschland ähnliche Verteilung erreicht (vgl. Unterkapitel 5.1). Die Stichprobenauswahl ist häufig mit problematischen Aspekten behaftet; so auch in der vorliegenden Studie. Beispielhaft sei an dieser Stelle neben der nicht vorhandenen Repräsentativität angeführt, dass eine repräsentative Abbildung der Grundgesamtheit auch deshalb nicht möglich gewesen wäre, da bisher keine Daten zur Grundgesamtheit der Studie vorliegen, die hätten zugrunde gelegt werden können. Diese Einschränkungen zur Stichprobenrepräsentativität sind jedoch zu relativieren. 
Aus der vergleichsweise großen Zahl an Studienteilnehmern $(n=930)$ resultierten aussagekräftige Hinweise auf die Forschungsfragen der Arbeit. Eine höhere Aussagekraft der Ergebnisse durch die Befragung einer konkreten Zielgruppe wurde in diesem Punkt einer statistisch repräsentativen Stichprobenauswahl ohne Einschränkung der Grundgesamtheit bewusst vorgezogen.

\subsection{Konstruktion des Fragebogens}

Im Rahmen der vorliegenden Studie wurde eine standardisierte schriftliche OnlineBefragung durchgeführt. Da es sich um eine Verbraucherbefragung handelte, wurde bei der Konstruktion des Fragebogens insgesamt Wert auf einen leicht verständlichen und logischen Aufbau gelegt. Vor dem Start der Befragung wurden, wie in Unterkapitel 4.1 zur Stichprobenwahl ausführlich beschrieben, zunächst zwei Screening-Fragen gestellt, um ausschließlich Fleischkäufer zu befragen, die ein gewisses Grundinteresse am Thema Nachhaltigkeit haben. Personen, die beide Screening-Fragen mit ,Ja' beantworteten, konnten an der Befragung teilnehmen. Der gesamte Fragebogen ist dem Anhang beigefügt. In diesem Abschnitt sollen daher bewusst nicht alle Fragen und Antwortmöglichkeiten vorgestellt werden, vielmehr soll lediglich auf die strategische Gestaltung des Fragebogens sowie auf einige Besonderheiten hingewiesen werden.

Wie in Tabelle 2 am Ende dieses Unterkapitels in der Übersicht ersichtlich wurde der Fragebogen in fünf Abschnitte untergliedert. Im ersten Abschnitt (A) wurden neun Fragen zum Einkaufs- und Ernährungsverhalten gestellt. Im zweiten Abschnitt (B) wurden zwölf Fragen zu Gütesiegeln gestellt, die den Verbrauchern beim Erkennen von Fleisch- und Wurstwaren aus einer artgerechteren Nutztierhaltung helfen sollen. Es folgten zwei weitere Abschnitte, die zum einen vier Fragen zum Informationsbedarf der Verbraucher beinhalteten (C) und zum anderen drei Fragen $\mathrm{zu}$ den politischen Präferenzen der Verbraucher (D). Abschließend wurden in Abschnitt E insgesamt 14 Fragen zur Soziodemografie der Befragten gestellt. Der Fragebogen war folglich mit 42 Fragen sehr umfangreich. 
Bei der Konstruktion des Fragebogens bestand das Ziel darin, Verbrauchern ausgewählte Fragen zum Tierwohl-Labeling zu stellen, die für Anbieter von Fleisch- und Wurstwaren aus einer artgerechteren und nachhaltigeren Nutztierhaltung von hoher Relevanz sind. Die drei ergänzenden Fragen zu den politischen Themen sollten der Politik ein Meinungsbild der Verbraucher zum Thema Tierwohl-Labeling widerspiegeln. Jede einzelne Frage zum Tierwohl-Labeling wurde so konstruiert, dass diese spezifischen Fragen bereits zu einem Erkenntnisgewinn für die Tierwohlforschung beitragen können. Zusätzlich sollten durch gezielte statistische Auswertungen einige der zentralen Fragen zum Tierwohl-Labeling (Abschnitte B, C, D) ergänzend zum Einkaufs- und Ernährungsverhalten (A) sowie zur Soziodemografie der Befragten (E) in Bezug gesetzt werden. Dieser Grundstrategie folgte die Konstruktion des gesamten Fragebogens. Die Fragen zum Tierwohl-Labeling, die den inhaltlichen Schwerpunkt des Fragebogens bildeten und die größte Konzentration der Befragten beanspruchten, wurden in der Mitte des Fragebogens gestellt.

Der erste Abschnitt zum Einkaufs- und Ernährungsverhalten (A) diente den Befragten als leichter Einstieg in den Fragebogen, da lediglich über das eigene Einkaufs- und Konsumverhalten berichtet wurde. Dieser Abschnitt sollte der Charakterisierung unterschiedlicher Käufertypen von Fleisch- und Wurstwaren dienen und die Grundlage für ausgewählte statistische Analysen im Hinblick auf das Tierwohl-Labeling bilden. Die Fragen bezogen sich allgemein auf den Einkauf von Fleisch- und Wurstwaren. Artgerechtere Tierhaltung oder Tierwohl-Labeling wurden in diesem Abschnitt (außer bei der Frage $\mathrm{zu}$ den Kaufkriterien) bewusst nicht thematisiert, um hier das Einkaufsverhalten gänzlich unabhängig vom Tierwohl-Labeling zu erfragen. Als Einstiegsfrage (Frage 1) sollten die Befragten zunächst angeben, für wen sie Fleisch- und Wurstwaren kaufen. Ziel dieser Frage war es, die Fleischesser von den Vegetariern bzw. Veganern unterscheiden zu können, um letzteren im weiteren Verlauf des Fragebogens z. B. keine konkreten Fragen zum Fleischverzehr bzw. zu Geschmacksfragen zu stellen. Vegetarier und Veganer sollten jedoch ausdrücklich mitbefragt werden, da vermutet wurde, dass bei diesen Personen das Interesse an einer artgerechteren und nachhaltigeren Nutztierhaltung und damit evtl. auch bei Tierwohl-Labels überdurchschnittlich groß ist. 
Als erster Schwerpunkt des Abschnitts zum Einkaufs- und Ernährungsverhalten wurden die Studienteilnehmer zu den genutzten Einkaufsstätten für den Einkauf von Fleisch- und Wurstwaren befragt (Frage 2). Hierfür wurden 24 unterschiedliche Einkaufsstätten zur Auswahl angeboten. Dabei wurde großen Wert auf eine möglichst umfassende Auswahl gelegt. Auf Basis statistischer Marktzahlen wurden zunächst die größten Lebensmitteleinzelhändler nach Umsatz in Deutschland ermittelt und in der Befragung berücksichtigt (LEBENSMITTELZEITUNG, 2020; EHI, 2020). Dazu zählten Supermärkte (Vollsortimenter) wie z. B. REWE oder EDEKA, Discounter wie ALDI oder LIDL und SB-Warenhäuser wie z. B. REAL.

Neben den konventionellen Lebensmitteleinzelhändlern wurden auch die führenden BioSupermärkte wie z. B. ALNATURA, DENN`S, BASIC BIO oder BIO COMPANY ermittelt und integriert. Als Einkaufsstätte für Fleisch- und Wurstwaren wurden daneben auch der Metzger, der Wochenmarkt, ein Hofladen, ein kleines Geschäft und ein OnlineShop als Antwortmöglichkeiten im Rahmen einer Mehrfachnennung zur Auswahl gestellt. Ergänzend konnten die Befragten andere Einkaufsstätten angeben.

Da die Haupteinkaufsstätte der befragten Fleischkäufer von großem Interesse für die diesbezüglich geplanten statistischen Auswertungen ist, wurde im Rahmen einer Einfachnennung (Frage 3) noch einmal gezielt nach der Haupteinkaufsstätte für Fleischund Wurstwaren gefragt. Den Studienteilnehmern wurden in dieser Frage nur die Einkaufsstätten angezeigt, die sie in der vorherigen Frage bei der Mehrfachnennung ausgewählt hatten. Insgesamt wurde in der vorliegenden Befragung eine Vielzahl unterschiedlicher Einkaufsstätten erfragt, um gezielt Unterschiede zwischen den Einkaufsstätten untersuchen zu können. Dies ist ein Punkt, in dem sich die vorliegende Studie von anderen Studien unterscheidet.

Den nächsten Schwerpunkt des ersten Abschnitts zum Einkaufs- und Ernährungsverhalten bildeten die Präferenzen der Befragten zu den Angebotsformen Frischetheke und Selbstbedienungsbereich. 
Hier wurden ebenfalls zwei separate Fragen gestellt, da bisherige Studien ergeben hatten, dass bei der Wahl der Frischetheke oder des SB-Bereichs Unterschiede zwischen Aufschnitt und Wurstwaren sowie frischen Fleischwaren zum Braten oder Grillen bestehen, und zudem vermutet wurde, dass es hier signifikante Unterschiede bzgl. der Präferenzen zu Tierwohl-Labels gibt. Diese beiden Fragen wurden daher in der statistischen Auswertung auch separat ausgewertet. Für die Beantwortung beider Fragen bzw. Produktvarianten wurde eine fünfstufige Likert-Skala verwendet (+2 fast nur an der Fleischtheke bis -2 fast nur im SB-Bereich).

Anschließend folgten zwei Fragen in Form von Einfachnennungen zum Konsum- bzw. Ernährungsverhalten der Studienteilnehmer. In der ersten Frage zum Konsumverhalten (Frage 6) stand die Verzehrhäufigkeit von Fleisch- und Wurstwaren im Haushalt der Befragten im Fokus und bei der zweiten Frage (Frage 7) die Reduktionsbereitschaft der Befragten. Bezüglich der Reduktionsbereitschaft wurde darauf geachtet, dass auch Antwortmöglichkeiten für Vegetarier oder Veganer gegeben waren.

Von zentraler Bedeutung ist die Frage zur Beachtung verschiedener Kaufkriterien beim Kauf von Fleisch- und Wurstwaren (Frage 8). Insgesamt 13 Kaufkriterien wurden dabei berücksichtigt. Hier wurde auf eine ausgewogene Mischung aus klassischen und ethischen Kaufkriterien Wert gelegt, deren Bedeutung bereits in anderen Studien belegt werden konnte. Ausgewählt wurden das Aussehen der Ware, bestimmte Marken, der Geschmack, Umweltfreundlichkeit, bestimmte Gütesiegel, faire Löhne für Landwirte, Sonderangebot/günstigster Preis, Gesundheitsaspekte, eine artgerechtere Tierhaltung, die Frische der Ware, eine ansprechende Verpackung, Bio-Qualität und die regionale Herkunft.

Im Unterschied zu den meisten anderen Studien wurde in Frage 8 gezielt danach gefragt, auf welche Kriterien die Befragten beim alltäglichen Einkauf von Fleisch- und Wurstwaren tatsächlich achten. Die Befragungsergebnisse anderer Verbraucherstudien zu derartigen Kriterien erschienen teilweise zu weit von den realen Marktdaten entfernt, wenn nach der Wichtigkeit einzelner Kriterien gefragt wurde. Hier wurde eine zu starke Zustimmungstendenz vermutet, der in der vorliegenden Studie durch die andere Fragenformulierung entgegengewirkt werden sollte. 
Abgefragt wurde die Beachtung der Kaufkriterien mit Hilfe einer fünfstufigen LikertSkala (+2 achte ich fast immer bis -2 achte ich nicht). Vegetarier und Veganer wurden bei dieser Frage nicht nach dem Geschmack gefragt. Von besonderer Bedeutung für die Vermarktung von Fleisch- und Wurstwaren mit Tierwohl-Labels sind die Kriterien artgerechtere Tierhaltung, Gesundheitsaspekte und Umweltfreundlichkeit. Hinsichtlich dieser drei Kriterien wurden in der vorliegenden Studie weitere statistische Auswertungen in Zusammenhang mit verschiedenen Tierwohl-Label-Fragen durchgeführt.

Abschließend für den Abschnitt des Einkaufs- und Ernährungsverhaltens wurde nach der Kaufhäufigkeit unterschiedlicher Sorten mit Hilfe einer fünfstufigen Likert-Skala $(+2$ mehrmals pro Woche bis -2 fast nie/nie) gefragt. Dabei wurden den Befragten drei verschiedene frische Fleischarten zur Auswahl gestellt (Geflügel, Rind, Schwein), Wurst und Aufschnitt sowie verschiedene Tiefkühlgerichte und Dosenware. Hier stellte sich im Nachhinein heraus, dass die Ergebnisse rein deskriptiv durchaus interessant sind, jedoch für die statistische Auswertung einige Interpretationsschwierigkeiten bestehen, da die Abfrage der Kaufhäufigkeit wie im Fragebogen konstruiert nur bedingt Rückschlüsse auf die Sortenpräferenzen der Befragten oder die eingekaufte Menge zulässt.

In Abschnitt B zum Thema Tierwohl-Labeling wurden zunächst Fragen zu konkreten Tierwohl-Labels gestellt (Frage 10 bis 15) und anschließend einkaufsrelevante Fragen mit eher begleitendem bzw. ergänzendem Charakter (Frage 16 bis 21). Im Rahmen einer Mehrfachnennung wurde als erstes der Bekanntheitsgrad von zwölf ausgewählten Tierwohl-Labels abgefragt. Dabei wurden sowohl die bekannten Bio-Labels als auch Tierwohl-Labels berücksichtigt, die keine ökologischen Aspekte bzw. Umweltfreundlichkeit berücksichtigen.

Zur Auswahl gestellte reine Tierwohl-Labels waren das NEULAND-Label, das Label des DEUTSCHEN TIERSCHUTZBUNDES und das Label von VIER PFOTEN. Als besondere Variante eines Tierwohl-Labels wurde zudem das geplante Label ,Mehr Tierwohl` vom Bundesministerium für Ernährung und Landwirtschaft abgefragt, das es zum Zeitpunkt der Erhebung noch gar nicht gab. 
Ein solches fiktives Label sollte integriert werden, um die Aussagekraft der Ergebnisse insgesamt kritisch zu betrachten bzw. zu relativieren oder zu stärken. Zum anderen wurde sich davon erhofft, bereits vor Einführung dieses staatlichen Tierwohl-Labels Hinweise auf die womöglich zu erwartende Akzeptanz in der Bevölkerung im Vergleich zu den anderen elf Tierwohl-Labels zu erhalten. Eine weitere Variante, die u. a. aufgrund der besonderen Kategorisierungsvariante und der großen Aktualität (Februar 2018) berücksichtigt wurde, war das Tierwohl-Label HALTUNGSKOMPASS durch LIDL, die sich in ihrer Umsetzung stark an die zu diesem Zeitpunkt diskutierte gesetzlich verpflichtende Haltungskennzeichnung anlehnte. Der als durchaus progressiv zu bezeichnende Vorstoß von LIDL mit dem HALTUNGSKOMPASS war das erste VierStufen-Modell mit einer Art Ampelkennzeichnung und legte den Grundstein für die später (nach der Befragung im Juni 2018) eingeführte ,Haltungsform“, der sich zahlreiche weitere Unternehmen anschlossen. Außerdem wurde das Label der Initiative Tierwohl berücksichtigt. Somit wurden alle zum Zeitpunkt der Befragung (Juni 2018) zentralen und aktuellen Tierwohl-Labels berücksichtigt. Die Befragten konnten zudem angeben, dass sie ,vielleicht‘ eines oder ,noch keins` dieser Gütesiegel gesehen haben.

Die folgenden Fragen im Abschnitt Tierwohl-Labeling fokussierten sich auf den konkreten Kauf von Produkten mit den entsprechenden Tierwohl-Labels. In Frage 11 wurden den Befragten alle Tierwohl-Labels angezeigt, die sie in der vorherigen Frage 10 angekreuzt hatten. Personen, die in Frage 10 zum Bekanntheitsgrad kein Gütesiegel angegeben, sondern die Antwortmöglichkeiten, vielleicht‘ oder ,noch keins ‘ gewählt hatten, sollten auch die Frage 11 zum Kauf der Tierwohl-Labels nicht beantworten. Aufbauend auf die beiden Fragen zum Bekanntheitsgrad und zum Kauf von TierwohlLabels wurde in Form einer Einfachnennung nach der Kaufhäufigkeit von Fleisch- und Wurstwaren mit den Gütesiegeln gefragt, die sie bereits gekauft haben. Alle Personen, die weder ein Tierwohl-Label bisher gesehen haben noch ein Produkt mit einem dieser Labels gekauft haben, wurden in Frage 13 nach den Gründen befragt, warum sie diese Tierwohl-Labels noch nicht gesehen oder gekauft haben. Den Befragten wurden neun Gründe vorgegeben, aus denen sie wählen konnten. Mehrfachnennungen waren dabei möglich. 
Aufgrund der häufig diskutierten Unklarheit der Bedeutung von Tierwohl-Labels, der geringen Studienlage bzgl. der vergleichenden Bewertung dieser zwölf Tierwohl-Labels sowie der hohen Relevanz der Erkenntnisse für die Vermarktung von Fleisch- und Wurstwaren aus einer artgerechteren und nachhaltigeren Nutztierhaltung sollten die Befragten in Frage 14 die Strenge der zwölf unterschiedlichen Tierwohl-Labels in den drei Kategorien Tierwohl, Umwelt und Gesundheit einschätzen. Ziel dieser Frage war es, herauszufinden, inwiefern die Verbraucher die Tierwohl-Labels unterschiedlich wahrnehmen bzw. beurteilen. Da hier das Bauchgefühl und nicht schwerpunktmäßig konkretes Wissen im Vordergrund stand, das für diese spezielle Frage als eher gering angenommen wurde, bestand das hohe Risiko, dass die große Mehrzahl der Befragten eine mittlere Kategorie (wie z. B. teils/teils) der verwendeten Skala wählen. Um dieses Risiko zu minimieren, wurde den Befragten bewusst keine mittlere Kategorie angeboten, d. h. sie mussten sich auf einer vierstufigen Skala für einen ,Strengegrad“ entscheiden $(+2$ sehr streng bis -2 gar nicht streng).

Eine der für die statistische Auswertung interessantesten Fragen war die Erfragung der allgemeinen Tierwohl-Label-Präferenz aller Befragten in Frage 15. Hier konnten die Studienteilnehmer angeben, welche Tierwohl-Labels sie konkret beim Kauf von Fleischund Wurstwaren aus einer artgerechteren Nutztierhaltung berücksichtigen würden und welche nicht. Hier wurde den Befragten eine mittlere Kategorie angeboten, da nicht damit zu rechnen war, dass die Studienteilnehmer bei dieser Frage stark zur Mitte tendieren.

Um zu erfassen, welche Probleme die Verbraucher am ehesten mit der intensiven Nutztierhaltung in Verbindung bringen, wurden in Frage 16 ausgewählte Probleme im Rahmen einer Mehrfachnennung zur Auswahl gestellt. Die Verbesserungen der am häufigsten genannten Herausforderungen können gleichzeitig als Vermarktungspotenziale für Fleisch- und Wurstwaren betrachtet werden, wenn es Unternehmen gelingt, in einem dieser Bereiche klare Vorteile hinsichtlich Nachhaltigkeit gegenüber Wettbewerbern zu präsentieren. Mit diesen Erkenntnissen könnte beispielsweise eine Priorisierung nachhaltiger Werbebotschaften erfolgen. 
Die Auswahl der einzelnen Herausforderungen orientierte sich an wissenschaftlichen Studien sowie an den wesentlichen Kritikpunkten der intensiven Landwirtschaft von Umweltschutz-, Tierschutz- und Gesundheitsorganisationen.

Mit Frage 17 sollte herausgefunden werden, welche weiteren Gütesiegel oder Logos sich positiv auf die Kaufentscheidung bei Tierwohlprodukten auswirken könnten, die bereits eines der vorgestellten Tierwohl-Labels tragen. Dafür wurden 24 Gütesiegel aus verschiedenen Bereichen in einer Mehrfachnennung zur Auswahl gestellt. Die Auswahl erfolgte nach verschiedenen Kriterien. Zum einen sollten alle zentralen Gütesiegel, die bereits auf Fleisch- und Wurstwaren zu finden sind, berücksichtigt werden. Zum anderen wurden Gütesiegel ergänzt, die sich womöglich für Produktverpackungen von Fleischund Wurstwaren eignen könnten, wie beispielsweise Testsiegel.

Außerdem wurden Logos verschiedener Organisationen integriert, um zu überprüfen, ob diese verkaufsfördernd sein können und evtl. die Möglichkeit zu einem Cause-RelatedMarketing-Projekt bieten. Frei erfunden wurden die Logos HORMONFREI, ANTIBIOTIKAFREI und MRSA-FREI als mögliche Kennzeichnungsidee der Zukunft. Anschließend wurde als eine Art Kontrollvariable in Frage 18 das generelle Interesse an Tierwohlprodukten erfragt, um sicherzustellen, dass die Befragten tatsächlich die richtige Zielgruppe bilden und die nötige Aufgeschlossenheit gegenüber Tierwohlprodukten besitzen.

Abschließend für den Abschnitt B des Fragebogens zum Tierwohl-Labeling wurden drei Fragen gestellt, die von großem Interesse für Anbieter von Fleisch- und Wurstwaren sind. Zunächst sollte die Zufriedenheit der Befragten mit dem Produkt- und Beratungsangebot für Fleisch- und Wurstwaren aus einer artgerechteren Tierhaltung in ihrer Haupteinkaufsstätte ermittelt werden. Auf einer fünfstufigen Likert-Skala (+2 rundum zufrieden bis -2 nicht zufrieden) konnten die Probanden sieben Aspekte bewerten, die womöglich ein Vermarktungspotenzial bzw. Optimierungspotenzial beinhalten. Da nicht bekannt war, ob die Befragten eine Beurteilung ihrer Haupteinkaufsstätte vornehmen können, wurde zusätzlich die Möglichkeit ,kann ich nicht beurteilen` ergänzt. 
Die Frage zur Kundenzufriedenheit wurde gestellt, um gezielt Verbesserungsmöglichkeiten bei der Vermarktung von Tierwohlprodukten feststellen zu können und entsprechende Handlungsempfehlungen differenziert nach der Haupteinkaufsstätte zu entwickeln. Ergänzend dazu wurde eine Frage mit rein explorativem Charakter gestellt, die im Rahmen der Dissertation und aufgrund privater Beobachtungen im Lebensmitteleinzelhandel aufkam. In Frage 20 konnten die Befragten angeben, welche Sortierung sie sich wünschen, wenn sie in einem Geschäft vor einem Kühlregal, einer Kühltruhe oder einer Fleischtheke mit verschiedenen Fleisch- und Wurstwaren stehen.

Als Antwortmöglichkeiten wurden zwei Alternativen angeboten: ein eigener Bereich (z. B. eigenes Regal) für alle Produkte mit sehr hohen Tierwohlstandards oder eine Sortierung nach Produktart (z. B. Salami), wobei bei letzter Variante herkömmliche Produkte und Tierwohlprodukte einer Produktart direkt nebeneinander platziert sind. Um die Relevanz des Produkt- und Beratungsangebots zum Thema artgerechtere Tierhaltung aus Perspektive der Verbraucher besser einschätzen zu können, wurde in Frage 21 unter Einsatz einer fünfstufigen Likert-Skala gefragt, ob die Befragten gegebenenfalls ihre Haupteinkaufsstätte wechseln würden, wenn sie bei ihrem Anbieter keine Verbesserungen in diesem Bereich feststellen würden.

In Abschnitt $\mathrm{C}$ des Fragebogens wurde der Informationsbedarf der Verbraucher mit Hilfe von vier Fragen ermittelt. Dabei wurde zum Anfang dieses Abschnitts eine Filterfrage gestellt, um herauszufinden, ob generell ein Informationsbedarf hinsichtlich der Haltungsbedingungen der Tiere und der Herkunft der Fleisch- und Wurstwaren besteht oder nicht. Die Frage wurde daher mit dichotom ausgeprägten Antwortkategorien $(1=$ ja, $2=$ nein) gestellt. Nur Personen, die diese Frage mit ,ja beantworteten, wurden die drei weiterführenden Fragen zum Informationsbedarf gestellt. Im Fokus standen hierbei die zentralen Fragen, wo sich die Befragten informieren möchten, welche Institutionen entsprechende Informationen bereitstellen sollten und wie hoch der Detaillierungsgrad von Herkunftsangaben sein sollte. 
Um die Frage zu beantworten, an welcher Stelle sich die Befragten gerne über die Haltungsbedingungen der Nutztiere und die Herkunft der von ihnen gekauften Fleischund Wurstwaren informieren möchten, wurden verschiedene Antwortmöglichkeiten vorgegeben. Als Antwortmöglichkeiten, die auf einer fünfstufigen Likert-Skala (+2 trifft voll und ganz zu bis -2 trifft überhaupt nicht zu) ausgewählt werden konnten, waren die Verkaufsräume der Einkaufsstätte (z. B. Broschüren), die Fleischtheke, die Verpackung der Produkte, kostenlose Werbeprospekte (Postwurfsendungen), ein zentrales Internetportal und die Medien möglich.

In Frage 24 konnten bei der Mehrfachnennung elf unterschiedliche Organisationen ausgewählt werden, die aus der Perspektive der Verbraucher weiterführende Informationen zu den Haltungsbedingungen der Tiere im Internet bereitstellen sollten. Als Anbieter von Fleisch- und Wurstwaren wurden Landwirte, Lebensmittelhersteller und der Lebensmitteleinzelhandel aufgeführt.

Aus dem Bereich Politik konnten die Befragten das Bundesministerium für Ernährung und Landwirtschaft sowie die Europäische Union (EU) auswählen. Als NGOs standen Verbraucher-, Natur- und Tierschutzorganisationen zur Auswahl. Außerdem konnten die Herausgeber der Gütesiegel, die Medien (TV, Radio, Print, Online etc.) sowie Verbände der Lebensmittelindustrie gewählt werden.

Abschließend für Abschnitt $\mathrm{C}$ wurde nach dem gewünschten Detaillierungsgrad der Herkunftsangaben gefragt, da eine möglichst detaillierte Angabe vermutlich besonders bei Tierwohlprodukten ein starkes Kaufargument darstellt. So wurden die Verbraucher gefragt, ob es für sie ein Kaufargument wäre, wenn ein Anbieter die Rückverfolgbarkeit von Fleisch- und Wurstwaren bis auf den konkreten Bauernhof zurück, von dem die Tiere stammen, transparent darstellen würde. Bei den Antwortmöglichkeiten (Einfachnennung) konnten die Befragten aus fünf abgestuften Antworten wählen, ob sie solche Produkte , auf jeden Fall' oder, wahrscheinlich` kaufen würden, ob sie sich unsicher sind, ob ihnen die Angabe der groben Region (z. B. Herkunftsland) reicht oder ob Herkunft gar keine Rolle für sie spielt. 
Tierwohlspezifische Fragen mit politischer Perspektive wurden in Abschnitt D des Fragebogens erfragt, mit dem Ziel, einen Beitrag zur politischen Diskussion zu leisten sowie das Thema der Arbeit kritisch zu reflektieren und in einen größeren Rahmen einzuordnen. Aus politischer Perspektive gibt es zahlreiche Fragestellungen, die im Hinblick auf das Thema Tierwohl diskutiert werden. Für die vorliegende Studie wurden drei Fragen ausgewählt.

In Frage 26 wurden den Befragten fünf verschiedene Ansätze vorgestellt, die in der Politik diskutiert werden, um das Wohl der Tiere in der Nutztierhaltung und die Kennzeichnung von Fleisch- und Wurstwaren aus einer artgerechteren Haltung zu verbessern. Diese Ansätze sollten die Befragten mit Hilfe einer fünfstufigen Skala nach Dringlichkeit/Wichtigkeit bewerten.

Hierbei mussten ein strengeres Tierschutzgesetz, eine gesetzlich verpflichtende Haltungskennzeichnung, freiwillige Gütesiegel, die staatliche Förderung von mehr Tierwohl und der Ausbau der Initiative Tierwohl in eine Reihenfolge von 1 bis 5 gebracht werden. So sollte eine Einordnung des Themas Tierwohl-Labeling im Vergleich zu anderen Lösungsansätzen für mehr Tierwohl ermöglicht werden.

Bei allen Tierwohl-Labels stellt sich die Frage nach der Kennzeichnungsform. Um ein aktuelles Meinungsbild der Verbraucher zu erlangen, wurde in Frage 27 erfragt, welche Form der Haltungskennzeichnung die Verbraucher bevorzugen würden, um beim Fleischkauf besser einschätzen zu können, ob die Tiere artgerecht gehalten werden. Bei dieser Einfachnennung mussten sich alle Befragten für eine Variante entscheiden. Zur Auswahl gestellt wurden u. a. Ampelfarben (rot, orange, grün), Sterne (je Verbesserungsstufe ein Stern), zwei Zahlenvarianten und Bronze-Silber-Gold.

Abschließend für den Block D konnten die Studienteilnehmer in Frage 28 sechs verschiedene Möglichkeiten, sich für eine verbesserte Tierhaltung einzusetzen, bewerten. Die Antworten konnten mit Hilfe einer fünfstufigen Likert-Skala (+2 stark beeinflussen bis -2 sehr wenig beeinflussen) angegeben werden. 
Neben dem persönlichen Einkaufs- und Konsumverhalten konnten die Befragten die Teilnahme an Petitionen, die Stimmabgabe für eine Partei bei politischen Wahlen, die Unterstützung von Tierschutz- oder Naturschutzorganisationen, die Teilnahme an Demonstrationen sowie die Unterstützung von Verbraucherschutzorganisationen auswählen.

Block E des Fragebogens umfasste 14 soziodemografische Kriterien, die zum einen der Stichprobenbeschreibung und zum anderen ausgewählten statistischen Analysen dienen sollten. Hier wurden Geschlecht, Alter, Bundesland, Stadt/Land (Ortsgröße), Haushaltseinkommen, Bildung, Haushaltsgröße/-zusammensetzung, Haustiere, Religion und politische Partei erfragt.

Die einzelnen Fragen $\mathrm{zu}$ den soziodemografischen Kriterien sowie die Antwortmöglichkeiten sind dem Fragebogen im Anhang zu entnehmen und werden an dieser Stelle daher nicht gesondert aufgeführt. Während im Rahmen der Fragebogenentwicklung die Fragen zur Soziodemografie an das Ende des Fragebogens gesetzt wurden, wurden die soziodemografischen Fragen bei der Online-Befragung von der RESPONDI AG bewusst an den Anfang der Befragung platziert, um die Quotierung (Unterkapitel 4.4) durchzuführen.

Innerhalb aller Abschnitte wurde bei der Konstruktion des Fragebogens und bei der Online-Befragung auf eine Durchmischung (Randomisierung) der Items geachtet, da möglichst keine Kontexteffekte wie beispielsweise Reihenfolgeneffekte auftreten sollten. Außerdem wurde berücksichtigt, möglichst neutrale, eindeutige, leicht verständliche und für die Ziele der Studie relevante Fragen- und Itemformulierungen zu wählen (BORG \& STAUFENBIEL, 2007).

In Tabelle 2 sind die übergreifenden Leitfragen abgebildet, aus denen die Fragen im Fragebogen (Anhang) entwickelt wurden. Zur möglichst differenzierten Beantwortung dieser Fragen waren teilweise mehrere Fragen im Fragebogen notwendig. 


\section{Tabelle 2: Konstruktion des Fragebogens}

\begin{tabular}{|c|c|}
\hline A. Einkaufs- und Ernährungsverhalten & Fragen-Nr. \\
\hline Wer kauft für wen ein? & $\mathrm{F} 1$ \\
\hline Wo werden Fleisch- und Wurstwaren gekauft? & $\mathrm{F} 2, \mathrm{~F} 3$ \\
\hline Wie sind die Präferenzen bzgl. Theke und Selbstbedienung? & $\mathrm{F} 4, \mathrm{~F} 5$ \\
\hline Wie ist die Verzehrhäufigkeit von Fleisch- und Wurstwaren im Haushalt? & F6 \\
\hline Wie ist die Reduktionsbereitschaft bzgl. des Fleischkonsums? & F7 \\
\hline Wie werden klassische und ethische Kaufkriterien beachtet? & F8 \\
\hline Welche Fleischarten (z. B. Rind) und Fleischsorten werden präferiert? & F9 \\
\hline \multicolumn{2}{|l|}{ B. Tierwohl-Labeling aus Verbraucherperspektive } \\
\hline Wie ist der Bekanntheitsgrad der ausgewählten Tierwohl-Labels? & $\mathrm{F} 10$ \\
\hline Wie ist die Kauferfahrung mit ausgewählten Tierwohl-Labels? & $\mathrm{F} 11, \mathrm{~F} 12$ \\
\hline Welche zentralen Kaufbarrieren bestehen bei Tierwohlprodukten? & F13 \\
\hline Wie streng werden die verschiedenen Tierwohl-Labels eingeschätzt? & $\mathrm{F} 14$ \\
\hline Wie sind die Kaufpräferenzen für verschiedene Tierwohl-Labels? & F15 \\
\hline Welches sind die kritischen Themen des Fleischkonsums? & F16 \\
\hline Wie sind die Präferenzen bzgl. weiterer Gütesiegel (Multi-Labeling)? & $\mathrm{F} 17$ \\
\hline Wie hoch ist das Interesse an Tierwohlprodukten? & $\mathrm{F} 18$ \\
\hline Gibt es Optimierungspotenziale beim Produkt- und Beratungsangebot? & $\mathrm{F} 19, \mathrm{~F} 20, \mathrm{~F} 21$ \\
\hline \multicolumn{2}{|l|}{ C. Informationsbedarf der Verbraucher } \\
\hline Wie viele Menschen wünschen sich bessere Tierwohlinformationen? & F22 \\
\hline Wo sollen diese Tierwohlinformationen bereitgestellt werden? & F23 \\
\hline Welche Institutionen sollen Tierwohlinformationen anbieten? & F24 \\
\hline Wie detailliert sollten Herkunftsangaben sein? & $\mathrm{F} 25$ \\
\hline \multicolumn{2}{|l|}{ D. Politikoptionen für mehr Tierwohl } \\
\hline Wie bewerten Verbraucher Politikoptionen für mehr Tierwohl? & F26 \\
\hline Wie sind die Präferenzen bei Kennzeichnungsformen? & $\mathrm{F} 27$ \\
\hline Wie bewerten Verbraucher ihren persönlichen Einfluss auf mehr Tierwohl? & $\mathrm{F} 28$ \\
\hline \multicolumn{2}{|l|}{ E. Soziodemografische Daten } \\
\hline $\begin{array}{l}\text { Geschlecht, Alter, } \begin{array}{c}\text { Bundesland, Stadt/Land (Ortsgröße), } \\
\text { Haushaltseinkommen, } \\
\text { Haustiere, Religion, politische Partei }\end{array} \text { Haushaltsgröße/-zusammensetzung, }\end{array}$ & F29-F42 \\
\hline
\end{tabular}

\section{Quelle: Eigene Darstellung}




\subsection{Pretest}

Nachdem die Fragebogenentwicklung bereits weit fortgeschritten war, wurde im September 2017 der entwickelte Fragebogen in Osnabrück im Rahmen eines Pretests von zufällig ausgewählten Personen $(\mathrm{N}=38)$ ausgefüllt. Das Ziel des schriftlichen Pretests war zunächst die Überprüfung der Verständlichkeit und der Eindeutigkeit der Fragen und Antwortmöglichkeiten des Fragebogens, um den Fragebogen weiter zu optimieren, bevor der Fragebogen durch das Marktforschungsinstitut RESPONDI online weiteren Pretests unterzogen werden sollte. Dabei wurde auch beobachtet, ob die Beantwortungszeit von ca. zwölf Minuten realistisch ist. Als Befragungsorte wurden schwerpunktmäßig verschiedene stadtnahe Supermärkte und Discounter ausgewählt. Weitere schriftliche Befragungen bzw. Pretests wurden auf dem Wochenmarkt, in einer traditionellen Osnabrücker Metzgerei und in einem Bio-Supermarkt durchgeführt.

Die Teilnehmer des Pretests beantworteten die Fragen selbstständig schriftlich an den beschriebenen Standorten in Osnabrück. Beim Pretest wurde Wert darauf gelegt, dass die Teilnehmer den Fragebogen alleine beantworten, und zur Reduzierung eines möglichen Interviewer-Bias hielten sich die Interviewer in einigen Metern Entfernung voneinander auf. Die Befragenden standen den Teilnehmern jedoch in einiger Entfernung als mögliche Ansprechpartner für eventuelle Rückfragen zur Verfügung. Alle relevanten Anmerkungen, Verständnisfragen und konstruktive Kritik der Befragten wurden zur weiteren Optimierung des Fragebogens genutzt, bevor der Fragebogen für die Durchführung der Online-Befragung fertiggestellt wurde, die im nächsten Abschnitt beschrieben wird.

\subsection{Durchführung der Befragung und Datenaufbereitung}

Die in Kapitel 5 dargestellten Ergebnisse der empirischen Studie basieren auf der Durchführung der standardisierten Online-Befragung im Juni 2018 durch die RESPONDI AG in Köln über das unternehmenseigene Online-Verbraucherpanel. 
Die Teilnehmer der Verbraucherpanels wurden per Zufallsverfahren via E-Mail zur Befragung eingeladen, jedoch ohne das Thema der Befragung zu nennen, um eine Vorselektion zu vermeiden. Dabei wurde ein Hinweis ergänzt, dass alle Aussagen anonym und vertraulich behandelt werden und die Aussagen ausschließlich statistischen und analytischen Zwecken dienen. Die Teilnahme an der Online-Studie wurde nicht entlohnt und war freiwillig (RESPONDI, 2019). Die Teilnahme an Befragungen wird durch die RESPONDI AG jedoch mit sogenannten mingle-Sammelpunkten belohnt, die bei verschiedensten Unternehmen wie Amazon, Tchibo, mymuesli und lieferando gegen Bargeld, Gutscheine oder Spenden eingetauscht werden können. Diese Incentivierung ist übliche Praxis bei kommerziellen Verbraucherpanels und kann durchaus kritisch betrachtet werden. Mögliche Effekte auf die Datenqualität werden jedoch von Anbietern wie der RESPONDI AG, die die Qualitätsrichtlinien des Marktforschungsverbands ESOMAR für Online-Befragungen einhalten, mit Hilfe zahlreicher Qualitätssicherungsmaßnahmen gering gehalten und erfüllen hohe Qualitätsstandards (RESPONDI, 2019).

Besondere Qualitätsmerkmale des Online-Panels der RESPONDI AG waren des Weiteren umfangreiche Scoring- und Kontrollverfahren im Rahmen des Panelmanagements. Bei der Befragung wurden die Qualitätsrichtlinien vom Marktforschungsverband ESOMAR für Online-Befragungen eingehalten (RESPONDI, 2019). Die Quotierung erfolgte auf Basis der Zahlen der Studie „Best for Planning“ der Gesellschaft für Integrierte Kommunikationsforschung und ist dem Anhang beigefügt (GIK, 2019).

Die Altersklassen wurden zu 18 bis 29 Jahre, 30 bis 39 Jahre, 40 bis 49 Jahre, 50 bis 59 Jahre und 60 bis 69 Jahre zusammengefasst. Die Einkommensklassen wurden von $<1250 ; 1250-2500 ; 2500-3500 ; 3500-4500 ;>4500$ festgelegt (jeweils in $€$...bis unter). Im Fragebogen wurden die Einkommensklassen in kleinere Abstände untergliedert, jedoch orientierte sich die Unterteilung an den gleichen Stufen wie die Basisdaten aus der Quotierung. Der Bildungsgrad wurde in drei Klassen eingeteilt, wobei eine niedrige Bildung für alle Befragten mit Hauptschulabschluss oder keinem Abschluss galt. Als mittlere Bildung wurde ein Realschulabschluss definiert. 
Als hohe Bildung galten die Fachhochschulreife und das Abitur sowie Hochschul- und Universitätsabschlüsse. Durch die Quotierung konnte eine Verteilung in der Befragung sichergestellt werden, die zwar nicht als repräsentativ bezeichnet werden kann, sich aber zu einem hohen Grad an den Verteilungen in der Gesamtbevölkerung bzgl. der Kriterien Geschlecht, Alter, Bundesland, Einkommen und Bildung orientiert. Eine Repräsentativität der Studie wurde, wie bereits beschrieben, aufgrund der beiden Screening-Fragen nicht angestrebt. In Tabelle 3 sind die wesentlichen Merkmale der Durchführung der Studie dargestellt.

\section{Tabelle 3: Durchführung der Befragung im Überblick}

\begin{tabular}{|c|c|}
\hline \multicolumn{2}{|l|}{ Durchführung der Befragung } \\
\hline Methode & Ad-hoc \\
\hline Land & Deutschland \\
\hline Zielgruppe & $\begin{array}{l}\text { Frauen und Männer im Alter zwischen } 18 \text { und } 69 \\
\text { Jahren }\end{array}$ \\
\hline Stichprobe & $\begin{array}{l}n=930 \\
\text { Personen, die 1.) in den letzten zwölf Monaten für sich } \\
\text { oder andere Personen Fleisch- oder Wurstwaren } \\
\text { gekauft haben und 2.) beim Einkauf von Lebensmitteln } \\
\text { schon einmal auf besondere Qualitätskriterien wie } \\
\text { beispielsweise Bio-Qualität, Gentechnikfreiheit, fairer } \\
\text { Handel, Umweltfreundlichkeit oder eine artgerechte } \\
\text { Tierhaltung geachtet haben. }\end{array}$ \\
\hline Inzidenzrate (IR) & IR im Feld $71 \%, 1310$ Fälle mit 930 echten completes. \\
\hline Erhebungsmethode & $\begin{array}{l}\text { Standardisierte schriftliche Online-Befragung mit halb- } \\
\text { offenen und geschlossenen Fragen bei strikter } \\
\text { Randomisierung zur Vermeidung von } \\
\text { Reihenfolgeneffekten }\end{array}$ \\
\hline Auswahlverfahren & $\begin{array}{l}\text { Rekrutierung der Befragten durch Online-Access- } \\
\text { Panel } \\
\text { Quotenvorgaben von Geschlecht, Alter, Region, } \\
\text { Einkommen und Bildung }\end{array}$ \\
\hline Befragungsdauer & ca. zwölf Minuten \\
\hline Zeitraum Feldphase & $07.06 .2018-12.06 .2018$ \\
\hline Zeitraum insgesamt & $\begin{array}{l}\text { Zwei Tage Programmierung Fragebogen und Testung, } \\
\text { sechs Tage Feldphase, ein Tag Bereinigung der } \\
\text { Rohdaten und Lieferung des Datensatzes, insgesamt } \\
\text { neun Tage }\end{array}$ \\
\hline Durchführung der Feldarbeit & RESPONDI AG, Köln \\
\hline Qualitätskontrolle & $\begin{array}{l}\text { Qualitätskontrolle durch Pretesting, Integration von } \\
\text { Plausibiliätskontrollen sowie Cleansing der Rohdaten, } \\
\text { insbesondere Exklusion von Straightlinern und } \\
\text { Teilnehmern mit zu kurzen Antwortzeiten }\end{array}$ \\
\hline
\end{tabular}

\section{Quelle: Eigene Darstellung}


Nach der Durchführung der Befragung wurde der bereinigte Datensatz als SPSS-Datei vom Marktforschungsinstitut RESPONDI bereitgestellt. Die Inzidenzrate lag im Feld bei $71 \%$. Von 1310 Fällen wurden insgesamt 930 Fragebögen vollständig ausgefüllt. Die Daten der quantitativen Befragung wurden nach der Datenerhebung mit Hilfe des Programms SPSS ausgewertet. Dabei wurden zunächst in Bezug auf die Auswertung der einzelnen Fragen Zusammenhangs- bzw. Abhängigkeitsstrukturen zwischen den Variablen mit Hilfe verschiedener uni- und bivariater statistischer Verfahren wie $\mathrm{Chi}^{2}$ Tests, Varianzanalysen und Korrelationsanalysen für die Beantwortung der Forschungsfragen untersucht. Anschließend wurde auf Basis der Tierwohl-LabelPräferenzen der Verbraucher eine hierarchische Faktorenanalyse durchgeführt, um erstmals eine Tierwohl-Label-Typologie zu erstellen. Auf Grundlage der erstellten Faktoren wurde eine hierarchische Clusteranalyse durchgeführt, um die Verbraucher bzgl. ihrer Tierwohl-Label-Präferenzen erstmals umfassend $\mathrm{zu}$ segmentieren. Die Unterschiede zwischen den gebildeten Gruppen wurden sowohl bei der Faktoren- als auch bei der Clusteranalyse mit der multinominalen logistischen Regressionsanalyse berechnet. Dafür waren verschiedene Klassenbildungen und Dichotomisierungen erforderlich, um ausreichend große Gruppen zu erhalten, die in Anhang A: Fragebogen dargestellt sind.

Es ist des Weiteren darauf hinzuweisen, dass das Skalenniveau von Rating- bzw. LikertSkalen mit messtheoretischen Problemen hinsichtlich der Frage behaftet ist, inwiefern eine Ratingskala als intervallskaliert angesehen werden kann und sich für weiterführende parametrische Verfahren eignet (BORG \& STAUFENBIEL, 2007). In der vorliegenden Studie wird die Position von BORTZ und DÖRING (2009) vertreten. Diese besagt, dass implizite messtheoretische Annahmen aufrechterhalten werden können, wenn die mit Ratingskalen erzielten Ergebnisse sinnvoll interpretierbar sind und sich seit Jahren in der Forschungspraxis bewährt haben. Daher wurden in der durchgeführten Studie ordinalskalierte Ratingskalen als metrisch bzw. intervallskaliert interpretiert, um aussagekräftigere Ergebnisse $\mathrm{zu}$ erzielen. Dennoch werden derartige Items in der Forschungspraxis oft als intervallskaliert verwendet, wenn die Annahme plausibel erscheint. 
Ein hohes Skalenniveau zu erreichen, ist bei komplexen Verbraucherbefragungen zum einen nicht immer möglich und zum anderen häufig mit einem aus forschungsökonomischer Perspektive nicht vertretbaren Aufwand verbunden (UNIVERSITÄT ZÜRICH, 2019). Bei der einfaktoriellen Varianzanalyse wurde zunächst die Homogenität der Varianzen mittels Levene-Test geprüft. Bei homogenen Varianzen wurde die einfaktorielle ANOVA gewählt. Sollten keine homogenen Varianzen vorliegen, könnte es zu einer Verzerrung des F-Tests kommen. Daher wurde in diesen Fällen der Welch-Test angewandt. Da es sich teilweise um unterschiedlich große Stichproben handelte, wurde als post-hoc-Test der konservative Bonferroni-posthoc-Test angewandt, der auch für unterschiedlich große Stichproben geeignet ist. Bei den nicht homogenen Varianzen wurde der Games-Howell-post-hoc-Test verwendet (UNIVERSITÄT ZÜRICH, 2019).

Für die Auswertungen wurden ausschließlich die vollständigen Datensätze ( $n=930)$ berücksichtigt und die übrigen Datensätze mit fehlenden Werten ignoriert. Nach GÖTHLICH (2009) stellt in großzahligen empirischen Erhebungen dieser Umgang mit fehlenden Werten am ehesten ein geeignetes Verfahren dar, um möglichst unverzerrte Parameter zu ermitteln. Die Ergebnisse der durchgeführten Befragung werden im folgenden Ergebnisteil der Arbeit in Form von Tabellen und Abbildungen dargestellt. 


\section{Ergebnisse der empirischen Studie}

In diesem Kapitel werden die zentralen Ergebnisse der durchgeführten Verbraucherbefragung präsentiert. Der Aufbau dieses Kapitels orientiert sich am Aufbau des Fragebogens. Es werden zunächst die Ergebnisse der deskriptiven und bivariaten Analysen vorgestellt und anschließend zusammengefasst. Daran schließt sich die Ergebnisdarstellung der Faktoren- und der Clusteranalyse an, die ebenfalls mit einem kompakten Überblick der Forschungsergebnisse endet.

\subsection{Beschreibung der Stichprobe}

Die Ergebnisse der Studie basieren auf insgesamt 930 vollständig ausgefüllten Fragebögen der Online-Befragung, die durch die RESPONDI AG durchgeführt wurde. In Tabelle 4 wird die Stichprobe der Studie anhand der zentralen Merkmale Geschlecht, Altersklassen, Bundesland, Wohnort (nach Größe), Einkommensklassen und Bildung dargestellt.

Tabelle 4: Soziodemografische Merkmale der Stichprobe im Überblick

\begin{tabular}{|c|c|c|c|}
\hline Merkmal & Ausprägung & $\begin{array}{l}\text { Absolute } \\
\text { Häufigkeit }\end{array}$ & $\begin{array}{l}\text { Häufigkeit } \\
\text { in } \%\end{array}$ \\
\hline \multirow[t]{2}{*}{ Geschlecht } & weiblich & 491 & 52,8 \\
\hline & männlich & 439 & 47,2 \\
\hline \multirow[t]{5}{*}{ Altersklasse } & $18-29$ & 175 & 18,8 \\
\hline & 30-39 & 160 & 17,2 \\
\hline & $40-49$ & 203 & 21,8 \\
\hline & $50-59$ & 237 & 25,5 \\
\hline & $60-69$ & 155 & 16,7 \\
\hline \multirow[t]{4}{*}{ Bundesland } & Baden-Württemberg & 125 & 13,4 \\
\hline & Bayern & 158 & 17,0 \\
\hline & Berlin & 36 & 3,9 \\
\hline & Brandenburg & 25 & 2,7 \\
\hline
\end{tabular}




\begin{tabular}{|c|c|c|c|}
\hline & Bremen & 7 & 0,8 \\
\hline & Hamburg & 17 & 1,8 \\
\hline & Hessen & 73 & 7,8 \\
\hline & Mecklenburg-Vorpommern & 17 & 1,8 \\
\hline & Niedersachsen & 94 & 10,1 \\
\hline & Nordrhein-Westfalen & 213 & 22,9 \\
\hline & Rheinland-Pfalz & 52 & 5,6 \\
\hline & Saarland & 10 & 1,1 \\
\hline & Sachsen & 32 & 3,4 \\
\hline & Sachsen-Anhalt & 24 & 2,6 \\
\hline & Schleswig-Holstein & 28 & 3,0 \\
\hline & Thüringen & 19 & 2,0 \\
\hline Einkommensklasse & $0 €$ bis unter $2500 €$ & 382 & 41,1 \\
\hline & $2500 €$ bis unter $4.500 €$ & 374 & 40,2 \\
\hline & $4.500 €$ und mehr & 174 & 18,7 \\
\hline Bildung & $\begin{array}{l}\text { Noch in schulischer Ausbildung } \\
\text { oder kein Bildungsabschluss }\end{array}$ & 14 & 1,6 \\
\hline & Volks- oder Hauptschule & 236 & 25,4 \\
\hline & Mittlere Reife/Realschule & 318 & 34,2 \\
\hline & (Fach-)Hochschulreife/Abitur & 146 & 15,7 \\
\hline & Fachhochschule/Hochschule & 96 & 10,3 \\
\hline & Universität & 120 & 12,9 \\
\hline
\end{tabular}

Quelle: Eigene Darstellung

\subsection{Einkaufs- und Ernährungsverhalten}

Bevor in Unterkapitel 5.3 die Forschungsergebnisse zu den Fragen vorgestellt werden, die sich konkret auf Tierwohl-Labels beziehen, sollen zunächst die Befragungsergebnisse zum allgemeinen Einkaufs- und Ernährungsverhalten der Befragten dargestellt werden. Das Einkaufs- und Ernährungsverhalten ist ein bedeutender Bestandteil der vorliegenden Arbeit, da ausgewählte Fragen zu den Tierwohl-Labels in Bezug auf das Einkaufs- und Ernährungsverhalten analysiert wurden. 
In der ersten Frage des Fragebogens wurde zunächst ermittelt, für wen die Befragten gewöhnlich Fleisch- und Wurstwaren einkaufen. Dabei konnte der Anteil an Vegetariern oder Veganern in der Stichprobe ermittelt werden. Die Frage diente als unkomplizierte Einstiegsfrage sowie als Kontrollvariable. Vegetarier und Veganer, die Fleisch- und Wurstwaren für andere Personen im Haushalt kaufen, sollten in der vorliegenden Studie explizit berücksichtigt werden, da alle Fleischkäufer befragt werden sollten. Die Auswertung zeigt, dass der Großteil der Befragten nicht nur für sich selbst einkauft, sondern auch für andere Menschen wie beispielsweise Partner, Kinder oder Freunde. $80 \%$ kaufen für sich selbst und andere Personen ein. $17 \%$ der Befragten kaufen Fleischoder Wurstwaren nur für sich selbst ein. Dies sind zum Beispiel Personen, die allein in ihrem Haushalt leben oder gemeinsam mit einer Person, die kein Fleisch isst. Rund $3 \%$ kaufen ausschließlich für andere Personen Fleisch- oder Wurstwaren. Diese Personen sind Vegetarier oder Veganer.

In den beiden anschließenden Fragen (Frage 2 und Frage 3) wurden die Präferenzen bei der Einkaufsstättenwahl für den Einkauf von Fleisch- und Wurstwaren in zwei verschiedenen Varianten erfragt, um diese wesentliche Frage für Hersteller und Händler von Fleisch- und Wurstwaren differenziert zu beantworten. Als Besonderheit wurden verschiedene genutzte Einkaufsstätten erfragt und zusätzlich wurde die Haupteinkaufsstätte für Fleisch- und Wurstwaren ermittelt.

\subsubsection{Einkaufsstättenwahl}

Wie im Fragebogen (Anhang) ersichtlich konnten die Befragten bei einer Mehrfachnennung zunächst aus 24 verschiedenen Einkaufsstätten alle Einkaufsstätten wählen, die sie in den letzten drei Monaten für ihren Fleisch- und Wursteinkauf genutzt haben. Dabei wurden neben den großen und bekannten Lebensmitteleinzelhändlern und dem Metzger auch zahlreiche andere Einkaufsstätten zur Auswahl angeboten, wie beispielsweise regionale Supermärkte, Bio-Supermärkte, Wochenmärkte, Hofläden und Online-Shops. Daneben hatten die Befragten die Möglichkeit, unter ,Andere Einkaufsstätten` weitere Einkaufsstätten einzutragen. 
Abbildung 6 zeigt im Überblick die Marktdurchdringung der bedeutendsten Einkaufsstätten für Fleisch- und Wurstwaren und gibt Aufschluss darüber, wieviel Prozent der Befragten welche Einkaufsstätten angegeben haben. Es wird deutlich, dass der Großteil der Menschen Fleisch- oder Wurstwaren in Supermärkten (EDEKA, REWE), Discountern (ALDI, LIDL, NETTO, PENNY) und SB-Warenhäusern (KAUFLAND und REAL) gekauft hat. Rund die Hälfte aller Befragten hat bei EDEKA, REWE, ALDI und LIDL Fleisch- und Wurstwaren eingekauft. ALDI NORD und ALDI SÜD wurden in der Befragung zunächst getrennt erfragt, um zukünftig die Möglichkeit für weitere detaillierte Analysen zu erhalten, jedoch wurden ALDI NORD und ALDI SÜD in dieser Auswertung zusammengefasst. Ein Drittel der Befragten hat den Metzger als Einkaufsstätte angegeben, was ungefähr der gleichen Größenordnung von NETTO oder auch KAUFLAND entspricht.

Rund $20 \%$ der Befragten haben in den letzten drei Monaten spezielle Supermärkte wie Combi oder tegut genutzt, die aufgrund ihrer eingeschränkten geografischen Verbreitung jedoch nur bedingt mit den bundesweit vertretenen Lebensmitteleinzelhändlern zu vergleichen sind. $14 \%$ haben ihren Einkauf im Hofladen oder auf dem Wochenmarkt direkt bei einem Landwirt getätigt. $6 \%$ aller Befragten haben in den letzten drei Monaten einen Bio-Supermarkt für den Einkauf von Fleisch- oder Wurstwaren genutzt und dort Fleisch- und Wurstwaren in Bio-Qualität gekauft.

Unter dem Punkt ,Bio-Supermärkte' wurden die Bio-Supermarktketten ALNATURA, DENN`S, BASIC BIO, SUPER BIO und BIO COMPANY zusammengefasst. Insgesamt bestätigten $20 \%$ der Befragten, in den letzten drei Monaten Fleisch- und Wurstwaren direkt beim Landwirt oder im Bio-Supermarkt gekauft zu haben.

Unter dem Punkt, Andere“ wurden von den Befragten noch einzelne besondere Einkaufsstätten erwähnt, wie beispielsweise Schlachthöfe mit Werksverkäufen oder türkische Lebensmittelhändler. Daneben wurden auch hauseigene Schlachtungen oder Auftragsschlachtungen (Crowdbutching) als Sondervarianten genannt. Auch wenn diese speziellen Einkaufsstätten durchaus interessant sind, finden sie im Rahmen dieser Studie keine weitere Berücksichtigung. 
Frage 2: Welche der folgenden Einkaufsstätten haben Sie in den letzten drei Monaten für Ihren Fleisch- und Wursteinkauf genutzt? Sie können mehrere Einkaufsstätten auswählen.

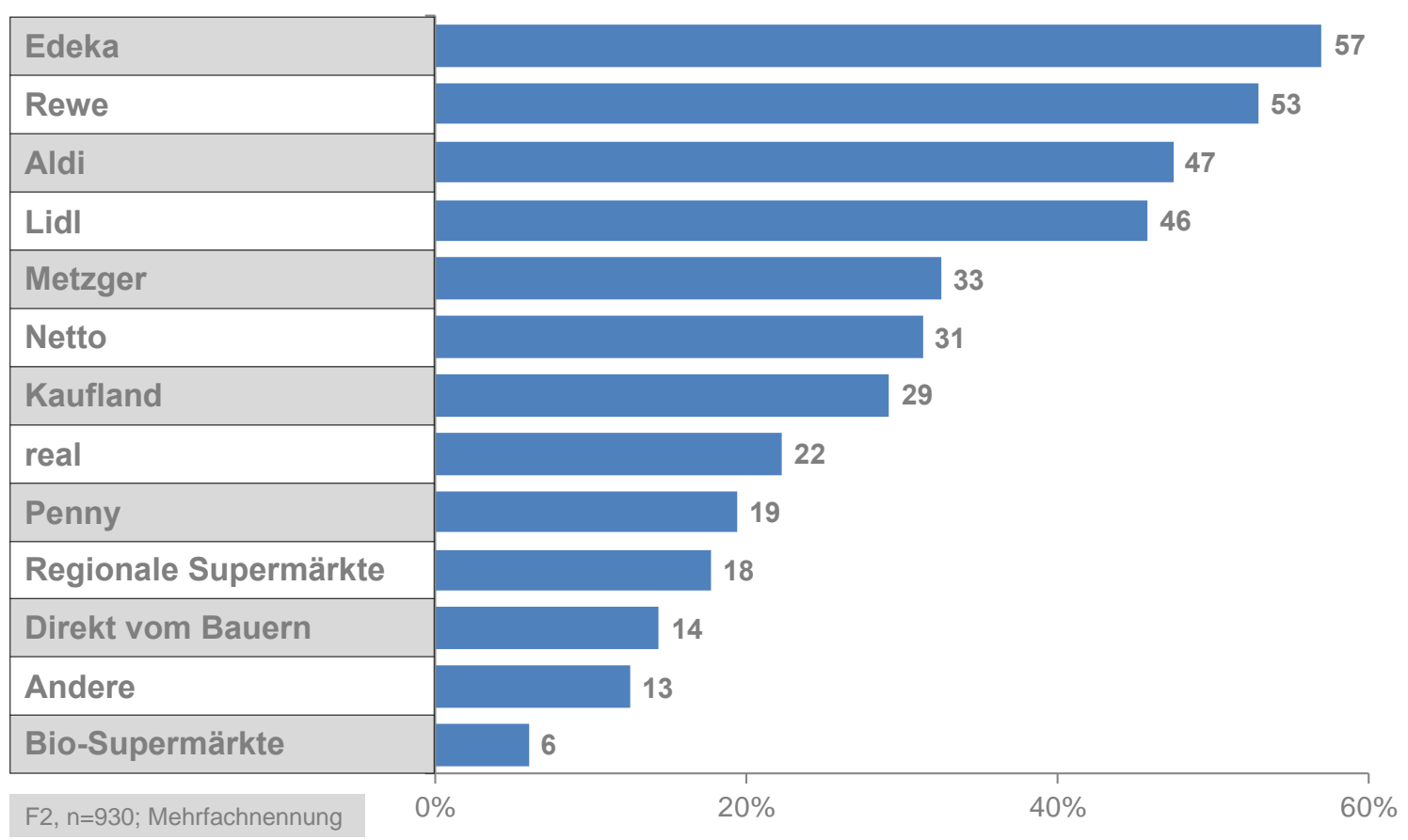

\section{Abbildung 6: Einkaufsstätten für Fleisch- und Wurstwaren (Quelle: Eigene Darstellung)}

Von größerem Interesse für die Betrachtung der tierwohlspezifischen Fragen ist jedoch die Angabe der Haupteinkaufsstätte für Fleisch- oder Wurstwaren (Frage 3). In Frage 3 sollten sich die Befragten auf eine einzige Haupteinkaufsstätte für Fleisch- und Wurstwaren festlegen. Mit dieser Einfachnennung ließen sich aussagekräftige Berechnungen durchführen.

In Abbildung 7 wird ersichtlich, dass $83 \%$ der Menschen in Deutschland ihre Fleischund Wurstwaren schwerpunktmäßig in sieben Einkaufsstätten einkaufen. Führend sind Discounter und SB-Warenhäuser (LIDL, KAUFLAND, ALDI, NETTO, REAL und PENNY) mit rd. $40 \%$ und die Supermarktketten EDEKA und REWE (rd. $35 \%$ ). $14 \%$ kaufen Fleisch- und Wurstwaren hauptsächlich beim Metzger. Nur $3 \%$ der befragten Personen kaufen Fleisch- oder Wurstwaren schwerpunktmäßig direkt beim Erzeuger (Wochenmarkt oder Hofladen). 
Frage 3: Sie haben die folgenden Einkaufsstätten ausgewählt, wo Sie Fleisch- und Wurstwaren kaufen. Welche dieser Einkaufsstätten ist Ihre Haupteinkaufsstätte für Ihren Fleisch- und Wursteinkauf? Nennen Sie nur eine Haupteinkaufsstätte.

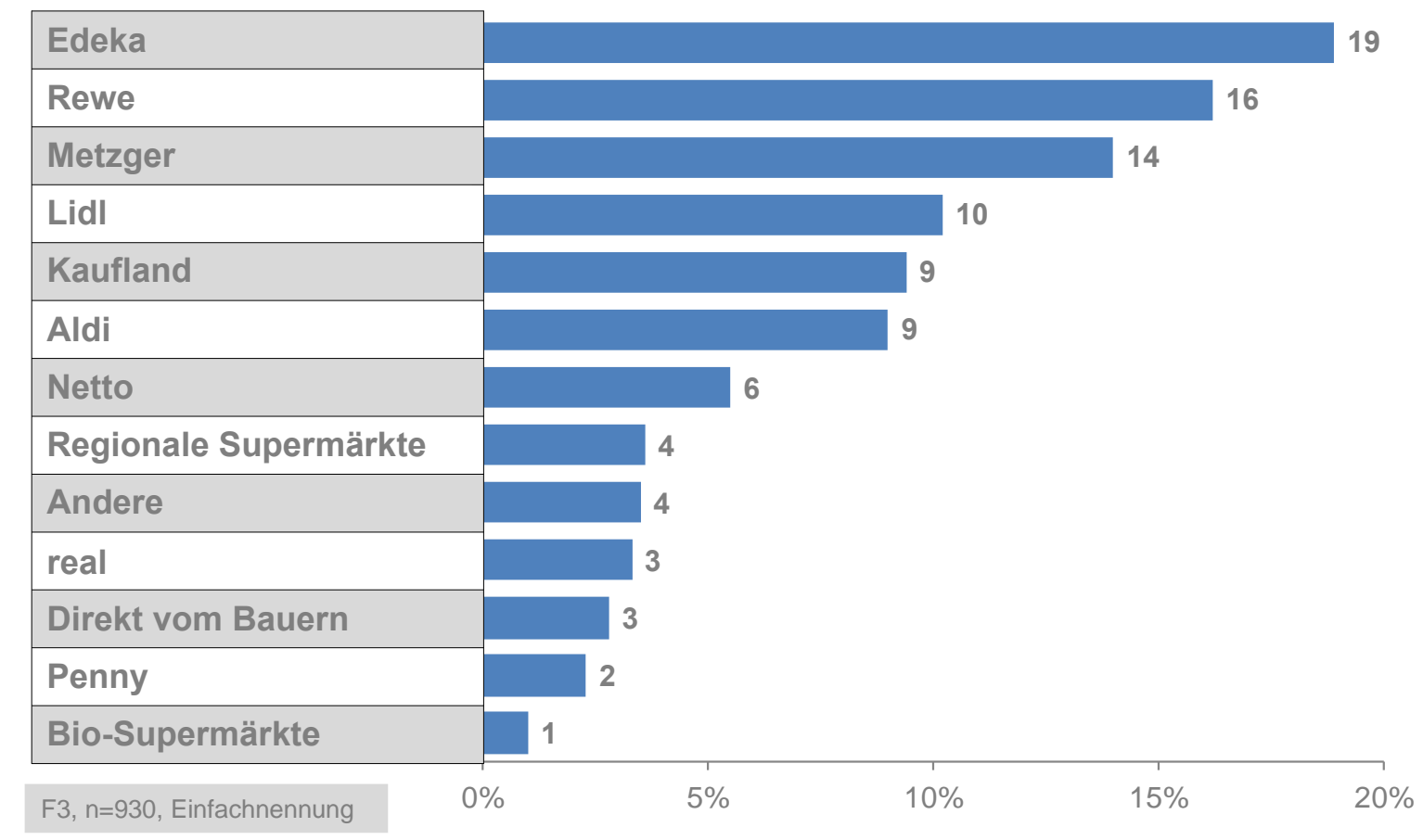

\section{Abbildung 7: Haupteinkaufsstätten für Fleisch- und Wurstwaren (Quelle: Eigene Darstellung)}

\subsubsection{Angebotsformen Fleischtheke oder Selbstbedienung}

In diesem Abschnitt werden zunächst die Ergebnisse der Präferenzen der Befragten für Theken- und SB-Ware dargestellt. Die Präferenzen der Menschen für die Frischetheke oder den Selbstbedienungsbereich wurden wie in Unterkapitel 3.1 beschrieben bereits in verschiedenen Studien untersucht. Die Ergebnisse der vorliegenden Arbeit sollen eine Ausgangsbasis für die Durchführung weiterführender Analysen hinsichtlich des Tierwohls schaffen. Daher werden die Ergebnisse an dieser Stelle nur kurz beschrieben. Zunächst wurde in Frage 4 erfragt, ob Wurstwaren oder Aufschnitt fürs Brot häufiger an der Fleischtheke oder im SB-Bereich gekauft werden. Die Ergebnisse sind in Tabelle 5 dargestellt. 
Bei Wurstwaren oder Aufschnitt teilen sich die Befragten der Studie ungefähr in zwei gleichgroße Gruppen an Thekenkäufern (36\%) und SB-Käufern (40 \%). Tabelle 5 zeigt, dass die Anzahl der Befragten, die die SB-Theke präferieren, geringfügig höher ist. Ein Viertel aller Befragten gab an, beide Varianten etwa gleich oft zu nutzen.

Tabelle 5: Präferierte Angebotsform für Wurstwaren oder Aufschnitt

Frage 4: Kaufen Sie Wurstwaren oder Aufschnitt fürs Brot häufiger beim Verkaufspersonal an der Fleischtheke oder im Selbstbedienungsbereich?

\begin{tabular}{|c|c|c|c|c|}
\hline $\begin{array}{c}\text { fast nur an der } \\
\text { Fleischtheke }\end{array}$ & $\begin{array}{c}\text { häufiger an der } \\
\text { Fleischtheke }\end{array}$ & ca. gleich viel & $\begin{array}{c}\text { häufiger im SB- } \\
\text { Bereich }\end{array}$ & $\begin{array}{c}\text { fast nur im SB- } \\
\text { Bereich }\end{array}$ \\
\hline $19 \%$ & $17 \%$ & $24 \%$ & $21 \%$ & $19 \%$ \\
\hline F4, n=930; Skala -2 bis +2; Mittelwert -0,04, Standardabweichung 1,376 & & \\
\hline
\end{tabular}

\section{Quelle: Eigene Darstellung}

Die Fleischtheke ist beim Einkauf von Fleisch zum Braten und Grillen demnach deutlich beliebter als der SB-Bereich. Etwa die Hälfte aller Befragten (49\%) kauft häufiger oder fast nur an der Fleischtheke und somit deutlich häufiger als im SB-Bereich (31\%). Ein Fünftel der 930 Befragten nutzt beide Varianten ungefähr gleich viel, wie in Tabelle $6 \mathrm{zu}$ erkennen.

Tabelle 6: Präferierte Angebotsform für Fleisch zum Braten und Grillen

Frage 5: Kaufen Sie Fleisch zum Braten oder Grillen häufiger beim Verkaufspersonal an der Fleischtheke oder im Selbstbedienungsbereich?

\begin{tabular}{|c|c|c|c|c|}
\hline $\begin{array}{c}\text { fast nur an der } \\
\text { Fleischtheke }\end{array}$ & $\begin{array}{c}\text { häufiger an der } \\
\text { Fleischtheke }\end{array}$ & ca. gleich viel & $\begin{array}{c}\text { häufiger im SB- } \\
\text { Bereich }\end{array}$ & $\begin{array}{c}\text { fast nur im SB- } \\
\text { Bereich }\end{array}$ \\
\hline $29 \%$ & $20 \%$ & $20 \%$ & $18 \%$ & $13 \%$ \\
\hline
\end{tabular}

F5, $n=930$; Skala -2 bis +2; Mittelwert 0,34, Standardabweichung 1,402

\section{Quelle: Eigene Darstellung}




\subsubsection{Verzehrmenge und Reduktionsbereitschaft}

Neben der Einkaufsstätte und der Angebotsform (Theke oder SB) im Lebensmitteleinzelhandel sind auch die Verzehrhäufigkeit im Haushalt und die Reduktionsbereitschaft der nachhaltigkeitsorientierten Fleischkäufer von Interesse für die Tierwohlforschung, wie in Kapitel 3 beschrieben. Daher wurden die Befragten in Frage 6 danach gefragt, wie oft in ihrem Haushalt Fleisch- oder Wurstwaren gegessen werden. $11 \%$ der Befragten gaben an, dass mehrmals täglich Fleisch oder Wurst im Haushalt gegessen werde. Diese Haushalte könnten demnach als ,Fleischfans-Haushalte“ charakterisiert werden. Bei rd. zwei Drittel der Befragten (68 \%) werden fast jeden Tag Fleisch- oder Wurstwaren gegessen (einmal täglich/drei bis fünf Mal wöchentlich). Diese Haushalte könnten somit als normale ,Durchschnittshaushalte" bezüglich des Fleischkonsums in Deutschland bezeichnet werden. Etwa $20 \%$ essen nur ein bis zwei Mal wöchentlich Fleisch- oder Wurstwaren oder weniger als ein Mal wöchentlich (5\%). Diese Haushalte könnten z. B. als ,Flexitarier-Haushalt‘ charakterisiert werden.

In Abbildung 8 ist die Reduktionsbereitschaft der befragten Personen dargestellt. Dabei wird deutlich, dass rd. $30 \%$ der Befragten ihren Fleischkonsum nicht reduzieren möchten. $26 \%$ haben ihren Fleischkonsum noch nicht reduziert, möchten aber in Zukunft etwas weniger Fleisch essen.

Rund $40 \%$ haben ihren Fleischkonsum bereits reduziert, möchten aber nicht ganz auf Fleisch verzichten. $5 \%$ der Studienteilnehmer sind Vegetarier oder Veganer oder möchten es gerne werden. Wie zu Beginn von Unterkapitel 5.2 bereits beschrieben sollten Vegetarier und Veganer explizit bei der Befragung berücksichtigt werden, da diese Personen häufig für andere Personen im Haushalt Fleisch- und Wurstwaren kaufen und der Fokus bei der Befragung auf alle Fleischkäufer gerichtet wurde. Rund ein Drittel der Befragten plant, in Zukunft weniger Fleisch zu essen, oder ist bereits Vegetarier/Veganer. Insgesamt haben rd. zwei Drittel aller Befragten ihren Fleischkonsum bereits reduziert oder möchten den Fleischkonsum reduzieren. 
Frage 7: Welche der folgenden Aussagen trifft am ehesten auf Sie zu?

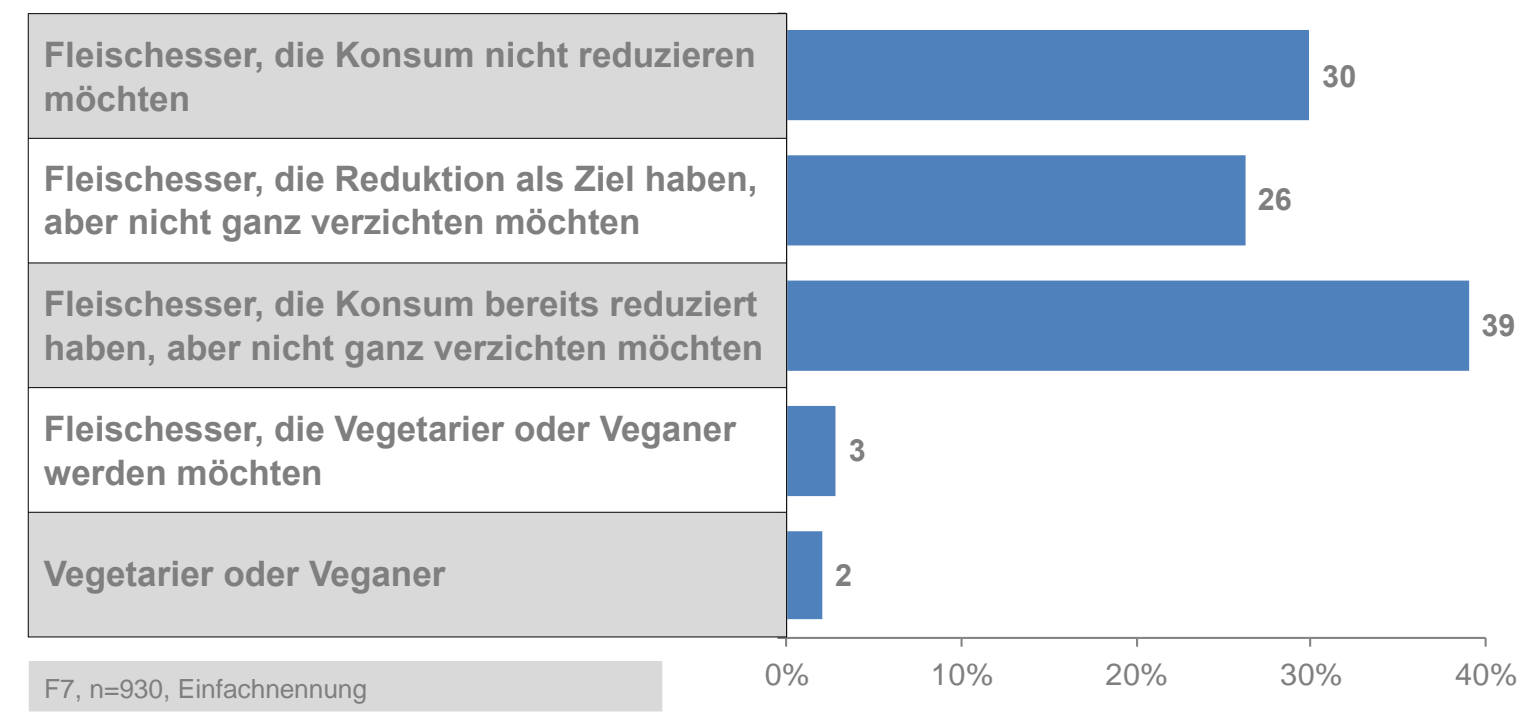

\section{Abbildung 8: Reduktion des Konsums von Fleisch- und Wurstwaren (Quelle: Eigene Darstellung)}

\subsubsection{Klassische und ethische Kaufkriterien}

Für die Vermarktung von Produkten spielt das Wissen über relevante Kaufkriterien eine zentrale Rolle. Anbieter, die die Gewichtung der einzelnen Kaufkriterien ihrer Kunden kennen, können die unterschiedlichen Kriterien gezielter im Marketing einsetzen. Wie in Kapitel 2 beschrieben haben sich die Anforderungen der Kunden an die Hersteller von Fleisch- und Wurstwaren in den letzten Jahren stark verändert. Es wird heute vermehrt auf Nachhaltigkeitskriterien wie Tierwohl oder Umweltfreundlichkeit geachtet. In Abbildung 9 werden die Mittelwerte der Kaufkriterien aufgeführt. Es kann festgestellt werden, dass die klassischen Kaufkriterien (hellblaue Balken) Frische, Geschmack und Aussehen für die meisten Befragten eine Grundvoraussetzung für den Kauf darstellen. Hinsichtlich der Nachhaltigkeitskriterien (dunkelblaue Balken) sind Regionalität und artgerechtere Tierhaltung die bedeutsamsten Kriterien, gefolgt von Umweltfreundlichkeit und Gesundheit. Auf eine ansprechende Verpackung, bestimmte Marken und faire Löhne für Landwirte achten die Befragten beim Fleischkauf vergleichsweise wenig. 
Frage 8: Worauf achten Sie persönlich beim alltäglichen Einkauf von Fleisch- oder Wurstwaren?

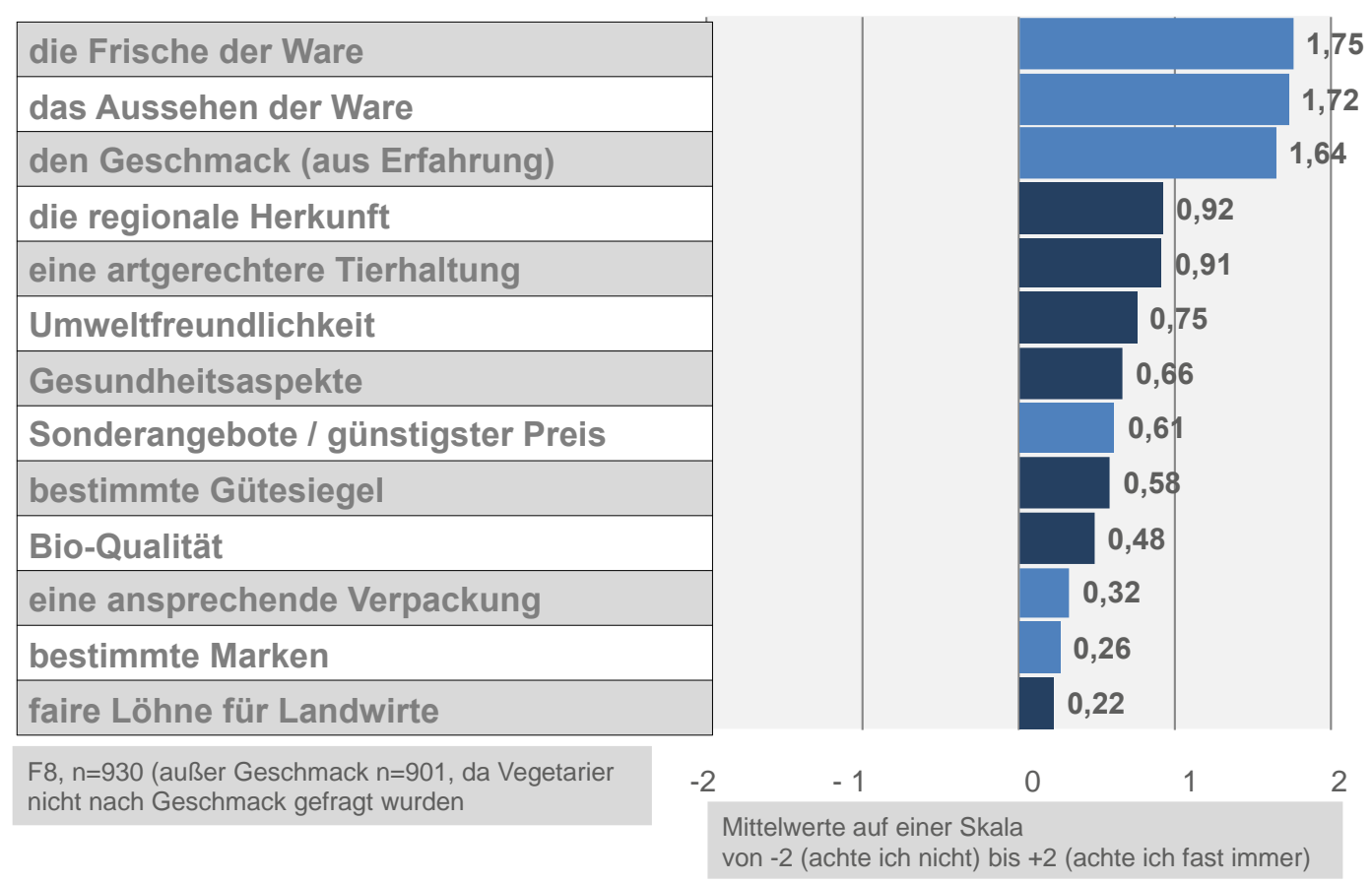

\section{Abbildung 9: Kaufkriterien beim Einkauf von Fleisch- und Wurstwaren (Quelle: Eigene Darstellung)}

Bei den Kaufkriterien artgerechtere Tierhaltung, Gesundheitsaspekte und Umweltfreundlichkeit handelt es sich um die drei zentralen Aspekte, die von TierwohlLabels abgebildet werden. Daher wurden die Zusammenhänge dieser drei Kriterien mit der Haupteinkaufsstätte, der Angebotsform (Theke/SB) und ausgewählten soziodemografischen Kriterien untersucht. Die Ergebnisse werden im Folgenden dargestellt.

Für die Auswertung wurden die meistgenannten Haupteinkaufsstätten in die Gruppen Supermarkt (EDEKA, REWE), Discounter (ALDI, LIDL, NETTO, PENNY), SBWarenhaus (KAUFLAND, REAL) und Metzger unterteilt. In Abbildung 10 lässt sich erkennen, dass ein hoch signifikanter Unterschied beim Kaufkriterium artgerechtere Tierhaltung $(\mathrm{F}(3,822)=8,272, \mathrm{p}<0,01, \mathrm{n}=826)$ zwischen den Haupteinkaufsstätten besteht. 
Im post-hoc-Test mit Bonferroni-Korrektur zeigten sich hoch signifikante Unterschiede ( $\mathrm{p}>0,01)$ zwischen Metzgerei-Käufern $(\mathrm{MW}=1,25$; SD =0,989) und SB-WarenhausKäufern $(\mathrm{MW}=0,67 ; \mathrm{SD}=1,071)$ sowie Metzgerei-Käufern $(\mathrm{MW}=1,25 ; \mathrm{SD}=0,989)$ und Discounter-Käufern (MW = 0,76; SD = 1,020).

Zudem konnte ein signifikanter Unterschied zwischen Metzgerei-Käufern (MW = 1,25; $\mathrm{SD}=0,989)$ und Supermarkt-Käufern $(\mathrm{MW}=0,91 ; \mathrm{SD}=1,060)$ ermittelt werden. Metzgerei-Käufer achten demnach häufiger auf das Kriterium artgerechtere Tierhaltung als Supermarkt-Käufer, SB-Warenhaus-Käufer und Discounter-Käufer.

Auch beim Kriterium Umweltfreundlichkeit $(\mathrm{F}(3,822)=5,152, \mathrm{p}<0,01, \mathrm{n}=826)$ besteht ein hoch signifikanter Unterschied zwischen den Haupteinkaufsstätten. Im posthoc-Test mit Bonferroni-Korrektur ergaben sich ein hoch signifikanter Unterschied $(\mathrm{p}<0,01)$ zwischen Metzgerei-Käufern $(\mathrm{MW}=0,98$; SD = 1,082) und SB-WarenhausKäufern $(\mathrm{MW}=0,47 ; \mathrm{SD}=1,076)$ sowie ein signifikanter Unterschied $(\mathrm{p}<0,5)$ zwischen Metzgerei-Käufern (MW $=0,98 ; \quad S D=1,082)$ und Discounter-Käufern (MW $=0,65 ; \mathrm{SD}=1,018)$. Metzgerei-Käufer achten demnach auch häufiger auf das Kriterium Umweltfreundlichkeit als SB-Warenhaus-Käufer und Discounter-Käufer.

Beim Kriterium Gesundheit $(\mathrm{F}(3,822)=3,795, \mathrm{p}=0,01, \mathrm{n}=826)$ zeigte sich ebenfalls ein signifikanter Unterschied zwischen den Haupteinkaufsstätten. Anhand von post-hocTests mit Bonferroni-Korrektur konnte ein signifikanter Unterschied $(\mathrm{p}<0,05)$ zwischen Metzgerei-Käufern (MW = 0,92; SD = 1,093) und SB-Warenhaus-Käufern ( $\mathrm{MW}=0,48$; $\mathrm{SD}=1,130)$ sowie Metzgerei-Käufern $(\mathrm{MW}=0,92 ; \mathrm{SD}=1,093)$ und DiscounterKäufern $(\mathrm{MW}=0,58 ; \mathrm{SD}=1,083)$ ermittelt werden.

Zudem existiert ein schwach signifikanter Unterschied $(\mathrm{p}<0,1)$ zwischen MetzgereiKäufern $(\mathrm{MW}=0,92 ; \mathrm{SD}=1,093)$ und Supermarkt-Käufern $(\mathrm{MW}=0,63 ; \mathrm{SD}=1,091)$. Mit Blick auf die Mittelwerte wird deutlich, dass Metzgerei-Käufer häufiger auf das Kriterium Gesundheit achten als Supermarkt-Käufer, SB-Warenhaus-Käufer und Discounter-Käufer. 
Hypothese 1: Es besteht ein Zusammenhang zwischen der Beachtung der Kaufkriterien artgerechtere Tierhaltung, Gesundheit sowie Umweltfreundlichkeit und der Haupteinkaufsstätte.

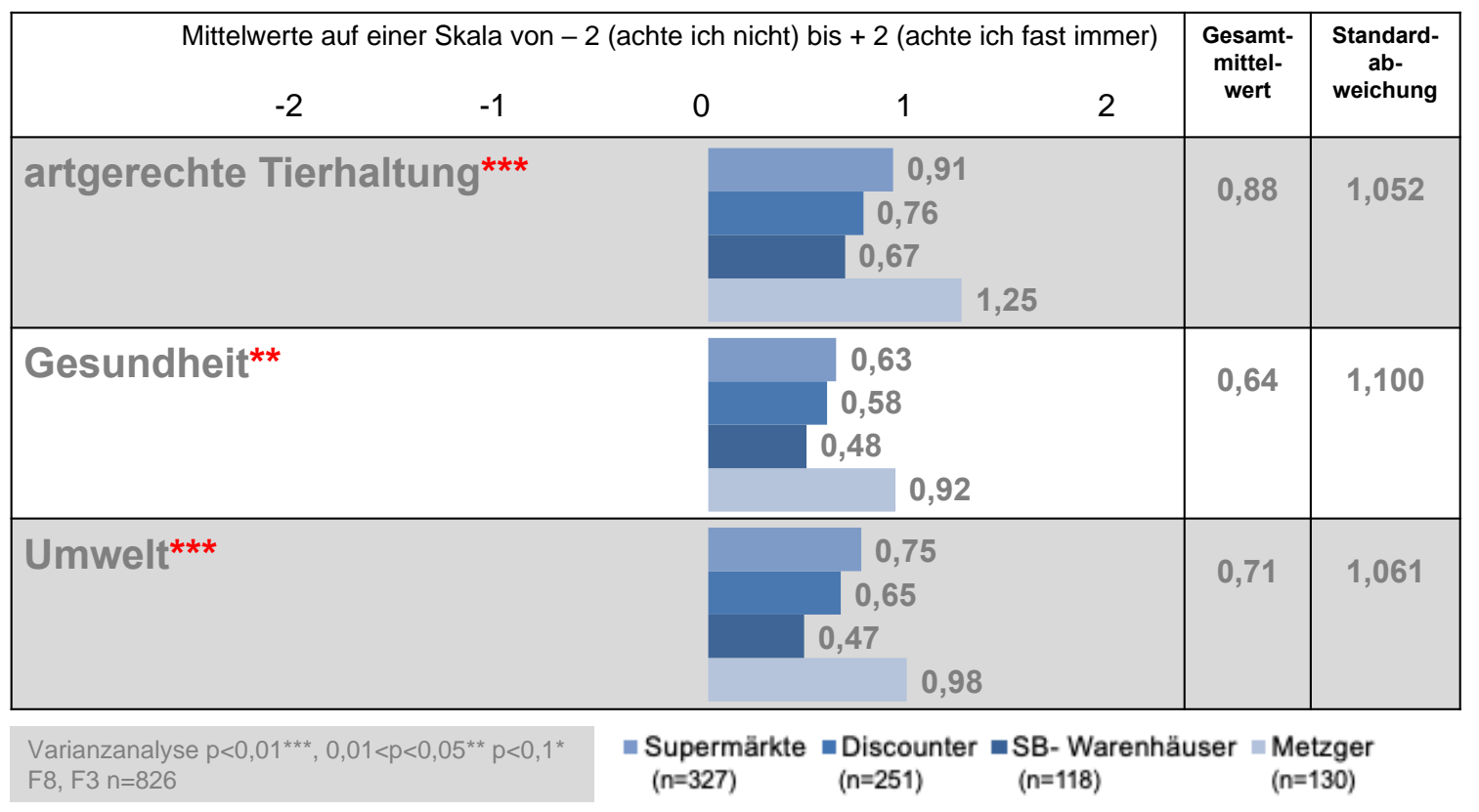

\section{Abbildung 10: Zusammenhang Kaufkriterien und Haupteinkaufsstätte (Quelle: Eigene Darstellung)}

Neben den oben dargestellten Auswertungen der Zusammenhänge zwischen den persönlichen Kaufkriterien der Befragten und der präferierten Haupteinkaufsstätte wurden in der vorliegenden Studie auch die Zusammenhänge zwischen den persönlichen Kaufkriterien und der präferierten Angebotsform (Fleischtheke oder Selbstbedienungsbereich) untersucht.

Wie in Tabelle 7 ersichtlich besteht eine hoch signifikante, positive Korrelation beim Kriterium artgerechtere Tierhaltung und Umweltfreundlichkeit hinsichtlich der Angebotsform (Theke oder SB-Bereich) für Wurst oder Aufschnitt. Hierbei handelt es sich um eine schwache Korrelation $(r<0,4)$. Dies bedeutet, dass Käufer, die häufiger an der Theke kaufen, eher auf eine artgerechtere Tierhaltung und Umweltfreundlichkeit achten als Käufer, die Wurst oder Aufschnitt häufiger im SB-Bereich kaufen. Zudem liegt eine signifikante, positive Korrelation beim Kriterium Gesundheit vor. Hierbei handelt es sich jedoch um eine sehr schwache Korrelation $(r<0,2)$. 
Hypothese 2: Personen, die auf eine artgerechtere Tierhaltung, Gesundheitsaspekte und Umweltfreundlichkeit achten, kaufen Wurstwaren oder Aufschnitt fürs Brot häufiger an der Theke.

Tabelle 7: Korrelation Kaufkriterien und Angebotsform - Wurst und Aufschnitt

\begin{tabular}{|l|l|}
\hline Angebotsform Wurst und Aufschnitt & Korrelationskoeffizient \\
\hline artgerechtere Tierhaltung & $0,223 * * *$ \\
\hline Gesundheitsaspekte & $0,156 * * *$ \\
\hline Umweltfreundlichkeit & $0,266 * * *$ \\
\hline
\end{tabular}

Korrelation, $p<0,01^{* \star *}, 0,01<p<0,05^{* *} p<0,1^{*}$

$F 4, F 8 n=930$

\section{Quelle: Eigene Darstellung}

Gemäß Tabelle 8 besteht eine hoch signifikante, positive Korrelation bei allen drei Kriterien hinsichtlich der Angebotsform für Fleisch zum Braten und Grillen. Hierbei handelt es sich um eine sehr schwache Korrelation $(r<0,2)$, weshalb konstatiert werden kann, dass Käufer, die häufiger an der Theke kaufen, eher auf die Kriterien artgerechtere Tierhaltung, Gesundheit und Umweltfreundlichkeit achten als Käufer, die Wurst oder Aufschnitt häufiger im SB-Bereich kaufen.

Hypothese 3: Personen, die auf eine artgerechtere Tierhaltung, Gesundheitsaspekte und Umweltfreundlichkeit achten, kaufen Fleisch zum Braten und Grillen häufiger an der Theke.

Tabelle 8: Korrelation Kaufkriterien und Angebotsform - Fleisch zum Braten

\begin{tabular}{|l|l|}
\hline Angebotsform Fleisch zum Braten und Grillen & Korrelationskoeffizient \\
\hline artgerechtere Tierhaltung & $0,116 * * *$ \\
\hline Gesundheitsaspekte & $0,156 * * *$ \\
\hline Umweltfreundlichkeit & $0,186 * * *$ \\
\hline
\end{tabular}

Korrelation, $p<0,01^{\star \star \star}, 0,01<p<0,05^{* \star} p<0,1^{*}$

$F 5, F 8 n=930$

\section{Quelle: Eigene Darstellung}


Tabelle 9 veranschaulicht den Zusammenhang zwischen den Kaufkriterien artgerechtere Tierhaltung, Gesundheit sowie Umweltfreundlichkeit und ausgewählten soziodemografischen Daten der Befragten. So lassen sich hinsichtlich des Geschlechts hoch signifikante Unterschiede beim Kriterium artgerechtere Tierhaltung $(\mathrm{F}(1,928)=7,034, \mathrm{p}<0,01, \mathrm{n}=930)$ feststellen.

Bei den Kriterien Umweltfreundlichkeit $(\mathrm{F}(1,928)=4,796, \mathrm{p}=0,029, \mathrm{n}=930)$ und Gesundheit $(\mathrm{F}(1,928)=4,460, \mathrm{p}=0,035, \mathrm{n}=930)$ liegen signifikante Unterschiede vor. Mit Blick auf die Mittelwerte lässt sich erkennen, dass Frauen mehr auf diese Kriterien achten als Männer.

Zur Auswertung der Altersklasse erfolgte die Unterteilung in drei Gruppen: ,Junge Befragte' im Alter von 18 bis 29 Jahren, Befragte im ,mittleren Alter' von 30 bis 59 Jahren sowie ,ältere Befragte‘ im Alter von über 60 Jahren.

Bei der Auswertung der Altersklassen ergaben sich hoch signifikante Unterschiede beim Kriterium artgerechtere Tierhaltung $(\mathrm{F}(2,927)=8,931, \mathrm{p}<0,01, \mathrm{n}=930)$. In post-hocTests mit Bonferroni-Korrektur erwiesen sich diese Unterschiede als hoch signifikant ( $\mathrm{p}<0,01)$ zwischen den älteren Befragten $(\mathrm{MW}=1,19 ; \mathrm{SD}=1,19)$ und den jungen Befragten $(\mathrm{MW}=0,78 ; \mathrm{SD}=1,124)$ sowie den Befragten im mittleren Lebensalter $(\mathrm{MW}=0,88 ; \mathrm{SD}=1,054)$. Demnach gaben signifikant mehr ältere Personen an, auf eine artgerechtere Tierhaltung zu achten, als Personen mittleren Alters oder junge Befragte. 
Hypothese 4: Es besteht ein Zusammenhang zwischen der Beachtung der Kaufkriterien artgerechtere Tierhaltung, Gesundheit sowie Umweltfreundlichkeit und der Soziodemografie.

Tabelle 9: Zusammenhang Kaufkriterien und Soziodemografie

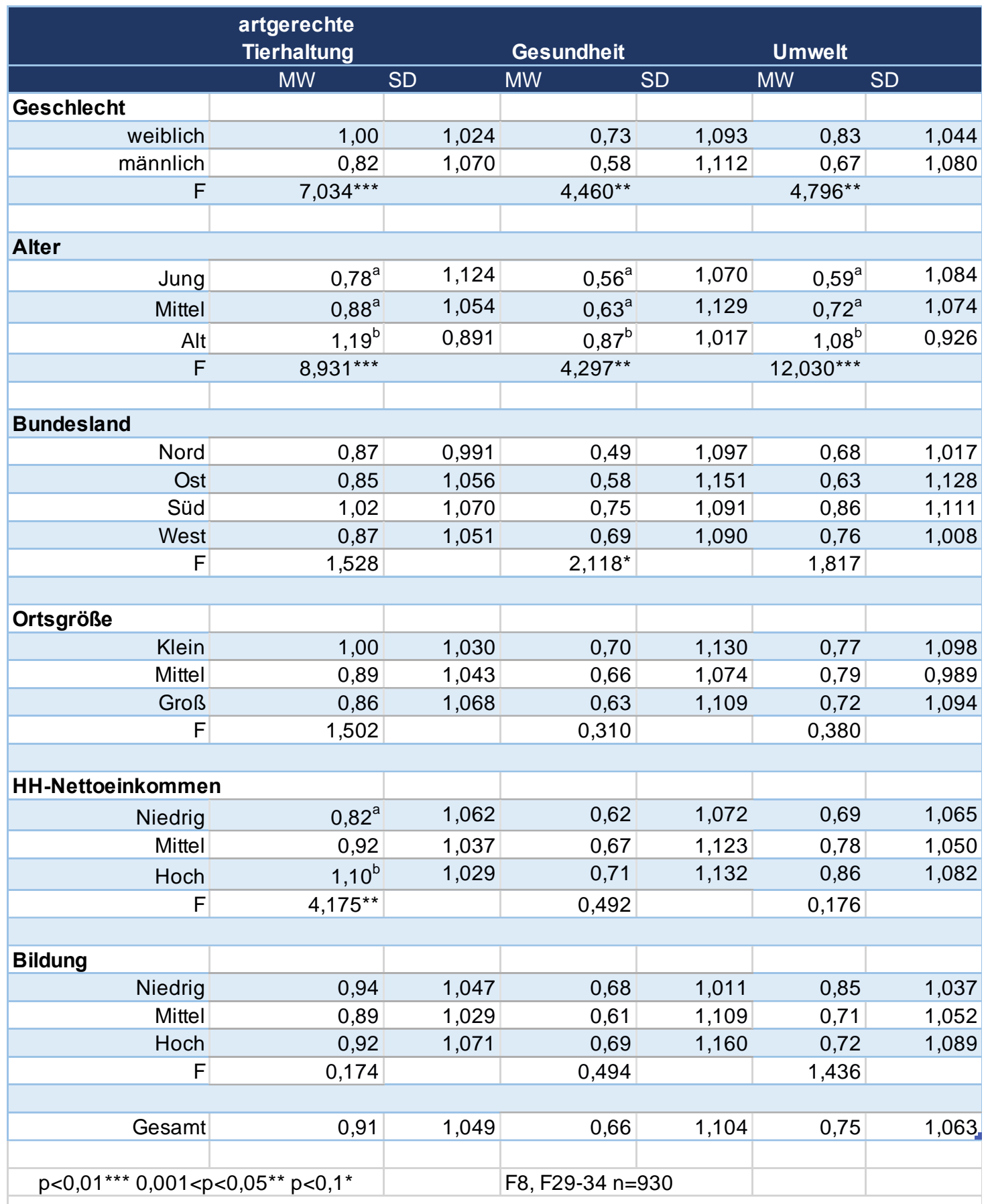

Anmerkung: Gruppen mit unterschiedlichen Kennbuchstaben $(a, b)$ unterscheiden sich mindestens signifikant auf dem 5\% Niveau; MW=Mittelwert / SD=Standardabweichung

\section{Quelle: Eigene Darstellung}


Ebenso besteht ein hoch signifikanter Unterschied beim Kriterium Umweltfreundlichkeit $(\mathrm{F}(2,927)=12,030, \mathrm{p}<0,01, \mathrm{n}=930)$. In den post-hoc-Tests mit Bonferroni-Korrektur ergaben sich signifikante Unterschiede $(\mathrm{p}<0,01)$ zwischen den älteren Befragten $(\mathrm{MW}=1,08 ; \mathrm{SD}=0,926)$ und den jungen Befragten $(\mathrm{MW}=0,59 ; \mathrm{SD}=1,084)$ und den Befragten im mittleren Alter ( $\mathrm{MW}=0,72 ; \mathrm{SD}=1,074)$.

Auch beim Kriterium Gesundheit $(\mathrm{F}(2,927)=4,297, \mathrm{p}=0,014, \mathrm{n}=930)$ liegt ein signifikanter Unterschied zwischen den Altersklassen vor. Im post-hoc-Test mit Bonferroni-Korrektur zeigten sich diese Unterschiede signifikant $(\mathrm{p}<0,05)$ zwischen den älteren Befragten $(\mathrm{MW}=0,87 ; \mathrm{SD}=1,017)$ und den jungen Befragten $(\mathrm{MW}=0,56$; $\mathrm{SD}=1,070)$ sowie Befragten im mittleren Alter $(\mathrm{MW}=0,63 ; \mathrm{SD}=1,129)$. Bei einem Vergleich der Mittelwerte lässt sich erkennen, dass die älteren Befragten angaben, mehr Wert auf alle drei untersuchten Kriterien (artgerechtere Tierhaltung, Umweltfreundlichkeit und Gesundheit) zu legen als die jungen und mittelalten Befragten.

Beim Herkunftsbundesland wurde vor der Auswertung ebenfalls eine Gruppenbildung vorgenommen, wobei zunächst eine Einteilung in die Nielsen-Gebiete erfolgte. Auf dieser Grundlage wurden die Bundesländer anschließend in die Bereiche Nord (Schleswig-Holstein, Hamburg, Niedersachsen und Bremen), Ost (Berlin, MecklenburgVorpommern, Brandenburg, Sachsen-Anhalt, Thüringen und Sachsen), Süd (BadenWürttemberg und Bayern) und West (Nordrhein-Westfalen, Hessen, Rheinland-Pfalz und Saarland) eingeteilt. Bei der Herkunft wurde lediglich ein schwach signifikanter Unterschied im Bereich Gesundheit ermittelt $(\mathrm{F}(2,927)=2,118 \mathrm{p}=0,096, \mathrm{n}=930)$. Im Süden Deutschlands wird demnach beim Fleischkauf vermutlich stärker auf Gesundheit geachtet als in Norddeutschland.

Bei der Auswertung der Ortsgröße wurde ebenfalls eine Gruppenbildung vorgenommen. Es erfolgte die Einteilung in ,Klein` (Dörfer und Städte bis 10.000 Einwohner), ,Mittel ‘ (Städte von 10.000 bis 100.000 Einwohner) und ,Groß‘ (Städte mit über 100.000 Einwohnern). Es konnte jedoch kein Zusammenhang zwischen der Ortsgröße und der Berücksichtigung der Kriterien artgerechtere Tierhaltung, Gesundheit und Umwelt festgestellt werden. 
Das Nettoeinkommen wurde vor der Auswertung in die Gruppen ,niedrig ‘ (<2500 Euro), ,mittel (2500 bis 4500 Euro) und ,hoch` (> 45000 Euro) eingeteilt. Beim Nettoeinkommen ließ sich ein signifikanter Unterschied beim Kriterium artgerechtere Tierhaltung ermitteln $(F(2,927)=4,175, p=0,016, n=930$.

Im post-hoc-Test mit Bonferroni-Korrektur zeigte sich ein signifikanter Unterschied zwischen Personen mit niedrigem $(\mathrm{MW}=0,82 ; \mathrm{SD}=0,82)$ und hohem $(\mathrm{MW}=1,10$; $\mathrm{SD}=1,029)$ Einkommen. Die Mittelwerte lassen erkennen, dass Personen mit höherem Einkommen häufiger auf eine artgerechtere Tierhaltung achten.

Um das Bildungsniveau der Befragten einzuteilen, wurde nach dem höchsten Abschluss gefragt. Diesbezüglich erfolgte eine Einteilung in die drei Gruppen ,niedrige Bildung ‘ (ohne allgemeinen Schulabschluss, noch in schulischer Ausbildung oder Volks- und Hauptschulabschluss), , mittlere Bildung' (Mittlere Reife, Realschule, Mittelschule) und ,hohe Bildung' (Fachhochschulreife, Abitur, abgeschlossenes Hochschul- oder Universitätsstudium). Hinsichtlich der Bildung konnte kein Zusammenhang mit der Berïcksichtigung der o. g. Kaufkriterien beobachtet werden.

Hypothese 5: Die Beachtung des Kaufkriteriums artgerechte Tierhaltung hängt damit zusammen, ob die Befragten ein Haustier besitzen.

Neben den soziodemografischen Kriterien wurde zusätzlich der Zusammenhang zwischen den drei soziodemografischen Kriterien Haustierbesitz, Religionszugehörigkeit und gewählte Partei bei der Bundestagswahl 2017 und dem Kaufkriterium artgerechtere Tierhaltung untersucht.

Dabei konnte festgestellt werden, dass ein hoch signifikanter Unterschied (F (1, 928) $=13,650, \mathrm{p}<0,01, \mathrm{n}=930$ ) zwischen Haustierbesitzern und Personen, die keine Haustiere besitzen, besteht. Mit Blick auf die Mittelwerte wird deutlich, dass Haustierbesitzer $(\mathrm{MW}=1,03 ; \mathrm{SD}=0,998)$ mehr auf eine artgerechtere Tierhaltung achten als die Befragten, die kein Haustier besitzen ( $\mathrm{MW}=0,78 ; \mathrm{SD}=1,089)$. 
Hypothese 6: Die Beachtung des Kaufkriteriums artgerechte Tierhaltung hängt damit zusammen, ob die Befragten einer Religion angehören.

Die Zugehörigkeit zu einer Religion hängt dagegen nicht signifikant mit dem Kaufkriterium artgerechtere Tierhaltung zusammen $(F(1,928)=1,369, p=0,242$, $\mathrm{n}=930)$.

Hypothese 7: Die Beachtung des Kaufkriteriums artgerechte Tierhaltung hängt damit zusammen, welche politische Partei die Befragten wählen.

Zur Auswertung des Zusammenhangs der gewählten Partei und der Berücksichtigung des Kaufkriteriums artgerechtere Tierhaltung wurden die sechs größten Parteien (CDU/CSU, SPD, AfD, FDP, Die Linken und Bündnis90/DieGrünen) berücksichtigt. Da auch ein besonderes Augenmerk auf die Wähler von Tierschutzparteien gelegt werden sollte, diese Gruppe für eine aussagekräftige Aussage jedoch zu klein ist, wurden diese Befragte in die Gruppe Bündnis90/DieGrünen integriert. Im Ergebnis lässt sich erkennen, dass die gewählte Partei einen hoch signifikanten Einfluss auf die Beachtung des Kaufkriteriums artgerechtere Tierhaltung $(\mathrm{F}(5,710)=3,236, \mathrm{p}<0,01, \mathrm{n}=716)$ hat. Im post-hoc-Test mit Bonferroni-Korrektur zeigten sich hoch signifikante Unterschiede zwischen Wählern von Grünen und Tierschutzparteien $(\mathrm{MW}=1,26 ; \quad \mathrm{SD}=0,858)$ und SPD-Wählern $(\mathrm{MW}=0,86 ; \mathrm{SD}=1,075)$ sowie AfD-Wählern $(\mathrm{MW}=0,81 ; \mathrm{SD}=1,024)$. Werden die Mittelwerte beurteilt, so achten Wähler von Bündnis90/DieGrünen und Tierschutzparteien häufiger auf eine artgerechtere Tierhaltung als SPD-Wähler und AfDWähler.

\subsubsection{Präferenzen für Fleischarten und -sorten}

Um die befragten Fleisch- und Wurstwarenkäufer möglichst umfassend zu charakterisieren, wurde neben den Kaufkriterien auch nach den Präferenzen für bestimmte Fleischarten und -sorten gefragt. Die unterschiedlichen Präferenzen der Befragten $\mathrm{zu}$ den verschiedenen Fleischarten und -sorten sind in Abbildung 11 dargestellt. 
Es zeigt sich, dass die befragten Personen am häufigsten Aufschnitt (wie z. B. Schinken) als Brotbelag und Wurstwaren (wie z. B. Mett oder Salami) kaufen. Bei frischem Fleisch wird am häufigsten Geflügel gekauft. Deutlich seltener kaufen die Befragten dagegen frisches Schweinefleisch und Rindfleisch. Am seltensten kaufen die Befragten TK-Ware, Dosen-, Tüten- und Glaswaren sowie Fleisch- oder Wurstsnacks.

Frage 9: Wie häufig kaufen Sie folgende Fleisch- und Wurstwaren?

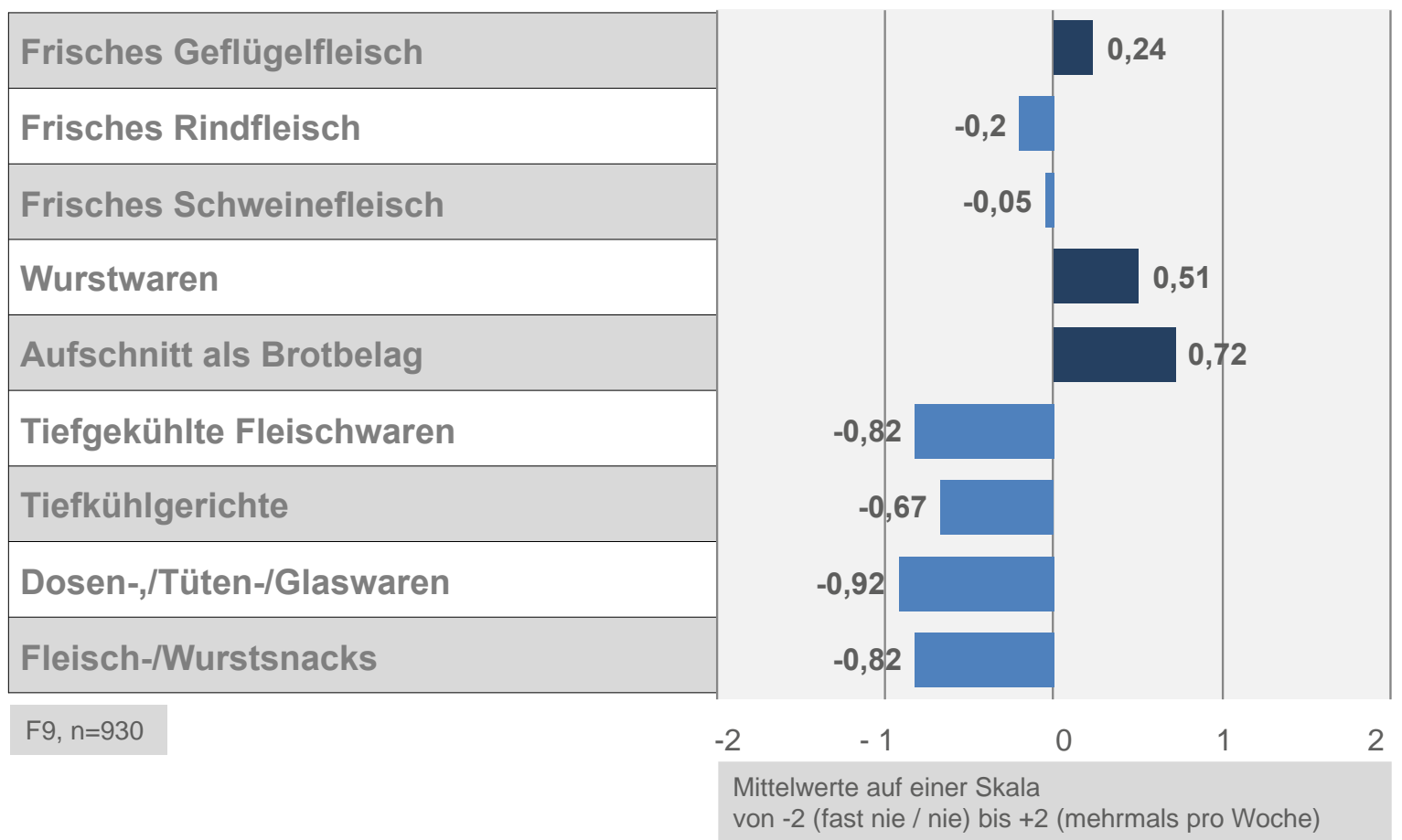

\section{Abbildung 11: Kaufhäufigkeit von Fleischarten und -sorten (Quelle: Eigene Darstellung)}




\subsection{Tierwohl-Labels als Einkaufshilfe}

In diesem Abschnitt werden die Ergebnisse zum Tierwohl-Labeling vorgestellt. Zunächst werden die Ergebnisse zur Beachtung von Tierwohl-Labels, wie zum Beispiel Bekanntheitsgrade der Labels und die Berücksichtigung beim Kauf von Fleisch- und Wurstwaren, dargestellt. Weitere Ergebnisse dieses Abschnitts betreffen verschiedene Hinderungsgründe für den Kauf, den Informationsbedarf bei Tierwohlprodukten sowie die politischen Präferenzen der Verbraucher.

\subsubsection{Beachtung von Tierwohl-Labels}

Wie in Kapitel 3 beschrieben besteht in der Bevölkerung ein hohes Interesse an Tierwohlprodukten. Daher sollte auch im Rahmen der durchgeführten Studie das Interesse an Fleisch- und Wurstwaren aus einer artgerechteren Tierhaltung evaluiert werden, um mögliche Unterschiede oder Gemeinsamkeiten zu bestehenden Studien zu identifizieren.

So wurde das Kaufinteresse von gut verfügbaren Produkten aus einer artgerechteren Tierhaltung mit einer vertrauenswürdigen Kennzeichnung erfragt, die kaum teurer sind als konventionelle Produkte. Die Frage wurde bewusst in dieser Form gestellt, um das generelle Interesse an Tierwohlprodukten zu ermitteln, unabhängig von den in der Frage beinhalteten Voraussetzungen.

Die Ergebnisse der Frage 18 sind in Tabelle 10 dargestellt. Es zeigt sich, dass, wenn die Kriterien Verfügbarkeit, Vertrauenswürdigkeit der Kennzeichnung und Preis aus Sicht der Befragten erfüllt sind, Fleisch- und Wurstwaren aus einer artgerechteren Haltung ein sehr hohes Marktpotenzial besitzen und mit hoher Wahrscheinlichkeit (92\%) gekauft würden. Nur $8 \%$ der Befragten sind sich unsicher, ob sie derartige Fleisch- und Wurstwaren überhaupt kaufen würden, und nur $2 \%$ der Befragten würden diese Produkte eher nicht kaufen. 


\section{Tabelle 10: Kaufinteresse an Tierwohlprodukten}

Frage 18: Angenommen, Fleisch- und Wurstwaren aus einer artgerechteren Tierhaltung mit einer vertrauenswürdigen Kennzeichnung wären für Sie gut verfügbar und kaum teurer als herkömmliche Produkte. Könnten Sie sich vorstellen, ab und zu diese Produkte zu kaufen?

\begin{tabular}{c|c|c|c|c|}
\hline $\begin{array}{c}\text { Ja, das kann ich } \\
\text { mir sehr gut } \\
\text { vorstellen }\end{array}$ & $\begin{array}{c}\text { Ja, diese } \\
\text { Produkte würde } \\
\text { ich zumindest } \\
\text { gelegentlich } \\
\text { kaufen }\end{array}$ & Das weiß ich nicht & $\begin{array}{c}\text { Nein, } \\
\text { wahrscheinlich } \\
\text { würde ich diese } \\
\text { Produkte eher } \\
\text { nicht kaufen }\end{array}$ & $\begin{array}{c}\text { Ich denke, dass } \\
\text { ich diese } \\
\text { Produkte sicher } \\
\text { nicht kaufen } \\
\text { werde }\end{array}$ \\
\hline $73 \%$ & $19 \%$ & $6 \%$ & $1 \%$ & $1 \%$ \\
\hline
\end{tabular}

$F 18, n=930$

\section{Quelle: Eigene Darstellung}

Ein nicht unwesentlicher Teil der geschlachteten Tiere wird zu Tierfutter für Haustiere verarbeitet. Vor diesem Hintergrund ist es für die Hersteller und Händler von Tierfutter von Bedeutung, ob ein Interesse an Produkten für Haustiere besteht, deren tierische Bestandteile aus einer artgerechteren Nutztierhaltung stammen, und wie viele Haustierbesitzer diesen Aspekt als ein Kaufkriterium ansehen. Letztlich bleiben von jedem geschlachteten Tier aus einer artgerechteren Haltung Teile, die in der Regel nicht von Menschen verzehrt werden, sich aber zur Herstellung von Heimtierfutter eignen oder weitere Verwendung finden.

Anhand von Abbildung 12 ist $\mathrm{zu}$ erkennen, dass der Großteil der Haustierbesitzer Tierfutter bevorzugen würde, wenn die im Tierfutter enthaltenen tierischen Bestandteile aus einer artgerechteren Haltung stammen (insgesamt 83,6 \%, wenn die Personen ohne Haustier und die Personen, die keine tierischen Bestandteile verfüttern, entsprechend abgerechnet werden). Tierwohl-Labels können demnach auch für einen Großteil der Heimtierfutter-Käufer ein positives Kaufargument beim Tierfutterkauf darstellen. 
Frage 40: Wenn Sie ein Haustier haben oder in Zukunft vielleicht gerne ein Haustier hätten, würden Sie Tierfutter bevorzugen, wenn die im Tierfutter enthaltenen tierischen Bestandteile aus einer artgerechteren Haltung stammen?

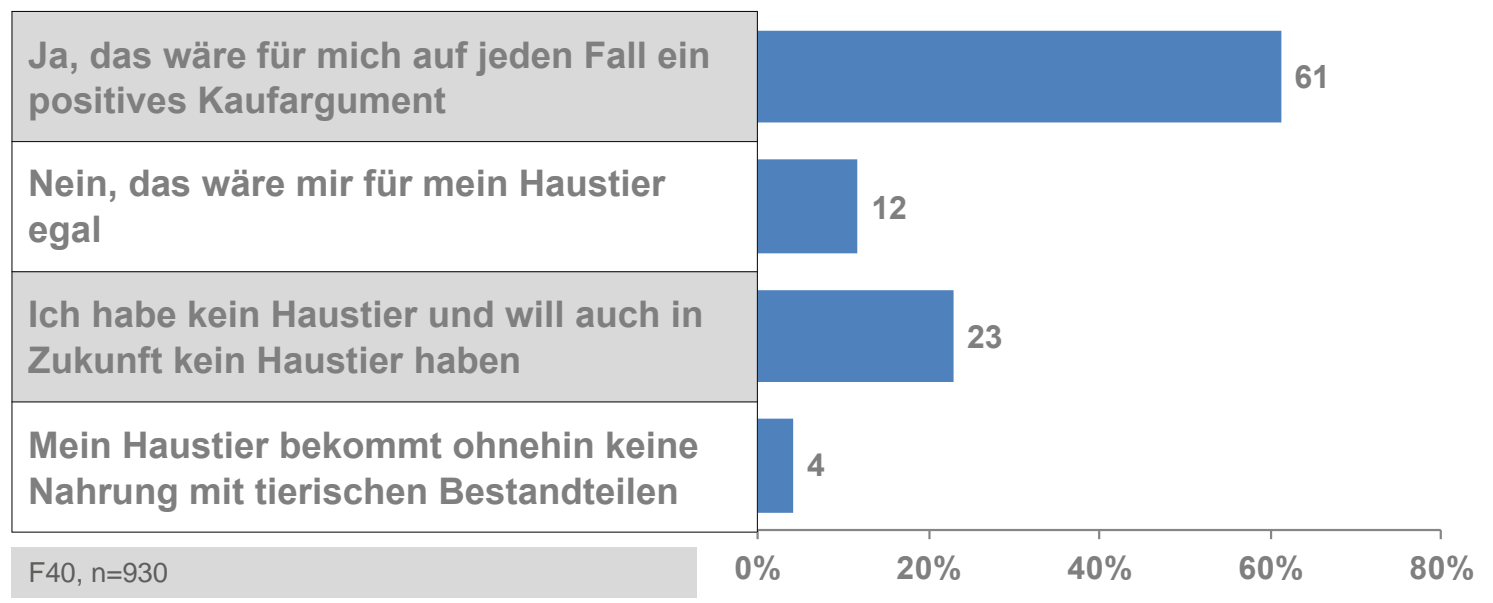

\section{Abbildung 12: Kaufinteresse an Tierwohlprodukten für Haustiere (Quelle: Eigene Darstellung)}

Zusammenfassend lässt sich sagen, dass für die meisten Befragten, die ein Haustier besitzen, Tierwohl auch beim Kauf von Heimtierfutter ein Kaufargument ist. Neben veganem Tierfutter hat insbesondere Bio-Heimtierfutter in den letzten Jahren stark zugenommen, und zahlreiche innovative Startups bieten im Internet ein breites Sortiment, schwerpunktmäßig für Hundebesitzer und Katzenbesitzer, an.

Wie in Abbildung $13 \mathrm{zu}$ sehen erreichen die Bio-Siegel von allen Tierwohl-Labels die höchsten Bekanntheitsgrade. Lediglich das Gütesiegel BIOKREIS fällt mit $6 \% \mathrm{im}$ Vergleich zu den anderen Bio-Siegeln weit ab, was damit zusammenhängen könnte, dass dieses Gütesiegel schwerpunktmäßig in Bio-Supermärkten angeboten wird, die nur eine Marktdurchdringung von $6 \%$ haben.

Das sechseckige deutsche BIO-Siegel ist das bekannteste Gütesiegel. Das BIOLANDLabel erreicht einen höheren Bekanntheitsgrad als das DE-BIO-Label. Neuere TierwohlLabels, die vor allem das Tierwohl in den Vordergrund stellen, erreichen im Vergleich zu den abgefragten Bio-Siegeln vergleichsweise geringe Bekanntheitsgrade. 
Das bekannteste Tierwohl-Label, das kein Bio-Siegel ist, ist NEULAND (21\%). Das Tierwohl-Label des DEUTSCHEN TIERSCHUTZBUNDES haben $11 \%$ der Befragten schon einmal gesehen. Sehr gering ist hingegen der Bekanntheitsgrad von VIER PFOTEN. Die Initiative Tierwohl von Herstellern und Händlern und den HALTUNGSKOMPASS von LIDL haben $16 \%$ der Befragten schon einmal gesehen. Das Label des Landwirtschaftsministeriums haben $13 \%$ der Befragten angegeben. $6 \%$ der Befragten waren sich nicht sicher, ob sie schon einmal ein Gütesiegel gesehen haben, oder haben tatsächlich noch keines gesehen (1\%). Insgesamt haben die Befragten durchschnittlich bereits vier der vorgestellten Tierwohl-Labels gesehen.

Frage 10: Bitte kreuzen Sie alle Gütesiegel an, die Sie schon einmal gesehen haben.

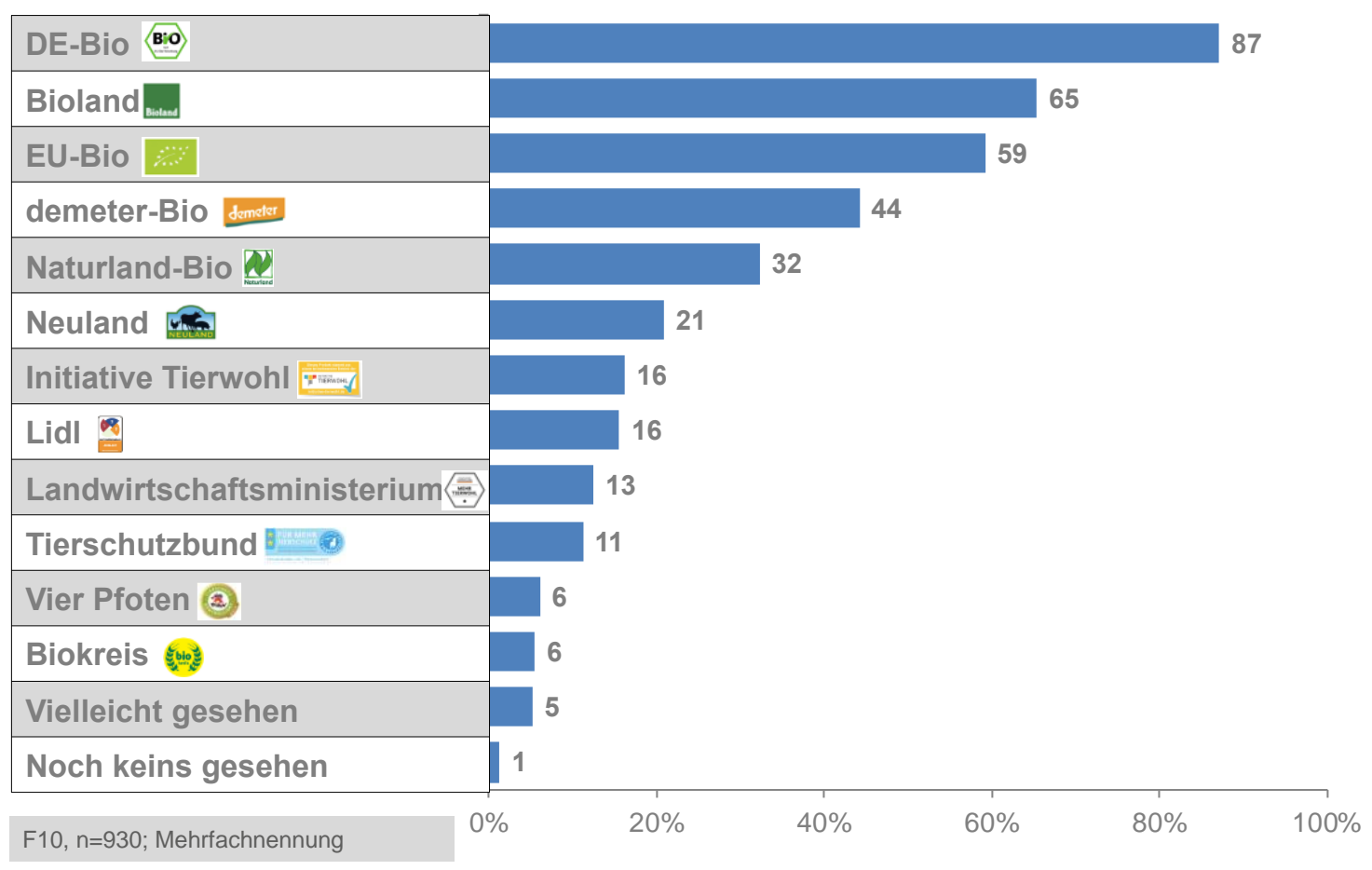

\section{Abbildung 13: Bekanntheitsgrad ausgewählter Tierwohl-Labels (Quelle: Eigene Darstellung)}

Von besonderem Interesse ist in der vorliegenden Studie der Zusammenhang zwischen dem Bekanntheitsgrad der untersuchten Tierwohl-Labels und der Haupteinkaufsstätte sowie soziodemografischen Kriterien. Abbildung 14 veranschaulicht die Ergebnisse. 
Bezüglich der Haupteinkaufsstätte wurde bei dem Label DEMETER ein hoch signifikanter Zusammenhang zwischen der Haupteinkaufsstätte und der Bekanntheit ermittelt (Chi-Quadrat(3) = 20,725, $\mathrm{p}<0,01, \mathrm{n}=826$ ). Der Zusammenhang ist allerdings nicht sehr stark (Cramer's V =0,158, p < 0,01). Das Gütesiegel ist bei 57,7 \% der Käufer mit der Haupteinkaufsstätte Metzger, bei 45 \% der Käufer mit der Haupteinkaufsstätte Supermarkt, bei 39,4 \% der Käufer mit der Haupteinkaufsstätte Discounter und lediglich bei 30,5 \% der Käufer mit der Haupteinkaufsstätte SB-Warenhaus bekannt. Zudem liegt ein signifikanter Zusammenhang zwischen dem Label NATURLAND und der Haupteinkaufsstätte vor (Chi-Quadrat (3) $F=3,597 \mathrm{p}=0,035 \mathrm{n}=826$ ). NATURLAND ist bei Supermarkt-Käufern (35,2\%) und Metzgerei-Käufern (36,2\%) bekannter als bei SB-Warenhaus-Käufern $(27,1 \%)$ und Discounter-Käufern $(27,1 \%)$.

Hypothese 8: Es besteht ein Zusammenhang zwischen dem Bekanntheitsgrad der TierwohlLabels für Fleisch- und Wurstwaren und der Haupteinkaufsstätte.

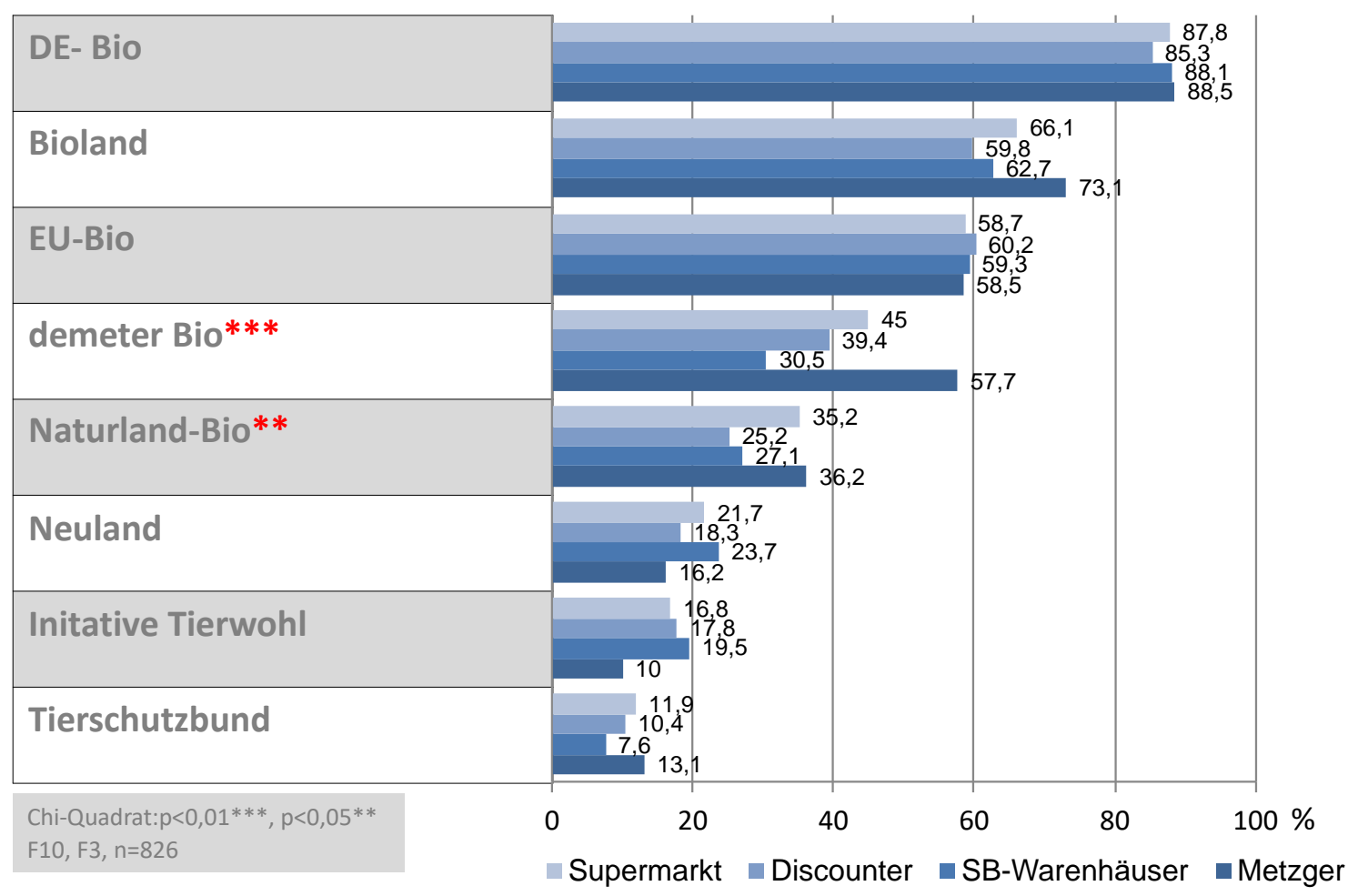

Abbildung 14: Zusammenhang Bekanntheitsgrad und Haupteinkaufsstätte (Quelle: Eigene Darstellung) 
In Tabelle 11 sind die Ergebnisse der Analysen zu den Zusammenhängen zwischen dem Bekanntheitsgrad der Tierwohl-Labels und ausgewählten soziodemografischen Kriterien ersichtlich. Demnach existiert ein signifikanter Unterschied bezüglich des Geschlechts bei DE-BIO (Chi-Quadrat (1) $\mathrm{F}=6,516 \mathrm{p}=0,011 \mathrm{n}=930$ ) und DEMETER (ChiQuadrat (1) $F=4,484 \mathrm{p}=0,034 \mathrm{n}=930$ ). Diese beiden Gütesiegel sind Frauen etwas bekannter als Männern. Bei beiden ist der Zusammenhang allerdings nicht sehr stark (Cramer's V =0,084 p =0,011 bzw. Cramer's V =0,069, p=0,034).

Beim Alter und Bekanntheitsgrad der Gütesiegel gibt es hoch signifikante Zusammenhänge bei BIOLAND (Chi-Quadrat (2) $\mathrm{F}=17,040, \mathrm{p}<0,01, \mathrm{n}=930$; Cramer's V = 0,014), DE-BIO (Chi-Quadrat (2) F = 23,462, $\mathrm{p}<0,01, \mathrm{n}=930$; Cramer's $\mathrm{V}=0,159$ ), DEMETER (Chi-Quadrat (2) $\mathrm{F}=34,790, \mathrm{p}<0,01, \mathrm{n}=930$; Cramer's $\mathrm{V}=0,069)$ und dem Tierwohl-Label des DEUTSCHEN TIERSCHUTZBUNDES (ChiQuadrat (2) $\mathrm{F}=9,988, \mathrm{p}<0,01, \mathrm{n}=930$; Cramer's V = 0,104).

Zudem besteht ein signifikanter Zusammenhang bei DE-BIO (Chi-Quadrat (2) F = 8,736, $\mathrm{p}=0,013, \mathrm{n}=930$; Cramer's $\mathrm{V}=0,097)$. Die Zusammenhänge sind allerdings alle schwach (Cramer's $\mathrm{V}<0,3$ ). Mit Blick auf die Prozentzahlen lässt sich erkennen, dass BIOLAND und DEMETER eher bei den älteren Befragten bekannt sind. DE-BIO, das Tierwohl-Label des DEUTSCHEN TIERSCHUTZBUNDES und DE-BIO sind hingegen bei den jungen Befragten etwas bekannter.

Anhand des Chi-Quadrat-Tests kann bestätigt werden, dass ein Zusammenhang zwischen dem Bundesland der Befragten und der Bekanntheit der Tierwohl-Labels BIOLAND (Chi-Quadrat (3) $\mathrm{F}=22,738, \mathrm{p}<0,01, \mathrm{n}=930$; Cramer's $\mathrm{V}=0$,156) und DEMETER (Chi-Quadrat (3) $\mathrm{F}=12,977, \mathrm{p}<0,01, \mathrm{n}=930$; Cramer's $\mathrm{V}=0,118$ ) besteht. Die Zusammenhänge sind allerdings schwach (Cramer's $\mathrm{V}<0,3$ ). Werden die Prozentwerte betrachtet, sind beide Labels im Süden und Westen Deutschlands bekannter als im Norden und im Osten Deutschlands. 
Hypothese 9: Es besteht ein Zusammenhang zwischen dem Bekanntheitsgrad der TierwohlLabels für Fleisch- und Wurstwaren und der Soziodemografie der Befragten.

Tabelle 11: Zusammenhang Bekanntheitsgrad Tierwohl-Label und Soziodemografie

\begin{tabular}{|c|c|c|c|c|c|c|c|c|}
\hline & DE-Bio & Bioland & Eu-Bio & $\begin{array}{c}\text { demeter } \\
\text { Bio }\end{array}$ & $\begin{array}{c}\text { Naturland } \\
\text { Bio }\end{array}$ & Neuland & $\begin{array}{l}\text { Initiative } \\
\text { Tierwohl }\end{array}$ & $\begin{array}{c}\text { Tierschutz } \\
\text { bund }\end{array}$ \\
\hline & in $\%$ & in $\%$ & in $\%$ & in $\%$ & in $\%$ & in $\%$ & in $\%$ & in $\%$ \\
\hline \multicolumn{9}{|l|}{ Geschlecht } \\
\hline weiblich & 88,2 & 64,2 & 63,1 & 47,5 & 32,4 & 20,4 & 17,5 & 11,4 \\
\hline männlich & 86,1 & 66,7 & 54,9 & 40,5 & 32,1 & 21,4 & 14,8 & 10,9 \\
\hline$x^{2}(1)$ & 0,901 & 0,686 & $6,516^{*}$ & $4,484^{*}$ & 0,007 & 0,154 & 1,251 & 0,052 \\
\hline Cramer-V & & & 0,084 & 0,069 & & & & \\
\hline \multirow{2}{*}{\multicolumn{9}{|c|}{ Alter }} \\
\hline & & & & & & & & \\
\hline Jung & 88,0 & 52,0 & 72,6 & 25,1 & 26,9 & 18,9 & 16,6 & 17,7 \\
\hline Mittel & 88,8 & 68,5 & 58,7 & 47,0 & 34,3 & 21,7 & 16,8 & 10,2 \\
\hline Alt & 80,0 & 68,4 & 46,5 & 54,8 & 30,3 & 20,0 & 13,5 & 7,7 \\
\hline$x^{2}(2)$ & $8,736^{*}$ & $17,040^{* *}$ & $23,462^{* *}$ & $34,790^{* *}$ & 3,784 & 0,731 & 0,995 & $9,988^{* *}$ \\
\hline Cramer-V & 0,097 & 0,014 & 0,159 & 0,069 & & & & 0,104 \\
\hline \multicolumn{9}{|l|}{ Bundesland } \\
\hline Nord & 87,7 & 61,0 & 55,5 & 40,4 & 35,6 & 23,3 & 17,1 & 8,9 \\
\hline Ost & 82,4 & 50,3 & 56,9 & 33,3 & 30,7 & 26,8 & 12,4 & 11,8 \\
\hline Süd & 88,3 & 70,7 & 62,2 & 50,5 & 32,2 & 15,5 & 14,5 & 13,4 \\
\hline West & 88,2 & 69,5 & 59,5 & 45,4 & 31,6 & 21,6 & 19,0 & 10,8 \\
\hline$x^{2}(3)$ & 3,903 & $22,738^{* *}$ & 2,242 & $12,977^{* *}$ & 0,988 & 8,727 & 4,267 & 2,695 \\
\hline Cramer-V & & 0,156 & & 0,118 & & & & \\
\hline \multicolumn{9}{|l|}{ Ortsgröße } \\
\hline Klein & 88,2 & 70,3 & 57,0 & 45,9 & 30,5 & 15,8 & 17,9 & 11,1 \\
\hline Mittel & 87,4 & 64,5 & 61,0 & 40,3 & 29,3 & 18,6 & 15,2 & 14,5 \\
\hline Groß & 86,1 & 62,3 & 59,6 & 46,0 & 36,0 & 26,6 & 15,8 & 8,6 \\
\hline$x^{2}(2)$ & 0,632 & 4,514 & 0,987 & 2,532 & 3,890 & $12,445^{\star *}$ & 0,877 & 5,630 \\
\hline Cramer-V & & & & & & 0,116 & & \\
\hline \multicolumn{9}{|l|}{ HH-Nettoeinkommen } \\
\hline Niedrig & 84,8 & 59,7 & 56,8 & 32,2 & 30,9 & 19,9 & 14,7 & 9,9 \\
\hline Mittel & 89,8 & 66,8 & 59,4 & 47,9 & 29,7 & 19,0 & 18,7 & 10,4 \\
\hline Hoch & 86,8 & 74,7 & 64,4 & 62,6 & 40,8 & 27,0 & 14,4 & 15,5 \\
\hline$x^{2}(2)$ & 4,307 & $12,522^{* *}$ & 2,834 & $48,340^{* *}$ & $7,282^{*}$ & 5,001 & 2,837 & 4,093 \\
\hline Cramer-V & & 0,116 & & 0,228 & 0,088 & & & \\
\hline \multicolumn{9}{|l|}{ Bildung } \\
\hline Niedrig & 82,0 & 58,0 & 50,4 & 29,6 & 24,0 & 18,8 & 12,0 & 12,8 \\
\hline Mittel & 87,4 & 63,5 & 54,4 & 40,6 & 32,4 & 18,2 & 17,6 & 11,9 \\
\hline Hoch & 90,6 & 72,1 & 69,6 & 57,5 & 37,8 & 24,6 & 18,0 & 9,4 \\
\hline$x^{2}(2)$ & 9,840 ** & $13,721^{\star * *}$ & $27,306^{\star *}$ & $49,112^{\star *}$ & $12,976^{\star *}$ & 5,010 & 4,527 & 2,015 \\
\hline Cramer-V & 0,103 & 0,121 & 0,171 & 0,230 & 0,118 & & & \\
\hline Signifikanzniveau ** & ${ }^{*} p<0,01,{ }^{*} p<1$ & F10, F29-3 & 30 & & & & & \\
\hline
\end{tabular}

\section{Quelle: Eigene Darstellung}


Bezüglich der Ortsgröße liegt nur ein signifikanter Unterschied beim NEULAND-Label (Chi-Quadrat (2) $\mathrm{F}=12,445, \mathrm{p}<0,01, \mathrm{n}=930$ ) vor, allerdings nur mit einem schwachen Zusammenhang (Cramer's $\mathrm{V}=0,116, \mathrm{p}=0,002$ ). Das NEULAND-Label ist in Großstädten $(26,6 \%)$ bekannter als in mittelgroßen Städten $(18,6 \%)$ sowie in kleinen Städten und auf dem Land $(26,6 \%)$.

Beim HH-Nettoeinkommen besteht ein signifikanter Zusammenhang bei den Labels BIOLAND (Chi-Quadrat (2) $\mathrm{F}=12,522, \mathrm{p}<0,01, \mathrm{n}=930$; Cramer's $\mathrm{V}=0,116$ ) und DEMETER (Chi-Quadrat (2) $\mathrm{F}=48,340, \mathrm{p}<0,01, \mathrm{n}=930$; Cramer's $\mathrm{V}=0,228$ ). Zudem ergab sich ein signifikanter Zusammenhang bei NATURLAND (Chi-Quadrat (2) $\mathrm{F}=7,282, \mathrm{p}<0,026, \mathrm{n}=930$; Cramer's $\mathrm{V}=0$,088). Diese drei Tierwohl-Label sind bei Personen mit höherem Einkommen bekannter.

Hinsichtlich des Kriteriums Bildung bestehen signifikante Unterschiede bei allen in der Tabelle betrachteten Bio-Labels: DE-BIO (Chi-Quadrat (2) $F=9,840 p<0,01 n=930$; Cramer's $V=0,103$ ), BIOLAND (Chi-Quadrat (2) $\mathrm{F}=13,721 \mathrm{p}<0,01 \mathrm{n}=930$; Cramer's V = 0,121), DE-BIO (Chi-Quadrat (2) F= 27,306 p < 0,01 n = 930; Cramer's $\mathrm{V}=0,171$ ), DEMETER (Chi-Quadrat (2) $\mathrm{F}=49,112 \mathrm{p}<0,01 \mathrm{n}=930$; Cramer's $\mathrm{V}=0,230$ ) und NATURLAND (Chi-Quadrat (2) $\mathrm{F}=12,976 \mathrm{p}<0,01 \mathrm{n}=930$; Cramer's $\mathrm{V}=0,118$ ); jeweils mit einem relativ schwachen Zusammenhang (Cramer's $\mathrm{V}<0,3$ ). Alle untersuchten Bio-Labels sind Personen mit höherer Bildung bekannter als Personen mit niedrigerer Bildung. Bei NEULAND, der Initiative Tierwohl und dem Label des DEUTSCHEN TIERSCHUTZBUNDES konnten indes keine signifikanten Unterschiede zwischen dem Bekanntheitsgrad der Tierwohl-Labels und der Bildung der Befragten festgestellt werden.

Ein weiterer Aspekt, der im Rahmen der Arbeit untersucht werden sollte, ist die konkrete Beachtung der ausgewählten Tierwohl-Labels beim Kauf von Fleisch- und Wurstwaren. Dazu wurden den Befragten die Tierwohl-Labels gezeigt, die sie schon einmal gesehen haben (Frage 10), um daraufhin die Frage zu stellen, welche dieser Tierwohl-Labels die Befragten schon einmal beim Fleisch- oder Wurstwarenkauf beachtet haben. 
In Abbildung 15 sind die Ergebnisse der Befragung dargestellt. Konkret beachtet bzw. gekauft wurden am häufigsten Fleisch- und Wurstwaren mit DE-BIO-Label und dem DEBIO-Label. Von den ökologischen Anbauverbänden kauften die meisten der Befragten Produkte mit BIOLAND-Label (32\%). DEMETER erreichte mit $18 \%$ der Nennungen nur ungefähr die Hälfte der Nennungen von BIOLAND. Bemerkenswert ist, dass sich fast ein Viertel der Befragten nicht sicher war, welches Gütesiegel sie schon einmal beim Fleisch- oder Wurstwarenkauf beachtet haben.

Frage 11: Welche dieser Gütesiegel haben Sie schon einmal beim Fleisch- oder Wurstwarenkauf beachtet bzw. damit gekennzeichnete Produkte gekauft?

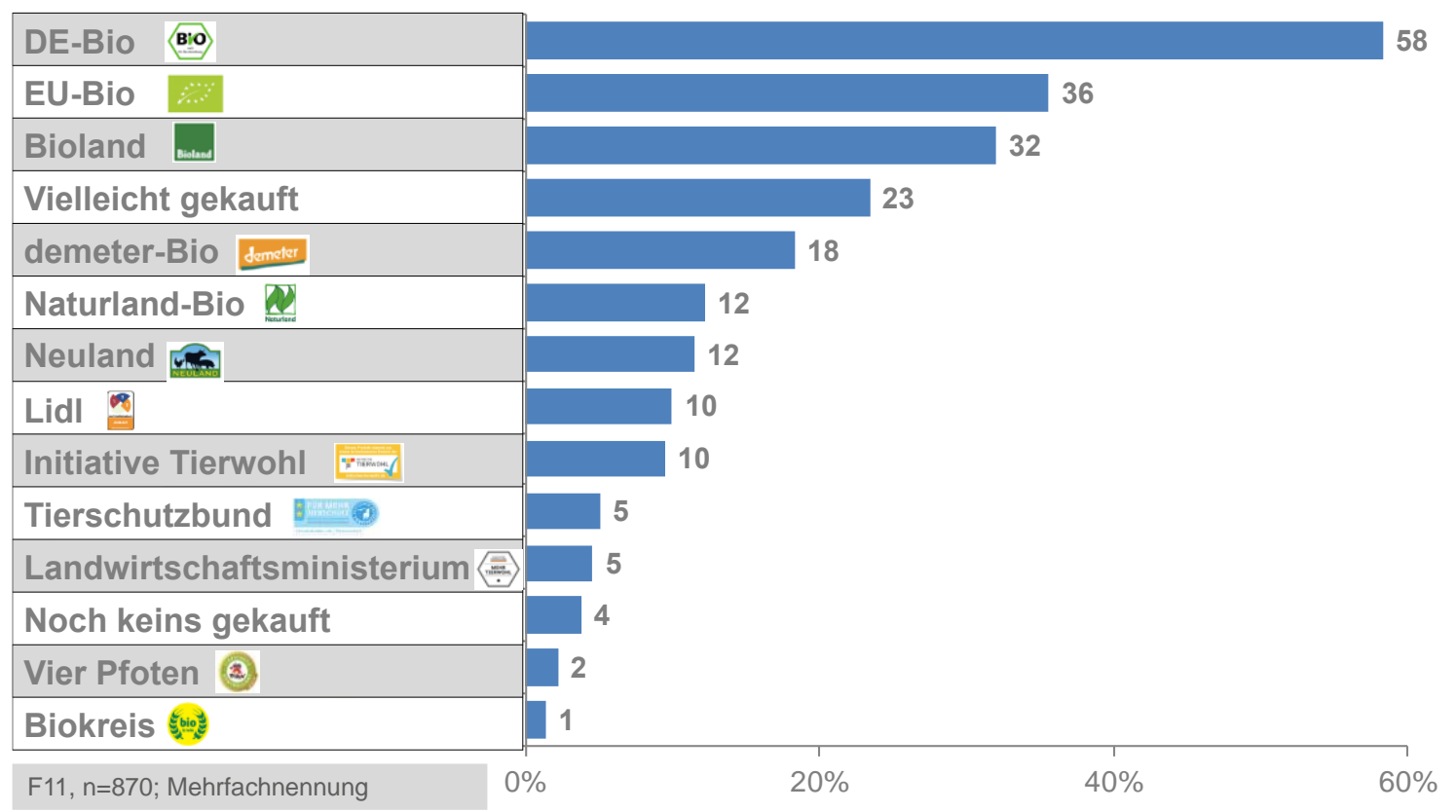

\section{Abbildung 15: Kauf von Produkten mit Tierwohl-Labels (Quelle: Eigene Darstellung)}

Um eine Gesamteinschätzung der Berücksichtigung von Tierwohl-Labels von allen Befragten $\mathrm{zu}$ erhalten, wurde in Frage 15 in Form einer fünfstufigen Likert-Skala abgefragt, welche der Gütesiegel die Teilnehmer der Studie beim Kauf von Fleisch- oder Wurstwaren aus einer artgerechteren Nutztierhaltung berücksichtigen würden und welche nicht. 
Die Ergebnisse der Frage 15 sind in Abbildung 16 dargestellt. Darin ist zu erkennen, dass die befragten Personen von den zwölf Tierwohl-Labels am ehesten die bekannten BioSiegel sowie das Gütesiegel des DEUTSCHEN TIERSCHUTZBUNDES berücksichtigen würden. Die Siegel von VIER PFOTEN, das Siegel des Landwirtschaftsministeriums und das NEULAND-Siegel würde hingegen nur weniger als die Hälfte der Befragten berücksichtigen. Und nur rd. $30 \%$ der Befragten würden Produkte kaufen, die mit dem Label der Initiative Tierwohl, BIOKREIS oder dem HALTUNGSKOMPASS von LIDL gekennzeichnet sind.

Frage 15: Welche der Gütesiegel würden Sie beim Kauf von Fleisch- oder Wurstwaren aus einer artgerechteren Nutztierhaltung berücksichtigen und welche nicht?

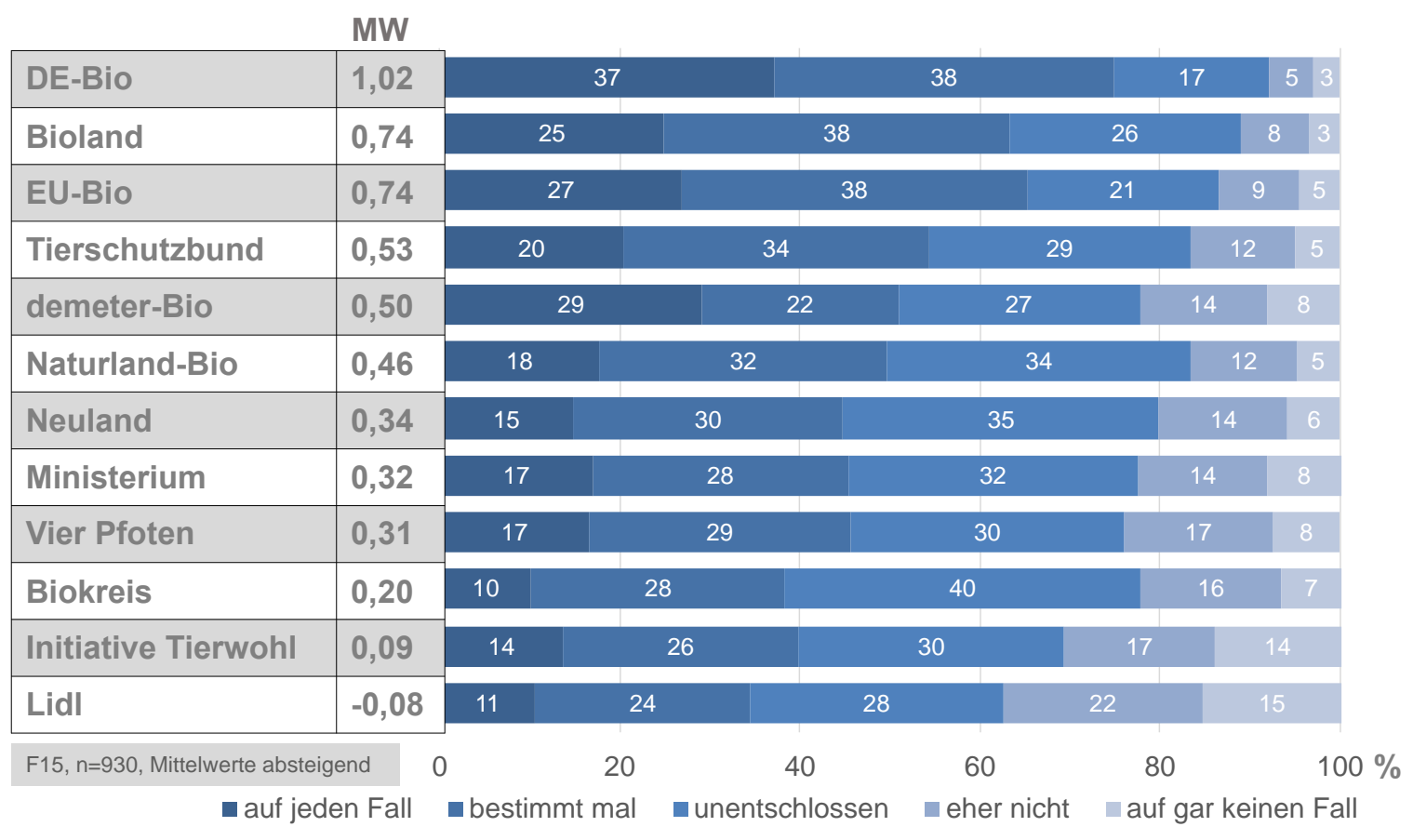

\section{Abbildung 16: Ranking der Tierwohl-Labels beim Fleischkauf (Quelle: Eigene Darstellung)}

Der Zusammenhang zwischen den Tierwohl-Labels DE-BIO, BIOLAND, DE-BIO, DEMETER, NATURLAND, NEULAND, TIERSCHUTZBUND sowie der Initiative Tierwohl und der Haupteinkaufsstätte wurde auf Basis der Frage 15 zusätzlich mit einer einfaktoriellen Varianzanalyse untersucht. 
Die Ergebnisse sind in Abbildung 17 dargestellt. Dabei zeigten sich schwach signifikante Unterschiede bei BIOLAND $(\mathrm{F}(3,822)=2,476, \mathrm{p}=0,061, \mathrm{n}=826)$. Der post-hoc-Test mit Bonferroni-Korrektur ergab, dass signifikante Unterschiede $(\mathrm{p}<0,05)$ zwischen Supermarkt-Käufern $(\mathrm{MW}=0,80 ; \mathrm{SD}=0,936)$ und Discounter-Käufern $(\mathrm{MW}=0,59$; $\mathrm{SD}=1,045)$ bestehen. Mit Blick auf die Mittelwerte wird deutlich, dass SupermarktKäufer beim Kauf von Fleisch- und Wurstwaren das Tierwohl-Label BIOLAND häufiger berücksichtigen als Discounter-Käufer. Ebenfalls zeigte sich ein schwach signifikanter Unterschied beim NEULAND-Label $(\mathrm{F}(3,822)=2,503, \mathrm{p}=0,058, \mathrm{n}=826)$. Einen signifikanten Unterschied ergab die Varianzanalyse auch bei NATURLAND $(\mathrm{F}(3,822)=3,312, \mathrm{p}=0,020, \mathrm{n}=826)$. Anhand des post-hoc-Tests mit BonferroniKorrektur konnte ermittelt werden, dass es einen signifikanten Unterschied $(\mathrm{p}<0,05)$ zwischen Supermarkt-Käufern (MW $=0,57 ; \quad \mathrm{SD}=0,994)$ und Discounter-Käufern $(\mathrm{MW}=0,29 ; \mathrm{SD}=1,066)$ gibt. Werden die Mittelwerte betrachtet, berücksichtigen Supermarkt-Käufer beim Kauf von Fleisch- und Wurstwaren häufiger das TierwohlLabel NATURLAND als Discounter-Käufer.

Das Tierwohl-Label des DEUTSCHEN TIERSCHUTZBUNDES unterscheidet sich ebenfalls signifikant $(\mathrm{F}(3,137)=3,236, \mathrm{p}=0,025, \mathrm{n}=826)$. Der post-hoc-Test mit Bonferroni-Korrektur zeigte, dass ein signifikanter Unterschied $(<0,05)$ zwischen Supermarkt-Käufern $(\mathrm{MW}=0,62 ; \mathrm{SD}=1,026)$ und Metzgerei-Käufern $(\mathrm{MW}=0,29$; $\mathrm{SD}=1,158)$ vorliegt. Werden die Mittelwerte betrachtet, berücksichtigen SupermarktKäufer beim Kauf von Fleisch- und Wurstwaren häufiger das Tierwohl-Label des DEUTSCHEN TIERSCHUTZBUNDES als Metzgerei-Käufer. Hoch signifikante Unterschiede ergaben sich bei DEMETER $(\mathrm{F}(3,822)=4,524, \mathrm{p}<0,01, \mathrm{n}=826)$. Anhand des post-hoc-Tests mit Bonferroni-Korrektur konnte ein signifikanter Unterschied $(\mathrm{p}<0,05)$ zwischen Supermarkt-Käufern (MW = 0,60; SD = 1,191) und SBWarenhaus-Käufern ( $\mathrm{MW}=0,23 ; \mathrm{SD}=1,270)$ sowie Discounter-Käufern ( $\mathrm{MW}=0,31$; $\mathrm{SD}=1,309)$ festgestellt werden. Mit Blick auf die Mittelwerte wird deutlich, dass Supermarkt-Käufer beim Fleischkauf häufiger das Tierwohl-Label von DEMETER berücksichtigen würden als SB-Warenhaus-Käufer und Discounter-Käufer. 
Es fällt zudem auf, dass Metzgerei-Käufer (MW =0,62; $\mathrm{SD}=1,290)$ am häufigsten das Tierwohl-Label DEMETER berücksichtigen würden, wenngleich kein statistisch signifikanter Unterschied zu Discountern und SB-Warenhäusern besteht.

Hoch signifikante Unterschiede zeigten sich bei der Initiative Tierwohl $(\mathrm{F}(3,822)=5,432, \mathrm{p}<0,01, \mathrm{n}=826)$. Der post-hoc-Test mit Bonferroni-Korrektur ergab, dass ein signifikanter Unterschied $(\mathrm{p}<0,05)$ zwischen Metzgerei-Käufern $(\mathrm{MW}=-0,22 ; \mathrm{SD}=1,234)$ und Supermarkt-Käufern $(\mathrm{MW}=0,13 ; \mathrm{SD}=1,200)$ sowie Discounter-Käufern (MW $=0,31 ; \quad \mathrm{SD}=1,202)$ existiert. Werden die Mittelwerte betrachtet, lässt sich erkennen, dass Metzgerei-Käufer das Label der Initiative Tierwohl weniger berücksichtigen würden als Supermarkt-Käufer und Discounter-Käufer.

Hypothese 10: Es besteht ein Zusammenhang zwischen den Tierwohl-Label-Präferenzen und der Haupteinkaufsstätte.

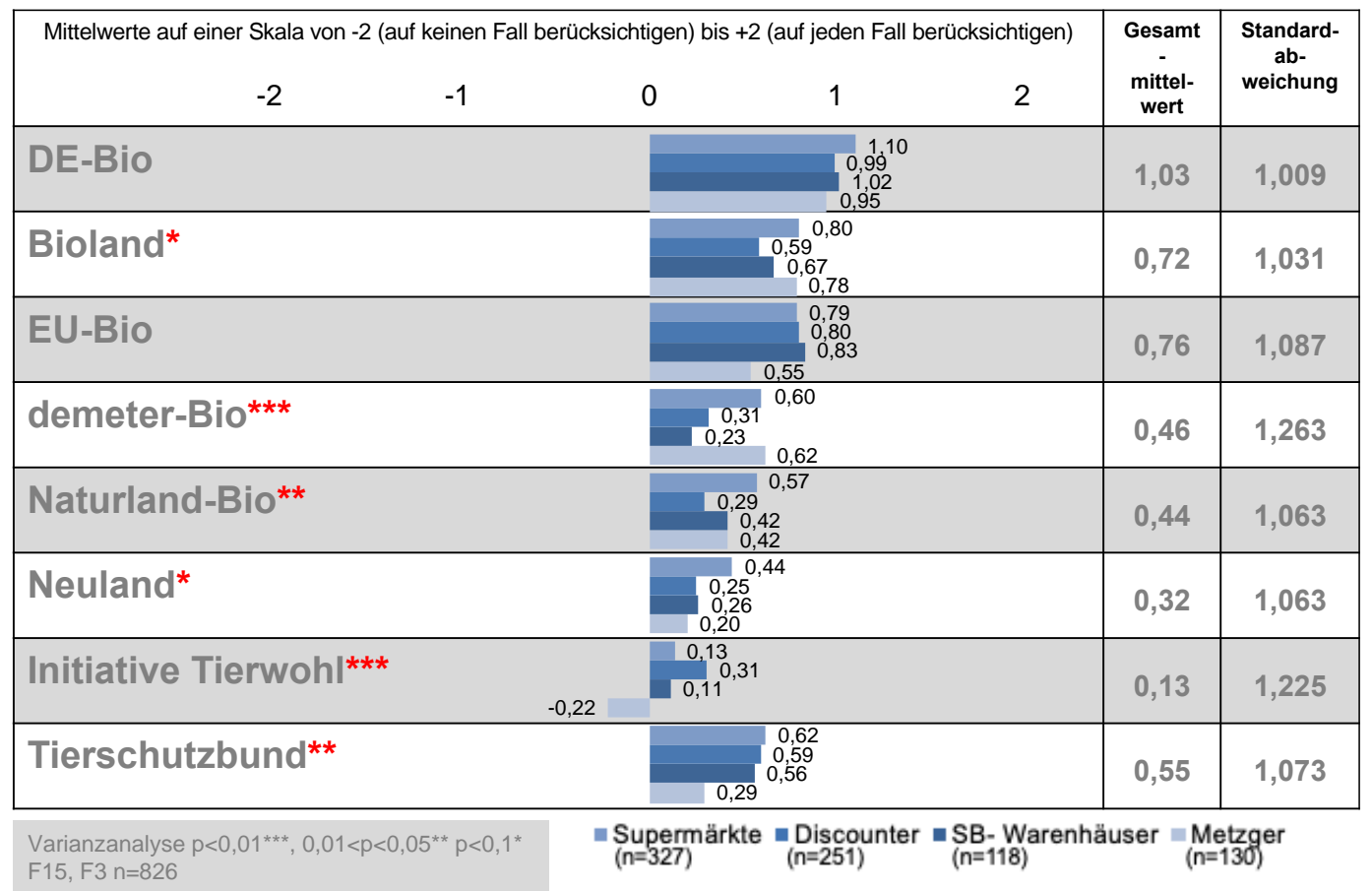

Abbildung 17: Zusammenhang Label-Präferenzen und Haupteinkaufsstätte (Quelle: Eigene Darstellung) 
Des Weiteren wurde die Korrelation zwischen den Label-Präferenzen und den Angebotsformen Fleischtheke und Selbstbedienungsbereich der Produktvarianten Wurst und Aufschnitt sowie frisches Fleisch zum Braten und Grillen untersucht. Die Ergebnisse sind in Tabelle 12 dargestellt. Es ergaben sich bei Wurst und Aufschnitt hoch signifikante positive Korrelationen bei BIOLAND, DEMETER, NATURLAND und NEULAND sowie eine signifikante positive Korrelation bei DE-BIO. Dies bedeutet, je häufiger die Befragten an der Fleischtheke kaufen, desto höher ist die Präferenz für die Labels BIOLAND, DEMETER, NATURLAND, NEULAND und DE-BIO. Anders ausgedrückt lässt sich ebenfalls sagen, je höher die Präferenz für die Labels BIOLAND, DEMETER, NATURLAND, NEULAND und DE-BIO ist, desto seltener kaufen die Befragten im SBBereich. Zudem besteht eine hoch signifikante negative Korrelation beim Label der Initiative Tierwohl bei sehr schwacher Korrelation $(r<0,2)$. Je häufiger die Befragten also im SB-Bereich kaufen, desto höher ist die Präferenz für das Label der Initiative Tierwohl. Oder: Je höher die Präferenz für das Label der Initiative Tierwohl ist, desto häufiger kaufen die Befragten im SB-Bereich.

Die Koeffizienten für Fleisch zum Braten und Grillen sind ebenfalls in Tabelle 12 dargestellt. Es ergaben sich hoch signifikante positive Korrelationen bei BIOLAND, DEMETER, NATURLAND und NEULAND sowie eine signifikante positive Korrelation bei DE-BIO. Die Korrelation war jedoch sehr schwach $(r<0,2)$. Dies bedeutet, je häufiger die Befragten Fleisch zum Braten und Grillen an der Fleischtheke kaufen, desto höher ist die Präferenz für die Labels BIOLAND, DEMETER, NATURLAND, NEULAND und DE-BIO. Oder: Je höher die Präferenz für die Labels BIOLAND, DEMETER, NATURLAND, NEULAND und DE-BIO ist, desto seltener kaufen die Befragten im SB-Bereich. Zudem konnte eine hoch signifikante negative Korrelation beim Label der Initiative Tierwohl bei sehr schwacher Korrelation $(r<0,2)$ festgestellt werden. Je häufiger die Befragten im SB-Bereich kaufen, desto höher ist demnach die Präferenz für das Label der Initiative Tierwohl. Vice versa kann gesagt werden, je höher die Präferenz für das Label der Initiative Tierwohl ist, desto häufiger kaufen diese Befragten im SB-Bereich. 
Hypothese 11: Personen, die bestimmte Tierwohl-Labels beim Kauf von Fleisch- und Wurstwaren berücksichtigen, kaufen Wurstwaren oder Aufschnitt fürs Brot häufiger an der Theke. Hypothese 12: Personen, die bestimmte Tierwohl-Labels beim Kauf von Fleisch- und Wurstwaren berücksichtigen, kaufen Fleisch zum Braten und Grillen häufiger an der Theke.

Tabelle 12: Korrelation Tierwohl-Label-Präferenzen und Angebotsform

\begin{tabular}{|l|l|l|}
\hline Tierwohl-Label & $\begin{array}{l}\text { Koeffizient } \\
\text { Wurst oder Aufschnitt }\end{array}$ & $\begin{array}{l}\text { Koeffizient } \\
\text { Fleisch zum Braten und Grillen }\end{array}$ \\
\hline DE- Bio & $0,076 *$ & $0,082^{*}$ \\
\hline Bioland & $0,160 * *$ & $0,165 * *$ \\
\hline EU-Bio & $-0,010$ & $-0,008$ \\
\hline demeter Bio & $0,146 * *$ & $0,111 * *$ \\
\hline Naturland-Bio & $0,122 * *$ & $0,104 * *$ \\
\hline Neuland & $0,130 * *$ & $0,110 * *$ \\
\hline Initiative Tierwohl & $-0,092 * *$ & $-0,124 * *$ \\
\hline Tierschutzbund & $-0,053$ & $-0,060$ \\
\hline Korrelation, $p<0,01 * *, p<0,05^{* *} F 4, F 15 n=930$ & \\
\hline
\end{tabular}

\section{Quelle: Eigene Darstellung}

Abbildung 18 veranschaulicht den Zusammenhang zwischen der Label-Präferenz und der Bereitschaft, den Fleischkonsum zu reduzieren. Hoch signifikante Unterschiede ergaben sich bei DEMETER $(\mathrm{F}(2,908)=7,623, \mathrm{p}<0,01, \mathrm{n}=911)$. Der post-hoc-Test mit Bonferroni-Korrektur brachte hervor, dass ein signifikanter Unterschied $(\mathrm{p}<0,05)$ zwischen den Befragten, die ihren Fleischkonsum bereits reduziert ( $M W=0,67$; $\mathrm{SD}=1,245)$ haben, und den Befragten, die keine Reduktionsbereitschaft ( $\mathrm{MW}=0,28$; $\mathrm{SD}=1,268)$ aufweisen, besteht.

Die Mittelwerte betrachtend lässt sich sagen, dass von den Befragten, die ihren Fleischkonsum bereits reduziert haben, das Tierwohl-Label DEMETER mehr berücksichtigt würde als von den Befragten, die keine Reduktionsbereitschaft aufweisen. Zudem existiert ein schwach signifikanter Unterschied bei NATURLAND $(\mathrm{F}(2,908)=2,453, \mathrm{p}=0,087, \mathrm{n}=911)$. 
Der post-hoc-Test ergab, dass es einen signifikanten Unterschied zwischen den Befragten, die ihren Fleischkonsum bereits reduziert haben ( $\mathrm{MW}=0,53 ; \mathrm{SD}=1,062$ ), und den Befragten, die keine Reduktionsbereitschaft aufweisen (MW=0,35; $\mathrm{SD}=1,042$ ), gibt. Werden die Mittelwerte betrachtet, würde von den Befragten, die ihren Fleischkonsum bereits reduziert haben, das Tierwohl-Label NATURLAND mehr berücksichtigt als von den Befragten, die keine Reduktionsbereitschaft aufweisen.

Hypothese 13: Es besteht ein Zusammenhang zwischen den Tierwohl-Label-Präferenzen und der persönlichen Reduktionsbereitschaft.

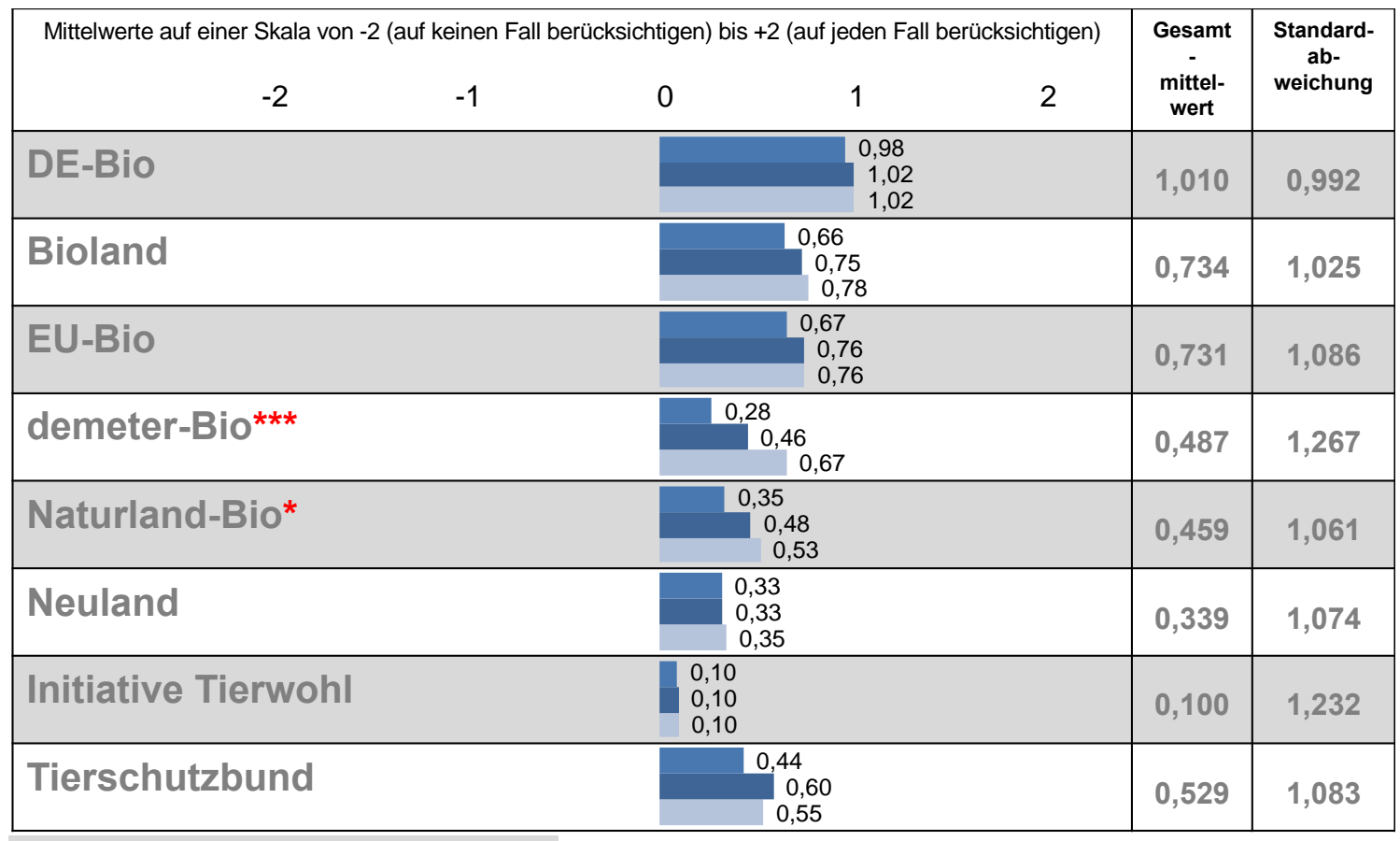

Varianzanalyse $p<0,01^{* * *}, 0,01<p<0,05^{* *} p<0,1^{*}$ $\mathrm{F} 15, \mathrm{~F} 7 \mathrm{n}=911$

= keine Reduktionsbereitschaft $=$ Reduktionsbereitschaft $=$ bereits reduziert

Abbildung 18: Zusammenhang Label-Präferenzen und Reduktionsbereitschaft (Quelle: Eigene Darstellung) 
In Tabelle 13 sind die unterschiedlichen Label-Präferenzen bei verschiedenen soziodemografischen Kriterien aufgeführt. Demnach bestehen hoch signifikante Unterschiede bei Männern und Frauen bzgl. der Label-Präferenzen bei DE-BIO $(\mathrm{F}(1,928)=9,269, \mathrm{p}<0,01, \mathrm{n}=930)$, DEMETER $\quad(\mathrm{F}(1,928)=12,441, \quad \mathrm{p}<0,01$, $\mathrm{n}=930)$, NEULAND $(\mathrm{F}(1,928)=11,510, \mathrm{p}<0,01, \mathrm{n}=930)$, Initiative Tierwohl $(\mathrm{F}(1,928)=45,983, \quad \mathrm{p}<0,01, \quad \mathrm{n}=930)$ und dem Label des DEUTSCHEN TIERSCHUTZBUNDES $(\mathrm{F}(1,928)=17,486, \mathrm{p}<0,01, \mathrm{n}=930)$.

Weitere signifikante Unterschiede bestehen bei DE-BIO $(\mathrm{F}(1,928)=4,341, \mathrm{p}<0,05$, $\mathrm{n}=930)$, NATURLAND $(\mathrm{F}(1,928)=4,165, \mathrm{p}<0,05, \mathrm{n}=930)$ und BIOLAND $(\mathrm{F}(1,928)=4,164, \mathrm{p}<0,05, \mathrm{n}=930)$. Mit Blick auf die Mittelwerte in der Tabelle kann konstatiert werden, dass alle Tierwohl-Labels von Frauen bevorzugt gekauft werden.

Beim Faktor Alter liegt ein hoch signifikanter Unterschied bei BIOLAND vor $(\mathrm{F}(2,927)=5,482, \mathrm{p}<0,01, \mathrm{n}=930)$. Mittels des post-hoc-Tests mit BonferroniKorrektur konnte ein signifikanter Unterschied zwischen den Kategorien ,junge Befragte‘ und ,ältere Befragte' ermittelt werden. Die Mittelwerte in der Tabelle betrachtend ist auffällig, dass ältere Befragte das Tierwohl-Label BIOLAND eher berücksichtigen als junge Befragte. Ein hoch signifikanter Unterschied zeigte sich auch beim Tierwohl-Label DE-BIO $(\mathrm{F}(2,927)=8,082, \mathrm{p}<0,01, \mathrm{n}=930)$ und DEMETER $(\mathrm{F}(2,927)=11,014$, $\mathrm{p}<0,01, \mathrm{n}=930$ ). Ein signifikanter Unterschied konnte für das Tierwohl-Label des DEUTSCHEN TIERSCHUTZBUNDES $\quad(\mathrm{F}(2,927)=3,837, \quad \mathrm{p}<0,05, \quad \mathrm{n}=930)$ identifiziert werden.

Die post-hoc-Tests mit Bonferroni-Korrektur brachten bei allen drei genannten TierwohlLabels einen signifikanten Unterschied zwischen den Kategorien der jungen Befragten und der Befragten mittleren und hohen Alters hervor. Die Mittelwerte in der Tabelle vergleichend berücksichtigen junge Befragte eher als ältere Befragte bevorzugt das Tierwohl-Label DE-BIO und das Label des DEUTSCHEN TIERSCHUTZBUNDES. Das Tierwohl-Label DEMETER wird jedoch eher von den Befragten im mittleren Lebensalter und älteren Befragten berücksichtigt. Zudem liegen hoch signifikante Unterschiede bei NATURLAND $(\mathrm{F}(2,927)=7,787, \mathrm{p}<0,01, \mathrm{n}=930)$ und NEULAND $(\mathrm{F}(2,927)=6,543, \mathrm{p}<0,01, \mathrm{n}=930)$ vor. 
Bei beiden zeigte sich in den post-hoc-Tests mit Bonferroni-Korrektur ein Unterschied zwischen jungen Befragten sowie Befragten im mittleren und hohen Alter. Mit Blick auf die Mittelwerte ist festzustellen, dass jüngere Käufer diese Tierwohl-Labels seltener berücksichtigen. Beim Label der Initiative Tierwohl $(F(2,927)=6,465, p<0,01$, $\mathrm{n}=930$ ) konnte ein signifikanter Unterschied zwischen allen drei Kriterien ermittelt werden. Die Mittelwerte betrachtend wird dieses Label am häufigsten von jungen Befragten und am seltensten von den älteren Befragten berücksichtigt.

Hinsichtlich des Herkunftsbundeslands gibt es signifikante Unterschiede bei DEMETER $(\mathrm{F}(3,926)=3,088, \mathrm{p}<0,05, \mathrm{n}=930)$. In den post-hoc-Tests mit Bonferroni-Korrektur zeigte sich ein signifikanter Unterschied zwischen Nord und West. Die Berücksichtigung des Labels von DEMETER ist im Westen höher als im Norden. Ebenfalls liegt ein signifikanter Unterschied beim Tierwohl-Label NEULAND $(F(3,926)=3,337, p<0,05$, $\mathrm{n}=930$ ) vor. Anhand der post-hoc-Tests mit Bonferroni-Korrektur konnte ein signifikanter Unterschied zwischen Ost und Süd festgestellt werden. Die Berücksichtigung des Labels von NEULAND ist im Osten größer als im Süden.

Mit Blick auf das Einkommen besteht ein hoch signifikanter Unterschiede bei BIOLAND $(\mathrm{F}(2,927)=6,546, \mathrm{p}<0,01, \mathrm{n}=930)$ und DEMETER $(\mathrm{F}(2,927)=10,443, \mathrm{p}<0,01$ $\mathrm{n}=930$ ). In den post-hoc-Tests mit Bonferroni-Korrektur zeigte sich ein signifikanter Unterschied zwischen niedrigem Einkommen und mittlerem sowie hohem Einkommen. Die Berücksichtigung der Labels BIOLAND und DEMETER ist bei den Befragten mit niedriger Bildung geringer. Zudem ergab sich ein schwach signifikanter Unterschied bei NATURLAND $(\mathrm{F}(2,927)=2,684, \mathrm{p}<0,1, \mathrm{n}=930)$.

Hypothese 14: Es besteht ein Zusammenhang zwischen den Tierwohl-LabelPräferenzen und der Soziodemografie der Befragten. 


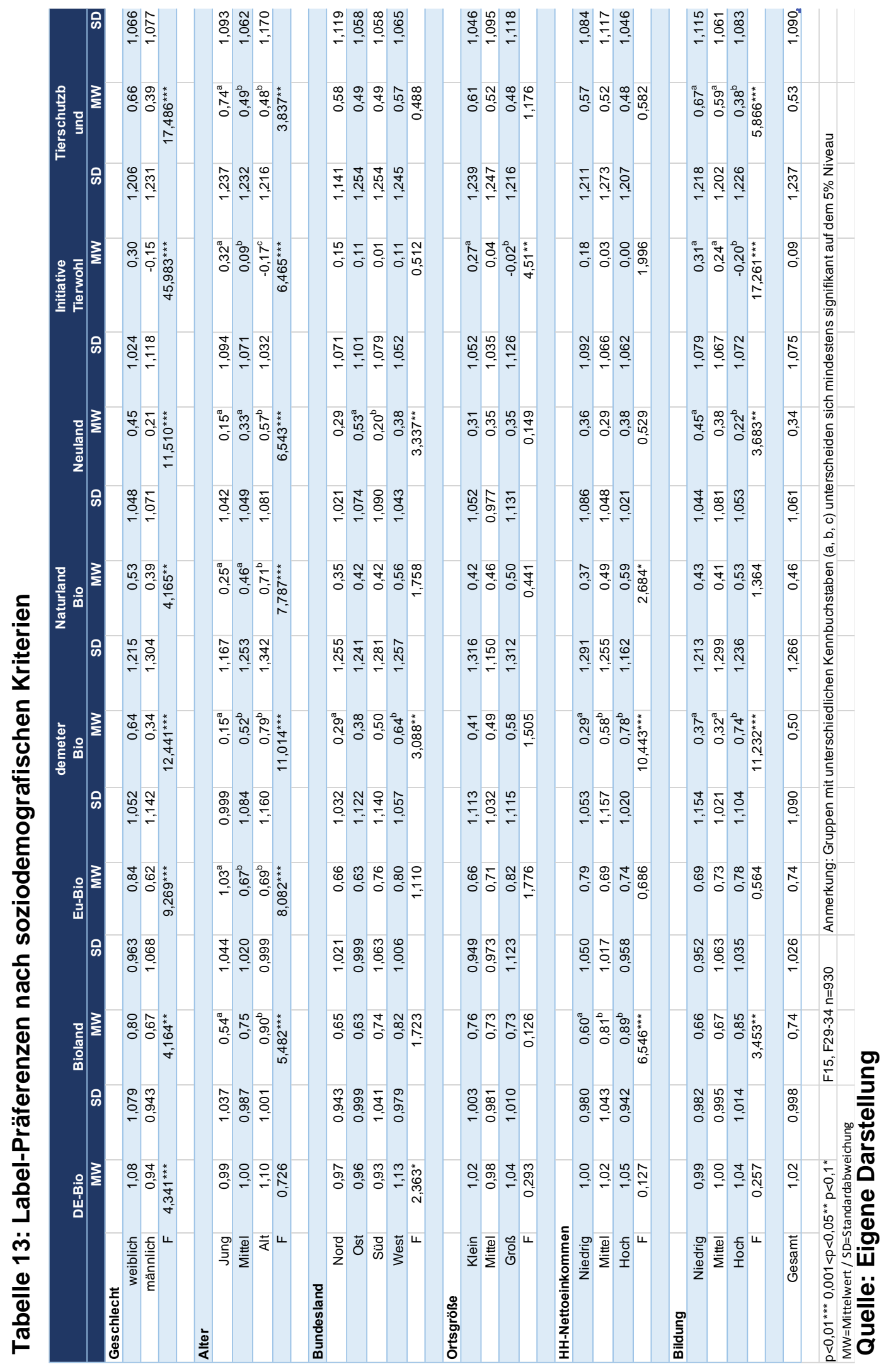


Hinsichtlich der Bildung liegen hoch signifikante Unterschiede bei DEMETER $(\mathrm{F}(2,927)=11,232, \quad \mathrm{p}<0,01, \quad \mathrm{n}=930), \quad$ dem Label der Initiative Tierwohl $(\mathrm{F}(2,927)=17,261, \quad \mathrm{p}<0,01, \quad \mathrm{n}=930) \quad$ und dem Label des DEUTSCHEN TIERSCHUTZBUNDES $(\mathrm{F}(2,927)=5,866, \mathrm{p}<0,01, \mathrm{n}=930)$ vor. Aus den post-hocTests mit Bonferroni-Korrektur geht ein signifikanter Unterschied zwischen Befragten mit niedriger sowie mittlerer Bildung und Befragten mit hoher Bildung hervor. Das Tierwohl-Label DEMETER wird von Befragten mit hoher Bildung häufiger berücksichtigt. Das Label Initiative Tierwohl und jenes des DEUTSCHEN TIERSCHUTZBUNDES werden von Befragten mit hoher Bildung weniger berücksichtigt. Das Tierwohl-Label BIOLAND $(\mathrm{F}(2,927)=3,453, \mathrm{p}<0,05, \mathrm{n}=930)$ wird ebenfalls von Befragten mit hoher Bildung signifikant häufiger berücksichtigt als von Befragten mit niedriger sowie mittlerer Bildung. Das Tierwohl-Label NEULAND $(\mathrm{F}(2,927)=3,683, \mathrm{p}<0,05, \mathrm{n}=930)$ wird von Befragten mit niedriger Bildung signifikant häufiger berücksichtigt als von Befragten mit hohem Bildungsniveau.

Die Ergebnisse der Fragen 10 (Bekanntheitsgrad), 11 (bereits beim Kauf beachtete Label) und 15 (Label-Präferenzen) geben Aufschluss darüber, welche Gütesiegel die Befragten schon einmal gesehen haben, welche sie bereits gekauft haben und welche Gütesiegel sie am ehesten beim Kauf berïcksichtigen würden. Aus diesen Ergebnissen geht jedoch nicht hervor, wie häufig die Befragten Fleisch- und Wurstwaren kaufen, die mit TierwohlLabels versehen sind. Mit Frage 12 zur Kaufhäufigkeit zertifizierter Tierwohlprodukte sollte diese Lücke geschlossen werden. Diese Frage wurde nur Personen gestellt, die bereits zertifizierte Tierwohlprodukte gekauft haben.

In Abbildung 19 lässt sich erkennen, dass $15 \%$ der Befragten meistens oder so gut wie immer Fleisch- oder Wurstwaren mit einer Tierwohlkennzeichnung kaufen. Der größte Anteil der Befragten in Höhe von $81 \%$ kauft Fleisch- oder Wurstwaren, die mit diesen Gütesiegeln gekennzeichnet sind, je nach Möglichkeit oder gelegentlich. Diese Gruppe könnte insgesamt als ,Gelegenheitskäufer' bezeichnet werden. Rund $4 \%$ kaufen nur sehr selten oder nur zu besonderen Anlässen Produkte mit Tierwohl-Labels. 
Frage 12: Wie häufig kaufen Sie Fleisch-oder Wurstwaren, die mit diesen Gütesiegeln gekennzeichnet sind? (nur für Käufer)

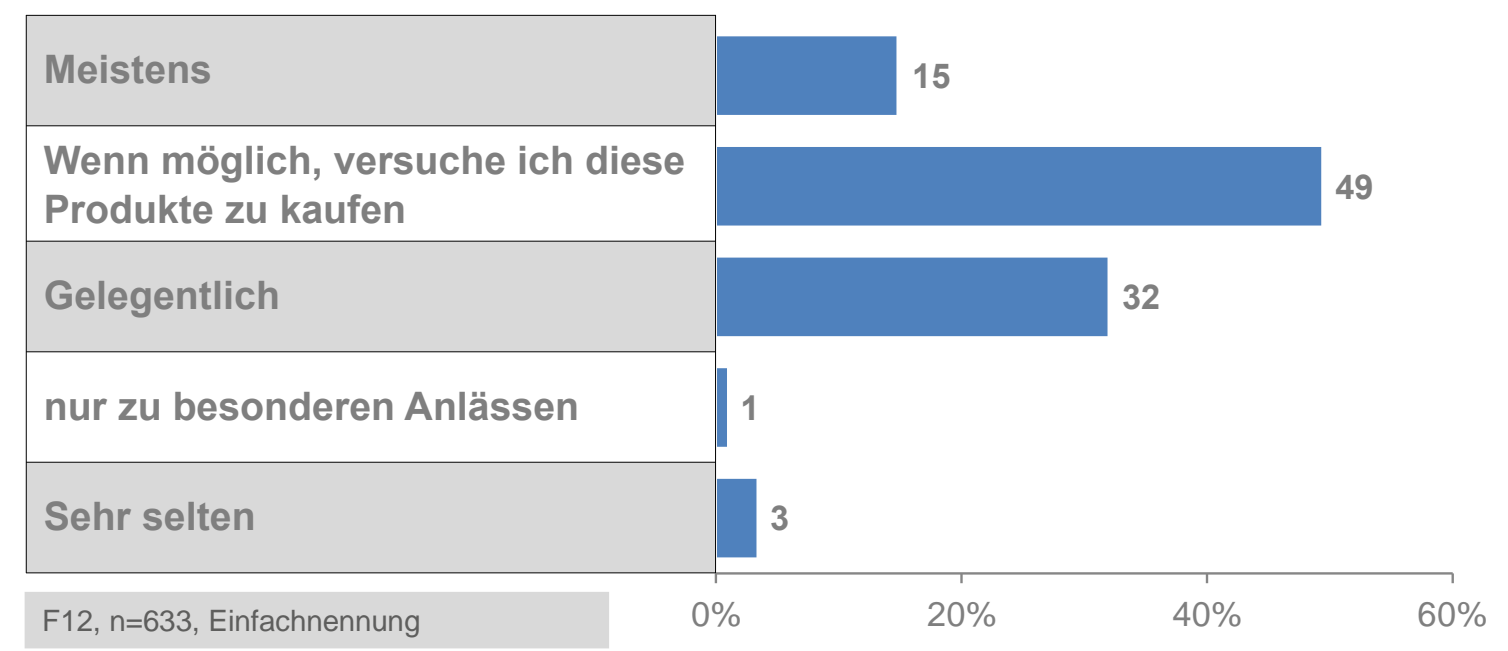

\section{Abbildung 19: Kaufhäufigkeit von Produkten mit Tierwohl-Labels (Quelle: Eigene Darstellung)}

Im Lebensmitteleinzelhandel steigt die Zahl der Produkte mit verschiedenen Gütesiegeln. Für Hersteller und Händler stellt sich hier die Frage, ob der Einsatz von Tierwohl-Labels zusätzlich mit anderen Gütesiegeln kombiniert werden sollte, um die Kaufwahrscheinlichkeit zu erhöhen, wobei ebenfalls herauszufinden wäre, welche zusätzlichen Gütesiegel sich positiv auf die Kaufentscheidung auswirken könnten. Daher wurden in Frage 17 allen Teilnehmern der Studie unterschiedliche Gütesiegel zur Auswahl gestellt.

Abbildung 20 veranschaulicht die Ergebnisse zum Thema Multi-Labeling. Der Großteil der befragten Personen befürwortet demnach den Einsatz mehrerer zusätzlicher Gütesiegel. Es wurden durchschnittlich fünf Gütesiegel angegeben. Die meisten Befragten nannten die Testsiegel von STIFTUNG WARENTEST und ÖKOTEST. Positiv auf die Kaufentscheidung wirken sich offensichtlich auch die ,Frei-von'-Siegel (Gentechnik, Hormone, Antibiotika oder MRSA) aus, die alle Menschen betreffen, und verschiedene Regionalsiegel. 
Frage 17: Welche dieser weiteren Gütesiegel oder Logos könnten sich positiv auf Ihre Kaufentscheidung beim Fleisch- und Wurstkauf auswirken? Sie können mehrere Gütesiegel/Logos auswählen?

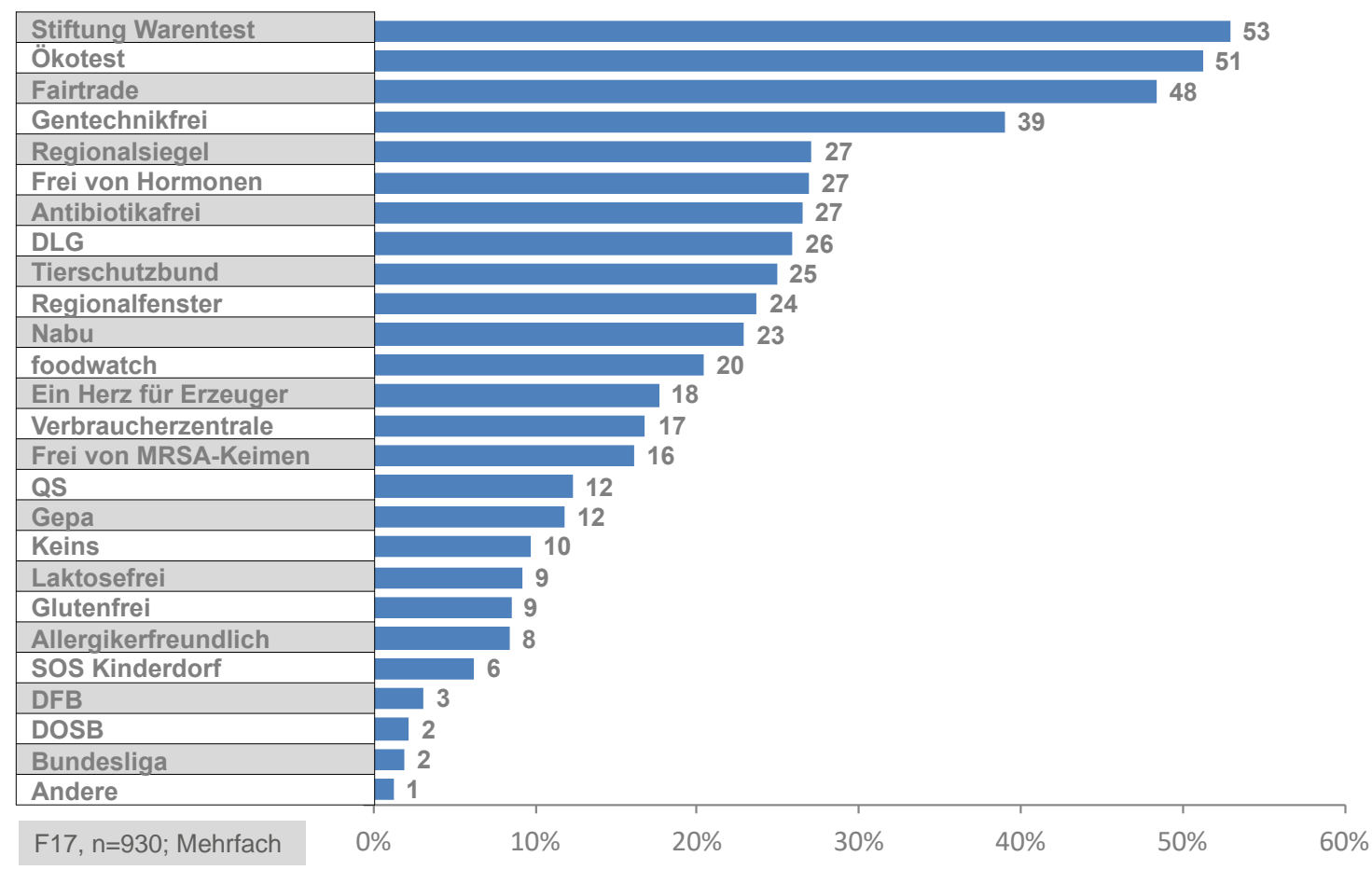

\section{Abbildung 20: Präferenzen zum Multi-Labeling von Tierwohlprodukten (Quelle: Eigene Darstellung)}

Die ,Frei-von'-Siegel, die nur einen kleinen Teil der Bevölkerung betreffen, wie z. B. ein Laktose- oder Gluten-Frei-Siegel, haben womöglich für alle gesunden Menschen nur einen sehr geringen bis gar keinen positiven Einfluss auf die Kaufentscheidung. Das weit verbreitete QS-Siegel könnte sich den Ergebnissen zufolge lediglich bei $12 \%$ der Befragten positiv auf die Kaufentscheidung auswirken.

Logos von Organisationen, die ebenfalls einen positiven Effekt auf die Kaufentscheidung haben könnten, werden je nach Organisation unterschiedlich bewertet. Die meisten Befürworter mit $48 \%$ aller Befragten findet das Logo der Organisation FAIRTRADE. 
Die Logos der Organisationen DLG, TIERSCHUTZBUND, NABU, FOODWATCH und die VERBRAUCHERZENTRALE konnten bei rd. einem Viertel der Befragten eine Kaufentscheidung positiv beeinflussen. Die zusätzliche Platzierung der Logos des DEUTSCHEN FUßBALLBUNDS (DFB), des DEUTSCHEN OLYMPISCHEN SPORTBUNDS (DOSB) oder der BUNDESLIGA scheint trotz hoher Sympathiewerte in der Bevölkerung für den Sport für die meisten Befragten keinen positiven Einfluss auf die Kaufentscheidung von Fleisch- und Wurstwaren zu haben.

Insgesamt kann konstatiert werden, dass es trotz der von einigen Verbrauchern wahrgenommenen ,Labelflut' (Kapitel 3) dennoch einen nicht unerheblichen Teil an Verbrauchern gibt, für die verschiedenste Gütesiegel bei Fleisch- und Wurstwaren einen positiven Effekt auf die Kaufentscheidung haben können.

\subsubsection{Hinderungsgründe für den Kauf}

Auf die Fragen 10 (Bekanntheitsgrad) und 11 (Kauf) des Fragebogens antworteten 297 Personen, noch keine Gütesiegel bewusst gesehen und gekauft zu haben. Diese Personen wurden in Frage 13 befragt, warum sie noch keine Fleisch- und Wurstwaren gekauft haben, die mit den vorgestellten Gütesiegeln gekennzeichnet sind. Dabei konnten mehrere Gründe genannt werden. Wie in Abbildung $21 \mathrm{zu}$ erkennen wurde das Vertrauen in die Metzger mit $45 \%$ am häufigsten als Grund aufgeführt, keine Fleisch- und Wurstwaren mit Tierwohl-Labels zu kaufen.

Auffällig ist, dass zahlreiche Befragte Gründe anführten, die auf Optimierungspotenziale in der Vermarktung der Tierwohlprodukte hindeuten. Etwa einem Drittel der Befragten sind die Produkte in der Einkaufsstätte noch nicht aufgefallen. Die Intransparenz der Preisgestaltung bei Tierwohlprodukten ist ein weiterer zentraler Grund (26\%). Ein Fünftel der Befragten gab als Grund an, dass keine ausreichenden Informationen zu den Haltungsbedingungen zur Verfügung stünden. $16 \%$ äußerten, dass ihnen diese Gütesiegel nicht bekannt seien. 
Insgesamt $5 \%$ haben die Produkte mit den höheren Tierwohlstandards noch nicht in der Werbung gesehen oder bereits nach diesen Produkten gesucht, sie aber nicht in ihrer Einkaufsstätte gefunden.

Zu hohe Preisunterschiede zum ,normalen“ Fleisch gaben $15 \%$ der Befragten als Grund an. Die Befragten hatten außerdem die Möglichkeit, bei dieser Frage zusätzlich andere Gründe anzuführen. Mehrfach genannt wurde dahingehend, dass es beim Metzger bzw. an der Frischetheke keine Fleisch- und Wurstwaren mit einer Tierwohlkennzeichnung gebe. Andere Personen äußerten, dass sie wenig Vertrauen in Gütesiegel hätten. Für einen weiteren kleinen Teil der Verbraucher sind Gütesiegel per se ,reine Augenwischerei ${ }^{\circ}$. Diese Verbrauchergruppe geht davon aus, dass Gütesiegel ein reines Marketinginstrument darstellen, um höhere Gewinne zu erzielen. Positive Effekte auf das Tierwohl, die Umwelt, die Gesundheit oder soziale Aspekte werden von dieser Gruppe nicht erwartet.

Frage 13: Warum haben Sie noch keine Fleisch- oder Wurstwaren mit diesen Gütesiegeln gekauft? Sie können mehrere Gründe angeben. (nur für Nichtkäufer)

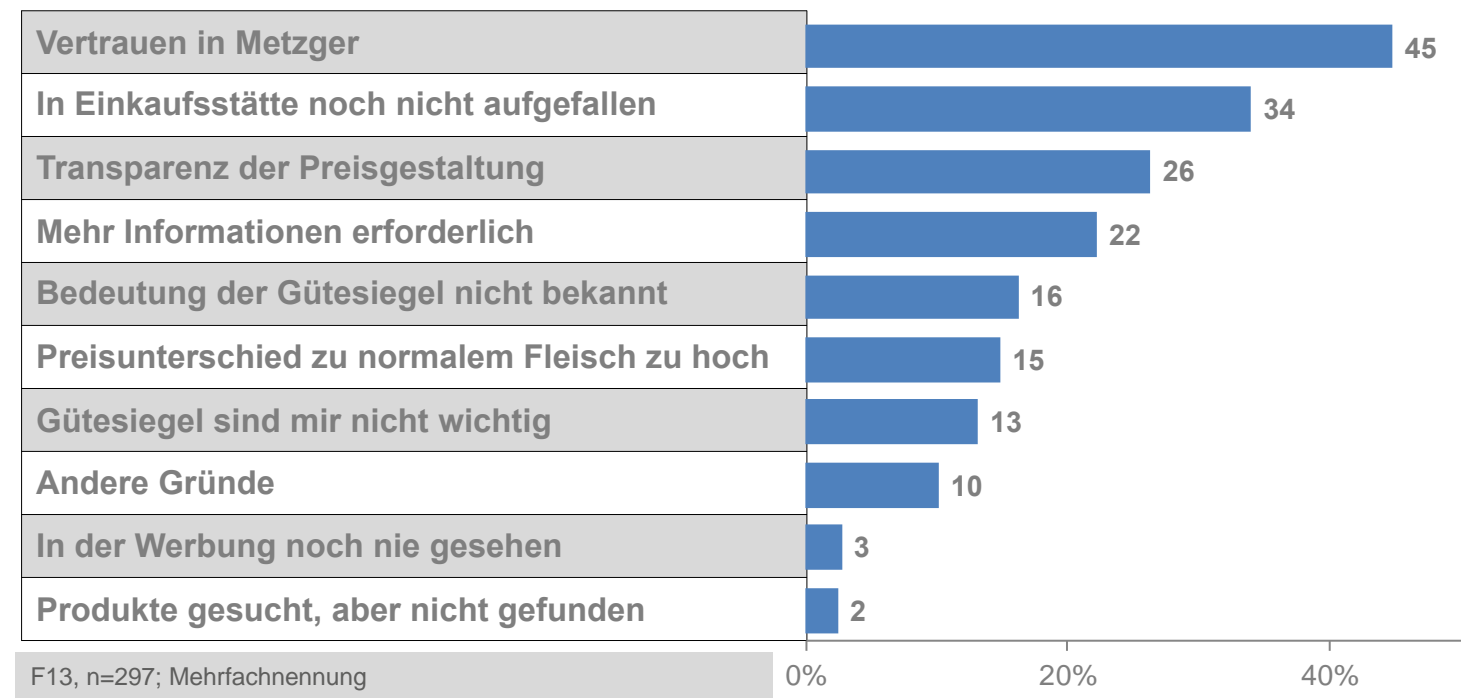

\section{Abbildung 21: Hinderungsgründe beim Kauf von Produkten mit Tierwohl-Labels (Quelle: Eigene Darstellung)}


Des Weiteren wurde der Zusammenhang zwischen den Hinderungsgründen beim Kauf von Produkten und den Haupteinkaufsstätten untersucht. In Abbildung 22 sind die dabei erzielten Ergebnisse ersichtlich. Demnach besteht ein hoch signifikanter Unterschied bei dem Kriterium Preisunterschied zum normalen Fleisch zu hoch (Chi-Quadrat (3) $\mathrm{F}=20,854, \mathrm{p}<0,01, \mathrm{n}=264)$. Hierbei handelt es sich jedoch um einen schwachen Zusammenhang (Cramer's V =0,282, p <0,01). Der zu hohe Preisunterschied zum normalen Fleisch stellt für fast ein Drittel $(29,3 \%)$ der Discounter-Käufer einen Hinderungsgrund dar; jedoch nur für $2 \%$ der Metzgerei-Käufer.

Hypothese 16: Es besteht ein Zusammenhang zwischen den Kaufbarrieren und der Haupteinkaufsstätte

\begin{tabular}{|c|c|c|}
\hline Im Laden noch nicht aufgefallen & $25,6^{33,7} 40$ & $\begin{array}{l}\text { Supermarkt } \\
\text { Discounter }\end{array}$ \\
\hline $\begin{array}{l}\text { Preisunterschied zum normalen } \\
\text { Fleisch zu hoch } * * *\end{array}$ & \begin{tabular}{|l|l|l|}
\multicolumn{3}{|c}{9,9} \\
\\
2 & 15,4 & 29,3
\end{tabular} & SB-Warenhäuser \\
\hline Transparenz der Preisgestaltung & \begin{tabular}{|l|l|} 
& 24,8 \\
14,3 & 30,8
\end{tabular} & Metzger \\
\hline In der Werbung noch nie gesehen & $\begin{array}{c}4 \\
2,6 \\
0\end{array}$ & \\
\hline Gütesiegel sind mir nicht wichtig & $\begin{array}{ll}12,9 \\
6,1\end{array} 25,6$ & \\
\hline $\begin{array}{l}\text { Mir ist nicht bekannt, was } \\
\text { Gütesiegel bedeuten }\end{array}$ & $\begin{array}{ll}13,9 & 21,3 \\
12,2 & 23,1\end{array}$ & \\
\hline Vertrauen in Metzger*** & $25,6,3$ & \\
\hline Mehr Informationen erforderlich & ${ }_{14,3}^{20,8} 23,1^{28}$ & \\
\hline Bereits gesucht, nicht gefunden & $\begin{array}{l}2 \\
2,7 \\
0\end{array}$ & \\
\hline $\begin{array}{l}\text { Chi-Quadrat: } p<0,01 * * * \\
F 13, F 3, n=264\end{array}$ & 20 & $100 \%$ \\
\hline
\end{tabular}

\section{Abbildung 22: Zusammenhang Kaufbarrieren und Haupteinkaufsstätte (Quelle: Eigene Darstellung)}

Für die meisten Metzgerei-Käufer $(91,8 \%)$ ist das Vertrauen in den Metzger ein Hinderungsgrund für den Kauf zertifizierter Fleisch- und Wurstwaren. Es ergab sich ein hoch signifikanter Zusammenhang (Chi-Quadrat (3) $\mathrm{F}=57,232, \mathrm{p}<0,01, \mathrm{n}=264$ ). Dieses Kriterium ist auch für 41,6 \% der Supermarkt-Käufer, 29,3\% der DiscounterKäufer und 25,6 \% der SB-Warenhaus-Käufer relevant. 
Frage 14 diente dazu, die untersuchten Tierwohl-Labels hinsichtlich ihrer Strenge für die drei Kriterien Tierwohl, Umwelt und Gesundheit einschätzen zu lassen. Abbildung 23 zeigt die Ergebnisse der Frage 14 in Form einer Positionierungsmatrix. Menschen schätzen demnach die Anforderungen bezüglich Tierwohl und Gesundheit bei BioProdukten am strengsten ein. Das Deutsche Bio-Siegel wird als strenger eingeschätzt als die Gütesiegel der deutschen Bio-Anbauverbände DEMETER, BIOLAND und NATURLAND. Für das Tierwohl-Label des DEUTSCHEN TIERSCHUTZBUNDES werden hohe Tierwohlstandards angenommen, jedoch wird von den Befragten vermutet, dass das Label hinsichtlich der gesundheitlichen Kriterien weniger streng ist. Als mittelmäßig streng werden u.a. das NEULAND-Label, VIER PFOTEN und BIOKREIS eingeschätzt. Die Strenge der Initiative Tierwohl und des HALTUNGSKOMPASSES von LIDL wird in beiden Kategorien als niedrig eingeschätzt.

Frage 14: Bitte schätzen Sie die folgenden Gütesiegel einmal grob hinsichtlich der Strenge ihrer unterschiedlichen Anforderungen ein.

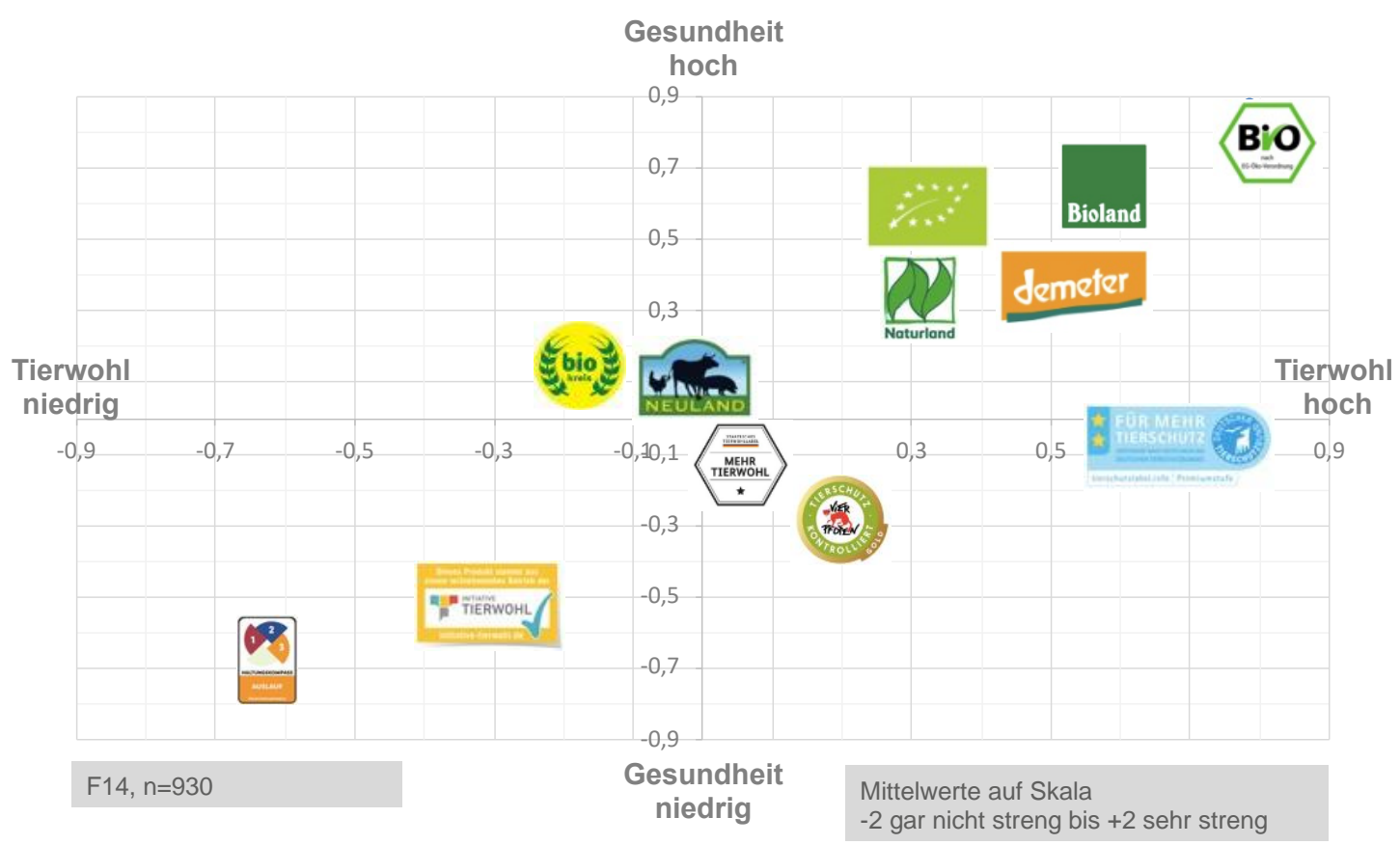

Abbildung 23: Einschätzung der Strenge von Gesundheit und Tierwohl (Quelle: Eigene Darstellung) 
In Abbildung 24 ist die Einschätzung der Befragten hinsichtlich der Strenge der Kriterien ebenfalls in einer Positionierungsmatrix dargestellt, dieses Mal jedoch in Bezug auf die Kategorien Umwelt und Gesundheit. Dabei ist ein ähnliches Bild erkennbar wie in Abbildung 23. Einige Unterschiede können dennoch wahrgenommen werden. Das Label des Tierschutzbundes wird in diesen beiden Kategorien eher als mittelmäßig streng eingeschätzt, und das NEULAND-Label sowie BIOKREIS werden hinsichtlich des Kriteriums Gesundheit als etwas strenger beurteilt.

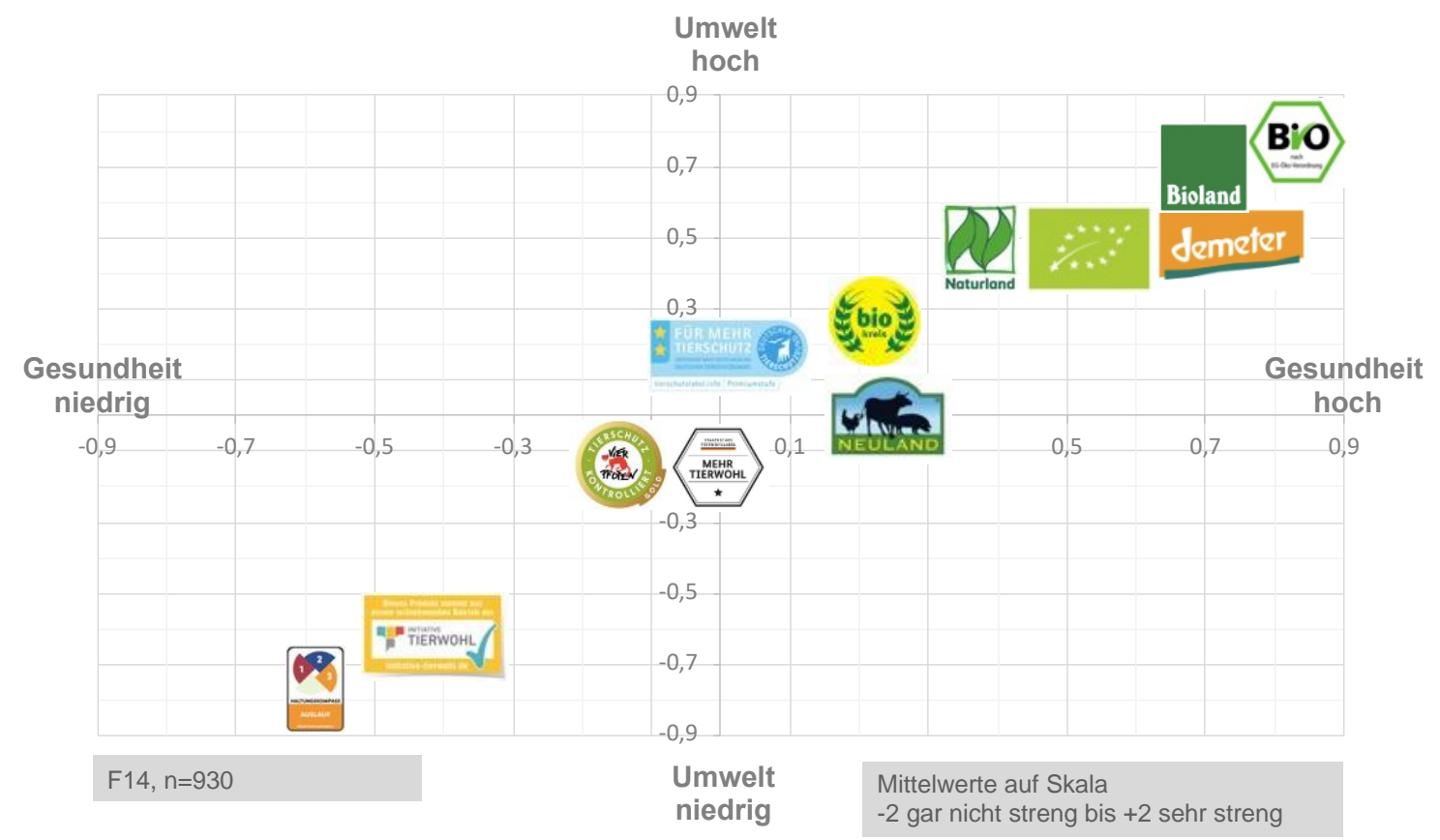

\section{Abbildung 24: Einschätzung der Strenge von Umwelt und Gesundheit (Quelle: Eigene Darstellung)}

Der Vergleich der Kategorien Tierwohl und Umwelt ist in Abbildung 25 veranschaulicht und zeigt, dass auch beim Vergleich der Kategorien Tierwohl und Umwelt die verschiedenen Tierwohl-Labels ähnlich bewertet werden. Auffällig ist vor allem, dass das Tierwohl-Label des DEUTSCHEN TIERSCHUTZBUNDES von den reinen TierwohlLabels als am strengsten eingeschätzt wird. 


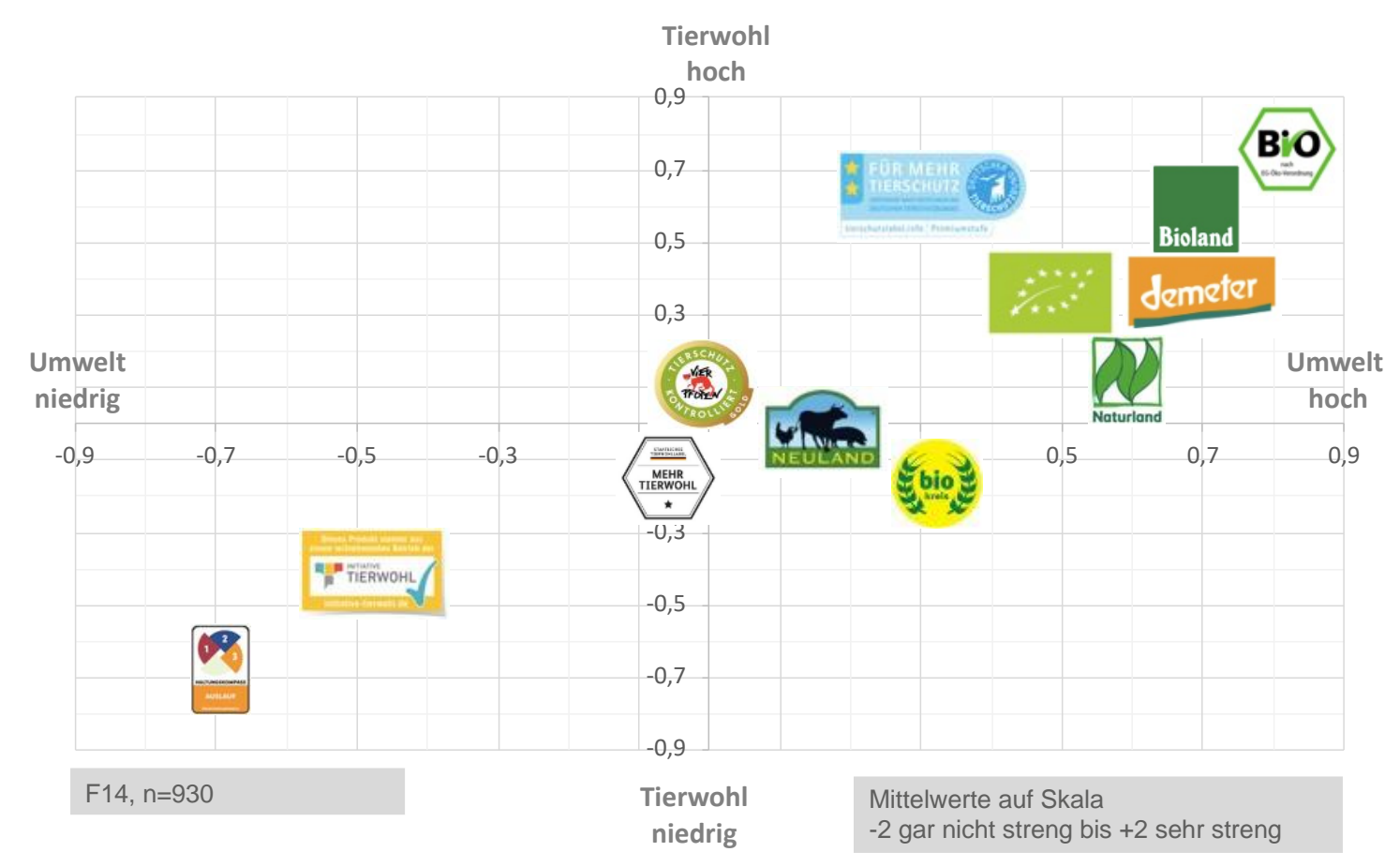

\section{Abbildung 25: Einschätzung der Strenge von Tierwohl und Umwelt (Quelle: Eigene Darstellung)}

Insgesamt wird deutlich, dass die verschiedenen Tierwohl-Labels hinsichtlich ihrer Strenge bezüglich der Kategorien Tierwohl, Umwelt und Gesundheit in drei unterschiedlichen ,Qualitätsklassen“ eingeordnet werden, wie in den Abbildungen 21 bis $23 \mathrm{zu}$ erkennen. In allen drei Kategorien werden Bio-Labels (mit Abweichungen beim Label BIOKREIS) als am strengsten eingeschätzt. Das deutsche Bio-Label gilt in allen Kategorien als strengstes Gütesiegel.

Ein weiterer Grund dafür, dass ein Teil der Bevölkerung keine Fleisch- und Wurstwaren aus einer artgerechteren Nutztierhaltung kauft, könnte das nicht ausreichende Wissen der Befragten über die Herausforderungen des übermäßigen Fleischkonsums in Deutschland sein. Daher wurden die Befragten zu ihrem Hintergrundwissen bezüglich der negativen Auswirkungen eines zu hohen Fleischkonsums befragt, um auch aus dieser allgemeineren Perspektive herauszufinden, welches die zentralen negativen Effekte des übermäßigen Fleischkonsums bzw. der übermäßigen Produktion von Fleisch- und Wurstwaren in Deutschland aus Sicht der Verbraucher sind. 
Dabei konnten die Befragten mehrere Gründe angeben. Die Ergebnisse sind in Abbildung 26 dargestellt und lassen erkennen, dass sich $95 \%$ der Befragten der negativen Auswirkungen des zu hohen Fleischkonsums in Deutschland bewusst sind. Es zeigen sich jedoch deutliche Unterschiede bei der Einschätzung der verschiedenen Aspekte.

Frage 16: Bitte kreuzen Sie nun alle für Sie richtig erscheinenden Aussagen an: Der übermäßige Verzehr bzw. die hohe Produktion von Fleisch- und Wurstwaren hat vermutlich negative Auswirkungen auf ...

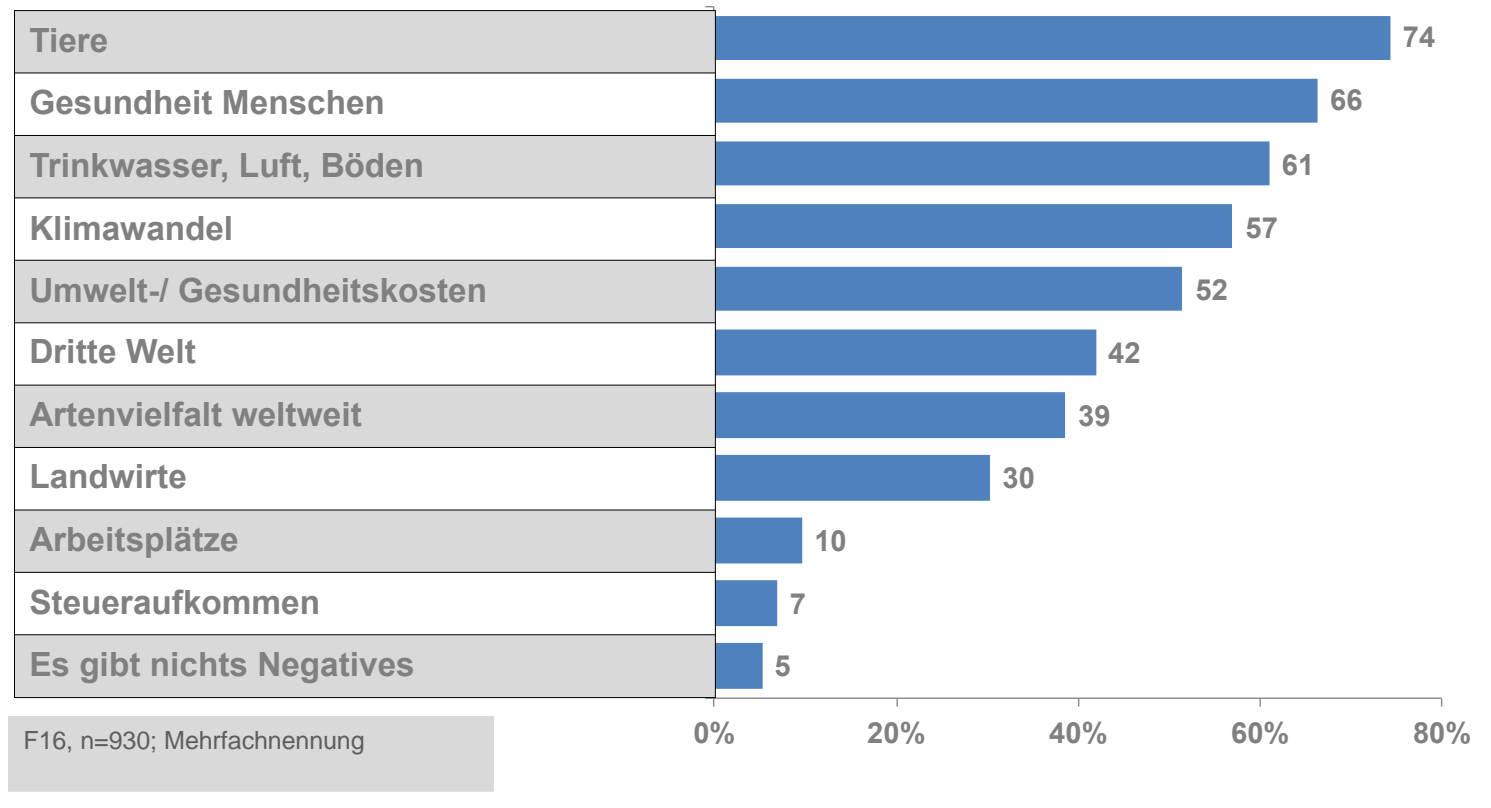

\section{Abbildung 26: Einschätzung negativer Auswirkungen des Fleischkonsums (Quelle: Eigene Darstellung)}

Die meisten Befragten (74\%) vermuten negative Auswirkungen auf die Tiere, die der Produktion von Fleisch- und Wurstwaren dienen. Am zweithäufigsten (66\%) wurden negative Auswirkungen auf die Gesundheit der Menschen in Deutschland genannt. Umweltbezogene Herausforderungen der intensiven Nutztierhaltung wie beispielsweise die Qualität des (Trink-)Wassers sowie die Qualität von Luft und Böden, der Klimawandel und die Artenvielfalt bilden den dritten Schwerpunkt aus Sicht der Verbraucher. Über die Hälfte der Befragten vermutet, dass die Höhe der Umwelt- und Gesundheitskosten für den Staat negativ durch den übermäßigen Fleischkonsum beeinflusst wird. 
Komplexere Zusammenhänge (die auch geographisch weiter entfernt sind), wie beispielsweise die negativen Auswirkungen des hiesigen Fleischkonsums bzw. der Fleischproduktion auf die Natur und die Menschen in Dritte-Welt-Ländern (z. B. auf die Artenvielfalt durch großflächigen Futtermittelanbau oder negative Effekte auf Kleinbauern oder indigene Völker), werden von $39 \%$ der Befragten richtig eingeschätzt. $30 \%$ der Befragten gehen von negativen Auswirkungen auf die Landwirte aus. Die geringsten negativen Auswirkungen werden bei der Anzahl der Arbeitsplätze und dem Steueraufkommen angenommen, und $5 \%$ gaben an, dass es keinerlei negative Auswirkungen gebe.

Abschließend für dieses Unterkapitel sollen die Ergebnisse über die Kundenzufriedenheit der Befragten im Hinblick auf das Produkt- und Beratungsangebot an zertifizierten Tierwohlprodukten vorgestellt werden. Mit dieser Frage wurde das Ziel verfolgt, aus Perspektive der Verbraucher festzustellen, in welchem Bereich der Vermarktung das größte Optimierungspotenzial zu verorten ist, um die Kundenzufriedenheit beim Produktund Beratungsangebot für Tierwohlprodukte zukünftig weiter zu erhöhen. Auf einer fünfstufigen Likert-Skala konnten die Befragten ihre Meinung zu verschiedenen Punkten kundtun.

Wie in Abbildung 27 ersichtlich sind die Befragten mit der Angebotsvielfalt an Fleischund Wurstwaren aus einer artgerechteren Haltung sowie der Auffindbarkeit bzw. der Erkennbarkeit im Geschäft recht zufrieden. Optimierungspotenzial besteht aus Perspektive der Befragten vor allem bei der Ästhetik der SB-Verpackungen, Fleischtheken oder Werbeprospekte sowie der Sichtbarkeit der Produkte in Werbeprospekten, Plakaten und Anzeigen. Hinsichtlich der persönlichen Beratung zu Tierwohlprodukten im Lebensmitteleinzelhandel und der Transparenz beim Engagement für eine artgerechtere Tierhaltung bestehen offensichtlich die größten Möglichkeiten, um die Kundenzufriedenheit zukünftig erhöhen zu können. In Unterkapitel 5.3.3 werden die Ergebnisse zum Informationsbedarf der Verbraucher präsentiert, wodurch die Wünsche der Verbraucher nach mehr Transparenz noch näher untersucht werden können. 
Frage 19: Wie zufrieden sind Sie mit dem Produkt- und Beratungsangebot für Fleischund Wurstwaren aus einer artgerechteren Tierhaltung in Ihrer Haupteinkaufsstätte?

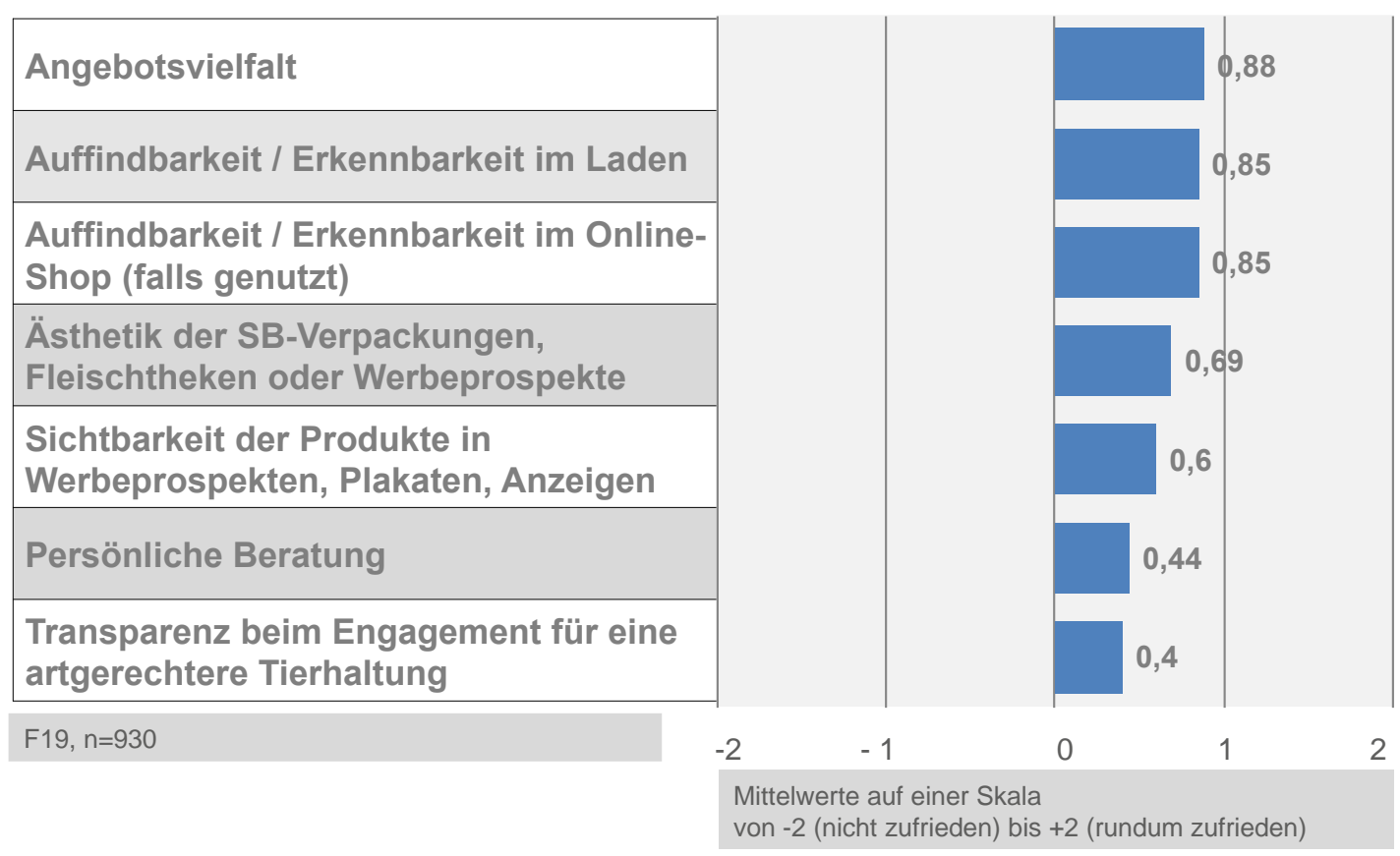

\section{Abbildung 27: Kundenzufriedenheit mit dem Angebot an Tierwohlprodukten (Quelle: Eigene Darstellung)}

Abbildung 28 zeigt die Zusammenhänge zwischen der Kundenzufriedenheit und der Haupteinkaufsstätte. Es lässt sich erkennen, dass beim Kriterium Angebotsvielfalt an Tierwohlprodukten $(\mathrm{F}(3,785)=19,473, \quad \mathrm{p}<0,01, \quad \mathrm{n}=789) \quad$ ein signifikanter Zusammenhang besteht.

Nach Auswertung der post-hoc-Tests mit Bonferroni-Korrektur lassen sich die Haupteinkaufsstätten in die drei Gruppen einteilen. Hierbei bilden die SB-WarenhausKäufer $(\mathrm{MW}=0,81 ; \mathrm{SD}=0,928)$ und die Discounter-Käufer $(\mathrm{MW}=0,58 ; \mathrm{SD}=1,063)$ eine gemeinsame Gruppe. Zwischen dieser Gruppe existiert ein signifikanter Unterschied zu Metzgerei-Käufern $(\mathrm{MW}=1,34 ; \mathrm{SD}=0,824)$ und Supermarkt-Käufern $(\mathrm{MW}=0,91$; SD $=0,901)$. Die Mittelwerte vergleichend wird deutlich, dass die Zufriedenheit bei Metzgerei-Käufern am höchsten und bei SB-Warenhaus-Käufern und DiscounterKäufern am geringsten ist. 
Hypothese 15: Es besteht ein Zusammenhang zwischen der Kundenzufriedenheit mit dem Produkt- und Beratungsangebot für Fleisch- und Wurstwaren aus einer artgerechteren Tierhaltung und der Haupteinkaufsstätte.

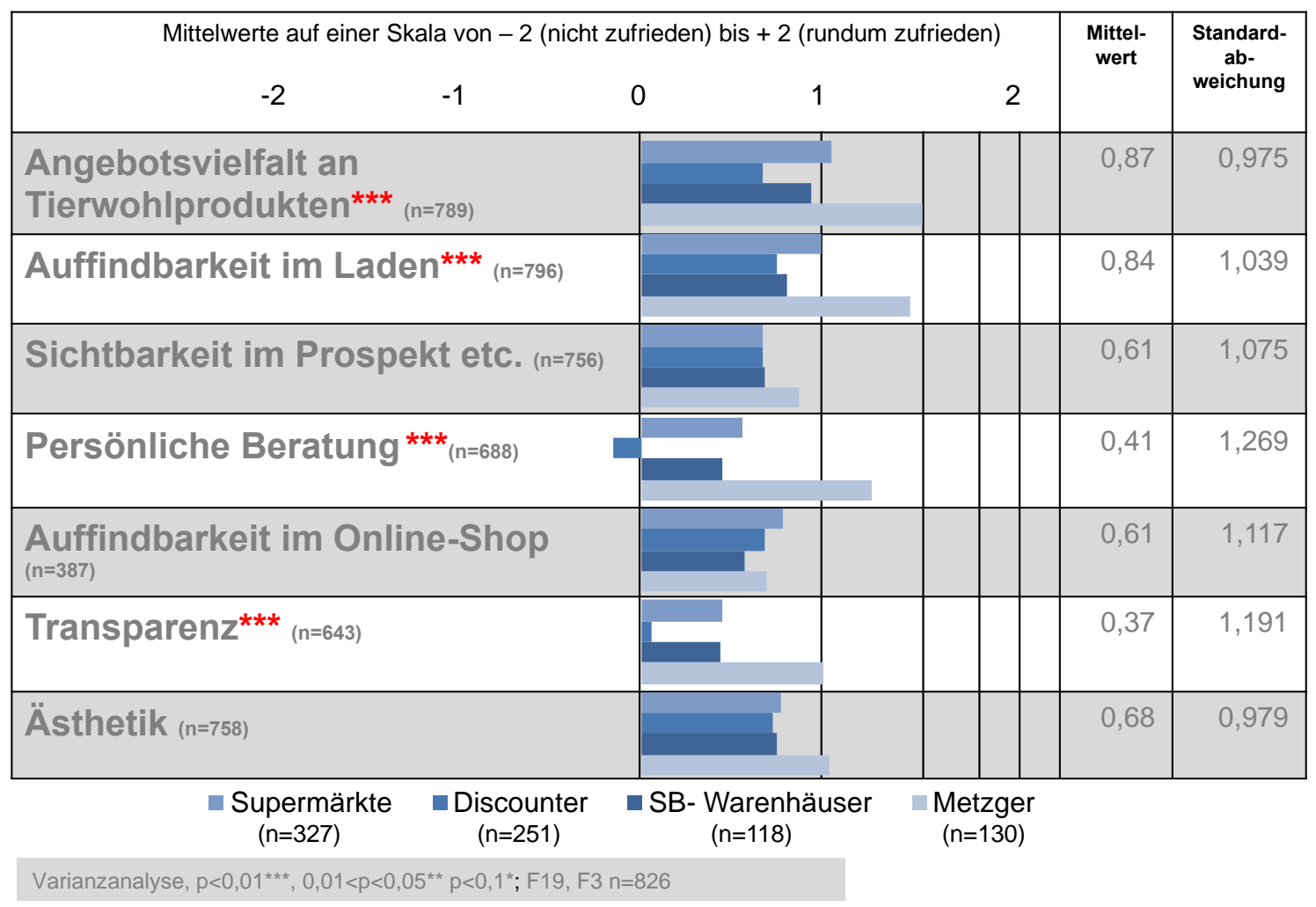

\section{Abbildung 28: Zusammenhang Kundenzufriedenheit und Haupteinkaufsstätte} (Quelle: Eigene Darstellung)

Es bestehen zudem hoch signifikante Unterschiede bei der Auffindbarkeit und Erkennbarkeit im Laden $(\mathrm{F}(3,792)=11,239, \mathrm{p}<0,01, \mathrm{n}=796)$. In den post-hoc-Tests zeigte sich ein signifikanter Unterschied zwischen Befragten, die den Metzger $(\mathrm{MW}=1,28 ; \mathrm{SD}=1,001)$ als Haupteinkaufsstätte angegeben haben, und SupermarktKäufern (MW = 0,86; SD = 0,981), Discounter-Käufern (MW =0,65; SD = 1,097) sowie SB-Warenhaus-Käufern (MW = 0,70; SD = 0,976).

Metzgerei-Käufer sind zufriedener hinsichtlich der Auffindbarkeit und Erkennbarkeit im Laden. Für das Kriterium der persönlichen Beratung $(\mathrm{F}(3,684)=25,884, p<0,01$, $\mathrm{n}=688)$ konnte ebenfalls ein hoch signifikanter Unterschied festgestellt werden. 
Nach Auswertung der post-hoc-Tests lassen sich die Haupteinkaufsstätten in drei Gruppen einteilen. Hierbei bilden die Supermarkt-Käufer (MW =0,48; SD = 1,205) und SB-Warenhaus-Käufer ( $\mathrm{MW}=0,39$; SD = 1,091) eine gemeinsame Gruppe. Zwischen diesen Gruppen gibt es einen signifikanten Unterschied bei Metzgerei-Käufern $(\mathrm{MW}=1,10 ; \mathrm{SD}=1,121)$ und Discounter-Käufern $(\mathrm{MW}=-0,13 ; \mathrm{SD}=1,310)$. Mit Blick auf die Mittelwerte ist zu erkennen, dass die Zufriedenheit mit der persönlichen Beratung bei Metzgerei-Käufern am höchsten und bei Discounter-Käufern am geringsten ist. Bei dem Kriterium Transparenz $(\mathrm{F}(3,639)=11,220, \mathrm{p}<0,01, \mathrm{n}=643)$ besteht ein hoch signifikanter Zusammenhang zwischen dem Kaufkriterium und den Haupteinkaufsstätten.

In den post-hoc-Tests zeigte sich ein signifikanter Unterschied zwischen MetzgereiKäufern $(\mathrm{MW}=0,87 ; \mathrm{SD}=1,142)$ und Supermarkt-Käufern $(\mathrm{MW}=0,39 ; \mathrm{SD}=1,124)$, SB-Warenhaus-Käufern $\quad(\mathrm{MW}=0,38 ; \quad \mathrm{SD}=1,176) \quad$ sowie $\quad$ Discounter-Käufern $(\mathrm{MW}=0,05 ; \mathrm{SD}=1,221)$. Zudem ergab der post-hoc-Test mit Bonferroni-Korrektur einen Unterschied zwischen Supermarkt-Käufern und Discounter-Käufern. MetzgereiKäufer sind am zufriedensten mit der Transparenz beim Engagement für eine artgerechte Tierhaltung. Discounter-Käufer weisen hingegen die geringste Zufriedenheit auf. 
In Frage 20 wurden zwei verschiedene Varianten einer Sortierung von Tierwohlprodukten zur Auswahl angeboten, zwischen denen sich die Befragten entscheiden sollten. Wie in Abbildung $29 \mathrm{zu}$ erkennen wünschen sich die meisten Befragten (64\%) im Lebensmitteleinzelhandel einen eigenen Bereich für alle Produkte mit besonders hohen Tierwohlstandards.

Frage 20: Sie stehen in einem Laden vor einem Kühlregal, einer Kühltruhe oder einer Fleischtheke mit verschiedenen Fleisch- und Wurstwaren. Welche Sortierung würden Sie sich persönlich wünschen? Wählen Sie bitte eine der zwei angebotenen Alternativen aus.

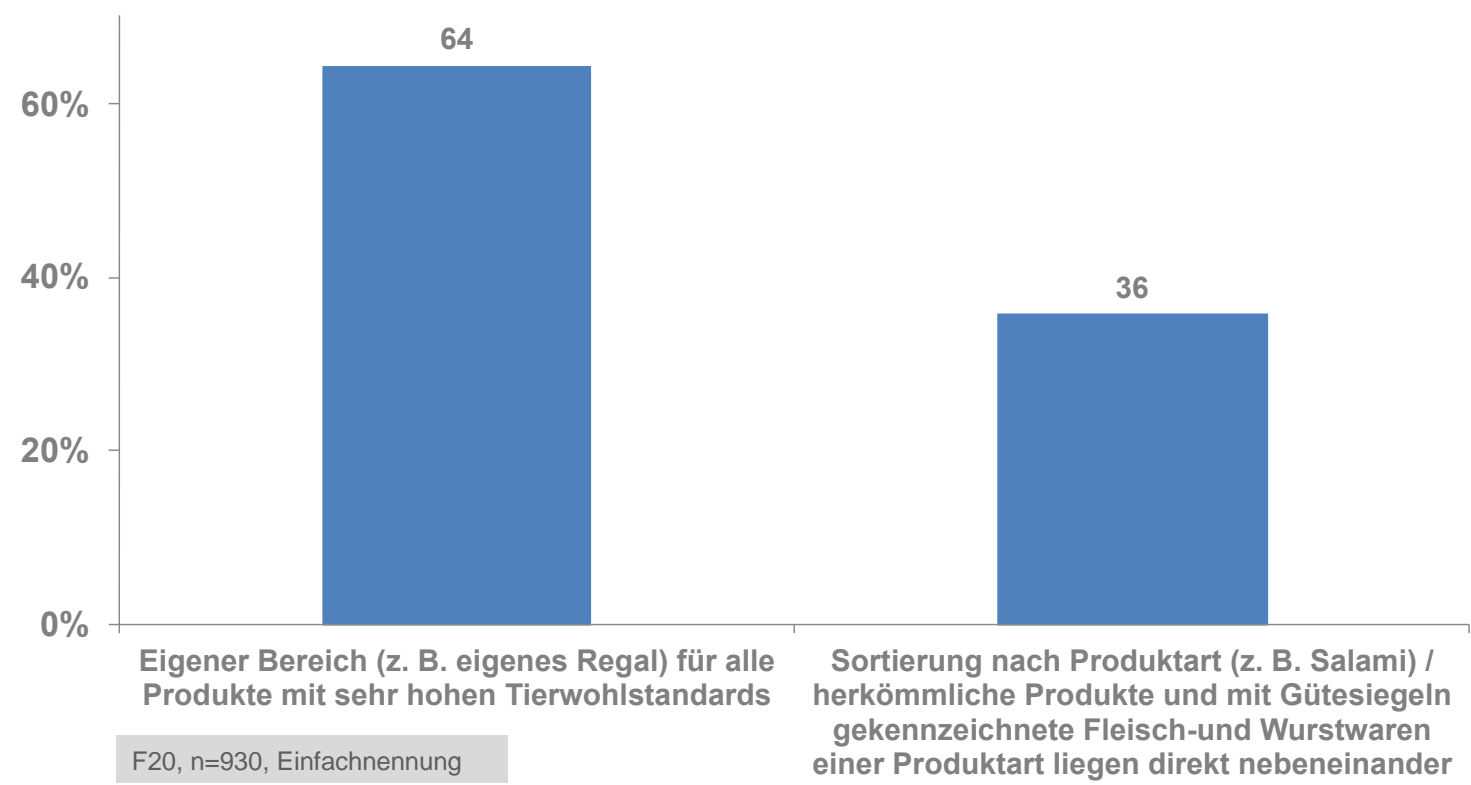

\section{Abbildung 29: Sortierung von Tierwohlprodukten im LEH (Quelle: Eigene Darstellung)}

Bei der Untersuchung des Zusammenhangs zwischen der Haupteinkaufsstätte und der bevorzugten Sortierung, deren Ergebnisse in Abbildung 30 dargestellt sind, zeigte sich kein signifikanter Zusammenhang (Chi-Quadrat (3) $F=4,103 \mathrm{p}<0,251 \mathrm{n}=826$ ). 
Hypothese17: Es besteht ein Zusammenhang zwischen den Präferenzen zur Sortierung der Tierwohlprodukte und der Haupteinkaufsstätte.

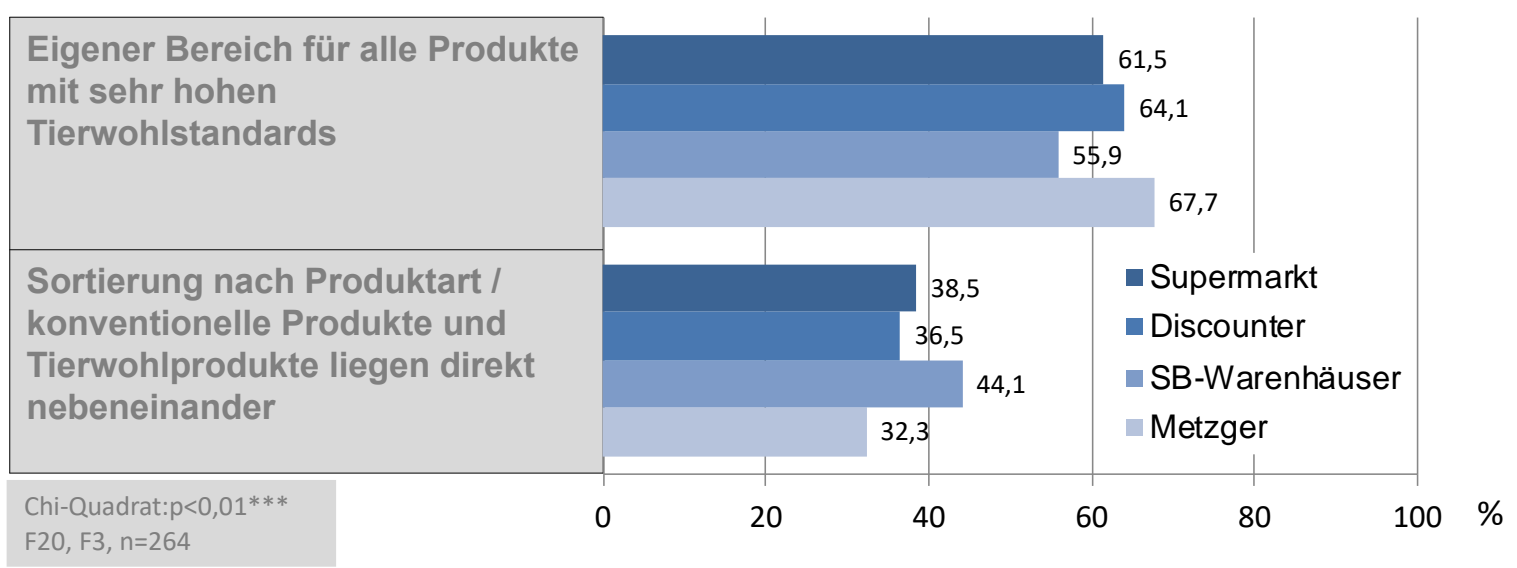

\section{Abbildung 30: Zusammenhang Sortierung und Haupteinkaufsstätte (Quelle: Eigene Darstellung)}

Im intensiven Wettbewerb des deutschen Lebensmitteleinzelhandels kann das Produktund Beratungsangebot zum Thema artgerechtere Tierhaltung womöglich ein bedeutsamer Faktor bei der Gewinnung neuer Kunden oder der Bindung bestehender Kunden sein. Daher sollte festgestellt werden, wie relevant das Thema Tierwohl für die Verbraucher ist und ob die Verbraucher aufgrund des Produkt- und Beratungsangebots an Tierwohlprodukten ihre Haupteinkaufsstätte für Fleisch- und Wurstwaren wechseln würden. Folglich wurde in Frage 21 die Wechselbereitschaft der Verbraucher hinsichtlich ihrer Haupteinkaufsstätte erfragt.

Abbildung 31 zeigt, dass der Großteil der Verbraucher (66\%) ihre Haupteinkaufsstätte wahrscheinlich (oder sicher) wechseln würde, wenn andere Anbieter von Fleisch- und Wurstwaren ihr Produkt- und Beratungsangebot beim Thema artgerechtere Tierhaltung verbessern und die Verbraucher bei ihrer Haupteinkaufsstätte keine Verbesserungen feststellen würden. Rund ein Drittel der Befragten sind unentschlossen, ob sie ihre Haupteinkaufsstätte wechseln würden. Nur $10 \%$ würden ihre Haupteinkaufsstätte wahrscheinlich nicht wechseln, sondern ihrer Haupteinkaufsstätte treu bleiben. 
Frage 21: Stellen Sie sich vor, viele Anbieter von Fleisch- und Wurstwaren verbessern zukünttig Ihr Produkt- und Beratungsangebot zum Thema artgerechtere Tierhaltung. Würden Sie Ihre Haupteinkaufsstätte wechseln, wenn Sie bei Ihrem Anbieter keine Verbesserungen feststellen?

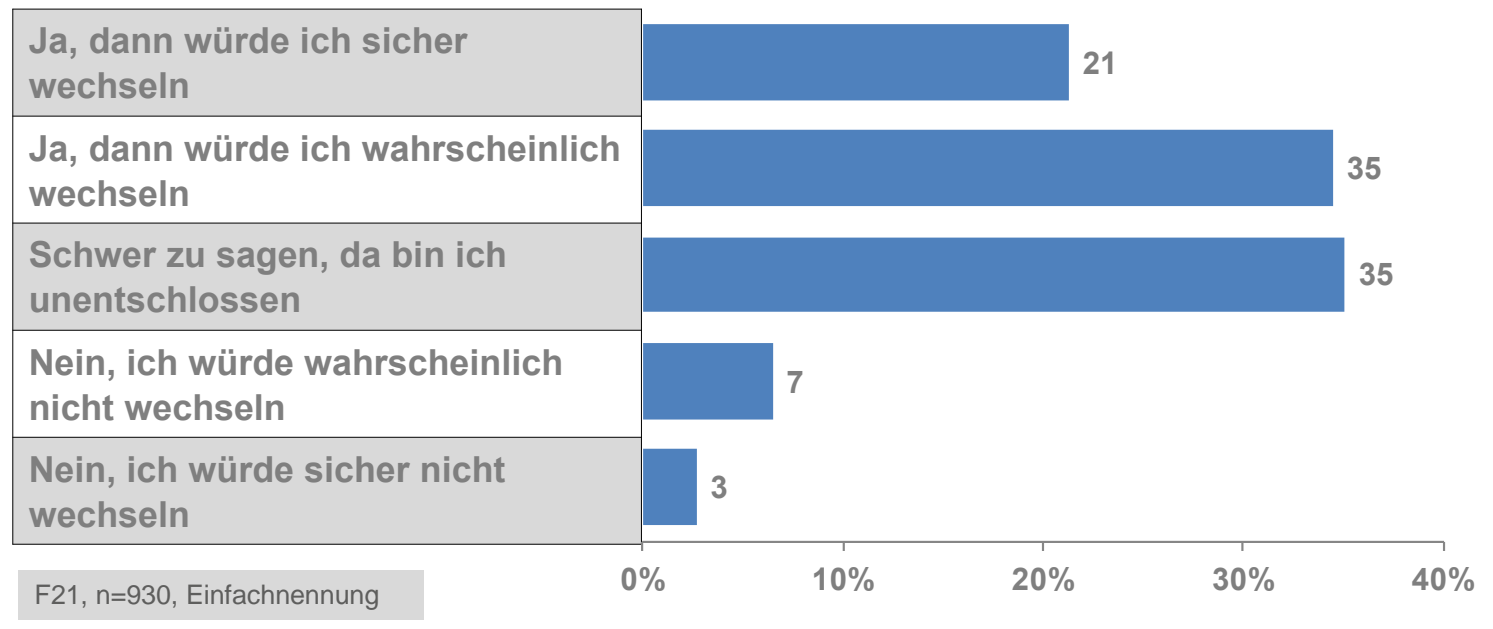

\section{Abbildung 31: Einkaufsstättenwechsel aufgrund von Tierwohlprodukten (Quelle: Eigene Darstellung)}

Des Weiteren wurde untersucht, ob die Wechselbereitschaft zwischen den Haupteinkaufsstätten variiert. In Abbildung 32 lässt sich erkennen, dass kein signifikanter Unterschied zwischen den Haupteinkaufstätten besteht $(\mathrm{F}(3,822)=1,145, \mathrm{p}=0,323$, $\mathrm{n}=826$ ). Es zeigte sich bei jeder Haupteinkaufsstätte eine Wechselbereitschaft, wenn andere Anbieter von Fleisch- und Wurstwaren im Gegensatz zur eigenen Haupteinkaufsstätte ihr Produkt- und Beratungsangebot beim Thema artgerechtere Tierhaltung verbessern würden. 
Hypothese18: Es besteht ein Zusammenhang zwischen der Wechselbereitschaft der Haupteinkaufsstätte bei fehlenden Verbesserungen hinsichtlich des Produkt- und Beratungsangebots für Fleisch- und Wurstwaren aus einer artgerechteren Tierhaltung und der Haupteinkaufsstätte.

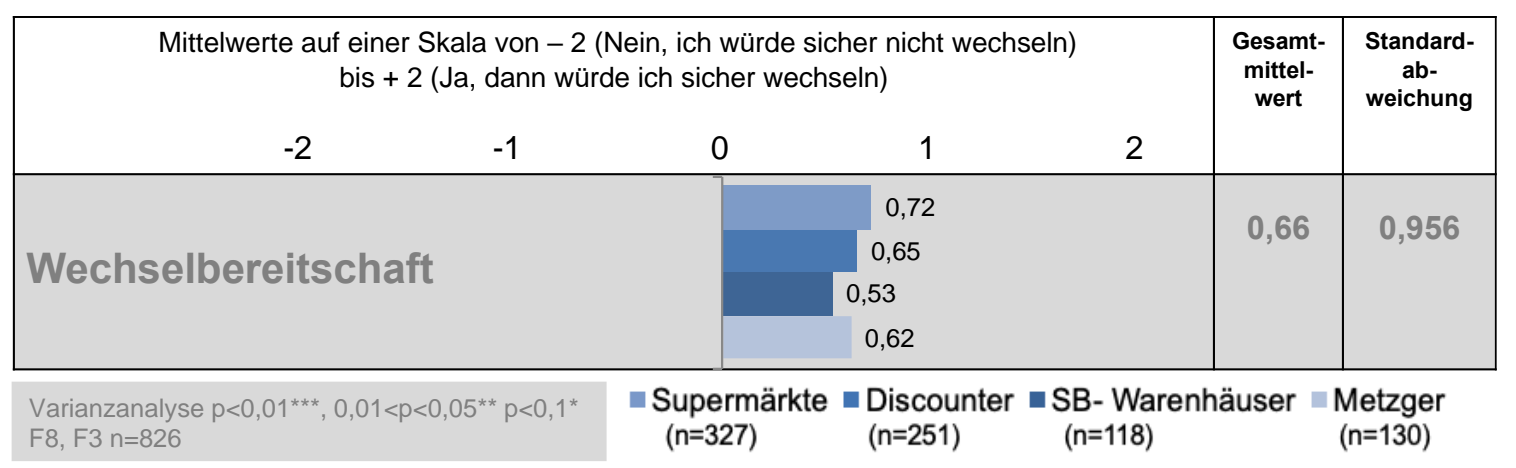

\section{Abbildung 32: Zusammenhang Wechselbereitschaft und Haupteinkaufsstätte (Quelle: Eigene Darstellung)}

\subsubsection{Informationsbedarf bei Tierwohlprodukten}

In Unterkapitel 3.4 zum Informationsbedarf bei Tierwohlprodukten wurde beschrieben, dass sich ein Großteil der Verbraucher vor allem bei Fleisch- und Wurstwaren mehr Transparenz hinsichtlich der artgerechteren Tierhaltung wünscht. Daher wurden im Fragebogen drei zentrale Fragen zum Informationsbedarf gestellt. Zunächst sollten die Befragten angeben, ob sie sich bessere Informationen zu den Haltungsbedingungen der Tiere und zur Herkunft der Fleisch- und Wurstwaren wünschen (Frage 22).

Personen $(\mathrm{N}=101)$, die kein Interesse an diesen Informationen angaben, wurde im Fragebogen daraufhin keine weitere Frage zum Informationsbedarf gestellt. Personen $(\mathrm{N}=829)$, die äußerten, sich mehr Hintergrundinformationen zu wünschen, wurden drei Fragen $\mathrm{zu}$ ihrem Informationsbedarf gestellt. Herausgefunden werden sollte diesbezüglich, wo sich die Befragten informieren möchten, wer entsprechende Hintergrundinformationen bereitstellen soll und ob detailliertere Herkunftsangaben ein Kaufargument für die Befragten darstellen. Im Folgenden werden die Ergebnisse dieser Fragen zum Informationsbedarf vorgestellt. 
Hinsichtlich der verschiedenen Tierwohl-Labels besteht aus Perspektive vieler Verbraucher ein Bedarf an weiterführenden Informationen (Unterkapitel 3.4). Mit Frage 22 sollte zunächst ermittelt werden, wie viel Prozent der Befragten nähere Informationen zu den Haltungsbedingungen der Tiere und zur Herkunft der Fleisch- und Wurstwaren erhalten möchten. Frage 22 hatte außerdem eine wesentliche Filterfunktion, da nur Personen zu ihrem Informationsbedarf befragt werden sollten, die sich entsprechende Informationen wünschen. Diesen Personen $(\mathrm{N}=829)$ wurden drei weitere spezifische Fragen zum Informationsbedarf gestellt.

In Abbildung 33 lässt sich zunächst erkennen, dass die deutliche Mehrheit (rd. $90 \%$ ) der Befragten für die Bereitstellung besserer Informationen zu den Haltungsbedingungen der Tiere und zur Herkunft der Fleisch- und Wurstwaren votieren.

Frage 22: Würden Sie gerne bessere Informationen zu den Haltungsbedingungen der Tiere und zur Herkunft der Fleisch- und Wurstwaren, die Sie kaufen, erhalten?

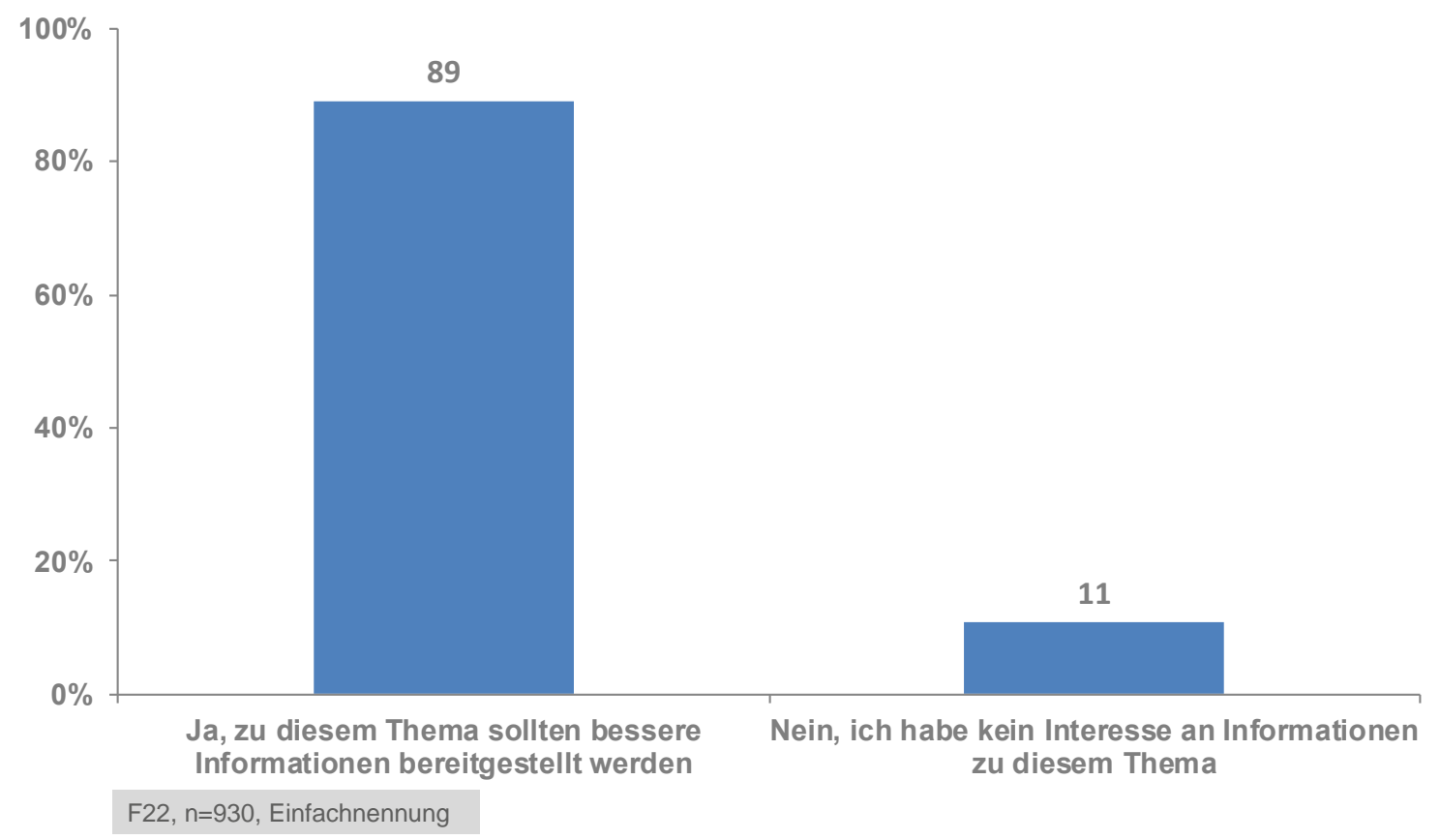

Abbildung 33: Informationsbedarf beim Kauf von Fleisch- und Wurstwaren (Quelle: Eigene Darstellung) 
Alle Personen, die Interesse an Informationen zu den Haltungsbedingungen der Tiere und zur Herkunft der Fleisch- und Wurstwaren angegeben hatten, wurden anschließend gefragt, wo sie sich gerne bezüglich der von ihnen gekauften Fleisch- und Wurstwaren über die Haltungsbedingungen der Tiere informieren möchten. Dabei konnten sie mehrere Antwortmöglichkeiten auswählen (Mehrfachnennung).

In Abbildung 34 sind die Antworten der Frage 23 dargestellt, wobei zwei zentrale Orte festgestellt werden können, an denen sich die meisten Verbraucher (> $80 \%$ ) informieren möchten. Die beiden bedeutendsten Orte sind die Produktverpackung und die Verkaufsräume. Daneben gaben über $60 \%$ der Befragten an, sich in den Werbeprospekten und beim Verkaufspersonal über die Haltungsbedingungen der Nutztiere und die Herkunft der von ihnen gekauften Fleisch- und Wurstwaren informieren zu wollen. Etwa die Hälfte der Befragten würde sich gerne auf einem Internetportal informieren.

Frage 23: Wo würden Sie sich gerne über die Haltungsbedingungen der Nutztiere und die Herkunft der von Ihnen gekauften Fleisch- und Wurstwaren informieren?

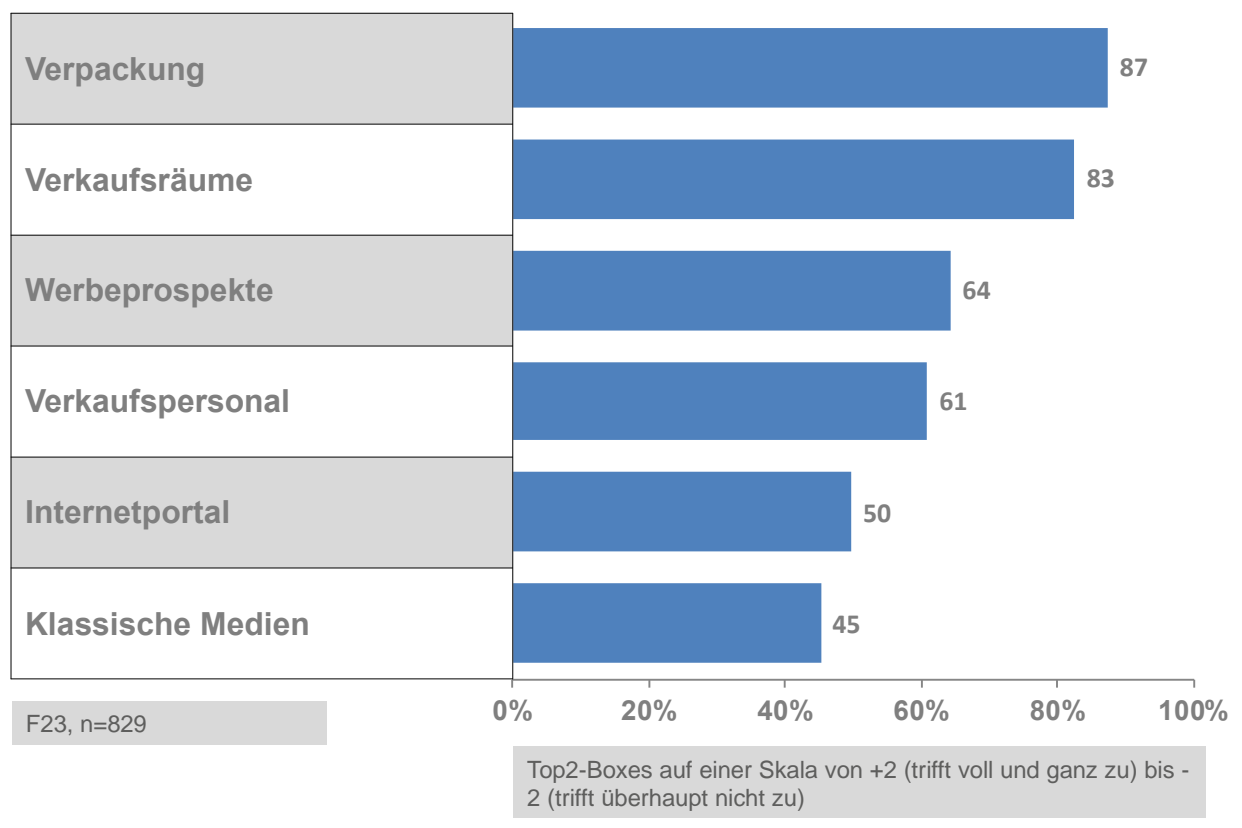

Abbildung 34: Bereitstellung von Tierwohlinformationen (Quelle: Eigene Darstellung) 
Zur weiteren Differenzierung wurde untersucht, ob ein Zusammenhang zwischen der Haupteinkaufsstätte und den präferierten Orten bzw. Medien für spezifische Tierwohlinformationen vorliegt. Wie in Abbildung $35 \mathrm{zu}$ erkennen bestehen hoch signifikante Unterschiede beim Kriterium Verkaufspersonal $(\mathrm{F}(3,738)=25,112$, $\mathrm{p}<0,01, \quad \mathrm{n}=742)$. Nach Auswertung der post-hoc-Tests lassen sich die Haupteinkaufsstätten in drei Gruppen einteilen. Hierbei bilden die Supermarkt-Käufer $(\mathrm{MW}=0,83 ; \mathrm{SD}=1,023)$ und die SB-Warenhaus-Käufer $(\mathrm{MW}=0,76 ; \mathrm{SD}=1,051)$ eine gemeinsame Gruppe, bei der ein signifikanter Unterschied zu Metzgerei-Käufern $(\mathrm{MW}=1,21 ; \quad \mathrm{SD}=0,899)$ und Discounter-Käufern $(\mathrm{MW}=0,22 ; \mathrm{SD}=1,212) \quad \mathrm{zu}$ verzeichnen ist. Die Mittelwerte betrachtend lässt sich feststellen, dass die persönliche Information beim Verkaufspersonal für Metzgerei-Käufer am wichtigsten ist. Zudem ergab sich ein schwach signifikanter Zusammenhang bei der Informationsbereitstellung in den Verkaufsräumen $(\mathrm{F}(3,738)=2,424, \mathrm{p}=0,065, \mathrm{n}=742)$.

Hypothese 19: Es besteht ein Zusammenhang zwischen den Präferenzen zur Informationsbereitstellung und der Haupteinkaufsstätte.

\begin{tabular}{|c|c|c|c|}
\hline $\begin{array}{l}\text { Mittelwerte auf einer Skala von }-2 \text { (trifft über } \\
\text { bis }+2 \text { (trifft voll und ganz zu) } \\
-2\end{array}$ & cht zu) & $\begin{array}{c}\text { Gesamt } \\
- \\
\text { mittel- } \\
\text { wert }\end{array}$ & $\begin{array}{l}\text { Standard- } \\
\text { ab- } \\
\text { weichung }\end{array}$ \\
\hline Verkaufsräume* & $\begin{array}{l}1,14^{128} \\
1,13_{1,36}\end{array}$ & 1,23 & 0,882 \\
\hline Verkaufspersonal*** & $\begin{array}{lll}0,22 & 0,83 & \\
& 0,76 & 1,21 \\
\end{array}$ & 0,69 & 1,123 \\
\hline Verpackung & $\begin{array}{r}1,40 \\
1,50 \\
1,34 \\
1,34 \\
\end{array}$ & 1,43 & 0,778 \\
\hline Werbeprospekte & $\begin{array}{r}0,81 \\
0,85 \\
0,62 \quad 0,97 \\
\end{array}$ & 0,81 & 1,146 \\
\hline Internetportal & $\begin{array}{l}0,50 \\
0,51 \\
0,40 \\
0,30\end{array}$ & 0,46 & 1,201 \\
\hline Klassische Medien & $\begin{array}{l}0,41 \\
0,27 \\
0,40 \\
0,21\end{array}$ & 0,33 & 1,204 \\
\hline
\end{tabular}

Abbildung 35: Zusammenhang Tierwohlinformationen und Haupteinkaufsstätte (Quelle: Eigene Darstellung) 
Im Rahmen der Analyse der Informationsbereitstellung im Zusammenhang mit der Angebotsform von Wurst und Aufschnitt zeigte sich, dass, je häufiger die Befragten an der Theke kaufen, desto eher bevorzugen sie, ihre Informationen über Broschüren und auf der Verpackung zu erhalten, und desto weniger wünschen sie sich eine persönliche Beratung durch das Verkaufspersonal. Hierbei handelt es sich um eine hoch signifikante, jedoch sehr schwache bzw. schwache Korrelation. Die Ergebnisse sind Tabelle $14 \mathrm{zu}$ entnehmen.

Hypothese 20: Die Präferenzen zur Informationsbereitstellung hängen mit der Angebotsform beim Kauf von Wurst und Aufschnitt fürs Brot zusammen (Theke/SB).

Tabelle 14: Korrelation Tierwohlinformationen und Angebotsform (Wurst)

\begin{tabular}{|l|l|}
\hline Angebotsform Wurst und Aufschnitt & Korrelationskoeffizient \\
\hline ...über Broschüren &, $093^{* *}$ \\
\hline ... persönlich beim Verkaufspersonal &,$- 112^{* *}$ \\
\hline ...auf der Verpackung &, $318^{* *}$ \\
\hline ... in den Werbeprospekten & $-0,032$ \\
\hline ...auf einem zentralen Internetportal & $-0,001$ \\
\hline ...in den Medien & 0,039 \\
\hline Korrelation, $p<0,01^{* *}, p<0,05^{*} F 4, F 23 n=829$ & \\
\hline
\end{tabular}

\section{Quelle: Eigene Darstellung}

Für die Angebotsform von Fleisch zum Braten oder Grillen konnte entgegen der Erwartung ermittelt werden, dass, je häufiger die Befragten an der Theke kaufen, desto eher möchten sie ihre Informationen über Broschüren und auf der Verpackung erhalten und desto weniger wünschen sie sich eine persönliche Beratung durch das Verkaufspersonal. Hierbei handelt es sich um eine signifikante, jedoch sehr schwache bzw. schwache Korrelation. Tabelle 15 zeigt das Ergebnis. 
Hypothese 21: Die Präferenzen zur Informationsbereitstellung hängen mit der Angebotsform beim Kauf von Fleisch zum Braten oder Grillen zusammen (Theke/SB).

Tabelle 15: Korrelation Tierwohlinformationen und Angebotsform (Fleisch)

\begin{tabular}{|l|l|}
\hline Angebotsform Fleisch zum Braten und Grillen & Korrelationskoeffizient \\
\hline$\ldots$...über Broschüren &, $084^{*}$ \\
\hline$\ldots$ persönlich beim Verkaufspersonal &,$- 099^{* *}$ \\
\hline ...auf der Verpackung &, $247^{* *}$ \\
\hline$\ldots$ in den Werbeprospekten & $-0,039$ \\
\hline$\ldots$...auf einem zentralen Internetportal & $-0,015$ \\
\hline$\ldots$ in den Medien & $-0,003$ \\
\hline Korrelation, $p<0,01^{* *}, p<0,05^{*}$ F5, F23 $n=829$ & \\
\hline
\end{tabular}

\section{Quelle: Eigene Darstellung}

Neben dem Ort der Bereitstellung von Informationen sollte zudem die Bedeutung der Herkunft für Fleisch- und Wurstwaren näher untersucht werden. Dass die Herkunft von Produkten für viele Verbraucher ein bedeutsames allgemeines Kaufkriterium darstellt, wurde bereits in verschiedenen Studien untersucht (Kapitel 3). Es herrscht jedoch Uneinigkeit darüber, wie detailliert diese Herkunftsangaben aus Perspektive der Verbraucher sein sollen. Der Detaillierungsgrad der Herkunftsangaben ist vor dem Hintergrund der geforderten Transparenz bei Tierwohlprodukten von besonderer Bedeutung und wurde daher in Frage 25 erfragt.

Abbildung 36 lässt sich entnehmen, dass die Herkunft der Fleisch- und Wurstwaren nur für 0,4\% der Befragten gar keine Rolle spielt. Die Angabe der groben Region (z. B. ,Made in Niedersachsen`) halten nur 1,8\% der Befragten für ausreichend. Rund $10 \%$ der Befragten sind sich unsicher, ob sie die Herkunft der Produkte beim Kauf berücksichtigen würden. Für fast $90 \%$ der Befragten wäre es jedoch ein Kaufargument, wenn Anbieter von Fleisch- und Wurstwaren die Herkunft bis auf einen konkreten Bauernhof, von dem die Tiere stammen, transparent darstellen würden. 
Frage 25: Wäre es für Sie ein Kaufargument, wenn ein Anbieter die Rückverfolgbarkeit von Fleisch- und Wurstwaren bis zurück auf den konkreten Bauernhof, von dem die Tiere stammen, transparent darstellen würde?

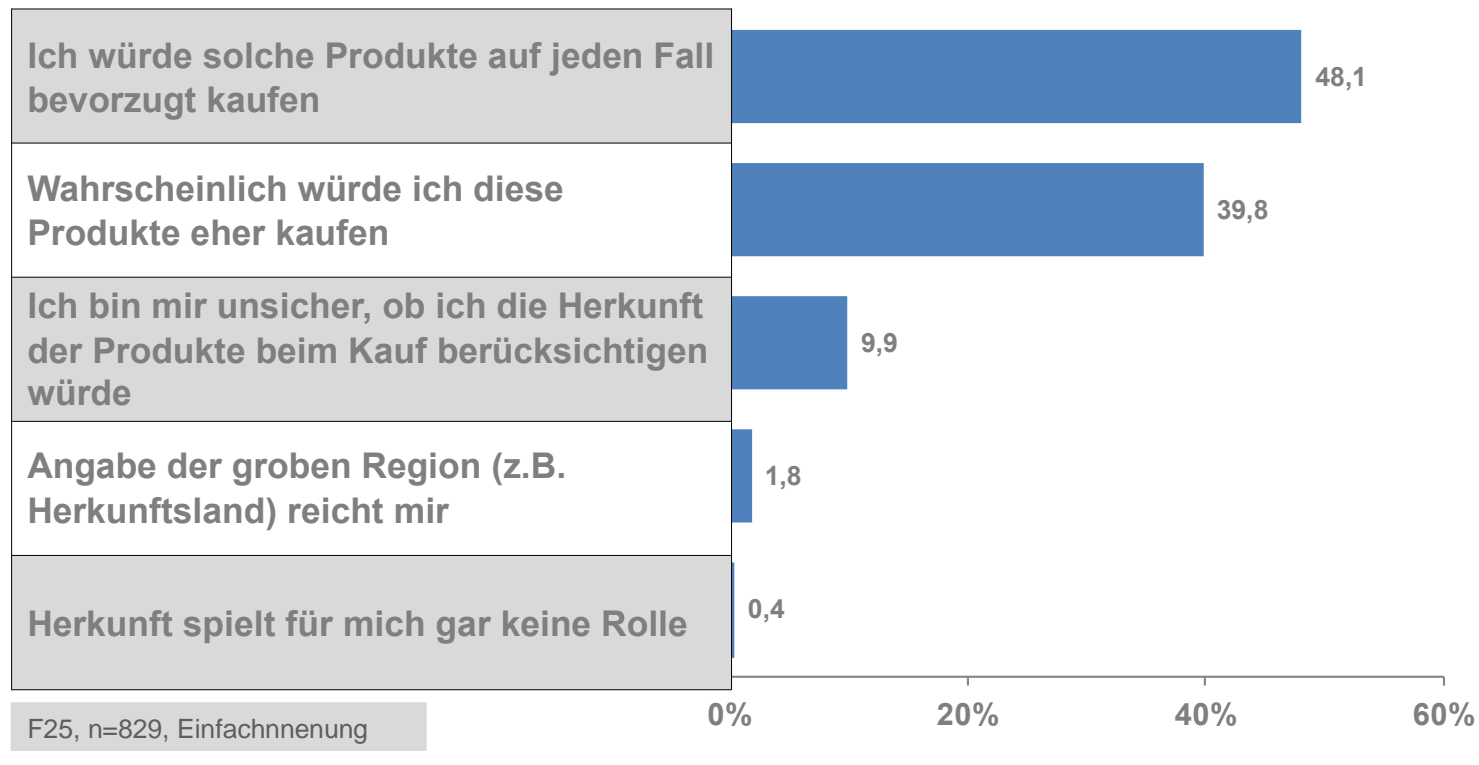

\section{Abbildung 36: Detaillierung von Herkunftsangaben als Kaufargument (Quelle: Eigene Darstellung)}

Vor dem Hintergrund der praktischen Umsetzung eines verbesserten Angebots an Tierwohlinformationen wurde außerdem erfragt, welche Institutionen aus der Perspektive der Verbraucher weiterführende Informationen zu den Haltungsbedingungen der Tiere im Internet bereitstellen sollen. Die Befragten konnten dabei in Frage 24 mehrere Institutionen auswählen.

Da diese Frage auf die Bereitstellung von Tierwohlinformationen im Internet abzielte, wurde diese Frage nur Personen gestellt, die sich im Internet zum Thema Tierwohl informieren würden $(\mathrm{N}=773)$. 56 Personen, die bei Frage 23 geäußert hatten, sich überhaupt nicht auf einem Internetportal zur artgerechteren Tierhaltung informieren zu wollen, wurde diese Frage nicht gestellt. 
Abbildung 37 zeigt, dass aus Sicht der Verbraucher vor allem die Anbieter von Fleischund Wurstwaren wie Lebensmittelhersteller (73\%), Landwirte (64\%) und der Lebensmitteleinzelhandel (59\%) Informationen zu den Haltungsbedingungen der Tiere bereitstellen sollten. Daneben erwarten die meisten Verbraucher die Bereitstellung dieser Informationen von den Herausgebern der Gütesiegel (70 \%). Weitere Institutionen, die nach den Angaben der Befragten Informationen zu diesem Thema bereitstellen sollten, sind das Bundesministerium für Ernährung und Landwirtschaft (58 \%) sowie NGOs aus den Bereichen Verbraucherschutz, Tierschutz und Naturschutz, die rd. von der Hälfte der Befragten genannt wurden. Nur rd. ein Drittel der Verbraucher erwartet die Bereitstellung weiterführender Informationen zum Tierwohl von Verbänden der Lebensmittelindustrie, der Europäischen Union, Verbänden von Landwirten und den Medien.

Frage 24: Welche der folgenden Institutionen oder Personen sollten weiterführende Informationen zu den Haltungsbedingungen der Tiere im Internet bereitstellen?

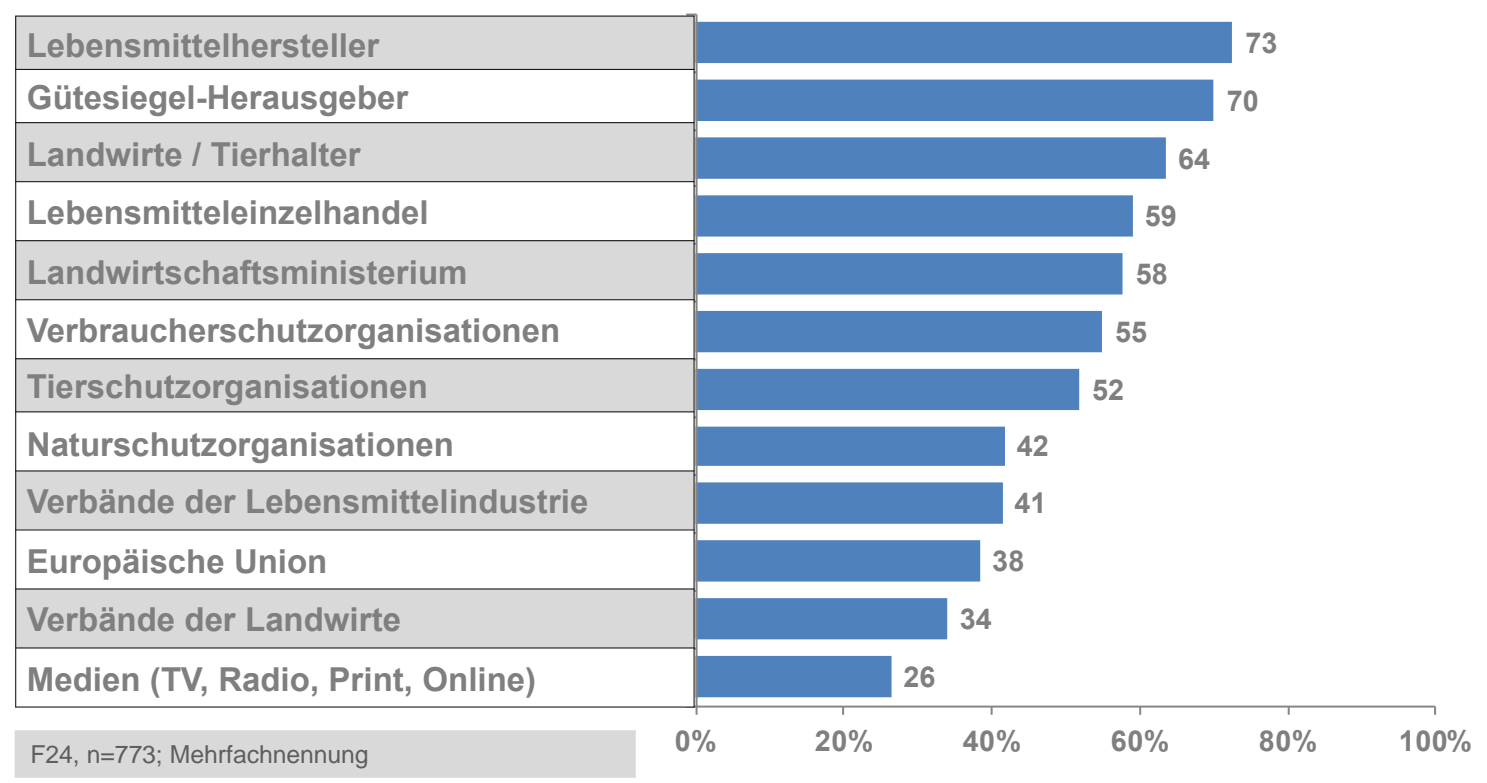

\section{Abbildung 37: Bereitsteller von Tierwohlinformationen (Quelle: Eigene Darstellung)}




\subsubsection{Politische Präferenzen und politische Partizipation}

In diesem Abschnitt werden zunächst die Ergebnisse zu den politischen Präferenzen der Befragten in Bezug auf die zentralen politischen Lösungsansätze für mehr Tierwohl dargestellt. Anschließend werden die Präferenzen der Befragten zu verschiedenen Kennzeichnungsformen deutlich gemacht. Zum Abschluss dieses Abschnitts werden die Ergebnisse zur Einschätzung der Verbraucher präsentiert, wie sie sich persönlich für eine verbesserte Tierhaltung einsetzen können.

In der gesellschaftlichen und politischen Diskussion zum Thema Tierwohl stehen in Deutschland, wie in Unterkapitel 3.5 beschrieben, verschiedene Lösungsansätze für mehr Tierwohl im Fokus. Neben einem strengeren Tierschutzgesetz wird über eine gesetzliche Haltungskennzeichnung diskutiert.

Ein weiterer Ansatzpunkt ist die finanzielle staatliche Förderung von mehr Tierwohl (z. B. durch Subventionen). Als weitere Lösungsmöglichkeiten werden private Initiativen wie die Initiative Tierwohl und Tierwohl-Labels betrachtet. Diese zentralen Lösungsansätze für mehr Tierwohl konnten in Frage 26 erstmals von deutschen Verbrauchern vergleichend bewertet werden.

In Abbildung 38 ist $\mathrm{zu}$ erkennen, dass mit deutlichem Abstand ein strengeres Tierschutzgesetz als besonders wichtig bewertet wird, um das Wohl der Tiere in der Nutztierhaltung zu verbessern.

Als weitere bedeutsame Lösungsansätze werden eine verpflichtende staatliche Haltungskennzeichnung sowie die staatliche Förderung von ,mehr Tierwohl` betrachtet. Der Ausbau der Initiative Tierwohl sowie der Einsatz freiwilliger Tierwohl-Labels werden im Vergleich zu den anderen Lösungsansätzen als weniger wichtig angesehen. 
Frage 26: Es gibt verschiedene Ansätze, die in der Politik diskutiert werden, um das Wohl der Tiere in der Nutztierhaltung und die Kennzeichnung von Fleisch- und Wurstwaren aus einer artgerechteren Haltung zu verbessern. Bitte bringen Sie die folgenden Ansätze in eine für Sie persönlich sinnvolle Reihenfolge nach Dringlichkeit/Wichtigkeit.

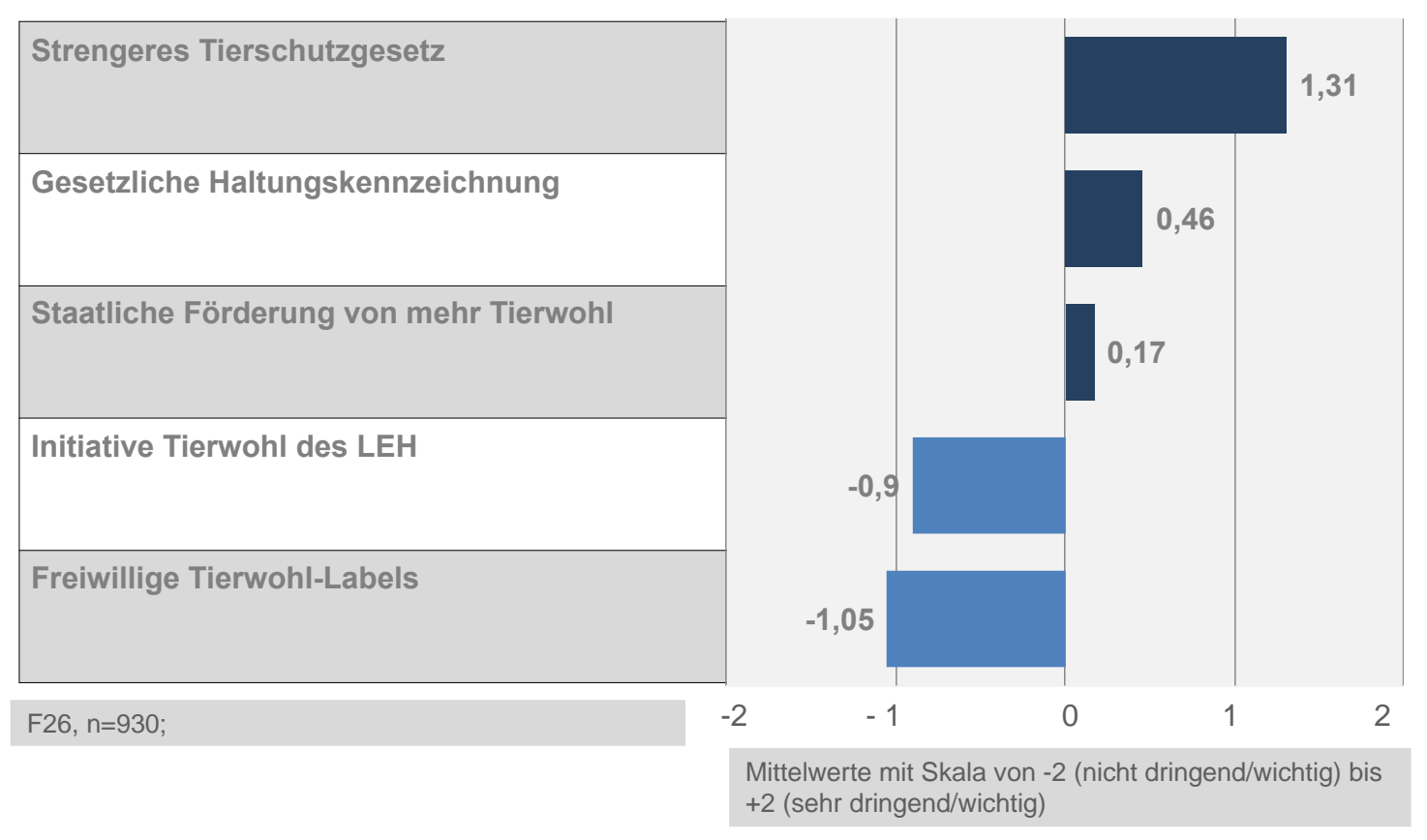

\section{Abbildung 38: Politikansätze für mehr Tierwohl aus Verbrauchersicht (Quelle: Eigene Darstellung)}

Des Weiteren wurde untersucht, inwiefern sich die Bewertung der verschiedenen politischen Ansätze hinsichtlich der unterschiedlichen Parteiwähler (Bundestagswahl 2017) unterscheidet. Abbildung 39 zeigt, dass hoch signifikante Unterschiede beim Kriterium strengeres Tierschutzgesetz $(\mathrm{F}(5,710)=3,150, \mathrm{p}<0,01, \mathrm{n}=716)$ vorliegen.

In den post-hoc-Tests mit Bonferroni-Korrektur ergab sich ein signifikanter Unterschied zwischen den Wählern von Bündnis90/DieGrünen und Tierschutzparteien (MW = 1,54; $\mathrm{SD}=0,828)$ und CDU-Wählern (MW = 1,12; SD = 1,218). Die Mittelwerte betrachtend lässt sich feststellen, dass die Einführung eines strengeren Tierschutzgesetzes von den Wählern von Bündnis90/DieGrünen und Tierschutzparteien als dringender bewertet wird. 
Zudem besteht ein hoch signifikanter Unterschied bezüglich des Einsatzes freiwilliger Tierwohl-Labels $(\mathrm{F}(5,710)=3,475, \mathrm{p}<0,01, \mathrm{n}=716)$. In den post-hoc-Tests mit Bonferroni-Korrektur ergab sich ein signifikanter Unterschied zwischen Wählern von Bündnis90/DieGrünen und Tierschutzparteien ( $\mathrm{MW}=-1,30 ; \mathrm{SD}=0,942)$ und CDUWählern (MW = -0,89; SD = 1,197). Mit Blick auf die Mittelwerte ist erkennbar, dass der Einsatz freiwilliger Tierwohl-Labels von CDU-Wählern als wichtiger beurteilt wird.

Hypothese 22: Die Bewertung politischer Ansätze zur Verbesserung des Tierwohls hängt mit der gewählten Partei zusammen.

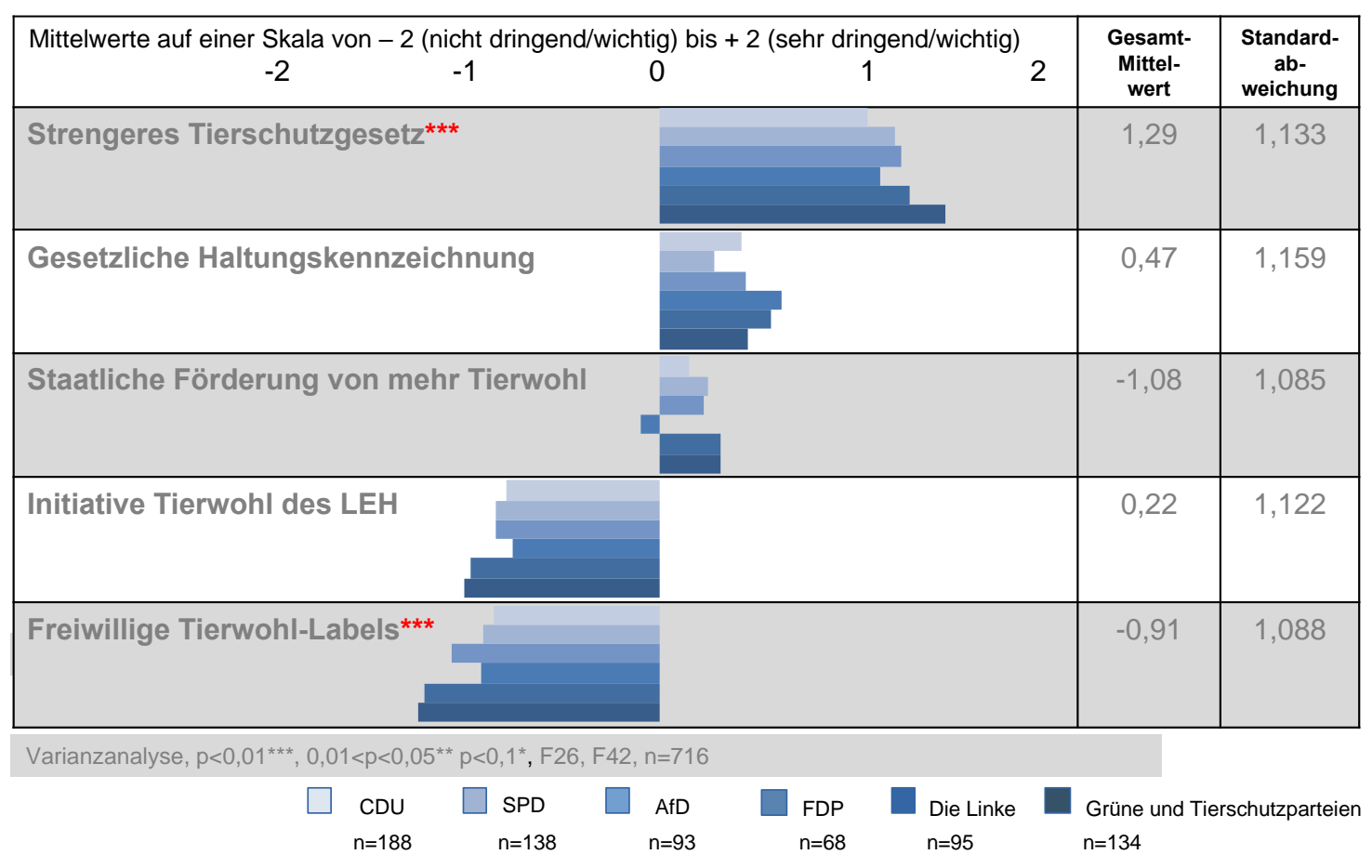

Abbildung 39: Bewertung politischer Ansätze nach gewählter Partei (Quelle: Eigene Darstellung)

Wie bereits in Kapitel 3 beschrieben existieren verschiedene Möglichkeiten, um Fleischund Wurstwaren aus einer artgerechteren und nachhaltigeren Nutztierhaltung zu kennzeichnen. Hinsichtlich der diskutierten gesetzlichen Haltungskennzeichnung stellt sich die Frage, in welcher Form diese Tierwohlkennzeichnung in der Praxis umgesetzt werden kann und welche Kennzeichnungsform die Verbraucher präferieren. 
Die Wahl einer passenden Kennzeichnungsform beeinflusst die Erkennbarkeit der unterschiedlichen Standards im Lebensmitteleinzelhandel wesentlich. Daher wurden den Befragten in Frage 27 verschiedene Kennzeichnungsformen zur Auswahl gestellt. Wie in Abbildung 40 veranschaulicht haben die Befragten bezüglich der Kennzeichnungsform klare Präferenzen.

Mit großem Abstand $\mathrm{zu}$ den anderen Kennzeichnungsformen votieren die meisten Verbraucher für eine farbliche Ampelkennzeichnung (rd. die Hälfte der Befragten). Nur ungefähr ein Viertel der Befragten befürwortet eine Kennzeichnung mit Sternen, die beispielsweise beim Tierwohl-Label des DEUTSCHEN TIERSCHUTZBUNDES eingesetzt wird und auch beim angedachten staatlichen Tierwohl-Label umgesetzt werden soll. Lediglich $14 \%$ der Befragten favorisieren eine Kennzeichnung mit Zahlen, die sich an der Kennzeichnung von Eiern in Deutschland orientiert. Nur sehr wenige Befragte stimmten für eine Kennzeichnung ohne Abstufungen, Zahlen in umgekehrter Reihenfolge im Vergleich zur Eierkennzeichnung oder eine Kennzeichnung in den drei Stufen Bronze, Silber und Gold, wie sie beim Label der Deutschen LandwirtschaftsGesellschaft (DLG) Verwendung finden.

Unter dem Punkt Andere Haltungskennzeichnung hatten die Befragten die Möglichkeit, kreativ $\mathrm{zu}$ werden und weitere Gestaltungsvorschläge $\mathrm{zu}$ unterbreiten. Gestaltungsvorschläge wurden zwar nicht formuliert, jedoch gaben einige Befragte an dieser Stelle konkrete Hinweise. So merkten zwei der Befragten an, sich klar definierte, vorgegebene Aussagen in Textform auf den Verpackungen zu wünschen. Mehrere weitere Befragte äußerten, dass die Kennzeichnungen vor allem nachvollziehbar, ehrlich und nicht manipulierbar sein sollten. 
Frage 27: Welche Form der Haltungskennzeichnung würden Sie persönlich bevorzugen, um beim Fleischkauf die Artgerechtheit der Tierhaltung besser einschätzen zu können?

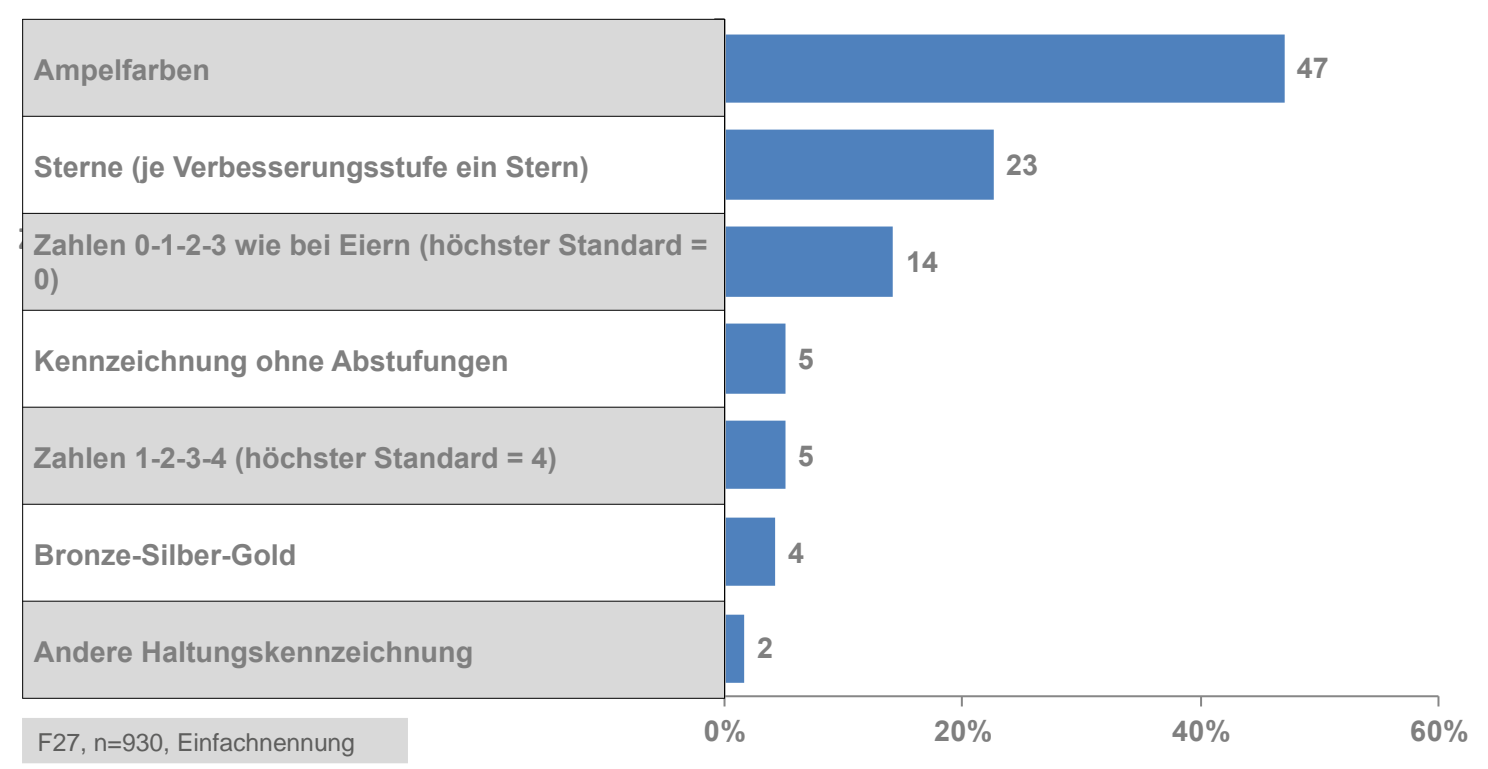

Abbildung 40: Verbraucherpräferenzen für Tierwohlkennzeichnungsformen (Quelle: Eigene Darstellung)

Darüber hinaus wurde untersucht, ob die Wähler bestimmter Parteien eine der drei meistgenannten Haltungskennzeichungen (Ampelfarben, Sterne und Zahlen) bevorzugen, um zu überprüfen, ob sich die Präferenzen der Wähler in diesem Punkt unterscheiden. Dadurch kann den politischen Parteien erstmals ein Meinungsbild ihrer Wähler zur oftmals geforderten gesetzlichen Haltungskennzeichnung präsentiert werden.

Hierbei ließ sich kein signifikanter Zusammenhang feststellen (Chi-Quadrat (10) $\mathrm{F}=9,031, \mathrm{p}=0,529 \mathrm{n}=574)$, d. h. alle Wähler präferieren in etwa gleichermaßen deutlich eine gesetzliche Ampelkennzeichnung bei Fleisch- und Wurstwaren, während eine Kennzeichnung mit Sternen oder Zahlen nur von wenigen Wählern befürwortet wird. Abbildung 41 enthält die Ergebnisse. 
Hypothese 23: Die präferierte Form einer gesetzlichen Haltungskennzeichnung hängt mit der gewählten Partei zusammen.

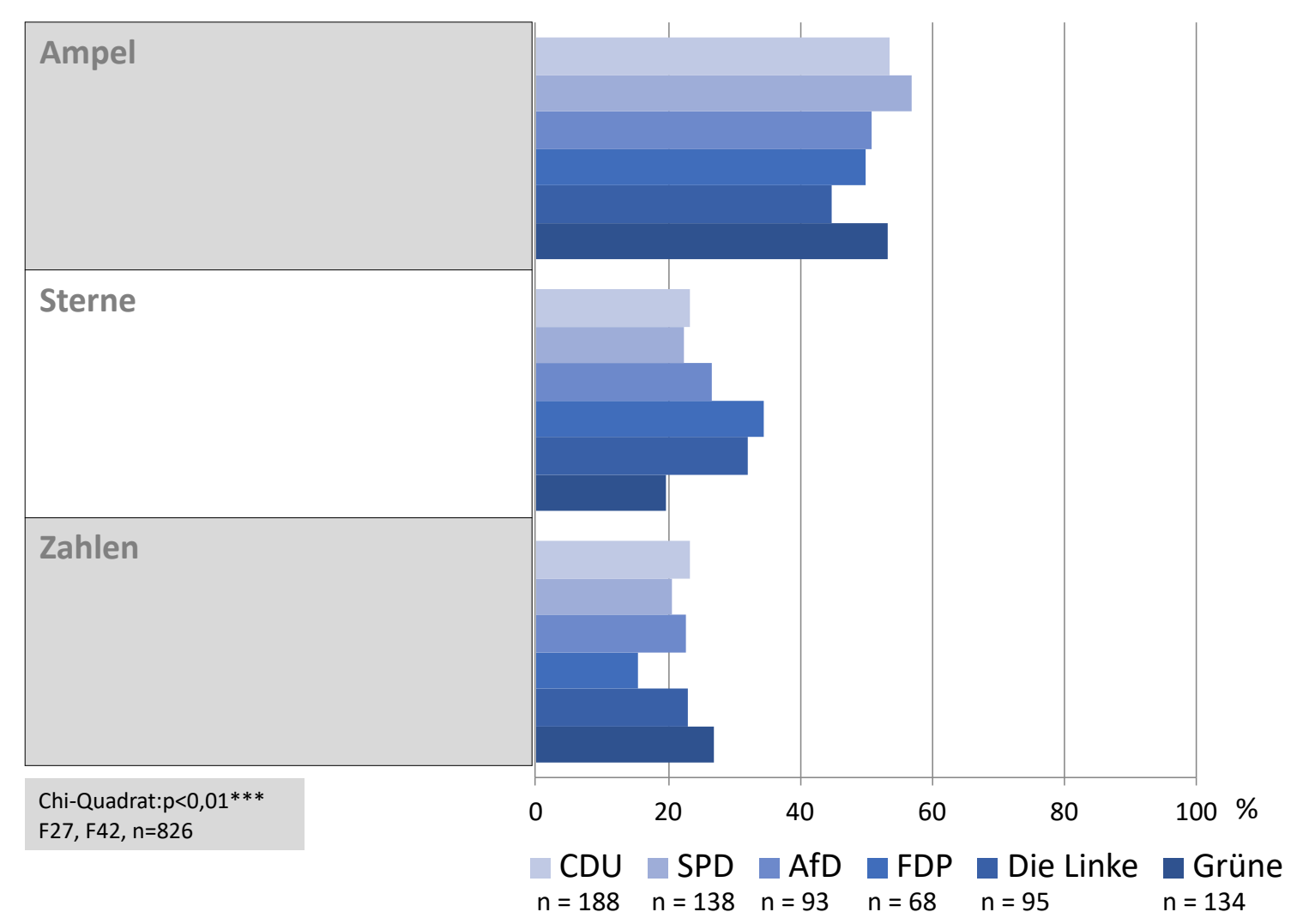

\section{Abbildung 41: Zusammenhang Kennzeichnungsformen und Partei} (Quelle: Eigene Darstellung)

Abschließend für die politischen Fragen der Studie zum Tierwohl-Labeling konnten die Befragten in Frage 28 noch einmal verschiedene Möglichkeiten, sich persönlich für eine verbesserte Tierhaltung in Deutschland einzusetzen, bewerten. Die in Abbildung 42 in Mittelwerten dargestellten Ergebnisse hierzu sind durchaus interessant.

An erster Stelle mit deutlichem Abstand zu den anderen aufgeführten Möglichkeiten vermuten die Befragten, dass sie sich durch ihr persönliches Einkaufs- oder Konsumverhalten für eine verbesserte Nutztierhaltung einsetzen können. Bereits an zweiter Stelle wurde die Möglichkeit der Unterstützung von NGOs in den Bereichen Tier, Natur- und Verbraucherschutz genannt. 
Auch Petitionen sehen manche Befragten als eine Möglichkeit an, sich persönlich für eine verbesserte Tierhaltung in Deutschland einzusetzen. Aus Sicht der Befragten könne durch die Stimmabgabe bei politischen Wahlen oder die Teilnahme an Demonstrationen die Verbesserung der Tierhaltung in Deutschland indes nur vergleichsweise wenig beeinflusst werden.

Frage 28: Es gibt verschiedene Möglichkeiten, wie sich Menschen für eine verbesserte Tierhaltung einsetzen können. Wie bewerten Sie die aufgeführten Möglichkeiten?

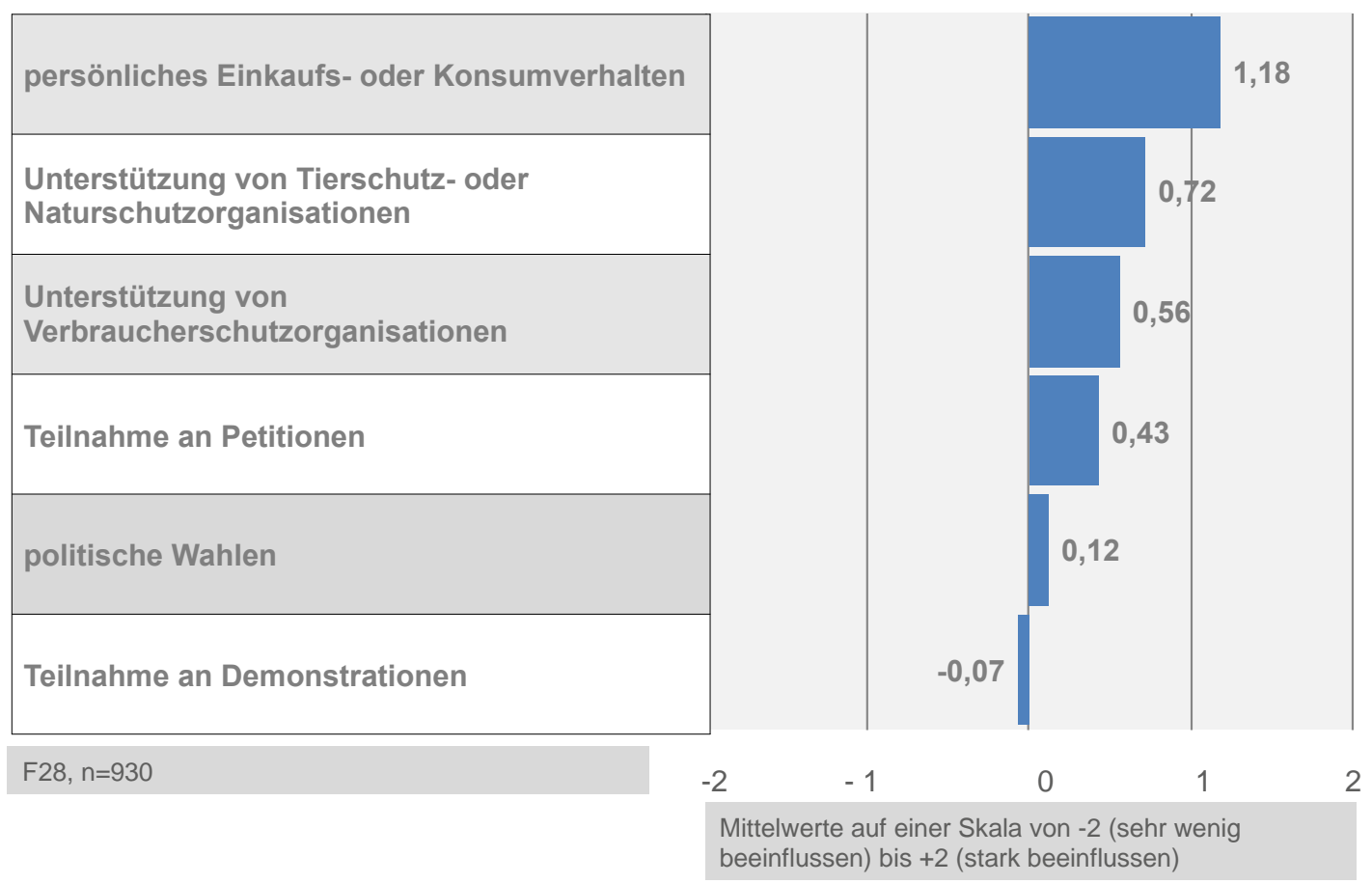

\section{Abbildung 42: Möglichkeiten der politischen Partizipation für mehr Tierwohl (Quelle: Eigene Darstellung)}

Wie in Abbildung 43 ersichtlich unterscheiden sich die Einschätzungen der Befragten hinsichtlich der Möglichkeiten, die Nutzierhaltung in Deutschland zu verbessern. Bei der gewählten Partei bestehen signifikante Unterschiede im Punkt Teilnahme an Petitionen $(\mathrm{F}(5,710)=2,789, \mathrm{p}<0,01, \mathrm{n}=716)$. 
In den post-hoc-Tests mit Bonferroni-Korrektur konnten signifikante Unterschiede zwischen Wählern der Partei Bündnis90/DieGrünen sowie Tierschutzparteien $(\mathrm{MW}=0,66 ; \mathrm{SD}=0,941)$ und CDU-Wählern ( $\mathrm{MW}=0,33 ; \mathrm{SD}=1,007)$ sowie AfDWählern $\quad(\mathrm{MW}=0,28 ; \quad \mathrm{SD}=1,004) \quad$ festgestellt werden. Wähler von Bündnis90/DieGrünen und Tierschutzparteien schätzen den Einfluss einer Teilnahme an Petitionen höher ein als CDU-Wähler und AfD-Wähler.

Es existiert zudem ein hoch signifikanter Zusammenhang für das Kriterium Stimmabgabe für eine Partei $(\mathrm{F}(5,710)=5,130, \mathrm{p}<0,01, \mathrm{n}=716)$. In den post-hoc-Tests mit Bonferroni-Korrektur ergaben sich signifikante Unterschiede zwischen AfD-Wählern $(\mathrm{MW}=-0,32 ; \mathrm{SD}=1,144)$ und Wählern der Parteien CDU $(\mathrm{MW}=0,18 ; \mathrm{SD}=1,145)$, SPD $\quad(M W=0,17 ; \quad S D=1,137), \quad$ Die Linke $\quad(M W=0,25 ; \quad S D=1,130) \quad$ sowie Bündnis90/DieGrünen und Tierschutzparteien ( $M W=0,43 ; S D=1,120)$. AfD-Wähler erachten den Einfluss der Stimmabgabe für eine Partei als geringer.

Der Einfluss auf die Verbesserung des Tierwohls in Deutschland durch die Unterstützung von Tier- $(\mathrm{F}(5,710)=4,611, \mathrm{p}<0,01, \mathrm{n}=716)$ und Verbraucherschutzorganisationen $(\mathrm{F}(5,710)=3,813, \mathrm{p}<0,01, \mathrm{n}=716)$ wird von SPD-Wählern $(\mathrm{MW}=0,78 ; \mathrm{SD}=0,826$ bzw. $\mathrm{MW}=0,67 ; \mathrm{SD}=0,831)$ und Wählern von Bündnis90/DieGrünen sowie Tierschutzparteien $\quad(\mathrm{MW}=0,99 ; \quad \mathrm{SD}=0,893 \quad$ bzw. $\mathrm{MW}=0,74 ; \quad \mathrm{SD}=0,831)$ als signifikant stärker eingeschätzt als von AfD-Wählern $(\mathrm{MW}=0,41 ; \mathrm{SD}=1,024$ bzW. $\mathrm{MW}=0,27 ; \mathrm{SD}=1,085)$.

Hoch signifikante Unterschiede ergaben sich bei den unterschiedlichen Wählergruppen auch in Bezug auf die Einschätzung des politischen Einflusses durch die Teilnahme an Demonstrationen (Varianzanalyse (5,710) $\mathrm{F}=3,627, \mathrm{p}<0,01 ; \mathrm{n}=716$ ). Nach Auswertung der post-hoc-Tests mit Bonferroni-Korrektur lassen sich CDU-Wähler $(\mathrm{MW}=-0,13 ; \mathrm{SD}=1,069)$ und AfD-Wähler $(\mathrm{MW}=-0,39 ; \mathrm{SD}=1,189)$ in eine Gruppe zusammenfassen, die sich signifikant von den Wählern der Partei Bündnis90/DieGrünen und der Tierschutzparteien ( $\mathrm{MW}=0,18 ; \mathrm{SD}=1,039)$ unterscheidet. CDU-Wähler und AfD-Wähler schätzen den Einfluss von Teilnahmen an Demonstrationen auf die Verbesserung des Tierwohls in Deutschland als geringer ein als die Wähler von Bündnis90/DieGrünen und die Wähler von Tierschutzparteien. 
Hypothese24: Die Einschätzung der persönlichen Einflussnahme auf das Tierwohl hängt mit der gewählten Partei zusammen.

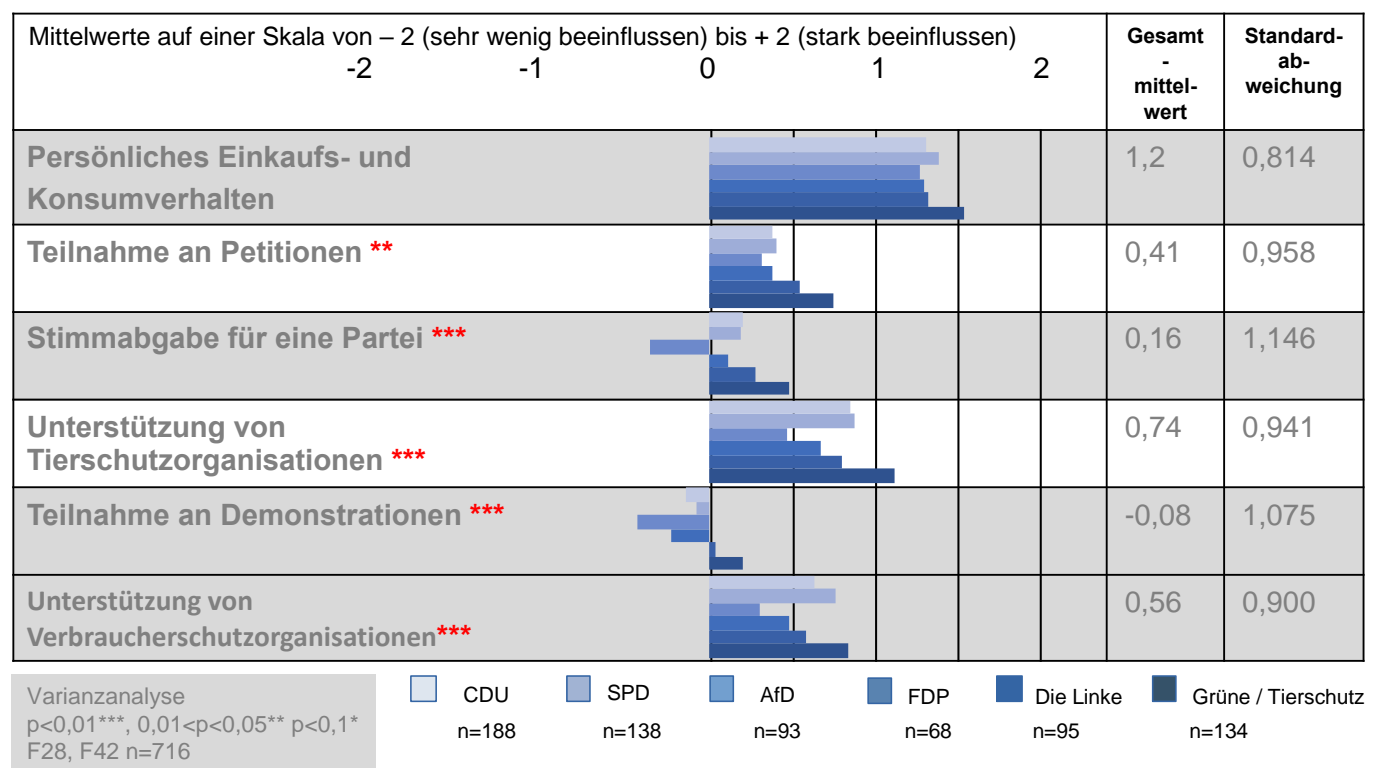

\section{Abbildung 43: Einschätzung politischer Partizipation nach Parteien} (Quelle: Eigene Darstellung)

\subsubsection{Zusammenfassung der deskriptiven und bivariaten Ergebnisse}

An dieser Stelle sollen die zentralen deskriptiven Ergebnisse sowie die Ergebnisse der uni- und bivariaten Analysen der Unterkapitel 5.2 und 5.3 im Überblick dargestellt werden.

Die Ergebnisse der Hypothesenprüfung für die einzelnen Analysen sind in Tabelle 16 zusammenfassend in einer Übersicht abgebildet. Es zeigt sich, dass die überwiegende Anzahl an Forschungshypothesen bestätigt werden konnte. 
Tabelle 16: Übersicht der Forschungsergebnisse

\begin{tabular}{|c|c|c|}
\hline Nr. & Hypothese & Ergebnis \\
\hline & Tierwohl-Labels als Einkaufshilfe & \\
\hline $\mathrm{H} 1$ & $\begin{array}{l}\text { Es besteht ein Zusammenhang zwischen der Beachtung der } \\
\text { Kaufkriterien artgerechtere Tierhaltung, Gesundheit sowie } \\
\text { Umweltfreundlichkeit und der Haupteinkaufsstätte. }\end{array}$ & bestätigt \\
\hline $\mathrm{H} 2$ & $\begin{array}{l}\text { Personen, die auf eine artgerechtere Tierhaltung, } \\
\text { Gesundheitsaspekte und Umweltfreundlichkeit achten, kaufen } \\
\text { Wurstwaren oder Aufschnitt fürs Brot häufiger an der Theke. }\end{array}$ & bestätigt \\
\hline $\mathrm{H} 3$ & $\begin{array}{l}\text { Personen, die auf eine artgerechtere Tierhaltung, } \\
\text { Gesundheitsaspekte und Umweltfreundlichkeit achten, kaufen } \\
\text { Fleisch zum Braten und Grillen häufiger an der Theke. }\end{array}$ & bestätigt \\
\hline $\mathrm{H} 4$ & $\begin{array}{l}\text { Es besteht ein Zusammenhang zwischen der Beachtung der } \\
\text { Kaufkriterien artgerechtere Tierhaltung, Gesundheit sowie } \\
\text { Umweltfreundlichkeit und der Soziodemografie der Befragten. }\end{array}$ & bestätigt \\
\hline H5 & $\begin{array}{l}\text { Die Beachtung des Kaufkriteriums artgerechte Tierhaltung hängt } \\
\text { damit zusammen, ob die Befragten ein Haustier besitzen. }\end{array}$ & bestätigt \\
\hline $\mathrm{H} 6$ & $\begin{array}{l}\text { Die Beachtung des Kaufkriteriums artgerechte Tierhaltung hängt } \\
\text { damit zusammen, ob die Befragten einer Religion angehören. }\end{array}$ & verworfen \\
\hline $\mathrm{H} 7$ & $\begin{array}{l}\text { Die Beachtung des Kaufkriteriums artgerechte Tierhaltung hängt } \\
\text { damit zusammen, welche politische Partei die Befragten wählen. }\end{array}$ & bestätigt \\
\hline $\mathrm{H} 8$ & $\begin{array}{l}\text { Es besteht ein Zusammenhang zwischen dem Bekanntheitsgrad } \\
\text { der Tierwohl-Labels für Fleisch- und Wurstwaren und der } \\
\text { Haupteinkaufsstätte. }\end{array}$ & bestätigt \\
\hline H9 & $\begin{array}{l}\text { Es besteht ein Zusammenhang zwischen dem Bekanntheitsgrad } \\
\text { der Tierwohl-Labels für Fleisch- und Wurstwaren und der } \\
\text { Soziodemografie der Befragten. }\end{array}$ & bestätigt \\
\hline $\mathrm{H} 10$ & $\begin{array}{l}\text { Es besteht ein Zusammenhang zwischen den Tierwohl-Label- } \\
\text { Präferenzen und der Haupteinkaufsstätte. }\end{array}$ & bestätigt \\
\hline $\mathrm{H} 11$ & $\begin{array}{l}\text { Personen, die bestimmte Tierwohl-Labels beim Kauf von Fleisch- } \\
\text { und Wurstwaren berücksichtigen, kaufen Wurstwaren oder } \\
\text { Aufschnitt fürs Brot häufiger an der Theke. }\end{array}$ & bestätigt \\
\hline $\mathrm{H} 12$ & $\begin{array}{l}\text { Personen, die bestimmte Tierwohl-Labels beim Kauf von Fleisch- } \\
\text { und Wurstwaren berücksichtigen, kaufen Fleisch zum Braten und } \\
\text { Grillen häufiger an der Theke. }\end{array}$ & bestätigt \\
\hline $\mathrm{H} 13$ & $\begin{array}{l}\text { Es besteht ein Zusammenhang zwischen den Tierwohl-Label- } \\
\text { Präferenzen und der persönlichen Reduktionsbereitschaft. }\end{array}$ & bestätigt \\
\hline $\mathrm{H} 14$ & $\begin{array}{l}\text { Es besteht ein Zusammenhang zwischen den Tierwohl-Label- } \\
\text { Präferenzen und der Soziodemografie der Befragten. }\end{array}$ & bestätigt \\
\hline $\mathrm{H} 15$ & $\begin{array}{l}\text { Es besteht ein Zusammenhang zwischen der } \\
\text { Kundenzufriedenheit mit dem Produkt- und Beratungsangebot für } \\
\text { Fleisch- und Wurstwaren aus einer artgerechteren Tierhaltung } \\
\text { und der Haupteinkaufsstätte. }\end{array}$ & bestätigt \\
\hline $\mathrm{H} 16$ & $\begin{array}{l}\text { Es besteht ein Zusammenhang zwischen den Kaufbarrieren und } \\
\text { der Haupteinkaufsstätte. }\end{array}$ & bestätigt \\
\hline $\mathrm{H} 17$ & $\begin{array}{l}\text { Es besteht ein Zusammenhang zwischen den Präferenzen zur } \\
\text { Sortierung der Tierwohlprodukte und der Haupteinkaufsstätte. }\end{array}$ & verworfen \\
\hline
\end{tabular}




\begin{tabular}{|c|c|c|}
\hline Nr. & Hypothese & Ergebnis \\
\hline \multirow[t]{2}{*}{$\mathrm{H} 18$} & $\begin{array}{l}\text { Es besteht ein Zusammenhang zwischen der Wechselbereitschaft } \\
\text { der Haupteinkaufsstätte bei fehlenden Verbesserungen } \\
\text { hinsichtlich des Produkt- und Beratungsangebots für Fleisch- und } \\
\text { Wurstwaren aus einer artgerechteren Tierhaltung und der } \\
\text { Haupteinkaufsstätte. }\end{array}$ & verworfen \\
\hline & Informationsbedarf der Verbraucher & \\
\hline $\mathrm{H} 19$ & $\begin{array}{l}\text { Es besteht ein Zusammenhang zwischen den Präferenzen zur } \\
\text { Informationsbereitstellung und der Haupteinkaufsstätte. }\end{array}$ & bestätigt \\
\hline $\mathrm{H} 20$ & $\begin{array}{l}\text { Die Präferenzen zur Informationsbereitstellung hängen mit der } \\
\text { Angebotsform beim Kauf von Aufschnitt und Wurst fürs Brot } \\
\text { zusammen (Theke/SB). }\end{array}$ & bestätigt \\
\hline \multirow[t]{2}{*}{$\mathrm{H} 21$} & $\begin{array}{l}\text { Die Präferenzen zur Informationsbereitstellung hängen mit der } \\
\text { Angebotsform beim Kauf von Fleisch zum Braten oder Grillen } \\
\text { zusammen (Theke/SB). }\end{array}$ & bestätigt \\
\hline & Bewertung von Politikoptionen & \\
\hline $\mathrm{H} 22$ & $\begin{array}{l}\text { Die Bewertung politischer Ansätze zur Verbesserung des } \\
\text { Tierwohls hängt mit der gewählten Partei zusammen. }\end{array}$ & bestätigt \\
\hline $\mathrm{H} 23$ & $\begin{array}{l}\text { Die präferierte Form einer gesetzlichen Haltungskennzeichnung } \\
\text { hängt mit der gewählten Partei zusammen. }\end{array}$ & verworfen \\
\hline $\mathrm{H} 24$ & $\begin{array}{l}\text { Die Einschätzung der persönlichen Einflussnahme auf das } \\
\text { Tierwohl hängt mit der gewählten Partei zusammen. }\end{array}$ & bestätigt \\
\hline
\end{tabular}

\section{Quelle: Eigene Darstellung}

Neben der Hypothesenprüfung konnten zahlreiche weitere interessante Forschungsergebnisse gewonnen werden. Wie in der Einleitung beschrieben diente die Erfassung des Einkaufs- und Ernährungsverhaltens (Unterkapitel 5.2) schwerpunktmäßig der weiterführenden Analyse spezifischer Tierwohlfragen. Diese Ergebnisse stellen zwar nur einige innovative Forschungserkenntnisse bereit. Sie unterstreichen jedoch die Praxisnähe der Studie, und zeigen, dass die Ergebnisse mit den Daten von Marktforschungsinstituten, staatlichen Einrichtungen und wissenschaftlichen Studien in hohem Maße übereinstimmen.

- Die fünf Lebensmitteleinzelhändler EDEKA, REWE, SCHWARZ-Gruppe, METRO und ALDI sind die größten Anbieter von Fleisch- und Wurstwaren in Deutschland. Obwohl EDEKA und REWE die meistgenutzten Einkaufsstätten für Fleisch- und Wurstwaren sind, kaufen insgesamt mehr Verbraucher Fleischwaren bei ALDI, LIDL, NETTO und PENNY.

- Besondere Einkaufsstätten wie Wochenmärkte, Hofläden und Bio-Supermärkte erreichen quantitativ zusammen eine ähnliche Bedeutung wie die Metzgereien. 
- Bezüglich der Angebotsform wird Fleisch zum Grillen und Braten von den Probanden häufiger an der Fleischtheke gekauft, während bei Wurst oder Aufschnitt der SBBereich etwas beliebter ist.

- Der Großteil der Befragten isst einmal täglich Fleisch- und Wurstwaren im Haushalt. Ungefähr ein Fünftel aller Haushalte der Befragten können als ,Flexitarier-Haushalte“ bezeichnet werden, da dort nur ein bis zwei Mal pro Woche Fleisch oder Wurst verzehrt wird.

- Hinsichtlich der Reduktionsbereitschaft beim Fleischkonsum konnten drei gleich große Verbrauchergruppen (rd. 30\%) identifiziert werden. Eine Gruppe hat ihren Fleischkonsum bereits bewusst reduziert (Flexitarier). Eine weitere Verbrauchergruppe möchte den Fleischkonsum zukünftig reduzieren. Die dritte Gruppe hat ihren Fleischkonsum ebenfalls noch nicht reduziert, möchte ihn aber auch in Zukunft nicht reduzieren.

- Die bedeutendsten Kaufmotive bei Fleisch- und Wurstwaren sind für die Befragten die Frische, das Aussehen der Ware und der Geschmack. Relevanter als der Preis der Produkte sind verschiedene ethische Kaufmotive: Regionalität, Tierwohl, Umweltfreundlichkeit sowie Gesundheitsaspekte.

- Die Soziodemografie betrachtend achten die weiblichen und die älteren Studienteilnehmer mehr auf diese Nachhaltigkeitsaspekte als die männlichen und jüngeren Probanden. Wider Erwarten bestehen keine Unterschiede bei der Beachtung ethischer Kaufmotive zwischen Verbrauchern bzgl. Ortsgröße (Stadt, Land), Bildung oder Religion. Es zeigte sich jedoch, dass Personen mit einem höheren Einkommen und Personen, die ein Haustier besitzen, deutlich häufiger auf Tierwohl achten als Verbraucher mit einem niedrigeren Einkommen bzw. Personen, die kein Haustier besitzen. Beim Fleischkauf achten zudem die Wähler der Partei Bündnis90/DieGrünen bzw. von Tierschutzparteien signifikant häufiger auf das Tierwohl als AfD-Wähler und SPD-Wähler.

- Bei der Analyse der einzelnen Kaufmotive wurde des Weiteren deutlich, dass Metzgerei-Käufer mehr auf Tierwohl, Umweltfreundlichkeit und Gesundheitsaspekte achten als Verbraucher, die Fleisch- und Wurstwaren im Supermarkt, beim Discounter oder im SB-Warenhaus einkaufen. 
- Ebenfalls konnte gezeigt werden, dass Verbraucher, die Fleisch und Wurst bevorzugt an der Fleischtheke kaufen, mehr auf eine artgerechtere Tierhaltung, Umweltfreundlichkeit und Gesundheitsaspekte achten als Personen, die Fleisch- und Wurstwaren im SB-Bereich kaufen.

- Abschließend für die Fragen zum Einkaufs- und Konsumverhalten gaben die Verbraucher in der Studie bzgl. ihrer Präferenzen von Fleischsorten und Fleischarten an, am häufigsten Aufschnitt und Wurstwaren einzukaufen. Von den Frischfleischprodukten kaufen die Befragten am meisten Hähnchen oder Pute, regelmäßig Schwein und seltener Rind. TK-, Dosen-, Tüten-/Glasware etc. wird dagegen aufgrund der längeren Haltbarkeit deutlich seltener gekauft.

Nach den Ergebnissen zum Einkaufs- und Ernährungsverhalten wurden die Ergebnisse zum Tierwohl-Labeling vorgestellt. Dabei wurden mit Hilfe verschiedener Analysen Zusammenhänge zum Einkaufsverhalten überprüft. So konnten in diesem Abschnitt zu einzelnen Aspekten des Tierwohl-Labelings zahlreiche neue Forschungserkenntnisse gewonnen werden. Die zentralen Erkenntnisse werden im Folgenden zusammengefasst.

- Das Kaufinteresse an Tierwohlprodukten bei Fleisch- und Wurstwaren ist in der vorliegenden Studie mit $90 \%$ als sehr hoch zu bezeichnen.

- Des Weiteren konnte festgestellt werden, dass das Thema Tierwohl auch für 83,6\% aller befragten Personen, die ein Haustier besitzen oder planen, sich ein Haustier anzuschaffen, in Bezug auf das Heimtierfutter kaufrelevant ist.

- Lediglich $15 \%$ der befragten Verbraucher kaufen schwerpunktmäßig und gezielt Tierwohlprodukte. Alle anderen Verbraucher sind überwiegend Gelegenheitskäufer.

- Als bedeutendste Kaufbarriere für den Kauf von Tierwohlprodukten konnte das Vertrauen in den Metzger identifiziert werden. Daneben wurden vor allem Vermarktungsdefizite genannt, wie etwa die geringe Verfügbarkeit, die schlechte Platzierung im Handel, die geringe Bewerbung der Produkte, die intransparenten Preise sowie die unzureichenden Hintergrundinformationen. Einem Drittel aller Discounter-Käufer ist der Preisunterschied zum konventionellen Fleisch zu hoch. Die meisten Metzgerei-Käufer stören diese Preisunterschiede dagegen nicht. 
- Eine weitere mögliche Kaufbarriere für Tierwohlprodukte ist ein zu geringes Wissen über die negativen Auswirkungen der intensiven Nutztierhaltung. Es stellte sich jedoch heraus, dass vielen Verbrauchern die zentralen Probleme (Tierwohl, Gesundheit, Umwelt) bewusst sind. Über die Hälfte der Verbraucher sieht zudem die hohen Umwelt- und Gesundheitskosten durch die intensive Nutztierhaltung. Positive Effekte werden für Fleischbranche, Arbeitsmarkt und Steueraufkommen erwartet.

- Um nicht genutzte Vermarktungspotenziale näher zu analysieren, wurde des Weiteren die Kundenzufriedenheit im Hinblick auf das Produkt- und Beratungsangebot von Tierwohlprodukten im deutschen Lebensmitteleinzelhandel untersucht. Die größten Verbesserungschancen bieten sich den Ergebnissen der Verbraucherbefragung zufolge für Supermärkte, Discounter und SB-Warenhäuser bzgl. der Transparenz der Produkte, der persönlichen Beratung zu Tierwohlstandards, der Produktwerbung sowie der Ästhetik von Produktverpackungen und Werbematerialien.

- Die Verbraucherpräferenzen zur Produktplatzierung von Tierwohlprodukten (,Regalsortierung') im Lebensmitteleinzelhandel wurden zudem untersucht, um Empfehlungen zum Category Management bei Tierwohlprodukten formulieren zu können. Es sprachen sich zwei Drittel aller Befragten für eine strikte Trennung von Tierwohlprodukten und konventionellen Produkten aus; ohne signifikante Unterschiede bei den verschiedenen Einkaufsstätten.

- Ein weiterers Ergebnis besteht darin, dass zwei Drittel aller Studienteilnehmer ihre Haupteinkaufsstätte für Fleisch- und Wurstwaren wechseln würden, wenn ein anderer Anbieter ein besseres Angebot an Tierwohlprodukten bereitstellt, und dass sich diese Wechselbereitschaft nicht nach Einkaufsstätten unterscheidet.

- Für die meisten befragten Verbraucher stellt es ein Kaufargument dar, wenn zusätzlich zu Tierwohl-Labels weitere Gütesiegel auf den Produkten angebracht sind. Besonders beliebt sind die Siegel von STIFTUNG WARENTEST, ÖKOTEST, FAIRTRADE und das GENTECHNIKFREI-Label. Positiv wurden auch Regionalsiegel, das DLG-Siegel, ausgewählte NGO-Logos und die frei erfundenen Labels HORMONFREI und ANTIBIOTIKAFREI bewertet. Nur wenige bewerteten das QS-Siegel positiv. Im weiteren Verlauf der Arbeit wird dieses Thema als ,MultiLabeling' bezeichnet. 
- Im ersten Teil der Arbeit wurden zudem die einzelnen Tierwohl-Labels mit Hilfe zahlreicher uni- und bivariater Analysen ( $\mathrm{Chi}^{2}$, Varianzanalyse, Korrelation) untersucht, wobei einige Unterschiede zwischen den einzelnen Tierwohl-Labels identifiziert werden konnten, die bisher in der wissenschaftlichen Forschung noch nicht so differenziert untersucht wurden. Tabelle 17 zeigt diese Forschungsergebnisse noch einmal in einer Übersicht.

Tabelle 17: Überblick der bivariaten Analysen zu einzelnen Tierwohl-Labels

\begin{tabular}{|c|c|c|c|c|c|c|c|c|}
\hline Variablen & demeter & |Bioland & & & Bio & & 3 & 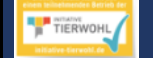 \\
\hline Geschlecht & $\begin{array}{l}\text { deutlich mehr } \\
\text { Frauen }\end{array}$ & \begin{tabular}{|l} 
etwas mehr \\
Frauen
\end{tabular} & \begin{tabular}{|l} 
etwas mehr \\
Frauen
\end{tabular} & $\begin{array}{l}\text { deutlich mehr } \\
\text { Frauen }\end{array}$ & $\begin{array}{l}\text { deutlich } \\
\text { mehr Frauen }\end{array}$ & $\begin{array}{l}\text { deutlich } \\
\text { mehr Frauen }\end{array}$ & $\begin{array}{l}\text { deutlich mehr } \\
\text { Frauen }\end{array}$ & $\begin{array}{l}\text { deutlich } \\
\text { mehr Frauen }\end{array}$ \\
\hline Alter & eher älter & eher älter & eher älter & eher älter & gemischt & eher jünger & eher jünger & eher jünger \\
\hline Bundesland & $\begin{array}{l}\text { Süd }>\text { Nord } \\
\text { West>Ost }\end{array}$ & $\begin{array}{l}\text { Süd }>\text { Nord } \\
\text { West>Ost }\end{array}$ & $\begin{array}{l}\text { kaum } \\
\text { Unterschied }\end{array}$ & Ost>Süd & West & $\begin{array}{l}\text { kaum } \\
\text { Unterschied }\end{array}$ & $\begin{array}{l}\text { kaum } \\
\text { Unterschiede }\end{array}$ & $\begin{array}{l}\text { kaum } \\
\text { Unterschied }\end{array}$ \\
\hline Ortsgröße & gemischt & gemischt & gemischt & eher Stadt & gemischt & gemischt & gemischt & eher Land \\
\hline Einkommen & sehr hoch & sehr hoch & etwas höher & $\begin{array}{l}\text { etwas } \\
\text { niedriger }\end{array}$ & gemischt & gemischt & $\begin{array}{l}\text { etwas } \\
\text { niedriger }\end{array}$ & sehr niedrig \\
\hline Bildung & sehr hoch & sehr hoch & hoch & niedrig & hoch & hoch & niedrig & sehr niedrig \\
\hline Bekanntheit & hoch & sehr hoch & hoch & hoch & sehr hoch & hoch & niedrig & niedrig \\
\hline $\begin{array}{l}\text { Label-Präferenz } \\
\text { (Kaufrelevanz) }\end{array}$ & hoch & sehr hoch & hoch & hoch & sehr hoch & sehr hoch & hoch & niedrig \\
\hline $\begin{array}{l}\text { Tierwohl, Umwelt } \\
\text { und Gesundheit }\end{array}$ & streng & sehr streng & streng & mittel & sehr streng & streng & $\begin{array}{l}\text { mittel, } \\
\text { Tierwohl sehr }\end{array}$ & nicht streng \\
\hline Einkaufsstätte & $\begin{array}{l}\text { Metzger (!) } \\
\text { Supermarkt }\end{array}$ & $\begin{array}{l}\text { Supermarkt } \\
\text { Metzger }\end{array}$ & $\begin{array}{l}\text { Supermarkt } \\
\text { Metzger }\end{array}$ & $\begin{array}{l}\text { Supermarkt } \\
\text { Metzger }\end{array}$ & gemischt & gemischt & $\begin{array}{l}\text { Supermarkt } \\
\text { kein Metzger }\end{array}$ & $\begin{array}{l}\text { Discounter } \\
\text { kein Metzger }\end{array}$ \\
\hline Angebotsform & Theke & Theke & Theke & Theke & Theke / SB & Theke / SB & SB & SB \\
\hline
\end{tabular}

\section{Quelle: Eigene Darstellung}


Wie in der Einleitung beschrieben sollten in der vorliegenden Studie der Informationsbedarf der Verbraucher bei Tierwohlprodukten sowie die politischen Präferenzen der Verbraucher beim Thema Tierwohl näher betrachtet werden. Im Folgenden sind die deskriptiven Ergebnisse zu diesen beiden Abschnitten dargestellt.

- Eine deutliche Mehrheit der Verbraucher forderte in der durchgeführten Studie bessere Informationen zu den Haltungsbedingungen der Tiere und zur Herkunft der Fleisch- und Wurstwaren, die sie vor allem auf Produktverpackungen, in den Verkaufsräumen im LEH, in Werbeprospekten, über das Verkaufspersonal und im Internet erhalten möchten. Dabei haben Discounter-Kunden im Gegensatz zu Metzgerei-Käufern eher wenig Interesse an Tierwohlinformationen seitens des Verkaufspersonals, sondern bevorzugen hierzu eher die Verpackung, Prospekte und das Internet. Vor allem Thekenkäufer wünschen sich Tierwohlinformationen über Broschüren und weitere Infomaterialien in der Einkaufsstätte und auf den Verpackungen, während sich SB-Käufer mehr persönliche Beratung bei Tierwohlfragen wünschen. Diese Informationen sollten der Verbrauchermeinung folgend sowohl von Lebensmittelherstellern und -händlern als auch von den Tierwohl-Label-Herausgebern bereitgestellt werden.

- Für fast $90 \%$ der Verbraucher würden konkrete Herkunftsangaben auf den Verpackungen von Fleisch- und Wurstwaren (bis auf den landwirtschaftlichen Betrieb) ein positives Kaufargument darstellen.

- Politisch sehen die Verbraucher in einem strengeren Tierschutzgesetz die beste Lösung, um das Wohl der Tiere in der Nutztierhaltung zu verbessern. Eine gesetzlich verpflichtende Haltungskennzeichnung sowie die stärkere Subventionierung von tierund umweltfreundlicheren Landwirten wurden ebenfalls als effektive Maßnahmen für mehr Tierwohl angeführt. Der politische Ansatz, das Tierwohl in Deutschland oder der Europäischen Union über freiwillige Tierwohlinititativen oder den Einsatz freiwilliger Tierwohl-Labels zu verbessern, wird dagegen nicht nur von den Wählern der Partei Bündnis90/DieGrünen und von Tierschutzparteien, sondern von den meisten Verbrauchern als wenig effektiv angesehen. 
- Hinsichtlich der (gestalterischen) Kennzeichung von Tierwohlprodukten sprachen sich mit großem Abstand die meisten Verbraucher für eine Ampelkennzeichnung aus. Nur ein Viertel der Verbraucher würde eine Kennzeichnung mit Sternen, wie beim Label des DEUTSCHEN TIERSCHUTZBUNDES oder dem geplanten staatlichen Tierwohl-Label, bevorzugen. Die Kennzeichnung von Fleisch- und Wurstwaren der Kennzeichnung von Eiern (0-1-2-3) anzugleichen, finden nur $14 \%$ der Verbraucher sinnvoll. Es zeigte sich jedoch, dass die Verbraucher eine grafische Tierwohlkennzeichnung den numerischen Varianten vorziehen.

- Was die Verbraucher selbst für mehr Tierwohl tun können, beantworteten die meisten Befragten mit ihrem eigenen Konsumverhalten. Als zweitwichtigste Möglichkeit, um sich für mehr Tierwohl in Deutschland einzusetzen, nannten die Verbraucher die Unterstiutzung von NGOs aus den Bereichen Tier-, Umwelt- und Verbraucherschutz. Petitionen werden insbesondere von Wählern der Partei Bündnis90/DieGrünen als Möglichkeit der politischen Partizipation betrachtet. Kaum ein Studienteilnehmer glaubt jedoch, dass er durch seine Stimmabgabe bei politischen Wahlen oder die Teilnahme an Demonstrationen zu einer verbesserten Tierhaltung in Deutschland beitragen kann.

Die Häufigkeitsauswertungen sowie die uni- und bivariaten Analysen zum Einkaufs- und Ernährungsverhalten der Verbraucher und zu einzelnen Aspekten des TierwohlLabelings haben bereits erste interessante Forschungsergebnisse hervorgebracht. Die Analyse der einzelnen Tierwohl-Labels, deren Ergebnisse in Tabelle 17 zusammenfassend dargestellt sind, lässt erkennen, dass es bzgl. der Berücksichtigung und Wahrnehmung von Tierwohl-Labels für Fleisch- und Wurstwaren bei deutschen Verbrauchern deutliche Unterschiede gibt.

Im weiteren Verlauf der vorliegenden Arbeit soll daher versucht werden, mit Hilfe einer explorativen Faktorenanalyse diese latenten Hintergrundkonstrukte von Tierwohl-Labels sichtbar zu machen und weiterführende Analysen durchzuführen. 


\subsection{Ergebnisse der explorativen Faktorenanalysen}

Detailfragen zum Einsatz von Tierwohl-Labels im Marketing wurden in der wissenschaftlichen Forschung bisher nur in geringem Umfang erforscht, insbesondere was die genauere Differenzierung von Käufergruppen anhand ihrer Label-Präferenzen betrifft.

Im Fragebogen wurden daher, wie im Methodenkapitel dargestellt, die Präferenzen der Verbraucher $\mathrm{zu}$ verschiedenen Tierwohl-Labels erfasst (F15), um erstmals eine differenzierte Typologisierung deutscher Verbraucher mit dem Fokus auf TierwohlLabels bei Fleisch- und Wurstwaren vorzunehmen, da anzunehmen ist, dass hier unterschiedliche Motivgruppen existieren.

Für Hersteller und Händler von Fleisch- und Wurstwaren handelt es sich hierbei um eine wesentliche Frage, da sie ihre Marketinginstrumente generell möglichst gezielt einsetzen sollten. Anhand der Faktorenanalyse soll verdeutlicht werden, dass es bei der Vermarktung von Tierwohlprodukten weniger auf ein einzelnes Tierwohl-Label ankommt, sondern dass sich hinter den zahlreichen unterschiedlichen Tierwohl-Labels eine gewisse Haltung einer Gruppe zu Tierwohl-Labels widerspiegelt.

Dies hat weitreichende Konsequenzen für den Einsatz der Marketinginstrumente von Herstellern und Händlern von Fleisch- und Wurstwaren, da sie ihre Marketinginstrumente nicht nach einzelnen Tierwohl-Labels differenziert gestalten müssen, sondern für eine Gruppe mit mehreren Tierwohl-Labels ähnliche Instrumente einsetzen können.

Zur Untersuchung, ob es bei Tierwohl-Labels verschiedene Motivgruppen gibt, wurde eine explorative Faktorenanalyse durchgeführt, um die hohe Anzahl der erfragten Tierwohl-Labels zusammenzufassen und entsprechenden Faktoren zuzuordnen. Die Zusammenfassung der einzelnen Tierwohl-Labels zu Faktoren bringt neben den bereits beschriebenen praktischen Vorteilen für Hersteller und Händler von Fleisch- und Wurstwaren einen weiteren Vorteil mit sich. Da die weiteren Berechnungen mit deutlich weniger Variablen durchgeführt werden können, reduziert sich die Komplexität der Analysen deutlich. 
Im weiteren Verlauf der Arbeit (Unterkapitel 5.5) werden auf Basis dieser extrahierten Faktoren, die auf den Verbraucherpräferenzen zu Tierwohl-Labels basieren, Cluster gebildet und analysiert. In diesem Abschnitt werden die Ergebnisse der multivariaten Faktorenanalyse beschrieben. Zunächst wird in Unterkapitel 5.4.1 die Faktorenbildung auf Basis der Label-Präferenzen (F15) beschrieben. Daran schließen sich die Ergebnisse ausgewählter Analysen mit den gebildeten Faktoren an. Dies sind im Wesentlichen die Ergebnisse $\mathrm{zu}$ soziodemografischen Unterschieden (Unterkapitel 5.4.2), vertriebsrelevanten Fragen (Einkaufsstätten, Angebotsformen und Produktplatzierung; Unterkapitel 5.4.3) sowie weiteren marketingrelevanten Analysen zu Fleischarten und sorten, zum Konsumverhalten, zum Multi-Labeling und zu Kaufkriterien (Unterkapitel 5.4.4).

\subsubsection{Faktorenextraktion aus Tierwohl-Label-Präferenzen}

Zielsetzung der explorativen Faktorenanalyse war es zunächst, die unterschiedlich ausgeprägte Motivation der Verbraucher gegenüber den verschiedenen Tierwohl-Labels zu erfassen, und herauszufinden, welches die ,motivbasierten' latenten Konstrukte sind. Um diese Konstrukte zu extrahieren, wurden die Label-Präferenzen der Verbraucher (F15) mit Hilfe der explorativen Faktorenanalyse untersucht.

Sowohl aus dem Bartlett-Test (Chi-Quadrat $(66)=5402.870, \mathrm{p}<.001)$ als auch dem Kaiser-Meyer-Olkin Measure of Sampling Adequacy (KMO = .86) ging hervor, dass sich die Variablen sehr gut für eine Faktorenanalyse eignen (UNIVERSITÄT ZÜRICH, 2020). Der hohe KMO-Wert lässt erkennen, dass das Antwortverhalten der Studienteilnehmer im Hinblick auf die unterschiedlichen Tierwohl-Labels sehr ähnlich ausfällt. Hinter den zahlreichen verschiedenen Tierwohl-Labels verbergen sich demzufolge nur wenige Label-Einstellungen. Es wurde eine Hauptkomponentenanalyse mit Varimax-Rotation durchgeführt. In der rotierten Komponentenmatrix, die die Basis für Tabelle 18 darstellt, konnten anhand der Höhe und Verteilung der Mittelwerte drei klar abgrenzbare Faktoren identifiziert werden, die 68,3 \% der Gesamtvarianz erklären. 
In Tabelle 18 sind die Ergebnisse der Faktorenanalyse enthalten, wobei die entsprechenden Mittelwerte, Standardabweichungen und Faktorladungen der verschiedenen Variablen bzw. Faktoren, die Kennzahlen des Gesamtmodells sowie die Ergebnisse der Reliabilitätsanalyse als Cronbachs Alpha (CA) dargestellt sind. Von besonderer Bedeutung ist in Tabelle 18, welche Tierwohl-Labels in welcher Höhe auf den gleichen Faktor laden. Im Ergebnis wird deutlich, dass sich die Label-Präferenzen der Verbraucher in drei verschiedene Faktoren aufteilen, die im Folgenden kurz beschrieben werden sollen.

Tabelle 18: Ergebnisse der Faktorenanalyse zu den Label-Präferenzen

\begin{tabular}{|c|c|c|}
\hline Faktoren und Items & MW (SD) & Faktorladung \\
\hline \multicolumn{3}{|l|}{$\mathrm{KMO}=\mathbf{0 , 8 6 0}$} \\
\hline Faktor 1 (CA 0,85) & $1,10(3,86)$ & \\
\hline TIERSCHUTZBUND & $0,53(1,09)$ & 0,795 \\
\hline VIER PFOTEN & $0,31(1,15)$ & 0,775 \\
\hline STAATLICHES TIERWOHL-LABEL & $0,32(1,15)$ & 0,808 \\
\hline LIDL HALTUNGSKOMPASS & $0,08(1,22)$ & 0,67 \\
\hline INITIATIVE TIERWOHL & $-0,09(1,24)$ & 0,817 \\
\hline Faktor 2 (CA 0,85) & $1,83(4,47)$ & \\
\hline DEMETER & $0,50(1,27)$ & 0,771 \\
\hline BIOLAND & $0,74(1,03)$ & 0,773 \\
\hline NATURLAND & $0,46(1,06)$ & 0,837 \\
\hline BIOKREIS & $0,20(1,03)$ & 0,737 \\
\hline NEULAND & $0,34(1,08)$ & 0,615 \\
\hline Faktor 3 (CA 0,78) & $1,25(1,47)$ & \\
\hline DE-BIO & $0,74(1,09)$ & 0,841 \\
\hline DE-BIO & $1,02(1,00)$ & 0,814 \\
\hline \multicolumn{3}{|c|}{$\begin{array}{l}\text { Kaiser-Meyer-Olkin-Kriterium }(K M O)=0,860 \text {, Bartlett-Test auf Sphärizität df }=66 \text {, Signifikanz } \\
\text { nach Bartlett,000. MW = Mittelwert. SD =Standardabweichung. Fünfstufige Likert-Skala von }+2 \\
\text { (würde ich auf jeden Fall berücksichtigen) bis }-2 \text { (würde ich auf keinen Fall berücksichtigen). } \\
68,3 \% \text { der Gesamtvarianz. Extraktionsmethode: Hauptkomponentenanalyse. } \\
\text { Rotationsmethode: Varimax mit Kaiser-Normalisierung in vier Iterationen konvergiert. } \\
C A=\text { Cronbachs Alpha. }\end{array}$} \\
\hline
\end{tabular}

\section{Quelle: Eigene Darstellung}


Die Tierwohl-Labels des DEUTSCHEN TIERSCHUTZBUNDES, VIER PFOTEN, INITIATIVE TIERWOHL und das STAATLICHE TIERWOHL-LABEL laden in ähnlichen Größenordnungen auf den Faktor 1.

Auf Faktor 1 laden im Wesentlichen Tierwohl-Labels, die einige Tierwohlkriterien beinhalten, jedoch im Gegensatz zu den beiden anderen Faktoren keine ökologischen Standards haben. Daher soll dieser Faktor als ,Reine Tierwohl-Labels ${ }^{6}$ bezeichnet werden. Dieser Faktor könnte auch als ,Tierwohl Light‘ charakterisiert werden, da diese Tierwohl-Labels nur auf relativ schwachen Tierwohlkriterien basieren. Insgesamt zeigt sich anhand der vergleichsweise niedrigen Mittelwerte der Items, dass diese TierwohlLabels im Vergleich zu den Bio-Labels deutlich weniger von Verbrauchern präferiert werden. Den höchsten Mittelwert erreichte das Label des DEUTSCHEN TIERSCHUTZBUNDES. Am wenigsten von allen abgefragten Labels würden die Verbraucher den Mittelwerten zufolge das Label der INITIATIVE TIERWOHL berücksichtigen.

Auf Faktor 2 laden die Tierwohl-Labels der deutschen ökologischen Anbauverbände DEMETER, BIOLAND, NATURLAND und BIOKREIS. Als Besonderheit lädt auch das nichtökologische Tierwohl-Label von NEULAND auf den Faktor 2. Dies zeigt, dass die Verbraucher NEULAND als etwas ähnliches wahrnehmen wie die ökologischen Anbauverbände. Hier wird offensichtlich, dass bei den latenten Größen bzw. Faktoren nicht die objektive Realität entscheidend ist, sondern vielmehr, wie Verbraucher die Dinge wahrnehmen. Der Faktor 2 wird der Einfachheit halber als ,Anbauverband-BioLabels' bezeichnet, auch wenn das nichtökologische Tierwohl-Label NEULAND beinhaltet ist.

Faktor 3 fasst ,Staatliche Bio-Labels' (DE-BIO und EU-BIO) zusammen, die wie die Tierwohl-Labels der ökologischen Anbauverbände neben verschiedenen Tierwohl- auch Umweltkriterien berücksichtigen, wenn auch in schwächerer Form als die TierwohlLabels der ökologischen Anbauverbände. 
Zusammenfassend können die Verbraucherpräferenzen zu den untersuchten TierwohlLabels in drei klar voneinander abgrenzbare Gruppen eingeteilt werden. Wie im Theorieteil beschrieben unterscheiden sich Tierwohl-Labels hinsichtlich ihrer Strenge bzw. Seriosität. Ebenso konnte anhand der Untersuchungen zur Einschätzung der unterschiedlichen Tierwohl-Labels durch die Verbraucher (F14) bereits belegt werden, dass die Tierwohl-Labels in ihrer Strenge unterschiedlich wahrgenommen werden (Abbildung 23, Abbildung 24, Abbildung 25). Durch Abbildung 44 wird bestätigt, dass die Verbraucherpräferenzen in Bezug auf die untersuchten Tierwohl-Labels stark differieren. Die relativ schwachen Tierwohl-Labels des DEUTSCHEN TIERSCHUTZBUNDES, VIER PFOTEN, das STAATLICHE TIERWOHL-LABEL, LIDL sowie der INITIATIVE TIERWOHL bilden eine eigene Motivgruppe (Faktor 1). Diese Tierwohl-Labels beinhalten im Gegensatz zu den anderen Tierwohl-Labels keine Umweltkriterien. Faktor 2 fasst die strengen Tierwohl-Labels der ökologischen Anbauverbände sowie das strenge Tierwohl-Label NEULAND zusammen. Faktor 3 wird von den staatlichen Bio-Labels (DE-BIO und EU-BIO) gebildet.

\begin{tabular}{|c|c|c|}
\hline $\begin{array}{l}\text { Reine Tierwohl-Labels } \\
\text { Schwache Tierwohl-Labels mit } \\
\text { geringen Unterschieden zum } \\
\text { Gesetz ohne Umweltkriterien }\end{array}$ & $\begin{array}{l}\text { Anbauverband-Bio-Labels } \\
\text { Strenge Tierwohl-Labels der } \\
\text { ökologischen Anbauverbände mit } \\
\text { Tierwohl- und Umweltkriterien }\end{array}$ & $\begin{array}{l}\text { Staatliche Bio-Labels } \\
\text { Mittelmäßig strenge Tierwohl- } \\
\text { Labels der BRD und der EU mit } \\
\text { Tierwohl- und Umweltkriterien }\end{array}$ \\
\hline 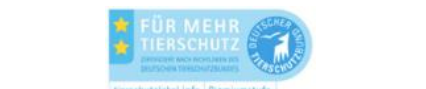 & demeter & \\
\hline rased & Bioland & $\mathrm{B}^{1} \mathbf{O}$ \\
\hline 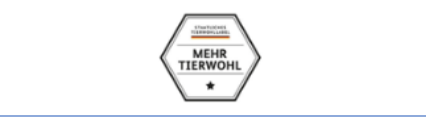 & & \\
\hline$=$ & bio & \\
\hline 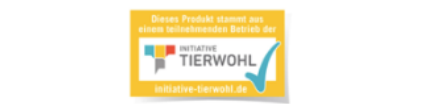 & & \\
\hline
\end{tabular}

Abbildung 44: Tierwohl-Label-Typologie nach Freckmann und Enneking (Quelle: Eigene Darstellung) 
Auf Basis der Faktorenanalyse zu den Label-Präferenzen (F15) wurden mehrere explorative Analysen mit Hilfe der Regressionsanalyse durchgeführt, um erstmals Unterschiede zwischen den identifizierten Tierwohl-Label-Präferenzgruppen sichtbar zu machen. Die Unterschiede zwischen den drei Gruppen bilden die Basis für gezielte praxisorientierte Handlungsempfehlungen. In den folgenden Abschnitten werden die Ergebnisse $\mathrm{zu}$ soziodemografischen Unterschieden (Unterkapitel 5.4.2), vertriebsrelevanten Fragen (Einkaufsstätten, Angebotsformen und Produktplatzierung; Unterkapitel 5.4.3) sowie weiteren marketingrelevanten Analysen zu Fleischarten und sorten, zum Konsumverhalten, Multilabeling und zu den Kaufkriterien (Unterkapitel 5.4.4) vorgestellt und die Ergebnisse anhand von Tabellen oder Abbildungen illustriert.

\subsubsection{Soziodemografische Unterschiede der Motivgruppen}

Zunächst wurden soziodemografische Unterschiede zwischen den extrahierten Faktoren analysiert. Im Fokus standen dabei die Variablen Geschlecht, Alter, Wohnortgröße, Haushaltseinkommen, Bildung sowie die Parteipräferenzen. Hierfür wurde die logistische Regressionsanalyse eingesetzt, um Zusammenhänge bzw. Korrelationen feststellen zu können, ohne jedoch Kausalitäten anzunehmen. Wie in Tabelle $19 \mathrm{zu}$ erkennen sind Personen, die Tierwohl-Labels bevorzugen, in allen drei Motivgruppen eher weiblich. Bei den Fans der ,Reinen Tierwohl-Labels` sind die Unterschiede hinsichtlich des Geschlechts besonders signifikant, während sich die beiden BioKäufergruppen nicht signifikant voneinander unterscheiden. Anbauverband-Bio-LabelFans finden sich am ehesten in der Altersgruppe der über 50-Jährigen. Jüngere Personen gehören eher zur Gruppe, die reine Tierwohl-Labels und staatliche Bio-Produkte präferieren.

Überraschenderweise konnten keine signifikanten Unterschiede zwischen den Bundesländern festgestellt werden, weder beim Nord-Süd- noch beim Ost-WestVergleich. Dabei ist anzumerken, dass zahlreiche soziodemografische Variablen analysiert wurden, jedoch aufgrund nicht signifikanter Unterschiede bzgl. der gebildeten Faktoren keine Berücksichtigung bei der Darstellung der Ergebnisse finden. 
$\mathrm{Zu}$ diesen nicht signifikanten Variablen gehören die Haushaltsgröße, die Anzahl der Kinder unter 14 Jahren, die Wohnsituation, der Besitz von Haustieren sowie die Religion der befragten Verbraucher.

Deutlich erkennbar ist in Tabelle 19 zudem, dass Anbauverband-Bio-Fans mit Abstand das höchste Haushaltseinkommen und die höchste Bildung aufweisen, während die reinen Tierwohl-Käufer, die eher auf dem Land als in der Stadt leben, das niedrigste Einkommen und mit großem Abstand die niedrigste Bildung haben. In dieser Gruppe befinden sich evtl. auch viele Schüler (noch ohne Schulabschluss), die der Kategorie ,niedrige Bildung ‘ zugeordnet wurden. Anbauverband-Bio-Fans wählen zudem eher die Linken, Bündnis90/DieGrünen und Tierschutzparteien als Käufer reiner Tierwohl-Labels oder staatlicher Bio-Labels.

Tabelle 19: Unterschiede Soziodemografie bei Tierwohl-Label-Präferenzgruppen

\begin{tabular}{|c|c|c|c|c|c|c|}
\hline & Reir & Tierwohl & Anba & arband-Bio & Sta & ch-Bio \\
\hline & 国 & Finitiony & demeler & D) raves & & $B: 0$ \\
\hline Variablen & B & Wald & B & Wald & B & Wald \\
\hline Geschlecht & $-0,364$ & $28,165^{\star \star \star}$ & $-0,117$ & 2,985 & $-0,118$ & 3,087 \\
\hline Alter & $-0,243$ & $12,546^{\star \star \star}$ & 0,363 & $26,344^{\star \star \star}$ & $-0,145$ & $4,447^{\star}$ \\
\hline Wohnortgröße & $-0,168$ & $6,125^{\star}$ & 0,081 & 1,423 & 0,068 & 1,004 \\
\hline HH-Einkommen & $-0,175$ & $6,749 * *$ & 0,318 & $21,535^{\star \star \star}$ & $-0,057$ & 0,708 \\
\hline Bildung & $-0,431$ & $36,688^{\star \star \star}$ & 0,201 & $8,326^{\star \star}$ & 0,094 & 1,834 \\
\hline Parteipräferenz & 0,102 & 1,673 & $-0,217$ & $7,243^{\star \star}$ & 0,079 & 1,032 \\
\hline $\begin{array}{l}\text { Logit-Modell. a } \\
\text { F_15_Faktor_2_Anba } \\
2=\text { männlich); Alter ( } \\
\text { Einwohnern = 1); HH } \\
\text { Abitur) }=1) ; \text { Parteiprä } \\
\text { Linke-Grüne-Tierschu } \\
\text { Wert }>6,66) ;{ }^{* * *} p<0,\end{array}$ & $\begin{array}{l}\text { Schri } \\
\text { erbandsBi } \\
-3=0 \text { (bis } \\
\text { lettoeinkom } \\
\text { renz (Rech } \\
\text {-Parteien } \\
1 \text { (Wald-W }\end{array}$ & $\begin{array}{l}1 \text { eingegeb } \\
F_{-} \text {15_Faktor_3 } \\
\text { Jahre }) \text { und } 4-5= \\
(<3000 €=0 \\
\text { nservative Parte } \\
\text { 0. Signifikanzn } \\
9,55) \text {. }\end{array}$ & $\begin{array}{l}\text { ene Va } \\
\text { Staatliches } \\
=1 \text { (älter a } \\
\text { und }>300 \\
\text { ien CDU, C } \\
\text { iveau }{ }^{*}=p\end{array}$ & $\begin{array}{l}\text { len: F_15_F } \\
\text { Kodierungen } \\
\text { ); Wohnortgröß } \\
\text { 1); Bildung (nie } \\
\text { SPD, AfD, FDP } \\
\text {,05 (Wald-Wert }\end{array}$ & $\begin{array}{l}\text { tor_1_Reir } \\
\text { Geschlec } \\
\text { (bis } 100.0 \\
\text { rig-mittel = } \\
\text { nd NPD mi } \\
3,84 \text { ); ** }\end{array}$ & $\begin{array}{l}\text { ierwohl-Label, } \\
(1=\text { weiblich, } \\
=0 ;>100.000 \\
\text { und hoch (ab } \\
6 \% \text { als } 1 \text { und } \\
0,01 \text { (Wald- }\end{array}$ \\
\hline
\end{tabular}

\section{Quelle: Eigene Darstellung}




\subsubsection{Vertriebsrelevante Unterschiede der Motivgruppen}

Die drei Label-Gruppen wurden, vor dem Hintergrund der in der Einleitung beschriebenen Ziele im Bereich der Vertriebspolitik, hinsichtlich ihrer Einkaufsstättenpräferenz, der Angebotsform (Theke/SB) und der Produktplatzierung beim Fleisch- und Wurstwarenkauf analysiert, um Antworten auf diese vertriebsrelevanten Fragestellungen $\mathrm{zu}$ erlangen. Die Kenntnis über die Einkaufsstättenpräferenzen der unterschiedlichen Tierwohl-Label-Fans hilft im Rahmen der Vertriebspolitik dabei, die richtigen Absatzwege für unterschiedliche Tierwohlprodukte auszuwählen. Die Wahl der richtigen Angebotsform für die verschiedenen Tierwohlprodukt-Käufer ist ebenfalls von großer Bedeutung für einen Hersteller oder Händler von Fleisch- und Wurstwaren. Sollten beispielsweise Fans von Bio-Produkten eindeutig die Theke präferieren, so verliert womöglich ein Supermarkt, der entsprechende Produkte nur als SB-Ware anbietet (z. B. DENN‘S Bio-Supermarkt), diese Kunden an einen Supermarkt mit Bio-Produkten in der Frischetheke (z. B. SUPER BIO). Die Produktplatzierung ist ebenfalls eine praktische Frage, die dem Category Management im Lebensmitteleinzelhandel zuzuordnen ist und Lebensmitteleinzelhändlern wertvolle Hinweise zur Strukturierung ihres Produktsortiments an Fleisch- und Wurstwaren geben kann. Eine bessere Strukturierung bietet sowohl den Kunden als auch den Lebensmitteleinzelhändlern einen Mehrwert. Kunden können Zeit und Mühe sparen, wenn sie nicht die einzelnen zertifizierten Tierwohlprodukte zwischen dem meist deutlich größeren Teil konventioneller Ware suchen müssen. Hier liegt ein beträchtliches Potenzial, die Kundenzufriedenheit zu erhöhen, was wiederum dem Lebensmitteleinzelhändler zugutekommt.

In Tabelle 20 sind die Unterschiede zwischen den drei Faktorgruppen bzw. den verschiedenen Tierwohl-Label-Fans hinsichtlich ihrer Einkaufsstättenpräferenzen (F2) dargestellt. Die Einkaufsstätten wurden nach Häufigkeit (in \%) absteigend sortiert, damit die Relevanz der einzelnen Einkaufsstätten für den Gesamtmarkt bei der Betrachtung deutlich wird. Die Beschreibung der Ergebnisse erfolgt der Übersichtlichkeit halber separat je Tierwohl-Label-Gruppe und nicht je Einkaufsstätte. 
Tabelle 20: Präferierte Einkaufsstätten unterschiedlicher Tierwohl-Label-Fans

\begin{tabular}{|c|c|c|c|c|c|c|c|}
\hline \multirow[b]{2}{*}{ Variablen } & \multirow[b]{2}{*}{$\%$} & \multicolumn{2}{|c|}{ 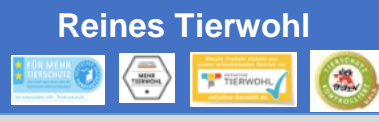 } & \multicolumn{2}{|c|}{ Anbauverband-Bio } & \multicolumn{2}{|c|}{ Staatlich-Bio } \\
\hline & & B & Wald & B & Wald & B & Wald \\
\hline EDEKA & 57 & 0,123 & 3,376 & 0,131 & 3,871 & 0,062 & 0,878 \\
\hline REWE & 53 & 0,034 & 0,26 & 0,158 & $5,723^{*}$ & 0,057 & 0,759 \\
\hline ALDI & 47 & 0,188 & $7,975^{\star \star}$ & 0,000 & 0,000 & 0,168 & $6,343^{*}$ \\
\hline LIDL & 46 & 0,349 & $25,449^{\star \star *}$ & $-0,215$ & $9,924^{\star \star *}$ & 0,152 & $4,976^{*}$ \\
\hline Metzger & 33 & $-0,156$ & $4,904^{\star}$ & 0,198 & $7,633^{\star \star}$ & $-0,019$ & 0,075 \\
\hline NETTO & 31 & 0,284 & $14,92^{\star \star \star}$ & $-0,142$ & $3,911^{*}$ & 0,083 & 1,295 \\
\hline KAUFLAND & 29 & 0,136 & 3,447 & $-0,04$ & 0,305 & 0,084 & 1,298 \\
\hline REAL & 22 & 0,174 & $4,66^{\star}$ & 0,024 & 0,092 & 0,136 & 2,729 \\
\hline PENNY & 19 & 0,286 & $10,857^{\star \star \star}$ & $-0,181$ & $4,637^{\star}$ & 0,141 & 2,65 \\
\hline Bio-Supermärkte & 14 & $-0,094$ & 0,689 & 0,746 & $29,564^{\star \star \star}$ & 0,436 & $9,784^{\star \star \star}$ \\
\hline Wochenmarkt & 12 & $-0,069$ & 0,471 & 0,535 & $22,62^{\star \star \star}$ & $-0,079$ & 0,566 \\
\hline Hofladen & 10 & $-0,169$ & 2,484 & 0,587 & $22,82^{\star \star \star}$ & 0,002 & 0 \\
\hline \multicolumn{8}{|c|}{ 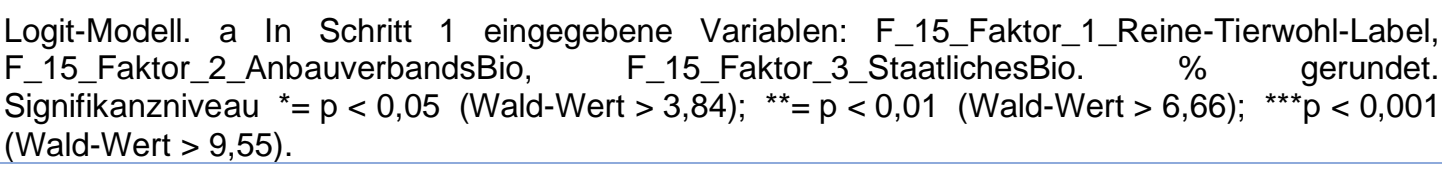 } \\
\hline
\end{tabular}

\section{Quelle: Eigene Darstellung}

Werden zunächst die Fans der reinen Tierwohl-Labels der Faktorgruppe 1 betrachtet, lässt sich erkennen, dass diese Verbrauchergruppe $\mathrm{zu}$ den klassischen DiscounterKäufern zählt. Dies zeigen vor allem die von allen Gruppen höchsten Koeffizienten bei LIDL, PENNY, NETTO und ALDI. Durch die hohen Wald-Werte werden die signifikanten Unterschiede zu den anderen Gruppen verdeutlicht. Die SB-Warenhäuser KAUFLAND und REAL werden von den Fans reiner Tierwohl-Labels ebenfalls am häufigsten genutzt. Diese Verbrauchergruppe kauft dagegen seltener beim Metzger Fleisch und Wurst ein als die beiden Bio-Käufergruppen, die hingegen erwartungsgemäß häufiger im Bio-Supermarkt einkaufen. Hofläden werden von dieser Gruppe am allerwenigsten genutzt und auch der Wochenmarkt zählt nicht zu den favorisierten Einkaufsstätten. Für EDEKA und REWE liegen keine signifikanten Unterschiede bei dieser Gruppe vor. Anhand der Koeffizienten kann abgelesen werden, dass EDEKA häufiger genutzt wird als REWE. 
Ein konträres Bild zeigt sich bei den Fans der ökologischen Anbauverbände und von NEULAND. LIDL, PENNY und NETTO werden von dieser Verbrauchergruppe am seltensten für den Fleischeinkauf genutzt und die Gruppe unterscheidet sich hier hoch signifikant von den anderen Gruppen. Die niedrigen Koeffizienten bei den SBWarenhäusern lassen erkennen, dass KAUFLAND und REAL am seltensten von dieser Gruppe genutzt werden. Des Weiteren wird deutlich, dass diese Personen von allen drei Gruppen am liebsten beim Metzger einkaufen. EDEKA und REWE werden von dieser Gruppe etwa gleich häufig für den Einkauf von Fleisch- und Wurstwaren gewählt, überraschenderweise auch häufiger als von den Fans staatlicher Bio-Produkte. Sehr deutliche Unterschiede zu den anderen Gruppen liegen jedoch bei der Nutzung von BioSupermärkten, Wochenmärkten und Hofläden vor. Diese eher speziellen Einkaufsstätten sind bei den Fans ökologischer Anbauverbände für den Fleischeinkauf von allen Gruppen am beliebtesten; insbesondere die Bio-Supermärkte. Was diese Einkaufsstätten betrifft, besteht auch ein deutlicher Unterschied zu den Fans staatlicher Bio-Produkte, die im Vergleich seltener Wochenmärkte und Hofläden nutzen als die Anbauverband-Bio-Fans.

Während die beiden ersten Faktorgruppen hinsichtlich ihrer Einkaufsstättenpräferenz eindeutig charakterisiert werden können, zeigt sich bei den Fans staatlicher Bio-Produkte ein heterogenes Bild. Am häufigsten werden Bio-Supermärkte für den Einkauf von Fleisch- und Wurstwaren bevorzugt, wenn auch nicht ganz so stark wie von den Anbauverband-Bio-Fans. Überraschend sind jedoch die anderen genutzten Einkaufsstätten dieser Verbrauchergruppe.

So nutzen die Fans staatlicher Bio-Produkte im Gegensatz zu den Anbauverband-BioFans außer den Bio-Supermärkten auch ALDI, LIDL, PENNY und REAL; teilweise auch NETTO und KAUFLAND. Seltener werden von dieser Gruppe auch EDEKA und REWE für den Einkauf von Fleisch- und Wurstwaren gewählt; beide Supermärkte etwa gleich häufig. Dies ist interessant, da es sich hier wie bei den reinen Tierwohlfans um Discounter-Käufer handelt, die ab und zu auch SB-Warenhäuser, REWE oder EDEKA nutzen. Der zentrale Unterschied liegt in der Präferenz für Bio-Produkte und der häufigen Nutzung von Bio-Supermärkten. 
Hinsichtlich der Einkaufsstättenpräferenz ist zu konstatieren, dass insbesondere Fans der ökologischen Anbauverbände in Bio-Supermärkten einkaufen. Daneben nutzen auch Käufer staatlicher Bio-Produkte ALNATURA, DENN`S, SUPER BIO, BASIC BIO oder BIO COMPANY. Käufer reiner (nichtökologischer) Tierwohlprodukte besuchen BioSupermärkte wie erwartet eher weniger.

In der Befragung wurden auch Metzger, Wochenmärkte und Hofläden als Einkaufsstätten berücksichtigt, da anhand der wissenschaftlichen Literatur angenommen wurde, dass die Affinität dieser Kundengruppen zu Tierwohlprodukten besonders hoch ist. Es zeigte sich, dass die Fans der ökologischen Anbauverbände den Metzger als Einkaufsstätte mehr präferieren als Fans staatlicher Bio-Labels und Fans reiner Tierwohl-Labels.

Wochenmarktkäufer sind den Ergebnissen zufolge am ehesten Fans der ökologischen Anbauverbände. Zudem existieren hoch signifikante Unterschiede zu den anderen beiden Gruppen. Andere Vertriebsschienen wie Discounter oder SB-Warenhäuser werden von dieser Gruppe eher weniger als Einkaufsstätte für den Fleischkauf genutzt. Dass in den Verkaufswagen auf dem Wochenmarkt die Fleisch- und Wurstwaren quasi in mobilen Frischetheken angeboten werden, passt zudem gut zur besonderen Präferenz dieser Zielgruppe für die Frischetheke bzw. den Metzger.

Unter den Hofladenkäufern sind Fans der ökologischen Anbauverbände ebenfalls am häufigsten vertreten. Es liegen hoch signifikante Unterschiede zu den anderen beiden Gruppen vor. Überraschenderweise kann ein Unterschied zu staatlichen Bio-Produkten konstatiert werden, was dafür spricht, dass die Bio-Käufer klar zwischen staatlichen BioProdukten und Produkten der ökologischen Anbauverbände differenzieren bzw. sich diese Gruppen in ihrem Kaufverhalten deutlich unterscheiden. Für diese Personen ist den Ergebnissen zufolge, Bio nicht gleich Bio‘. Die Anbauverband-Bio-Fans nutzen Hofläden signifikant häufiger als Fans staatlicher Bio-Produkte, die vor allem im konventionellen Handel (in Supermärkten, Discountern etc.) einkaufen. Die Fans reiner Tierwohl-Labels (Faktorgruppe 1) nutzen Hofläden hingegen nur selten. 
Bei EDEKA und REWE fallen die Unterschiede zwischen den unterschiedlichen Tierwohl-Label-Fans im Vergleich zu den Metzgern, Wochenmärkten, Hofläden und Bio-Supermärkten deutlich geringer aus. Das heißt, dass EDEKA und REWE mehr von allen drei Käufergruppen genutzt werden. Dennoch unterscheidet sich die Gruppe der reinen Tierwohlkäufer und der Anbauverband-Bio-Fans geringfügig von den staatlichen Bio-Käufern, wenn die Höhe der Koeffizienten betrachtet wird. Kunden von EDEKA und REWE haben demnach die höchste Affinität zu den Tierwohl-Labels DEMETER, NATURLAND, BIOLAND, BIOKREIS und NEULAND und teilweise auch zu staatlichen Bio-Produkten, jedoch eine geringere Affinität zu reinen Tierwohl-Labels. Die Präferenzen zu Tierwohl-Labels unterscheiden sich bei den Kunden der beiden führenden Supermärkte in Deutschland nur in relativ geringem Ausmaß. Beide Kundengruppen zeigen sich insgesamt als sehr aufgeschlossen gegenüber TierwohlLabels. Bei näherer Betrachtung der Koeffizienten lässt sich feststellen, dass die Affinität zu den Tierwohl-Labels der Anbauverbände bei EDEKA-Kunden etwas höher sein könnte als bei REWE und die Präferenz für reine Tierwohl-Labels dagegen etwas niedriger als bei REWE-Kunden.

Eine weitere zentrale Erkenntnis bei der Betrachtung der drei unterschiedlichen Gruppen besteht darin, dass sich die Tierwohl-Label-Präferenzen der Kunden von Discountern und SB-Warenhäusern nur marginal unterscheiden. In der Folge ist für Hersteller und Händler von Fleisch- und Wurstwaren bei der Gestaltung des Angebots an Tierwohlprodukten bei diesen Vertriebsformen nur wenig differenziertes Vorgehen erforderlich. Die Unterschiede fallen bei Discountern und SB-Warenhäusern vor allem im Vergleich zu Metzgern, Hofläden, Wochenmärkten und Bio-Supermärkten deutlich geringer aus, was anhand der insgesamt niedrigen Wald-Werte ersichtlich ist. Bei ALDI, LIDL, NETTO, PENNY und den SB-Warenhäusern KAUFLAND und REAL kaufen den Ergebnissen zufolge Personen ein, die vor allem reine Tierwohl-Labels und teilweise auch staatliche Bio-Produkte bevorzugen. Personen, die Fleisch- und Wurstwaren von DEMETER, BIOLAND, NATURLAND oder NEULAND präferieren, kaufen dagegen eher weniger in diesen Einkaufsstätten ein. 
Bei Betrachtung der Koeffizienten ist anzunehmen, dass Tierwohl für Kunden von KAUFLAND und REAL im Vergleich zu den Discounter-Käufern (und in noch deutlich größerem Ausmaß zu den Nutzern anderer Einkaufstätten) insgesamt eine geringere Bedeutung hat. Dieses Ergebnis überrascht, da aufgrund der ausgeprägten ,Billig-billigeram-billigsten-Mentalität‘ der Discounter davon ausgegangen wurde, dass die Gruppe der Discounter-Käufer am wenigsten auf ethische Kaufkriterien achtet. Sie erwiesen sich jedoch als deutlich interessierter an ethischen Kaufkriterien als die SB-WarenhausKunden. Des Weiteren ist die Gruppe, die staatliche Bio-Produkte präferiert, unter den REAL-Kunden größer als unter den KAUFLAND-Kunden. Daraus könnte der Schluss gezogen werden, dass den Kunden von REAL ethische Kaufkriterien etwas wichtiger sind als den Kunden von KAUFLAND, wenn auch auf einem vergleichsweise niedrigen Niveau.

Zusammenfassend lässt sich sagen, dass Personen, die reine Tierwohlprodukte bevorzugen, vor allem bei Discountern und SB-Warenhäusern einkaufen. Ein Teil dieser Käufer würde auch staatliche Bio-Produkte (DE-BIO oder EU-BIO) kaufen, legt jedoch auf eine Zertifizierung mit DEMETER, BIOLAND, NATURLAND oder NEULAND weniger Wert. Dies könnte daran liegen, dass sie diese Tierwohl-Labels nicht so gut kennen, wie die Ergebnisse zur Bekanntheitsgradmessung nahegelegt haben.

Als weiteres vertriebsrelevantes Thema wurden die Unterschiede zwischen den gebildeten Faktoren bzgl. der präferierten Angebotsformen (Theke und SB) untersucht. Hinsichtlich der Angebotsform unterscheiden sich die drei Gruppen hoch signifikant voneinander, wie in Tabelle 21 ersichtlich. Personen, die in der Befragung überwiegend reine Tierwohlprodukte wählten, die nicht aus ökologischer Produktion stammen, bevorzugen sowohl Wurst und Aufschnitt als auch Fleisch zum Braten und Grillen tendenziell eher im SB-Bereich. Die Fans der ökologischen Anbauverbände kaufen dagegen alle Fleisch- und Wurstwaren deutlich lieber an der Frischetheke und unterscheiden sich in diesem Punkt am stärksten von allen anderen Gruppen. Verbraucher, die staatliche Bio-Produkte bevorzugen, haben weder bei Wurst und Aufschnitt noch bei Fleisch zum Braten und Grillen eine klare Tendenz zu einer Angebotsform. 
Tabelle 21: Präferierte Angebotsformen unterschiedlicher Tierwohl-Label-Fans

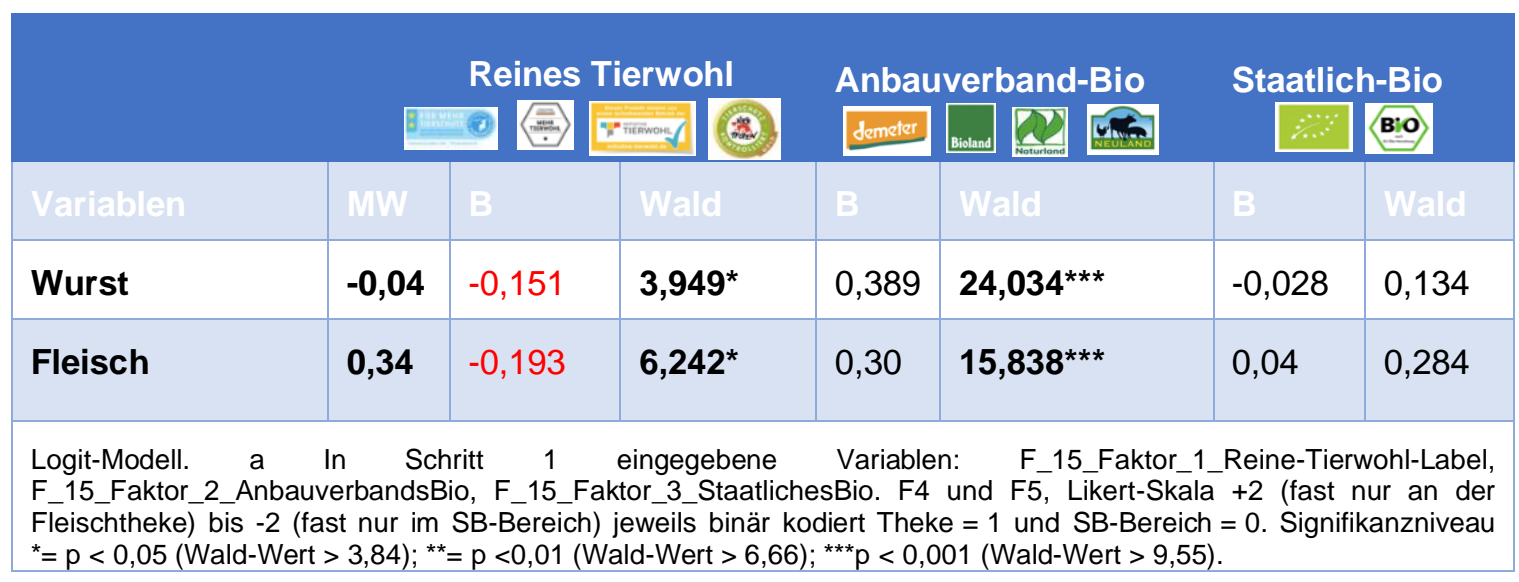

\section{Quelle: Eigene Darstellung}

Des Weiteren wurde aufgrund der hohen Praxisimplikation als weiteres vertriebsrelevantes Thema untersucht, ob sich die Präferenzen der Tierwohl-LabelKäufer-Gruppen bei der Produktplatzierung im Einzelhandel unterscheiden. Für Lebensmitteleinzelhändler stellt sich die Frage, wo bzw. wie die besonderen Tierwohlprodukte im Supermarkt platziert werden sollen. Bisher wurde noch nicht wissenschaftlich untersucht, ob Tierwohlprodukte getrennt von konventionellen Produkten angeboten werden sollten bzw. wie diese platziert werden sollten, und ob diesbezüglich Unterschiede zwischen verschiedenen Tierwohl-Label-Fans bestehen.

In der vorliegenden Studie wurden den Befragten zwei Varianten zur Auswahl angeboten: zum einen die strikte (räumliche) Trennung von konventionellen Fleisch- und Wurstwaren und zum anderen die Sortierung lediglich nach Sorten (z. B. Schinken), wobei konventionelle und zertifizierte Fleisch- und Wurstwaren direkt nebeneinander angeboten werden. Insgesamt sprachen sich in der Befragung $70 \%$ für eine klare räumliche Trennung von konventionellen und ökologischen Produkten bzw. Tierwohlprodukten in den Einkaufsstätten aus. Die Mehrheit der Verbraucher wünscht sich demnach einen eigenen Bereich für Tierwohlprodukte am Point of Sale. Mit Hilfe der Faktorenanalyse konnte zudem festgestellt werden, dass sich insbesondere die Fans der Tierwohl-Labels DEMETER, BIOLAND, NATURLAND und NEULAND für einen getrennten Bereich im Einzelhandel aussprechen, wie Tabelle 22 zeigt. 
Tabelle 22: Motivbasierte Unterschiede bei Präferenzen zur Produktplatzierung

\begin{tabular}{|l|l|l|l|l|}
\hline \multicolumn{7}{c}{ Reines Tierwohl } \\
\hline
\end{tabular}

Quelle: Eigene Darstellung

\subsubsection{Marketingrelevante Unterschiede der Motivgruppen}

Neben den soziodemografischen und den vertriebsrelevanten Aspekten (Einkaufsstätten, Angebotsform und Produktplatzierung) wurden im Rahmen der Arbeit des Weiteren Unterschiede zwischen verschiedenen Produktarten bzw. Fleischsorten (F9) in Bezug auf die drei Faktorgruppen analysiert. Dabei stellte sich heraus, dass die Motivgruppe 1 (reine Tierwohl-Labels) signifikant mehr Geflügel, Aufschnitt, Rind und Schwein kauft als die beiden anderen Gruppen. Außerdem konnte herausgefunden werden, dass diese Gruppe am häufigsten TK-, Dosen-, Tüten- und Glaswaren sowie Snacks kauft. AnbauverbandsBio-Käufer kaufen dagegen am seltensten TK-Ware.

Von Interesse war zudem, in welche Label-Gruppe sich die sogenannten Flexitarier einordnen lassen, die ihren Fleischkonsum bewusst reduzieren und auf verschiedene besondere Produktqualitäten achten. In Tabelle 23 lässt sich erkennen, dass Flexitarier am ehesten in der Label-Gruppe der ökologischen Anbauverbände zu verorten sind. Die Reduktionsbereitschaft der Faktorgruppe 1 (reine Tierwohl-Labels) wie auch der Käufer staatlicher Bio-Produkte (Faktorgruppe 3) ist deutlich niedriger. Faktorgruppe 1 besteht demzufolge überwiegend aus ,normalen Fleischessern', die täglich Fleisch- und Wurstwaren verzehren und keinen besonderen Wert auf Tierwohl oder Umwelt legen. 
Tabelle 23: Reduktionsbereitschaft unterschiedlicher Tierwohl-Label-Fans

\begin{tabular}{|c|c|c|c|c|c|c|}
\hline & Reine & ierwohl & Anbal & erband-Bio & & aatlich-Bio \\
\hline & $\mathrm{Fin}$ & (2) $\div$ & demeler & ND & & BंO \\
\hline Variablen & B & Wald & B & Wald & B & Wald \\
\hline Reduktionsbereitschaft & 0,065 & 0,948 & $-0,191$ & $8,109^{\star \star}$ & 0,016 & 0,058 \\
\hline
\end{tabular}

\section{Quelle: Eigene Darstellung}

Im deutschen Lebensmitteleinzelhandel finden sich häufig unterschiedliche Gütesiegel gleichzeitig auf Verpackungen. Daher stellt sich aus Händler- und Herstellerperspektive die Frage, ob und wie Tierwohl-Labels mit anderen Gütesiegeln sinnvoll kombiniert werden können. In Tabelle 24 sind die statistischen Auswertungen der Analyse der drei Faktorengruppen (F15) mit den verschiedenen abgefragten Gütesiegeln (F17) dargestellt, um erstmals die Präferenzen der Verbraucher bzgl. des Multi-Labelings bei Fleisch- und Wurstwaren aufzuzeigen.

Festzustellen ist, dass Regional-Labels für alle drei Motivgruppen ein zusätzliches positives Kaufargument darstellen. Jedoch bestehen hoch signifikante Unterschiede zwischen den drei Gruppen. Besonders relevant sind Regional-Labels für Personen, die Anbauverband-Bio-Produkte kaufen. Für Personen, die reine Tierwohl-Labels bevorzugen, stellen Regional-Labels überraschenderweise deutlich häufiger ein positives Kaufargument dar als für Käufer staatlicher Bio-Labels.

STIFTUNG WARENTEST ist besonders für Käufer reiner Tierwohlprodukte und staatlicher Bio-Produkte ein zusätzliches positives Kaufargument. Für die Gruppe der Anbauverband-Bio-Fans stellt STIFTUNG WARENTEST hingegen nur bedingt einen Zusatznutzen dar. Es bestehen hoch signifikante Unterschiede zwischen den drei Gruppen. Natürlich sollte das Testurteil dabei positiv ausfallen, relevante Kaufargumente abbilden (z. B. nicht nur die Verpackung bewerten) und aktuell sein, um einen positiven Einfluss auf die Kaufentscheidung zu erzielen. 
Tabelle 24: Multi-Labeling-Präferenzen unterschiedlicher Tierwohl-Label-Fans

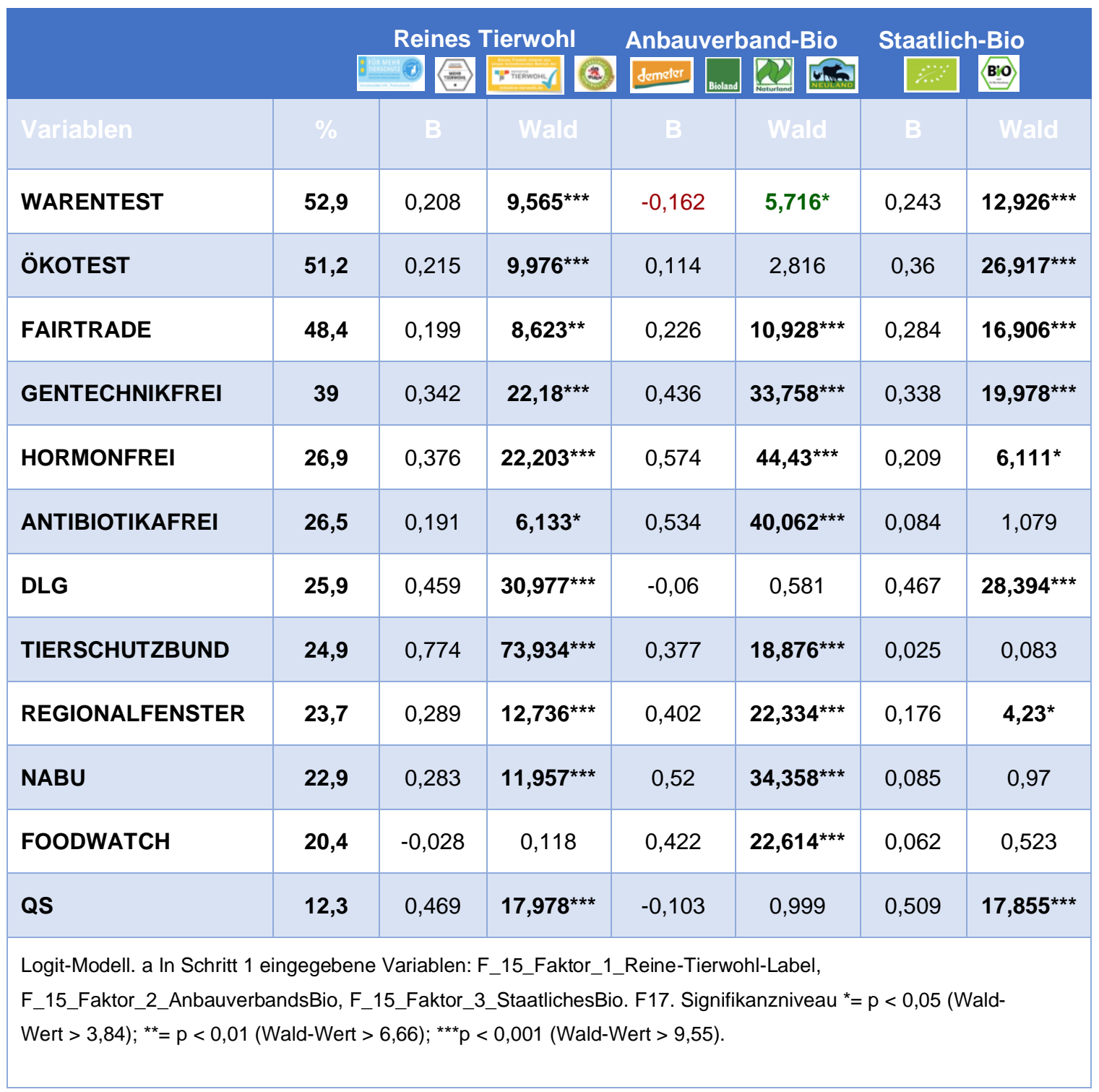

Quelle: Eigene Darstellung

Das ÖKOTEST-Label findet die meisten Fans unter den Käufern staatlicher BioProdukte und stellt auch für Käufer reiner Tierwohlprodukte ein zusätzliches positives Kaufargument dar. Für die Gruppe der Anbauverband-Bio-Fans bringt das ÖKOTESTSiegel überraschenderweise nur einen vergleichsweise geringen Zusatznutzen. Zwischen den Motivgruppen bestehen teilweise hoch signifikante Unterschiede. Wie bei STIFTUNG WARENTEST bzw. generell allen Prüfsiegeln sollte das Testurteil dabei positiv ausfallen, relevante Kaufargumente abbilden und möglichst aktuell sein. 
Das Logo des NABU findet mit Abstand die meisten Befürworter unter den Käufern von Anbauverband-Bio-Produkten und stellt für diese Motivgruppe ein zusätzliches positives Kaufargument dar. Die Gruppe der reinen Tierwohl-Label-Käufer bewertet das NABULogo auf Verpackungen ebenfalls positiv, während Käufer staatlicher Bio-Produkte den geringsten Zusatznutzen in der zusätzlichen Anbringung des NABU-Logos bei Tierwohlprodukten sehen. Das NABU-Logo auf Fleisch- und Wurstwaren eignet sich demzufolge als Ergänzung zu DEMETER, BIOLAND, NATURLAND und NEULAND und auch als Ergänzung zu reinen Tierwohl-Labels wie dem Label des DEUTSCHEN TIERSCHUTZBUNDES.

Das Logo des DEUTSCHEN TIERSCHUTZBUNDES findet mit Abstand die meisten Fans unter den Käufern reiner Tierwohl-Labels und stellt für diese ein positives zusätzliches Kaufargument dar. Die Gruppe der reinen Tierwohlkäufer bewertet das Logo des DEUTSCHEN TIERSCHUTZBUNDES auf Verpackungen sehr positiv, während auch Anbauverband-Bio-Fans in diesem Logo ein zusätzliches positives Kaufargument sehen. Staatliche Bio-Käufer sehen den geringsten Zusatznutzen in der Anbringung des TIERSCHUTZBUND-Logos auf Fleisch- und Wurstwaren.

Das Logo des DEUTSCHEN TIERSCHUTZBUNDES auf Fleisch- und Wurstwaren eignet sich daher am ehesten als Ergänzung zu den reinen Tierwohl-Labels. Bedingt eignet sich das Logo des DEUTSCHEN TIERSCHUTZBUNDES evtl. auch als Ergänzung zu den Siegeln der ökologischen Anbauverbände, auch wenn deren Kriterien deutlich höher sind als die Kriterien des DEUTSCHEN TIERSCHUTZBUNDES.

Das Logo der Verbraucherschutzorganisation FOODWATCH findet mit Abstand die meisten Fans unter den Käufern von Tierwohl-Labels der ökologischen Anbauverbände DEMETER, BIOLAND, NATURLAND sowie NEULAND und stellt für diese Gruppe ein besonders positives zusätzliches Kaufargument dar. Daher eignet es sich für diese Motivgruppe am besten. Womöglich ist der Bekanntheitsgrad dieser Organisation in dieser Gruppe auch am höchsten. 
Das Logo der Organisation FAIRTRADE des TRANSFAIR E. V. wird von allen Käufergruppen als sehr positiv bewertet, findet aber womöglich die meisten Fans unter den Bio-Käufern. Das Logo von FAIRTRADE eignet sich daher am ehesten auf ökologischen Fleisch- und Wurstwaren als Ergänzung zu den Siegeln von DEMETER, BIOLAND, NATURLAND, DE-BIO, EU-BIO sowie NEULAND und zu staatlichen Bio-Labeln.

Das DLG-Siegel, das von Landwirten herausgegeben wird und klassische Kaufkriterien wie Frische, Geschmack, Aussehen (also die ,innere‘ Produktqualität) abbildet und keine Kriterien für eine artgerechtere Tierhaltung oder eine höhere Umweltfreundlichkeit enthält, eignet sich am ehesten als Ergänzung zu reinen Tierwohlprodukten oder staatlichen Bio-Produkten. Wenig sinnvoll erscheint die Anbringung des DLG-Siegels auf nach den ökologischen Anbauverbänden zertifizierten Fleisch- und Wurstwaren.

Das QS-Siegel, das von der Fleischindustrie herausgegeben wird und die Umsetzung gesetzlicher Vorgaben im Bereich der Lebensmittelgesundheit abbildet, eignet sich am ehesten als Ergänzung zu reinen Tierwohlprodukten oder staatlichen Bio-Produkten. Wenig sinnvoll erscheint die Anbringung des QS-Siegels auf nach den ökologischen Anbauverbänden zertifizierten Produkten.

Ein ANTIBIOTIKAFREI-Siegel, das den Befragten als neue Idee in der vorliegenden Studie präsentiert wurde, findet den mit Abstand größten Anklang bei Personen, die bevorzugt Produkte der ökologischen Anbauverbände kaufen würden. Weniger sinnvoll erscheint die Anbringung des ANTIBIOTIKAFREI-Siegels auf reinen Tierwohlprodukten oder staatlichen Bio-Produkten.

Ein HORMONFREI-Siegel, das ebenfalls als neue Idee in der vorliegenden Studie den Befragten präsentiert wurde, findet ebenso den größten Anklang bei Personen, die bevorzugt Produkte der ökologischen Anbauverbände kaufen würden, und wird auch von vielen Käufern reiner Tierwohl-Labels begrüßt. Weniger sinnvoll erscheint die Anbringung des HORMONFREI-Siegels auf staatlichen Bio-Produkten, auch wenn es insgesamt positiv bewertet wird. 
Das GENTECHNIKFREI-Siegel hat für alle drei Käufergruppen einen positiven Einfluss auf die Kaufentscheidung und ist daher für alle drei Käufergruppen ein großes Anliegen. Die mit Abstand größte Zustimmung findet das GENTECHNIKFREI-Siegel bei Personen, die bevorzugt Produkte der ökologischen Anbauverbände kaufen würden. Daher erscheint die Integration dieses Siegels vor allem als Zusatzlabel bei Produkten von DEMETER, BIOLAND, NATURLAND und NEULAND sinnvoll. Das GENTECHNIKFREI-Siegel eignet sich daneben auch als Ergänzung zu reinen TierwohlLabels und staatlichen Bio-Siegeln.

Im Hinblick auf die Gestaltung des Marketing-Mix für Fleisch- und Wurstwaren ist zudem die Gewichtung der einzelnen Kaufkriterien von zentraler Bedeutung. Im Folgenden werden die Unterschiede bzgl. der Kaufmotive bei Fleisch- und Wurstwaren bei den drei gebildeten Motivgruppen zunächst einzeln analysiert und beschrieben, bevor anschließend aus den verschiedenen Kaufkriterien ebenfalls Faktoren extrahiert werden.

Die Unterschiede bei der Beachtung von Kaufkriterien beim Einkauf von Fleisch- und Wurstwaren sind in Tabelle 25 dargestellt, wobei deutliche Unterschiede zwischen den drei gebildeten Motivgruppen zu erkennen sind. Käufer reiner Tierwohl-Labels achten am meisten auf Marken und den Geschmack, während Käufer von Anbauverband-BioLabels am häufigsten auf Umweltfreundlichkeit, Gütesiegel und Fairtrade achten. Fans reiner Tierwohl-Labels und staatlicher Bio-Labels achten ebenfalls auf Gütesiegel, jedoch nicht so stark.

Käufer reiner Tierwohlprodukte achten vergleichsweise stark auf Sonderangebote, während die Gruppe Anbauverband-Bio am wenigsten auf günstige Preise achtet. Käufern von Anbauverband-Bio sind die Frische der Produkte, Gesundheitsaspekte und eine artgerechtere Tierhaltung am wichtigsten. Unerwarteterweise gaben Personen, die reine Tierwohl-Labels bevorzugen, häufiger an, auf Tierwohl zu achten, als Käufer staatlicher Bio-Produkte. Käufer reiner Tierwohlprodukte achten zudem am meisten auf die Verpackung, die auch für Käufer staatlicher Bio-Produkte relevant ist. 
Tabelle 25: Gewichtung von Kaufkriterien unterschiedlicher Tierwohl-Label-Fans

\begin{tabular}{|c|c|c|c|c|c|c|c|}
\hline \multirow{2}{*}{\begin{tabular}{|l} 
\\
Variablen
\end{tabular}} & & \multicolumn{2}{|c|}{$\begin{array}{l}\text { Reines Tierwohl } \\
\text { (20) } 0\end{array}$} & demender & \multicolumn{2}{|c|}{ Anbauverband-Bio } & $\begin{array}{l}\text { itaatlich-Bio } \\
\ldots . \\
\text { Bo }\end{array}$ \\
\hline & MW & B & Wald & B & Wald & B & Wald \\
\hline Frische & 1,75 & 0,313 & 1,179 & 0,486 & 3,25 & 0,228 & 0,814 \\
\hline Aussehen & 1,72 & 0,213 & 0,841 & $-0,298$ & 1,477 & 0,191 & 0,707 \\
\hline Geschmack & 1,64 & $-0,053$ & 0,037 & $-0,098$ & 0,124 & 0,552 & $4,604^{*}$ \\
\hline Regionalität & 0,92 & $-0,039$ & 0,112 & 0,727 & $37,261^{\star \star \star}$ & 0,05 & 0,2 \\
\hline Tierwohl & 0,91 & 0,367 & $10,211^{\star \star \star}$ & 0,598 & $29,321^{\star \star \star}$ & 0,066 & 0,415 \\
\hline Umwelt & 0,75 & 0,262 & $6,159^{\star \star}$ & 0,597 & $34,618^{\star \star \star}$ & 0,13 & 1,789 \\
\hline Gesundheit & 0,66 & 0,183 & 3,551 & 0,519 & $29,447^{\star \star \star}$ & 0,187 & $4,129^{*}$ \\
\hline Preis & 0,61 & 0,287 & $8,998^{\star \star}$ & $-0,335$ & $12,291^{\star \star \star}$ & 0,142 & 2,32 \\
\hline Gütesiegel & 0,58 & 0,395 & $17,193^{\star \star \star}$ & 0,453 & $23,841^{\star \star *}$ & 0,337 & $13,205^{\star \star \star}$ \\
\hline Bio-Qualität & 0,48 & 0,001 & 0 & 0,803 & $60,613^{\star \star \star}$ & 0,496 & $26,963^{\star \star \star}$ \\
\hline Verpackung & 0,32 & 0,291 & $12,727^{\star \star \star}$ & 0,098 & 1,459 & 0,231 & $8,566^{* *}$ \\
\hline Marken & 0,26 & 0,311 & $14,477^{\star * \star}$ & 0,107 & 1,797 & 0,172 & $4,558^{*}$ \\
\hline faire Löhne & 0,22 & 0,185 & $5,23^{*}$ & 0,465 & $31,202^{\star \star \star}$ & $-0,014$ & 0,028 \\
\hline $\begin{array}{l}\text { Logit-Modell. a In } \\
\text { F_15_Faktor_2 } \\
\text { (achte ich fast ni } \\
\text { (Wald-Wert }>6,6\end{array}$ & 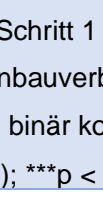 & 201 & iablen: & nzniv & $\begin{array}{l}\text { Reine-Tierw } \\
\text { F8, Likert- }\end{array}$ & $\begin{array}{l}\text { bel, } \\
-2(a c\end{array}$ & $\begin{array}{l}\text { st immer) bis } \\
{ }^{* *}=p<0,01\end{array}$ \\
\hline
\end{tabular}

\section{Quelle: Eigene Darstellung}

Käufer von Anbauverband-Bio achten am meisten auf Bio-Qualität, und zwar deutlich mehr als Käufer staatlicher Bio-Labels. Käufer reiner Tierwohlprodukte achten nicht auf Bio-Qualität, da ihnen Umwelt- und Gesundheitsaspekte womöglich weniger wichtig sind als das Tierwohl und sie nicht wissen, dass die Tierwohlstandards bei Bio-Produkten in der Regel höher sind als bei reinen Tierwohlprodukten.

Außerdem berücksichtigt die Anbauverband-Bio-Gruppe am meisten die Regionalität, während das Thema für Fans reiner Tierwohlprodukte und staatlicher Bio-Produkte (auch, aber) weniger bedeutsam ist. 
Insgesamt zeigen die Koeffizienten, dass der Faktorgruppe 1 mit wenigen Ausnahmen (z. B. Verpackung) viele Punkte generell nicht so wichtig sind wie den anderen Gruppen. So lange das Fleisch frisch und günstig ist und schmeckt, ist Gruppe 1 womöglich schon zufrieden. Mehr Tierwohl fänden Mitglieder dieser Gruppe ebenfalls gut, wenn dadurch keine Mehrkosten entstehen. Die anspruchsvollste Gruppe scheinen Anbauverband-BioFans zu sein.

Auf Basis der Kaufkriterien wurde eine weitere Faktorenanalyse zur Reduzierung der vielfältigen Kaufkriterien beim Fleisch- und Wurstwarenkauf durchgeführt, die im nächsten Abschnitt beschrieben wird. Dabei werden wie bei der Faktorenanalyse auf Basis der Label-Präferenzen zunächst das Vorgehen bei der Faktorenbildung sowie die Gütekriterien des Gesamtmodells beschrieben, bevor dargestellt wird, welche Kaufkriterien auf welchen Faktor laden.

\subsubsection{Faktorenextraktion aus Kaufkriterien}

In Unterkapitel 5.4.1 wurde die Faktorenanalyse zu den Tierwohl-Label-Präferenzen der Verbraucher beschrieben und in den Folgekapiteln weiterführend analysiert. Dabei standen die Präferenzen der Verbraucher zu konkreten Tierwohl-Labels (welche Tierwohl-Labels die Verbraucher kaufen würden) im Vordergrund. In diesem Unterkapitel wird eine weitere durchgeführte Faktorenanalyse beschrieben, die auf den allgemeinen Kaufkriterien bei Fleisch- und Wurstwaren basiert und sich demnach deutlich von der Faktorenanalyse zu den Tierwohl-Label-Präferenzen unterscheidet.

Diese Analyse wird für weitere Berechnungen im Rahmen der Clusteranalyse (Unterkapitel 5.5) benötigt. Die möglichst genaue Kenntnis der für die Kunden bedeutsamen Kaufkriterien ist ein wesentlicher Erfolgs- oder auch Misserfolgsfaktor für ein Unternehmen. Je besser ein Anbieter diese Kundenbedürfnisse kennt und vor allem erfüllt, desto eher wird eine gute Position im Wettbewerb um die Kundengruppe erreicht. 
Daher basieren die meisten Verbrauchertypologien auf den Kaufmotiven bzw. -kriterien. In der vorliegenden Studie wurde dagegen der Ansatz verfolgt, eine Verbrauchertypologie auf Basis der Tierwohl-Label-Präferenzen der Verbraucher zu erstellen. Dennoch sollten dabei auch die Kaufmotive eine zentrale Rolle für weiterführende Analysen in dieser Arbeit einnehmen.

Der Kaufentscheidung der Verbraucher liegt eine Vielzahl an Kriterien zugrunde, die auch im Rahmen wissenschaftlicher Arbeiten nicht umfassend berücksichtigt werden können. In der vorliegenden Studie wurde jedoch versucht, die bedeutendsten Kaufkriterien beim Einkauf von Fleisch- und Wurstwaren aus der wissenschaftlichen Literatur abzuleiten und von den Verbrauchern bewerten zu lassen. Insgesamt 13 Kaufkriterien wurden dabei untersucht. Als Besonderheit wurden nicht nur die klassischen Kaufkriterien (wie z. B. Frische oder der Preis) berücksichtigt, sondern ergänzend zahlreiche ethische Kaufkriterien, da im Rahmen der vorliegenden Studie schwerpunktmäßig die ethischen Aspekte des Fleischkonsums untersucht werden sollten. Die große Anzahl an Kriterien sollte mit Hilfe einer Faktorenanalyse für weiterführende Analysen reduziert werden, um die Komplexität für die statistischen Auswertungen zu reduzieren. Außerdem wurde davon ausgegangen, dass verschiedenen Kaufkriterien ähnliche latente Hintergrundkonstrukte bzw. ähnliche Präferenzen zugrunde liegen.

Die Ergebnisse der Faktorenanalyse sind in Tabelle 26 dargestellt. Demnach weisen sowohl der Bartlett-Test (Chi-Quadrat $(78)=3786,925, \mathrm{p}<.001$ ) als auch das KaiserMeyer-Olkin Measure of Sampling Adequacy $(\mathrm{KMO}=.88)$ darauf hin, dass sich die Variablen für eine Faktorenanalyse sehr gut eignen (UNIVERSITÄT ZÜRICH, 2020). Es wurde eine Hauptkomponentenanalyse mit Varimax-Rotation durchgeführt, die eine Drei-Faktor-Lösung ergab, die 57,58 \% der Gesamtvarianz erklärt. Tabelle 26 enthält die Ergebnisse der Faktorenanalyse und stellt die entsprechenden Mittelwerte, Standardabweichungen und Faktorladungen der verschiedenen Variablen bzw. Faktoren sowie die Kennzahlen des Gesamtmodells und die Ergebnisse der Reliabilitätsanalyse als Cronbachs Alpha (CA) dar. 
Auf Faktor 1 laden die Kaufmotive Umweltfreundlichkeit, Gütesiegel, faire Löhne, Gesundheitsaspekte, artgerechtere Tierhaltung, Bio-Qualität und Regionalität. Diese Kriterien sind allesamt eng mit einem Bewusstsein für nachhaltiges Handeln verknüpft. Umweltfreundlichkeit und eine artgerechtere Tierhaltung laden am stärksten auf diesen Faktor. Bereits die Mittelwertberechnungen der einzelnen Variablen (Abbildung 9) ergaben, dass die Umweltfreundlichkeit der Produkte und eine artgerechtere Tierhaltung neben der Regionalität auch insgesamt die bedeutendsten Kaufkriterien für deutsche Verbraucher im Hinblick auf nachhaltiges Handeln darstellen. Dieser Faktor soll im weiteren Verlauf der Arbeit als ,Nachhaltigkeitsbewusstsein‘ bezeichnet werden.

Auf Faktor 2 laden die Kaufkriterien Aussehen der Ware, Geschmack (aus Erfahrung) sowie Frische der Ware; allesamt Punkte, die für die meisten Verbraucher wesentliche Kaufkriterien sind, wie die hohen Mittelwerte der Items zeigen. Diese drei Kriterien können daher als Grundnutzen betrachtet werden, der bei allen Produkten generell gegeben sein muss, während die Nachhaltigkeitsmotive als Zusatznutzen betrachtet werden können, dem mittlerweile immer mehr Verbraucher einen hohen Stellenwert beimessen. Diese Kriterien werden im weiteren Verlauf der Arbeit als ,Klassische Kaufkriterien` bezeichnet, da sie für alle Verbraucher traditionell eine hohe Bedeutung besitzen.

Die Kaufkriterien Marken, Sonderangebot/günstigster Preis und ansprechende Verpackung laden zusammen auf den Faktor 3 hoch und können als ,Äußere Merkmale“ bezeichnet werden, die Verbraucher direkt beim Kauf sehen können und damit klassische Sucheigenschaften darstellen. Im Gegensatz dazu stellen die Kriterien des Faktors 1, wie bspw. eine artgerechtere Tierhaltung, Vertrauenseigenschaften dar, falls kein seriöses Tierwohl-Label zur Kennzeichnung der Produkte verwendet wird. 
Tabelle 26: Ergebnisse der Faktorenanalyse zu Kaufkriterien beim Fleischkauf

\begin{tabular}{|c|c|c|}
\hline Faktoren und Items & MW (SD) & Faktorladung \\
\hline \multicolumn{3}{|l|}{$\mathrm{KMO}=\mathbf{0 , 8 8}$} \\
\hline Faktor 1: Nachhaltigkeitsbewusstsein $(C A=0,88)$ & $3,75(5,29)$ & \\
\hline F8: Kaufkriterium - Umweltfreundlichkeit & $0,75(1,06)$ & 0,825 \\
\hline F8: Kaufkriterium - Gütesiegel & $0,57(1,11)$ & 0,758 \\
\hline F8: Kaufkriterium - faire Löhne für Landwirte & $0,21(1,21)$ & 0,769 \\
\hline F8: Kaufkriterium - Gesundheitsaspekte & $0,64(1,10)$ & 0,656 \\
\hline F8: Kaufkriterium - artgerechtere Tierhaltung & $0,9(1,05)$ & 0,806 \\
\hline F8: Kaufkriterium - Bio-Qualität & $0,47(1,11)$ & 0,738 \\
\hline F8: Kaufkriterium - Regionalität & $0,91(1,04)$ & 0,726 \\
\hline Faktor 2: Klassische Qualitätskriterien $(C A=0,62)$ & $3,96(1,15)$ & \\
\hline F8: Kaufkriterium - Aussehen der Ware & $1,74(0,63)$ & 0,73 \\
\hline F8: Kaufkriterium - Geschmack (aus Erfahrung) & $1,64(0,66)$ & 0,726 \\
\hline F8: Kaufkriterium - Frische der Ware & $1,76(0,59)$ & 0,762 \\
\hline Faktor 3: Äußere Merkmale $(C A=0,42)$ & $0,98(1,91)$ & \\
\hline F8: Kaufkriterium - Marken & $0,26(1,23)$ & 0,632 \\
\hline F8: Kaufkriterium - Sonderangebot/günstigster Preis & $0,63(1,12)$ & 0,664 \\
\hline F8: Kaufkriterium - ansprechende Verpackung & $0,32(1,22)$ & 0,668 \\
\hline \multicolumn{3}{|c|}{$\begin{array}{l}\text { Kaiser-Meyer-Olkin-Kriterium (KMO) =0,877, Bartlett-Test auf Sphärizität df }=78 \text {, Signifikanz nach Bartlett } \\
, 000 \text { (Chi2 } 3786,925) \text {, fünfstufige Likert-Skala von }+2 \text { (achte ich fast immer) bis }-2 \text { (achte ich nicht). } 57,58 \% \\
\text { der Gesamtvarianz. Extraktionsmethode: Hauptkomponentenanalyse. Rotationsmethode: Varimax mit } \\
\text { Kaiser-Normalisierung in vier Iterationen konvergiert. CA = Cronbachs Alpha. } N=930 \text {. }\end{array}$} \\
\hline
\end{tabular}

Quelle: Eigene Darstellung

\subsubsection{Zusammenfassung der Ergebnisse der Faktorenanalyse}

Die einzelnen Ergebnisse der Faktorenanalyse auf Basis der Tierwohl-Label-Präferenzen der Verbraucher sollen in diesem Abschnitt in einer Gesamtübersicht dargestellt werden. Die Ergebnisse dienen als Grundlage für die Diskussion der erstellten Tierwohl-LabelTypologie sowie für die Ableitung von Handlungsempfehlungen in Kapitel 6.

Die in Tabelle 27 dargestellten Ergebnisse wurden bereits im Ergebnisteil ausführlich beschrieben. Im Rahmen dieser Zusammenfassung sollen die Ergebnisse der Faktorenanalyse bzw. der Logit-Modelle daher noch einmal aus einer allgemeineren Perspektive betrachtet werden. 
Die drei abgeleiteten ,Tierwohlpräferenzmotivgruppen‘ können als Grundlage für generelle Zielsetzungen der Produktpolitik in drei ,Tierwohlqualitätsstufen“ dienen; je nach Anspruchsniveau der Präferenzgruppe. Im Hinblick auf das Tierwohl stellen die Bio-Labels von DEMETER, BIOLAND, NATURLAND und BIOKREIS sowie das nichtökologische Tierwohl-Label von NEULAND die höchste Stufe dar (Zielsetzung: Premium-Tierwohlprodukte anbieten). Mit einer Premium-Tierwohlstrategie wird die Käufergruppe mit dem höchsten Einkommen erreicht. Darauf folgen an zweiter Stelle die staatlichen Bio-Labels DE-BIO und EU-BIO für Anbieter, die quasi ,MediumTierwohlprodukte' anbieten möchten bzw. eine mittlere Positionierung bei Tierwohlprodukten anstreben.

Ein Mindestmaß an Tierwohl garantieren den Kunden z. B. die reinen Tierwohl-Labels von VIER PFOTEN oder dem DEUTSCHEN TIERSCHUTZBUND (vor allem in der Premiumstufe) und sind Anbietern zu empfehlen, die das Ziel verfolgen, im Hinblick auf Tierwohlprodukte wenig anspruchsvolle Zielgruppen zu bedienen. Die reinen TierwohlLabels können als dritte und unterste Tierwohlqualitätsstufe betrachtet werden. Die konventionellen Fleisch- und Wurstwaren, die im Lebensmitteleinzelhandel häufig mit der Haltungskennzeichnung der INITIATIVE TIERWOHL (Stufe 1 bis Stufe 3) oder der HALTUNGSFORM (Stufe 1 bis Stufe 3) ausgezeichnet sind, können quasi als Referenzkategorie betrachtet werden.

Insgesamt ist die Kaufkraft der Verbraucher hoch mit dem präferierten Tierwohlniveau korreliert. Die einkommensstärksten Zielgruppen erreichen Anbieter von Fleisch- und Wurstwaren mit dem Angebot von Bio-Produkten der ökologischen Anbauverbände; teilweise auch mit staatlichen Bio-Produkten. Lebensmittelherstellern und einzelhändlern bietet sich mit dem Angebot dieser anspruchsvollen Tierwohlprodukte die Chance, überaus attraktive Kundengruppen zu gewinnen. 
Tabelle 27: Kompakte Charakterisierung der Tierwohl-Label-Präferenzgruppen

\begin{tabular}{|c|c|c|c|}
\hline & Reine Tierwohl-Labels & Anbauverband-Bio & Staatlich-Bio \\
\hline & 200 & demeter & B⿺辶一 \\
\hline Soziodemografie & $\begin{array}{l}\text { - vor allem Frauen } \\
\text { - jüngste Gruppe } \\
\text { - eher vom Land } \\
\text { - geringstes Einkommen } \\
\text { - niedrigste Bildung } \\
\text { - konservative Wähler } \\
\text { (CDU, AFD, FDP etc.) }\end{array}$ & $\begin{array}{l}\text { - etwas mehr Frauen } \\
\text { - mittelalt bis älter } \\
\text { - eher aus Städten } \\
\text { - höchstes Einkommen } \\
\text { - höchste Bildung } \\
\text { - grün-linke Wähler }\end{array}$ & $\begin{array}{l}\text { - etwas mehr Frauen } \\
\text { - jung bis mittelalt } \\
\text { - eher aus Städten } \\
\text { - gutes Einkommen } \\
\text { - hohe Bildung } \\
\text { - gemischte Wähler }\end{array}$ \\
\hline $\begin{array}{l}\text { Favorisierte } \\
\text { Einkaufsstätten }\end{array}$ & $\begin{array}{l}\text { LIDL, NETTO, PENNY, } \\
\text { ALDI } \\
\text { REAL, KAUFLAND, } \\
\text { EDEKA }\end{array}$ & $\begin{array}{l}\text { Bio-Supermarkt, } \\
\text { Wochenmarkt, } \\
\text { Hofladen, Metzger, } \\
\text { REWE, EDEKA }\end{array}$ & $\begin{array}{l}\text { Bio-Supermarkt, } \\
\text { ALDI, LIDL, PENNY, } \\
\text { REAL, KAUFLAND, } \\
\text { NETTO }\end{array}$ \\
\hline $\begin{array}{l}\text { Präferierte } \\
\text { Angebotsform }\end{array}$ & SB-Bereich & Theke & Theke/SB rd. 50/50 \\
\hline $\begin{array}{l}\text { Produktplatzierung } \\
\text { Tierwohlprodukte }\end{array}$ & $\begin{array}{l}\text { Eher eigener Bereich } \\
\text { präferiert }\end{array}$ & $\begin{array}{l}\text { Sehr starke Präferenz } \\
\text { für eigenen Bereich }\end{array}$ & $\begin{array}{l}\text { Eher eigener Bereich } \\
\text { präferiert }\end{array}$ \\
\hline $\begin{array}{l}\text { Flexitarier-Anteil } \\
\text { mit Schätzung }\end{array}$ & $\begin{array}{l}\text { Sehr gering } \\
<10 \%\end{array}$ & $\begin{array}{l}\text { Höchster Anteil } \\
>80 \%\end{array}$ & $\begin{array}{l}\text { Mittel } \\
50 \%\end{array}$ \\
\hline $\begin{array}{l}\text { Präferenzen } \\
\text { Multi-Labeling }\end{array}$ & $\begin{array}{l}\text { TIERSCHUTZBUND, } \\
\text { QS, DLG, } \\
\text { HORMONFREI, } \\
\text { GENTECHNIKFREI, } \\
\text { REGIONALFENSTER, } \\
\text { NABU, ÖKOTEST, } \\
\text { WARENTEST, } \\
\text { FAIRTRADE }\end{array}$ & $\begin{array}{l}\text { HORMON-, } \\
\text { ANTIBIOTIKA-, UND } \\
\text { GENTECHNIKFREI, } \\
\text { REGIONALFENSTER, } \\
\text { NGOS wie NABU, } \\
\text { FOODWATCH, } \\
\text { FAIRTRADE, } \\
\text { TIERSCHUTZBUND }\end{array}$ & $\begin{array}{l}\text { QS, DLG, ÖKOTEST, } \\
\text { GENTECHNIKFREI, } \\
\text { FAIRTRADE, } \\
\text { WARENTEST, } \\
\text { HORMONFREI, } \\
\text { REGIONALFENSTER }\end{array}$ \\
\hline $\begin{array}{l}\text { Bedeutsame } \\
\text { Kaufkriterien }\end{array}$ & $\begin{array}{l}\text { Gütesiegel, Tierwohl, } \\
\text { Frische, Marken, Preis, } \\
\text { Verpackung }\end{array}$ & $\begin{array}{l}\text { Bio-Qualität, } \\
\text { Regionalität, Tierwohl, } \\
\text { Umwelt, Gesundheit, } \\
\text { Frische, Gütesiegel, } \\
\text { faire Löhne }\end{array}$ & $\begin{array}{l}\text { Geschmack, Bio- } \\
\text { Qualität, Gütesiegel, } \\
\text { Verpackung, Frische }\end{array}$ \\
\hline
\end{tabular}

Quelle: Eigene Darstellung 


\subsection{Ergebnisse der hierarchischen Clusteranalyse}

Ein weiteres zentrales Ziel der vorliegenden Studie besteht, wie in der Einleitung der Arbeit beschrieben, darin, eine auf Tierwohl-Label-Präferenzen basierende Verbrauchertypologie zu erstellen. Im Rahmen der in Unterkapitel 5.4 dargestellten Faktorenanalysen waren die ,Fans der drei Faktorgruppen' streng genommen keine Personen, sondern akkumulierte Label-Präferenzen. Es kann daher vorkommen, dass z. B. eine Person zwei Label-Präferenzen vereint (gleichzeitig zwei unterschiedliche Vorlieben hat). Durch die Anwendung der hierarchischen Clusteranalyse können dagegen alle befragten Verbraucher konkret einem einzelnen Cluster zugeordnet werden.

In Deutschland werden auf zahlreichen Fleisch- und Wurstwaren unterschiedlichste Tierwohl-Labels in verschiedenen Einkaufsstätten angebracht. Jedoch scheinen die Auswahl der Tierwohl-Labels sowie entsprechende Marketingmaßnahmen wenig strategisch bzw. zielgruppenorientiert zu sein. Aus diesem Grund wurde in der vorliegenden Studie eine hierarchische Clusteranalyse durchgeführt, um Marktsegmente auf Basis der bedeutendsten Tierwohl-Labels zu identifizieren und diese Marktsegmente so gut wie möglich zu charakterisieren und zu quantifizieren. Im Wesentlichen sollen die zentralen Unterschiede zwischen den Marktsegmenten, also den unterschiedlichen Verbrauchergruppen, herausgearbeitet werden.

Herstellern und Händlern von Fleisch- und Wurstwaren soll es damit ermöglicht werden, die möglichst passenden Tierwohl-Labels für ihre Zielgruppe strategischer auszuwählen und den Einsatz von Marketinginstrumenten gezielter an die unterschiedlichen Verbrauchergruppen und deren Bedürfnisse anzupassen. Es soll insgesamt ein tieferer Einblick in die vielfältigen Unterschiede im Konsum- und Kaufverhalten der Verbraucher bei Fleisch- und Wurstwaren in Bezug auf Tierwohl sowie weitere ethische Kaufkriterien ermöglicht werden. Insbesondere die ,Label-Welt‘ der Fleischkäufer soll somit besser durchleuchtet werden. 


\subsubsection{Clusterbildung auf Basis der Tierwohl-Label-Präferenzen}

Clusteranalysen dienen dem Erkennen von Mustern und Strukturen in Datensätzen und werden daher auch als strukturentdeckende Verfahren bezeichnet. Die identifizierten Gruppen ,ähnlicher' Objekte werden dabei als Cluster bezeichnet. Die aktiven Variablen der Clusteranalyse stellen in der vorliegenden Studie die drei auf Basis der LabelPräferenzen (F15) extrahierten Faktoren dar, die im vorherigen Abschnitt ausführlich beschrieben wurden. Aus diesem Grund kann hier von einer motivbasierten Clusteranalyse gesprochen werden, durch die die Erstellung einer Verbrauchertypologie ermöglicht wird, die auf den Tierwohl-Label-Präferenzen basiert.

Insgesamt gingen $\mathrm{n}=930$ Datensätze in die Clusteranalyse ein. Als Clustermethode wurde die Ward-Methode gewählt, da diese im Gegensatz zu partitionierenden Methoden keine Festlegung einer Startpartition erfordert. Damit wird das Risiko, dass bedeutsame Zusammenhänge aufgrund einer suboptimalen Clusteranzahl verloren gehen, minimiert (BOSTANCI, 2011).

Basierend auf dem Dendrogramm und mit Hilfe des Elbow-Kriteriums wurden in SPSS bei der hierarchischen Clusteranalyse zunächst verschiedene Cluster-Anzahlen (von 4 bis 6) eingetragen und die Lösungen näher analysiert. Aufgrund des Trade-Offs zwischen Komplexitätsreduktion und Informationsverlust wurden Lösungen mit weniger als vier sowie mehr als sechs Clustern ausgeschlossen.

Bei der Festlegung von drei Clustern ist der Informationsverlust zu groß. Bei der Festlegung von mehr als sechs Clustern wird dagegen die Komplexität zu hoch. Wesentliche Faktoren bei der Festlegung der Anzahl auf fünf Cluster in der vorliegenden Studie waren zudem die Beschreibbarkeit der Cluster, die theoretische und praktische Plausibilität sowie die Verteilung der Grundgesamtheit $(n=930)$ auf die gebildeten Cluster. Als aktive Variablen wurden die drei extrahierten Faktoren aus den TierwohlLabel-Präferenzen (Unterkapitel 5.4.1) für die Clusteranalyse verwendet. Die ClusterWerte im Datensatz gingen als abhängige Variablen in multinominale Logit-Modelle ein. 
Die gebildeten Cluster können anhand ihrer immanenten Motive beschrieben werden, wozu die Mittelwerte der einzelnen Cluster, die im Cluster-Modell in Tabelle 28 dargestellt sind, interpretiert werden sollen, um ein besseres Verständnis der Cluster zu erhalten. Des Weiteren sind in der Tabelle unter den Mittelwerten der Cluster die jeweiligen Regressionskoeffizienten (B) sowie in Klammern die entsprechende WaldStatistik dargestellt. Im weiteren Verlauf sollen die fünf Cluster anhand der Tabelle 28 zunächst beschrieben werden.

Tabelle 28: Stärke der Einflüsse der Motivfaktoren auf die gefundenen Cluster

\begin{tabular}{|c|c|c|c|c|c|}
\hline & $\begin{array}{c}\text { CLUSTER } \\
1\end{array}$ & $\begin{array}{c}\text { CLUSTER } \\
2\end{array}$ & $\begin{array}{c}\text { CLUSTER } \\
3\end{array}$ & $\begin{array}{c}\text { CLUSTER } \\
4\end{array}$ & $\begin{array}{c}\text { CLUSTER } \\
5\end{array}$ \\
\hline & MW/B/Wald & MW/B/Wald & MW/B/Wald & MW/B/Wald & MW/B/Wald \\
\hline Faktor_1_Tierwohl & $\begin{array}{c}0,92 \\
9,027^{\star \star \star} \\
(94,404)\end{array}$ & $\begin{array}{c}0,55 \\
6,187^{\star \star \star} \\
(80,922)\end{array}$ & $\begin{array}{c}-0,71 \\
-0,72^{*} \\
(4,447)\end{array}$ & $\begin{array}{c}-0,56 \\
0 \\
(0)\end{array}$ & 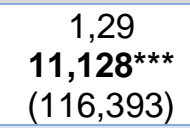 \\
\hline Faktor_2_Anbau-Bio & $\begin{array}{c}0,84 \\
5,478^{\star \star \star} \\
(85,141)\end{array}$ & $\begin{array}{c}-1,19 \\
-4,449^{\star \star \star} \\
(62,075)\end{array}$ & $\begin{array}{c}0,23 \\
1,781^{\star \star *} \\
(30,032)\end{array}$ & $\begin{array}{c}-0,25 \\
0 \\
(0)\end{array}$ & $\begin{array}{c}-0,38 \\
-0,141 \\
(0,062)\end{array}$ \\
\hline Faktor_3_StaatlichBio & $\begin{array}{c}0,16 \\
4,388^{\star \star \star} \\
(58,073)\end{array}$ & $\begin{array}{c}0,59 \\
5,832^{\star \star *} \\
(107,918)\end{array}$ & $\begin{array}{c}0,55 \\
6,488^{\star \star \star} \\
(124,521)\end{array}$ & $\begin{array}{c}-1,29 \\
0 \\
(0)\end{array}$ & $\begin{array}{c}-1,12 \\
-0,662 \\
(1,69)\end{array}$ \\
\hline $\begin{array}{l}\text { Kaufinteresse bei } \\
\text { Tierwohl-Labeln }\end{array}$ & $\begin{array}{l}\text { +staatl. Bio } \\
\text { ++Anbau-Bio } \\
\text { ++reinTW }\end{array}$ & $\begin{array}{l}\text { +staatl. Bio } \\
\text {---Anbau-Bio } \\
\text { +reinTW }\end{array}$ & $\begin{array}{l}\text { +++staatl. Bio } \\
\text { +Anbau-Bio } \\
\text {--reinTW }\end{array}$ & $\begin{array}{l}\text {---staatl . } \\
\text {-Anbau-Bio } \\
\text {--reinTW }\end{array}$ & $\begin{array}{l}\text {-staatl. Bio } \\
\text {-Anbau-Bio } \\
+++ \text { reinTW }\end{array}$ \\
\hline Segmentgröße & $21 \%$ & $15 \%$ & $38 \%$ & $19 \%$ & $8 \%$ \\
\hline Gesamtbevölkerung & 11,45 Mio. & 8,18 Mio. & 20,7 Mio. & 10,36 Mio. & 4,36 Mio. \\
\hline \multicolumn{6}{|c|}{$\begin{array}{l}\text { Darstellung der Mittelwerte der Cluster, Regressionskoeffizienten und Wald-Werte in Klammern. Berechnung mit } \\
\text { multinominalem Logit-Modell. Referenzkategorie: } 4 \text {; ohne Koeffizienten und Wald-Statistik. Datenbasis der Faktoren: } \\
\text { F15 Abfrage zum Kaufinteresse einzelner Tierwohl-Labels mit Likert-Skala }+2 \text { (würde ich auf jeden Fall } \\
\text { berücksichtigen) bis }-2 \text { (würde ich auf keinen Fall berücksichtigen). }{ }^{*}=p<0,05 \text { (Wald-Wert }>3,84 \text { ); }{ }^{* *}=p<0,01 \text { (Wald- } \\
\text { Wert }>6,66) ;{ }^{* * *}=p<0,001 \text { (Wald-Wert }>9,55 \text { ). }\end{array}$} \\
\hline
\end{tabular}

\section{Quelle: Eigene Darstellung}

Im Rahmen der Faktorenanalyse wurden bereits beschrieben, wie sich die Faktoren bilden. Faktor 1 steht für die reinen Tierwohl-Labels des DEUTSCHEN TIERSCHUTZBUNDES, VIER PFOTEN und als zwei Sondervarianten das Kennzeichen der INITIATIVE TIERWOHL sowie des geplanten staatlichen TierwohlLabels. Faktor 2 steht für die Tierwohl-Labels der ökologischen Anbauverbände DEMETER, BIOLAND, NATURLAND sowie das NEULAND-Label. Faktorgruppe 3 bildet sich aus den staatlichen Bio-Labels (DE-BIO und EU-BIO). 
Bei Betrachtung der Mittelwerte in Tabelle 28 fällt auf, dass Cluster 1 aus Verbrauchern besteht, die ungefähr gleichermaßen reine Tierwohl-Labels und auch Tierwohl-Labels der ökologischen Anbauverbände präferieren. Auffällig ist des Weiteren, dass in dieser Gruppe die ökologischen Anbauverbände von allen fünf Clustern am beliebtesten sind. Daneben zeigen sich diese Personen offen gegenüber staatlichen Bio-Produkten. Die Verbraucher des Clusters 1 sind generell Fans von Nachhaltigkeitssiegeln. Alle Vorzeichen sind positiv. Daher kann diese Gruppe als ,Enthusiastische Nachhaltigkeitsfans ' bezeichnet werden.

In Cluster 2 zeigt sich, dass diese Gruppe an Verbrauchern weniger enthusiastisch auf Gütesiegel reagiert als die Personen in Cluster 1. Reine Tierwohl-Labels und staatliche Bio-Labels werden jedoch durchaus als positives Kaufargument beim Einkauf von Fleisch- und Wurstwaren bewertet. Des Weiteren würde diese Gruppe die TierwohlLabels der ökologischen Anbauverbände weniger berücksichtigen. Insgesamt könnte diese Gruppe als ,Unentschlossene Verbraucher' bezeichnet werden, die zwischen reinen Tierwohl-Labels und staatlichen Bio-Produkten schwanken.

Verbraucher, die sowohl Fleisch- und Wurstwaren mit staatlichen Bio-Labels als auch mit Tierwohl-Labels der ökologischen Anbauverbände kaufen würden, finden sich in Cluster 3. Diese Gruppe lehnt jedoch im Gegensatz zu Cluster 1 und 2 reine TierwohlLabels eher ab. Staatliche Bio-Produkte werden von dieser Gruppe etwas mehr präferiert als Fleisch- und Wurstwaren der ökologischen Anbauverbände. Diese Gruppe stellt von allen Clustern die größte Gruppe dar und soll im weiteren Verlauf der Arbeit als , ̈̈berzeugte Bio-Fans ‘ bezeichnet werden.

Relativ pessimistisch bzw. ablehnend gegenüber Tierwohl-Labels sind die Verbraucher des Clusters 4 eingestellt. Keine der drei Tierwohl-Label-Gruppen findet bei diesen Personen wirklich Anklang. Beim Kauf von Fleisch- und Wurstwaren werden TierwohlLabels deutlich weniger berücksichtigt als bei allen anderen Verbrauchergruppen. Dies zeigen die durchweg negativen Vorzeichen aller Mittelwerte des Clusters 4. 
Fleisch- und Wurstwaren, die mit staatlichen Bio-Labels gekennzeichnet sind, möchte diese Gruppe am wenigsten kaufen. Auch an Fleisch- und Wurstwaren, die mit reinen Tierwohl-Labels gekennzeichnet sind, hat diese Gruppe wenig Interesse. Diese Gruppe soll daher als ,Desinteressierte Label-Ablehner' charakterisiert werden und eignet sich am besten als Referenzkategorie für die multinominalen Logit-Modelle, die im nächsten Abschnitt vorgestellt werden. Diese Gruppe stellt rd. $20 \%$ des Gesamtmarkts dar und besteht womöglich überwiegend aus Fleischessern ohne besondere Ansprüche an ethische Aspekte bei Fleisch- und Wurstwaren und hat keinen Bezug zum Thema Nachhaltigkeit.

Das Cluster 5 ist ebenfalls klar zu charakterisieren. Die diesem Cluster zugeordneten Personen präferieren eindeutig die reinen Tierwohl-Labels. Von allen Clustern haben diese Personen die stärkste Präferenz für die reinen Tierwohl-Labels, die keine ökologischen Kriterien beinhalten (DEUTSCHER TIERSCHUTZBUND, VIER PFOTEN, STAATLICHES TIERWOHL-LABEL, HALTUNGSKOMPASS (LIDL) und INITIATIVE TIERWOHL).

Womöglich handelt es sich hierbei um Verbraucher, die sich in jedem Fall mehr Tierwohl wünschen, aber nicht wissen, dass die staatlichen Bio-Labels und die Bio-Labels der ökologischen Anbauverbände die strengsten Tierwohl-Labels in Deutschland sind. Diese Gruppe könnte Bio-Produkte auch aufgrund eines generell geringen Vertrauens in die Bio-Branche ablehnen, weshalb sie als die Gruppe ,Tierwohl ja - Bio nein“ charakterisiert werden kann. Sie stellt jedoch mit nur $8 \%$ das kleinste aller Marktsegmente dar.

In Tabelle 29 ist die Zusammensetzung der einzelnen Cluster dargestellt, die als Segmentgröße oder potenzieller Marktanteil angesehen werden können. Demnach verteilt sich die Grundgesamtheit $(n=930)$ relativ ausgewogen auf die gebildeten Cluster. Das größte Cluster bilden die ,Überzeugten Bio-Fans“ (37,5 \%; Cluster 3), gefolgt von den ,Enthusiastischen Nachhaltigkeitsfans' (rd. $20 \%$; Cluster 1). Diese beiden Gruppen können als Kernzielgruppe von Tierwohlprodukten betrachtet werden und machen rd. $60 \%$ der befragten Verbraucher aus, die eine hohe Affinität zu den Themen Nachhaltigkeit und Tierwohl auszeichnet. 
Cluster 2 (,Unentschlossene Verbraucher") und Cluster 5 (Gruppe, Tierwohl ja - Bio nein') stehen Tierwohl-Labels nicht so positiv gegenüber wie die Kernzielgruppe, sind jedoch generell auch offen gegenüber Tierwohl-Labels eingestellt. Diese beiden Gruppen erreichen zusammen einen Anteil von ungefähr einem Viertel (rd. 23\%) aller Studienteilnehmer. Rund $20 \%$ der befragten Verbraucher lehnen generell alle Arten an Tierwohl-Labels ab (Cluster 4; ,Desinteressierte Label-Ablehner').

Tabelle 29: Zusammensetzung der Cluster

\begin{tabular}{|l|l|l|l|}
\hline Cluster & N & \multicolumn{2}{l|}{ Kumulierte \% } \\
\hline 1 - Enthusiastische Nachhaltigkeitsfans & 192 & 20,6 & 20,6 \\
\hline 2 - Unentschlossene Verbraucher & 143 & 15,4 & 36 \\
\hline 3 - Überzeugte Bio-Fans & 349 & 37,5 & 73,5 \\
\hline 4 - Desinteressierte Label-Ablehner & 176 & 18,9 & 92,5 \\
\hline 5 - Gruppe ,Tierwohl ja - Bio nein` & 70 & 7,5 & 100 \\
\hline Gesamt & $\mathbf{9 3 0}$ & & \\
\hline
\end{tabular}

Quelle: Eigene Darstellung

\subsubsection{Soziodemografische Unterschiede zwischen den Clustern}

Vor dem Hintergrund der Gestaltung eines möglichst zielgruppenorientierten Angebots an Fleisch- und Wurstwaren aus einer artgerechteren und nachhaltigeren Nutztierhaltung ist eine möglichst konkrete Kenntnis der (potenziellen) Kunden erforderlich. Je besser ein Anbieter von Fleisch- und Wurstwaren seine Kunden kennt, desto gezielter kann das Produktangebot an die Kundenbedürfnisse angepasst werden und desto gezielter können die passenden Marketinginstrumente eingesetzt werden.

$\mathrm{Zu}$ den grundlegenden Kenntnissen zählt die Soziodemografie der Zielgruppe. In diesem Abschnitt sollen daher zunächst die fünf Cluster mit Hilfe soziodemografischer Daten analysiert werden. Zum besseren Verständnis der Analysen soll zuvor noch kurz auf die methodische Vorgehensweise bei den Analysen eingegangen werden. 
Die Beschreibung der fünf Cluster basierte ausschließlich auf den Variablen, die in die Clusteranalyse integriert wurden bzw. mit deren Hilfe die Cluster gebildet worden sind: den drei mittels Faktorenanalyse ermittelten Tierwohl-Label-Gruppen. Um die Cluster näher zu beschreiben, wurde die multinominale logistische Regressionsanalyse angewendet. Mit Hilfe multinominaler Logit-Modelle lässt sich feststellen, mit welcher Wahrscheinlichkeit eine Person einem konkreten Cluster zugeordnet werden kann. Darüber hinaus kann die Stärke ermittelt werden, mit der die unabhängigen passiven Variablen (Kovariaten) diese Wahrscheinlichkeit beeinflussen. Der Regressionskoeffizient B wird dabei hinsichtlich seiner Wirkungsrichtung anhand des Vorzeichens interpretiert und die Unterschiede zwischen den Clustern werden mit Hilfe der Wald-Statistik ermittelt, wobei der Wald-Wert dem quadrierten t-Wert entspricht.

Bei der Durchführung der multinominalen logistischen Regression wurde das Cluster 4 der Desinteressierten Label-Ablehner als Referenzkategorie festgelegt, da sich dieses Cluster am deutlichsten von allen anderen Clustern unterscheidet. Außerdem werden die Beschreibung und die Interpretation der Regressionskoeffizienten durch die negativen Vorzeichen aller Mittelwerte in Cluster 4 erleichtert. Das Referenzcluster 4 wurde bei allen multinominalen Logit-Modellen beibehalten. Die Interpretation der Ergebnisse bezieht sich daher vor allem auf die signifikanten Unterschiede der gebildeten Cluster zum Referenzcluster der Desinteressierten Label-Ablehner.

Daneben werden die Cluster jedoch auch anhand der Höhe und Vorzeichen der Regressionskoeffizienten verglichen. Dahingehend muss bedacht werden, dass hierbei keine Prüfung auf signifikante Unterschiede erfolgt. Alle statistisch abgesicherten Signifikanzen, die mit Wald-Werten und Sternchen an den Koeffizienten angegeben sind, beziehen sich auf das Referenzcluster. Für die Anwendung der multinominalen logistischen Regression wurden einige kategoriale Variablen vorab in Klassen eingeteilt bzw. verdichtet und dichotomisiert, um zwei Ausprägungen zu erhalten (ja/nein bzw. 1/0). Durch die Dichotomisierung wird eine Zerlegung von Mehrfachantworten in einen binären Code ermöglicht: sogenannte Dummy-Variablen. 
Diese Variablen werden anschließend für die Regressionsmodelle verwendet, wobei je eine Dummy-Variable nicht in das Regressionsmodell eingehen darf, da sie den einfließenden Dummy-Variablen als Referenzkategorie dient.

Daneben muss darauf hingewiesen werden, dass bei der folgenden Ergebnisdarstellung der multinominalen Logit-Modelle ausschließlich signifikante Ergebnisse $(\mathrm{p}<0,05)$ berücksichtigt werden, um nur statistisch abgesicherte Unterschiede zwischen den Clustern aufzuzeigen. Zudem wurden gezielt Themenblöcke gebildet, um Redundanzen bei den Analysen zu vermeiden.

Der Zielsetzung der Arbeit folgend sind dies soziodemografische, vertriebsrelevante und marketingrelevante Unterschiede zwischen den Clustern. Des Weiteren wird der Einsatz verschiedener Gütesiegel als Ergänzung zu den Tierwohl-Labels je Cluster untersucht, da das Thema Multi-Labeling im deutschen Lebensmitteleinzelhandel eine immer größere Rolle spielt. Zunächst werden die soziodemografischen Unterschiede zwischen den Clustern vorgestellt.

Tabelle 30 zeigt die signifikanten Unterschiede zwischen den Clustern in Bezug auf die ausgewählten soziodemografischen Variablen Geschlecht, Alter, Ortsgröße, Einkommen und Bildung. Wie in der Tabelle ersichtlich sind nur vier von insgesamt fünf Clustern dargestellt, da das Cluster 4 der Desinteressierten Label-Ablehner als Referenzkategorie diente.

Letztlich kann für dieses Cluster eine weitere Spalte hinzugedacht werden, in der alle Elemente den Wert 0 aufweisen. Die Wald-Statistik ist unter den Regressionskoeffizienten in Klammern angegeben und entsprechend gekennzeichnete Signifikanzen in der Tabelle beziehen sich immer auf das Referenzcluster 4. 
Tabelle 30: Soziodemografische Charakterisierung der Cluster

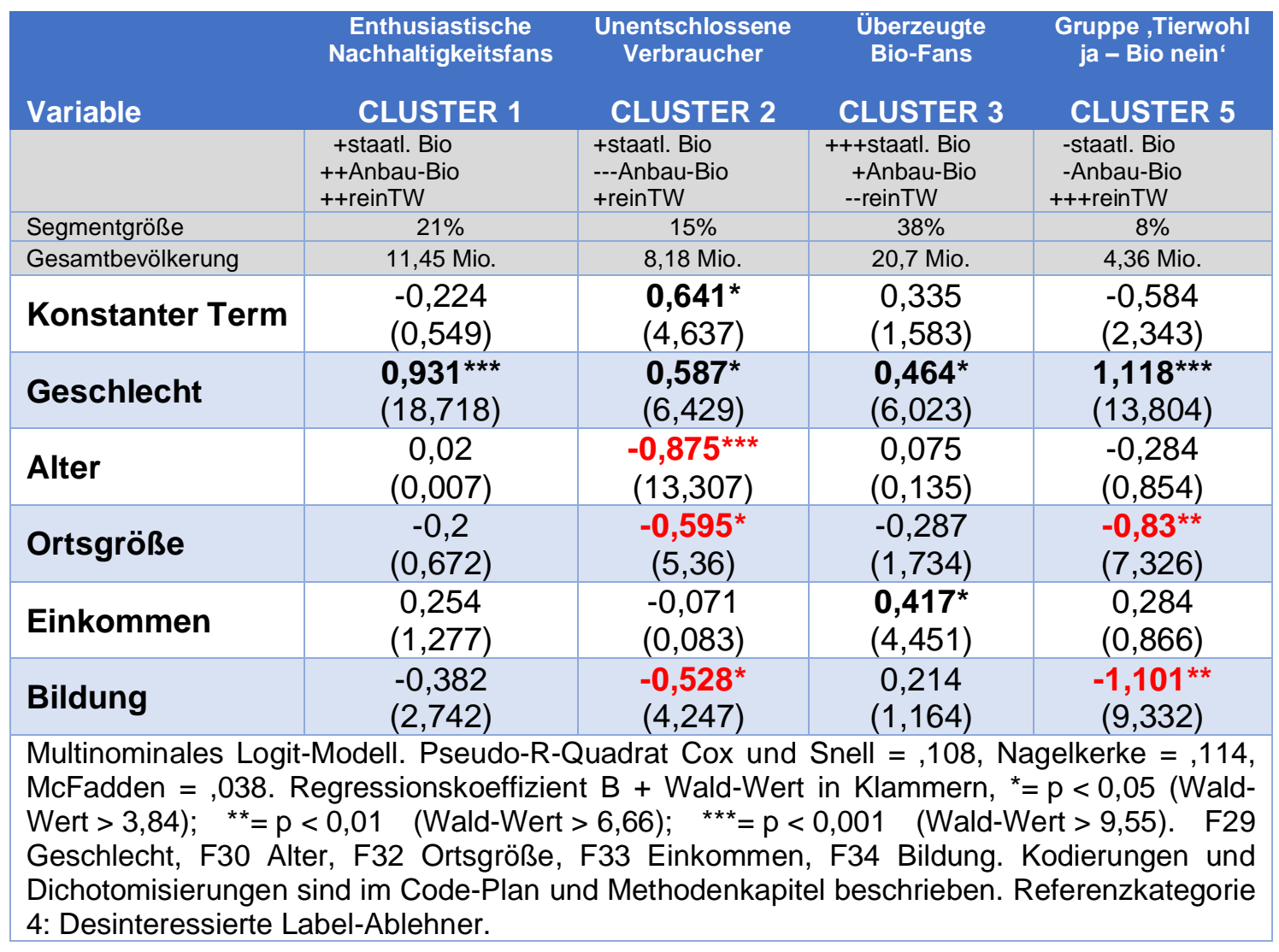

\section{Quelle: Eigene Darstellung}

In Cluster 1, das aus den ,Enthusiastischen Nachhaltigkeitsfans ' besteht, finden sich in Bezug auf die Soziodemografie der Personen verhältnismäßig wenig signifikante Unterschiede. Im Hinblick auf das Geschlecht sind in diesem Cluster signifikant mehr Frauen vertreten als im Referenzcluster 4 der ,Desinteressierten Label-Ablehner`. Mit Blick auf die Regressionskoeffizienten zeigt sich zudem, dass der Anteil der Frauen deutlich höher ist als bei den ,Unentschlossenen Verbrauchern' in Cluster 2 und den ,Überzeugten Bio-Fans` in Cluster 3. Der Frauenanteil in Cluster 1 ist ähnlich hoch wie in Cluster 5 (Gruppe, Tierwohl ja - Bio nein'), das ebenfalls einen hohen Frauenanteil aufweist. Die beiden Cluster 1 und 5 unterscheiden sich vom Referenzcluster mit den höchsten Wald-Werten. Im Referenzcluster ist demzufolge der Männeranteil besonders hoch. 
Bezüglich des Alters lässt sich anhand des Regressionskoeffizienten feststellen, dass die ,Enthusiastischen Nachhaltigkeitsfans “ in Cluster 1 eher älter sind als die ,Unentschlossenen Verbraucher' und die Gruppe ,Tierwohl ja - Bio nein` in Cluster 5. Die Nachhaltigkeitsfans unterscheiden sich altersmäßig jedoch nur unwesentlich von den ,Überzeugten Bio-Fans` in Cluster 3. Des Weiteren leben die ,Enthusiastischen Nachhaltigkeitsfans، am ehesten in größeren Städten und verfügen über ein gutes Einkommen, das etwa dem Einkommen der Gruppe ,Tierwohl ja - Bio nein“ (Cluster 5) entspricht. Das Einkommen der Personen in Cluster 1 ist jedoch deutlich niedriger als das Einkommen der ,Überzeugten Bio-Fans‘. Im Vergleich zu den ,Unentschlossenen Verbrauchern“ (Cluster 2) und vor allem im Vergleich zur Gruppe, Tierwohl ja - Bio nein“ (Cluster 5) weisen die ,Enthusiastischen Nachhaltigkeitsfans' in Cluster 1 eine höhere Bildung auf, auch wenn diese niedriger ist als die Bildung der ,Überzeugten BioFans'.

Die ,Unentschlossenen Verbraucher" unterscheiden sich dagegen gleich in mehreren Punkten vom Referenzcluster der ,Desinteressierten Label-Ablehner'. Signifikante Unterschiede konnten bzgl. des Geschlechts, des Alters, der Ortsgröße und der Bildung festgestellt werden. So weisen die ,Unentschlossenen Verbraucher' einen signifikant höheren Anteil an Frauen als das Referenzcluster auf. Der Männeranteil in dieser Gruppe ist jedoch höher als bei den ,Enthusiastischen Nachhaltigkeitsfans ‘ und der Gruppe ,Tierwohl ja - Bio nein“ in Cluster 5. Besonders auffällig ist, dass die ,Unentschlossenen Verbraucher" von allen Clustern mit Abstand die jüngste Gruppe darstellen, die sich in diesem Punkt hoch signifikant vom Referenzcluster unterscheidet. Außerdem lebt diese Zielgruppe signifikant häufiger auf dem Land oder in Kleinstädten als die Personen im Referenzcluster. Der niedrige Koeffizient zeigt zudem, dass die Personen in Cluster 2 über das geringste Einkommen von allen Segmenten verfügen, was aufgrund des jungen Alters plausibel erscheint. Hinsichtlich des Einkommens unterscheidet sich das Cluster jedoch nicht signifikant vom Referenzcluster. Darüber hinaus unterscheiden sich die ,Unentschlossenen Verbraucher' in Cluster 2 bzgl. ihrer Bildung signifikant vom Referenzcluster. Sie verfügen über eine eher niedrige Bildung. Lediglich die Verbraucher in Cluster 5 weisen eine noch niedrigere Bildung auf. 
Die Gruppe der ,Überzeugten Bio-Fans ' in Cluster 3 besteht ebenso wie die anderen Cluster aus signifikant mehr Frauen als das Referenzcluster der ,Desinteressierten LabelAblehner", auch wenn der Unterschied zum Referenzcluster nicht so hoch ist wie bei Cluster 1 und Cluster 5. Ähnlich wie bei den ,Enthusiastischen Nachhaltigkeitsfans“ gehören zu den Bio-Fans mehr ältere Personen. Hinsichtlich der Ortsgröße lässt sich konstatieren, dass die, Überzeugten Bio-Fans' häufiger in Städten leben als die ,Desinteressierten Label-Ablehner‘. Ein signifikanteres Merkmal dieser Zielgruppe ist jedoch das Einkommen. Die Gruppe ,Überzeugte Bio-Fans‘ verfügt mit Abstand über das höchste Einkommen aller betrachteten Cluster und unterscheidet sich in diesem Punkt signifikant vom Referenzcluster. Das hohe Einkommen liegt womöglich in der sehr hohen Bildung dieser Personen begründet. Bei Betrachtung der Koeffizienten wird deutlich, dass die ,Überzeugten Bio-Käufer` eine deutlich höhere Bildung haben als die ,Unentschlossenen Verbraucher', die ,Enthusiastischen Nachhaltigkeitsfans` und vor allem die Gruppe ,Tierwohl ja - Bio nein` in Cluster 5. Die ,Überzeugten Bio-Käufer‘ unterscheiden sich hinsichtlich Bildung jedoch nicht signifikant vom Referenzcluster.

Die Gruppe ,Tierwohl ja - Bio nein' (Cluster 5) unterscheidet sich ebenfalls in einigen Punkten hoch signifikant vom Referenzcluster. So besteht beispielsweise ein signifikant höherer Anteil an Frauen als bei den ,Desinteressierten Label-Ablehnern“ im Referenzcluster. Personen dieses Segments sind dem Koeffizienten zufolge deutlich jünger als die Nachhaltigkeitsfans oder die Bio-Fans. Sie leben zudem signifikant häufiger auf dem Land als die Personen im Referenzcluster. Was das Einkommen betrifft, so ist dies dem Koeffizienten zufolge deutlich niedriger als das Einkommen der Bio-Fans, was womöglich vor allem in der niedrigeren Bildung dieser Personen begründet liegt, die sich auch hoch signifikant vom Referenzcluster unterscheidet. Das Einkommen ist jedoch höher als das Einkommen der ,Unentschlossenen Verbraucher“ in Cluster 2. Verbraucher in Cluster 5 geben womöglich deutlich weniger Geld für Lebensmittel aus als die ,Enthusiastischen Nachhaltigkeitsfans ‘ oder die ,Überzeugten Bio-Fans', obwohl sie auch über ein solides Einkommen verfügen und daher nicht aus finanziellen Gründen auf die teureren zertifizierten Fleisch- und Wurstwaren verzichten müssen. 


\subsubsection{Vertriebsrelevante Unterschiede zwischen den Clustern}

Wie in der Einleitung beschrieben besteht eines der zentralen Ziele der vorliegenden Arbeit darin, Vertriebsentscheidungen von Herstellern und Händlern im Hinblick auf den Verkauf von mit Tierwohl-Labels gekennzeichneten Fleisch- und Wurstwaren zu unterstützen. Über welche Vertriebskanäle bestimmte Produkte am besten verkauft werden können, ist eine der zentralen Fragen im Vertrieb. Daher wurde diesem Thema in der Befragung ein besonderer Stellenwert eingeräumt. Es sollte festgestellt werden, welche Tierwohlprodukte sich die unterschiedlichen Verbrauchergruppen wünschen und wo diese Personen Fleisch- und Wurstwaren einkaufen. Für die gebildeten Cluster wurden sowohl besondere Vertriebsformen wie Metzger, Wochenmärkte, Hofläden und Bio-Supermärkte auf Basis von Frage 2 (gelegentliche Nutzung von Einkaufsstätten als Mehrfachnennung) als auch klassische Vertriebsformen wie Supermärkte oder Discounter auf Basis von Frage 3 (Haupteinkaufsstätten als Einfachnennung) mit multinominalen Logit-Modellen untersucht. Für Lebensmitteleinzelhändler und Anbieter von Fleisch- und Wurstwaren ist es von großer Bedeutung, zu wissen, ob Verbraucher zu den Stammkunden zählen, die hauptsächlich in ihrer Einkaufsstätte Fleisch und Wurst einkaufen, oder ob sie dies nur gelegentlich tun und schwerpunktmäßig woanders einkaufen.

Die unterschiedlichen Fragevarianten erforderten eine getrennte Analyse im multinominalen Logit-Modell. Daher wurden zwei Logit-Modelle in SPSS berechnet, deren Ergebnisse in zwei Tabellen (Tabelle 31 und Tabelle 32) dargestellt sind. In beiden Modellen zu den Einkaufsstätten wurde zudem die Wechselbereitschaft der vier Gruppen im Zusammenhang mit ihrer Einkaufsstättennutzung untersucht. Zum einen soll es dadurch möglich werden, die Treue der unterschiedlichen Einkaufstypen zu ihrer Einkaufsstätte besser einzuschätzen. Zum anderen kann damit auch die Wichtigkeit eines Sortiments an Tierwohlprodukten für die Verbrauchergruppen beurteilt werden. Dies ist vor dem Hintergrund der Gewinnung neuer Kunden bzw. der Haltung bestehender Kunden über ein gutes Angebot an Tierwohlprodukten bedeutsam. Die Ergebnisse zu Bio-Supermärkten, Metzger, Wochenmarkt und Hofladen sind in Tabelle 31 dargestellt. 
Tabelle 31: Segmentspezifische Unterschiede nach genutzten Einkaufsstätten I

\begin{tabular}{|c|c|c|c|c|}
\hline \multirow[b]{2}{*}{ Variable } & $\begin{array}{l}\text { Enthusiastische } \\
\text { Nachhaltigkeitsfans }\end{array}$ & $\begin{array}{l}\text { Unentschlossene } \\
\text { Verbraucher }\end{array}$ & $\begin{array}{l}\text { Überzeugte } \\
\text { Bio-Fans }\end{array}$ & \multirow{2}{*}{$\begin{array}{c}\text { Gruppe ,Tierwohl } \\
\text { ja - Bio nein' } \\
\text { CLUSTER } 5\end{array}$} \\
\hline & CLUSTER 1 & CLUSTER 2 & CLUSTER 3 & \\
\hline & $\begin{array}{l}\text { +staatl. Bio } \\
\text { ++Anbau-Bio } \\
\text { ++reinTW }\end{array}$ & $\begin{array}{l}\text { +staatl. Bio } \\
\text {---Anbau-Bio } \\
\text { +reinTW }\end{array}$ & $\begin{array}{l}\text { +++staatl. Bio } \\
\text { +Anbau-Bio } \\
\text {-reinTW }\end{array}$ & $\begin{array}{l}\text {-staatl. Bio } \\
\text {-Anbau-Bio } \\
\text { +++reinTW }\end{array}$ \\
\hline Segmentgröße & $21 \%$ & $15 \%$ & $38 \%$ & $8 \%$ \\
\hline Gesamtbevölkerung & 11,45 Mio. & 8,18 Mio. & 20,7 Mio. & 4,36 Mio. \\
\hline Konstanter Term & $\begin{array}{l}-0,9^{*} \\
(5,555)\end{array}$ & $\begin{array}{l}-0,363 \\
(1,187)\end{array}$ & $\begin{array}{l}-0,003 \\
(0)\end{array}$ & $\begin{array}{l}-1,773^{\star \star \star} \\
(10,399)\end{array}$ \\
\hline $\begin{array}{l}\text { Bio- } \\
\text { Supermärkte }\end{array}$ & $\begin{array}{l}\mathbf{0 , 9 6 3}^{*} \\
(4,123)\end{array}$ & $\begin{array}{l}0,312 \\
(0,27)\end{array}$ & $\begin{array}{l}0,856 \\
(3,497)\end{array}$ & $\begin{array}{l}-1,1 \\
(0,998)\end{array}$ \\
\hline Metzger & $\begin{array}{r}-0,287 \\
(0,952)\end{array}$ & $\begin{array}{r}-0,104 \\
(0,103)\end{array}$ & $\begin{array}{l}0,13 \\
(0,242)\end{array}$ & $\begin{array}{c}-0,14 \\
(0,122)\end{array}$ \\
\hline Wochenmarkt & $\begin{array}{c}-0,15 \\
(0,157)\end{array}$ & $\begin{array}{l}-1,159^{*} \\
(4,419)\end{array}$ & $\begin{array}{r}-0,515 \\
(2,088)\end{array}$ & $\begin{array}{r}-0,626 \\
(1,047)\end{array}$ \\
\hline Hofladen & $\begin{array}{l}0,054 \\
(0,017)\end{array}$ & $\begin{array}{r}-0,901 \\
(2,174)\end{array}$ & $\begin{array}{l}0,314 \\
(0,71)\end{array}$ & $\begin{array}{r}-0,492 \\
(0,505)\end{array}$ \\
\hline $\begin{array}{l}\text { Wechsel- } \\
\text { bereitschaft }\end{array}$ & $\begin{array}{l}1,406^{\star \star *} \\
(12,323)\end{array}$ & $\begin{array}{l}0,522 \\
(2,102)\end{array}$ & $\begin{array}{l}\mathbf{0 , 7 6 1 ^ { * }} \\
(6,188)\end{array}$ & $\begin{array}{l}1,389^{*} \\
(5,923)\end{array}$ \\
\hline \multicolumn{5}{|c|}{$\begin{array}{l}\text { Multinominales Logit-Modell. Pseudo-R-Quadrat Cox und Snell }=, 086, \text { Nagelkerke }=, 091 \text {, } \\
\text { McFadden }=, 030 \text {. Regressionskoeffizient } \mathrm{B}+\text { Wald-Wert in Klammern, }{ }^{*}=p<0,05 \text { (Wald- } \\
\left.\text { Wert }>3,84 \text { ); }{ }^{* *}=p<0,01 \quad \text { (Wald-Wert }>6,66\right) ; \quad{ }^{* * *}=p<0,001 \quad \text { (Wald-Wert }>9,55 \text { ). } \\
\text { Referenzkategorie 4: Desinteressierte Label-Ablehner. F2 und F21. Bio-Supermärkte = } \\
\text { ALNATURA, DENN`S, BASIC BIO, SUPER BIO, BIO COMPANY. }\end{array}$} \\
\hline
\end{tabular}

\section{Quelle: Eigene Darstellung}

Anhand von Tabelle 31 lässt sich erkennen, dass zwischen den untersuchten Clustern einige interessante Unterschiede bei diesen Einkaufsstätten vorliegen. Die ,Enthusiastischen Nachhaltigkeitsfans ' unterscheiden sich vom Referenzcluster signifikant hinsichtlich der Nutzung von Bio-Supermärkten und hinsichtlich ihrer Wechselbereitschaft.

Sie besuchen Bio-Supermärkte deutlich häufiger als die ,Desinteressierten Verbraucher des Clusters 4. Sie haben zudem eine deutlich höhere Bereitschaft, ihre Einkaufsstätte zu wechseln, falls in einer anderen Einkaufsstätte ein artgerechter und nachhaltiger gestaltetes Sortiment an Fleisch- und Wurstwaren angeboten wird. 
Die ,Unentschlossenen Verbraucher' dagegen unterscheiden sich vom Referenzcluster nur in einem Punkt signifikant: Sie kaufen signifikant seltener Fleisch- und Wurstwaren auf dem Wochenmarkt als die ,Desinteressierten Verbraucher'. Werden die weiteren Regressionskoeffizienten näher betrachtet, so nutzen diese Personen im Vergleich zu allen anderen Verbrauchergruppen am seltensten Wochenmärkte und Hofläden für den Fleischkauf. Bio-Supermärkte werden von dieser Gruppe ebenfalls seltener als Einkaufsstätte genutzt. Die Wechselbereitschaft ist in dieser Gruppe zudem am niedrigsten von allen Clustern. Dies bedeutet, dass diesen Verbrauchern ein breites Sortiment an Tierwohlprodukten weniger wichtig ist und sie nur schwer von Wettbewerbern, die ein besseres Angebot an Tierwohlprodukten haben, ,abgeworben` werden können.

Die Gruppe der, ̈̈̈berzeugten Bio-Fans ‘ unterscheidet sich in einem Punkt signifikant vom Referenzcluster, und zwar bzgl. der Wechselbereitschaft. Diese ist bei den Bio-Fans deutlich stärker ausgeprägt als bei den ,Desinteressierten Label-Ablehnern“ im Referenzcluster 4. Lediglich die Nachhaltigkeitsfans (Cluster 1) und die Tierwohl-Fans (Cluster 5) zeigen eine noch höhere Wechselbereitschaft. Die übrigen Koeffizienten betrachtend lässt sich feststellen, dass die Bio-Fans neben den Nachhaltigkeitsfans von allen Gruppen Bio-Supermärkte am häufigsten nutzen, was plausibel erscheint. Diese Gruppe präferiert auch am meisten den Metzger und Hofläden als Einkaufsstätte für Fleisch- und Wurstwaren.

Die Fraktion, Tierwohl ja - Bio nein ' hebt sich vom Referenzcluster ebenfalls durch eine sehr hohe Wechselbereitschaft ab. Demzufolge können diese Verbraucher mit einem verbesserten Angebot an Fleisch- und Wurstwaren aus einer artgerechteren Nutztierhaltung ebenso wie die Nachhaltigkeitsfans durchaus zu einem Wechsel ihrer Einkaufsstätte motiviert werden, jedoch weniger mit Bio-Produkten. Auffällig ist des Weiteren, dass diese Gruppe Bio-Supermärkte am wenigsten nutzt, was aufgrund der klaren Ablehnung von Bio-Produkten plausibel erscheint. Wochenmärkte und Hofläden gehören ebenfalls nicht zu den favorisierten Einkaufsstätten dieser Gruppe. 
Tabelle 32: Segmentspezifische Unterschiede nach genutzten Einkaufsstätten II

\begin{tabular}{|c|c|c|c|c|}
\hline & $\begin{array}{l}\text { Enthusiastische } \\
\text { Nachhaltigkeitsfans }\end{array}$ & $\begin{array}{l}\text { Unentschlossene } \\
\text { Verbraucher }\end{array}$ & $\begin{array}{l}\text { Überzeugte } \\
\text { Bio-Fans }\end{array}$ & $\begin{array}{l}\text { Gruppe ,Tierwohl } \\
\text { ja-Bio nein' }\end{array}$ \\
\hline \multirow[t]{2}{*}{ Variable } & CLUSTER 1 & CLUSTER 2 & CLUSTER 3 & CLUSTER 5 \\
\hline & $\begin{array}{l}\text { +staatl. Bio } \\
\text { ++Anbau-Bio } \\
\text { ++reinTW }\end{array}$ & $\begin{array}{l}\text { +staatl. Bio } \\
\text {---Anbau-Bio } \\
\text { +reinTW }\end{array}$ & $\begin{array}{l}\text { +++staatl. Bio } \\
\text { +Anbau-Bio } \\
\text {-reinTW }\end{array}$ & $\begin{array}{l}\text {-staatl. Bio } \\
\text {-Anbau-Bio } \\
+++ \text { reinTW }\end{array}$ \\
\hline Segmentgröße & $21 \%$ & $15 \%$ & $38 \%$ & $8 \%$ \\
\hline Gesamtbevölkerung & 11,45 Mio. & 8,18 Miо. & 20,7 Mio. & 4,36 Mio. \\
\hline Konstanter Term & $\begin{array}{l}-1,221^{\star *} \\
(8,905)\end{array}$ & $\begin{array}{l}-1,172^{\star *} \\
(8,409)\end{array}$ & $\begin{array}{l}-0,033 \\
(0,012)\end{array}$ & $\begin{array}{l}-2,197^{\star \star *} \\
(13,726)\end{array}$ \\
\hline EDEKA & $\begin{array}{l}0,187 \\
(0,26)\end{array}$ & $\begin{array}{l}0,025 \\
(0,003)\end{array}$ & $\begin{array}{l}-0,253 \\
(0,568)\end{array}$ & $\begin{array}{l}0,374 \\
(0,585)\end{array}$ \\
\hline REWE & $\begin{array}{l}0,486 \\
(1,459)\end{array}$ & $\begin{array}{l}\mathbf{1 , 0 4 8}^{*} \\
(4,993)\end{array}$ & $\begin{array}{l}0,199 \\
(0,287)\end{array}$ & $\begin{array}{l}-0,351 \\
(0,284)\end{array}$ \\
\hline ALDI & $\begin{array}{l}1,105 \\
(3,815)\end{array}$ & $\begin{array}{l}1,565^{*} \\
(6,172)\end{array}$ & $\begin{array}{l}0,564 \\
(1,056)\end{array}$ & $\begin{array}{l}0,766 \\
(1,034)\end{array}$ \\
\hline LIDL & $\begin{array}{l}0,606 \\
(1,456)\end{array}$ & $\begin{array}{l}\mathbf{1}, \mathbf{8} 7^{\star \star \star} \\
(13,113)\end{array}$ & $\begin{array}{l}0,216 \\
(0,21)\end{array}$ & $\begin{array}{l}0,1 \\
(0,017)\end{array}$ \\
\hline NETTO & $\begin{array}{l}-1,097 \\
(2,306)\end{array}$ & $\begin{array}{l}0,74 \\
(1,499)\end{array}$ & $\begin{array}{l}-0,597 \\
(1,261)\end{array}$ & $\begin{array}{l}0,299 \\
(0,181)\end{array}$ \\
\hline SB-Warenhäuser & $\begin{array}{l}0,599 \\
(1,39)\end{array}$ & $\begin{array}{l}\mathbf{1 , 1 4 8}^{*} \\
(4,135)\end{array}$ & $\begin{array}{l}0,646 \\
(2,037)\end{array}$ & $\begin{array}{l}1,139 \\
(3,541)\end{array}$ \\
\hline $\begin{array}{l}\text { Wechsel- } \\
\text { bereitschaft }\end{array}$ & 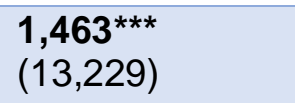 & $\begin{array}{l}0,345 \\
(0,868)\end{array}$ & $\begin{array}{l}\mathbf{0 , 8 4 ^ { \star \star }} \\
(7,467)\end{array}$ & 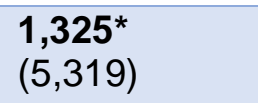 \\
\hline $\begin{array}{l}\text { Multinominales Logi } \\
\text { McFadden }=, 036 . R \\
\text { Wert }>3,84) ;{ }^{* *}=p< \\
\text { SB-Warenhäuser }= \\
\text { Ablehner. }\end{array}$ & $\begin{array}{l}\text { dell. Pseudo-R } \\
\text { essionskoeffizie } \\
1 \text { (Wald-Wert > } \\
\text { UFLAND und }\end{array}$ & $\begin{array}{l}\text { drat Cox un } \\
+ \text { Wald-We } \\
;{ }^{* * *}=p<0,0 \\
\text { Referenzk }\end{array}$ & $\begin{array}{l}\text { ll }=, 101, \uparrow \\
\text { Klammern, } \\
\text { Vald-Wert > } \\
\text { rie 4: Desil }\end{array}$ & $\begin{array}{l}\text { elkerke }=, 107, \\
p<0,05 \text { (Wald- } \\
55 \text { ). F3 und F21. } \\
\text { ressierte Label- }\end{array}$ \\
\hline
\end{tabular}

\section{Quelle: Eigene Darstellung}

Bezüglich der Einkaufsstättennutzung der verschiedenen Verbrauchergruppen bzgl. Supermärkten, Discountern und SB-Warenhäusern, die in Tabelle 32 dargestellt ist, wird deutlich, dass die meisten signifikanten Unterschiede zum Referenzcluster in der Gruppe der ,Unentschlossenen Verbraucher' vorliegen. Diese Gruppe unterscheidet sich vom Referenzcluster vor allem signifikant dadurch, dass sie ALDI, LIDL und SBWarenhäuser (KAUFLAND und REAL) häufiger nutzt. Auch REWE wird signifikant häufiger genutzt. Werden die Koeffizienten im Vergleich $\mathrm{zu}$ den anderen Clustern betrachtet, nutzt diese Gruppe auch am meisten NETTO. Es kann festgestellt werden, dass dieses Cluster überwiegend aus Discounter-Käufern und SB-Warenhaus-Käufern besteht. 
Die Wechselbereitschaft ist bei dieser Gruppe am niedrigsten, was bedeutet, dass dies womöglich die treueste Kundengruppe darstellt. Diese Erkenntnis konnte auch bei der Analyse der Wechselbereitschaft hinsichtlich der Vertriebsformen Metzger, Wochenmärkte, Hofläden und Bio-Supermärkte in Tabelle 31 gewonnen werden.

Die Gruppe der ,Enthusiastischen Nachhaltigkeitsfans ' unterscheidet sich lediglich hinsichtlich der Wechselbereitschaft vom Referenzcluster, die deutlich höher ist als die der ,Desinteressierten Verbraucher‘. Diese Gruppe weist die höchste Wechselbereitschaft von allen Kundengruppen auf. Daraus kann geschlossen werden, dass die Nachhaltigkeitsfans durch ein artgerechteres und nachhaltigeres Produktsortiment an Fleisch- und Wurstwaren am ehesten als neue Kunden zu gewinnen sind. Im Vergleich der Koeffizienten zwischen den Clustern lässt sich ferner erkennen, dass bei dieser Gruppe EDEKA und REWE beliebte Einkaufsstätten sind. NETTO und SB-Warenhäuser nutzt diese Gruppe dagegen am wenigsten als Einkaufsstätte für Fleisch- und Wurstwaren. Überraschenderweise ist ALDI dagegen bei dieser Gruppe relativ beliebt.

Die ,Überzeugten Bio-Fans' zeichnen sich ebenfalls durch eine höhere Wechselbereitschaft als die ,Desinteressierten Verbraucher' (Referenzcluster) aus, was die hohe Bedeutung eines guten Tierwohlsortiments für diese Gruppe verdeutlicht. Werden die Koeffizienten zwischen den Clustern verglichen, zeigt sich, dass diese Gruppe eher weniger SB-Warenhäuser (KAUFLAND, REAL) und Discounter (ALDI, LIDL, NETTO) nutzt. Von den drei Discountern wird ALDI von Bio-Käufern am häufigsten genutzt, während NETTO am seltensten von Bio-Käufern für den Fleischkauf besucht wird. LIDL erzielt beim Vergleich dieser drei Discounter eine mittlere Position.

Für die Gruppe ,Tierwohl ja - Bio nein' (Cluster 5) ist ein gutes Angebot an Tierwohlprodukten ebenfalls so bedeutsam, dass sie ihre Haupteinkaufsstätte für ein umfassenderes Fleisch- und Wurstwarensortiment aus artgerechterer Haltung wechseln würden. Sie unterscheiden sich im Punkt Wechselbereitschaft signifikant vom Referenzcluster. REWE-Märkte werden von dieser Gruppe von allen Clustern am wenigsten genutzt. EDEKA und NETTO sind dagegen deutlich beliebter. 
Insgesamt präsentiert sich diese Gruppe heterogen, was die Einkaufsstättenwahl betrifft, da im Gegensatz zu anderen Clustern keine klaren Tendenzen für eine Vertriebsform zu identifizieren sind. Die hohen Koeffizienten bei ALDI und SB-Warenhäusern bei dieser Gruppe lassen jedoch vermuten, dass diese Gruppe den Verbrauchern des Clusters 2 bei der Einkaufsstättenwahl sehr ähnlich ist und sie tendenziell eher zu den Discounter- und SB-Warenhaus-Käufern gehören, jedoch mit dem Unterschied, dass sich in dieser Gruppe deutlich mehr EDEKA-Kunden befinden und weniger REWE- und LIDL-Kunden.

\subsubsection{Marketingrelevante Unterschiede zwischen den Clustern}

Neben den vertriebsrelevanten Unterschieden zwischen den identifizierten Verbrauchergruppen wurden zudem ausgewählte marketingrelevante Aspekte untersucht, um entsprechende Handlungsempfehlungen für Hersteller und Händler ableiten zu können. Neben den zuvor beschriebenen soziodemografischen Unterschieden zwischen den Clustern sollen in diesem Kapitel ausgewählte psychographische Unterschiede analysiert und vorgestellt werden, die für die spezielle Vermarktung von mit Tierwohl-Labels zertifizierten Fleisch- und Wurstwaren bedeutsam sind. Von besonderer Bedeutung für das Marketing sind Kenntnisse über die Kaufkriterien der Verbraucher beim Einkauf von Fleisch- und Wurstwaren, ihre Kaufabsichten bei Tierwohlprodukten, die Kundenbedürfnisse hinsichtlich Herkunftsangaben, das Konsumverhalten, der Bekanntheitsgrad der Tierwohl-Labels sowie die Multi-LabelingPräferenzen der Verbraucher. Die Verbrauchersegmente wurden daher hinsichtlich dieser Punkte analysiert, um eine gezieltere Segmentbearbeitung zu ermöglichen und den Einsatz von Marketinginstrumenten besser an den identifizierten Zielgruppen auszurichten.

In Tabelle 33 sind ausgewählte segmentspezifische Unterschiede mit Tierwohlbezug dargestellt, die sich auf die Beachtung der Kaufkriterien (F8), der Tierwohlproduktaffinität (F18), der Bedeutung von Herkunftsangaben (F25), der Konsumhäufigkeit im Haushalt (F6) und der Reduktionsbereitschaft (F7) beziehen. 
Tabelle 33: Ausgewählte segmentspezifische Unterschiede mit Tierwohlbezug

\begin{tabular}{|c|c|c|c|c|}
\hline \multirow[b]{2}{*}{ VARIABLE } & $\begin{array}{l}\text { Enthusiastische } \\
\text { Nachhaltigkeitsfans }\end{array}$ & $\begin{array}{l}\text { Unentschlossene } \\
\text { Verbraucher }\end{array}$ & $\begin{array}{l}\text { Überzeugte } \\
\text { Bio-Fans }\end{array}$ & $\begin{array}{l}\text { Gruppe, Tierwohl } \\
\text { ja-Bio nein' }\end{array}$ \\
\hline & CLUSTER 1 & CLUSTER 2 & CLUSTER 3 & CLUSTER 5 \\
\hline & $\begin{array}{l}\text { +staatl. Bio } \\
\text { ++Anbau-Bio } \\
\text { ++reinTW }\end{array}$ & $\begin{array}{l}\text { +staatl. Bio } \\
\text {---Anbau-Bio } \\
\text { +reinTW }\end{array}$ & $\begin{array}{l}\text { +++staatl. Bio } \\
\text { +Anbau-Bio } \\
\text {--reinTW }\end{array}$ & $\begin{array}{l}\text {-staatl. Bio } \\
\text {-Anbau-Bio } \\
\text { +++reinTW }\end{array}$ \\
\hline Segmentgröße & $21 \%$ & $15 \%$ & $38 \%$ & $8 \%$ \\
\hline Gesamtbevölkerung & 11,45 Mio. & 8,18 Mio. & 20,7 Mio. & 4,36 Mio. \\
\hline Konstanter Term & $\begin{array}{l}-2,89^{* * *} \\
(14,612)\end{array}$ & $\begin{array}{l}-1,63^{* *} \\
(7,153)\end{array}$ & $\begin{array}{l}-0,767 \\
(2,657)\end{array}$ & $\begin{array}{l}-1,601^{*} \\
(5,185)\end{array}$ \\
\hline $\begin{array}{l}\text { Faktor } 1 \\
\text { Nachhaltigkeit }\end{array}$ & $\begin{array}{l}\mathbf{0 , 5 1 4} 4^{\star * \star} \\
(14,405)\end{array}$ & $\begin{array}{l}-0,091 \\
(0,432)\end{array}$ & $\begin{array}{l}\mathbf{0 , 2 7 ^ { * }} \\
(5,265)\end{array}$ & $\begin{array}{l}-0,023 \\
(0,019)\end{array}$ \\
\hline $\begin{array}{l}\text { Faktor } 2 \\
\text { Innere Qualität }\end{array}$ & $\begin{array}{l}-0,124 \\
(0,646)\end{array}$ & $\begin{array}{l}-0,221 \\
(2,117)\end{array}$ & $\begin{array}{l}-0,342^{* *} \\
(7,345)\end{array}$ & $\begin{array}{l}-0,225 \\
(1,568)\end{array}$ \\
\hline $\begin{array}{l}\text { Faktor } 3 \\
\text { Äußere Merkmale }\end{array}$ & $\begin{array}{l}\mathbf{0 , 4 1 6} \mathbf{6}^{\star \star \star} \\
(11,459)\end{array}$ & $\begin{array}{l}0,218 \\
(2,596)\end{array}$ & $\begin{array}{l}-0,025 \\
(0,053)\end{array}$ & $\begin{array}{l}0,237 \\
(2,149)\end{array}$ \\
\hline $\begin{array}{l}\text { Tierwohlprodukt- } \\
\text { affinität }\end{array}$ & $\begin{array}{l}1_{1,978}^{* \star} \\
(8,826)\end{array}$ & $\begin{array}{l}0,839 \\
(2,61)\end{array}$ & $\begin{array}{l}0,61 \\
(2,439)\end{array}$ & $\begin{array}{l}0,595 \\
(0,967)\end{array}$ \\
\hline Herkunft & $\begin{array}{l}\mathbf{0 , 9 6 5 ^ { \star }} \\
(5,678)\end{array}$ & $\begin{array}{l}0,306 \\
(0,757)\end{array}$ & $\begin{array}{l}\mathbf{0 , 7 ^ { * }} \\
(5,019)\end{array}$ & $\begin{array}{l}0,375 \\
(0,755)\end{array}$ \\
\hline $\begin{array}{l}\text { Fleischkonsum } \\
\text { im Haushalt }\end{array}$ & 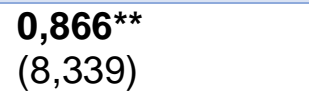 & $\begin{array}{l}\mathbf{1}^{1,023^{\star \star}} \\
(8,641)\end{array}$ & $\begin{array}{l}\mathbf{0 , 8 1 4} 4^{\star \star} \\
(9,38)\end{array}$ & $\begin{array}{l}0,662 \\
(2,94)\end{array}$ \\
\hline $\begin{array}{l}\text { Reduktions- } \\
\text { bereitschaft }\end{array}$ & $\begin{array}{l}-0,59^{*} \\
(5,15)\end{array}$ & $\begin{array}{l}-0,553^{*} \\
(3,964)\end{array}$ & $\begin{array}{l}-0,384 \\
(2,678)\end{array}$ & $\begin{array}{l}-0,868^{\star *} \\
(6,97)\end{array}$ \\
\hline $\begin{array}{l}\text { Multinominales Logit-M } \\
, 045 . \text { Wald-Wert in } \\
* *=p<0,01 \text { (Wald-We } \\
\text { Umweltfreundlichkeit, G } \\
\text { Geschmack, Frische. F } \\
\text { F6 Konsum HH, F7 Re }\end{array}$ & $\begin{array}{l}\text { II. Pseudo-R-Quac } \\
\text { mmern unter R } \\
6,66) ; \quad{ }^{* * *}=p<0 \\
\text { siegel, Fairtrade, } \\
\text { or 3: Marken, Prei } \\
\text { ionsbereitschaft. F }\end{array}$ & $\begin{array}{l}\text { dheit, Tierwoh } \\
\text { rpackung. F18 } \\
\text { enzkategorie } 4 \text { : }\end{array}$ & $\begin{array}{l}\text { 27, Nagelkerke = } \\
\text {, } \quad{ }^{*}=p<0,05 \\
5) \text {. Faktoren a } \\
\text { io, Regionalität. } \\
\text { erwohlproduktaffi } \\
\text { esinteressierte Le }\end{array}$ & $\begin{array}{l}\text { 133, McFadden = } \\
\text { Wald-Wert }>3,84) \text {; } \\
\text { us F8. Faktor 1: } \\
\text { aktor 2: Aussehen, } \\
\text { ität, F25 Herkunft, } \\
\text { bel-Ablehner. }\end{array}$ \\
\hline
\end{tabular}

\section{Quelle: Eigene Darstellung}

Die Kaufkriterien wurden dabei in Form der drei Faktoren berücksichtigt, die aus der in Unterkapitel 5.4.5 beschriebenen Faktorenanalyse zu den Kaufkriterien stammen. Dabei konnten drei Faktoren identifiziert werden. Faktor 1 fasst die Nachhaltigkeitsmotive (Umweltfreundlichkeit, Gütesiegel, Fairtrade, Gesundheit, Tierwohl, Bio, Regionalität) zusammen, während Faktor 2 die innere Qualität (Aussehen, Geschmack, Frische) und Faktor 3 die äußeren Merkmale (Preis, Marken, Verpackung) aggregiert. 
Für die Gruppe der ,Enthusiastischen Nachhaltigkeitsfans ' ist festzustellen, dass sich die Gruppe in fast allen Punkten signifikant von der Referenzgruppe der ,Desinteressierten Label-Ablehner' unterscheidet. Diese beiden Gruppen stellen daher in Bezug auf die untersuchten Merkmale zwei Extreme dar. Das Interesse am Thema Tierwohl und Nachhaltigkeit ist bei den Nachhaltigkeitsfans am höchsten und bei den ,Desinteressierten Label-Ablehnern“ im Referenzcluster 4 am niedrigsten. Am deutlichsten ist der Unterschied zur Referenzgruppe bezüglich der Beachtung ethischer Kaufkriterien beim Fleischkauf, die im Faktor 1 (Nachhaltigkeit) integriert sind. Diese Gruppe achtet demnach beim Einkauf von Fleisch- und Wurstwaren deutlich mehr auf Umweltkriterien, Gütesiegel, Fairtrade, Tierwohl, Bio-Qualität, Regionalität und Gesundheitsaspekte als die ,Desinteressierten Verbraucher' in Cluster 4.

Die Koeffizienten aller Cluster zeigen zudem, dass die Verbraucher in Cluster 1 (Nachhaltigkeitsfans) und Cluster 3 (Bio-Fans) am meisten auf Nachhaltigkeitsaspekte beim Fleischkauf achten, während die ,Unentschlossenen Verbraucher' (Cluster 2) und die Gruppe ,Tierwohl ja - Bio nein“ (Cluster 5) solchen Aspekten einen geringeren Stellenwert einräumen. Überraschenderweise berücksichtigen die Nachhaltigkeitsfans auch am meisten die äußeren Merkmale der Produkte wie beispielsweise eine ansprechende Verpackung, bestimmte Marken und den Preis. Kein Unterschied zum Referenzcluster besteht hinsichtlich der ,inneren Qualität‘, die die klassischen Qualitätskriterien (Aussehen der Ware, Geschmack und Frische) umfasst. Auf diese Kriterien achten demnach die Nachhaltigkeitsfans und die ,Desinteressierten LabelAblehner' etwa gleichermaßen. Die Erfüllung der klassischen Kaufkriterien aus Perspektive der Verbraucher stellt daher eine Grundvoraussetzung für den erfolgreichen Produktverkauf dar.

Zudem ist die Affinität zu Tierwohlprodukten in dieser Gruppe signifikant höher als in der Referenzgruppe und stellt insgesamt die höchste von allen Verbrauchergruppen dar. Nachhaltigkeitsfans unterscheiden sich von den ,Desinteressierten Label-Ablehnern“ außerdem darin, dass ihnen Herkunftsangaben deutlich wichtiger sind. 
Sie essen signifikant weniger Fleisch- und Wurstwaren als die Referenzgruppe, jedoch etwas mehr als die Bio-Fans. Der Anteil der Flexitarier ist in dieser Gruppe höher als in der Gruppe der ,Unentschlossenen Verbraucher', jedoch deutlich niedriger als bei den ,Überzeugten Bio-Fans‘.

Die ,Unentschlossenen Verbraucher' (Cluster 2) unterscheiden sich in zwei Punkten signifikant von der Referenzgruppe, und zwar bzgl. der Konsumhäufigkeit im Haushalt und der Reduktionsbereitschaft. Der Fleischkonsum ist höher als in der Referenzgruppe, die Reduktionsbereitschaft jedoch auch. Diese Gruppe, die überwiegend aus jüngeren Discounter-Käufern und SB-Warenhaus-Käufern besteht, isst von allen Gruppen am meisten Fleisch- und Wurstwaren. Die Koeffizienten aller Cluster vergleichend fällt auf, dass diese Gruppe sehr wenig auf Nachhaltigkeitskriterien achtet. Äußere Merkmale wie der Preis sind ihnen dagegen deutlich wichtiger als den Bio-Fans, was womöglich mit dem deutlich niedrigeren Einkommen dieser Gruppe zusammenhängt.

Die Affinität zu Tierwohlprodukten ist jedoch in dieser Gruppe ähnlich hoch wie bei den Bio-Fans (Cluster 3) und auch bei der Gruppe ,Tierwohl ja - Bio nein“ (Cluster 5). Dies zeigt sich anhand der Aufgeschlossenheit dieser Gruppe gegenüber Tierwohlprodukten (Tierwohlproduktaffinität; F18). Solange entsprechende Produkte eine vertrauenswürdige Kennzeichnung vorweisen, gut verfügbar sind und zu einem fairen Preis angeboten werden, würde diese Gruppe diese Produkte auch konventioneller Ware vorziehen. Die konkreten Herkunftsangaben sind dieser Gruppe im Vergleich zu den Nachhaltigkeitsfans (Cluster 1) und den Bio-Fans (Cluster 3) nicht so wichtig.

Die ,ய̈berzeugten Bio-Fans' heben sich von der Referenzgruppe bzgl. der Beachtung von Nachhaltigkeitskriterien und klassischen Kaufkriterien beim Einkauf sowie ihren Präferenzen zu Herkunftsangaben ab. Die Bio-Fans berücksichtigen signifikant häufiger Nachhaltigkeitskriterien, jedoch weniger klassische Kaufkriterien (innere Qualität) als die Referenzgruppe. Sie legen außerdem von allen Gruppen den geringsten Wert auf äußere Merkmale wie den Preis, bestimmte Marken oder die Verpackung. 
Auf möglichst konkrete Herkunftsangaben achten die Bio-Fans deutlich mehr als die ,Desinteressierten Verbraucher' des Referenzclusters (Cluster 4). Bezüglich der Konsumhäufigkeit im Haushalt (F6) lässt sich sagen, dass die Bio-Fans signifikant weniger Fleisch im Haushalt essen als die Personen des Referenzclusters. Hinsichtlich ihres eigenen Konsumverhaltens (F7) bilden die Bio-Fans von allen Clustern die Gruppe mit den meisten Flexitariern.

Die Gruppe ,Tierwohl ja - Bio nein' (Cluster 5) ist von der Referenzgruppe signifikant hinsichtlich der Reduktionsbereitschaft für den eigenen Fleischkonsum zu differenzieren, die in dieser Gruppe deutlich stärker ausgeprägt ist. Bei einem Vergleich der Koeffizienten aller Cluster fällt auf, dass diese Gruppe beim Kauf von Fleisch- und Wurstwaren wenig auf Nachhaltigkeitskriterien achtet. Verbraucher in Cluster 5 berücksichtigen demzufolge eher weniger Nachhaltigkeitskriterien wie Umweltfreundlichkeit, Gesundheitsaspekte, Fairtrade oder Bio-Qualität. Von allen Nachhaltigkeitskriterien ist ihnen das Tierwohl am wichtigsten. In diesem Punkt ähnelt die Gruppe ,Tierwohl ja - Bio nein` der Gruppe der ,Unentschlossenen Verbraucher` des Clusters 2.

Unwesentlich unterscheidet sich das Cluster 5 von den anderen Clustern bzgl. der Beachtung klassischer Kaufkriterien (innere Qualität) und äußerer Produktmerkmale. Auch wenn mehr Tierwohl befürwortet wird, ist die Affinität zu Tierwohlprodukten geringer als bei allen anderen Gruppen. Ebenso wie bei den ,Unentschlossenen Verbrauchern' in Cluster 2 sind konkrete Herkunftsangaben auch für die Gruppe ,Tierwohl ja - Bio nein' (Cluster 5) wenig relevant. Der Konsum im Haushalt ist den Angaben zufolge etwas niedriger als bei den anderen Gruppen, während die Reduktionsbereitschaft von allen Clustern am geringsten ist. Daher ist davon auszugehen, dass sich in den Clustern 2 und 5 (und ebenfalls im Referenzcluster) die meisten normalen Fleischesser befinden, die derzeit ihren Fleischkonsum nicht reduzieren möchten, was einem ungefähren Anteil von $40 \%$ der Bevölkerung entspricht. 
Ein weiterer zentraler Aspekt im Marketing, der bei der Vermarktung von Tierwohlprodukten von besonderer Bedeutung ist, ist der Bekanntheitsgrad der Tierwohl-Labels bei den Verbrauchern. Tierwohl-Labels, die die Verbraucher kennen und denen sie vertrauen (aufgrund einer seriösen Zertifizierung und eines guten Images), wirken sich am ehesten positiv auf die Kaufentscheidung interessierter Verbrauchergruppen aus. Im Ergebnisteil zu den uni- und bivariaten Analysen wurden bereits einige Erkenntnisse zum Bekanntheitsgrad der unterschiedlichen Tierwohl-Labels vorgestellt.

Dabei zeigte sich, dass insbesondere die staatlichen Bio-Labels DE-BIO und EU-BIO und die Bio-Labels der ökologischen Anbauverbände (BIOLAND, DEMETER, NATURLAND) den höchsten Bekanntheitsgrad in der Bevölkerung aufweisen. Von den reinen Tierwohl-Labels erreichte das NEULAND-Label den höchsten Bekanntheitsgrad, gefolgt von der Kennzeichnung der INITIATIVE TIERWOHL und dem DEUTSCHEN TIERSCHUTZBUND. Um diese Tierwohl-Labels strategisch für die identifizierten Marktsegmente einsetzen zu können, wurden Unterschiede im Bekanntheitsgrad der Tierwohl-Labels zwischen den neu identifizierten Clustern untersucht und in Tabelle 34 dargestellt.

Im Ergebnis der Analyse wird deutlich, dass große Unterschiede hinsichtlich des Bekanntheitsgrads zwischen den Verbrauchergruppen bestehen. Wird zunächst die Tabelle insgesamt betrachtet, lässt sich erkennen, dass insbesondere die ,Unentschlossenen Verbraucher` und die Gruppe ,Tierwohl ja - Bio nein`weniger Tierwohl-Labels kennen als die anderen Verbrauchergruppen. Dies zeigt sich an den negativen Vorzeichen der Koeffizienten sowie den hoch signifikanten Unterschieden zum Referenzcluster; vor allem in Bezug auf die Tierwohl-Labels der ökologischen Anbauverbände. Während die Bio-Fans diese Tierwohl-Labels am besten kennen, sind den anderen Gruppen die reinen Tierwohl-Label besser bekannt. 
Tabelle 34: Tierwohl-Label-Bekanntheit in den verschiedenen Segmenten

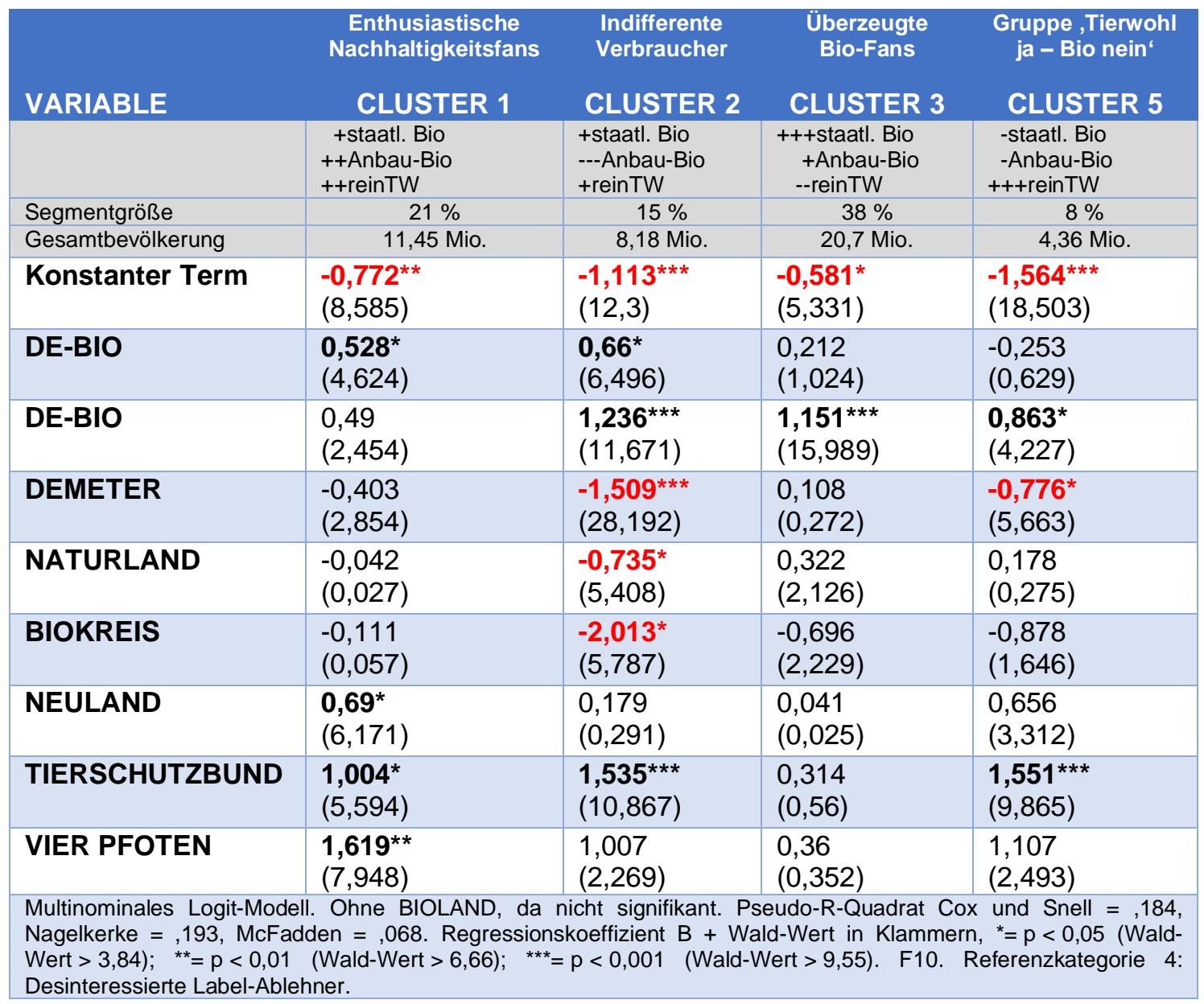

\section{Quelle: Eigene Darstellung}

Die ,Enthusiastischen Nachhaltigkeitsfans ' unterscheiden sich bei der Betrachtung des Bekanntheitsgrads der Tierwohl-Labels vom Referenzcluster 4 darin, dass die Labels EUBIO, NEULAND, TIERSCHUTZBUND und VIER PFOTEN in dieser Gruppe einen deutlich höheren Bekanntheitsgrad aufweisen. Insgesamt kennen die Nachhaltigkeitsfans überdurchschnittlich viele Gütesiegel gut. Mit Blick auf die Koeffizienten lässt sich jedoch feststellen, dass ihnen die Labels der ökologischen Anbauverbände weniger bekannt sind als den Bio-Fans. Dagegen erreichen die Labels von NEULAND, TIERSCHUTZBUND und VIER PFOTEN in dieser Gruppe einen deutlich höheren Bekanntheitsgrad als bei den Bio-Fans. 
Die ,Unentschlossenen Verbraucher' interessieren sich am wenigsten für TierwohlLabels bzw. bewegen sich überwiegend in einer Einkaufswelt, in der Nachhaltigkeitssiegel eher weniger in Erscheinung treten, wie beispielsweise bei Discountern und SB-Warenhäusern. Von allen Gruppen weisen sie die geringsten Kenntnisse von Tierwohl-Labels auf.

Vom Referenzcluster unterscheidet sich diese Gruppe bzgl. des Bekanntheitsgrads bei fast allen Tierwohl-Labels. Einen höheren Bekanntheitsgrad als in der Referenzgruppe erreichen bei den ,Unentschlossenen Verbrauchern“ nur die Labels EU-BIO, DE-BIO und das Label des DEUTSCHEN TIERSCHUTZBUNDES. Signifikant niedriger als bei den ,Desinteressierten Verbrauchern“ des Referenzclusters ist der Bekanntheitsgrad der Tierwohl-Labels der ökologischen Anbauverbände DEMETER, NATURLAND und BIOKREIS.

Die ,Überzeugten Bio-Fans ' heben sich vom Referenzcluster überraschenderweise nur in ihrer Kenntnis des DE-BIO-Siegels ab, die deutlich höher ist als bei den ,Desinteressierten Verbrauchern“. Ein Grund hierfür könnte darin bestehen, dass der Großteil der Bio-Fans vor allem das DE-BIO-Siegel kennt und nur einem eher kleineren Teil der Bio-Fans die Siegel der ökologischen Anbauverbände bekannter sind als der Gruppe der ,Desinteressierten Verbraucher' (Referenzcluster 4). Dennoch ist der Bekanntheitsgrad fast aller Tierwohl-Labels, insbesondere von DEMETER und NATURLAND, bei den ,Überzeugten Bio-Fans“ höher als bei den Gruppen ,Unentschlossene Verbraucher' (Cluster 2) und der Gruppe ,Tierwohl ja - Bio nein` (Cluster 5).

Eine Ausnahme stellt lediglich der höhere Bekanntheitsgrad von EU-BIO in der jüngeren Gruppe der ,Unentschlossenen Verbraucher“ (Cluster 2) und der Nachhaltigkeitsfans (Cluster 1) dar. DE-BIO weist bei allen Clustern einen hohen Bekanntheitsgrad auf, was durch die Ergebnisse der Häufigkeitsauswertungen bereits nahegelegt wurde. Auffällig sind des Weiteren die niedrigeren Koeffizienten im Vergleich zur Gruppe der Nachhaltigkeitsfans bei den Tierwohl-Labels von NEULAND, TIERSCHUTZBUND und VIER PFOTEN. 
Die Gruppe ,Tierwohl ja - Bio nein` unterscheidet sich vom Referenzcluster signifikant durch eine höhere Kenntnis des DE-BIO-Siegels und des Siegels des DEUTSCHEN TIERSCHUTZBUNDES sowie eine geringere Kenntnis von DEMETER. Cluster 5 weist dabei zusammen mit Cluster 2 die höchste Kenntnis des Labels des DEUTSCHEN TIERSCHUTZBUNDES auf, das auf zahlreichen Fleisch- und Wurstwaren mit geringen Tierwohlstandards in ihren favorisierten Einkaufstätten (Discounter und SBWarenhäuser) angeboten wird.

NATURLAND, DEMETER und BIOKREIS weisen in dieser Gruppe einen ähnlich niedrigen Bekanntheitsgrad auf wie bei der Gruppe der ,Unentschlossenen Verbraucher ‘ (Cluster 2). Das Label von NEULAND ist in dieser Gruppe dagegen relativ bekannt und erreicht einen ähnlich hohen Bekanntheitsgrad wie bei den Nachhaltigkeitsfans in Cluster 1. Insgesamt zeigt sich, dass die Verbraucher in Cluster 5 einen eher niedrigen Wissensstand bei Tierwohl-Labels haben und nur wenige Tierwohl-Labels kennen. Abgesehen vom DE-BIO-Logo, das in Deutschland den höchsten Bekanntheitsgrad besitzt, sind dieser Gruppe die meisten Bio-Labels fremd.

Neben den verschiedenen ausgewählten marketingrelevanten Kriterien (Tabelle 33) und der Analyse des Bekanntheitsgrads (Tabelle 34) wurden segmentspezifische Unterschiede für den strategischen Einsatz ergänzender Gütesiegel zu den TierwohlLabels im Rahmen des Multi-Labelings untersucht. Angesichts der Zielsetzung der Arbeit sollen Unterschiede bei den Verbrauchergruppen herausgefunden werden, um entsprechende Handlungsempfehlungen ableiten $\mathrm{zu}$ können, welche zusätzlichen Gütesiegel sich für welche Verbrauchergruppen am besten eignen.

Im Lebensmitteleinzelhandel wird das Multi-Labeling häufig eingesetzt, erfolgt jedoch bisher nur wenig strategisch bzw. zielgruppenorientiert. Mit der vorliegenden Studie soll eine wissenschaftliche Basis geschaffen werden, auf deren statistisch abgesicherten Erkenntnissen verschiedene Gütesiegel für ausgewählte Kundengruppen sinnvoll miteinander kombiniert werden können. Es ist indes wenig sinnvoll, Gütesiegel auf Lebensmitteln anzubringen, die die anvisierte Verbrauchergruppe nicht kennt oder die kein positives Kaufargument für diese Käufergruppe darstellen. 
In Tabelle 35 sind die Unterschiede bzgl. der Multi-Labeling-Präferenzen bei den Verbrauchergruppen dargestellt. Bei Betrachtung der Ergebnisse lässt sich zunächst feststellen, dass zahlreiche signifikante Unterschiede bzgl. der Multi-LabelingPräferenzen zwischen den verschiedenen Verbrauchergruppen bestehen. Die Gruppe der ,Unentschlossenen Verbraucher' (Cluster 2) unterscheidet sich bei fünf Gütesiegeln signifikant vom Referenzcluster, während sich die ,Enthusiastischen Nachhaltigkeitsfans` (Cluster 1), die Gruppe ,Tierwohl ja - Bio nein“ (Cluster 5) und die ,Überzeugten Bio-Fans‘ (Cluster 3) bei jeweils drei Gütesiegeln signifikant vom Referenzcluster unterscheiden.

In Cluster 1 (Nachhaltigkeitsfans) sind bereits deutliche Unterschiede zum Referenzcluster bzgl. der Präferenz für das GENTECHNIKFREI-Siegel, das REGIONALFENSTER und das DLG-Siegel zu erkennen. Dies bedeutet, dass alle drei Gütesiegel für die Nachhaltigkeitsfans ein stärkeres Kaufargument darstellen als für die ,Desinteressierten Verbraucher‘ im Referenzcluster 4.

Die ,Unentschlossenen Verbraucher" (Cluster 2) unterscheiden sich vom Referenzcluster bezüglich der deutlich positiveren Bewertung des ÖKO-TEST-Siegels, des Labels des DEUTSCHEN TIERSCHUTZBUNDES, des DLG-Siegels und des QSSiegels. Für das QS-Siegel und das DLG-Siegel wurden in dieser Gruppe die höchsten Koeffizienten erreicht, d. h. diese beiden Siegel erzielen in dieser Gruppe den stärksten Effekt.

Während das ÖKOTEST-Siegel deutlich positiver bewertet wird als von der Referenzgruppe, bestehen bei STIFTUNG WARENTEST keine Unterschiede zu den ,Desinteressierten Verbrauchern'. Das Logo vom NABU hat für diese Gruppe offensichtlich keinen positiven Einfluss auf die Kaufentscheidung und kann sich womöglich sogar negativ auf die Kaufentscheidung auswirken. 
Tabelle 35: Multi-Label-Präferenzen in den verschiedenen Segmenten

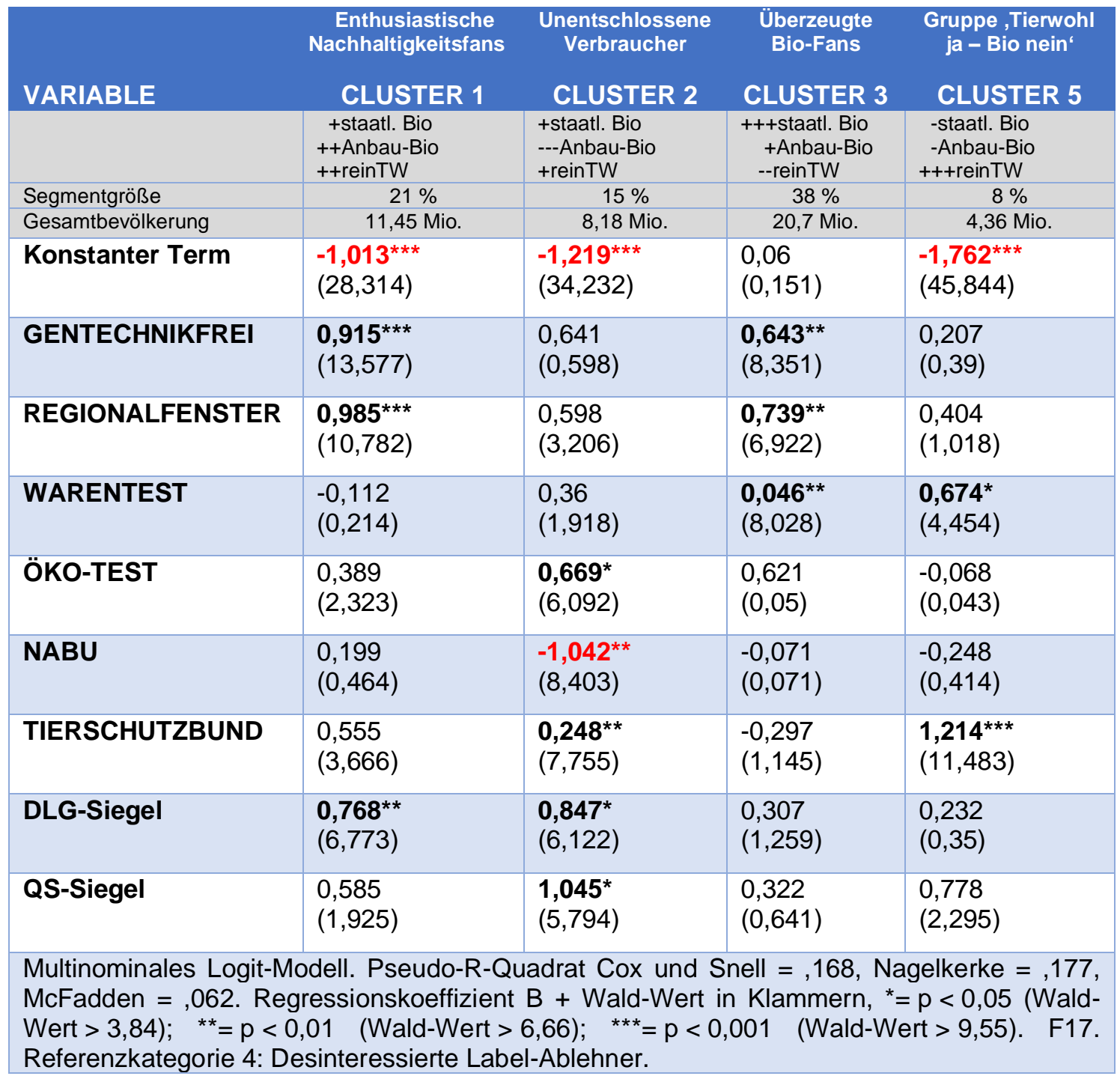

Quelle: Eigene Darstellung

Die ,Überzeugten Bio-Fans ' unterscheiden sich positiv vom Referenzcluster bzgl. der Bewertung des Labels GENTECHNIKFREI, des REGIONALFENSTERS und STIFTUNG WARENTEST. Dabei ist anzumerken, dass sich STIFTUNG WARENTEST im Vergleich zu den anderen beiden Siegeln nur auf einem geringen Niveau positiv auf die Kaufentscheidung auswirkt. In den hohen Koeffizienten von REGIONALFENSTER, des GENTECHNIKFREI-Siegels und von ÖKOTEST äußert sich die besonders hohe Bedeutung der Regionalität und Gesundheit für die Gruppe der Bio-Fans. 
Die Label vom NABU und DEUTSCHEN TIERSCHUTZBUND sind überraschenderweise für die Bio-Fans relativ unbedeutend und haben nur einen ähnlich niedrigen positiven Einfluss auf die Kaufentscheidung wie das DLG-Siegel oder das QSSiegel, wobei aufgrund des starken Bezugs von Bio-Fans zum Thema Tier- und Naturschutz hier eine positivere Bewertung hätte erwartet werden können.

Cluster 5 ,Tierwohl ja - Bio nein' unterscheidet sich signifikant positiv vom Referenzcluster hinsichtlich der Siegel von STIFTUNG WARENTEST und des Siegels des DEUTSCHEN TIERSCHUTZBUNDES, d. h. diese beiden Siegel stellen für diese Gruppe ein deutlich positiveres Kaufargument dar als für die Referenzgruppe. Auffällig ist, dass STIFTUNG WARENTEST von dieser Gruppe von allen Clustern am häufigsten gewählt wurde und es sich daher für diese Gruppe am besten eignet. Anders verhält es sich mit dem ÖKOTEST-Siegel, NABU-Logo und DLG-Siegel, die sich allesamt für diese Gruppe nur wenig eignen. Gentechnikfreiheit ist dieser Gruppe im Clustervergleich zudem am wenigsten wichtig von allen Gruppen, während das QS-Siegel und vor allem das Label des DEUTSCHEN TIERSCHUTZBUNDES bei dieser Gruppe besonders gut ankommen.

\subsubsection{Zusammenfassung der Ergebnisse der Clusteranalyse}

In diesem Abschnitt sollen die Ergebnisse der hierarchischen Clusteranalyse bzw. der verschiedenen multinominalen Logit-Modelle in einer Gesamtübersicht in Tabelle 36 präsentiert werden. Insgesamt konnten sehr viele Unterschiede zwischen den identifizierten Verbrauchergruppen festgestellt werden, die an dieser Stelle nicht noch einmal detailliert beschrieben werden sollen.

Eine ausführliche Interpretation dieser Ergebnisse erfolgt in Kapitel 6 im Rahmen der Diskussion. Neben der weiterführenden Interpretation der einzelnen Cluster werden dort auch beispielhaft Handlungsempfehlungen für die einzelnen Segmente im Hinblick auf die Anwendung zentraler Marketinginstrumente ausgesprochen. 
Für eine übersichtlichere Darstellung der Verbrauchertypologie in Tabelle 36 wird bewusst auf die Angabe der statistischen Kennzahlen verzichtet, die bereits im Ergebnisteil erläutert wurden. Die ermittelten signifikanten Unterschiede und Tendenzen zwischen den Verbrauchergruppen werden so dargestellt, dass sie leicht erfassbar und vergleichbar sind. Dabei sind alle soziodemografischen sowie vertriebs- und marketingrelevanten Unterschiede dargestellt, die im Rahmen der Clusteranalyse und in den Regressionsanalysen ermittelt werden konnten.

Die Wechselbereitschaft in der Tabelle meint, wie bereits in Unterkapitel 5.3 beschrieben, die Bereitschaft, die eigene Haupteinkaufsstätte für Fleisch- und Wurstwaren aufgrund eines besseren Angebots an Tierwohlprodukten in einer anderen Einkaufsstätte dauerhaft zu wechseln. Der Aspekt Nachhaltigkeit in der Tabelle umfasst Umweltfreundlichkeit, Gütesiegel, Fairtrade, Gesundheitsaspekte, Tierwohl, Bio und Regionalität. Aussehen, Geschmack und Frische verbergen sich hinter der inneren Qualität. Die äußere Qualität definiert sich aus den hohen Präferenzen für Marken, Preis und Verpackung.

Die Tierwohlaffinität wurde aus der Kaufrelevanz von Tierwohlprodukten berechnet und der Flexitarier-Anteil wurde aus der Reduktionsbereitschaft der Verbraucher ermittelt. Die Label-Kenntnis wurde anhand der Anzahl bekannter Gütesiegel charakterisiert. Die Cause-Related-Marketingaffinität wurde aus der Bewertung der NGO-Labels abgeleitet. 
Tabelle 36: Überblick über die Ergebnisse der hierarchischen Clusteranalyse

\begin{tabular}{|c|c|c|c|c|}
\hline & $\begin{array}{l}\text { Enthusiastische } \\
\text { Nachhaltigkeitsfans }\end{array}$ & $\begin{array}{l}\text { Unentschlossene } \\
\text { Verbraucher }\end{array}$ & $\begin{array}{l}\text { Überzeugte } \\
\text { Bio-Fans }\end{array}$ & $\begin{array}{c}\text { Gruppe } \\
\text {,Tierwohl ja - } \\
\text { Bio nein' }\end{array}$ \\
\hline \multirow[t]{2}{*}{ VARIABLE } & CLUSTER 1 & CLUSTER 2 & CLUSTER 3 & CLUSTER 5 \\
\hline & $\begin{array}{l}\text { +staatl. Bio } \\
\text { ++Anbau-Bio } \\
\text { ++reinTW }\end{array}$ & $\begin{array}{l}\text { +staatl. Bio } \\
\text {---Anbau-Bio } \\
\text { +reinTW }\end{array}$ & $\begin{array}{l}\text { +++staatl. Bio } \\
\text { +Anbau-Bio } \\
\text {--reinTW }\end{array}$ & $\begin{array}{l}\text {-staatl. Bio } \\
\text {-Anbau-Bio } \\
+++ \text { reinTW }\end{array}$ \\
\hline Segmentgröße & $21 \%$ & $15 \%$ & $38 \%$ & $8 \%$ \\
\hline Gesamtbevölkerung & 11,45 Mio. & 8,18 Mio. & 20,7 Mio. & 4,36 Mio. \\
\hline Geschlecht & sehr viele Frauen & mehr Frauen & mehr Frauen & $\begin{array}{l}\text { sehr viele } \\
\text { Frauen }\end{array}$ \\
\hline Alter & mittel bis hoch & jung bis mittel & mittel bis hoch & jung bis mittel \\
\hline Lebensraum & eher Stadt & eher Land & eher Stadt & eher Land \\
\hline Einkommen & hoch & sehr niedrig & sehr hoch & mittelmäßig \\
\hline Bildungsgrad & hoch & niedrig & sehr hoch & sehr niedrig \\
\hline $\begin{array}{l}\text { Beliebte } \\
\text { Einkaufsstätten }\end{array}$ & $\begin{array}{l}\text { Edeka, Rewe, Bio- } \\
\text { Supermarkt, Aldi, } \\
\text { Wochenmarkt }\end{array}$ & $\begin{array}{l}\text { Lidl, Netto, Aldi, } \\
\text { real, Kaufland }\end{array}$ & $\begin{array}{l}\text { Bio-Supermarkt, } \\
\text { Metzger, Rewe, } \\
\text { W-Markt/Hofladen }\end{array}$ & $\begin{array}{l}\text { Aldi, Netto, } \\
\text { Kaufland, real }\end{array}$ \\
\hline $\begin{array}{l}\text { Weniger beliebte } \\
\text { Einkaufsstätten }\end{array}$ & $\begin{array}{l}\text { Kaufland, real, } \\
\text { Netto }\end{array}$ & $\begin{array}{l}\text { Bio-Supermarkt, } \\
\text { Wochenmarkt, } \\
\text { Hofladen }\end{array}$ & $\begin{array}{l}\text { Lidl, Netto, } \\
\text { Kaufland, real, } \\
\text { Edeka }\end{array}$ & $\begin{array}{l}\text { Bio- } \\
\text { Supermarkt, } \\
\text { Metzger }\end{array}$ \\
\hline Wechselbereitschaft & sehr hoch & sehr niedrig & mittelmäßig & hoch \\
\hline Nachhaltigkeit & sehr wichtig & sehr unwichtig & sehr wichtig & $\begin{array}{l}\text { sehr unwichtig } \\
\text { (nur Tierwohl) }\end{array}$ \\
\hline Innere Qualität & mittelmäßig & mittelmäßig & unwichtig & mittelmäßig \\
\hline Äußere Qualität & sehr wichtig & sehr wichtig & sehr unwichtig & wichtig \\
\hline Tierwohlaffinität & hoch & hoch & hoch & niedrig \\
\hline Herkunft & sehr wichtig & sehr unwichtig & sehr wichtig & unwichtig \\
\hline Flexitarier-Anteil & hoch & sehr niedrig & sehr hoch & sehr niedrig \\
\hline Label-Kenntnis & sehr hoch & sehr niedrig & hoch & niedrig \\
\hline $\begin{array}{l}\text { Bekannteste } \\
\text { Tierwohl-Labels }\end{array}$ & $\begin{array}{l}\text { EU/DE-BIO, } \\
\text { Neuland, Vier } \\
\text { Pfoten }\end{array}$ & $\begin{array}{l}\text { EU/DE-BIO, } \\
\text { Tierschutzbund }\end{array}$ & $\begin{array}{l}\text { EU/DE-BIO, } \\
\text { Demeter, Bioland, } \\
\text { Naturland }\end{array}$ & $\begin{array}{l}\text { EU/DE-BIO, } \\
\text { Neuland, Tier- } \\
\text { schutzbund }\end{array}$ \\
\hline $\begin{array}{l}\text { Defizite Tierwohl- } \\
\text { Label-Bekanntheit }\end{array}$ & $\begin{array}{l}\text { Anbauverband- } \\
\text { Bio-Labels }\end{array}$ & $\begin{array}{l}\text { Anbauverband- } \\
\text { Bio-Labels }\end{array}$ & $\begin{array}{l}\text { reine Tierwohl- } \\
\text { Labels }\end{array}$ & $\begin{array}{l}\text { Anbauverband } \\
\text {-Bio-Labels }\end{array}$ \\
\hline $\begin{array}{l}\text { Multi-Labeling- } \\
\text { Präferenzen }\end{array}$ & $\begin{array}{l}\text { Regionalsiegel, } \\
\text { Gentechnikfrei }\end{array}$ & QS, DLG & $\begin{array}{l}\text { Gentechnikfrei, } \\
\text { Regionalsiegel, } \\
\text { Ökotest }\end{array}$ & $\begin{array}{l}\text { QS, Stiftung } \\
\text { Warentest }\end{array}$ \\
\hline $\begin{array}{l}\text { Cause-Related- } \\
\text { Marketing? }\end{array}$ & gerne & nein & warum nicht & nein \\
\hline
\end{tabular}

Quelle: Eigene Darstellung 


\section{Diskussion und Implikationen der Ergebnisse}

Das Hauptanliegen dieser Arbeit bestand, wie in der Einleitung beschrieben, in einer empirischen Überprüfung der Bedeutung von Tierwohl-Labels für Fleisch- und Wurstwaren-Käufer in Deutschland. Im Ergebnisteil wurden zunächst die deskriptiven Ergebnisse der Verbraucherbefragung sowie verschiedene uni- und bivariate Analysen zu den vier Themenschwerpunkten des Fragebogens beschrieben (Unterkapitel 5.2 und 5.3). Dabei standen das Einkaufs- und Ernährungsverhalten bei Fleisch- und Wurstwaren, die Bedeutung von Tierwohl-Labels als Einkaufshilfe, der Informationsbedarf der Verbraucher bei Tierwohlprodukten und die politischen Präferenzen der Befragten im Mittelpunkt. Anschließend wurden die Ergebnisse der explorativen Faktorenanalysen (Unterkapitel 5.4) und der hierarchischen Clusteranalyse (Unterkapitel 5.5) sowie verschiedene gruppenspezifische Auswertungen mit Hilfe der multinominalen logistischen Regression beschrieben, die die Erstellung einer Tierwohl-Label-Typologie und einer Verbrauchertypologie auf Basis der Tierwohl-Label-Präferenzen der Verbraucher ermöglichten.

Die präsentierten Forschungsergebnisse sollen in diesem Kapitel, der Struktur des Ergebniskapitels folgend, interpretiert und diskutiert werden. Der Diskussion der Tierwohl-Label- bzw. Verbraucher-Typologie (Unterkapitel 6.3 und 6.4) wird dabei ein größerer Stellenwert eingeräumt als der Diskussion der uni- und bivariaten Analysen (Unterkapitel 6.1 und 6.2), da sich aus diesen Ergebnissen Handlungsempfehlungen für Anbieter von Fleisch- und Wurstwaren ableiten lassen, die sich gezielt auf die identifizierten Gruppen beziehen. An geeigneten Stellen werden im Rahmen der Diskussion auch Handlungsempfehlungen zum Tierwohl-Labeling für die Politik gegeben. Es werden in diesem Abschnitt bewusst nicht alle Teilergebnisse diskutiert, sondern schwerpunktmäßig die Erkenntnisse, die für die Praxis den größten Nutzen darstellen und das Potenzial haben, die Vermarktung von Fleisch- und Wurstwaren aus einer artgerechteren und nachhaltigeren Nutztierhaltung in Deutschland zu verbessern, um den Marktanteil an Tierwohlprodukten zukünftig zu erhöhen. 


\subsection{Einkaufs- und Ernährungsverhalten}

Das allgemeine Einkaufs- und Ernährungsverhalten der Verbraucher beim Fleischkauf diente in der vorliegenden Studie der weiterführenden Analyse der Fragen, die sich konkret auf die Bedeutung von Tierwohl-Labels beim Kauf von Fleisch- und Wurstwaren bezogen und stand daher nicht im Fokus der Untersuchung, sondern hatte nur eine unterstützende (wenn auch für die weiterführenden Analysen bedeutsame) Funktion. Aktuelle Daten zum Einkaufs- und Ernährungsverhalten finden sich zudem bei zahlreichen kommerziellen Anbietern wie beispielsweise der GESELLSCHAFT FÜR KONSUMFORSCHUNG (GFK) oder STATISTA (NIER, 2019). Daher sollen an dieser

Stelle nur die wesentlichen Resultate der Online-Befragung zum Einkaufs- und Ernährungsverhalten interpretiert und diskutiert werden, bevor die Ergebnisse zu den tierwohlbezogenen Fragen (Unterkapitel 6.2 bis 6.4) ausführlicher diskutiert werden.

Die deskriptiven Ergebnisse der Befragung zur Wahl der Einkaufsstätte beim Kauf von Fleisch- und Wurstwaren (vgl. Unterkapitel 5.2.1) spiegelten die hohe Konzentration im deutschen Lebensmitteleinzelhandel wider. Lediglich fünf Handelsunternehmen (EDEKA mit NETTO, REWE mit PENNY, die SCHWARZ-Gruppe mit LIDL und KAUFLAND, ALDI und METRO) teilen sich den Großteil des deutschen Marktes für Fleisch- und Wurstwaren.

Die deutschen Verbraucher (mit Ausnahme der Metzgerei-Käufer) kaufen Fleisch- oder Wurstwaren regelmäßig mit anderen Lebensmitteln zusammen vor allem in Supermärkten, Discountern und SB-Warenhäusern. Dieses Verbraucherverhalten ist vor allem durch das stetig gestiegene Bedürfnis begründet, aus Gründen der Zeitersparnis und Bequemlichkeit möglichst viele Einkäufe gebündelt an einem Ort zu tätigen. In Wissenschaft und Praxis wird dieser Trend als ,One-Stop-Shopping ‘ bezeichnet und seit der Jahrtausendwende in verschiedenen Lebensbereichen untersucht (BAYE et al., 2018; JOHANSEN, 2012; MARQUES, 2007; SPILLER \& SCHULZE, 2008; WENZL et al., 2019). 
Nicht nur die Konzepte von Einkaufszentren bzw. Shoppingmalls begründen sich durch den Trend des ,One-Stop-Shoppings', sondern auch die Bündelung unterschiedlicher Geschäfte und Dienstleister an bestimmten Orten geht hierauf zurück (Convenience Center).

Vor diesem Hintergrund wird Supermärkten wie EDEKA oder REWE oder auch SBWarenhäusern wie KAUFLAND und REAL beim Verkauf von Fleisch- und Wurstwaren aufgrund des breiteren Sortiments und der Fleischtheke ein Wettbewerbsvorteil gegenüber Discountern und dem Fleischerhandwerk bzw. lokalen Metzgern zugesprochen. Da Verbraucher in Supermärkten und SB-Warenhäusern sowohl Lebensmittel als auch eine Fleischtheke und viele andere Güter des täglichen Bedarfs vorfinden, müssen sie keine anderen Einkaufsstätten aufsuchen.

Die Einkaufsstättenwahl wurde in zwei unterschiedlichen Varianten erfragt: zum einen als Mehrfachnennung und ergänzend als Einfachnennung bzgl. der Haupteinkaufsstätte für Fleisch- und Wurstwaren, um aussagekräftigere Handlungsempfehlungen geben zu können. Eine interessante Erkenntnis der Befragung besteht darin, dass die Metzger bei der Mehrfachnennung (gelegentliche Nutzung) lediglich $8 \%$ erreichten.

Bei der Frage nach der Haupteinkaufsstätte gaben allerdings $14 \%$ den Metzger an. Der Metzger war damit nach EDEKA und REWE die meistgenannte Haupteinkaufsstätte für Fleisch- und Wurstwaren in Deutschland. Dieser Befund kann evtl. damit erklärt werden, dass insgesamt weniger Menschen den Metzger als Einkaufsstätte wählen als beispielsweise ALDI, die Metzgerei-Käufer jedoch einen höheren Anteil an Käufern haben, die überwiegend beim Metzger einkaufen. Ein besonders hoher Stammkundenanteil bei Metzgern wurde u. a. von INDERHEES et al. (2004) ermittelt.

Bio-Supermärkte waren ebenfalls von großem Interesse für diese Studie, da sie zu $100 \%$ Fleisch mit Tierwohl-Labels (Bio-Labels) anbieten und in den meisten Studien und Statistiken zum Fleischkonsum in Deutschland häufig außer Acht gelassen werden. BioSupermarkt-Kunden sind eine bedeutsame Zielgruppe für Tierwohlprodukte und BioSupermärkte sind die Spezialisten bei der Vermarktung von Bio-Produkten. 
Ein weiterer Grund war, dass sich das häufig zweigeteilte Bild in die ,guten“ (Bio-Läden) und die ,bösen“ Lebensmitteleinzelhändler (Supermärkte, Discounter und SBWarenhäuser) in den letzten Jahren immer mehr aufgelöst hat. Dies ist vor allem durch das stetig gestiegene Angebot von Bio-Lebensmitteln bei konventionellen Lebensmitteleinzelhändlern begründet (HABLESREITER \& STUMMERER, 2020). In der vorliegenden Befragung konnten insgesamt 132 Personen berücksichtigt werden, die gelegentlich Fleisch und Wurst in den Bio-Supermärkten DENN'S, ALNATURA, BASIC BIO, SUPER BIO und BIO COMPANY einkaufen. Die Idee zur Berücksichtigung dieser Zielgruppe geht zudem auf eine Studie von STAUS (2010) zurück, der nachweisen konnte, dass eine Präferenz für Bio-Produkte maßgeblich die Einkaufsstättenwahl beeinflusst. HUDDLESTON et al. (2009) stellte zudem fest, dass Bio-Kunden zufriedener sind als Verbraucher konventioneller Märkte.

Ein weiterer Schwerpunkt der Befragung zum Einkaufs- und Ernährungsverhalten betraf die Wahl der Angebotsformen Fleischtheke und Selbstbedienungsbereich. Die Ergebnisse zu den Präferenzen hinsichtlich dieser Angebotsformen wurden dabei zu zwei verschiedenen Produktgruppen erfragt. Im Rahmen der SGS-Verbraucherstudie (SGS GERMANY, 2014) konnte herausgefunden werden, dass rd. zwei Drittel der deutschen Verbraucher Fleisch- und Wurstwaren an der Fleischtheke bzw. beim Metzger kaufen und nur ein Drittel im SB-Bereich.

Die in Unterkapitel 5.2.2 präsentierten Ergebnisse legen nahe, dass der Großteil der Verbraucher bei Fleisch zum Braten und Grillen auch die Frischetheke gegenüber dem SB-Bereich präferiert, während bei den meisten Verbrauchern bei Wurst oder Aufschnitt fürs Brot jedoch eine Tendenz zum SB-Bereich vorliegt. Hier gibt es offensichtlich deutliche Unterschiede je nach Produktgruppe. Da laut SCHULZE und SPILLER (2008) die Fleischtheke besonders für das Kaufkriterium der Frische steht, wird Fleisch zum Braten und Grillen womöglich bevorzugt an der Fleischtheke gekauft. Die Präferenzen $\mathrm{zu}$ den Angebotsformen werden in Unterkapitel 6.2 und 6.3 sowohl hinsichtlich verschiedener tierwohlrelevanter Fragestellungen als auch im Rahmen der Verbrauchertypologie diskutiert. 
Weiterhin war das Konsumverhalten der Befragten von Interesse, das in der Befragung in Form der Verzehrhäufigkeit im Haushalt und der Reduktionsbereitschaft des Fleischkonsums erfragt wurde (Unterkapitel 5.2.3). Hinsichtlich der Verzehrhäufigkeit im Haushalt zeigte sich, dass in den meisten Haushalten deutscher Verbraucher mehrmals oder zumindest einmal täglich Fleisch- und Wurstwaren gegessen werden (rd. $80 \%$ ).

Die ,Flexitarier-Haushalte', in denen nur wenig Fleisch- und Wurstwaren gegessen werden, kamen in der vorliegenden Verbraucherstudie auf einen Anteil von $20 \%$. Diese Ergebnisse können indes nur schwer interpretiert werden, da nicht genau gesagt werden kann, wie sich die Haushalte zusammensetzen und welche Personen im Haushalt wie häufig Fleisch- und Wurstwaren essen. So ist beispielsweise nicht bekannt, ob in einer modernen Familie beispielsweise der Hausmann und der Sohn vegan leben, während die berufstätige Frau und Tochter große Fleischfans sind. Daher war die Reduktionsbereitschaft der befragten Personen von größerem Interesse, da hier eindeutig auf das Konsumverhalten der Befragten geschlossen werden konnte.

In der Einleitung wurde bereits beschrieben, dass zahlreiche Wissenschaftler und verschiedenste Organisationen (z. B. UN, IPCC, UBA) eine stärkere Reduktion des Fleischkonsums fordern. Als Begründung werden z. B. gesundheitliche Gründe (DGE, 2019; THE LANCET, 2019), Tierwohlaspekte und vor allem zahlreiche ökologische Aspekte wie Ressourcenknappheit, der Verlust der Artenvielfalt sowie der stetig fortschreitende Klimawandel aufgeführt (GREENPEACE, 2020; HARWATT et al., 2017; HEDENUS et al., 2014; NIJDAM et al., 2012; STEHFEST et al., 2009; WWF, 2019).

Auch der Wissenschaftliche Beirat für Agrarpolitik, Ernährung und gesundheitlichen Verbraucherschutz (WBAE) des Bundesministeriums für Ernährung und Landwirtschaft fordert in seinem Gutachten ,Politik für eine nachhaltigere Ernährung ' insbesondere vor dem Hintergrund der ehrgeizigen Nachhaltigkeitsziele im Bereich Klima und Umwelt dazu auf, den Fleischkonsum stark zu reduzieren (WBAE, 2020). 
Die Ergebnisse zur Reduktionsbereitschaft, die in Unterkapitel 5.2.3 vorgestellt wurden, lassen den Schluss zu, dass erhebliche Unterschiede zwischen den Verbrauchergruppen bestehen. Ein Drittel aller Befragten möchte den Fleischkonsum nicht reduzieren. Ungefähr ein weiteres Drittel der Verbraucher möchte den Fleischkonsum zukünftig reduzieren. Die größte Gruppe (rd. $40 \%$ ) gab an, den Fleischkonsum bereits reduziert zu haben, aber nicht auf Fleisch- und Wurstwaren verzichten zu wollen. $5 \%$ der Befragten gaben an, vegetarisch oder vegan zu leben oder zukünftig ganz auf Fleisch- und Wurstwaren verzichten zu wollen.

Hier bestehen einige Unterschiede zur Befragung in der Studie von CORDTS et al. (2013), auf der die Grundidee dieser Frage zur Reduktionsbereitschaft beruht. Während die ,Unbekümmerten Fleischesser ${ }^{\star}$ in der Studie von CORDTS et al. (2013) rd. $75 \%$ der Probanden ausmachten, sind in der vorliegenden Studie nur $30 \%$ der Befragten nicht bereit, ihren Fleischkonsum zu reduzieren. Die ,Reduktionswilligen Fleischesser` sind in der vorliegenden Studie mit $26 \%$ deutlich mehr vertreten als in der Studie von CORDTS et al. (2013), die hierzu nur rd. $10 \%$ der Befragten ermittelten.

Flexitarier, die ihren Konsum bereits reduziert haben und stärker auf besondere Produktqualitäten wie mehr Tierwohl achten (GRUBER, 2013), sind in der vorliegenden Studie mit $39 \%$ ebenfalls stärker vertreten als in der Studie von CORDTS et al. (2013) beschrieben (11,6\%). Diese vergleichsweise hohe Differenz könnte u. a. auch aus der besonderen Stichprobe der vorliegenden Arbeit resultieren, bei der explizit nur Verbraucher mit einem gewissen Grundinteresse am Thema Nachhaltigkeit befragt wurden.

Die Ergebnisse der beiden Studien vergleichend zeigt sich, dass der Anteil der Menschen, die ihren Fleischkonsum bereits reduziert haben oder ihren Fleischkonsum reduzieren möchten, in den letzten Jahren größer geworden ist. Diese Entwicklung ist im Wesentlichen auf den in Kapitel 2 beschriebenen gesellschaftlichen Wertewandel zurückzuführen und drückt sich in Deutschland und vielen weiteren westlichen Industriestaaten im rückläufigen Fleischkonsum aus. 
Der vom ZUKUNFTSINSTITUT beschriebene langfristige Megatrend der NeoÖkologie wird zukünftig zu einer noch stärkeren Abnahme des Fleischkonsums führen, als bisher vermutet wird. Ein besonderer Treiber der Entwicklung hin zu einer fleischlosen Ernährung (Vegetarismus und Veganismus) ist der zunehmende Anteil der Ernährung mit proteinreichen Getreidearten wie Fingerhirse und unterschiedlichen Arten an Hülsenfrüchten (Leguminosen) wie z. B. Bohnen, Erbsen, Erdnüssen, Kichererbsen, Linsen, Platterbsen (Wicken) sowie Sojabohnen oder Lupinen (HANDSCHUCH, 2014; LEMKEN et al., 2018).

Daneben trägt auch die Entwicklung alternativer Proteinquellen bzw. von Fleischersatzprodukten (z. B. BEYOND MEAT BURGER, vegane Salami von RÜGENWALDER, indonesische Tempeh-Schnitzel) zu dieser Entwicklung bei.

Zahlreiche Hersteller und Händler haben mittlerweile erkannt, dass sie mit Fleischersatzprodukten höhere Gewinne erzielen können als mit Fleisch- und Wurstwaren, und weiten daher das Produktangebot an Fleischersatzprodukten stetig aus und verbessern die Rezepturen, wodurch die Produktvielfalt an veganen Produkten im deutschen Lebensmitteleinzelhandel für die Verbraucher stark erhöht wird. Des Weiteren beschleunigen auch die zahlreichen innovativen veganen Kochrezepte in Kochbüchern und im Internet (z. B. auf der Website EAT THIS) diesen Wandel.

Aufgrund des stetig steigenden Bewusstseins für Tierschutz-, Umweltschutz- und Gesundheitsaspekte in der jungen FRIDAYS-FOR-FUTURE-Generation ist davon auszugehen, dass sich die Anzahl der Veganer bzw. Vegetarier der Anzahl an Fleischessern immer mehr annähern wird.

Eventuell wird in Deutschland im Jahr 2050 mehr als die Hälfte aller Menschen in Deutschland auf den Verzehr von Fleisch- und Wurstwaren verzichten, mit entsprechenden Vorteilen für die Gesundheit der Menschen, die Umwelt und gegebenenfalls auch für die Nutztiere (ZUKUNFTSINSTITUT, 2019). Bis die Empfehlungen der Deutschen Gesellschaft für Ernährung (DGE), den Fleischkonsum pro Person und Jahr von rd. 60 auf $30 \mathrm{~kg}$ zu halbieren, erreicht werden, wird es demnach wahrscheinlich noch einige Jahrzehnte dauern. 
Die DGE begründet ihre Empfehlungen vor allem mit den positiven Effekten für die Gesundheit der Menschen z. B. im Hinblick auf Blutdruck, Cholesterinwerte, Krebserkrankungen, Adipositas per magna, Herz-Kreislauf-Erkrankungen oder DiabetesTyp-II (DGE, 2019; WCRF, 2020).

Zusammenfassend lässt sich zur Reduktion des Fleischkonsums anhand der Ergebnisse der vorliegenden Studie konstatieren, dass immer mehr Verbraucher ihren Fleischkonsum bereits reduziert haben oder diesen künftig reduzieren möchten. Insgesamt nimmt der Fleischkonsum daher in Deutschland seit ein paar Jahren leicht ab, jedoch laut WBAE in einem nicht ausreichenden Tempo (WBAE, 2020). Daher empfiehlt der WBAE der Politik, deutlich stärker in den Markt einzugreifen als in den letzten Jahrzehnten und eine schnellere Reduktion des Fleischkonsums zu unterstützen.

Da die Politik von der Nachkriegszeit bis heute die Erhöhung des Fleischkonsums und der Fleischproduktion in Deutschland durch hohe Subventionen an die Landwirte sehr stark forciert und Aspekte des Tier- und Umweltschutzes bisher den ökonomischen Wachstumszielen stetig untergeordnet hat, wird mit den Forderungen nach Reduktion nun ein konträres Handeln erwartet. Für die Reduktion des Fleischkonsums empfiehlt der WBAE zunächst eine Mehrwertsteuersenkung für Obst, Gemüse und Hülsenfrüchte, Informationskampagnen über den ökologischen Fußabdruck ausgewählter Lebensmittel sowie ein spezielles Klimalabel.

Des Weiteren wird aus umwelt- und klimapolitischen Gründen eine Anhebung des Regelsteuersatzes von 7 auf $19 \%$ gefordert, im Hinblick auf die durch Mehrwertsteuersenkung subventionierten Lebensmittel wie Fleisch- und Wurstwaren (WBAE, 2020). Die Effekte dieser empfohlenen Maßnahmen zur Reduktion des Fleischkonsums werden jedoch womöglich durch die parallel stetige Intensivierung der Nutztierhaltung und die Erhöhung der Produktionsmengen von Fleisch- und Wurstwaren in Deutschland für den Export substituiert. Insbesondere auf größere Fleischproduzenten und Tierhalter werden diese Maßnahmen daher wahrscheinlich nur wenige negative ökonomische Effekte haben und sie werden die intensive Tierhaltung in Deutschland für die globalen Märkte auch mittelfristig weiter ausbauen können. 
Aktuell steigt, trotz der zahlreichen Nachhaltigkeitstrends, die weltweite Nachfrage nach Fleisch- und Wurstwaren insgesamt für die meisten Fleisch- bzw. Tierarten an (steigend vor allem für Geflügel, sinkend für Schweinefleisch). Dies zeigt, dass der steigende Fleischkonsum in Entwicklungs- und Schwellenländern quantitativ ein deutlich größeres Gewicht hat als die Reduktion des Fleischkonsums in den Industriestaaten. Langfristig ist allerdings davon auszugehen, dass mit Produkten aus pflanzlichen Proteinen ein stärkeres Wachstum und eine höhere Wertschöpfung erzielt werden kann als mit tierischen Produkten (FAO, 2019).

Im Ergebnisteil wurde im Hinblick auf die Kaufkriterien deutlich, dass die meisten Befragten auf drei zentrale Kaufkriterien achten: die Frische der Waren, das Aussehen der Waren sowie den Geschmack. Die (regionale) Herkunft, eine artgerechtere Tierhaltung, Umweltfreundlichkeit und Gesundheitsaspekte stellen die bedeutsamsten ethischen Kaufkriterien dar (SGS GERMANY, 2014). Da diese ethischen Kaufkriterien zu den Hauptzielen einer nachhaltigeren Ernährung gehören, kann festgestellt werden, dass die Befragten bereits in hohem Maße versuchen, durch ihr eigenes Konsumverhalten zu diesen Nachhaltigkeitszielen beizutragen (WBAE, 2020). Interessant ist hierbei, dass die Befragten angaben, weniger auf den Preis $\mathrm{zu}$ achten als auf diese ethischen Kaufkriterien.

Die große Bedeutung ethischer Kaufkriterien wird auch durch das ÖKOBAROMETER bestätigt (BMEL, 2016). Im Unterschied zu dieser Studie wurden in der vorliegenden Arbeit jedoch sowohl klassische als auch ethische Kaufkriterien berücksichtigt. Zudem wurde in der vorliegenden Studie herausgefunden, dass viele Verbraucher weniger auf Gütesiegel und Bio-Qualität achten als auf artgerechtere Tierhaltung, Umweltfreundlichkeit oder Gesundheitaspekte. Dies ist ein leicht irritierender Befund, da z. B. Bio-Labels in diesen drei Bereichen vergleichsweise strenge Anforderungen besitzen. Auf Gütesiegel wird jedoch mehr geachtet als auf Marken. Viele der beschriebenen Tierwohl-Labels weisen einen höheren Bekanntheitsgrad auf als die meisten Marken für Fleisch- und Wurstwaren. Wenige Ausnahmen für bekannte Marken bei Fleisch- und Wurstwaren sind beispielsweise WIESENHOF oder die RÜGENWALDER MÜHLE. 
Daher könnten die Gütesiegel bis zu einem gewissen Grad als Ersatz für Marken dienen, was eine deutliche Kosteneinsparung für Hersteller und Händler zertifizierter Fleischund Wurstwaren bedeuten würde. Imagewerbung für die Gütesiegel wird beispielsweise von den Herausgebern der Gütesiegel betrieben. Dadurch müssen Hersteller oder Händler diese Werbung nicht selbst finanzieren.

Die drei Kaufkriterien artgerechte Tierhaltung, Umweltfreundlichkeit und Gesundheitsaspekte wurden in der vorliegenden Studie näher analysiert, da sie eine besonders hohe Bedeutung für die zentralen Ziele einer nachhaltigeren Ernährung haben und insbesondere für die Vermarktung von Fleisch- und Wurstwaren mit TierwohlLabels relevant sind (WBAE, 2020). Dies stellten u. a. auch ZENNER et al. (2004) und STAUS (2010) fest. Wie im Ergebnisteil in Unterkapitel 5.2.4 beschrieben konnten bei allen drei Kriterien teils hoch signifikante Unterschiede zwischen den verschiedenen Käufertypen nach Haupteinkaufsstätten ermittelt werden.

Metzgerei-Käufer achten den Ergebnissen zufolge am häufigsten auf Tierwohl, Umwelt und Gesundheit und unterscheiden sich häufig signifikant von SB-Warenhaus-Käufern, Discounter-Käufern und Supermarkt-Käufern. Die SB-Warenhaus-Käufer beachten diese drei ethischen Kaufkriterien am wenigsten. Bei Betrachtung der Mittelwerte kann insgesamt vermutet werden, dass die Beachtung der Kaufkriterien Tierwohl, Umwelt und Gesundheit in der Reihenfolge Metzgerei-Käufer, Supermarkt-Käufer, DiscounterKäufer und SB-Warenhaus-Käufer abnimmt. Die hohe Beachtung der Kriterien durch die Metzgerei-Käufer ist erwartungskonform mit den Ergebnissen von SPILLER und SCHULZE (2008) sowie WEINRICH et al. (2015), die eine Präferenz für artgerechte Tierhaltung am ehesten bei Metzgerei-Käufern sehen.

Aus den Ergebnissen der vorliegenden Studie kann geschlossen werden, dass die Metzgerei-Käufer am aufgeschlossensten gegenüber den drei Kaufargumenten Tierwohl, Umwelt und Gesundheit sind und Metzgereien demnach von allen untersuchten Einkaufsstätten das höchste Vermarktungspotenzial für Tierwohlprodukte haben. Metzgerei-Käufer gehen jedoch grundsätzlich eher davon aus, dass die Fleisch- und Wurstwaren vom Metzger aus einer artgerechteren Haltung stammen. 
Dies lässt sich ohne ein glaubwürdiges Tierwohl-Label mit einer Third-PartyZertifizierung oder ohne eine Hofbesichtigung gleichwohl nicht unbedingt nachvollziehen. Die Aussprache von Handlungsempfehlungen bzgl. des Einsatzes von Tierwohl-Labels beim Metzger kann daher erst erfolgen, nachdem im Rahmen weiterer Forschungsprojekte in Kaufexperimenten die Frage beantwortet werden konnte, ob Metzgerei-Käufer höherpreisige zertifizierte Tierwohlprodukte kaufen würden und vor allem welche Tierwohl-Labels Metzgerei-Käufer präferieren. Welche konkreten Tierwohl-Labels Metzgerei-Käufer am ehesten berücksichtigen würden, konnte in der vorliegenden Studie ermittelt werden und spezifiziert dadurch die Ergebnisse von SPILLER und SCHULZE (2008) sowie WEINRICH et al. (2015).

Des Weiteren wurden signifikante Unterschiede bei der Beachtung der drei Kriterien Tierwohl, Umwelt und Gesundheit im Hinblick auf die Angebotsform, sowohl für Wurst und Aufschnitt als auch für Fleisch zum Braten und Grillen, festgestellt. Käufer, die häufiger an der Fleischtheke kaufen, achten demnach häufiger auf eine artgerechtere Tierhaltung und Umweltfreundlichkeit als SB-Bereich-Käufer. Tendenziell eignen sich Tierwohlprodukte somit mehr für die Fleischtheke. Dieser Befund ist ebenfalls konform mit den Ergebnissen von SPILLER und SCHULZE (2008) sowie WEINRICH et al. (2015). Die Korrelation war jedoch so schwach, dass nicht interpretiert werden kann, dass im SB-Bereich keine Tierwohlprodukte angeboten werden sollten.

Hinsichtlich der soziodemografischen Kriterien konnten weitere Auffälligkeiten identifiziert werden. So achten Frauen signifikant häufiger auf die Kriterien Tierwohl, Umwelt und Gesundheit als Männer. Des Weiteren berücksichtigen die älteren Befragten diese drei Kriterien beim Einkauf von Fleisch- und Wurstwaren häufiger als die jüngeren Studienteilnehmer. $\mathrm{Zu}$ ähnlichen Ergebnissen kamen auch SPILLER und SCHULZE (2008). Interessanterweise ließen sich bezüglich der Beachtung dieser Kaufkriterien in den Regionen (Nord, Süd, Ost, West) sowie nach Ortsgröße keine nennenswerten Unterschiede eruieren. Hier wurde erwartet, dass Verbraucher in Großstädten stärker auf diese Kriterien achten als Verbraucher auf dem Land. Daher bedarf es bzgl. dieser drei Kriterien z. B. für die Werbung in Deutschland keiner geographischen Differenzierung. 
Hinsichtlich des Einkommens ergaben sich nur beim Kriterium artgerechte Tierhaltung signifikante Unterschiede. So achten Verbraucher mit höherem Einkommen beim Fleischkauf mehr auf eine artgerechte Tierhaltung. Dies unterstreicht die Vermutung, dass es sich bei nachhaltigkeitsinteressierten Käufergruppen um eher einkommensstarke Personen handelt, die eine attraktive Zielgruppe für den Lebensmitteleinzelhandel darstellen. Obwohl das Einkommen üblicherweise hoch mit der Bildung korreliert, konnten in der vorliegenden Studie keine signifikanten Unterschiede zwischen den drei Kaufkriterien Tierwohl, Umwelt und Gesundheit und dem Kriterium Bildung festgestellt werden.

Zusätzlich wurde in der vorliegenden Studie herausgefunden, dass Haustierbesitzer signifikant häufiger auf eine artgerechtere Tierhaltung achten als Verbraucher, die kein Haustier besitzen. Hier bietet sich folglich die Möglichkeit, das Problem des Absatzes teurerer Kuppelprodukte (durch die besseren Haltungsbedingungen) durch eine potenzielle Mehrzahlungsbereitschaft von Haustierbesitzern für Tierwohl-Heimtierfutter abzumildern. Da immer mehr Verbraucher auch bei Heimtierfutter auf besondere Produktqualitäten wie z. B. eine Bio-Zertifizierung achten, werden weitere Forschungsprojekte benötigt, um herausfinden zu können, inwiefern die in dieser Arbeit diskutierten Tierwohl-Labels differenziert für die Vermarktung von Premium-TierwohlHeimtierfutter eingesetzt werden können (DUARTE, 2019; PIRSICH et al., 2017; SCHLEICHER et al., 2019). Teilweise übertragen Haustierbesitzer die Bedürfnisse ihrer Heimtiere auch auf die Tiere in der Nutztierhaltung (SCHULZE \& SPILLER, 2008).

Abschließend für den Abschnitt Einkaufs- und Ernährungsverhalten wurden die Probanden zu ihren Sortenpräferenzen befragt. Diesbezüglich wurde deutlich, dass die Befragten am häufigsten Aufschnitt und Wurstwaren kaufen. Demzufolge scheinen Aufschnitt und Wurst als Brotbelag für die meisten Befragten zum täglichen Frühstück oder Abendbrot dazuzugehören und trotz relativ langer Haltbarkeit weniger auf Vorrat gekauft zu werden. Frische Fleischwaren werden dagegen von den meisten Befragten nur zwischen einmal in der Woche und alle vier Wochen gekauft. 
Von den frischen Fleischwaren kaufen die Befragten mit Abstand am häufigsten Geflügel und eher seltener Schweine- und Rindfleisch. Tiefgekühlte Fleischwaren, Tiefkühlgerichte, Dosen-, Tüten- und Glaswaren sowie Wurstsnacks werden aufgrund ihrer langen Haltbarkeit dagegen in deutlich größeren zeitlichen Abständen gekauft. Zu den gekauften Mengen kann in der vorliegenden Studie keine konkrete Aussage getroffen werden. Womöglich kaufen die meisten Menschen Wurstwaren und Aufschnitt eher in kleineren Mengen ein und frisches Fleisch in deutlich größeren Mengen. Im Fokus der Frage zu den Sortenpräferenzen stand jedoch nicht die Menge, sondern es waren lediglich die unterschiedlichen präferierten Sorten und deren Kaufhäufigkeit von Interesse. Insgesamt gehört Schweinefleisch in Deutschland zu den Fleischarten, die immer weniger gekauft werden, während Rind und Geflügel leichte Zuwächse verzeichnen können. Dagegen wächst die Nachfrage nach ökologisch produzierten frischen Fleischwaren weiter stark an.

Zusammenfassend lässt sich sagen, dass die Erkenntnisse zum allgemeinen Einkaufs- und Ernährungsverhalten, die primär für die Analyse der Bedeutung von Tierwohl-Labels in der Befragung erhoben wurden (Unterkapitel 6.2), in zahlreichen Studien bestätigt wurden, was die Aussagekraft der vorliegenden Studienergebnisse insgesamt stärkt. Unterschiede zu bestehenden Studien wurden in diesem Abschnitt ebenfalls beschrieben.

\subsection{Tierwohl-Labels als Einkaufshilfe}

In diesem Abschnitt werden die Ergebnisse der Befragung zu Tierwohl-Labels als Einkaufshilfe (Unterkapitel 5.3) interpretiert und diskutiert. Die Struktur dieses Abschnitts orientiert sich dabei an der Struktur des Ergebniskapitels. Es werden die verschiedenen Aspekte der Beachtung von Tierwohl-Labels, Hinderungsgründe für den Kauf sowie das Produkt- und Beratungsangebot an Tierwohlprodukten diskutiert. Handlungsempfehlungen und weiterer Forschungsbedarf werden an geeigneter Stelle aus der Ergebnisinterpretation abgeleitet. 
Die Ergebnisse der Studie verdeutlichen, dass über $90 \%$ der befragten Verbraucher Interesse an Tierwohlprodukten haben. In der Fragestellung (Frage 18) waren mit einer guten Verfügbarkeit, einer vertrauenswürdigen Kennzeichnung sowie einem Preis, der nicht zu stark vom Preis konventioneller Produkte abweicht, bewusst drei Voraussetzungen vorgegeben, die für die meisten Verbraucher von zentraler Bedeutung sind. Die Ergebnisse sind demzufolge nicht als Marktpotenziale zu interpretieren, sondern zeigen, dass die Befragten Tierwohlprodukte womöglich anderen Produkten vorziehen würden, wenn alle drei dargestellten Voraussetzungen erfüllt sind. Damit konnte belegt werden, dass die Verfügbarkeit der Produkte und deren vertrauenswürdige Kennzeichnung sowie der Preis drei wesentliche Erfolgskriterien für die Vermarktung von Tierwohlprodukten sind. Diese drei Faktoren haben eine große Bedeutung für die Einkaufsstättenwahl (STAUS, 2010; WEINRICH et al., 2015). Daher ist die Gestaltung eines adäquaten Produktangebots, in dem diese drei Faktoren berücksichtigt werden, von zentraler Bedeutung. Die Ergebnisse der vorliegenden Befragung zu den Kaufkriterien (Abbildung 9) legen nahe, dass Verbraucher ethische Kriterien als bedeutsamer bewerten als den Preis der Produkte. ENNEKING et al. (2019) konnten jedoch nachweisen, dass nur rd. 15 bis $30 \%$ der Verbraucher mehr Geld für Tierwohlprodukte ausgeben.

Ein zentrales Ziel der vorliegenden Studie war zudem die Messung der Bekanntheitsgrade der ausgewählten Tierwohl-Labels, da dem Bekanntheitsgrad u. a. von SPILLER und SCHULZE (2008) eine große Bedeutung zugesprochen wird. Dahingehend konnte festgestellt werden, dass die Bio-Siegel von allen Tierwohl-Labels die höchsten Bekanntheitsgrade aufweisen. Diese Ergebnisse stimmen mit der in Kapitel 3 vorgestellten Studie von ZÜHLSDORF et al. (2016) überein. Da die Bio-Labels neben Fleisch- und Wurstwaren auch auf vielen anderen Produkten, wie etwa Obst oder Gemüse, vorzufinden sind und seit vielen Jahren existieren, sind die Bekanntheitsgrade deutlich höher als bei den nichtökologischen Tierwohl-Labels. Das bekannteste Gütesiegel ist das deutsche DE-BIO-Siegel mit $87 \%$. Das BIOLAND-Label erreichte in der vorliegenden Studie, wie bei ZÜHLSDORF et al. (2016), den zweithöchsten Bekanntheitsgrad und somit einen höheren Bekanntheitsgrad als das deutlich weiter verbreitete EU-BIO-Siegel. 
Erklärungsansätze hierfür könnten sein, dass sich die Verbraucher das BIOLAND-Label aufgrund der Label-Gestaltung besser einprägen können als das EU-BIO-Logo und dass der BIOLAND-Verband (teilweise gemeinsam mit Lebensmitteleinzelhändlern wie LIDL) regelmäßig Werbekampagnen durchführt. Das NEULAND-Label kannte rd. ein Fünftel aller Befragten (21\%). Damit ist NEULAND das bekannteste Tierwohl-Label, das kein Bio-Label ist. Dies bestätigen auch ZÜHLSDORF et al. (2016), die ebenfalls einen Bekanntheitsgrad von $21 \%$ ermittelten. Das Label der INITIATIVE TIERWOHL sowie den HALTUNGSKOMPASS von LIDL kennen rd. $16 \%$ der Befragten.

ZÜHLSDORF et al. (2016) hatten in ihrer Studie für die INITIATIVE TIERWOHL einen Bekanntheitsgrad (ebenfalls gestützt) von nur knapp $4 \%$ ermittelt. Beim Vergleich der beiden Studien kann festgestellt werden, dass sich der Bekanntheitsgrad der INITIATIVE TIERWOHL innerhalb weniger Jahre vervierfacht hat, während der Bekanntheitsgrad der anderen Tierwohl-Labels ungefähr gleich geblieben ist (ZÜHLSDORF et al., 2016). Dies ist womöglich auf die stark gewachsene Verfügbarkeit des Labels der INITIATIVE TIERWOHL im deutschen Lebensmitteleinzelhandel zurückzuführen. Hieraus könnte ein starker Zusammenhang zwischen der Verfügbarkeit der Produkte und dem Bekanntheitsgrad abgeleitet werden.

Interessant ist ebenfalls, dass das geplante Tierwohl-Label der Bundesregierung in der vorliegenden Studie einen Bekanntheitsgrad von $13 \%$ erreichte, obwohl dieses Gütesiegel noch gar nicht existiert. Ein möglicher Erklärungsansatz hierfür wäre, dass sich die Gestalter des geplanten staatlichen Tierwohl-Labels offensichtlich stark an der sechseckigen Form des deutschen Bio-Siegels orientiert haben.

Die ähnliche Erscheinungsform könnte somit dazu geführt haben, dass es in dieser Studie bei der Befragung womöglich zu einigen Verwechslungen dieser beiden Gütesiegel auf Seiten der Verbraucher kam. Infolgedessen wäre dem Bundesministerium für Ernährung und Landwirtschaft zu empfehlen, dass sich die Gestaltung des staatlichen TierwohlLabels deutlicher vom deutschen BIO-Siegel absetzen sollte, um eine Verwirrung der deutschen Verbraucher in diesem Punkt zu vermeiden. 
Das Tierwohl-Label des DEUTSCHEN TIERSCHUTZBUNDES erreichte einen Bekanntheitsgrad von $11 \%$. ZÜHLSDORF et al. (2016) ermittelten ebenfalls $11 \%$ für das Tierwohl-Label des DEUTSCHEN TIERSCHUTZBUNDES, was für eine Stagnation des Bekanntheitsgrads spricht. Die übrigen Tierwohl-Labels von VIER PFOTEN und BIOKREIS erreichten mit rd. $5 \%$ nur sehr geringe Bekanntheitsgrade. BIOKREIS-Produkte finden sich vorzugsweise in Bio-Supermärkten, die insgesamt nur eine geringe Marktdurchdringung haben, wie in Unterkapitel 5.2.1 beschrieben. Überraschend sind die hohen Übereinstimmungen der Bekanntheitsgrade mit den Ergebnissen von ZÜHLSDORF et al. (2016). Dies spricht für die Aussagekraft der statistischen Analysen der vorliegenden Arbeit.

In der vorliegenden Studie wurde des Weiteren ein signifikanter Zusammenhang zwischen dem Bekanntheitsgrad der Tierwohl-Labels und der Einkaufsstätte festgestellt. Das DEMETER-Siegel und das NATURLAND-Siegel sind beispielsweise bei Metzgerei-Käufern deutlich bekannter als bei SB-Warenhaus-Käufern. Des Weiteren konnte eruiert werden, dass signifikant mehr Frauen DEMETER und EU-BIO kennen als Männer. Bei den anderen Tierwohl-Labels bestehen beim Geschlecht keine signifikanten Unterschiede. Signifikante Unterschiede ließen sich jedoch hinsichtlich des Alters der Befragten bei den Tierwohl-Labels BIOLAND, EU-BIO, DEMETER, dem TierwohlLabel des DEUTSCHEN TIERSCHUTZBUNDES sowie DE-BIO erheben. Ältere Personen kennen eher BIOLAND und DEMETER, während jüngere Personen eher EUBIO, DE-BIO und das Label des DEUTSCHEN TIERSCHUTZBUNDES kennen. Zudem hat die Analyse ergeben, dass BIOLAND und DEMETER im Süden und im Westen bekannter sind als im Norden und im Osten. Bezüglich der Ortsgröße ist NEULAND in Großstädten signifikant bekannter als in mittleren oder kleinen Städten bzw. auf dem Land.

Ein für die Vermarktung von Tierwohlprodukten interessantes Ergebnis ist, dass die Tierwohl-Labels DEMETER, BIOLAND und NATURLAND bei Personen mit höherem Einkommen (> $4500 € \mathrm{HH}-$ Nettoeinkommen im Monat) eine höhere Bekanntheit haben. Beim Kriterium Bildung zeigten sich bei allen acht untersuchten Tierwohl-Labels signifikante Unterschiede. Bio-Labels sind bei Personen mit höherer Bildung bekannter. 
Diese Aspekte sollten Hersteller und Händler von Fleisch- und Wurstwaren bei der Vermarktung von Tierwohlprodukten bzw. der Auswahl von Tierwohl-Labels für bestimmte Zielgruppen beachten.

Insgesamt können aus der Bekanntheitsgradmessung zudem verschiedene weitere Schlussfolgerungen gezogen werden. Zum einen eignen sich Bio-Labels und das NEULAND-Label durch die hohen Bekanntheitsgrade am ehesten für den Einsatz bei der Vermarktung von Tierwohlprodukten. Zum anderen erzielen die eher unbekannten Tierwohl-Labels derzeit vor allem aufgrund der geringen Verfügbarkeit noch nicht die notwendige Bekanntheit. Die kleineren Gütesiegelherausgeber müssten womöglich mit mehreren großen Lebensmitteleinzelhändlern oder großen Fleisch- und Wurstwarenhersteller kooperieren, da sie aus eigenen Mitteln nicht die hohen Investitionen in den Aufbau eines relevanten Bekanntheitsgrads aufbringen können. Der Bekanntheitsgrad hängt von zahlreichen Faktoren ab, so etwa von der Verfügbarkeit in den verschiedenen Einkaufsstätten, der Zeit am Markt und davon, ob es das TierwohlLabel neben Fleisch- und Wurstwaren auch für andere Lebensmittel oder gar andere Produktkategorien (z. B. Naturkosmetik oder Mode) gibt.

In Anbetracht der insgesamt hohen Anzahl an Tierwohl-Labels, von denen in dieser Studie zwölf Kennzeichnungen untersucht wurden, und der überwiegend niedrigen Bekanntheitsgrade der reinen Tierwohl-Labels wäre evtl. eine Art Label-Konsolidierung zu empfehlen (Reduktion auf eine geringe Anzahl strenger Tierwohl-Label). Neuere Tierwohl-Labels bedürfen sehr hoher Werbeinvestitionen in den Bekanntheitsaufbau. Bei zu vielen Labels (,Label-Flut') ergibt sich zudem ein negativer Effekt auf die Bekanntheitsgrade (FRANZ et al., 2012; MALHOTRA, 1984).

Neben dem Bekanntheitsgrad der Tierwohl-Labels wurde auch die Berücksichtigung beim Kauf erfragt. Mit Abbildung 15 des Ergebnisteils wurde veranschaulicht, dass von den Tierwohl-Labels, die die Befragten bereits gesehen haben, vor allem Bio-Labels beim Kauf berücksichtigt werden. Interessant ist auch, dass fast ein Viertel der Befragten angab, dass sie Tierwohl-Labels nur, vielleicht gekauft‘ hätten. 
Dies macht deutlich, dass dieser Teil der Verbraucher beim Einkauf nicht genau auf Tierwohl-Labels geachtet bzw. sich diese nicht gemerkt hat oder sie nicht zuordnen konnte. Nur etwa jeder zehnte Verbraucher gab an, die weniger bekannten TierwohlLabels beim Kauf berücksichtigt zu haben.

Insgesamt haben jedoch $70 \%$ der Befragten beim Kauf von Fleisch- und Wurstwaren bereits einmal bewusst auf Bio-Labels geachtet. Angesichts der niedrigen Marktanteile von Bio-Produkten bei Fleisch- und Wurstwaren erscheint dieser Anteil hoch. Eine Erklärung für diese Diskrepanz lässt sich nur schwer finden. Gegebenenfalls haben die Befragten überlesen, dass es in der Frage explizit um die tatsächliche Berücksichtigung der vorgestellten Gütesiegel beim Kauf von Fleisch- und Wurstwaren ging. Eventuell hatte auch die vorhergehende Frage 10 zum Bekanntheitsgrad der Gütesiegel einen Einfluss auf das Antwortverhalten, da sich diese Frage bewusst auf den allgemeinen Lebensmitteleinkauf bezog.

Um eine Gesamteinschätzung der Label-Präferenzen aller Befragten zu den untersuchten Tierwohl-Labels zu erhalten, wurden alle Studienteilnehmer in Frage 15 mit Hilfe einer fünfstufigen Likert-Skala gefragt, welche Tierwohl-Labels sie beim Einkauf von Fleischund Wurstwaren berücksichtigen würden und welche nicht.

In Abbildung 16 waren im Ergebnis die beliebtesten Tierwohl-Labels in absteigender Reihenfolge gelistet. Hier zeigten sich Parallelen zu den Ergebnissen hinsichtlich der Frage, welche der Tierwohl-Labels die Befragten bereits beim Kauf berücksichtigt haben (Frage 12), sowie zur Messung des Bekanntheitsgrads (Frage 10).

Das DE-BIO-Siegel, das BIOLAND-Siegel sowie das EU-BIO-Siegel sind diejenigen Tierwohl-Labels, die die meisten Befragten beim Kauf von Fleisch- und Wurstwaren am ehesten berücksichtigen würden (ZÜHLSDORF et al., 2016). Im Unterschied zum Bekanntheitsgrad und dem Kauf der Label würden die Befragten jedoch das TierwohlLabel des DEUTSCHEN TIERSCHUTZBUNDES insgesamt eher berücksichtigen als die Bio-Siegel von DEMETER und NATURLAND. 
Diese Besonderheit könnte evtl. damit erklärt werden, dass der Begriff ,Tierschutzbund“ im Namen des Tierwohl-Labels des DEUTSCHEN TIERSCHUTZBUNDES mehr dem Wunsch der Befragten nach mehr Tierwohl entspricht, als dies bei den Namen DEMETER oder NATURLAND der Fall ist.

Diese Ergebnisse können Herstellern und Händlern wertvolle Hinweise darüber liefern, welche der untersuchten Tierwohl-Labels sich für welche Zielgruppen und für welche Einkaufsstätten eignen. In der Studie konnten diesbezüglich zahlreiche signifikante Unterschiede festgestellt werden. Bei Betrachtung der Mittelwerte in Abbildung 17 würden Supermarkt-Käufer z. B. eher BIOLAND, NATURLAND und NEULAND kaufen als Discounter-Käufer.

Das Label des DEUTSCHEN TIERSCHUTZBUNDES würden Supermarkt-Käufer eher berücksichtigen als Metzgerei-Käufer. DEMETER würden Supermarkt-Käufer eher berücksichtigen als SB-Warenhaus-Käufer und Discounter-Käufer. Interessant ist, dass Metzgerei-Käufer am ehesten DEMETER berücksichtigen würden. Des Weiteren würden das Label der INITIATIVE TIERWOHL weniger Metzgerei-Käufer berücksichtigen als Supermarkt-Käufer und Discounter-Käufer.

Darüber hinaus stellt sich Anbietern von Fleisch- und Wurstwaren die vermarktungsrelevante Frage, welche der in Deutschland bekannten Tierwohl-Labels sich eher für die Fleischtheke oder den SB-Bereich eignen. Die Ergebnisse der Studie können dabei helfen, hierauf eine Antwort zu finden. Je häufiger die Verbraucher an der Fleischtheke kaufen, desto höher ist beispielsweise die Präferenz für Bio-Labels und das NEULAND-Label vice versa (WEINRICH et al., 2015).

Bei SB-Käufern steigt die Präferenz für das Label der INITIATIVE TIERWOHL, je häufiger die Befragten im SB-Bereich Fleisch- und Wurstwaren kaufen. Dabei macht es keinen wesentlichen Unterschied, ob es sich um Wurst oder Aufschnitt oder Fleisch zum Braten oder Grillen handelt. NATURLAND und DEMETER würden zudem von Personen, die ihren Fleischkonsum bereits reduziert haben (z. B. Flexitariern), eher berücksichtigt werden als von ,Fleischfans', die keine Reduktionsbereitschaft aufweisen. 
Hinsichtlich der Kaufhäufigkeit von Fleisch- und Wurstwaren mit Tierwohl-Labels ist den Befragungsergebnissen aus Unterkapitel 5.3.1 zu entnehmen, dass nur $15 \%$ der Befragten meistens zertifizierte Tierwohlprodukte kaufen. Die Nachfrage tierschutzbewusster Konsumenten hat demnach einen eher geringen Einfluss auf die Angebotsseite, was auch NORWOOD und LUSK (2009) bestätigen. Jedoch kauft fast die Hälfte aller Befragten je nach Möglichkeit Tierwohlprodukte und ein weiteres Drittel kauft zumindest gelegentlich Tierwohlprodukte.

Diese Ergebnisse könnten derart interpretiert werden, dass es bisher nur einen relativ kleinen Teil an Verbrauchern gibt, die gezielt auf bestimmte Tierwohl-Labels beim Einkauf von Fleisch- und Wurstwaren achten und diese Produkte auch meistens kaufen. Der Großteil der Verbraucher (rd. $80 \%$ ) kauft Tierwohlprodukte wahrscheinlich eher spontan, wenn diese Produkte in der Einkaufsstätte entsprechend angeboten werden und das Gesamtpaket der Kaufkriterien den Kundenerwartungen entspricht.

Hinsichtlich des Multi-Labelings von Tierwohlprodukten beinhalten die Befragungsergebnisse (Abbildung 20), dass der Einsatz mehrerer zusätzlicher Gütesiegel zu den vorgestellten Tierwohl-Labels für viele Verbraucher ein positives Kaufargument darstellt. Die Befragten wählten durchschnittlich fünf unterschiedliche Gütesiegel von 25 möglichen Gütesiegeln aus. Die beliebtesten Gütesiegel waren die Gütesiegel von STIFTUNG WARENTEST, ÖKOTEST und FAIRTRADE, die rd. von der Hälfte aller Befragten angegeben wurden. Weitere beliebte Gütesiegel sind die ,Frei-von'-Siegel, die schwerpunktmäßig die Gesundheit aller Befragten betreffen, sowie verschiedene Regionalsiegel. Des Weiteren wählte rd. ein Viertel der Befragten Logos von Organisationen wie beispielsweise der DLG, dem TIERSCHUTZBUND oder dem NABU.

Den Studienergebnissen zufolge, jedoch entgegen der ursprünglichen Vermutung, haben die Logos von sportbezogenen Organisationen vermutlich keinen verkaufsfördernden Effekt. Hieraus kann geschlussfolgert werden, dass große Unterschiede zwischen den einzelnen Organisationen bestehen. 
Es kann demzufolge nicht die allgemeingültige Aussage getroffen werden, dass sich Logos von Organisationen grundsätzlich positiv auf die Kaufentscheidung auswirken. Die Auswahl der Organisation sowie die Passung zum konkreten Produkt können vermutlich stark unterschiedliche Effekte auf die Kaufentscheidung haben.

Das Risiko, dass sich zusätzliche Gütesiegel auf Produktverpackungen negativ auf die Kaufentscheidung auswirken, ist jedoch in den meisten Fällen als eher gering einzuschätzen. Es kann eher davon ausgegangen werden, dass zusätzliche Gütesiegel, die für die Verbraucher vergleichsweise unbedeutend sind, lediglich keinen Effekt auf die Kaufentscheidung haben und keine Beachtung finden.

,Frei-von'-Siegel, die nur einen eingeschränkten Personenkreis betreffen, wie beispielsweise Allergiker (z. B. bei Unverträglichkeiten von Gluten oder Laktose), haben der vorliegenden Studie zufolge für alle Nichtallergiker keinen verkaufsfördernden Effekt.

Insgesamt lässt sich aus diesen Ergebnissen ableiten, dass die Kombination von Tierwohl-Labels mit passend ausgewählten anderen Gütesiegeln die Kaufwahrscheinlichkeit von Fleisch- und Wurstwaren aus einer artgerechteren und nachhaltigeren Nutztierhaltung erhöhen kann. So könnte beispielsweise die Verpackung eines Tierwohlprodukts zusätzlich mit einem ÖKOTEST-Siegel, den verschiedenen beliebten ,Frei-von'-Siegeln, einem Regionalsiegel und einem Logo einer ausgewählten Organisation wie dem NABU (z. B. im Rahmen eines Cause-Related-MarketingProjekts) versehen werden.

Um die Effekte näher zu überprüfen, sollten auf Basis dieser Erkenntnisse verschiedene weitere Forschungsprojekte in Form von realen Kaufexperimenten durchgeführt werden. Letztlich bestehen dahingehend viele verschiedene Kombinationsmöglichkeiten. Welche konkreten Tierwohl-Labels mit welchen zusätzlichen Gütesiegeln kombiniert werden sollten, muss im Einzelfall von den Anbietern von Fleisch- und Wurstwaren entschieden werden. Als Hilfestellung für die Auswahlentscheidungen zum Multi-Labeling bieten die in Abbildung 20 dargestellten Ergebnisse eine erste Orientierung. 
Wie anhand der Ergebnisse in Unterkapitel 5.3.2 ersichtlich existieren zahlreiche Hinderungsgründe beim Kauf zertifizierter Tierwohlprodukte. Als größte Kaufbarriere für Fleisch- und Wurstwaren, die mit den vorgestellten Tierwohl-Labels gekennzeichnet sind, wurde im Rahmen der Befragung das Vertrauen in die Metzger identifiziert. Fast die Hälfte aller Studienteilnehmer (45 \%) nannte den Einkauf beim Metzger als Grund dafür, dass sie keine Produkte mit Tierwohl-Labels kaufen.

Weitere Gründe sind vor allem Vermarktungsdefizite (Produktplatzierung, Werbung etc.) und eine zu geringe Transparenz bzgl. Preisgestaltung und Informationen. Manchen Verbrauchern fehlt auch einfach das Geld für Tierwohlprodukte, wie die vorliegende Studie zeigt. Diese Gründe verhindern die so oft geforderte höhere Wertschätzung von Lebensmitteln mit besonderen Produktqualitäten, was auch der Bundesverband des Deutschen Lebensmittelhandels in einer Studie zur Wertschätzung von Lebensmitteln bestätigen konnte (BVLH, 2020).

Dass der Metzger manchen Verbrauchern als besonderes Qualitätskriterium ausreicht, lässt sich durch die Ergebnisse der Studie ÖKOBAROMETER bestätigen (BMEL, 2016). Dort hatten $71 \%$ der Befragten, die Bio-Lebensmittel konsumieren (aber nicht ausschließlich Bio-Fleisch kaufen) angegeben, kein Bio-Fleisch zu kaufen, weil sie ihrem Metzger vertrauen würden. Der Metzger wurde außerdem als bevorzugter Ort für den Kauf von Bio-Fleisch angegeben (BMEL, 2016). Obwohl sich die Befragtengruppe des ÖKOBAROMETERS und die Art der Produkte (nur Bio-Produkte) von der vorliegenden Studie unterscheiden, wird dennoch die besondere Wahrnehmung des Metzgers deutlich.

An dieser Stelle soll der Versuch unternommen werden, das Ergebnis der vorliegenden Studie zu interpretieren. Zunächst erscheinen diese Ergebnisse vor dem Hintergrund interessant, dass die meisten Metzger in Deutschland kein bzw. nur sehr wenig BioFleisch anbieten. Die Tiere, die für die Fleischwaren vom Metzger verarbeitet werden, stammen oftmals von ähnlichen Großbetrieben wie die Tiere, aus denen Fleischwaren für Discounter oder SB-Warenhäusern hergestellt werden. 
Daher sind die Fleischwaren bei einem Metzger, der keine zertifizierten Tierwohlprodukte anbietet, nicht allgemein mit höheren Tierwohlstandards für die Tiere gleichzusetzen, wie dies beispielsweise bei einer zertifizierten NEULAND-Metzgerei der Fall wäre. Dennoch scheint ein Teil der Verbraucher beim Metzgerkauf davon auszugehen, dass die Tiere artgerechter gehalten wurden (SCHULZE \& SPILLER, 2008). Dabei stellt das Vertrauen in den Metzger laut den Studienergebnissen auch für rd. $40 \%$ der Supermarkt-Käufer sowie rd. $30 \%$ der SB-Warenhaus-Käufer und DiscounterKäufer eine Kaufbarriere für Tierwohlprodukte dar.

Wie im Ergebnisteil in Unterkapitel 5.3.1 beschrieben äußerten einige Studienteilnehmer Kritik, dass beim Metzger keine Fleisch- und Wurstwaren mit einem Tierwohl-Label angeboten würden. Insgesamt könnte daher vermutet werden, dass das Angebot zertifizierter Tierwohlprodukte beim Metzger ausbaufähig ist. Die in dieser Befragung und in anderen Befragungen (SCHULZE \& SPILLER, 2008; WEINRICH et al., 2015) identifizierten Präferenzen für Tierwohl-Labels und die gleichzeitig bestehenden Präferenzen für die Frischetheke werden offensichtlich von den Metzgern derzeit noch nicht optimal bedient.

Von zentraler Bedeutung ist die Vertrauenswürdigkeit der Tierwohl-Labels, die eng mit der eingeschätzten Strenge der Tierwohl-Labels zusammenhängt. Die Ergebnisse der durchgeführten Befragung zur Einschätzung der Strenge der Gütesiegel (Unterkapitel 5.3.2) zeigen, dass die Verbraucher die unterschiedlich strengen Standards der TierwohlLabels in den drei Kategorien Tierwohl, Umwelt und Gesundheit überraschenderweise gut einschätzen konnten. Besonderheiten sind beispielsweise, dass das DE-BIO-Label als strenger eingeschätzt wurde als die tatsächlich strengeren Bio-Labels DEMETER, BIOLAND und NATURLAND.

Insgesamt wurden in den drei Kategorien Tierwohl, Umwelt und Gesundheit die BioLabels als strengste Tierwohl-Labels eingeschätzt. Als Besonderheit wurde das TierwohlLabel des DEUTSCHEN TIERSCHUTZBUNDES in der Kategorie Tierwohl als strenger eingeschätzt als DEMETER oder NATURLAND. 
Alle übrigen Tierwohl-Labels wurden als nur mittelmäßig oder gar nicht streng eingeschätzt (vgl. Unterkapitel 5.3.2). Als am wenigsten streng wurden die INITIATIVE TIERWOHL und der HALTUNGSKOMPASS von LIDL eingeschätzt. $\mathrm{Zu}$ ähnlichen Ergebnissen kamen auch ZÜHLSDORF et al. (2016).

Zusammenfassend lässt sich aus den Ergebnissen zur Einschätzung der unterschiedlichen Tierwohl-Labels schlussfolgern, dass die meisten Verbraucher Tierwohl-Labels in zwei (streng, nicht streng) bis maximal drei ,Qualitätsstufen“ wahrnehmen (streng, mittel, nicht streng). Die Mehrzahl der Verbraucher beurteilt die Unterschiede jedoch evtl. nur in diesen übergeordneten, Qualitätsstufen“. Eine klare Differenzierung der Verbraucher bezüglich der zentralen Kriterien Tierwohl, Umwelt und Gesundheit ist aus den Studienergebnissen nicht erkennbar. Abzuleiten ist hier folglich weiterer Forschungsbedarf, wobei eine umfassende und detaillierte kriterienbasierte Bewertung aller in dieser Studie untersuchten Tierwohl-Labels vorgenommen werden sollte.

Das Wissen der Befragten zu den Auswirkungen eines übermäßigen Fleischkonsums wurde erhoben, um festzustellen, welche kritisch diskutierten Themen die Verbraucher am ehesten mit dem übermäßigen Fleischkonsum in Deutschland verbinden.

Die in Unterkapitel 5.3.2 beschriebenen Ergebnisse legen nahe, dass die meisten Befragten negative Auswirkungen auf die Tiere, die Gesundheit der Menschen und die Umwelt vermuten. Diese Aspekte bilden demzufolge aus Verbraucherperspektive die drei wesentlichen Problemfelder der intensiven Nutztierhaltung (BMEL, 2015).

Die vorgestellten Ergebnisse untermauern die Fokussierung der durchgeführten Studie auf diese drei zentralen Aspekte, wie beispielsweise bei der näheren Analyse der Kaufkriterien (Unterkapitel 5.2.4) oder der Einschätzung der Strenge der TierwohlLabels (Unterkapitel 5.3.2). Bei der Vermarktung von Fleisch- und Wurstwaren aus einer artgerechteren und nachhaltigeren Nutztierhaltung sind daher die wesentlichen Vorteile für die Tiere, die menschliche Gesundheit und die Umwelt herauszuarbeiten, die als Grundlage für die Produktdifferenzierung von konventionellen Fleisch- und Wurstwaren dienen und in der Produktkommunikation eingesetzt werden können. 
Abschließend für diesen Abschnitt sollen die Ergebnisse der Befragung zum Produktund Beratungsangebot an Tierwohlprodukten im Lebensmitteleinzelhandel interpretiert und diskutiert werden. In Unterkapitel 5.3.2 wurde ersichtlich, dass mehrere Optimierungspotenziale bei der Vermarktung von mit Tierwohl-Labels gekennzeichneten Fleisch- und Wurstwaren vorhanden sind. Die größten Optimierungspotenziale bieten sich dabei hinsichtlich der Transparenz beim Engagement für eine artgerechtere Tierhaltung. Dieser identifizierte Themenkomplex betrifft schwerpunktmäßig das Informationsangebot $\mathrm{zu}$ Tierwohlprodukten und wird daher ausführlich in Unterkapitel 6.3 diskutiert.

Ein weiterer Bereich mit Optimierungspotenzial, der bzgl. des Produkt- und Beratungsangebots identifiziert werden konnte, ist die persönliche Beratung bei Tierwohlprodukten. Lebensmitteleinzelhändler, die Wert auf eine kompetente Beratung ihrer Kunden legen, sollten ihr Personal im Bereich Fleisch- und Wurstwaren gezielter hinsichtlich der unterschiedlich strengen Kriterien der verschiedenen Tierwohl-Labels schulen, um in diesem Bereich eine bestmögliche Beratung gewährleisten zu können.

Damit das Personal nicht nur die theoretischen Unterschiede zwischen den Standards kennt, wären Exkursionen zu unterschiedlichen landwirtschaftlichen Betrieben zu empfehlen. Um einen besseren Überblick zu erhalten, könnten die Mitarbeiter z. B. einen Betrieb der ökologischen Anbauverbände DEMETER, NATURLAND oder BIOLAND, einen Betrieb von NEULAND und einen konventionellen Betrieb besichtigen. Auf diesem Weg könnten ein deutlich höherer Wissenstand der Mitarbeiter und eine fundiertere Beratung der Kunden erreicht werden.

Weiteres Optimierungspotenzial konnte aus den Ergebnissen zur Kundenzufriedenheit (Abbildung 27) für die Sichtbarkeit der Tierwohlprodukte in Werbeprospekten, Plakaten und Anzeigen ermittelt werden. Statt nur Produkte im Lebensmitteleinzelhandel mit Tierwohl-Labels zu versehen, sollten diese Produkte stärker beworben werden. Während konventionelle Fleisch- und Wurstwaren, ebenso wie Alkohol und Süßwaren, stark beworben werden, finden sich vergleichsweise wenig Tierwohlprodukte in der Werbung. 
Unternehmen, die sich mit dem Angebot von Fleisch- und Wurstwaren aus einer artgerechteren und nachhaltigeren Nutztierhaltung von Wettbewerbern abgrenzen möchten, können in diesem Bereich die Kundenzufriedenheit noch weiter erhöhen. Daneben ist aus der Perspektive der Verbraucher die Ästhetik von SB-Verpackungen, Fleischtheken und Werbeprospekten verbesserungswürdig. Die Vermarktung von Fleisch- und Wurstwaren aus einer artgerechteren und nachhaltigeren Nutztierhaltung sollte nicht nur hinsichtlich der Produkte, sondern auch hinsichtlich der Ästhetik einen hohen Anspruch verfolgen. Die Anbringung eines anspruchsvollen Tierwohl-Labels und die gleichzeitige Vermarktung als reine Massenware (Bulk Product) unterstreichen nicht in ausreichendem Maße die Ernsthaftigkeit eines Engagements für mehr Tierwohl.

Bezüglich der Auffindbarkeit von Tierwohlprodukten in der Einkaufsstätte bestehen den Studienergebnissen zufolge ebenfalls noch Verbesserungsmöglichkeiten. So sind Metzgerei-Käufer diesbezüglich deutlich zufriedener als Supermarkt-Käufer, Discounter-Käufer oder SB-Warenhaus-Käufer. Dies ist hinsichtlich der eher geringen Angebotsvielfalt im Vergleich zu den anderen Betriebsformen leicht nachzuvollziehen.

In diesem Ergebnis drückt sich wiederum die Vermutung von SCHULZE und SPILLER (2008) aus, dass Metzgerei-Käufer Fleisch- und Wurstwaren vom Metzger generell als Tierwohlprodukte ansehen. In der vorliegenden Studie lag der Fokus jedoch auf mit Tierwohl-Labels gekennzeichneten Tierwohlprodukten, die in den meisten konventionellen Fleischereifachgeschäften derzeit noch selten auffindbar sind (HUDDLESTON et al., 2009).

Als Ergebnis der Frage 20 gaben die meisten Verbraucher an, dass sie sich einen eigenen Bereich für Tierwohlprodukte im Lebensmitteleinzelhandel wünschen würden. Eine weitere Analyse ergab in diesem Punkt überraschenderweise keine signifikanten Unterschiede über alle untersuchten Betriebsformen bzw. Einkaufsstätten. Dies bedeutet, dass sich zwei Drittel aller Verbraucher, unabhängig von der genutzten Einkaufsstätte, eine striktere räumliche Trennung von Tierwohlprodukten und konventionellen Produkten in den Einkaufsstätten wünschen. 
Beispielsweise könnte ein Bereich einer Fleischtheke oder eines SB-Regals ausschließlich für Fleisch- und Wurstwaren mit besonderen Tierwohl-Labels eingerichtet werden. Hieraus ergäbe sich der Vorteil, dass Kunden, die überwiegend oder ausschließlich Tierwohlprodukte kaufen, diese Produkte deutlich schneller finden, als wenn diese Produkte zusammen mit konventionellen Fleisch- und Wurstwaren in einem Bereich angeboten werden. Diesbezüglich empfehlen sich Forschungsprojekte, in deren Rahmen die Implementierung beider Varianten in der Praxis getestet wird sowie Vorund Nachteile im Hinblick auf die Vermarktung analysiert werden.

Das Produkt- und Beratungsangebot zum Thema artgerechtere Tierhaltung ist offensichtlich ein bedeutsamer Faktor bei der Gewinnung neuer Kunden oder der Bindung bestehender Kunden. Um herauszufinden, wie wichtig den Verbrauchern das Thema ist, wurden die Probanden in der Studie gefragt, ob sie ihre Haupteinkaufsstätte für Fleisch- und Wurstwaren wechseln würden, wenn andere Einkaufsstätten ein besseres Produkt- und Beratungsangebot zu Fleisch- und Wurstwaren aus einer artgerechteren Haltung anbieten.

Im Ergebnis wurde deutlich, dass der Großteil der Verbraucher (66\%) ihre Haupteinkaufsstätte tatsächlich wechseln würden, während rd. ein Drittel der Verbraucher unentschlossen war. Bekräftigt wird dieses Ergebnis dadurch, dass diese stark ausgeprägte Wechselbereitschaft bei allen untersuchten Einkaufsstätten gegeben war. Bei Betrachtung der Mittelwerte könnte die Vermutung aufgestellt werden, dass Supermarkt-Käufer die höchste Wechselbereitschaft besitzen. Discounter-Käufer und Metzgerei-Käufer weisen ungefähr eine gleichhohe Wechselbereitschaft auf. SBWarenhaus-Käufer würden am wenigsten ihre Haupteinkaufsstätte wechseln. Daraus kann geschlussfolgert werden, dass das Produkt- und Beratungsangebot an Tierwohlprodukten für zahlreiche Fleischkäufer eine große Rolle bei der Auswahl der Haupteinkaufsstätte spielt. Lebensmitteleinzelhändler sollten daher das Produkt- und Beratungsangebot für Tierwohlprodukte weiter ausbauen, um bedeutende Kundengruppen zu binden bzw. zu gewinnen. 


\subsection{Informationsbedarf bei Tierwohlprodukten}

Aus der Perspektive vieler Verbraucher besteht insbesondere bei Fleisch- und Wurstwaren ein hoher Bedarf an weiterführenden Informationen. Laut TNS EMNID (2014) sind dabei besonders Informationen $\mathrm{zu}$ den Haltungsbedingungen und zur Umweltfreundlichkeit erwünscht. SPILLER und NITZKO (2014) führen als Grund in diesem Zusammenhang die hohe Verunsicherung der Verbraucher durch die ,Consumer Confusion' auf.

Ziel der Frage 22 zum allgemeinen Informationsbedarf war es, zunächst herauszufinden, wieviel Prozent der Befragten nähere Informationen zu den Haltungsbedingungen der Tiere und zur Herkunft der Fleisch- und Wurstwaren erhalten möchten. Anhand der diesbezüglich gewonnenen Ergebnisse kann konstatiert werden, dass sich die deutliche Mehrheit der Verbraucher (rd. $90 \%$ ) für die Bereitstellung besserer Informationen zu den Haltungsbedingungen der Tiere und zur Herkunft der Fleisch- und Wurstwaren ausspricht (Abbildung 33). Das hohe Interesse an weiterführenden Informationen wird von ZÜHLSDORF et al. (2016) bestätigt, die diesbezüglich einen Anteil von $65 \%$ ermittelten.

Daraus könnte abgeleitet werden, dass der Informationsbedarf in den letzten Jahren noch weiter angestiegen ist. Die Informationsdefizite sollten folglich abgebaut werden und den Verbrauchern sollten bessere Informationen zur Verfügung gestellt werden. Nur wenn es gelingt, die Transparenz bei Tierwohlprodukten mit Hilfe der Bereitstellung zusätzlicher Informationen deutlich zu erhöhen, können Verbraucher die Unterschiede in der Tierhaltung differenzierter bewerten und dieses Wissen in ihre Kaufentscheidung bei Tierwohlprodukten miteinfließen lassen (LANCASTER, 1971).

Nach ZÜHLSDORF et al. (2016) ist der Wunsch nach mehr Informationen zudem in hohem Maße mit einer höheren Zahlungsbereitschaft korreliert. Ein höherer Preis würde jedoch nur gezahlt, wenn sich die Verbraucher wirklich sicher sein können, dass die Haltungsbedingungen für die Tiere bei höherpreisigen Produkten wirklich besser sind (ZÜHLSDORF et al., 2016). Bei der Bereitstellung von Informationen sollte gleichwohl ein ,Informationsoverload‘ verhindert werden. 
$\mathrm{Zu}$ viele Informationen, die womöglich zudem noch schwer verständlich und nicht nachvollziehbar sind, können statt zu einer höheren Transparenz lediglich zu mehr Verwirrung und Überforderung der Verbraucher führen. Generell würden Verbraucher laut ZÜHLSDORF et al. (2016) lieber einheitlichen gesetzlichen Standards vertrauen wollen, als sich mit freiwilligen Gütesiegeln und umfangreichen Informationen auseinandersetzen zu müssen. Hierauf wird in Unterkapitel 6.4 im Rahmen der Diskussion zu den Politikoptionen näher eingegangen.

In der vorliegenden Studie wurde die Übernahme von Kosten für diese zusätzlichen Informationen nicht explizit erfragt. Hier soll auf die Studie von SPILLER und NITZKO (2014) verwiesen werden, der zufolge rd. die Hälfte der Verbraucher es als Selbstverständlichkeit ansieht, dass Anbieter diese Informationen beim Verkauf der Produkte kostenlos bereitstellen. Die andere Hälfte der Verbraucher würde für diese zusätzlichen Informationen womöglich auch einen geringen Geldbetrag bezahlen.

Von Interesse war außerdem die Frage, wie das Informationsangebot aus der Perspektive der Verbraucher in der Praxis gestaltet werden sollte. Um diesbezüglich weitere Erkenntnisse zu gewinnen, wurden alle Verbraucher, die ein Interesse an Informationen zu den Haltungsbedingungen der Tiere und zur Herkunft der Fleisch- und Wurstwaren geäußert haben, in der Studie gefragt, wo sie sich gerne bezüglich der von ihnen gekauften Fleisch- und Wurstwaren über die Haltungsbedingungen der Tiere informieren möchten. Im Ergebnis konnten zwei zentrale Orte identifiziert werden, an denen sich die meisten Verbraucher (> $80 \%)$ am ehesten informieren möchten.

Als die zwei wesentlichen Orte wurden die Produktverpackung und die Verkaufsräume der Einkaufsstätte genannt. Die zentrale Bedeutung der Produktverpackung wurde u. a. auch in der Studie von TNS EMNID (2014) sowie von SPILLER und NITZKO (2014) bestätigt. Daneben gaben über $60 \%$ der Befragten an, dass sie sich gerne in Werbeprospekten und beim Verkaufspersonal über die Haltungsbedingungen der Nutztiere informieren möchten. Rund die Hälfte aller Befragten möchte sich zudem gerne auf einem Internetportal zur artgerechteren Tierhaltung informieren. 
Zur weiteren Differenzierung wurde in der vorliegenden Studie untersucht, ob sich diese Präferenzen hinsichtlich der genutzten Einkaufsstätte für Fleisch- und Wurstwaren unterscheiden. Dabei konnten lediglich signifikante Unterschiede bei der Beratung durch das Verkaufspersonal ermittelt werden. Nach Betrachtung der Mittelwerte würden sich Metzgerei-Käufer lieber beim Verkaufspersonal über die Haltungsbedingungen der Nutztiere und die Herkunft der von ihnen gekauften Fleisch- und Wurstwaren informieren als Discounter-Käufer. Dass Metzgerei-Käufer ein allgemein höheres Interesse an Informationen haben, konnten bereits WEINRICH et al. (2015) bestätigen. Da die persönliche Beratung in den meisten Discountern, im Gegensatz zu Metzgern, nicht im Vordergrund steht, sind diese Ergebnisse leicht nachvollziehbar. Des Weiteren ergab sich ein signifikanter Unterschied zwischen Metzgerei-Käufern und SB-Warenhaus-Käufern hinsichtlich der Informationsbereitstellung in der Einkaufsstätte (z. B. über ausgelegte Broschüren). Die Mittelwerte zeigten, dass sich Metzgerei-Käufer eher Informationen in der Einkaufsstätte wünschen als SB-Warenhaus-Käufer. Hinsichtlich der anderen Möglichkeiten wie beispielsweise Produktverpackungen, Werbeprospekte, Internetportale oder Medien konnten überraschenderweise keine signifikanten Unterschiede bei den Einkaufsstätten beobachtet werden.

Ein weiteres interessantes Ergebnis der Studie besteht darin, dass, je häufiger die Befragten an der Theke kaufen, desto eher möchten sie Tierwohlinformationen über Broschüren in der Einkaufsstätte oder auf der Produktverpackung erhalten und desto weniger wünschen sie sich eine persönliche Beratung durch das Verkaufspersonal. Diese Ergebnisse verwundern, da vermutet wurde, dass insbesondere Thekenkäufer sich lieber persönlich beim Verkaufspersonal informieren möchten.

Interpretiert werden könnten diese Ergebnisse evtl. so, dass die beiden Käufergruppen (Theke/SB) bei der Beantwortung der Frage den Fokus auf ein mögliches Optimierungspotenzial bei der Informationsbereitstellung gelegt haben. Theken-Käufer, die generell mit der persönlichen Beratung an der Theke zufrieden sind, wünschen sich demnach noch zusätzliche Informationen in den Verkaufsräumen oder auf der Produktverpackung. 
Eine Schwerpunktsetzung bei der Bereitstellung von Tierwohlinformationen auf Produktverpackungen und begleitende Informationsmaterialien in den Verkaufsräumen erscheint den Ergebnissen zufolge sinnvoll. Dabei sollten diese Informationen eher im Thekenbereich bereitgestellt werden als im SB-Bereich. Jedoch sollte auch das Verkaufspersonal im SB-Bereich potenzielle Rückfragen der Verbraucher zu besonderen tierwohlbezogenen Fragen kompetent beantworten können. Alle Mitarbeiter, die potenziell für derartige Rückfragen von Kunden konsultiert werden, sollten entsprechend geschult werden.

Insgesamt kann anhand der Ergebnisse empfohlen werden, dass sich die Bereitstellung von Tierwohlinformationen nicht auf die Anbringung eines Tierwohl-Labels auf der Produktverpackung beschränken sollte. Hier könnte ein integriertes TierwohlKommunikationskonzept entwickelt werden, das die präferierten Informationsorte in einem Gesamtkonzept sinnvoll und pragmatisch miteinander kombiniert.

Zunächst könnten auf der (möglichst umweltfreundlichen) Produktverpackung von SBProdukten, auf der sich die meisten Verbraucher generell am liebsten informieren möchten, neben dem Tierwohl-Label zusätzlich in kurzen Stichpunkten die wesentlichen Verbesserungen für Tierwohl, Umwelt und Gesundheit aufgeführt werden (Stufe 1: kürzeste Form an Infos). Ergänzt werden könnten auf jeder Verpackung ein Internetlink und/oder ein QR-Code, der zu detaillierteren Informationen auf einer Website (Stufe 3: sehr detaillierte Informationen) führt und direkt mit dem Smartphone abgerufen werden kann (KHEIRAVAR \& RICHTER, 2016; MAY, 2016; RIEBER, 2017; THOMAS et al., 2020). Um die Kaufwahrscheinlichkeit zu erhöhen, sollten die Tierwohl-Labels mit beliebten anderen Gütesiegeln (vgl. Unterkapitel 5.3.1) kombiniert werden.

In den Verkaufsräumen, in denen sich die meisten Verbraucher ebenfalls zusätzliche Informationen wünschen, könnten die Anbieter ergänzende Informationen zu den Haltungsbedingungen und zur Herkunft der Fleisch- und Wurstwaren mit Broschüren, Lieferantenlisten, Aufstellern oder Displays bereitstellen (Stufe 2: umfassendere Informationen als auf der Verpackung). 
Für die Fleischtheke könnten beispielsweise die Tüten, die für die Verpackung der Fleisch- und Wurstwaren verwendet werden, die Funktion der Produktverpackung als Informationsmedium übernehmen und mit Tierwohlinformationen bedruckt werden. Zusätzlich könnten mit kleinen Fähnchen oder Schildern die Tierwohlprodukte an der Fleischtheke mit den entsprechenden Tierwohl-Labels gekennzeichnet werden. Dabei wünschen sich den Studienergebnissen zufolge, wie bereits zur Sortierung der Produkte beschrieben, die meisten Verbraucher einen eigenen Bereich für konventionelle Produkte und einen eigenen Bereich für Tierwohlprodukte.

In den Werbeprospekten, der drittwichtigsten Informationsquelle für die Verbraucher, sollten die Tierwohlprodukte entsprechend beworben werden (z. B. auf einer eigenen Seite in den Prospekten). In diesem Rahmen sollten auch nähere Informationen zu den Vorteilen der Produkte für die Tiere, die Umwelt und die Gesundheit aufgeführt werden. Der Detaillierungsgrad der Tierwohlinformationen in den Prospekten sollte sich dabei an den Broschüren in der Einkaufsstätte orientieren (Stufe 2: umfassendere Information).

Hinsichtlich der eingesetzten Werbematerialien wäre es generell empfehlenswert, alle hier aufgeführten Informationskanäle hinsichtlich des Designs und des Inhalts im Rahmen eines 360-Grad-Kommunikationskonzepts besser aufeinander abzustimmen und zu vernetzen, um die Wiedererkennung der Tierwohl-Labels bzw. der entsprechenden Tierwohlprodukte im Lebensmitteleinzelhandel zu erleichtern. Die Vorteile für die Tiere, die Umwelt und die Gesundheit sollten verständlicher aufbereitet werden. Die Verwendung eines glaubwürdigen Tierwohl-Labels, das auch von Tierschutz- und Naturschutzorganisationen positiv bewertet wird, ist dabei für den Gesamterfolg der bedeutsamste Faktor, da sich an der Strenge eines Tierwohl-Labels ein ,GreenwashingKonzept $^{`}$ von einem seriösen Marketingkonzept für mehr Tierwohl unterscheidet.

Das Verkaufspersonal von Fleisch- und Wurstwaren sollte außerdem durch regelmäßige Tierwohl-Schulungen und Exkursionen $\mathrm{zu}$ landwirtschaftlichen Betrieben mit unterschiedlichen Haltungsstandards die Besonderheiten der einzelnen Tierwohl-Labels besser kennenlernen. 
Auf diesem Wege könnten detailliertere und kritischere Verbraucherfragen, die womöglich zukünftig verstärkt zu erwarten sind, in der Einkaufsstätte adäquater beantwortet werden. Wie beschrieben sollten alle Verkäufer im Lebensmittelbereich als kompetente Ansprechpartner in Tierwohlfragen zur Verfügung stehen und Auskunft zu den Unterschieden zwischen den Tierwohl-Labels geben können. Hierfür bietet sich eine Tierwohl-Schulung für die entsprechenden Mitarbeiter an.

Auf einem zentralen Tierwohl-Internetportal könnten außerdem für alle Hersteller und Händler von Fleisch- und Wurstwaren sowie die Verbraucher detaillierte Informationen zu den einzelnen Tierwohl-Labels in den Bereichen Tierwohl, Umwelt und Gesundheit mit den entsprechenden Unterschieden bereitgestellt werden (Stufe 3: sehr detaillierte Informationen). Diese Internetseite könnte auf allen Produktverpackungen sowie in Werbematerialien am Verkaufsort angegeben werden. Integriert werden könnte der Link zu einem derartigen Tierwohl-Portal auch in Werbeprospekte und auf den Internetseiten der Lebensmittelhersteller und -einzelhändler. Einzelne Landwirte und Metzgereien könnten ebenso von einem derartigen Internetportal profitieren. Sie könnten beispielsweise in eigenen Broschüren oder Postern auf die besonderen Tierwohlstandards der gewählten Zertifizierung ihres Betriebs (z. B. Neuland) aufmerksam machen und für weiterführende Informationen auf ein derartiges Tierwohl-Portal verweisen, um ihren Kunden maximal transparente Informationen zu den Tierwohlstandards anzubieten.

Ein derartiges zentrales deutsches oder europäisches Informationsportal rund um das Thema Tierwohl könnte einen Beitrag für mehr Transparenz schaffen und würde zudem verschiedene weitere Vorteile bieten. Zum einen könnten die Kosten für die Informationsbereitstellung für alle Marktteilnehmer reduziert werden, die derzeit alle für sich selbst Informationen rund um das Tierwohl aufbereiten, die jedoch meist unvollständig sind. Um einen Überblick über die vorgestellten Tierwohl-Labels zu erhalten, müssen Verbraucher daher eine Vielzahl an unterschiedlichen Internetquellen auswerten, die jedoch allesamt nur unvollständige Informationen anbieten. 
Beispielsweise beziehen sich die Informationen der Gütesiegelherausgeber in der Regel nur auf das eigene Tierwohl-Label. Des Weiteren fokussieren Informationen von NGOs häufig auf ein bestimmtes Schwerpunktthema (z. B. Umwelt) und Anbieter von Fleischund Wurstwaren beschränken sich lediglich auf ihr eigenes Produktangebot. Neben dem Aspekt des Tierwohls sollten auch die Vor- und Nachteile der verschiedenen TierwohlLabels in den Bereichen Umwelt- und Naturschutz und Gesundheit aufgeführt werden. Hierzu zählt auch die Herkunft des verwendeten Tierfutters.

Ein derartiges Tierwohl-Portal könnte im Rahmen eines Gemeinschaftsprojekts von Tierwohl-Label-Herausgebern, Lebensmittelherstellern und -einzelhändlern, Landwirten sowie Verbraucherschutz-, Tierschutz- und Naturschutzorganisationen unter wissenschaftlicher Beratung von Universitäten und Forschungseinrichtungen gemeinsam entwickelt und von Bundesministerien und der EU gefördert werden. Die Mitarbeit von Verbraucherschutz-, Tierschutz- und Naturschutzorganisationen ist für die Glaubwürdigkeit der bereitgestellten Informationen rund um das Thema Tierwohl von zentraler Bedeutung. Die Ergebnisse der Studie (Abbildung 37) unterstreichen einen derartigen interdisziplinären Ansatz, da diese Akteure aus Sicht der Verbraucher Informationen zu den Haltungsbedingungen der Tiere im Internet bereitstellen sollten.

Durch ein derartiges 360-Grad-Tierwohl-Informationskonzept in drei Stufen könnte sich jeder Verbraucher je nach Interesse über Tierwohl-Themen informieren; von oberflächlichen Informationen (Stufe 1) über umfassendere Überblicke (Stufe 2) bis hin zu fundierten Vergleichen einzelner Standards (Stufe 3). Verbraucher, die sich nicht für Tierwohl oder Nachhaltigkeit interessieren, können im Rahmen dieser Konzeption die angebotenen Informationen zudem leicht ignorieren.

Auch wenn davon auszugehen ist, dass die bereitgestellten Informationen nicht so stark genutzt werden, wie sie von den Verbrauchern gefordert werden, ermöglicht die Bereitstellung der Informationen dennoch, dass sich interessierte Verbraucher einfacher detailliert informieren können, als es derzeit der Fall ist. Allein durch die Bereitstellung umfassender, vergleichbarer und qualitativ hochwertiger Informationen kann ein Beitrag dazu geleistet werden, die Transparenz bei Tierwohlprodukten für die Verbraucher zu erhöhen. 
Abschließend für den Abschnitt Informationsbedarf wurden in der Befragung die Verbraucherpräferenzen zu Herkunftsangaben näher untersucht. Der Detaillierungsgrad der Herkunftsangaben ist vor dem Hintergrund der geforderten Transparenz bei Tierwohlprodukten von besonderer Bedeutung.

Anhand der Studienergebnisse (Abbildung 36) wird deutlich, dass die Angabe der groben Region (z. B. ,Hergestellt in Deutschland oder Niedersachsen') überraschenderweise nur für 1,8\% der Befragten eine ausreichende Information darstellt. Für fast $90 \%$ der Befragten wäre es jedoch ein starkes Kaufargument, wenn Anbieter von Fleisch- und Wurstwaren die Herkunft bis auf einen konkreten Bauernhof, von dem die Tiere stammen, transparent darstellen würden. Die Erkenntnis, dass die Rückverfolgbarkeit von Produkten eine große Bedeutung hat, bestätigten auch SPILLER und SCHULZE (2008).

Anbieter von Fleisch- und Wurstwaren, die mit einer artgerechteren und nachhaltigeren Nutztierhaltung werben, können die konkreten Anschriften von Bauernhöfen in die Unternehmens- und Produktkommunikation einbinden, um sich mit der hohen Transparenz bei der Herkunft der Tierwohlprodukte positiv von Wettbewerbern abzuheben. Dabei ist gleichwohl darauf hinzuweisen, dass Herkunftsangaben auch negative Effekte haben können (LAZO GALDOS, 2011). Daneben könnten Hersteller und Händler von Fleisch- und Wurstwaren aus einer artgerechteren Tierhaltung Kunden beispielsweise auch im Rahmen eines Gewinnspiels auf den Bauernhof zu einer Betriebsbesichtigung einladen, damit sie sich persönlich ein Bild von den besseren Haltungsbedingungen machen und über ihre Erfahrungen im Internet berichten können. Richtig umgesetzt kann die konkrete Angabe der Herkunft bis auf Hofebene das Vertrauen bei zahlreichen Verbrauchern erhöhen, sodass auf diesem Weg womöglich zahlreiche neue Kunden gewonnen werden können. 


\subsection{Politische Präferenzen und politische Partizipation}

In der gesellschaftlichen und politischen Diskussion zum Thema Tierwohl stehen in Deutschland verschiedene Lösungsansätze für mehr Tierwohl im Fokus, die im Rahmen der vorliegenden Arbeit von den befragten Verbrauchern bewertet wurden (Unterkapitel 5.3.4). Mit deutlichem Abstand sehen die meisten Verbraucher in einem strengeren Tierschutzgesetz mit strengeren Kontrollen und strengeren Sanktionen den besten und dringendsten Lösungsansatz für mehr Tierwohl in Deutschland.

Als weitere hilfreiche Lösungsansätze wurden eine gesetzlich verpflichtende Haltungskennzeichnung sowie die staatliche Förderung von ,mehr Tierwohl` z. B. durch Subventionen genannt. Der Ausbau der INITIATIVE TIERWOHL sowie der Einsatz freiwilliger Tierwohl-Labels wurden von den Verbrauchern dagegen als weniger relevant betrachtet, um das Wohl der Tiere in der Nutztierhaltung zu verbessern. Die verschiedenen politischen Ansätze werden von den Verbrauchern sehr unterschiedlich bewertet, wobei eine klare Tendenz zu strengeren marktregulierenden Eingriffen durch den Gesetzgeber zu erkennen ist.

Hoch signifikante Unterschiede ergaben sich bei dem Kriterium strengeres Tierschutzgesetz. Im post-hoc-Test zeigten sich signifikante Unterschiede zwischen den Wählern der Partei Bündnis90/DieGrünen und CDU-Wählern (Abbildung 39). Nach Betrachtung der Mittelwerte ist eine Gesetzesänderung aus Sicht der Partei Bündnis90/DieGrünen dringender und bedeutsamer als für CDU-Wähler. Interessant ist, dass CDU-Wähler gleichzeitig freiwillige Gütesiegel als relevanter beurteilen als die Wähler der Partei Bündnis90/DieGrünen. Trotz dieser Unterschiede sehen CDU-Wähler freiwillige Tierwohl-Labels nicht als bedeutendes Instrument für eine Verbesserung der Nutztierhaltung an.

Eine gesetzlich verpflichtende Haltungskennzeichnung für Fleisch- und Wurstwaren stufen die Verbraucher als zweitwichtigsten politischen Lösungsansatz für mehr Tierwohl ein. Eine derartige Haltungskennzeichnung könnte ergänzend die unterschiedlich strengen freiwilligen Standards in einem bundesweit einheitlichen und klar definierten Kategorisierungssystem mit Ampelfarben zuordnen und so für mehr Transparenz sorgen. 
Die staatliche Förderung von mehr Tierwohl wird als relevanter eingestuft als freiwillige Tierwohl-Labels und die private INITIATIVE TIERWOHL. Beispielhaft für eine staatliche Förderung von mehr Tierwohl seien an dieser Stelle die Zahlungen der EU für Maßnahmen zur Entwicklung des ländlichen Raums (ELER) genannt, die Betriebe für eine artgerechtere und nachhaltigere Nutztierhaltung belohnen sollen.

Dabei erhalten in der Regel zunächst die Bundesländer die finanziellen Mittel und zahlen die Gelder über eigene Förderprogramme an Landwirte aus. So vergütet das Förderprogramm für Agrarumwelt, Klimaschutz und Tierwohl des Landes BadenWürttemberg verschiedenen Landwirten den Mehraufwand für weiche Liegeflächen, Beschäftigungsmöglichkeiten und Auslauf bei Mastschweinen. Natur- und Tierschützer fordern diesbezüglich eine noch stärkere Umschichtung der EU-Subventionen von der 1. Säule (Direktzahlungen durch Flächenprämien) in die 2. Säule (ELER), um auf diesem Weg mehr Tierwohl und Naturschutz zu erreichen.

Zusammenfassend lässt sich schlussfolgern, dass die meisten Verbraucher in einem strengeren Tierschutzgesetz und der gesetzlich verpflichtenden Haltungskennzeichnung die beiden bedeutsamsten politischen Ansätze für eine verbesserte Nutztierhaltung sehen. Als relevant wird auch die Zahlung von Subventionen an Landwirte für mehr Tierwohl und Umweltfreundlichkeit betrachtet. Von freiwilligen Gütesiegeln und privaten Initiativen werden dagegen keine Verbesserungen in der deutschen Nutztierhaltung erwartet. Freiwillige Tierwohl-Labels und private Initiativen als Einkaufshilfe für Fleisch- und Wurstwaren werden jedoch von vielen Verbrauchern gerne genutzt. Hier gilt es klarer zu differenzieren.

In Unterkapitel 5.3.4 wurden die Ergebnisse hinsichtlich der Verbraucherpräferenzen zu verschiedenen Kennzeichnungsformen von Tierwohl-Labels vorgestellt. Diesbezüglich wurde deutlich, dass die meisten Verbraucher eine farbliche Ampel bevorzugen (Abbildung 40). Eine Kennzeichnung mit Ampelfarben ist demnach zu empfehlen. Nur ungefähr ein Viertel der Befragten befürwortet eine Kennzeichnung mit Sternen, wie beispielsweise beim Tierwohl-Label des DEUTSCHEN TIERSCHUTZBUNDES. Lediglich $14 \%$ der Befragten favorisieren eine Kennzeichnung mit Zahlen, die sich an der Kennzeichnung von Eiern in Deutschland orientiert. 
Die Verbrauchermeinung widerspricht damit der Meinung verschiedener Akteure, die die Angleichung der Fleischkennzeichnung an die Eierkennzeichnung fordern, wie z. B. der BUND ÖKOLOGISCHE LEBENSMITTELWIRTSCHAFT (BÖLW, 2018).

Insgesamt ist aus der Studie ein deutlicher Wunsch der Verbraucher nach einer möglichst klar nachvollziehbaren, ehrlichen und nicht manipulierbaren Tierwohlkennzeichnung erkennbar. Diesem Anspruch werden viele Tierwohl-Labels heute noch nicht gerecht. Weiterführende Analysen haben ergeben, dass es bei allen untersuchten Parteien keinen signifikanten Unterschied hinsichtlich der Präferenzen zu den Kennzeichnungsformen gibt.

Abschließend für die politikbezogenen Fragen der Studie konnten die Befragten Möglichkeiten bewerten, wie sie ihren persönlichen Einfluss auf eine verbesserte Tierhaltung in Deutschland einschätzen (Frage 28). Die in Abbildung 42 dargestellten Ergebnisse sind durchaus interessant. An erster Stelle mit deutlichem Abstand zu den anderen aufgeführten Möglichkeiten vermuten die Befragten, dass sie sich am ehesten durch ihr persönliches Einkaufs- oder Konsumverhalten für eine verbesserte Nutztierhaltung einsetzen können. Wie groß der Einfluss des Fleischkonsums der Verbraucher auf die Produktionsseite jedoch tatsächlich ist, lässt sich schwer ermitteln. Mit Blick auf den insgesamt sinkenden Fleischkonsum in Deutschland und die gleichzeitig steigenden Exportzahlen, kann konstatiert werden, dass ein verändertes Konsumverhalten deutscher Verbraucher einen geringeren Effekt auf die Nutztierhaltung in Deutschland hat, als die meisten Verbraucher annehmen.

Bereits an zweiter Stelle wurde die Möglichkeit der Unterstützung von NGOs in den Bereichen Tierschutz, Naturschutz und Verbraucherschutz angegeben. Dies ist insofern interessant, als die Unterstützung von NGOs als sinnvollere Möglichkeit zur Verbesserung der Nutztierhaltung angesehen wird als die Unterstützung einer Partei. Einige der Befragten sehen die Teilnahme an Petitionen als eine Möglichkeit an, sich persönlich für eine verbesserte Nutztierhaltung in Deutschland einzusetzen. Aus Sicht der meisten Befragten kann durch die Stimmabgabe bei politischen Wahlen oder die Teilnahme an Demonstrationen die Tierhaltung in Deutschland kaum positiv beeinflusst werden. 
Dass die Verbraucher die Teilnahme an politischen Wahlen als eine der letzten Möglichkeiten sehen, die Nutztierhaltung in Deutschland zu verbessern, könnte als Politikverdrossenheit der Verbraucher beim Thema Tierwohl interpretiert werden. Die politische Berücksichtigung des Themas Tierwohl hat, ebenso wie das Thema Umweltschutz, für viele Menschen in Deutschland eine immer größere Bedeutung, die sich in Zukunft noch weiter verstärken wird. Die mangelhafte politische Berücksichtigung des Themas Tierwohl hat in den letzten Jahrzehnten zu immer mehr Problemen in der Nutztierhaltung geführt. Dennoch wurde entgegen der Forderung nach mehr Tierwohl vieler Verbraucher (Wähler) nicht vom Wachstumskurs abgewichen und es wurden jedes Jahr immer noch mehr Fleisch- und Wurstwaren in Deutschland für den Export in über 100 Länder der Welt produziert. Das konsequente Außerachtlassen der Forderungen der deutlichen Mehrheit der Gesellschaft birgt jedoch das hohe Risiko eines weiter zunehmenden Vertrauensverlustes in die Demokratie.

\subsection{Motiv- und segmentspezifische Unterschiede}

Ein zentrales Ziel der vorliegenden Studie war es, ein differenziertes Bild zu den Tierwohl-Label-Präferenzen der Verbraucher zu gewinnen. In der gesellschaftlichen Diskussion zum Thema Tierwohl werden mit den ,Ökos` und den ,Billigfleischkäufern` häufig zwei Extremgruppen betrachtet. Um zu zeigen, dass es in der Gesellschaft ein vielfältigeres und differenziertes Meinungsbild hinsichtlich des Tierwohls gibt, sollte im Rahmen der vorliegenden Arbeit wissenschaftlich untersucht werden, welche Verbrauchergruppen diesbezüglich in Deutschland existieren. Damit soll den häufig stark verallgemeinernden Darstellungen in den Medien entgegengewirkt werden. Um dieses Ziel zu erreichen, wurde zunächst die Wahrnehmung zahlreicher Tierwohl-Labels mit Hilfe einer explorativen Faktorenanalyse untersucht. Anschließend wurden auf Basis der Tierwohl-Label-Präferenzen mit Hilfe einer Clusteranalyse unterschiedliche Verbrauchergruppen identifiziert und es wurde eine Verbrauchertypologie erstellt. In diesem Abschnitt sollen die Ergebnisse der Faktoren- und der Clusteranalyse interpretiert und diskutiert werden. Abschließend für diesen Abschnitt werden Handlungsempfehlungen je Marktsegment ausgesprochen. 


\subsubsection{Interpretation und Diskussion der Faktorenanalyse}

Die Bundesregierung, NGOs, Medien und weitere Institutionen sprechen den Verbrauchern regelmäßig Empfehlungen für die Berücksichtigung von Tierwohl-Labels beim Kauf von Fleisch- und Wurstwaren aus. Beispielhaft genannt seien hier die Angaben auf dem Tierwohl-Portal ,Tierwohl stärken“ des BMEL, in den ,Fleischführern` von GREENPEACE oder WWF, von Verbraucherschutzorganisationen (z. B. STIFTUNG WARENTEST, VERBRAUCHERZENTRALE etc.) sowie von zahlreichen Websites (BMEL, 2019; GREENPEACE, 2020; STIFTUNG WARENTEST, 2020; UTOPIA, 2019; VERBRAUCHERZENTRALE, 2020; WWF, 2020).

Mit Hilfe der in der vorliegenden Studie durchgeführten Faktorenanalyse konnten drei klar voneinander abgrenzbare Motivgruppen für verschiedene Arten von TierwohlLabels identifiziert werden. Die erste Gruppe der eher schwachen reinen Tierwohl-Labels ohne ökologische Kriterien besteht aus dem Label des DEUTSCHEN TIERSCHUTZBUNDES, dem Label von VIER PFOTEN, dem STAATLICHEN TIERWOHL-LABEL, dem HALTUNGSKOMPASS und der INITIATIVE TIERWOHL. Die zweite Gruppe umfasst die Premium-Tierwohl-Labels der ökologischen Anbauverbände DEMETER, BIOLAND, NATURLAND, BIOKREIS sowie NEULAND. Die staatlichen Bio-Labels (DE-BIO und EU-BIO) bilden die dritte klar abgrenzbare Gruppe. Gemäß den Ergebnissen der vorliegenden Studie lassen sich die drei Label-Motivgruppen deutlich voneinander unterscheiden. Die Gruppen wurden im Hinblick auf Soziodemografie, Einkaufsstättenwahl, Angebotsformen, (Tierwohl)Produktplatzierung, Reduktionsbereitschaft beim Fleischkonsum, Multi-LabelingPräferenzen und Kaufmotive untersucht.

Soziodemografisch ist ein Fan reiner Tierwohl-Labels eher weiblich, hat eine geringe Bildung und verfügt nur über ein geringes Einkommen. Ein Anbauverband-Bio-Fan ist ebenfalls etwas wahrscheinlicher eine Frau, die jedoch bereits etwas älter ist, eine sehr hohe Bildung aufweist und über ein sehr hohes Einkommen verfügt. Ein Fan von staatlichen Bio-Produkten ist dagegen soziodemografisch eine etwas jüngere Frau, die eher in einer Stadt wohnt und durch ihre hohe Bildung ein hohes Einkommen erzielt. 
Bei Betrachtung der Einkaufsstättenpräferenzen dieser drei Gruppen zeigten sich interessante und hoch signifikante Unterschiede. Die,Fans der reinen Tierwohl-Labels“ konnten als klassische Discounter-Käufer identifiziert werden. Dies zeigte sich an den von allen Gruppen höchsten Koeffizienten bei LIDL, PENNY, NETTO und ALDI. Die hohen Wald-Werte verdeutlichen die großen Unterschiede zu den anderen Gruppen.

Auch die SB-Warenhäuser KAUFLAND und REAL werden von den Fans reiner Tierwohl-Labels am häufigsten von allen drei Gruppen genutzt. Metzgereien werden von dieser Gruppe dagegen signifikant weniger genutzt als von den beiden BioKäufergruppen, die dagegen häufiger im Bio-Supermarkt einkaufen. Hofläden und Wochenmärkte werden von dieser Gruppe so gut wie gar nicht besucht, sodass der Einsatz reiner Tierwohl-Labels für diese beiden Einkaufsstätten nicht empfohlen werden kann.

Bei EDEKA und REWE ergaben sich keine signifikanten Unterschiede. Anhand der Koeffizienten kann jedoch vermutet werden, dass EDEKA von den Fans reiner TierwohlLabels etwas häufiger als Einkaufsstätte genutzt wird als REWE.

Diese Verbrauchergruppe besteht überwiegend aus Personen, die eine hohe Sonderangebotsaffinität aufweisen. Die Fans reiner Tierwohl-Labels sind jedoch nicht mit den sogenannten ,Smart Shoppern`zu verwechseln, die womöglich eher versuchen würden eine besonders hohe Qualität (z.B. DEMETER) zu einem relativ günstigen Preis zu erhalten (DAVID \& HILPERT, 2016; GÓMEZ-SUÁREZ et al., 2020; REDLER, 2018).

SPILLER et al. (2019) beziffern den Anteil der eher preisorientierten Verbraucher an der Gesamtbevölkerung bei den Fleischkäufern auf nur etwa $20 \%$. Vor diesem Hintergrund scheint die starke Sonderangebotsfixierung im Lebensmitteleinzelhandel, insbesondere der Vollsortimenter, an den Bedürfnissen der meisten Verbraucher vorbeizugehen. Die häufige Behauptung, die deutschen Verbraucher würden nur ,billiges Fleisch' kaufen wollen, ist der Studie von SPILLER et al. (2019) zufolge nur für einen kleinen Teil der Gesellschaft zutreffend. 
Der Anteil der Verbraucher, die größeren Wert auf besondere Produktqualitäten legen als auf den Preis, ist in Deutschland deutlich höher als der Anteil der eher preisorientierten Verbraucher. Zudem verfügen die Qualitätskäufer über ein deutlich höheres Einkommen und stellen daher die attraktivere Kundengruppe für den LEH dar. Dies konnte in der vorliegenden Studie ebenfalls bestätigt werden. Der Anteil der eher preisorientierten Verbraucher wird jedoch in der vorliegenden Studie mit $41 \%$ (Cluster 2, 4 und 5) als etwas höher eingeschätzt. Dennoch waren die Qualitätskäufer (Cluster 1 und Cluster 3) auch in der vorliegenden Studie in der Überzahl, was insgesamt eher für eine Qualitätspositionierung als für eine reine Preispositionierung der Anbieter von Fleischund Wurstwaren spricht. Das Angebot an Tierwohlprodukten im LEH für die Qualitätskäufer deckte demzufolge in den letzten Jahren womöglich nicht die Nachfrage nach Tierwohlprodukten. Bei Betrachtung der SB-Regale und Fleischtheken entsteht auch heute noch der Eindruck, dass $95 \%$ der angebotenen Fleisch- und Wurstwaren aus konventioneller Haltung stammen. Zudem wurde in der Studie herausgefunden, dass sich die Tierwohl-Label-Präferenzen von Discounter-Käufern nur unwesentlich von den Präferenzen von SB-Warenhaus-Käufern unterscheiden. Für beide Vertriebsformen können am ehesten reine Tierwohlprodukte und staatliche Bio-Produkte empfohlen werden. In der Folge ist für Hersteller und Händler von Fleisch- und Wurstwaren bei der Gestaltung des Angebots an Tierwohlprodukten bei diesen Einkaufsstätten ein nur wenig differenziertes Vorgehen erforderlich.

Ein konträres Bild zu den Fans reiner Tierwohl-Labels zeigte sich bei den Fans der ökologischen Anbauverbände und von NEULAND. LIDL, PENNY und NETTO werden von dieser Verbrauchergruppe am seltensten für den Fleischeinkauf genutzt und die Gruppe unterscheidet sich hier signifikant von den anderen beiden Gruppen. Ein Grund für die geringe Nutzung von Discountern könnte das fehlende Angebot an Fleisch- und Wurstwaren der ökologischen Anbauverbände sein. Des Weiteren ist in den meisten Märkten der Discounter keine Frischetheke vorhanden, die dieser Gruppe besonders wichtig ist. Sollten die Discounter die einkommensstarken Fans der ökologischen Anbauverbände zukünftig als Kunden gewinnen wollen, müssten diese Punkte folglich verbessert werden. 
Alternativ zur Einrichtung von Frischetheken sollte zumindest eine Standardauswahl an mit den Tierwohl-Labels von DEMETER, BIOLAND, NATURLAND, BIOKREIS oder NEULAND zertifizierten Produkten im SB-Bereich angeboten werden, wie es bei ALDI mit einigen NEULAND-Produkten bereits der Fall ist.

Die niedrigen Koeffizienten bei den SB-Warenhäusern zeigen, dass KAUFLAND und REAL am seltensten von dieser Gruppe genutzt werden. Ebenfalls achten die SBWarenhaus-Kunden weniger auf Nachhaltigkeitsaspekte als die Discounter-Käufer. Dieses Ergebnis wurde in dieser Form nicht erwartet, da vielmehr davon ausgegangen wurde, dass die ,Billig-billiger-am-billigsten-Mentalität“ bei den Discounter-Käufern am stärksten ausgeprägt sein würde. REAL-Kunden achten dabei etwas mehr auf Nachhaltigkeitsaspekte als KAUFLAND-Kunden.

Des Weiteren konnte herausgefunden werden, dass diese Personen von allen drei Gruppen am liebsten beim Metzger einkaufen, sodass ein Angebot von AnbauverbandBio-Produkten am ehesten für den Metzger oder die Frischetheke zu empfehlen ist. In der Praxis finden sich an der Fleischtheke oder beim klassischen Metzger jedoch kaum Tierwohlprodukte, die nach diesen strengen Standards produziert wurden.

EDEKA und REWE werden von dieser Gruppe etwa gleich häufig für den Einkauf von Fleisch- und Wurstwaren genutzt, überraschenderweise auch deutlich häufiger als von den Fans staatlicher Bio-Produkte. Daher sollten EDEKA und REWE eher Produkte der ökologischen Anbauverbände anbieten als staatliche Bio-Produkte und die Fleisch- und Wurstwaren schwerpunktmäßig an der Fleischtheke verkaufen.

Deutliche Unterschiede zu den anderen Gruppen zeigten sich bei der Nutzung von BioSupermärkten, Wochenmärkten und Hofläden. Diese eher speziellen Einkaufsstätten sind bei den Fans ökologischer Anbauverbände für den Fleischeinkauf von allen Gruppen am beliebtesten; insbesondere die Bio-Supermärkte. Die Erfolgsgeschichte der BioSupermarktketten in Deutschland resultiert womöglich daraus, dass die konventionellen Lebensmitteleinzelhändler in den letzten 20 Jahren den unterschiedlichen Bio-Kunden kein adäquates Produkt- und Beratungsangebot (auch bei Fleisch- und Wurstwaren) angeboten haben. 
Was die Wochenmärkte und Hofläden betrifft, konnte ebenfalls ein deutlicher Unterschied zu den Fans staatlicher Bio-Produkte ermittelt werden, die diese beiden Einkaufsstätten deutlich seltener nutzen als die Anbauverband-Bio-Fans. Demzufolge sollten Anbieter von Fleisch und Wurst auf Wochenmärkten und in Hofläden schwerpunktmäßig Anbauverband-Bio-Produkte (inkl. NEULAND) anbieten. Dafür spricht auch, dass auf den Wochenmärkten Fleisch- und Wurstwaren überwiegend in Verkaufswagen mit mobiler Frischetheke angeboten werden, die zusätzlich die starke Präferenz der Anbauverband-Bio-Fans für die Frischetheke erfüllen.

Aufgrund der hohen Präferenz der Anbauverband-Bio-Fans für Hofläden könnte vermutet werden, dass bei dieser Gruppe die starke Beachtung von Tierwohl, Umweltfreundlichkeit und Gesundheitaspekten eng mit dem Wunsch nach regionalen Fleisch- und Wurstwaren von persönlich bekannten Produzenten verbunden ist. Die etwas romantische Vorstellung von einem kleinbäuerlichen, möglichst extensiv und ökologisch wirtschaftenden landwirtschaftlichen Betrieb passt zur Vertriebsform des Hofladens womöglich am besten. Hinsichtlich der Präferenzen für Einkaufsstätten zeigte sich insgesamt mehrfach in dieser Arbeit, dass es sich bei den beiden Label-Motiv-Gruppen (Anbauverband-Bio und Staatlich-Bio) um sehr unterschiedliche Personen handelt.

Während die beiden ersten Faktorgruppen hinsichtlich ihrer Einkaufsstättenpräferenz eindeutig charakterisiert werden konnten, erwies sich die Gruppe der Fans staatlicher Bio-Produkte als unerwartet heterogen.

Am häufigsten werden Bio-Supermärkte für den Einkauf von Fleisch- und Wurstwaren genutzt, wenn auch nicht so stark wie von den Anbauverband-Bio-Fans. Wider Erwarten konnte festgestellt werden, dass die Fans staatlicher Bio-Produkte im Gegensatz zu den Anbauverband-Bio-Fans außer den Bio-Supermärkten Fleisch- und Wurstwaren auch häufig bei ALDI, LIDL, PENNY und REAL, teilweise auch bei NETTO und KAUFLAND einkaufen. Seltener werden von dieser Gruppe auch EDEKA und REWE für den Einkauf von Fleisch- und Wurstwaren genutzt (in etwa gleichen Teilen). Interessanterweise handelt es sich bei den meisten Fans staatlicher Bio-Produkte wie auch bei den Fans reiner Tierwohl-Labels um Discounter-Käufer, die ab und zu auch SBWarenhäuser sowie seltener REWE oder EDEKA besuchen. 
Demzufolge sollten am ehesten Discounter und SB-Warenhäuser schwerpunktmäßig staatliche Bio-Produkte anbieten, während EDEKA und REWE, wie bereits erwähnt, schwerpunktmäßig Anbauverband-Bio-Produkte anbieten sollten. Der Metzger wird ebenfalls von einigen Fans staatlicher Bio-Produkte genutzt, jedoch nicht so stark wie von den Anbauverband-Bio-Fans.

Der zentrale Unterschied zu den Fans reiner Tierwohl-Labels besteht darin, dass ein Teil der Fans staatlicher Bio-Produkte gerne in Bio-Supermärkten einkauft. Innerhalb der Gruppe der Fans staatlicher Bio-Labels scheint es eine Art ,Zweiklassengesellschaft ${ }^{`} \mathrm{zu}^{\mathrm{z}}$ geben. Eine Gruppe besteht aus klassischen Discounter-Käufern, die für günstige Fleischund Wurstwaren mit DE-BIO- oder EU-BIO-Siegel zu begeistern wären. Die andere Gruppe scheinen eher, Qualitätskäufer' zu sein und ähneln sowohl soziodemografisch als auch hinsichtlich ihres Einkaufsverhaltens den Anbauverband-Bio-Fans.

Die durch den globalen Megatrend der Neo-Ökologie gestiegene Nachfrage bzgl. nachhaltigerer Produktalternativen hat mittlerweile zu einem immer größeren Angebot dieser Produkte im LEH geführt. Dies zeigt sich nicht nur in der stetig wachsenden Zahl an Bio-Supermärkten, sondern auch anhand des Sortiments von Supermärkten, Discountern und SB-Warenhäusern. Die meisten Anbieter stellen immer mehr Informationen zum Thema Tierwohl und Nachhaltigkeit auf der unternehmenseigenen Website bereit und formulieren strategische Nachhaltigkeitsziele.

Insgesamt handelt es sich dabei vor dem Hintergrund der zahlreichen globalen Herausforderungen in den Bereichen Tierwohl, Umweltschutz, Gesundheit und Soziales um eine begrüßenswerte Entwicklung. Dennoch stammt der Großteil des gesamten Produktsortiments der konventionellen Lebensmitteleinzelhändler nach wie vor aus einer nichtnachhaltigen Produktion. Gleichwohl scheinen die meisten Lebensmitteleinzelhändler mittlerweile erkannt zu haben, dass insbesondere mit BioProdukten der ökologischen Anbauverbände die einkommensstärksten Zielgruppen erreicht werden können, und passen ihr Produktsortiment entsprechend an. Beispielhaft genannt seien hier die Kooperationen zwischen LIDL und BIOLAND oder EDEKA und DEMETER. Am stärksten fokussieren sich die Bio-Supermärkte auf diese Zielgruppe. 
REWE bietet ein entsprechendes Produktangebot an zertifizierten Fleisch- und Wurstwaren von NATURLAND, um diese Zielgruppe zu erreichen. EDEKA bietet jedoch bis heute kaum Fleisch- und Wurstwaren für die Zielgruppe der AnbauverbandBio-Fans an, wobei dies EDEKA auf Grundlage der vorliegenden Studie durchaus empfohlen werden kann.

Für die bestehenden Discounter-Kunden ist Anbauverband-Bio nicht zu empfehlen, es sei denn, die Fans von Anbauverband-Bio sollen als Neukunden gewonnen werden. Mittlerweile ist mit dem herkömmlichen Fleisch- und Wurstwarensortiment aus konventioneller Haltung kein stetiges Wachstum mehr im gesättigten Markt zu erzielen. Voraussichtlich wird der Konsum der konventionellen Standardfleischwaren in Deutschland zukünftig weiter stetig abnehmen, da der Großteil der deutschen Verbraucher seinen Fleischkonsum eher weiter reduziert und sich insbesondere konventionelle Fleisch- und Wurstwaren aus industrieller Produktion immer weniger Beliebtheit erfreuen.

Als weiteres vertriebsrelevantes Thema wurden in der vorliegenden Studie die Unterschiede zwischen den drei Label-Motiv-Gruppen bzgl. der präferierten Angebotsform (Theke und SB) untersucht. Dabei konnten hoch signifikante Unterschiede festgestellt werden.

Personen, die am ehesten Tierwohlprodukte kaufen möchten, die nach den Standards der ökologischen Anbauverbände produziert werden, gaben in der Befragung an, sowohl Wurst und Aufschnitt als auch Fleisch zum Braten und Grillen schwerpunktmäßig an der Theke zu kaufen, und präferieren die Theke von allen drei Gruppen am meisten.

Personen, die am ehesten reine Tierwohlprodukte kaufen möchten, bevorzugen Wurst und Aufschnitt als auch Fleisch zum Braten und Grillen von allen Gruppen am meisten im SB-Bereich. Fleisch zum Grillen und Braten wird von dieser Gruppe dabei etwas häufiger an der Theke gekauft als Wurst und Aufschnitt, für die eher der SB-Bereich präferiert wird. 
Personen, die staatliche Bio-Produkte bevorzugen, zeigten weder bei Wurst und Aufschnitt noch bei Fleisch zum Braten und Grillen eine wirklich klare Tendenz zu einer Angebotsform. Beide Angebotsformen werden zu etwa ähnlichen Anteilen genutzt. Demzufolge kann empfohlen werden, die reinen Tierwohlprodukte schwerpunktmäßig im SB-Bereich anzubieten und Fleisch- und Wurstwaren, die nach den ökologischen Anbauverbänden zertifiziert sind, vor allem an der Frischetheke. Die staatlichen BioFleischwaren sollten in ungefähr gleichen Teilen sowohl im SB-Bereich als auch an der Fleischtheke angeboten werden.

Aufgrund der hohen Praxisimplikation und des hohen Potenzials, die Kundenzufriedenheit beim Angebot von Tierwohlprodukten zu verbessern, wurde als weiteres vertriebsrelevantes Thema untersucht, ob sich die Präferenzen der drei LabelGruppen bei der Platzierung von Tierwohlprodukten im Einzelhandel unterscheiden. Wie in Unterkapitel 5.4.3 beschrieben sprach sich in der durchgeführten Befragung der Großteil der Befragten (70 \%) für einen eigenen Bereich für Tierwohlprodukte aus.

Eine klare räumliche Trennung von konventionellen Fleisch- und Wurstwaren und Tierwohlprodukten bzw. ökologischen Fleisch- und Wurstwaren in den Einkaufsstätten stellt demnach aus Sicht der meisten Verbraucher einen deutlichen Vorteil beim Fleischkauf dar. Mit Hilfe der logistischen Regression konnte zudem bei den drei LabelGruppen festgestellt werden, dass den Fans der Tierwohl-Labels DEMETER, BIOLAND, NATURLAND und NEULAND ein getrennter Bereich besonders wichtig ist.

Ein Vorteil für die Kunden bestünde beispielsweise darin, dass die Suche nach entsprechenden Tierwohlprodukten im Lebensmitteleinzelhandel dadurch erleichtert würde. Fleisch- und Wurstwaren mit den besonderen Produktqualitäten müssten nicht zwischen dem großen Sortiment an konventionellen Fleischwaren gesucht werden. Bei anderen Produktkategorien ist der Lebensmitteleinzelhandel bereits teilweise dazu übergegangen und hat spezielle Ecken für Bio-Obst, glutenfreie Nahrungsmittel, vegane Spezialitäten oder auch internationale Delikatessen eingerichtet. 
Bei Fleisch- und Wurstwaren zeigte sich bei eigenen Recherchen im LEH, dass es diese Bereiche für Tierwohlprodukte häufig noch nicht gibt. Fleisch- und Wurstwaren werden bisher meistens nach Sorte bzw. Produktart nebeneinander angeboten, was die Suche nach den entsprechenden Tierwohlprodukten aufgrund ihres bisher sehr geringen Anteils am konventionellen Gesamtsortiment erschwert. In der vorliegenden Studie konnte jedoch nachgewiesen werden, dass über zwei Drittel der Verbraucher in Deutschland eine klare räumliche Trennung von Tierwohlprodukten und konventioneller Ware wünschen. Demzufolge kann empfohlen werden, dass die Lebensmitteleinzelhändler ihr Sortiment an Fleisch- und Wurstwaren getrennt nach besonderen Tierwohlprodukten (z. B. BIOLAND-Sortiment) und konventionellen Produkten anbieten sollten. Vorstellbar wären eigene Abschnitte in Kühlregalen oder auch Tiefkühltruhen ebenso wie ein getrennter Bereich an der Fleischtheke. Dabei sollte das Personal an der Fleischtheke im Hinblick auf die unterschiedlichen Tierwohlstandards gezielt geschult werden, um mögliche Fragen der Kunden zum Thema Tierwohl kompetent beantworten zu können. Im Idealfall sollten sich die Angestellten die verschiedenen Haltungsformen der unterschiedlichen Tierwohl-Labels auf landwirtschaftlichen Betrieben persönlich anschauen (z. B. im Rahmen eines Betriebsausflugs).

Des Weiteren sollte in der vorliegenden Studie analysiert werden, in welche der drei Tierwohl-Label-Gruppen sich Flexitarier, die ihren Fleischkonsum bewusst reduzieren und auf verschiedene besondere Produktqualitäten achten, am ehesten einordnen lassen. Wie in Tabelle 23 im Ergebnisteil ersichtlich lassen sich die meisten Flexitarier unter den Fans der ökologischen Anbauverbände verorten. Die Reduktionsbereitschaft sowohl der reinen Tierwohl-Label-Fans als auch der Fans staatlicher Bio-Produkte war deutlich niedriger als in der Anbauverband-Bio-Gruppe. Fans reiner Tierwohl-Labels essen am häufigsten Fleisch- und Wurstwaren und viele Personen in dieser Gruppe möchten ihren Fleischkonsum auch zukünftig nicht reduzieren. Auch die Fans staatlicher Bio-Produkte essen unerwartet häufig Fleisch und Wurst. Die Erkenntnis, dass derart starke Unterschiede im Hinblick auf den Fleischkonsum zwischen den beiden identifizierten unterschiedlichen Bio-Label-Gruppen bestehen, konnte in der bestehenden Literatur bisher noch nicht festgestellt werden, da eine derart spezifische Label-GruppenUntersuchung dieser Art noch nicht durchgeführt wurde. 
Zusammenfassend kann aus diesen Ergebnissen geschlossen werden, dass Flexitarier am ehesten Fleisch- und Wurstwaren von DEMETER, BIOLAND, NATURLAND, BIOKREIS oder NEULAND bevorzugen, was eine differenziertere Definition des Konsumverhaltens von Flexitariern nach dem ,Less-but-better-Prinzip“ ermöglicht. Möchten Anbieter von Fleisch- und Wurstwaren die klassischen Flexitarier als Zielgruppe ansprechen, so sollten am ehesten die Tierwohl-Labels der ökologischen Anbauverbände oder NEULAND gewählt werden.

In der vorliegenden Studie wurde zudem das Thema Multi-Labeling bei Fleisch- und Wurstwaren wissenschaftlich untersucht. Hintergrund war die im deutschen Lebensmitteleinzelhandel gängige Praxis, einzelne Produkte gleich mit mehreren Gütesiegeln unterschiedlichster Art zu kennzeichnen. Aus Händler- und Herstellerperspektive stellt sich die Frage, ob und wie Tierwohl-Labels mit anderen Gütesiegeln bei Fleisch- und Wurstwaren möglichst sinnvoll, also optimal an den Präferenzen der Verbraucher ausgerichtet, kombiniert werden können.

In Tabelle 23 des Ergebnisteils sind die Ergebnisse der Multi-Labeling-Analyse ausführlich dargestellt. An dieser Stelle sollen die Kernergebnisse kurz interpretiert werden. Während im Ergebnisteil der Einsatz der einzelnen Gütesiegel dargestellt wurde, werden im Folgenden die Multi-Label-Präferenzen je Verbrauchergruppe zusammengefasst. Die Ausführungen sind gleichzeitig als Handlungsempfehlungen zu verstehen, welche zusätzlichen Gütesiegel Anbieter von Fleisch- und Wurstwaren für welche Label-Gruppe am besten einsetzen sollten. Die (abgefragten) Gütesiegel, die bei den einzelnen Gruppen nicht aufgeführt sind, stellen kein positives Kaufargument für diese Gruppe dar, weshalb Anbieter von Tierwohlprodukten auf diese Gütesiegel verzichten sollten.

Für die Fans reiner Tierwohl-Labels (DEUTSCHER TIERSCHUTZBUND, STAATLICHES TIERWOHL-LABEL, INITIATIVE TIERWOHL und VIER PFOTEN) eignen sich vor allem die Gütesiegel des DEUTSCHEN TIERSCHUTZBUNDES, das QS-Siegel sowie das DLG-Siegel. Diese Gruppe erweist sich insgesamt als relativ aufgeschlossen gegenüber zusätzlichen Gütesiegeln. 
So wurden auch die getesteten Labels HORMONFREI, GENTECHNIKFREI, REGIONALFENSTER, NABU, FAIRTRADE sowie die beiden Testsiegel von STIFTUNG WARENTEST und ÖKOTEST positiv bewertet und können als Ergänzung zu den reinen Tierwohl-Labels verwendet werden.

Ein differenzierteres Bild ergab sich hingegen bei den Anbauverband-Bio-Fans. Diese Gruppe bewertete alle Gütesiegel besonders positiv, die einen starken Bezug zur eigenen Gesundheit besitzen, wie z. B. die Labels HORMONFREI, ANTIBIOTIKAFREI und GENTECHNIKFREI.

Daraus kann abgeleitet werden, dass diese Gruppe womöglich am besorgtesten um ihre eigene Gesundheit ist. Vor diesem Hintergrund ist Anbietern zu empfehlen, noch stärker als bisher die gesundheitlichen Vorteile der entsprechenden Produkte in den Vordergrund zu stellen. Angesichts der positiven Resonanz der frei erfundenen Siegel ANTIBIOTIKAFREI und HORMONFREI könnten entsprechende neue Kennzeichnungen oder Werbeslogans speziell für Fleisch- und Wurstwaren der ökologischen Anbauverbände entwickelt werden, wenn sich diese Produkte in Bezug auf Antibiotikarückstände bzw. die Absenz von MRSA-Keimen positiv von konventionellen Fleisch- und Wurstwaren unterscheiden, was in Studien des Robert-Koch-Instituts regelmäßig für verschiedene Fleischwaren nachgewiesen wurde. Derartige ,Frei-von“Siegel beinhalten gleichwohl ein Risiko für die Anbieter, sollte beispielsweise nachgewiesen werden, dass die Produkte doch nicht frei von MRSA-Keimen sind.

Des Weiteren erhöhen auch die Gütesiegel von Organisationen wie FOODWATCH oder dem DEUTSCHEN TIERSCHUTZBUND das Vertrauen dieser Gruppe in die angebotenen Produkte, und auch einer FAIRTRADE-Zertifizierung wird ein positiver Effekt auf die Kaufentscheidung zugesprochen. Die besonders hohe Bedeutung des Themas Regionalität für die Anbauverband-Bio-Fans drückt sich in der positivsten Bewertung des Siegels REGIONALFENSTER aus. Regionalsiegel können den Ergebnissen folgend zwar auf allen Produkten mit Tierwohl-Labels für alle drei LabelGruppen zusätzlich angebracht werden, eignen sich jedoch besonders als Ergänzung zu DEMETER, BIOLAND, NATURLAND und NEULAND. 
Bei der Vermarktung von Fleisch- und Wurstwaren für diese Gruppe sollte die Regionalität ergänzend zur reinen Anbringung des Regionalsiegels stets in der Kommunikation hervorgehoben werden, wenn die Produkte tatsächlich aus der unmittelbaren Region stammen (z. B. Produktion im Umkreis von 100 km). Umsetzen lässt sich dies beispielsweise mit einer möglichst konkreten Herkunftsangabe, entsprechend gewählten regionalen Markennamen oder Werbeslogans mit Regionalbezug. Die besonderen Vorteile der regionalen Produkte sollten deutlich, z. B. mit Spiegelstrichen, hervorgehoben werden. Beispielhafte Vorteile regionaler Fleischund Wurstwaren umfassen die besondere Frische der Produkte, die wirtschaftliche Stärkung der heimischen Landwirte, eine höhere Transparenz oder auch kürzere Transportwege als Beitrag zum Klimaschutz und zum Vorteil für die Tiere.

Die Verwendung von Logos von Umwelt- und Naturschutzorganisationen wurde von den Anbauverband-Bio-Fans in der Befragung ebenfalls am positivsten bewertet, sodass Cause-Related-Marketing-Maßnahmen in Kooperation mit Umwelt- und Naturschutzorganisationen am ehesten für diese Gruppe zu empfehlen sind. In diesem Rahmen könnten kreative verkaufsfördernde Konzepte umgesetzt werden, wie etwa die Durchführung von Gewinnspielen (z. B. Wochenende auf einem DEMETERBauernhof). Dabei sollten, wie bei allen Produkten der ökologischen Anbauverbände, die besonderen Vorteile für Umwelt, Tier und Mensch hervorgehoben werden. Die Gütesiegel von STIFTUNG WARENTEST, ÖKOTEST, QS und DLG eignen sich bei Fleisch- und Wurstwaren dagegen weniger für die Anbauverband-Bio-Fans.

Die Analyse der Fans staatlicher Bio-Produkte ergab hinsichtlich des Themas MultiLabeling ebenfalls einige unerwartete Befunde. So wurden die Labels QS und DLG von den meisten Käufern staatlicher Bio-Produkte positiv bewertet. In diesem Punkt ähnelt diese Gruppe den reinen Tierwohl-Label-Fans und unterscheidet sich wiederum von den Anbauverband-Bio-Fans. Weitere Gütesiegel, die Anbieter von Fleisch- und Wurstwaren für diese Gruppe verwenden können, sind ÖKOTEST, GENTECHNIKFREI, FAIRTRADE, STIFTUNG WARENTEST, HORMONFREI und REGIONALFENSTER. Wenig eignen sich für diese Gruppe die Siegel ANTIBIOTIKAFREI, NABU und FOODWATCH. 
Unabhängig von der Zusammenstellung des ,Multi-Label-Mix ‘ sollten Anbieter die konkreten Vorteile der verwendeten Gütesiegel ergänzend erklären, idealerweise direkt auf der Produktverpackung und, falls dies nicht möglich sein sollte, durch ergänzende Werbematerialien oder einen Verweis auf eine Website.

Anbieter sollten sich im Klaren darüber sein, dass hinter den Präferenzen für die verschiedenen Gütesiegel oftmals die Wichtigkeit einzelner Themen für die Verbraucher steht, wie beispielsweise die Affinität zur Entwicklungshilfe beim FAIRTRADE-Logo, der Bezug zur Heimatregion bei Regional-Labels etc. Diese Aspekte sollten im Idealfall in die gesamte Marketingkommunikation integriert werden, um die Kunden besser anzusprechen, als wenn lediglich Gütesiegel,wortlos‘ auf der Produktverpackung angebracht werden.

Neben den Präferenzen zum Multi-Labeling wurden die drei Label-Gruppen näher bzgl. der Beachtung von Kaufkriterien beim Einkauf von Fleisch- und Wurstwaren untersucht, um Unterschiede zwischen den drei Gruppen identifizieren zu können. Im Hinblick auf die Gestaltung des Marketing-Mix ist die Berücksichtigung der für Verbraucher bedeutsamen Kaufkriterien von grundlegender Bedeutung für den erfolgreichen Verkauf der Produkte. Im Ergebnisteil (Tabelle 24) wurde dargestellt, dass bzgl. der Kaufkriterien deutliche Unterschiede zwischen den drei Label-Gruppen bestehen.

Es zeigte sich, dass Käufer reiner Tierwohl-Labels am meisten auf Marken, die Verpackung, den Geschmack und am stärksten auf Sonderangebote achten. Unerwartet viele Personen, die reine Tierwohl-Labels bevorzugen, gaben an, besonders auf Gütesiegel zu achten. Bei näherer Betrachtung wurde jedoch ersichtlich, dass es vor allem Gütesiegel waren, die keine Nachhaltigkeitskriterien beinhalten, wie z. B. QS oder DLG. Interessant ist des Weiteren, dass diese Gruppe häufiger angab, Tierwohl- und Umweltaspekte zu berücksichtigen als Käufer staatlicher Bio-Produkte, im Widerspruch dazu jedoch nicht auf Bio-Qualität zu achten. Dies kann evtl. damit erklärt werden, dass diese Gruppe nicht weiß, dass der Tierwohlstandard und die Umweltfreundlichkeit bei Bio-Produkten in der Regel deutlich höher sind als bei reinen Tierwohlprodukten. 
Wenn für sie Tierwohl besonders relevant ist, müssten sie eher Bio-Produkte präferieren als reine Tierwohlprodukte. Insgesamt zeigte sich anhand der generell niedrigen Koeffizienten, dass den Fans reiner Tierwohl-Labels (Faktorgruppe 1) die meisten Kaufkriterien nicht so wichtig sind wie den beiden Bio-Gruppen. Solange das Fleisch frisch und günstig ist und schmeckt, sind die Fans reiner Tierwohl-Labels schon recht zufrieden. Mehr Tierwohl würde diese Gruppe sicherlich ebenfalls begrüßen, jedoch sollte ein Mehr an Tierwohl aus Sicht dieser preissensiblen Gruppe der DiscounterKäufer möglichst nicht viel mehr Kosten verursachen als konventionelle Fleisch- und Wurstwaren.

Käufer von Anbauverband-Bio-Labels achten dagegen am häufigsten auf eine artgerechtere Tierhaltung, Umwelt- und Gesundheitsaspekte, Gütesiegel und Fairtrade und vor allem auf die Frische der Ware, jedoch mit Abstand am wenigsten auf die Verpackung und den Preis. Dass diese Gruppe wenig auf die Verpackung achtet, erscheint plausibel, da die Frische der Ware eine besonders hohe Bedeutung für diese Gruppe hat und sie weniger verpackte SB-Ware kaufen als die anderen beiden Motivgruppen. Die Anbauverband-Bio-Fans besitzen schließlich von allen Gruppen die höchste Präferenz für die Fleischtheke. Der Preis hat für diese Gruppe mit Abstand die geringste Bedeutung. Die Zahlungsbereitschaft für Tierwohlprodukte wird daher bei dieser Gruppe womöglich signifikant höher sein als bei den anderen beiden Motivgruppen.

Die Gruppe Anbauverband-Bio achtet zudem am meisten auf Bio-Qualität, und zwar deutlich mehr als die Gruppe der Fans staatlicher Bio-Labels. Außerdem berücksichtigt die Anbauverband-Bio-Gruppe am meisten von allen Gruppen das Thema Regionalität, während dieses für Fans reiner Tierwohlprodukte und staatlicher Bio-Produkte (auch, aber) deutlich weniger bedeutsam ist. Insgesamt bilden die Anbauverband-Bio-Fans die anspruchsvollste Gruppe, die sich bei der Beachtung der Kaufkriterien deutlich und häufig von den anderen beiden Gruppen unterscheidet. Wider Erwarten unterscheiden sich die Anbauverband-Bio-Fans auch stark von den Fans staatlicher Bio-Produkte.

Die Gruppe der Fans staatlicher Bio-Produkte gab in der vorliegenden Studie an, beim Fleischkauf auf den Geschmack, Bio-Qualität, Gütesiegel, die Verpackung und die Frische zu achten. 
Eher unwichtig sind für diese Gruppe faire Löhne, Regionalität, Tierwohl und Umweltfreundlichkeit. Diese Ergebnisse verdeutlichen die nicht konsequente Beachtung ethischer Kaufkriterien. Stark auf Bio-Qualität zu achten und gleichzeitig wenig auf Tierwohl und Umweltfreundlichkeit, passt nicht zusammen. Dieser Befund lässt sich jedoch erklären. Die Gruppe der staatlichen Bio-Fans ähnelt in Bezug auf ihre Soziodemografie und ihr Einkaufsverhalten der Gruppe der Fans reiner Tierwohl-Labels. Im Fragebogen wurde danach gefragt, welche Tierwohl-Labels die Verbraucher am ehesten bei Fleisch und Wurst berücksichtigen würden. Die Kaufkriterien wurden an anderer Stelle erfragt und bezogen sich auf die Beachtung von Kaufkriterien beim alltäglichen Fleischkauf. Die Personen sind lediglich Fans staatlicher Bio-Produkte und definieren sich nicht als Bio-Käufer.

\subsubsection{Interpretation und Diskussion der Clusteranalyse}

Nachdem die Ergebnisse der Clusteranalyse und der logistischen Regressionsanalysen im Ergebnisteil ausführlich dargestellt und beschrieben wurden, sollen sie in diesem Abschnitt nun näher interpretiert und diskutiert werden. Abschließend für diesen Abschnitt sollen für die Bearbeitung der identifizierten Marktsegmente Handlungsempfehlungen für Hersteller und Händler von Fleisch- und Wurstwaren abgeleitet werden, die mit Tierwohl-Labels zertifizierte Fleisch- und Wurstwaren aus einer artgerechteren und nachhaltigeren Nutztierhaltung anbieten. Ein vollständiges Bild der Marktsegmente ergibt sich aus der gleichzeitigen Betrachtung der clusterbildenden Variablen und der clusterbeschreibenden Variablen. In Unterkapitel 5.5.1 wurde die Clusterbildung auf Basis der identifizierten Motivfaktoren (Unterkapitel 5.4) beschrieben. Die Untersuchung der Cluster im Hinblick auf soziodemografische, vertriebsrelevante und marketingrelevante Unterschiede erfolgte in den Unterkapiteln 5.5.2 bis 5.5.4. Dabei wurden ausschließlich signifikante bzw. heterogene Variablen (Wald-Wert > 3,84) dargestellt, da nur diese Variablen aussagekräftige Unterschiede zwischen den gebildeten Clustern bzw. Marktsegmenten aufzeigen. In Tabelle 37 sind die signifikanten Ergebnisse der sechs multinominalen Logit-Modelle als Interpretationsund Diskussionsgrundlage zusammengefasst. 
Tabelle 37: Übersicht der heterogenen Items beim Vergleich der Marktsegmente

\begin{tabular}{|c|c|c|c|c|}
\hline & $\begin{array}{l}\text { Enthusiastische } \\
\text { Nachhaltigkeitsfans }\end{array}$ & $\begin{array}{l}\text { Unentschlossene } \\
\text { Verbraucher }\end{array}$ & $\begin{array}{l}\text { Überzeugte } \\
\text { Bio-Fans }\end{array}$ & $\begin{array}{l}\text { Gruppe } \\
\text {,Tierwohl ja- } \\
\text { Bio nein' } \\
\text { Ci uSTFR } 5\end{array}$ \\
\hline VARIABLE & $\begin{array}{l}\text { CLUSTER } 1 \\
\text { +staatl. Bio } \\
\text { ++Anbau-Bio }\end{array}$ & $\begin{array}{l}\text { CLUSTER } 2 \\
\text { +staatl. Bio } \\
--- \text { Anbau-Bio }\end{array}$ & $\begin{array}{l}\text { CLUSTER } 3 \\
\text { +++staatl. Bio } \\
\text { +Anbau-Bio }\end{array}$ & $\begin{array}{l}\text { CLUSTER } 5 \\
\text {-staatl. Bio } \\
\text {-Anbau-Bio }\end{array}$ \\
\hline Segmentgröße & $\begin{array}{l}++ \text { reinTW } \\
21 \%\end{array}$ & $\begin{array}{l}+ \text { reinTW } \\
15 \%\end{array}$ & $\begin{array}{r}- \text {-reinTW } \\
38 \%\end{array}$ & $\begin{array}{c}+++ \text { reinTW } \\
\quad 8 \%\end{array}$ \\
\hline Gesamtbevölkerung & 11,45 Mio. & 8,18 Mio. & 20,7 Mio. & 4,36 Mio. \\
\hline \multicolumn{5}{|l|}{ Soziodemografie } \\
\hline Geschlecht & +++ & + & + & +++ \\
\hline Alter & & -- & & \\
\hline Ortsgröße & & - & & -- \\
\hline Einkommen & & & + & \\
\hline Bildung & & - & & - \\
\hline \multicolumn{5}{|l|}{ Einkaufsstättenwahl } \\
\hline Bio-Supermärkte & + & & & \\
\hline Wochenmarkt & & - & & \\
\hline REWE & & + & & \\
\hline ALDI & & + & & \\
\hline LIDL & & +++ & & \\
\hline \multicolumn{5}{|l|}{ NETTO } \\
\hline SB-Warenhäuser & & + & & \\
\hline Wechselbereitschaft & +++ & & ++ & + \\
\hline \multicolumn{5}{|c|}{ Weitere ausgewählte marketingrelevante Variablen } \\
\hline $\begin{array}{l}\text { Beachtung } \\
\text { Nachhaltigkeit }\end{array}$ & +++ & & + & \\
\hline $\begin{array}{l}\text { Beachtung innerer } \\
\text { Qualität }\end{array}$ & & & - - & \\
\hline $\begin{array}{l}\text { Beachtung äußerer } \\
\text { Merkmale }\end{array}$ & +++ & & & \\
\hline $\begin{array}{l}\text { Kaufabsicht } \\
\text { Tierwohlprodukte }\end{array}$ & ++ & & & \\
\hline Bedeutung Herkunft & + & & + & \\
\hline $\begin{array}{l}\text { Fleischkonsum im } \\
\text { Haushalt }\end{array}$ & ++ & ++ & ++ & \\
\hline $\begin{array}{l}\text { Reduktions- } \\
\text { bereitschaft }\end{array}$ & - & - & & - \\
\hline
\end{tabular}




\begin{tabular}{|c|c|c|c|c|}
\hline \multirow[t]{2}{*}{ VARIABLE } & $\begin{array}{c}\text { Enthusiastische } \\
\text { Nachhaltigkeitsfans } \\
\text { CLUSTER } 1\end{array}$ & $\begin{array}{c}\begin{array}{c}\text { Unentschlossene } \\
\text { Verbraucher }\end{array} \\
\text { CLUSTER } 2\end{array}$ & $\begin{array}{l}\text { Überzeugte } \\
\text { Bio-Fans } \\
\text { CLUSTER } 3\end{array}$ & $\begin{array}{c}\text { Gruppe } \\
\text {,Tierwohl ja - } \\
\text { Bio nein' } \\
\text { CLUSTER } 5\end{array}$ \\
\hline & $\begin{array}{l}\text { +staatl. Bio } \\
\text { ++Anbau-Bio } \\
\text { ++reinTW }\end{array}$ & $\begin{array}{l}\text { +staatl. Bio } \\
\text {---Anbau-Bio } \\
\text { +reinTW }\end{array}$ & $\begin{array}{l}\text { +++staatl. Bio } \\
\text { +Anbau-Bio } \\
\text {--reinTW }\end{array}$ & $\begin{array}{l}\text {-staatl. Bio } \\
\text {-Anbau-Bio } \\
+++ \text { reinTW }\end{array}$ \\
\hline Segmentgröße & $21 \%$ & $15 \%$ & $38 \%$ & $8 \%$ \\
\hline Gesamtbevölkerung & 11,45 Mio. & 8,18 Mio. & 20,7 Mio. & 4,36 Mio. \\
\hline \multicolumn{5}{|c|}{ Tierwohl-Label-Bekanntheit } \\
\hline EU-BIO & + & + & & \\
\hline DE-BIO & & +++ & +++ & + \\
\hline DEMETER & & --- & & - \\
\hline NATURLAND & & - & & \\
\hline BIOKREIS & & - & & \\
\hline NEULAND & + & & & \\
\hline TIERSCHUTZBUND & + & +++ & & +++ \\
\hline VIER PFOTEN & ++ & & & \\
\hline \multicolumn{5}{|l|}{ Multilabel-Präferenzen } \\
\hline GENTECHNIKFREI & +++ & & ++ & \\
\hline REGIONALFENSTER & +++ & & ++ & \\
\hline $\begin{array}{l}\text { STIFTUNG } \\
\text { WARENTEST }\end{array}$ & & & ++ & + \\
\hline ÖKO-TEST & & + & & \\
\hline NABU & & -- & & \\
\hline TIERSCHUTZBUND & & ++ & & +++ \\
\hline DLG-Siegel & ++ & + & & \\
\hline QS-Siegel & & + & & \\
\hline \multicolumn{5}{|c|}{$\begin{array}{l}\text { Zusammenfassende Darstellung der Ergebnisse der sechs einzeln gerechneten multinomianlen Logit-Modelle } \\
\text { (Unterkapitel 5.5.2 bis 5.5.4). SB-Warenhäuser=KAUFLAND+REAL. Faktor Nachhaltigkeitskriterien: Umweltkriterien, } \\
\text { Gütesiegel, Fairtrade, Bio-Qualität, Regionalität und Gesundheitsaspekte. Faktor Äußere Merkmale: Preis, } \\
\text { Verpackung und Marken. Faktor Innere Qualität: Frische, Aussehen und Geschmack. } \\
+=p<0,05 \text { (Wald-Wert }>3,84 \text { ); }++=p<0,01 \text { (Wald-Wert }>6,66 \text { ); }+++=p<0,001 \text { (Wald-Wert }>9,55 \text { ) Negative } \\
\text { Vorzeichen der Regressionskoeffizienten werden in dieser Tabelle mit Minuszeichen dargestellt. Referenzkategorie 4: } \\
\text { Desinteressierte Label-Ablehner. }\end{array}$} \\
\hline
\end{tabular}

\section{Quelle: Eigene Darstellung}

Alle in der Tabelle dargestellten Unterschiede beziehen sich auf das Referenzcluster der ,Desinteressierten Label-Ablehner' (Cluster 4). Im Ergebniskapitel wurden die Unterschiede zwischen den Clustern neben der Wald-Statistik auch hinsichtlich der Regressionskoeffizienten detailliert beschrieben. Diese Aspekte sind aus Tabelle 37 nicht abzulesen, da in diesem Abschnitt eine eher allgemeine Interpretation der Marktsegmente erfolgen soll. Dabei werden zunächst die einzelnen Ergebnisse der Analysen je Cluster interpretiert, bevor Handlungsempfehlungen erfolgen. 
Wird in Tabelle 37 zunächst das Cluster 1 der ,Enthusiastischen Nachhaltigkeitsfans “ betrachtet, zeigt sich, dass in dieser Verbrauchergruppe der Frauenanteil signifikant höher ist als in der Referenzgruppe. Außer dem Geschlecht konnten entgegen der Erwartungen keine weiteren signifikanten soziodemografischen Unterschiede zwischen den Nachhaltigkeitsfans und den ,Desinteressierten Label-Ablehnern' im Referenzcluster festgestellt werden.

Es kann demnach konstatiert werden, dass das Geschlecht das bedeutendste soziodemografische Unterscheidungskriterium dieser Gruppe darstellt. Die anderen soziodemografischen Kriterien eignen sich indes weniger für eine fundierte Charakterisierung dieser Gruppe. Im Ergebnisteil wurden jedoch auch die nichtsignifikanten soziodemografischen Unterschiede zwischen den Clustern anhand der Höhe und der Vorzeichen der Regressionskoeffizienten analysiert. Demnach können die Nachhaltigkeitsfans als sehr weibliche Verbrauchergruppe charakterisiert werden, die älter ist als die ,Unentschlossenen Verbraucher` (Cluster 2) und die Gruppe ,Tierwohl ja - Bio nein“ (Cluster 5) und ungefähr gleich alt wie die ,Überzeugten Bio-Fans`(Cluster 3); der Großteil ist älter als 30 Jahre. Sie leben häufiger in Städten und verfügen über ein gutes Einkommen, das jedoch nicht so hoch ist wie das sehr hohe Einkommen der BioFans. Die Nachhaltigkeitsfans weisen eine höhere Bildung als die ,Unentschlossenen Verbraucher' (Cluster 2) und die Gruppe ,Tierwohl ja - Bio nein' (Cluster 5) auf; jedoch ist ihre Bildung nicht so hoch wie die Bildung der Bio-Fans. Hier zeigt sich, dass es zwar Schnittstellen zwischen den Nachhaltigkeitsfans und den Bio-Fans gibt, es sich jedoch um zwei unterschiedliche Verbrauchergruppen handelt.

Was die favorisierten Einkaufsstätten für den Fleisch- und Wurstwarenkauf der Nachhaltigkeitsfans betrifft, unterscheidet sich diese Gruppe vom Referenzcluster vor allem in der häufigeren Nutzung von Bio-Supermärkten. Es konnte zudem festgestellt werden, dass bei den Nachhaltigkeitsfans EDEKA und REWE relativ beliebt sind, während NETTO, KAUFLAND und REAL von dieser Gruppe am wenigsten für den Fleischeinkauf genutzt werden. Die Nachhaltigkeitsfans wechseln vergleichsweise schnell ihre Haupteinkaufsstätte aufgrund eines besseren Angebots an Tierwohlprodukten in einer anderen Einkaufsstätte. 
Sie sind also eine eher untreue Kundengruppe, wenn ihre Bedürfnisse hinsichtlich Tierwohl und Nachhaltigkeit in ihrer Einkaufsstätte nicht zufriedenstellend erfüllt werden. Vice versa kann diese Verbrauchergruppe am ehesten durch ein ihren Bedürfnissen angepasstes Produktsortiment als neue Kundengruppe gewonnen werden. In der hohen Wechselbereitschaft der Nachhaltigkeitsfans drückt sich der starke Wunsch nach mehr Tierwohl und Nachhaltigkeit aus.

Verschiedenen Studien (ALBRECHT, 2014; BROOKS, 2001; CHOI, 2020; COHENPFISTER, 2015; FÖRSTER \& LORING, 2014; WEISSINGER, 2020) zufolge gehört die Berücksichtigung von Nachhaltigkeitsaspekten beim Konsumverhalten für die Nachhaltigkeitsfans zur Lebensphilosophie bzw. zum Lifestyle. Die meisten Nachhaltigkeitsfans leben vegan, vegetarisch oder zumindest ,flexitarisch “ und versuchen, auch in weiteren Lebensbereichen möglichst nachhaltig zu handeln. Dazu gehören häufig neben einem generell reduzierten Konsum oder dem weitestgehenden Verzicht auf Plastikprodukte auch der bevorzugte Kauf von zertifizierten Lebensmitteln, Papier-Produkten, Holzmöbeln, Pflegeprodukten, Second-Hand-Bekleidung oder auch die bewusste Auswahl von Dienstleistern, die ethische Aspekte in ihrer Unternehmensphilosophie verankert haben, wie beispielsweise Nachhaltigkeits- und Umweltbanken, Ökostromanbieter oder umweltfreundliche Suchmaschinen wie ECOSIA. Daneben werden auch das Fahrrad und die Bahn häufiger genutzt als das Auto oder das Flugzeug.

Die Gruppe der in dieser Studie identifizierten Nachhaltigkeitsfans besteht mutmaßlich aus einer bunten Mischung der zahlreichen Neo-Ökologie-affinen sozialen Gruppierungen wie den Lohas, den Bobos, den Hipstern, BIONADE-Biedermeiern, Grünen, Alternativen, Hippies und einer Vielzahl weiterer Personen, die dem Nachhaltigkeitstrend folgen (ALBRECHT, 2014; BROOKS, 2001; CHOI, 2020; COHEN-PFISTER, 2015; FÖRSTER \& LORING, 2014; IKRATH, 2015; WEISSINGER, 2020).

Insgesamt wurde bei der Analyse deutlich, dass sich die Nachhaltigkeitsfans in vielen Punkten deutlich vom Referenzcluster der ,Desinteressierten Verbraucher ${ }^{6}$ unterscheiden. 
Daher können diese beiden Gruppen als zwei ,Extremgruppen‘ betrachtet werden. Während die Beachtung von Tierwohl, Umweltschutz, Gütesiegeln, Fairtrade, BioQualität, Regionalität und Gesundheitsaspekten (Faktor Nachhaltigkeitsbewusstsein) beim Fleischkauf bei den Nachhaltigkeitsfans von allen Gruppen am höchsten war, achtete die Referenzgruppe am allerwenigsten auf diese Themen.

Unerwartet stark achten die Nachhaltigkeitsfans auch auf, äußere Merkmale‘ der Produkte, wie etwa eine ansprechende Verpackung, bestimmte Marken und den Preis. Dieser Gruppe ist die ,Ästhetik‘ bei der Gestaltung von Produktverpackungen, die möglichst umweltfreundlich sein sollte, deutlich wichtiger als den ,Desinteressierten Verbrauchern'.

Die Nachhaltigkeitsfans sind zudem markenaffiner als die Referenzgruppe und günstige Preise haben für sie eine höhere Bedeutung als für die ,Überzeugten Bio-Fans‘. Aus den Präferenzen der Nachhaltigkeitsfans lässt sich schließen, dass sich für diese anspruchsvolle Kundengruppe die gesamte Vermarktung von Fleisch- und Wurstwaren aus einer artgerechteren und nachhaltigeren Nutztierhaltung deutlich von der ,0815Vermarktung' (die z. B. für Verbraucher des Referenzclusters vermutlich ausreicht) unterscheiden sollte.

Kein signifikanter Unterschied zum Referenzcluster konnte hinsichtlich des Aussehens der Ware, des Geschmacks oder der Frische (Innere Qualität) festgestellt werden. Auf diese klassischen Kaufkriterien achten die Nachhaltigkeitsfans und die ,Desinteressierten Label-Ablehner' in etwa gleichermaßen, sodass es diesbezüglich keiner differenzierten Segmentbearbeitung bedarf.

Die Kaufabsicht von Tierwohlprodukten ist bei den Nachhaltigkeitsfans am höchsten von allen Gruppen, wobei sie großen Wert auf möglichst konkrete Herkunftsangaben legen. Was den Fleischkonsum im eigenen Haushalt betrifft, so essen die Nachhaltigkeitsfans signifikant weniger Fleisch- und Wurstwaren als die Referenzgruppe, den Koeffizienten zufolge jedoch etwas mehr als die Bio-Fans. Nach den Bio-Fans ist der Anteil an Flexitariern, also der Menschen, die ihren Fleischkonsum bereits bewusst reduziert haben, in dieser Gruppe am höchsten. 
Was ihre Kenntnis von Tierwohl-Labels betrifft, so kennen sich die Nachhaltigkeitsfans insgesamt sehr gut mit Gütesiegeln aus. EU-BIO, NEULAND, TIERSCHUTZBUND und VIER PFOTEN kennen deutlich mehr Nachhaltigkeitsfans als ,Desinteressierte Label-Ablehner'. NEULAND, TIERSCHUTZBUND und VIER PFOTEN kennen den Koeffizienten zufolge mehr Nachhaltigkeitsfans als Bio-Fans, während den Bio-Fans die Siegel der ökologischen Anbauverbände bekannter sind als den Nachhaltigkeitsfans. Dies lässt sich damit erklären, dass die Siegel der ökologischen Anbauverbände insbesondere bei Fleisch- und Wurstwaren überwiegend im Bio-Fachhandel angeboten werden, während dort wiederum keine reinen Tierwohl-Label angeboten werden.

Interessant ist ebenfalls, dass für die Nachhaltigkeitsfans die Siegel GENTECHNIKFREI, REGIONALFENSTER und DLG einen positiveren Einfluss auf die Kaufentscheidung haben als für die Verbraucher des Referenzclusters. Hinsichtlich des GENTECHNIKFREI-Siegels und des REGIONALFENSTERS erscheint dieses Ergebnis plausibel. Bezüglich des DLG-Siegels, das keine Nachhaltigkeitsaspekte berücksichtigt, überrascht das Ergebnis hingegen.

Für die Nachhaltigkeitsfans scheinen nicht nur alle Tierwohl-Labels, sondern auch zahlreiche weitere Gütesiegel einen positiven Effekt auf die Kaufentscheidung zu haben. Weitere Gütesiegel, die besonders empfohlen werden können, sind das ÖKOTESTSiegel, das NABU-Logo und das Siegel des DEUTSCHEN TIERSCHUTZBUNDES. Die Anbringung des Siegels von STIFTUNG WARENTEST hat bei dieser Gruppe dagegen eher keinen positiven Effekt.

Das Cluster 2 der ,Unentschlossenen Verbraucher' unterscheidet sich nicht nur vom Referenzcluster, sondern auch stark $\mathrm{zu}$ den anderen Verbrauchergruppen. Die ,Unentschlossenen Verbraucher' entstammen womöglich einem anderen sozialen Milieu als die Nachhaltigkeitsfans und die Bio-Fans. Sie unterscheiden sich bzgl. ihrer Soziodemografie in mehreren Punkten von den ,Desinteressierten Label-Ablehnern“ im Referenzcluster. Signifikante Unterschiede konnten bzgl. des Geschlechts, des Alters, der Ortsgröße und der Bildung festgestellt werden. Die ,Unentschlossenen Verbraucher ‘ weisen einen signifikant höheren Anteil an Frauen als das Referenzcluster auf. 
Der Männeranteil in dieser Gruppe ist jedoch höher als bei den ,Enthusiastischen Nachhaltigkeitsfans` und der Gruppe ,Tierwohl ja - Bio nein` in Cluster 5. Besonders auffällig ist, dass die ,Unentschlossenen Verbraucher' von allen Clustern die jüngste Gruppe darstellen. Außerdem lebt diese Verbrauchergruppe häufiger auf dem Land oder in Kleinstädten. Der niedrige Regressionskoeffizient lässt zudem erkennen, dass die Personen in Cluster 2 über das geringste Einkommen von allen Segmenten verfügen, was aufgrund des relativ jungen Alters plausibel erscheint.

Es ließ sich zudem feststellen, dass sich die ,Unentschlossenen Verbraucher ' in Cluster 2 bzgl. ihrer Bildung signifikant vom Referenzcluster unterscheiden. Sie verfügen über eine deutlich niedrigere Bildung als die ,Desinteressierten Label-Ablehner ${ }^{`}$. Lediglich die Verbraucher in Cluster 5 (Gruppe, Tierwohl ja - Bio nein') haben eine noch niedrigere Bildung (die niedrigste von allen Gruppen). Die Verbraucher der Cluster 2 und 5 bilden demzufolge eher eine relativ junge Verbrauchergruppe mit sehr niedriger Bildung.

Hinsichtlich der Einkaufsstättenwahl konnte für die ,Unentschlossenen Verbraucher (Cluster 2) herausgefunden werden, dass sie signifikant seltener Fleisch- und Wurstwaren auf dem Wochenmarkt kaufen als die ,Desinteressierten Verbraucher' des Referenzclusters. Mit Blick auf die Regressionskoeffizienten wird deutlich, dass diese Personen am seltensten von allen Verbrauchergruppen Wochenmärkte und Hofläden für den Fleischkauf nutzen. Bio-Supermärkte werden von dieser Gruppe ebenfalls so gut wie gar nicht als Einkaufsstätte genutzt.

Die Wechselbereitschaft der Einkaufsstätte aufgrund eines besseren Angebots an Tierwohlprodukten in einer anderen Einkaufsstätte ist in dieser Gruppe am niedrigsten von allen Clustern. Daraus kann geschlussfolgert werden, dass diesen Verbrauchern ein breites Sortiment an Tierwohlprodukten weniger wichtig ist und sie nur schwer von Wettbewerbern, die ein besseres Angebot an Tierwohlprodukten haben, ,abgeworben“ werden können. Sie stellen in diesem Sinne die treueste Kundengruppe dar. 
Besonders viele signifikante Unterschiede zum Referenzcluster zeigten sich bei der Nutzung von Supermärkten, Discountern und SB-Warenhäusern. Die jungen ,Unentschlossenen Verbraucher' vom Land mit niedriger Bildung (Cluster 2) bevorzugen für ihren Fleisch- und Wurstkauf besonders LIDL. Daneben ist auch ALDI sehr beliebt. Für den Fleischkauf werden teilweise auch KAUFLAND, REAL und REWE genutzt.

Bei diesen vier Einkaufsstätten unterscheidet sich das Cluster 2 signifikant vom Referenzcluster. Die Koeffizienten vergleichend lässt sich erkennen, dass EDEKA von dieser Verbrauchergruppe nur selten genutzt wird, womöglich da ihnen die Fleisch- und Wurstwaren bzw. das Gesamtsortiment dort zu teuer sind. Auch NETTO erreicht bei dieser Gruppe von allen Gruppen den höchsten Koeffizienten. Daraus kann abgeleitet werden, dass die ,Unentschlossenen Verbraucher' in Cluster 2 überwiegend DiscounterKäufer und teilweise auch SB-Warenhaus-Käufer sind.

Hinsichtlich ihres Konsumverhaltens unterscheiden sich die ,Unentschlossenen Verbraucher' (Cluster 2) in zwei Punkten signifikant von der Referenzgruppe, und zwar bzgl. der Konsumhäufigkeit im Haushalt und der Reduktionsbereitschaft. Der Fleischkonsum ist höher als in der Referenzgruppe, die Reduktionsbereitschaft jedoch ebenfalls. Diese Gruppe, die überwiegend aus jüngeren Discounter-Käufern und SBWarenhaus-Käufern besteht, scheint demzufolge von allen Gruppen am meisten Fleischund Wurstwaren zu essen, möchte aber zukünftig weniger Fleisch- und Wurstwaren verzehren.

Im Hinblick auf die Kaufkriterien, die für die Verbraucher des Clusters 2 beim Fleischkauf eine zentrale Rolle spielen, zeigt der niedrige Koeffizient beim Faktor Nachhaltigkeit an, dass diese Gruppe am wenigsten auf Nachhaltigkeitskriterien achtet. Äußere Merkmale wie der Preis sind ihnen deutlich wichtiger als den Bio-Fans, was auch die hohe Präferenz für Discounter und SB-Warenhäuser plausibel erscheinen lässt. Der Preis stellt für diese Verbrauchergruppe offensichtlich das zentrale Motiv bei der Einkaufsstättenwahl dar, wahrscheinlich auch deswegen, da ihnen von allen gesellschaftlichen Gruppen nur das geringste Einkommen zur Verfügung steht. 
Das niedrige Einkommen könnte womöglich auch mit dem jungen Altersdurchschnitt zusammenhängen (es handelt sich hierbei um die jüngste Gruppe). Die Verbraucher des Clusters 2 kaufen also gerne viel Fleisch und Wurst zu günstigen Preisen und legen insgesamt wenig Wert auf besondere Produktqualitäten. Die konkreten Herkunftsangaben sind dieser Gruppe im Vergleich zu den Nachhaltigkeitsfans (Cluster 1) und den BioFans (Cluster 3) ebenfalls nicht so wichtig, d. h. die meist ungenauen Herkunftsangaben bei Fleisch- und Wurstwaren (z. B. ,Hergestellt in Deutschland') reichen diesen Verbrauchern aus.

Deutschen Verbrauchern wird häufig von zahlreichen gesellschaftlichen Gruppen (Landwirten, NGOs, Medien, Wissenschaftlern, LEH etc.) pauschal vorgeworfen, sie würden mehr Tierwohl fordern, sich aber widersprüchlich dazu verhalten und vor allem billiges Fleisch kaufen. Dieser Vorwurf trifft jedoch nur auf Teile des Clusters 2 und Teile des Clusters 5 (Gruppe ,Tierwohl ja - Bio nein`) zu, die gemeinsam nur rd. ein Viertel der Gesellschaft ausmachen. Die Affinität zu Tierwohlprodukten ist in der Gruppe der ,Unentschlossenen Verbraucher' überraschenderweise ähnlich hoch wie bei den BioFans (Cluster 3) und der Gruppe ,Tierwohl ja - Bio nein“(Cluster 5). Dies zeigt sich in der Aufgeschlossenheit dieser Gruppe gegenüber Tierwohlprodukten (Tierwohlproduktaffinität; F18). Würden Tierwohlprodukte eine vertrauenswürdige Kennzeichnung vorweisen, wären sie gut verfügbar und würden sie zu einem fairen Preis angeboten, würde diese Gruppe diese Produkte auch bevorzugt kaufen. Anders ausgedrückt befürworten die Verbraucher der Cluster 2 und 5 prinzipiell mehr Tierwohl, es soll jedoch klar nachvollziehbar sein, dass die Mehrkosten tatsächlich dem Wohl der Tiere zugutekommen, und die Produkte sollen nicht deutlich mehr kosten als konventionelle Ware.

Die ,Desinteressierten Verbraucher' des Referenzclusters kaufen zwar ebenfalls überwiegend günstige Fleisch- und Wurstwaren aus konventioneller Haltung, fordern aber weniger eine bessere Berücksichtigung des Tierwohls oder eine bessere Berücksichtigung von Umweltschutz- oder Gesundheitsaspekten als die Verbraucher in den Clustern 2 und 5. 
Insgesamt kann demzufolge der Anteil der Verbraucher, die derzeit noch einer ,Hauptsache-billig-Mentalität‘ beim Fleischkauf folgen, in der Gesellschaft auf ca. $40 \%$ geschätzt werden. Dieser Anteil erscheint im Hinblick auf den in Kapitel 2 beschriebenen gesellschaftlichen Wandel hin zu mehr Nachhaltigkeit zwar immer noch hoch, relativiert jedoch die Pauschalaussage, dass ,die Verbraucher' nur billig kaufen würden. Der Großteil der Bevölkerung (ca. 60 \%) in Deutschland achtet mittlerweile auch auf ethische Kriterien beim Fleischkauf und schränkt den Fleischkonsum bewusst ein; mit stark zunehmender Tendenz.

Aus der geringen Kenntnis von Tierwohl-Labels der ,Unentschlossenen Verbraucher könnte geschlussfolgert werden, dass sich die Verbraucher in Cluster 2 wenig für Tierwohl-Labels interessieren. Dies könnte jedoch auch daran liegen, dass sie sich überwiegend in einer ,Einkaufswelt‘ bewegen, in der viele Tierwohl-Labels bzw. Nachhaltigkeitssiegel eher weniger in Erscheinung treten. Die Analyse zu den Einkaufsstättenpräferenzen ergab, dass diese Gruppe überwiegend aus klassischen Discounter-Käufern und SB-Warenhaus-Käufern besteht. Von allen Gruppen kennen sie die wenigsten Tierwohl-Labels. Vom Referenzcluster unterscheidet sich diese Gruppe bzgl. des Bekanntheitsgrads bei fast allen Tierwohl-Labels.

Einen höheren Bekanntheitsgrad als in der Referenzgruppe erreichen bei den ,Unentschlossenen Verbrauchern“ nur die Labels EU-BIO, DE-BIO und das Label des DEUTSCHEN TIERSCHUTZBUNDES. Dies erscheint plausibel, da die staatlichen BioLabels generell einen sehr hohen Bekanntheitsgrad bei allen Gruppen aufweisen und Produkte mit dem Siegel des DEUTSCHEN TIERSCHUTZBUNDES häufig bei Discountern angeboten werden.

Der sehr niedrige Bekanntheitsgrad der Tierwohl-Labels der ökologischen Anbauverbände DEMETER, NATURLAND und BIOKREIS ist womöglich dadurch zu begründen, dass diese Produkte in den letzten Jahrzehnten kaum von Discountern oder SB-Warenhäusern angeboten wurden. 
Ergänzende Gütesiegel wie das ÖKO-TEST-Siegel, das Label des DEUTSCHEN TIERSCHUTZBUNDES, das DLG-Siegel und das QS-Siegel bewerten die ,Unentschlossenen Verbraucher' positiver als das Referenzcluster, d. h. diese Siegel sollten am ehesten für die Verbraucher in Cluster 2 angeboten werden. Das NABU-Logo hat dagegen für diese Gruppe offensichtlich keinen positiven Einfluss auf die Kaufentscheidung. Hersteller und Händler können demzufolge auf den Einsatz dieses Logos sowie wahrscheinlich auch auf Siegel weiterer NGOs für diese Gruppe verzichten, da kein positiver Effekt auf die Kaufentscheidung nachgewiesen werden konnte. Dies lässt den Schluss $\mathrm{zu}$, dass diese Gruppe womöglich auch für Cause-RelatedMarketingmaßnahmen nicht zu begeistern ist.

Die Gruppe der ,Überzeugten Bio-Fans ' in Cluster 3 besteht, ebenso wie die anderen Cluster, signifikant mehr aus Frauen als das Referenzcluster der ,Desinteressierten LabelAblehner'. Ein besonders signifikantes Merkmal dieser Gruppe ist das Einkommen. Die Gruppe der ,Überzeugten Bio-Fans“ verfügt mit Abstand über das höchste Einkommen aller betrachteten Cluster. Das hohe Einkommen ist vermutlich durch die sehr hohe Bildung dieser Personen begründet, die sie für Berufe qualifiziert, die bessere Einkommenserzielungschancen bieten. Die Koeffizienten zeigen, dass die ,Überzeugten Bio-Käufer` eine deutlich höhere Bildung besitzen als die ,Unentschlossenen Verbraucher', die ,Enthusiastischen Nachhaltigkeitsfans“ und vor allem die Gruppe ,Tierwohl ja - Bio nein“ (Cluster 5). Hier wird deutlich, dass der Konsum von BioLebensmitteln auch heute noch in erstaunlich hohem Maße mit der sozialen Schicht (bzgl. Einkommen, Bildung und Beruf) der Verbraucher zusammenhängt. Der regelmäßige Konsum von Bio-Lebensmitteln ist häufig ein Privileg der Oberschicht bzw. der oberen Mittelschicht.

Eine stärkere staatliche Förderung des ökologischen Landbaus bei gleichzeitigen politischen Maßnahmen zur Erhöhung der Preise konventioneller Fleischwaren könnte dazu beitragen, die Preisabstände zwischen ökologischen und konventionellen Fleischwaren zu verringern und dieser ,Zweiklassengesellschaft ${ }^{\star}$ beim Fleischkonsum entgegenzuwirken. 
Beispielhaft genannt seien hier die Anpassung des Mehrwertsteuersatzes für Fleisch- und Wurstwaren auf $19 \%$, die Erhebung einer Fleischsteuer, eine Beschäftigung der Mitarbeiter in der Wertschöpfungskette zu ordentlichen Löhnen oder die Verbesserung von tier- und umweltschutzrechtlichen Vorschriften (WBAE, 2020). Andererseits könnten sich bei einer entsprechenden Reduktion des Fleischkonsums und einer höheren Prioritätensetzung für Lebensmittel im Hinblick auf das Haushaltseinkommen die meisten Personen in Deutschland ein- bis zweimal pro Woche Fleisch- und Wurstwaren aus einer ökologischen Haltung leisten.

Ähnlich wie bei den ,Enthusiastischen Nachhaltigkeitsfans` finden sich unter den BioFans mehr ältere Personen. Dieser Befund ist schwierig zu interpretieren. Zum einen könnte aufgrund des fortgeschrittenen Alters vermutet werden, dass diese Zielgruppen reflektierter sind und mehr auf Gesundheitsaspekte achten und daher eher Bio-Produkte kaufen und ihnen ihr relativ hohes Einkommen auch den regelmäßigeren Konsum von Bio-Lebensmitteln ermöglicht. Zum anderen überraschen diese Ergebnisse jedoch, da vermutet wurde, dass sich heute vor allem junge Verbraucher deutlich stärker für mehr Tierwohl und mehr Nachhaltigkeit (Klimaschutz etc.) einsetzen als ältere Verbraucher; gerade im Hinblick auf die immer häufigeren Proteste von FRIDAYS FOR FUTURE, ENDE GELÄNDE oder EXTINCTION REBELLION.

Ferner könnte es auch sein, dass diese Personen in der vorliegenden Studie unterrepräsentiert sind, weil sich der Großteil der aktivistischen jüngeren Personen ohnehin vegan oder zumindest vegetarisch ernährt oder diese Gruppe seltener an OnlineBefragungen teilnimmt. Ein weiterer Erklärungsansatz könnte darin bestehen, dass der Konsum von Bio-Lebensmitteln bei diesen Personen weniger im Vordergrund steht als andere Nachhaltigkeitsthemen, wie beispielsweise Klima- oder Artenschutz, und dass Parallelen zwischen diesen Themen nicht immer gesehen werden. Womöglich ist der Konsum von Bio-Lebensmitteln für diese junge Gruppe auch einfach zu teuer oder eine andere Person mit geringerem Nachhaltigkeitsinteresse tätigt noch die Einkäufe. 
In der vorliegenden Befragung konnte jedoch mit dem Cluster 2 der, Unentschlossenen Verbraucher' eine große Gruppe jüngerer Personen identifiziert werden, die wenig Wert auf das Thema Nachhaltigkeit (zumindest im Hinblick auf ihr Einkaufsverhalten bei Fleisch- und Wurstwaren) legen. Daher kann vermutet werden, dass es in Deutschland zwei Extremgruppen an jüngeren Verbrauchern gibt. Zum einen die nachhaltigkeitsaffinen und aktivistischen Personen, die häufiger aus gut situierten Familien stammen, jedoch in der Gruppe der ,Überzeugten Bio-Käufer ‘ unterrepräsentiert sind und eher zur Gruppe der Nachhaltigkeitsfans zählen. Diesen jungen Nachhaltigkeitsfans steht ein mindestens ebenso großer Teil an jüngeren Menschen aus sozial schwächeren und bildungsferneren Schichten (Cluster 2 und teilweise auch Cluster 5) gegenüber, die generell weniger Wert auf Nachhaltigkeit legen und auch beim Einkauf von Fleisch- und Wurstwaren nur wenig auf ethische Kaufkriterien achten, obgleich sie mehr Tierwohl befürworten.

Hinsichtlich der Ortsgröße ließ sich zudem anhand der Koeffizienten ablesen, dass die ,Überzeugten Bio-Fans‘ häufiger in Städten leben als die ,Desinteressierten LabelAblehner' des Referenzclusters, wenn auch nicht signifikant. Ein signifikantes Merkmal der ,Überzeugten Bio-Fans“ ist zudem die Bereitschaft, ihre Haupteinkaufsstätte aufgrund eines besseren Angebots an Tierwohlprodukten in einer anderen Einkaufsstätte zu wechseln. Die Wechselbereitschaft ist bei den Bio-Fans deutlich stärker ausgeprägt als bei den ,Desinteressierten Label-Ablehnern“. Lediglich die Nachhaltigkeitsfans (Cluster 1) und die Fans reiner Tierwohl-Labels (Cluster 5) weisen eine noch höhere Wechselbereitschaft auf.

Anhand dieser Ergebnisse kann auf die hohe Bedeutung eines guten Angebots an Tierwohlprodukten für diese drei Gruppen geschlossen werden. Anders ausgedrückt ist das Risiko, diese überdurchschnittlich einkommensstarken Käufergruppen zu verlieren, am höchsten, wenn die Auswahl des Angebots an zertifizierten Fleisch- und Wurstwaren zu gering ist oder auf die falschen Tierwohl-Label-Standards gesetzt wird. Außerdem ist zu beachten, dass sich die Frage auf die Haupteinkaufsstätte bezieht. 
Die meisten Personen würden vor dem Hintergrund des beliebten ,One-Stop-Shopping ‘ dann auch alle anderen Güter des täglichen Bedarfs bei einem Wettbewerber kaufen, der ein breiteres und hochwertigeres Sortiment an Tierwohlprodukten führt. Dem für viele Menschen relevanten Angebot an Tierwohlprodukten kann insgesamt eine zentrale Rolle beim Kampf um die attraktivsten Käufergruppen im Lebensmitteleinzelhandel zugesprochen werden.

Hinsichtlich der Einkaufsstättenpräferenzen der überzeugten Bio-Käufer lässt sich außerdem feststellen, dass die Bio-Fans neben den Nachhaltigkeitsfans von allen Gruppen Bio-Supermärkte am meisten nutzen, was plausibel erscheint. Diese Gruppe präferiert als Einkaufsstätte für Fleisch- und Wurstwaren auch den Metzger und Hofläden. Es zeigte sich, dass die Bio-Fans eher weniger SB-Warenhäuser (KAUFLAND, REAL) und Discounter (ALDI, LIDL, NETTO) für den Fleisch- und Wurstkauf nutzen. Von den drei Discountern wird ALDI von Bio-Käufern noch am häufigsten genutzt, während NETTO am seltensten von Bio-Käufern für den Fleischkauf genutzt wird. Außerdem ist REWE offensichtlich bei den Bio-Käufern beliebter als EDEKA. Eigenen Recherchen zufolge stellte REWE seinen Bio-Kunden frühzeitiger ein hochwertigeres und umfangreicheres Angebot an Tierwohlprodukten bereit als EDEKA. Die mit dem relativ hohen NATURLAND-Standard ausgezeichnete Produktlinie im SBBereich umfasst seit vielen Jahren neben Aufschnitt und Wurstwaren auch unterschiedliche zertifizierte ökologische Fleisch- und Wurstwaren wie Schweineschnitzel, Rindersteak oder Hähnchenbrust. Gemäß den Ergebnissen der vorliegenden Studie sollten entsprechende Produkte allerdings im Idealfall an der Frischetheke in einem separaten Bereich angeboten werden.

Wie zu erwarten war, unterscheiden sich die, Überzeugten Bio-Fans' von der Referenzgruppe bzgl. der Beachtung von Nachhaltigkeitskriterien und klassischen Kaufkriterien besonders deutlich. Die Bio-Fans achten signifikant häufiger auf Nachhaltigkeitskriterien, jedoch weniger auf klassische Kaufkriterien (innere Qualität) als die Referenzgruppe. Sie legen außerdem von allen Gruppen den geringsten Wert auf äußere Merkmale wie den Preis, bestimmte Marken oder die Verpackung. 
Möglichst konkrete Herkunftsangaben sind den Bio-Fans ebenfalls wichtiger als den ,Desinteressierten Verbrauchern' des Referenzclusters (Cluster 4). Hieran zeigt sich die hohe Bedeutung des Themas Regionalität für die Bio-Fans. Bezugnehmend auf die Konsumhäufigkeit im Haushalt (F6) essen die Bio-Fans signifikant weniger Fleisch als die Personen des Referenzclusters. Hinsichtlich ihres eigenen Konsumverhaltens (F7) bilden die Bio-Fans zudem die Gruppe mit den meisten Flexitariern von allen Clustern, d. h. in der Gruppe der Bio-Fans haben die meisten Menschen ihren Fleischkonsum bereits bewusst reduziert. Es wird deutlich weniger Fleisch und Wurst gegessen, aber dafür in Bio-Qualität (,Less-but-better-Prinzip‘).

Die ,Überzeugten Bio-Fans ' unterscheiden sich jedoch vom Referenzcluster nur in ihrer Kenntnis des DE-BIO-Siegels signifikant, die deutlich höher ist als bei den ,Desinteressierten Verbrauchern'. Ursächlich hierfür könnte sein, dass der Großteil der Bio-Fans vor allem das DE-BIO-Siegel kennt und nur einem kleineren Teil der Bio-Fans die Siegel der ökologischen Anbauverbände bekannter sind als der Gruppe der ,Desinteressierten Verbraucher' (Referenzcluster 4). Die Koeffizienten zeigten jedoch, dass nahezu alle Tierwohl-Labels, insbesondere DEMETER und NATURLAND, den ,Überzeugten Bio-Fans` bekannter sind als der Gruppe der ,Unentschlossenen Verbraucher' (Cluster 2) und der Gruppe ,Tierwohl ja - Bio nein“ (Cluster 5). Eine Ausnahme bildet lediglich der höhere Bekanntheitsgrad von EU-BIO in der jüngeren Gruppe der ,Unentschlossenen Verbraucher' (Cluster 2) und bei den Nachhaltigkeitsfans (Cluster 1).

Dies liegt vermutlich daran, dass Cluster 3 eher aus traditionellen und älteren BioKäufern besteht und vielen EU-BIO nicht so bekannt ist wie DE-BIO. Obwohl die beiden staatlichen Bio-Siegel in den letzten Jahren häufig gleichzeitig auf Produktverpackungen abgebildet wurden, ist der Bekanntheitsgrad von DE-BIO insgesamt immer noch höher, wie die Ergebnisse der Studie zeigen. Auffällig sind des Weiteren bei den Bio-Fans die niedrigeren Koeffizienten bei den Tierwohl-Labels von NEULAND, TIERSCHUTZBUND und VIER PFOTEN im Vergleich zur Gruppe der Nachhaltigkeitsfans. 
Dies kann gegebenenfalls damit erklärt werden, dass die überzeugten Bio-Fans keine reinen Tierwohlprodukte in Bio-Fachgeschäften oder Bio-Supermärkten vorfinden und diese Produkte normalerweise nicht kaufen. Dennoch überrascht dieses Ergebnis, da von den ,Überzeugten Bio-Fans', die die höchste Bildung aller Cluster vorweisen, eine bessere Allgemeinbildung in Bezug auf die Kenntnis von Tierwohl-Labels erwartet wurde. Insgesamt scheint die Gruppe der Nachhaltigkeitsfans (Cluster 1) mit Abstand das höchste Wissen über Tierwohl-Labels bzw. Nachhaltigkeitssiegel zu besitzen.

Des Weiteren unterscheiden sich die ,Überzeugten Bio-Fans` vom Referenzcluster bzgl. der Bewertung der verschiedenen Labels, die im Rahmen des Multi-Labelings abgefragt wurden. Die Labels GENTECHNIKFREI, REGIONALFENSTER und STIFTUNG WARENTEST haben für die Bio-Fans einen positiveren Einfluss auf die Kaufentscheidung als für die desinteressierten Verbraucher. Dabei ist jedoch anzumerken, dass sich insbesondere STIFTUNG WARENTEST im Vergleich zu den beiden anderen Siegeln nur auf einem sehr geringen Niveau positiv auf die Kaufentscheidung der Bio-Fans auswirkt und STIFTUNG WARENTEST daher keine große Bedeutung für Bio-Fans zugesprochen werden sollte; auch wenn sich die Gruppe in diesem Punkt signifikant vom Referenzcluster unterscheidet. In den hohen Koeffizienten von REGIONALFENSTER, GENTECHNIKFREI-Siegel und ÖKOTEST spiegelt sich dagegen die besonders hohe Bedeutung von Regionalität und Gesundheitsaspekten für die Bio-Fans wider.

Etwas überraschender ist der Befund, dass die Labels des NABU und DEUTSCHEN TIERSCHUTZBUNDES für die Bio-Fans nur einen ähnlich niedrigen positiven Einfluss auf die Kaufentscheidung bei Fleisch- und Wurstwaren haben wie das DLG-Siegel oder das QS-Siegel, wobei aufgrund des starken Bezugs von Bio-Fans zum Thema Tier- und Naturschutz eine deutlich positivere Bewertung erwartet wurde. Möglicherweise ist für einen Großteil der Bio-Käufer die Erhaltung der eigenen Gesundheit ein bedeutenderes Motiv für den Kauf von Bio-Lebensmitteln als der Natur- oder Tierschutz. Wahrscheinlicher ist jedoch, dass diese Organisationen von Bio-Fans in anderen Kontexten überaus positiv bewertet werden, jedoch nicht im Kontext mit Fleisch- und Wurstwaren (als Logo auf einer Fleischverpackung). 
Insbesondere bei Fleisch- und Wurstwaren erscheint es daher für die bessere Ansprache von Bio-Fans weniger sinnvoll zu sein, Logos von NGOs in der Vermarktung einzusetzen, um besondere Umweltvorteile oder Vorteile für die Tiere mit Hilfe dieser Logos hervorzuheben. Womöglich sind ihnen die negativen Effekte des Fleischkonsums auf die Umwelt und die Tiere auch bekannter als den anderen Gruppen und sie empfinden aus diesem Grund die Verwendung dieser Logos als unpassend.

Abschließend soll versucht werden, die besondere Gruppe ,Tierwohl ja-Bio nein • (Cluster 5) zu charakterisieren. Die Verbraucher dieses Clusters unterscheiden sich bzgl. ihrer Soziodemografie in einigen Punkten hoch signifikant vom Referenzcluster. Es besteht zunächst ein signifikant höherer Anteil an Frauen als bei den ,Desinteressierten Label-Ablehnern' im Referenzcluster. Sie leben zudem deutlich häufiger auf dem Land und sind im Durchschnitt jünger als die Nachhaltigkeitsfans oder die Bio-Fans. Das Einkommen dieser Gruppe ist dem Koeffizienten zufolge jedoch deutlich niedriger als das Einkommen der Bio-Fans. Dies liegt womöglich an der sehr niedrigen Bildung dieser Personengruppe; der niedrigsten Bildung aller Verbrauchergruppen. Das Einkommen ist jedoch höher als das Einkommen der ,Unentschlossenen Verbraucher' in Cluster 2, was etwas überrascht, da deren Bildung zwar ebenfalls niedrig ist, aber deutlich höher als die Bildung der Verbraucher in Cluster 5. Ein Erklärungsansatz für das höhere Einkommen der Personen in Cluster 5 im Vergleich zu den Personen in Cluster 2 könnte sein, dass die Verbraucher in Cluster 5 im Durchschnitt älter sind und daher ein höheres Einkommen aufweisen.

Beim Einkaufsverhalten ließ die Gruppe, Tierwohl ja - Bio nein` eine sehr hohe Wechselbereitschaft bzgl. ihrer Haupteinkaufsstätte erkennen. Demzufolge können diese Verbraucher mit einem verbesserten Angebot an Fleisch- und Wurstwaren aus einer artgerechteren Nutztierhaltung ebenso wie die Nachhaltigkeitsfans oder die Bio-Fans leicht zu einem Wechsel ihrer Haupteinkaufsstätte motiviert werden, jedoch nicht mit Bio-Produkten. Auch wenn sie sehr ähnliche Einkaufsstätten nutzen wie die Verbraucher des Clusters 2, scheint den Verbrauchern in Cluster 5 das Tierwohl deutlich wichtiger zu sein. 
Diese Gruppe nutzt Bio-Supermärkte am seltensten, was aufgrund der Ablehnung von Bio-Produkten plausibel ist. Wochenmärkte und Hofläden gehören ebenfalls nicht zu den favorisierten Einkaufsstätten dieser Gruppe. Bemerkenswert bei dieser Gruppe ist deren Angabe in der Befragung, dass ihnen Tierwohl durchaus wichtig sei, sie jedoch BioProdukte ablehnen. Dies kann evtl. damit erklärt werden, dass großen Teilen dieser Gruppe nicht bekannt ist, dass Bio-Produkte meistens die höchsten Tierwohlstandards aufweisen, was wohl mit dem sehr niedrigen Bildungsniveau zusammenhängt. Ein weiterer Grund könnte sein, dass diese preisorientierte Gruppe, die überwiegend aus Discounter-Käufern besteht, generell nur eine geringe Zahlungsbereitschaft für Fleischund Wurstwaren besitzt und nicht bereit ist, die höheren Preise für Bio-Produkte zu bezahlen.

Insgesamt präsentiert sich diese Gruppe als heterogen, was die Einkaufsstättenwahl betrifft, da im Gegensatz zu anderen Clustern keine klaren Tendenzen für eine Vertriebsform zu identifizieren sind. Die hohen Koeffizienten bei ALDI und SBWarenhäusern lassen jedoch vermuten, dass diese Gruppe hinsichtlich der Einkaufsstättenwahl den Verbrauchern des Clusters 2 am ähnlichsten ist und sie tendenziell eher zu den Discounter- und SB-Warenhaus-Käufern gehören, jedoch mit dem Unterschied, dass zu dieser Gruppe mehr EDEKA- und NETTO-Kunden und weniger REWE- und LIDL-Kunden gehören.

Die Verbraucher in Cluster 5 (,Tierwohl ja - Bio nein') achten beim Kauf von Fleischund Wurstwaren weniger auf Nachhaltigkeitskriterien wie Umweltfreundlichkeit, Gesundheitsaspekte, Fairtrade oder Bio-Qualität. Von allen Nachhaltigkeitskriterien scheint ihnen nur das Tierwohl wichtig zu sein. Auch in diesem Punkt ähnelt die Gruppe ,Tierwohl ja - Bio nein` der Gruppe der, Unentschlossenen Verbraucher` des Clusters 2. Auch wenn Tierwohl als Kaufkriterium (F7) stark befürwortet wird, ist die Kaufabsicht von Tierwohlprodukten (F18) geringer als bei den anderen Gruppen. Es könnte vermutet werden, dass ein Teil der Verbraucher in Cluster 5 zu den Verbrauchern zählt, die sich für mehr Tierwohl aussprechen, jedoch ihr eigenes Handeln nicht an ihren Worten ausrichten. Sie kaufen Fleisch- und Wurstwaren überwiegend bei ALDI und in SBWarenhäusern. 
Diese Einkaufsstätten sind bisher nicht für ein breites Angebot an strengen Tierwohlprodukten bekannt, sondern bieten zum Großteil konventionelle Fleisch- und Wurstwaren auf dem untersten Niveau der INITIATIVE TIERWOHL (Stufe 1) an. Ebenso wie die ,Unentschlossenen Verbraucher' in Cluster 2 sind konkrete Herkunftsangaben für die Gruppe ,Tierwohl ja - Bio nein“ (Cluster 5) wenig relevant, d. h. ihnen reichen grobe Herkunftsangaben wie ,Wurst aus Deutschland“ aus. Die Reduktionsbereitschaft bzgl. des Fleischkonsums ist von allen Clustern am geringsten, d. h. es handelt sich um eine Gruppe, die mehr Tierwohl befürwortet, jedoch nicht weniger Fleisch essen möchte.

Hinsichtlich ihrer Kenntnis von Tierwohl-Labels unterscheidet sich die Gruppe ,Tierwohl ja - Bio nein' vom Referenzcluster durch eine höhere Bekanntheit des DE-BIO-Siegels und des Siegels des DEUTSCHEN TIERSCHUTZBUNDES sowie einer geringeren Bekanntheit von DEMETER. Cluster 5 weist dabei zusammen mit Cluster 2 die höchste Kenntnis des Labels des DEUTSCHEN TIERSCHUTZBUNDES auf. Dies liegt womöglich daran, dass in ihren favorisierten Einkaufstätten (Discountern und SBWarenhäusern) zahlreiche Fleisch- und Wurstwaren angeboten werden, die das TierwohlLabel des DEUTSCHEN TIERSCHUTZBUNDES tragen. NATURLAND, DEMETER und BIOKREIS weisen in dieser Gruppe, wie in der Gruppe der ,Unentschlossenen Verbraucher' (Cluster 2), einen sehr niedrigen Bekanntheitsgrad auf, was wahrscheinlich ebenfalls mit den Einkaufsstättenpräferenzen der Gruppe zusammenhängt. Discounter und SB-Warenhäuser führen kaum Produkte mit diesen Tierwohl-Labels.

Bei den weiteren abgefragten Gütesiegeln (Multi-Labeling) ergab sich, dass für die Verbraucher des Clusters 5 (Gruppe ,Tierwohl ja - Bio nein') das Siegel von STIFTUNG WARENTEST und das Siegel des DEUTSCHEN TIERSCHUTZBUNDES ein deutlich positiveres Kaufargument darstellen als für die Referenzgruppe. Auffällig ist, dass STIFTUNG WARENTEST von dieser Gruppe von allen Clustern am häufigsten gewählt wurde und es sich daher für diese Gruppe am besten eignet. Auch das QS-Siegel wird von dieser Gruppe positiv bewertet. 
Gegenteilig dazu verhält es sich mit dem ÖKOTEST-Siegel, dem NABU-Logo und dem DLG-Siegel, die sich allesamt für diese Gruppe nur wenig eignen. Gentechnikfreiheit ist dieser Gruppe am unwichtigsten. In den Präferenzen zum Multi-Labeling spiegeln sich zum einen die hohe Affinität zum Thema Tierwohl und zum anderen die niedrige Affinität zum Natur- und Umweltschutz wider. Zusammenfassend kann festgestellt werden, dass zahlreiche Unterschiede zwischen den Verbrauchergruppen bzgl. ihrer Soziodemografie bestehen. Die interessantere Differenzierung und Charakterisierung ist jedoch bzgl. des beobachtbaren Kaufverhaltens (z. B. Einkaufsstättenwahl) und der psychographischen Merkmale (z. B. Beachtung von Kaufkriterien, Label-Bekanntheit etc.) möglich.

\subsubsection{Handlungsempfehlungen für die identifizierten Marktsegmente}

Nachdem alle Marktsegmente bzw. Verbrauchergruppen, die mit der Clusteranalyse identifiziert wurden, detailliert beschrieben wurden, können Anbieter von Fleisch- und Wurstwaren die Zielsegmente, die sie aufgrund der Label-Präferenzen und Charakterisierung der Verbraucher ,bearbeiten`wollen, auswählen. Diese Entscheidung sollte bei den Anbietern von Fleisch- und Wurstwaren, unabhängig davon, ob sie Hersteller oder Lebensmitteleinzelhändler sind, vom strategischen Management getroffen werden.

Da die Auswahl der Marktsegmente eine der bedeutendsten strategischen Entscheidungen im Marketing darstellt, sollte diese Entscheidung möglichst auf wissenschaftlichen Erkenntnissen beruhen, mit einer mittel- bis langfristigen Perspektive mit Bedacht getroffen und konsequent umgesetzt werden. Die Entscheidung für oder gegen die Bearbeitung eines Marktsegments orientiert sich an den strategischen Zielen eines Unternehmens und ist von zahlreichen Faktoren abhängig (BÖCKENHOLT et al., 2018; DAVIDSON \& LA ROCHE, 2018; DEEKELING \& BARGHOP, 2017; ERRICHIELLO \& ZSCHIESCHE, 2017; HEINRICH, 2017, KREUTZER, 2018; RUTSCHMANN, 2018; STEHR \& STRUVE, 2017; THEOBALD, 2017; THEOBALD \& HAISCH, 2017). 
Das dies für jedes Unternehmen individuell zu prüfen ist, kann an dieser Stelle einzelnen Anbietern keine konkrete Empfehlung bzgl. der Auswahl von Marktsegmenten ausgesprochen werden. Im Folgenden wird jedoch beispielhaft erläutert, welche Konsequenzen die Auswahl eines bestimmten Marktsegments bzw. einer bestimmten Verbrauchergruppe für die Gestaltung des Marketing-Mix im Hinblick auf das Angebot von Tierwohlprodukten haben kann. Die Ausführungen erheben dabei keinen Anspruch auf Vollständigkeit, da dieser Themenkomplex zu umfangreich für eine ausführliche Beschreibung aller existierenden Marketinginstrumente ist.

Was die Produktstrategie betrifft, so ist für das Cluster 1, das von den ,Enthusiastischen Nachhaltigkeitsfans' ${ }^{6}$ gebildet wird, eine Premiumstrategie für die gesamte Vermarktung zu empfehlen, da die Qualität der Produkte eine sehr hohe Bedeutung für diese Zielgruppe hat. Da sich die Nachhaltigkeitsfans besonders für die ethischen Aspekte der Produktqualität interessieren, sollten Anbieter dieser kritisch-reflektierten Zielgruppe Fleisch- und Wurstwaren anbieten, die mit möglichst strengen Tierwohl-Labels gekennzeichnet sind. Das Aussehen der Ware, der Geschmack und die Frische sind für diese Gruppe ebenfalls sehr wichtig. Diese klassischen Kaufkriterien eignen sich gleichwohl weniger gut als Differenzierungsmerkmale wie die ethischen Kaufkriterien, da nahezu alle Verbrauchergruppen gleichermaßen auf diese klassischen Kaufkriterien achten.

Je mehr Nachhaltigkeitsaspekte hinsichtlich der ,ethischen Produktqualität‘ berücksichtigt werden, desto besser werden die Produkte von den Nachhaltigkeitsfans bewertet. Dies bedeutet zunächst, dass möglichst strenge Tierwohl-Labels verwendet werden sollten. Teile dieser Gruppe würden im Gegensatz zu den ,Überzeugten BioFans' jedoch auch Produkte kaufen, die mit reinen Tierwohl-Labels wie dem Label von VIER PFOTEN oder dem Label des DEUTSCHEN TIERSCHUTZBUNDES ausgezeichnet sind. Die Nachhaltigkeitsfans stehen den verschiedenen Arten von Tierwohlprodukten insgesamt sehr aufgeschlossen gegenüber. Um weitere positive Kaufargumente für die Nachhaltigkeitsfans zu schaffen, können Anbieter die Fleisch- und Wurstwaren mit den Labels GENTECHNIKFREI und ÖKOTEST (,sehr gut ${ }^{\star}$ ) auszeichnen. 
Eine besondere Bedeutung kommt auch dem Angebot regionaler Produkte zu, die mit einer möglichst konkreten Herkunftskennzeichnung (idealerweise der Angabe der Adresse des Bauernhofs) versehen werden sollten. Hierfür eignen sich Regionalsiegel wie z. B. das REGIONALFENSTER (JANSSEN et al., 2014). Je breiter und tiefer bzw. vielfältiger das Produktsortiment an Tierwohlprodukten dabei ist, desto besser. Da den Nachhaltigkeitsfans auch die, äußeren Produktmerkmale ' besonders wichtig sind, sollten die besonders umweltfreundlich und ästhetisch verpackten Produkte im Rahmen einer einheitlich vermarkteten nachhaltigen Produktlinie mit hohem Wiedererkennungswert angeboten werden, mit dessen Image sich die Nachhaltigkeitsfans identifizieren können. Ein gutes Beispiel hierfür ist die NATURLAND-Produktlinie von REWE.

Die Vermarktung der Fleisch- und Wurstwaren sollte sich eng am nachhaltigen Lifestyle dieser Verbraucher orientieren und sich in diesem Punkt deutlich von der Vermarktung konventioneller Fleisch- und Wurstwaren unterscheiden. Anbieter von Fleisch- und Wurstwaren können sich hinsichtlich der Vermarktung nachhaltigerer Produktalternativen auch mittels ,Benchmarking' an anderen Branchen oder Produkten orientieren wie z. B. ,Meine Lieblings Forelle، von KRONE FISCH aus zertifizierter Aquakultur (Aqua Stewardship Council Standard; ASC) oder der Vermarktung nachhaltiger Modemarken, die diese Zielgruppe evtl. kauft (z. B. Knowledge Cotton Apparel etc.). Auf diesem Weg könnten die Anbieter ein besseres Gefühl für die Zielgruppe erhalten.

Nachhaltiger zu leben ist für viele Nachhaltigkeitsfans ein Lifestyle, aber keine reine Modeerscheinung bzw. ein Trend, der wieder vergeht. Dem Trend liegt bei den meisten Nachhaltigkeitsfans (Lohas, Bobos, Hipster, Hippies etc.) ein ernsthaftes Interesse an einer nachhaltigeren Wirtschaftsweise zugrunde. Der Werteindex 2020 vom Marktforschungsinstitut KANTAR bestätigt diese Entwicklung weg vom reinen Lifestyle hin zu mehr Politik (WUV, 2020). Die Menschen werden insgesamt reflektierter und Diskussionen werden kritischer, politischer und handfester geführt als noch vor einigen Jahren. Insbesondere jüngere Nachhaltigkeitsfans erwarten von Entscheidern in Wirtschaft und Politik eine bessere Berücksichtigung von Nachhaltigkeitsaspekten und werden diese Forderungen in Zukunft immer stärker ausdrücken (DFV, 2020). 
Bezüglich der Kommunikation ergaben sich in der vorliegenden Studie überraschenderweise keine relevanten Unterschiede bei der Nutzung von Kommunikationsmedien (Prospekte, Internet, Verpackung) unter den sehr unterschiedlichen Verbrauchergruppen, sodass diesbezüglich keine zielgruppenspezifische Differenzierung vorzunehmen ist. Da sich die Nachhaltigkeitsfans von allen Gruppen jedoch am besten mit dem Thema Nachhaltigkeit auskennen und eine höhere Bildung als die Käufer konventioneller Ware vorweisen, sollte sich die Kommunikation im Niveau und Tonalität (die sich eher an Personen mittleren Alters richten sollte) unterscheiden.

Statt diese Gruppe mit längst bekannten Fakten zu konfrontieren, sollten im Rahmen der Kommunikation besser neue interessante Erkenntnisse und Zusammenhänge im Hinblick auf die konkreten Produktvorteile für die Tiere oder die Umwelt für diese wissbegierige Zielgruppe beschrieben werden. Die gesamte Charakterisierung der Nachhaltigkeitsfans deutet darauf hin, dass sich neben der Auswahl der passenden Tierwohl-Labels die gesamte Kommunikation für Fleisch- und Wurstwaren aus einer artgerechteren und nachhaltigeren Nutztierhaltung deutlich von der standardisierten Massenwarenvermarktung unterscheiden sollte.

Im Gegensatz zu den meisten anderen Gruppen sind die Nachhaltigkeitsfans auch für Cause-Related-Marketing-Maßnahmen bzw. Kooperationen mit NGOs zu begeistern. Die kombinierte Verwendung von Tierwohl-Labels und dem NABU-Logo wurde von dieser Gruppe sehr positiv bewertet. Derartige Kooperationen könnten im Zusammenhang mit entsprechenden Werbekampagnen und Gewinnspielen kreativ umgesetzt werden. Da Fleisch- und Wurstwaren jedoch im Gegensatz zu vielen anderen Produkten besonders kritisch betrachtet werden, besteht die Gefahr, dass es bei Teilen der Nachhaltigkeitsfans sowie Teilen der NGO-Unterstützern bei einer derartigen Kooperation zu Reaktanzen kommt, selbst wenn das übergeordnete strategische Ziel einer Kooperation darin bestünde, den Anteil an artgerechter und nachhaltiger produzierten Fleisch- und Wurstwaren in Deutschland zu erhöhen und mehr Menschen zu einem nachhaltigeren Konsum aufzurufen. 
Im Hinblick auf die Preispolitik ist aufgrund des sehr hohen Interesses an Nachhaltigkeitsaspekten und des relativ hohen Einkommens davon auszugehen, dass die Nachhaltigkeitsfans eine höhere Zahlungsbereitschaft für Fleisch- und Wurstwaren aus einer artgerechteren und nachhaltigeren Nutztierhaltung haben als die Verbraucher der Cluster 2, 4 und 5. Um konkreter zu ermitteln, wie viel die Nachhaltigkeitsfans für unterschiedlich strenge Tierwohlstandards bezahlen würden, sind dennoch konkrete Zahlungsbereitschaftsanalysen notwendig. Dabei wird es in der Gruppe der Nachhaltigkeitsfans voraussichtlich sehr heterogene Zahlungsbereitschaften je nach Soziodemografie und untersuchten Tierwohl-Labels geben, die in weiteren wissenschaftlichen Studien differenziert untersucht werden müssen. Der Preis der Tierwohlprodukte eignet sich als Differenzierungskriterium zu konventioneller Ware, da höhere Preise für höhere seriöse Tierwohlstandards erwartet werden und einen Einfluss auf die Wahrnehmung des Tierwohlniveaus haben (Preis als Imagefaktor).

Nachhaltigkeitsfans reagieren dabei, wie die ,nachhaltigkeitsfernen“ Verbraucher, überraschenderweise auch auf besondere Preisangebote (mehr als die Bio-Fans). Daher ist den Anbietern neben der Verfolgung einer differenzierten Premiumpreisstrategie zu empfehlen, weitere Instrumente der Preispolitik einzusetzen und kreativ zu gestalten, wie beispielsweise Mengenrabatte durch eine gemischte Preisbündelung oder Staffelpreise (z. B. zwei Tierwohlprodukte für $10 €$ bei einem Einzelpreis von $6 €$ ) oder ein TierwohlTreuepunktesystem (z. B. 1 Treue-Punkt je Euro Umsatz; bei 100 Punkten gibt es ein Tierwohlprodukt oder eine Grillzange etc. gratis). Begrüßenswert sind aus Sicht dieser Zielgruppe womöglich auch Incentives wie Gutscheine oder besondere TierwohlAngebote an bestimmen Wochentagen.

Hinsichtlich der Vertriebspolitik legen die Ergebnisse nahe, dass sich die Nachhaltigkeitsfans vom Referenzcluster vor allem in der häufigeren Nutzung von BioSupermärkten unterscheiden. Daher sollten Anbieter von Fleisch- und Wurstwaren aus einer besonders artgerechten und nachhaltigen Nutztierhaltung diesem Absatzweg eine besondere Bedeutung einräumen. Dabei müssen die Produkte jedoch mindestens BioStandard haben, da Bio-Supermärkte ausschließlich Bio-Produkte verkaufen. 
Beim Vergleich der Regressionskoeffizienten zwischen den Clustern konnte zudem festgestellt werden, dass bei den Nachhaltigkeitsfans EDEKA und REWE relativ beliebt sind, während NETTO und die SB-Warenhäuser KAUFLAND und REAL von dieser Gruppe am wenigsten von allen Gruppen für den Fleischeinkauf genutzt werden. Diesen Erkenntnissen folgend sollten möglichst die passenden Absatzwege für die Nachhaltigkeitsfans gewählt werden.

Nachhaltigkeitsfans wechseln zudem deutlich schneller ihre Haupteinkaufsstätte (aufgrund eines besseren Angebots an Tierwohlprodukten in einer anderen Einkaufsstätte) als die meisten anderen Verbraucher. Es handelt sich somit um eine eher untreue Kundengruppe, wenn ihre Bedürfnisse hinsichtlich Tierwohl und Nachhaltigkeit in ihrer Einkaufsstätte nicht zufriedenstellend erfüllt werden. Vice versa kann diese Verbrauchergruppe am ehesten durch ein möglichst gut an ihre Bedürfnisse angepasstes Produktsortiment als neue Kundengruppe gewonnen werden. In der sehr hohen Wechselbereitschaft der Nachhaltigkeitsfans drückt sich die besonders hohe Bedeutung von Tierwohls und Nachhaltigkeit für diese Gruppe aus. Daraus kann die Empfehlung abgeleitet werden, dass die Wechselbewegungen dieser Verbrauchergruppe zwischen den Einkaufsstätten regelmäßig beobachtet werden sollten.

Insgesamt leben Nachhaltigkeitsfans zudem mehr in größeren Städten und weniger auf dem Land, was ebenfalls bei der strategischen Ausrichtung der Distributionspolitik berücksichtigt werden sollte. Weitere vertriebsrelevante Empfehlungen umfassen das schwerpunktmäßige Angebot von Tierwohlprodukten an Fleischtheken statt im SBBereich mit einer möglichst kompetenten Beratung zu den unterschiedlichen Standards bei einem möglichst getrennten Bereich in den Einkaufsstätten für Tierwohlprodukte bzw. für diese Zielgruppe.

Hersteller sollten bei der Auswahl von Einkaufsstätten darauf achten, dass die Märkte eine möglichst angenehme Einkaufsatmosphäre (stilvolle Inneneinrichtung, Beleuchtung, Musik, Ruhe, Freundlichkeit etc.) haben, da die Ästhetik diesen Verbrauchern häufig wichtiger ist als anderen Verbrauchergruppen (z. B. Discounter-Käufern). 
Manche Bio-Supermärkte haben dies bereits berücksichtigt und unterscheiden sich hinsichtlich der Gestaltung der Einkaufsstätte deutlich von den meisten konventionellen Einkaufsstätten durch höherwertigere und stilvollere Einrichtungen z. B. mit mehr Holz und Metall. Die klassischen Vertriebsziele, wie eine starke Präsenz vor Ort, eine gute Erreichbarkeit sowie kurze Lieferzeiten, müssen natürlich ebenfalls über die von der Zielgruppe gewählten Einkaufsstätten realisierbar sein.

Als nächstes soll für die Gruppe der ,Unentschlossenen Verbraucher ${ }^{6}$ (Cluster 2) versucht werden, auf Basis der ermittelten besonderen Charakteristika dieser Gruppe Handlungsempfehlungen für die Gestaltung des Marketing-Mix zu formulieren. Dabei wird der gleichen Struktur gefolgt wie bei den Nachhaltigkeitsfans und zunächst die Gestaltung der Produktpolitik betrachtet. Wie anhand der Studienergebnisse ersichtlich ist diese Gruppe deutlich schwieriger zu charakterisieren als die Nachhaltigkeitsfans, da sie dem Thema Tierwohl heterogen bzw. unentschlossen gegenüberstehen. Generell achtet diese Gruppe bei Fleisch- und Wurstwaren wenig auf Nachhaltigkeitsaspekte. Dennoch ist das Interesse an Tierwohlprodukten in dieser Gruppe hoch. Wenn ihnen also Tierwohlprodukte angeboten werden sollen, so sollten auf Basis der Studienergebnisse am ehesten einige Fleisch- und Wurstwaren mit staatlichem Bio-Siegel angeboten werden (DE-BIO und EU-BIO) und ergänzend dazu Produkte mit den Tierwohl-Labels des DEUTSCHEN TIERSCHUTZBUNDES und von VIER PFOTEN sowie von NEULAND. Anbauverband-Bio-Produkte (z. B. DEMETER, BIOLAND oder NATURLAND) sind dieser Gruppe nur geringfügig bekannt, weshalb sie diese Produkte eher weniger kaufen würden. Die derzeit mangelnde Kaufabsicht dieser Produkte könnte sich gleichwohl bei den ,Unentschlossenen Verbrauchern“ mit zunehmendem Bekanntheitsgrad dieser Labels und einem entsprechenden Vertrauensaufbau mit der Zeit verändern.

Positiv wirken sich auch eine Zertifizierung der Produkte mit DLG- und QS-Siegel sowie die Kennzeichnung der Produkte als GENTECHNIKFEI auf die Kaufentscheidung dieser Verbraucher aus. Überraschenderweise hat auch das ÖKOTEST-Siegel für größere Teile dieser Verbraucher eine positive Wirkung auf die Kaufabsicht, ebenso (wenn auch deutlich weniger) wie STIFTUNG WARENTEST. 
Die Tierwohlprodukte müssen für die ,Unentschlossenen Verbraucher' (im Gegensatz zu den Nachhaltigkeitsfans oder den Bio-Fans) zudem nicht unbedingt aus der näheren Region stammen. Den meisten dieser Verbraucher reichen grobe Herkunftsangaben aus, z. B. dass die Fleisch- und Wurstwaren in Deutschland hergestellt wurden. Hierfür kann das REGIONALFENSTER mit einer entsprechenden Herkunftsangabe verwendet werden. Die Sortimentsbreite und -tiefe betreffend reicht diesen Verbrauchern womöglich eher ein klassisches Standardsortiment an Fleisch- und Wurstwaren. An die Ästhetik werden insgesamt deutlich geringere Ansprüche gestellt als von den Nachhaltigkeitsfans oder den Bio-Fans, und eine umweltfreundlichere Produktverpackung hat für diese Gruppe womöglich eher eine geringe Bedeutung.

Vor dem Hintergrund der bekannten Umweltherausforderungen durch Verpackungsmüll sollten Anbieter dieser Zielgruppe dennoch möglichst umweltfreundliche Verpackungen anbieten, auch wenn diese Gruppe keinen besonderen Wert darauf legt (KETELSEN et al., 2020). Die Markenaffinität dieser Gruppe ist vermutlich ebenfalls als eher niedrig einzuschätzen, sodass keine besondere Markenführung für diese Zielgruppe erforderlich ist. Eine Premiumproduktstrategie für diese Verbrauchergruppe ist vor dem Hintergrund der Studienergebnisse insgesamt wenig sinnvoll.

Bezüglich der Kommunikation konnten in der vorliegenden Studie, wie bereits beschrieben, keine relevanten Unterschiede hinsichtlich der Nutzung von Kommunikationsmedien (Prospekte, Internet, Verpackung) bei den unterschiedlichen Verbrauchergruppen beobachtet werden, sodass diesbezüglich keine zielgruppenspezifische Differenzierung vorzunehmen ist. Da die ,Unentschlossenen Verbraucher' zu den Verbrauchergruppen gehören, die sich am wenigsten mit dem Thema Tierwohl und Nachhaltigkeit auskennen, bietet die Produktkommunikation jedoch die Chance, das relativ geringe Wissen dieser Verbrauchergruppe zu erhöhen. Dies kann durch entsprechende Zusatzinformationen $\mathrm{zu}$ den verschiedenen Tierwohl- bzw. Nachhaltigkeitsstandards über die verschiedenen abgefragten Kommunikationsmedien erfolgen. Neben den Produktverpackungen (diese Gruppe kauft am ehesten SB-Ware) eignen sich hierfür vor allem Prospekte, Broschüren oder das Internet. 
Aufgrund der eher niedrigen Bildung dieser Verbrauchergruppe sollten die entsprechenden Inhalte möglichst einfach und klar verständlich dargestellt werden. Da das Interesse an Tierwohl deutlich größer ist als das Interesse an generellen Nachhaltigkeitsthemen sollte im Rahmen der Kommunikationspolitik vor allem das Tierwohl in den Fokus gestellt werden. Im Gegensatz zu den Nachhaltigkeitsfans sind die ,Unentschlossenen Verbraucher' nicht für Cause-Related-Marketing-Maßnahmen bzw. Kooperationen mit NGOs zu begeistern. Von allen Gruppen wird die in der vorliegenden Studie beispielhaft kombinierte Verwendung von Tierwohl-Labels und dem NABU-Logo am stärksten abgelehnt. Daher sollte auf derartige Kooperationen für diese Zielgruppe, die womöglich nur wenig NGO-Unterstützer beinhaltet, verzichtet werden.

Im Hinblick auf die Preispolitik ist aufgrund des sehr niedrigen Einkommens der ,Unentschlossenen Verbraucher ${ }^{\star}$ davon auszugehen, dass eine ,Mehrzahlungsbereitschaft' für Fleisch- und Wurstwaren aus einer artgerechteren und nachhaltigeren Nutztierhaltung aufgrund der Tierwohlaffinität zwar durchaus vorhanden ist, jedoch nur in einem begrenzten Rahmen. Aufgrund der Studienergebnisse ist zu vermuten, dass die sehr jungen Verbraucher mit sehr geringem Einkommen zu den Gruppen gehören, für die ein Preisaufschlag für mehr Tierwohl bis zu einer Höhe von ca. $30 \%$ noch akzeptabel ist. Die Verbraucher der Cluster 5 und 4 haben womöglich eine ähnlich niedrige Mehrzahlungsbereitschaft. Deutlich höhere Preisaufschläge für Premiumtierwohlprodukte z. B. von BIOLAND werden vom Großteil dieser Gruppe wahrscheinlich nicht bezahlt. Diese Vermutungen sind lediglich aus dem soziodemografischen Hintergrund dieser Gruppe abgeleitet und sollten daher mit genaueren Zahlungsbereitschaftsanalysen untersucht werden. Eine Premiumpreisstrategie wie bei den Nachhaltigkeitsfans und den Bio-Fans ist somit für diese Gruppe an Verbrauchern nicht zu empfehlen. Da die ,Unentschlossenen Verbraucher' des Clusters 2 häufig auf besondere Preisangebote achten, ist der Einsatz weiterer preispolitischer Instrumente für diese Gruppe äußerst sinnvoll. Mengenrabatte, Treuepunkte oder Couponing werden von dieser Zielgruppe sicherlich begrüßt, ebenso wie weitere Preisvorteile bei Tierwohlprodukten. 
Hinsichtlich der Vertriebspolitik zeigen die Ergebnisse, dass die Gruppe der ,Unentschlossenen Verbraucher' häufig ALDI, LIDL, NETTO und SB-Warenhäuser (KAUFLAND und REAL) als Einkaufsstätte für Fleisch- und Wurstwaren nutzt und diese Gruppe somit überwiegend aus Discounter-Käufern und SB-Warenhaus-Käufern besteht. Die ,Unentschlossenen Verbraucher' kaufen dagegen nur sehr selten Fleisch- und Wurstwaren in Bio-Supermärkten, auf dem Wochenmarkt oder in Hofläden. Dementsprechend sollten die möglichst passenden Absatzwege für diese Gruppe gewählt werden. Dabei ist auch zu berïcksichtigen, dass diese Gruppe schwerpunktmäßig auf dem Land lebt. Ein weiteres besonderes Kennzeichen dieser Gruppe stellt zudem die niedrige Wechselbereitschaft dar. Das Angebot günstiger Tierwohlprodukte in ihrer Einkaufsstätte wird von dieser Gruppe zwar begrüßt, aber sie würden aufgrund eines besseren Angebots an Tierwohlprodukten in einer anderen Einkaufsstätte nicht von ihrer Einkaufstätte dorthin wechseln. Daraus lässt sich schließen, dass es sich hierbei um die treueste Kundengruppe handelt, die nur schwer mit einem guten Sortiment an Tierwohlprodukten ,abgeworben' werden kann. Anders ausgedrückt würde diese Gruppe Tierwohlprodukte nur kaufen, falls diese in ihrer Einkaufsstätte zu günstigen Preisen angeboten werden. Weitere vertriebsrelevante Empfehlungen, die aus der vorliegenden Arbeit abgeleitet werden können, umfassen das schwerpunktmäßige Angebot von Tierwohlprodukten im SB-Bereich; möglichst getrennt von konventionellen Fleisch- und Wurstwaren.

Als nächstes sollen aus den Forschungsergebnissen Handlungsempfehlungen für die Gestaltung des Marketing-Mix für Anbieter von Fleisch- und Wurstwaren ausgesprochen werden, die Produkte für die Gruppe der , Überzeugten Bio-Fans` (Cluster 2) anbieten möchten. Dabei wird der gleichen Struktur gefolgt wie bei den anderen Gruppen und zunächst die Gestaltung der Produktpolitik betrachtet. Die Studienergebnisse legen nahe, dass die ,Überzeugten Bio-Fans' beim Kauf von Fleisch- und Wurstwaren stark auf Nachhaltigkeitskriterien wie beispielsweise Umweltfreundlichkeit, Tierwohl, Gütesiegel, Fairtrade, Regionalität und natürlich Bio-Qualität achten. Anbieter von Bio-Produkten sollten demnach möglichst viele dieser Aspekte bei der Gestaltung ihres Produktangebots berücksichtigen. 
Wesentlich für die Gestaltung der Produktpolitik ist des Weiteren, dass die meisten BioKäufer relativ wenig Wert auf bestimmte Marken oder die Verpackung legen. Hohe Investitionen in den Aufbau von besonderen Marken sind daher nicht unbedingt notwendig. Die Bio-Siegel ersetzen womöglich für die meisten Bio-Käufer Marken. Die Verpackung sollte für Bio-Käufer aus möglichst umweltfreundlichen Materialien bestehen, jedoch ist es den Bio-Käufern wichtiger, dass die Produkte eine hohe BioQualität aufweisen, als dass die Produkte besonders verpackt sind. MARKEN et al. (2020) stellten fest, dass viele Bio-Käufer Fans von ,Unverpackt-Konzepten“ sind. Die geringe Bedeutung der Verpackung ist wahrscheinlich in der hohen Präferenz der BioFans für Frischetheke begründet. Die Herkunftsangaben auf den Verpackungen oder an den Fleischtheken sollten dagegen für Bio-Käufer besonders konkret sein (je konkreter desto besser) und nachweisen, dass die Produkte möglichst aus der näheren Region kommen, da die Regionalität den Bio-Käufern ein großes Anliegen ist.

Sowohl die Konsumhäufigkeit im Haushalt als auch die große Reduktionsbereitschaft beim Konsum von Fleisch und Wurst verdeutlichen, dass die Bio-Fans die Gruppe mit den meisten Flexitariern bilden. Für Anbieter bedeutet dies, dass sie zwar mengenmäßig weniger Fleisch und Wurst an Bio-Käufer verkaufen können als an die Verbraucher der Cluster 2, 4 und 5, aber in Bio-Qualität zu höheren Preisen. Hier bietet sich die Chance, dass das ,Buy-less-but-better-Prinzip“ dieser Konsumenten auch ein Art ,Sell-less-earnmore-Modell' für die Produzenten sein kann; bei gleichzeitigen Vorteilen für die Tiere und die Umwelt.

Den ,Überzeugten Bio-Fans` sind die meisten Bio-Siegel bekannt und sie kennen die spezielleren Bio-Siegel der ökologischen Anbauverbände deutlich besser als die ,Unentschlossenen Verbraucher' (Cluster 2) und die Gruppe ,Tierwohl ja - Bio nein“ (Cluster 5). Auch wenn DE-BIO und EU-BIO oft gleichzeitig auf den Verpackungen angebracht sind, sollten Anbieter in jedem Fall das deutsche Bio-Siegel nicht durch das EU-BIO-Siegel ersetzen, sondern beide Logos verwenden, da insbesondere mehr ältere Verbraucher das DE-BIO-Logo besser kennen als das EU-BIO-Logo. 
Auf die zusätzliche Zertifizierung mit reinen Tierwohl-Labels kann bei Bio-Produkten verzichtet werden, da viele Bio-Käufer z. B. die Tierwohl-Labels des DEUTSCHEN TIERSCHUTZBUNDES oder von VIER PFOTEN nicht kennen und auch nicht kaufen würden. Die Bio-Käufer lehnen reine Tierwohl-Labels überwiegend ab.

Weitere Gütesiegel, die für Bio-Käufer ergänzend zu den Bio-Siegeln angebracht werden sollten, sind die Label GENTECHNIKFREI, REGIONALFENSTER und ÖKOTEST. In der positiven Bewertung dieser Gütesiegel äußert sich die hohe Bedeutung von Regionalität, Gesundheitsaspekten und Umweltfreundlichkeit für die Bio-Fans. Überraschend ist, dass die Labels des NABU und DEUTSCHEN TIERSCHUTZBUNDES für die meisten Bio-Fans keinen positiven Einfluss auf die Kaufentscheidung bei Fleisch- und Wurstwaren haben. Aufgrund des starken Bezugs von Bio-Fans zum Thema Tier- und Naturschutz hätte eine deutlich positivere Bewertung erwartet werden können. Im Rahmen der Diskussion wurde bereits versucht, Erklärungsansätze hierfür zu finden, die hier daher nicht wiederholt werden sollen.

Als Handlungsempfehlung ist festzuhalten, dass Anbieter von Fleisch- und Wurstwaren für Bio-Käufer bei Fleisch- und Wurstwaren (im Gegensatz zu anderen Produkten wie beispielsweise Honig) keine Logos von Natur- und Umweltschutzorganisationen anbringen sollten, da Cause-Related-Marketing-Maßnahmen bei Bio-Fleischwaren wenig sinnvoll erscheinen. Anbieter ökologischer Fleisch- und Wurstwaren können ebenfalls auf die Anbringung des DLG-Siegels und des QS-Siegels verzichten, da diese beiden Siegel keinen positiven Effekt auf die Kaufentscheidung der meisten Bio-Fans haben.

Im Hinblick auf die Kommunikationspolitik kann für Bio-Fans aufgrund der nicht signifikanten Unterschiede im Hinblick auf die Informationsmedien keine differenzierte Handlungsempfehlung ausgesprochen werden. Bio-Fans nutzen wie die anderen Verbrauchergruppen die wesentlichen Informationsmedien wie die Produktverpackung oder Prospekte. Daneben ist jedoch besonderer Wert auf die Qualität der persönlichen Beratung zu Tierwohlfragen an der Fleischtheke zu legen. 
Das allgemeine Interesse an Tierwohlinformationen ist bei den Bio-Käufern (und den Nachhaltigkeitsfans) am höchsten von allen Verbrauchergruppen. Lebensmitteleinzelhändler, die Bio-Fans als Zielgruppe auswählen, könnten gezielt ,Tierwohlschulungen“ für das Verkaufspersonal anbieten. Insgesamt sollte auf ein hohes Niveau in der Kommunikation geachtet werden.

Was die Preispositionierung betrifft, sind die ,Überzeugten Bio-Fans' wohl am ehesten dazu bereit, höhere Preise für Tierwohlprodukte zu bezahlen. Diese Gruppe verfügt mit Abstand über das höchste Einkommen aller betrachteten Cluster, die Bio-Fans achten weniger auf den Preis und Tierwohl und Nachhaltigkeit sind ihnen besonders wichtig. Daher ist insgesamt eine Premiumpreisstrategie für strenge Tierwohlprodukte (im Idealfall mit einem Anbauverband-Bio-Label ausgezeichnet) empfehlenswert. Aufgrund der vergleichsweise geringen Bedeutung der Preise für Bio-Fans ist auch davon auszugehen, dass der Großteil der Bio-Fans eher weniger mit Rabattaktionen, Couponing oder Treuepunkten zu begeistern ist als beispielsweise die, Unentschlossenen Verbraucher' oder die Gruppe ,Tierwohl ja - Bio nein`.

Im Hinblick auf die Vertriebspolitik können als Absatzweg die favorisierten Einkaufsstätten der Bio-Fans empfohlen werden. Die starke Präferenz für die Fleischtheke zeigte sich auch bei einer Gesamtbetrachtung aller genutzten Einkaufsstätten. So werden überwiegend Einkaufsstätten mit Fleischtheke genutzt. Besonders viele Bio-Fans nutzen Bio-Supermärkte, Metzger sowie REWE. Daneben werden gelegentlich auch Wochenmärkte und Hofläden als Einkaufsstätte genutzt.

Da die Wechselbereitschaft der Bio-Kunden hoch ist, können Lebensmitteleinzelhändler davon ausgehen, dass sich Bio-Kunden eine andere Einkaufsstätte suchen, wenn sie mit dem Angebot an Tierwohlprodukten nicht zufrieden sind. Abgesehen von den BioSupermärkten ist zudem allen anderen konventionellen Lebensmitteleinzelhändlern zu empfehlen, möglichst ausgewiesene Bereiche für das Bio-Sortiment an Fleisch- und Wurstwaren räumlich getrennt von den konventionellen Fleischwaren einzurichten und dabei einen starken Fokus auf Regionalität zu legen. 
Abschließend sollen bzgl. des Einsatzes der Marketinginstrumente beispielhaft einige Handlungsempfehlungen für die Gruppe, Tierwohl ja - Bio nein' ausgesprochen werden. Aus der Beachtung der Kaufkriterien dieser besonderen Verbrauchergruppe kann abgeleitet werden, dass Anbieter dieser Gruppe am ehesten schön (nicht nachhaltig) verpackte Markenfleischwaren zu günstigen Preisen anbieten sollten. Passende Vermarktungsbeispiele für diese Gruppe sind womöglich die RÜGENWALDER MÜHLE oder auch GUTFRIED. Wie bereits im Ergebnisteil und der Diskussion beschrieben präsentiert sich diese Verbrauchergruppe als höchst ambivalent, da sie eine der höchsten Präferenzen für reine Tierwohl-Labels vorweist, dagegen jedoch beim alltäglichen Fleisch- und Wurstkauf nicht auf eine artgerechtere Tierhaltung achtet und auch insgesamt die geringste, Tierwohlaffinität ${ }^{\star}$ erkennen lässt. Ursächlich hierfür könnte eine Kombination aus fehlendem Wissen über Tierwohl-Labels mit einer geringen Verfügbarkeit der entsprechenden Produkte in den von dieser Gruppe genutzten Einkaufsstätten sein. Nachhaltigkeitsaspekte müssen für diese Zielgruppe nicht in der Produktpolitik berücksichtigt werden, da diese Gruppe keinen Wert auf Nachhaltigkeit legt. Als Tierwohl-Labels sollte für diese Gruppe das Label des DEUTSCHEN TIERSCHUTZBUNDES oder von NEULAND ausgewählt und mit dem QS-Siegel und STIFTUNG WARENTEST ergänzt werden. Zudem müssen keine regionalen Produkte angeboten werden, da der Regionalität in dieser Gruppe keine große Bedeutung zukommt.

Bezüglich der Kommunikationspolitik kann aufgrund der starken Präferenz für TierwohlLabels und der geringen Bedeutung des Themas Nachhaltigkeit empfohlen werden, in der gesamten Kommunikation schwerpunktmäßig das Tierwohl in den Vordergrund zu stellen. Die Kommunikation von Umweltthemen bzw. -vorteilen der Produkte wird diese Gruppe vermutlich eher langweilen. Da diese Gruppe aus Discounter-Käufern und SBWarenhaus-Käufern besteht, die überwiegend im SB-Bereich einkaufen, sollten sich entsprechende Kommunikationsmaßnahmen auf die Produktverpackungen und den SBBereich in der Einkaufsstätte konzentrieren. Eine besondere Aufgabe kommt der Kommunikationspolitik bei dieser Gruppe im Hinblick auf die Bereitstellung zusätzlicher Informationen $\mathrm{zu}$. 
Die Informationen zu den Vorteilen für die Tiere von Tierwohlprodukten sollten für diese Gruppe besonders leicht verständlich aufbereitet werden. Aufgrund der niedrigen Affinität zu NGOs sollte von Cause-Related-Marketing-Maßnahmen abgesehen werden. Für diese Zielgruppe eignen sich anstelle von Cause-Related-Marketing-Maßnahmen eher klassische Gewinnspiele (Handy, Auto, Reisen etc.).

Im Hinblick auf die Preispolitik kann Anbietern, die diese Zielgruppe bedienen möchten, keine Premiumpreisstrategie empfohlen werden, da diese Gruppe nur ein relativ geringes Einkommen zur Verfügung hat und eine starke Sonderangebotsorientierung vorweist. Dies zeigen auch die Einkaufsstättenpräferenzen (ALDI, NETTO etc.). Aus der hohen Tierwohl-Label-Präferenz könnte jedoch der Schluss gezogen werden, dass Anbieter von Tierwohlprodukten dennoch mit einer Mehrzahlungsbereitschaft von bis zu ca. $30 \%$ rechnen können. Außerdem empfehlen sich für diese Gruppe die vielfältigen Sonderangebotsvarianten, die bereits bei den anderen sonderangebotsaffinen Zielgruppen beispielhaft aufgezählt wurden.

Als Absatzwege können für die Zielgruppe ,Tierwohl ja - Bio nein‘ im Rahmen der Distributionspolitik vor allem die Discounter und die SB-Warenhäuser empfohlen werden. Ein kleinerer Teil dieser Gruppe nutzt auch EDEKA als Einkaufsstätte für Fleisch- und Wurstwaren. Tendenziell sollten die Tierwohlprodukte schwerpunktmäßig im SB-Bereich angeboten werden. Dass diese Gruppe überwiegend auf dem Land lebt, sollte ebenfalls bei den Vertriebsentscheidungen berücksichtigt werden. Wie die anderen Verbrauchergruppen präferieren auch die Verbraucher der Gruppe, Tierwohl ja - Bio nein` einen eigenen Bereich für Tierwohlprodukte in den Einkaufsstätten. Daher sollten Anbieter in den SB-Regalen den meist weiblichen Käuferinnen ein kleines Sortiment an Fleisch- und Wurstwaren anbieten, das z. B. nach den Standards des DEUTSCHEN TIERSCHUTZBUNDES produziert wurde und gleichzeitig als Markenprodukt präsentiert wird. 


\section{Zusammenfassung}

In der kontrovers geführten gesellschaftlichen Diskussion zu den Herausforderungen der intensiven Nutztierhaltung in Deutschland fordern immer größere Teile der Gesellschaft von der Fleischbranche und der Politik eine stärkere Berücksichtigung des Tier- und Umweltschutzes, der Gesundheit und des Arbeitnehmerschutzes. Um den gesellschaftlichen Forderungen nachzukommen, bestehen aus politischer Perspektive verschiedene Möglichkeiten. Differenziert werden können dahingehend verpflichtende gesetzliche Vorschriften und auf freiwilliger Basis nutzbare Marktlösungen. Die Bundesregierung bzw. die EU könnte theoretisch Tierschutz- und Umweltschutzgesetze verschärfen und die Einhaltung der Gesetze in der Praxis stärker kontrollieren.

Den Gesetzesänderungen stehen jedoch ökonomische Ziele, wie z. B. die Steigerung des Exports von Fleisch- und Wurstwaren oder der Erhalt von Arbeitsplätzen, entgegen. Daher werden gesetzliche Beschränkungen der intensiven Nutztierhaltung in Deutschland von der Wirtschaft mehrheitlich abgelehnt. Eine ähnliche ,Blockadehaltung ‘ der Wirtschaft gegenüber dem Gesetzgeber findet sich auch in anderen Branchen, wenn Gesetzesänderungen private Gewinne zu stark mindern, auch wenn mit den Gesetzesänderungen Vorteile für die Mehrheit der Menschen verbunden wären, wie z. B. Verbesserungen für die Gesundheit der Menschen, die Umwelt oder das Tierwohl.

Als alternative Strategie zur Abmilderung der negativen Auswirkungen der intensiven Nutztierhaltung werden den Verbrauchern daher eine Reduktion des Fleischkonsums und der Kauf von Fleisch- und Wurstwaren aus einer artgerechteren und nachhaltigeren Nutztierhaltung empfohlen. Verschiedene freiwillige Tierwohl-Labels sollen den Verbrauchern im Lebensmitteleinzelhandel zudem als Einkaufshilfe dienen.

In der vorliegenden Studie wurde die Bedeutung dieser verschiedenen Tierwohl-Labels beim Einkauf von Fleisch- und Wurstwaren aus der Perspektive der Verbraucher untersucht. Einzelne Aspekte des Tierwohl-Labelings wurden in der Vergangenheit bereits in verschiedenen interessanten Studien untersucht. 
Eine aktuelle Analyse der Verbraucherpräferenzen bzgl. der vielfältigen Formen an Tierwohl-Labels und eine umfassendere Charakterisierung der unterschiedlichen LabelKäufertypen standen jedoch noch aus. Insgesamt sollten Anbieter von Fleisch- und Wurstwaren durch die Erkenntnisse der vorliegenden Studie den Einsatz von TierwohlLabels bei der Vermarktung von Fleisch- und Wurstwaren aus einer artgerechteren und nachhaltigeren Nutztierhaltung nun strategischer vornehmen können.

Im Juni 2018 wurden bundesweit 930 nachhaltigkeitsinteressierte Fleischkäufer im Rahmen einer standardisierten Online-Befragung $\mathrm{zu}$ ihrem Einkaufs- und Ernährungsverhalten sowie zu ihren Präferenzen zu Tierwohl-Labels befragt. Hinsichtlich der Verbraucherpräferenzen standen die Bedeutung von Tierwohl-Labels als Einkaufshilfe, der Informationsbedarf bei Tierwohlprodukten und die politischen Präferenzen der Befragten zum Thema Tierwohl im Fokus. Daneben wurden von allen Studienteilnehmern soziodemografische Daten erfasst und mit der Software IBM SPSS 26 ausgewertet. Dabei wurden zunächst verschiedene uni- und bivariate Analysen zu einzelnen Aspekten des Tierwohl-Labelings durchgeführt, wie beispielsweise Chi-2Tests sowie Korrelations- und Varianzanalysen. Des Weiteren wurden verschiedene multivariate Methoden angewendet, um erstmals eine Tierwohl-Label-Typologie sowie eine Verbrauchertypologie auf der Basis von Tierwohl-Label-Präferenzen zu erstellen und gruppenspezifische Auswertungen vorzunehmen. Hierfür wurden mehrere explorative Faktorenanalysen mit entsprechenden Reliabilitätsanalysen sowie eine hierarchische Clusteranalyse durchgeführt, um anschließend mit Hilfe der multinominalen logistischen Regressionsanalyse Unterschiede zwischen den identifizierten Gruppen zu analysieren.

Die Studie beinhaltet wertvolle Erkenntnisse zur Bedeutung zahlreicher Tierwohl-Labels beim Kauf von Fleisch- und Wurstwaren. So kann beispielsweise nachgelesen werden, welche Verbrauchergruppen besonders auf die ,Big Four' einer nachhaltigeren Ernährung (Tierwohl, Umweltschutz, Gesundheit, Soziales) achten. Positionierungsmatrizen zeigen zudem, wie Verbraucher die verschiedenen Tierwohl-Labels im Hinblick auf Nachhaltigkeitsaspekte bewerten. 
Daneben werden umfassende Erkenntnisse zum Einfluss von Einkaufsstätten und Angebotsformen (Theke/SB) und weiteren marketing- und vertriebsrelevanten Aspekten auf die Präferenzen der Verbraucher vorgestellt.

Die Ergebnisse der Verbrauchertypologie machen erstmals unterschiedliche Verbrauchergruppen auf Basis ihrer Tierwohl-Label-Präferenzen sichtbar. Die verschiedenen Verbrauchergruppen werden in der vorliegenden Arbeit umfassend charakterisiert und es wird gezeigt, dass das Verbraucherverhalten in unserer Gesellschaft deutlich heterogener ausgeprägt ist, als es häufig in den Medien dargestellt wird.

Wie Hersteller von Fleisch- und Wurstwaren und der Lebensmitteleinzelhandel sowie die Politik das Tierwohl in Deutschland weiter voranbringen können, kann den aus den Forschungsergebnissen abgeleiteten Handlungsempfehlungen entnommen werden. Die Ergebnisse der Arbeit sind von hoher Relevanz für die Verbraucherforschung zum Thema Tierwohl-Labeling und für Anbieter von Fleisch- und Wurstwaren, die die Ergebnisse für die bessere Vermarktung von Tierwohlprodukten nutzen können.

Es werden konkrete Vorschläge präsentiert und diskutiert, wie die Präferenzen der Verbraucher bei der Vermarktung von Fleisch- und Wurstwaren mit Tierwohl-Labels besser berücksichtigt werden können. Der Politik können die Ergebnisse bei der Bewertung unterschiedlicher Lösungsstrategien für mehr Tierwohl in Deutschland helfen.

\subsection{Limitationen der Forschung}

Nicht vergessen werden dürfen einige Limitationen der Studie, auf die in diesem Abschnitt eingegangen werden soll. Zunächst wurde hinsichtlich der Auswahl an Tierwohl-Labels versucht, möglichst viele Tierwohl-Labels in Deutschland zu berücksichtigen. Um einer Überforderung der Studienteilnehmer vorzubeugen, musste die Anzahl jedoch auf zwölf unterschiedliche Tierwohl-Labels begrenzt werden. 
Es gibt jedoch eine Vielzahl weiterer spezieller Tierwohlkennzeichnungen oder auch besondere Marken wie beispielsweise BIOPARK, PRO ETHIKA Freilandgeflügel, KIKOK Maishähnchen oder CHIEMGAUER NATURFLEISCH, die in der vorliegenden Befragung aus forschungsökonomischen Gründen nicht berücksichtigt werden konnten.

Des Weiteren waren einige der untersuchten Tierwohl-Labels mehrstufige Gütesiegel. Beispielhaft genannt seien hier die mehrstufigen Tierwohl-Labels des DEUTSCHEN TIERSCHUTZBUNDES sowie das geplante STAATLICHE TIERWOHL-LABEL. Ein weiteres Beispiel für ein erfolgreiches mehrstufiges Tierwohl-Label ist das Gütesiegel BETER LEVEN der niederländischen Supermarktkette ALBERT HEIJN. Eine nähere Differenzierung der unterschiedlichen Stufen erfolgte in der vorliegenden Studie jedoch nicht, um die Komplexität des Fragebogens gering zu halten. Das abgefragte ,TierwohlLabel'von LIDL (HALTUNGSKOMPASS) ist zudem im engeren Sinne kein TierwohlLabel, sondern eine Art ,Kategorisierungslabel ‘, das Tierwohl-Labels in Stufen bewertet integriert. Diese Kennzeichnung wird von Verbrauchern jedoch womöglich wie ein Tierwohl-Label wahrgenommen. LIDL führte diese Haltungskennzeichnung als erster Lebensmitteleinzelhändler kurz vor dem Start der Online-Befragung im Frühjahr 2018 ein. Daher wurde der HALTUNGSKOMPASS kurzfristig bei der Befragung berücksichtigt, um möglichst viele der zum Befragungszeitpunkt im deutschen LEH existierenden Tierwohlkennzeichnungen in der Studie zu berücksichtigen. Nach der Befragung wurde der HALTUNGSKOMPASS durch die HALTUNGSFORM ersetzt.

Bei der hierarchischen Clusteranalyse bzw. den multinominalen Logit-Modellen musste zudem darauf geachtet werden, dass alle Verbrauchergruppen ausreichend groß waren, um die Daten mit SPSS auswerten zu können. Dafür mussten häufig Gruppen gebildet werden, wodurch teilweise detaillierte Analysen nicht möglich waren oder aufgrund $z u$ geringer Stichprobengrößen nicht durchgeführt werden konnten. Beispielhaft genannt seien detaillierte Analysen bzgl. der Religion, der Nutzung von Online-Portalen für den Fleischkauf, der Nutzung einzelner Bio-Supermärkte, zu verschiedenen Labeln (z. B. VIER PFOTEN), einzelnen Bundesländern (wie Bremen, Hamburg, MecklenburgVorpommern, Saarland oder Thüringen) oder auch einzelnen kleinen Parteien. 
Während in anderen Studien häufig gezielt einzelne Bio-Labels (meist EU-BIO) oder reine Tierwohl-Labels untersucht wurden, wurden in der vorliegenden Studie beide Varianten berücksichtigt. Zum einen waren dies Tierwohl-Labels, die deutliche Verbesserungen für das Wohl der Tiere beinhalten, jedoch als eine Art ,Gold-Standard“ nur ein relativ kleines Marktsegment bedienen (Bio-Labels). Zum anderen waren dies Tierwohl-Labels, die eine Massenmarktstrategie verfolgen, aber nur marginale Verbesserungen gegenüber dem gesetzlichen Standard erzielen. Durch die Berücksichtigung beider Arten von Tierwohl-Labels und der hohen Anzahl unterschiedlicher Tierwohl-Labels konnten keine detaillierten Forschungsfragen beantwortet werden, wie sie in spezifischeren Studien vorkommen, die sich auf eine Angebotsform, eine Vertriebsform, eine Tierart oder ein Tierwohl-Label konzentrieren. Die Konzeption der vorliegenden Studie wurde jedoch bewusst so gewählt, um ein möglichst umfassendes Bild über die Tierwohl-Label-Präferenzen zu erhalten.

Eine weitere Limitation der Studie besteht darin, dass die Zielgruppe der bundesweiten Befragung durch zwei Screening-Fragen eingeschränkt wurde und die Befragung somit trotz repräsentativer Quotierung durch die RESPONDI AG im engsten Sinne nicht als repräsentativ für die Gesamtbevölkerung bezeichnet werden kann. Der Fragebogen wurde bewusst für Fleischkäufer entwickelt, die ein gewisses Grundinteresse am Thema Nachhaltigkeit haben, da ansonsten aufgrund des starken Fokus auf Nachhaltigkeitsfragen im Fragebogen mit einer zu hohen Abbruchquote zu rechnen gewesen wäre.

Weitere Limitationen betreffen bei Online-Befragungen häufig das unsachgemäße oder zu schnelle Ausfüllen von Online-Fragebögen, Mehrfachteilnahmen oder unvollständig ausgefüllte Fragebögen. Die Aussagekraft der vorgestellten Ergebnisse wird zudem durch Panel-Effekte beeinflusst. Diesen nachteiligen Effekten wurde jedoch von der RESPONDI AG mit geeigneten Qualitätsmaßnahmen begegnet, sodass diese Limitationen für die vorliegende Studie weitestgehend nicht gelten. Vorstellbar ist gleichwohl, dass in Online-Panels bestimmte Personengruppen unterrepräsentiert sind, ohne dass dies anhand der soziodemografischen Merkmale erkennbar wäre, z. B. weil bestimmte Personen generell nicht an Online-Befragungen teilnehmen. 
An dieser Stelle ist erneut darauf hinzuweisen, dass die meisten Mechanismen bei einer Kaufentscheidung von Verbrauchern unbewusst ablaufen. Diese Prozesse sind im Rahmen empirischer Studien nicht genau zu erfassen. Zudem können nicht alle Einflussfaktoren auf die Kaufentscheidung im Rahmen einer Studie ermittelt werden. So wurde in der vorliegenden Studie beispielsweise nicht erhoben, ob bestimmte Einkaufsstätten lediglich aus Bequemlichkeit genutzt werden, da sie sich in unmittelbarer Nähe des Wohnortes befinden, auch wenn es dort kein optimales Angebot an Tierwohlprodukten gibt.

Dennoch wurde versucht, mit der größten Sorgfältigkeit die bedeutsamsten Aspekte im Hinblick auf den Kauf von Tierwohlprodukten zu berücksichtigen. Da jedoch nicht alle offenen Forschungsfragen zum Tierwohl-Labeling im Rahmen einer Studie beantwortet werden können, kann die vorliegende Arbeit eine Basis für weitere Forschungsarbeiten darstellen, indem aus den neu gewonnenen Erkenntnissen weiterführende Theorien bzw. Hypothesen abgeleitet werden können. Im nächsten Abschnitt sollen zusammenfassend einige Ideen für neue Forschungsprojekte in Form weiteren Forschungsbedarfs präsentiert werden, die aus der vorliegenden Studie abgeleitet wurden. Gegebenenfalls finden zukünftige Doktoranden, Wissenschaftler oder Praktiker einen spannenden Forschungsansatz.

\subsection{Weiterer Forschungsbedarf}

Aus den Forschungsergebnissen zur Einschätzung der Strenge der unterschiedlichen Tierwohl-Labels kann abgeleitet werden, dass die meisten Verbraucher Tierwohl-Labels in zwei (streng, nicht streng) bis maximal drei ,Qualitätsstufen' wahrnehmen (streng, mittel, nicht streng). Eine klare Differenzierung der Verbraucher bezüglich der für Tierwohlprodukte zentralen Kriterien Tierwohl, Umwelt und Gesundheit zwischen den Labels ist aus den Studienergebnissen hingegen nicht immer erkennbar. Den untersuchten Tierwohl-Labels liegen jedoch die unterschiedlichsten Kriterienkataloge zugrunde. 
Diese Kriterienkataloge sind auf den Websites der Gütesiegelherausgeber einzusehen und von großer Bedeutung für die Bewertung und die Glaubwürdigkeit der einzelnen Tierwohl-Labels. Bisher liegt jedoch keine umfassende wissenschaftliche Untersuchung vor, die die Kriterien dieser vorgestellten Tierwohl-Labels prüft und vergleichend analysiert sowie ein Ranking der strengsten bzw. seriösesten Gütesiegel erstellt. Daher sollte eine umfassende kriterienbasierte Tierwohl-Label-Bewertung in Form einer MetaStudie erfolgen, um die Transparenz bei Tierwohl-Labels zu erhöhen. Diese Ergebnisse könnten z. B. in die Erstellung von Tierwohl-Label-Ratgebern für die Verbraucher einfließen.

Aus den Angaben der Befragten in der vorliegenden Studie zum Thema Reduktionsbereitschaft ist außerdem nicht erkennbar, wie die Definition der Reduktionsbereitschaft beim Fleischkonsum konkret aussieht. Unklar bleibt beispielsweise auch, welche Produkte weniger gegessen werden und ob den geäußerten Absichten zur Reduktion des Fleischkonsums tatsächlich im Alltag gefolgt wird. Der ,Speck in der Linsensuppe‘ wird zudem evtl. auch von einigen Verbrauchern vergessen. Aufgrund der besonderen Bedeutung der Reduktion des Fleischkonsums sowohl für die Umwelt als auch für die Ökonomie ist eine genauere Analyse bzw. Quantifizierung der Reduktionsbereitschaft zu empfehlen. Dabei sollte auch der Zusammenhang zwischen Kochkünsten und Reduktionsbereitschaft untersucht werden, da davon ausgegangen wird, dass die meisten Fleischfans nur selten vegane Gerichte kochen können, die ihnen gut schmecken, und bisher auch eher selten derartige Gerichte gegessen haben.

Weitere Problemfelder der Nutztierhaltung wie beispielsweise Aspekte der Sozialverträglichkeit (z. B. faire Erzeugerpreise für hiesige Landwirte, Beschäftigungsbedingungen in Schlachtbetrieben, negative Auswirkungen auf Entwicklungsländer durch den Außenhandel) oder Raumnutzungskonflikte wurden in der vorliegenden Studie nur teilweise im Rahmen der Kaufkriterienanalyse und der Beachtung besonderer Gütesiegel betrachtet. Aus forschungsökonomischen Gründen wurden diese sozialen Problemfelder jedoch nicht tiefergehend analysiert, da die sozialen Aspekte nicht im Fokus der vorliegenden Arbeit standen. 
Obwohl die sozialen Aspekte als Teil der, Big Four' einer nachhaltigeren Ernährung ebenfalls von großer Bedeutung sind, existieren vergleichsweise wenige Forschungsarbeiten zu diesem Themenkomplex. Daher sollten die sozialen Effekte der intensiven Nutztierhaltung in zukünftigen Forschungsprojekten untersucht werden; beispielsweise anhand einer komparativen Bewertung der positiven und negativen sozialen Effekte der intensiven Nutztierhaltung.

Der von LEA und WORSLEY (2001) identifizierte Einfluss von Bezugsgruppen beim Fleischkonsum sollte vor dem Hintergrund der bzgl. der Konsumtypen heute immer unterschiedlicher zusammengesetzten Haushalte (Veganer, Vegetarier, Flexitarier, Fleischfans etc.) noch einmal detailliert untersucht werden. Zukünftig werden immer mehr Veganer bzw. Vegetarier in ihren Haushalten für ein verändertes Konsumverhalten aller Personen sorgen; vor allem in den zukünftigen Familien der ,Millenials“ oder jüngeren Generationen. Dies wird vermutlich $\mathrm{zu}$ einer stärkeren Abnahme des Fleischkonsums in Deutschland führen, als bisher vermutet wird, was sowohl aus gesundheitlicher als auch aus Umwelt- und tierethischer Perspektive begrüßenswert wäre. Vor diesem Hintergrund sollte sich eine zukunftsorientierte Forschung an Universitäten und Hochschulen strategisch verstärkt auf die umweltverträgliche Produktion und Vermarktung von pflanzlichen Proteinprodukten konzentrieren und den Wandel hin zu mehr Nachhaltigkeit proaktiv mitgestalten.

Forscher der Universität Kassel untersuchten verschiedene Vermarktungsansätze für Fleisch- und Wurstwaren von alternativen Nutztierrassen, die vom Aussterben bedroht sind (BLE, 2020; MENGER et al., 2020). Vor dem Hintergrund der in der vorliegenden Studie ermittelten großen Unterschiede zwischen Tierwohl-Labels und Verbrauchergruppen, ist ein Folgeprojekt zu empfehlen, in dessen Rahmen detailliert untersucht wird, welche Tierwohl-Labels für welche Tierart bzw. Fleisch- oder Wurstsorte am ehesten verwendet werden sollten und in welchen Einkaufsstätten diese Produkte am ehesten anzubieten sind. Es wird vermutet, dass für eine zielgruppengerechte Preis- und Kommunikationspolitik die Auswahl eines passenden Tierwohl-Labels und einer passenden Einkaufsstätte entscheidend für den Markterfolg ist. 
Des Weiteren basieren die Anmerkungen bzw. Handlungsempfehlungen zur Preispolitik in der vorliegenden Arbeit lediglich auf den soziodemografischen Hintergründen der Befragten (z. B. Einkommen) und deren Präferenzen (z. B. Preisorientierung). Für die identifizierten Marktsegmente sollten gezielte Zahlungsbereitschaftsanalysen durchgeführt werden, um noch fundiertere Handlungsempfehlungen bzgl. der Preispolitik aussprechen zu können. Vor dem Hintergrund der vorliegenden Studie sollte die Zahlungsbereitschaft der Verbraucher differenziert nach den einzelnen in dieser Studie untersuchten Tierwohl-Labels oder zumindest differenziert nach den drei Gruppen der Tierwohl-Label-Typologie untersucht werden. Dabei sollten die Unterschiede der spezifischen Zahlungsbereitschaften für die einzelnen Tierwohl-Labels herausgearbeitet werden. Von Bio-Käufern schwerpunktmäßig genutzte Einkaufsstätten (Bio-Supermärkte mit und ohne Theke, BioFachgeschäfte, Metzger, Wochenmärkte, Hofläden) sollten ebenfalls Berücksichtigung finden.

Die Vermarktung von Fleisch- und Wurstwaren aus einer artgerechteren und nachhaltigeren Nutztierhaltung sollte ferner nicht nur hinsichtlich der Produkte, sondern auch hinsichtlich der Ästhetik einen hohen Anspruch verfolgen. Die Anbringung eines anspruchsvollen Tierwohl-Labels und die gleichzeitige Vermarktung als reine Massenware (Bulk Product) unterstreichen nicht in ausreichendem Maße die Ernsthaftigkeit eines besonderen Engagements für mehr Tierwohl. Insbesondere im konventionellen Lebensmitteleinzelhandel werden bei den meisten Fleisch- und Wurstwaren keine besonderen Aspekte bei der Ästhetik beachtet. Für Nachhaltigkeitsfans ist eine umweltfreundlichere und ästhetisch anspruchsvollere Produktverpackung bei zertifizierten Fleisch- und Wurstwaren jedoch von Bedeutung (sowohl im SB-Bereich als auch für Thekenware). Die Gestaltung nachhaltigerer Verpackungen und Kommunikationsmaterialien könnte z. B. von Studienteilnehmern der beiden identifizierten Verbrauchergruppen ,Überzeugte Bio-Fans ‘ und ,Nachhaltigkeitsfans ‘ im Rahmen von Forschungsprojekten bewertet werden. In diesem Rahmen können von dieser Gruppe auch weitere zielgruppengerecht gestaltete Werbemaßnahmen bewertet werden. 
Die Einschätzung der Befragten zu den einzelnen Tierwohl-Labels und zu den Auswirkungen eines übermäßigen Fleischkonsums in der vorliegenden Studie offenbart, dass insbesondere die beiden Gruppen ,Unentschlossene Verbraucher' und ,Tierwohl ja - Bio nein‘ einige Defizite bzgl. ihrer Kenntnis von Tierwohl-Labels und ihrer Allgemeinbildung beim Thema Nachhaltigkeit aufweisen. Hier könnte die Wissenschaft für den deutschen Lebensmitteleinzelhandel ein ,Tierwohl-Umwelt-Bildungskonzept * entwickeln, um diese Verbrauchergruppen besser über Tierwohl-, Gesundheits- und Nachhaltigkeitsaspekte beim Fleischkauf zu informieren.

Vor dem Hintergrund der ermittelten starken Präferenzen der meisten Verbraucher bzgl. des räumlich getrennten Angebots von Tierwohlprodukten im deutschen LEH empfiehlt es sich, die Implementierung der zwei untersuchten Category-Management-Varianten (Tierwohlprodukte getrennt von konventionellen Produkten oder zusammen anzubieten) in Praxisforschungsprojekten zu testen und Vor- und Nachteile im Hinblick auf Vermarktung und Abverkäufe systematisch zu analysieren.

Die Forschungsergebnisse zum Multi-Labeling zeigen, dass Gütesiegel, die ergänzend zu Tierwohl-Labels angebracht sind, einen positiven Einfluss auf die Kaufentscheidung vieler Verbraucher haben. Auf Basis der differenzierten Erkenntnisse aus der durchgeführten Online-Befragung sollten weiterführende Analysen z. B. in Form von Kaufexperimenten im LEH oder in Form von Discrete-Choice-Analysen durchgeführt werden, um die besten Kombinationen verschiedener Gütesiegel für Fleisch- und Wurstwaren im Hinblick auf die identifizierten Marktsegmente zu ermitteln.

Bei der Betrachtung der ausführlich beschriebenen Marktsegmente wurde vermutet, dass sich die unterschiedlichen Verbrauchergruppen hinsichtlich der Prioritätensetzung bei der Verwendung des Haushaltseinkommens stark unterscheiden, auch wenn diese Priorisierung nicht Thema der vorliegenden Arbeit war. Für weitere Forschungsarbeiten sind hier womöglich interessante Ergebnisse mit einigem kontroversen Verhalten zu erwarten. Ziel wäre eine deutlich umfassendere Charakterisierung der unterschiedlichen Fleischkäufer anhand ihres weiteren Konsumverhaltens. 
So gibt es vermutlich beispielsweise auf der einen Seite Verbraucher, die sich mit dem Fahrrad ein BIOLAND-Steak für $13 €$ kaufen und auf einem Grill für 5,99 $€$ zubereiten, während sich andere Verbraucher mit dem Porsche ein Steak von ,GUT \& GÜNSTIG، für 1,99 € kaufen und auf einem 600-€-Grill zubereiten. Ziel weiterer Forschungsarbeiten sollte es folglich sein, Muster bzw. Unterschiede zwischen dem Einkaufs- und Konsumverhalten bei Fleisch- und Wurstwaren und dem Einkaufs- und Konsumverhalten sowie konkreten Markenaffinitäten bei Produkten und Dienstleistungen in anderen Bereichen (Auto, Handy, Bekleidung, Urlaub, Möbel, Drogerieartikel, Banken etc.) zu untersuchen, um ein noch umfassenderes Bild von den einzelnen Verbrauchergruppen zu erhalten. Dafür sollten nicht nur repräsentativ angelegte Studien durchgeführt werden, sondern auch spezielle Zielgruppenstudien zu den ,Nachhaltigkeitsfans “ und ,Überzeugten Bio-Käufern` erfolgen, z. B. durch eine gezielte Befragung von BioSupermarkt-Kunden, UTOPIA-Lesern oder Mitgliedern verschiedener Naturschutzorganisationen, um einen aktuellen und noch besseren Überblick zum allgemeinen nachhaltigen Konsumverhalten $\mathrm{zu}$ erhalten und die Fleischkäufertypen dadurch noch besser zu verstehen.

Abschließend konnte in der vorliegenden Studie festgestellt werden, dass Haustierbesitzer signifikant häufiger auf eine artgerechtere Tierhaltung achten als Verbraucher, die kein Haustier besitzen. In einem weiteren interessanten neuen Forschungsprojekt könnte daher untersucht werden, inwiefern die in dieser Arbeit diskutierten unterschiedlichen Tierwohl-Labels für die Vermarktung von PremiumTierwohl-Heimtierfutter eingesetzt werden können und wie entsprechende Vermarktungskonzepte hierzu zu entwickeln wären (z. B. BIOLAND-BREKKIES).

Es gibt bereits mit Tierwohl-Labels zertifiziertes Heimtierfutter, jedoch wurde noch nicht wissenschaftlich untersucht, welche Tierwohl-Labels sich am besten für welche Heimtierprodukte, Zielgruppen und Vertriebswege eignen. Aus den Erkenntnissen der vorliegenden Arbeit wären potenzielle Kunden vor allem die identifizierten ,Enthusiastischen Nachhaltigkeitsfans ‘ sowie die ,Überzeugten Bio-Käufer', die selbst einen Hund oder eine Katze besitzen. 
Neben veganem Tierfutter wird insbesondere Bio-Heimtierfutter immer häufiger angeboten und zahlreiche innovative Startups bieten im Internet ein breites Sortimentsangebot schwerpunktmäßig für Hunde- und Katzenbesitzer an. Dabei werden jedoch womöglich strategische Marketingentscheidungen in Bezug auf den TierwohlLabel-Einsatz selten auf fundierter wissenschaftlicher Basis getroffen. Durch den stärkeren Absatz von ökologischem Heimtierfutter bietet sich zudem die Möglichkeit, das Problem des Absatzes teurerer Kuppelprodukte (aufgrund der besseren Haltungsbedingungen) durch die Mehrzahlungsbereitschaft von Haustierbesitzern für zertifiziertes Tierwohl-Heimtierfutter etwas abzumildern, indem mehr Teile von BioTieren (die der Mensch nicht verzehrt) für Bio-Heimtierfutter verarbeitet werden. 


\section{Literaturverzeichnis}

AKERLOF, G. A. (1970). The Market for Lemons, Qualitative Uncertainty and the Market Mechanism, in: Quarterly Journal of Economics, Vol. 84 (1970), S. 488-500.

ALBERSMEIER, F. \& SPILLER, A. (2009). Das Ansehen der Fleischwirtschaft: Zur Bedeutung einer stufenübergreifenden Perspektive. In: Böhm J, Albersmeier F, Spiller A (Hrsg.) Die Ernährungswirtschaft im Scheinwerferlicht der Öffentlichkeit. Eul Verlag, Lohmar, S. 213-250.

ALBERSMEIER, F. \& SPILLER, A. (2010). Die Reputation der Fleischwirtschaft: eine Kausalanalyse. In: German Journal of Agricultural Economics 60(4): 258-270.

ALBRECHT, J. (2014). Vom „Kohlrabi-Apostel“ zum „Bionade-Biedermeier“. In: Martina Löw (Hrsg.): Vielfalt und Zusammenhalt: Verhandlungen des 36. Kongresses der Deutschen Gesellschaft für Soziologie in Bochum und Dortmund 2012, Teil 1 Campus Verlag, 2014. Leipzig. Campus Verlag, 2014.

ALI, A. \& CHENG, K. M. (1985). Early egg production in genetically blind (rc/rc) chickens in comparison with sighted $(\mathrm{Rc}+/ \mathrm{rc})$ controls. Poultry Science 64: 789-794.

ALONSO, M. E., GONZÁLEZ-MONTAÑA, J. R., \& LOMILlOS, J. M. (2020). Consumers' concerns and perceptions of farm animal welfare. Animals, 10(3), 385.

AMINE, A. \& CADENAT, S. (2003). Efficient retailer assortment: a consumer choice evaluation perspective. In: International Journal of Retail and Distribution Management, 31(10): 486-497.

ANDERSEN, H. J., OKSBJERG, N. \& THERKILDSEN, M. (2005). Potential quality control tools in the production of fresh pork, beef and lamb demanded by the European society. Livestock Production Science 94: 105-124.

ARMSTRONG, S. \& BOTZLER, R. (2008). The animal ethics reader. Routledge, London and New York.

BAUER, M. W. (2009). The evolution of public understanding of science. Discourse and comparative evidence. Science Technology \& Society 14: 221-240.

BÄURLE, H. \& TAMÁSY, C. (2012). Regionale Konzentrationen der Nutztierhaltung in Deutschland. ISPA Mitteilungsheft 79. Vechta. 
BAYE, I., VON SCHLIPPENBACH, V., \& WEY, C. (2018). One-Stop Shopping Behavior, Buyer Power and Upstream Merger Incentives. Journal of Industrial Economics, 66(1), 66-94, https://doi.org/10.1111/joie.12160 [abgerufen am 13.12.2018].

BEARDSWORTH, A. \& BRYMAN, A. (1999). Meat consumption and vegetarianism among young adults in the UK. British Food Journal 101: 289-300.

BEARDSWORTH, A. \& BRYMAN, A. (2004). Meat consumption and meat avoidance among young people. British Food Journal 106: 313-327.

BEARDSWORTH, A. \& KEIL, E. T. (1991). Vegetarianism, veganism, and meat avoidance: recent trends and findings. British Food Journal 93: 19-24.

BLE (2013). Ökobarometer 2013. Repräsentative Bevölkerungsbefragung im Auftrag des Bundesministeriums für Ernährung, Landwirtschaft und Verbraucherschutz (BMELV),https://www.oekolandbau.de/fileadmin/redaktion/dokumente/journaliste n/130817_Oekobarometer_2013_final.pdf [abgerufen am 12.05.2019].

BLE (2020). Pressemitteilung: Studie gibt Vermarktungsideen für alte Nutztierrassen, https://www.ble.de/SharedDocs/Pressemitteilungen/DE/2020/200518_Nutztierrasse n.html [abgerufen am 20.08.2020].

BLUE HORIZON (2020). Report: The meat industry is unsustainable. Blue Horizon Corporation AG, https://www.bluehorizon.com/report-the-meat-industry-isunsustainable [abgerufen am 23.09.2020].

BMEL (2012). Politikstrategie Food Labelling. September 2011. Erschienen in: Berichte über Landwirtschaft. Zeitschrift für Agrarpolitik und Landwirtschaft. Band 90 (1) · 001-172 . April 2012 ISSN 0005-9080, https://www.researchgate.net/publication/291074727_Politikstrategie_Food_Labell ing_Gemeinsame_Stellungnahme_der_Wissenschaftlichen_Beirate_fur_Verbrauc her_und_Ernahrungspolitik_sowie_Agrarpolitik_beim_Bundesministerium_fur_Er nahrung_Landwirtschaft_[abgerufen am 12.03.2020].

BMEL (2015). Wege zu einer gesellschaftlich akzeptierten Nutztierhaltung. Bundesministerium für Ernährung und Landwirtschaft (BMEL), Berlin, https://www.bmel.de/SharedDocs/Downloads/DE/_Ministerium/Beiraete/agrarpolit ik/GutachtenNutztierhaltung.pdf?_blob=publicationFile\&v=2 [abgerufen am 10 . Dezember 2016]. 
BMEL (2016). Ökobarometer 2016, infas Institut für angewandte Sozialwissenschaft, https://www.bmel.de/SharedDocs/Downloads/DE/_Landwirtschaft/BiologischerLandbau/Oekobarometer2016.pdf?_blob=publicationFile\&v=3 [abgerufen am 12.05.2019].

BMEL (2018). Deutschland wie es isst - Der BMEL-Ernährungsreport 2018, https://www.bmel.de/SharedDocs/Downloads/DE/Broschueren/Ernaehrungsreport2 018.pdf;jsessionid=99D8E3785F7BB151E102ED4AE080BCA6.live831?_blob=p ublicationFile \&v=4 [abgerufen am 20.11.2019].

BMEL (2019a). Deutschland wie es isst - Der BMEL-Ernährungsreport 2019, https://www.bmel.de/SharedDocs/Downloads/DE/Broschueren/Ernaehrungsreport2 019.pdf?_blob=publicationFile\&v=4 [abgerufen am 10.07.2020].

BMEL (2019b). Label für mehr Tierwohl, https://www.tierwohlstaerken.de/einkaufshilfen/tierwohl-label [abgerufen am 27.06.2020].

BOAITEY, A. \& MINEGISHI, K. (2020). Who are farm animal welfare conscious consumers? British Food Journal.

BÖCKENHOLT, I., MEHN, A. \& WESTERMANN, A. (2018). Konzepte und Strategien für Omnichannel-Exzellenz : innovatives Retail-Marketing mit mehrdimensionalen Vertriebs- und Kommunikationskanälen. Springer Gabler. http://search.ebscohost.com/login.aspx?direct=true $\& \mathrm{db}=$ cat03594a\&AN=HSO.102 3312840\&lang=de\&site=eds-live [abgerufen am 05.03.2020].

BÖLW (2018). Pressemitteilung BÖLW: Fleischkennzeichnung muss sich am Ei orientieren, $\quad$ https://www.boelw.de/presse/meldungen/artikel/boelwfleischkennzeichnung-muss-sich-am-ei-orientieren/page/41 [abgerufen am 24.02.2020].

BORG, I. \& STAUFENBIEL T. (2007). Theorien und Methoden der Skalierung. Huber, Bern.

BORTZ, J. \& DÖRING, N. (2009). Forschungsmethoden und Evaluation für Humanund Sozialwissenschaftler.

BOSSERT, L. (2014). Tierethik. Die verschiedenen Positionen und ihre Auswirkungen auf die Mensch - nichtmenschliches Tier - Beziehung. In: Voget-Kleschin L, Bossert L, Ott K (Hrsg.) Nachhaltige Lebensstile. Metropolis-Verlag, Marburg, S. 32-57. 
BOSTANCI, H. (2011). Clusterbasierte Datenanalyse auf Grundlage genetischer Algorithmen in SAP-BI: Ein Verfahren zur selbstständigen Ermittlung der optimalen Anzahl Cluster. Diplomatica Verlag, Hamburg.

BOVENKERK, B., BROM, F., VAN DEN BERGH, B. (2002). Brave new birds: the use of "animal integrity" in animal ethics. The Hastings Center Report 32: 16-22.

BROOKS, D. (2001). Die Bobos: der Lebensstil der neuen Elite. Ullstein.

BRUHN, M., KIRCHGEORG, M. \& MEFFERT, H. (2018). Marketing Weiterdenken : Zukunftspfade für eine marktorientierte Unternehmensführung; Herrn Prof. Dr. Dr. h.c. mult. Heribert Meffert zum 80. Geburtstag. Springer Gabler.

BUNDESTAG (2016). Staatliche Tierschutzlabels in ausgewählten europäischen Staaten,https://www.bundestag.de/resource/blob/488970/68678190e6787d9a893a2 18b3f81c0c0/wd-5-101-16-pdf-data.pdf [abgerufen am 24.06.2017].

BUSCH, G., \& SPILLER, A. (2020). Warum wir eine Tierschutzsteuer brauchen: Die Bürger-Konsumenten-Lücke (No. 2001). Diskussionsbeitrag.

BUXEL H. \& SCHULZ S. (2010). Akzeptanz und Nutzung von Güte- und Qualitätssiegeln auf Lebensmitteln, Münster 2010, https://docplayer.org/20659156Akzeptanz-und-nutzung-von-guete-und-qualitaetssiegeln-auf-lebensmittelnergebnisse-einer-empirischen-untersuchung.html [abgerufen am 07.02.2017].

BUXEL, H. (2018). Prüf- und Gütesiegel auf Lebensmitteln: Verbrauchereinstellungen, Bekanntheit und Einfluss auf die Produktwahrnehmung sowie die Kauf- und Zahlungsbereitschaft, Münster 2018, https://docplayer.org/106576091-Vertraulichmuenster-prof-dr-holger-buxel.html [abgerufen am 27.06.2019].

BVLH (2020). Wertschätzung und Mehrzahlungsbereitschaft für Lebensmittel - Eine qualitative Verbraucherbefragung im Auftrag des Bundesverbandes des Deutschen Lebensmittelhandels,https://www.bvlh.net/fileadmin/redaktion/downloads/pdf/2020 /BVLH-ZHAW_Wertschätzung-MZB_LDR_2020.pdf [abgerufen am 15.10.2020].

CASWELL, J. A. \& Mojduszka, E. M. (1996). "Using Informational Labeling to Influence the Market for Quality in Food Products." American Journal of Agricultural Economics 78(5):1248-53.

CHOI, S. Y. (2020). LOHAS (Lifestyle of Health and Sustainability) scale development and validation (Doctoral dissertation, Purdue University Graduate School). 
CHRISTOPH-SCHULZ et al. (2018). SocialLab - Nutztierhaltung im Spiegel der Gesellschaft. J Consum Prot Food Saf 13, 145-236 (2018), https://doi.org/10.1007/s00003-017-1144-7 [abgerufen am 07.06.2020].

CLARK, B., STEWART, G. B., PANZONE, L. A., KYRIAZAKIS, I., \& FREWER, L. J. (2017). Citizens, consumers and farm animal welfare: A meta-analysis of willingness-to-pay studies. Food Policy, 68, 112-127.

COHEN-PFISTER, L. (2015). Social Consciousness in the Bionade-Biedermeier: An Interview with Filmmakers Marc Bauder and Dörte Franke. In J. Twark \& A. Hildebrandt (Eds.), Envisioning Social Justice in Contemporary German Culture (pp. 186-202). Boydell \& Brewer.

CORDTS, A., WITTIG, F., SCHUlZE, B., EISINGER-WATZL, M., HEUER, T., SPILLER, A. \& HOFFMANN, I. (2013). Eine Typologisierung männlicher BioKäufer und Nicht-Bio-Käufer: Ernährungs-, Gesundheits- und Kaufverhalten, in: Ernährungs Umschau 60 (3), S. 36-42.

CRESPI, J. M. \& MARETTE, S. (2005). Eco-labelling economics: Is public involvement necessary? In S. Krarup \& C. S. Russell (Eds.), Environment, information and consumer behavior (pp. 93-110). Northampton, MA: Edward Elgar.

DARBY, M. \& KARNI, E. (1973). Free competition and the optimal amount of fraud. Journal of Europäische Union 2014.

DAVID, T., \& HILPERT, M. (2016). Der unberechenbare Kunde. Standort, 40(3), 189193.

DAVIDSON, A. \& LAROCHE, M. (2018). Consumer preferences for human uniqueness in marketing communications, Journal of Marketing Communications, 24(5), p. 506.

DBV (2019). Deutscher Bauernverband. Unseren Kühen geht es gut, https://media.repromayr.de/31/595531.pdf [abgerufen am 16.04.2019].

DE BOER, J., HOOGLAND, C. T. \& BOERSEMA, J. J. (2007). Towards more sustainable food choices: value priorities and motivational orientations. Food Quality and Preference 18: 985-996.

DE JONGE, J., VAN DER LANS, I. O. \& VAN TRIJP, H. C. M. (2015). Different shades of grey: Compromise products to encourage animal friendly consumption. In: Food Quality and Preference, 45: 87-99. 
DE JONGE, J. \& VAN TRIJP, H. C. M. (2013). Meeting heterogeneity in consumer demand for animal welfare. A reflection on existing knowledge and implications for the meat sector. In: Journal of Agricultural and Environmental Ethics, 26(3), S. 629661.

DEEKELING, E. \& BARGHOP, D. (2017). Kommunikation in der digitalen Transformation. Springer Gabler, http://search.ebscohost.com/login.aspx?direct=true $\& \mathrm{db}=$ cat03594a\&AN=HSO.890 841748\&lang=de\&site=eds-live [abgerufen am 05.03.2020].

DEIMEL, I., FRANZ, A., FRENTRUP, M., VON MEYER, M., SPILLER, A. \& THEUVSEN, L. (2010). Perspektiven für ein Europäisches Tierschutzlabel. Gutachten, Georg-August-Universität Göttingen.

DEUTSCHER TIERSCHUTZBUND (2019). Bauernhöfe statt Agrarfabriken, https://www.tierschutzbund.de/organisation/partner/bauernhoefe-statt-agrarfabriken [abgerufen am 13.05.2019].

DFV (2020). Werte-Index 2020, https://www.dfv-fachbuch.de/handel-fmcg/4949-werteindex-2020.html [abgerufen am 13.08.2020].

DGE (2019). Orientierungswerte, http://www.dgeernaehrungskreis.de/orientierungswerte [abgerufen am 23.07.2019].

DRZE (2019). Ethische Aspekte - DRZE, http://www.drze.de/imblickpunkt/biodiversitaet/ethische-aspekte [abgerufen am 04.08.2019].

DUARTE, R. (2019). Blue buffalo-organic pet food (Doctoral dissertation).

EDWARDS \& SCHNEIDER (2005): The World Veterinary Association and animal welfare. In: Revenue Scientifique et Technique (International Office of Epizootics), 24(2): 639-646.

EHI (2020). Ranking der größten Lebensmitteleinzelhändler in Deutschland nach Umsatz, https://www.ehi.org/de/pressemitteilungen/lebensmittelhandel-stabileswachstum [abgerufen am 10.10.2020].

ENNEKING, U., KLEINE-KALMER, R., DAUERMANN, A. \& VOIGT, R. (2019). Kaufbereitschaft bei verpackten Schweinefleischprodukten, https:/www.hsosnabrueck.de/fileadmin/HSOS/Homepages/Personalhomepages/Personalhomepag es-AuL/Enneking/Tierwohlstudie-HS-Osnabrueck_Teil-Realdaten_17-Jan2019.pdf [abgerufen am 12.11.2019]. 
ERRICHIELLO, O. C. \& ZSCHIESCHE, A. (2017). Grüne Markenführung: Erfolgsfaktoren und Instrumente nachhaltiger Brands. Springer Gabler, http://search.ebscohost.com/login.aspx?direct=true $\& \mathrm{db}=$ cat03594a\&AN=HSO 869 747088\&lang=de\&site=eds-live [abgerufen am 05.03.2020].

EUROPEAN COMMISSION (2016). Attitudes of Europeans towards Animal Welfare, Special Eurobarometer

442 , http://www.izs.it/IZS/Engine/RAServeFile.php/f/pdf_vari_grafica_/Attitudes_of_E uropeans_towar ds_Animal_Welfare.pdf [abgerufen am 05.10.2017].

EUROPEAN COMMISSION (2005). Attitudes of consumers towards the welfare of farmed animals, Special Eurobarometer 229, http://ec.europa.eu/commfro ntoffice/publicopinion/archives/ebs/ebs_229_en.pdf [abgerufen am 05.08.2016].

FAO (2019). Food Outlook - Biannual Report on Global Food Markets. Rome, http://www.fao.org/3/ca4526en/ca4526en.pdf [abgerufen am 02.11.2020].

FEINDT, P. H., CANENBLEY, C., GOTTSCHICK, M., MÜLLER, C. \& ROEDENBECK, I. (2004). Konflikte des Agrarsektors - eine Landkarte. Empirische Ergebnisse einer konflikttheoretischen Fundierung der Nachhaltigkeitsforschung. In: BIOGUM-Forschungsbericht, 12.

FÖRSTER, C. \& LORING, J. (2014). Der moderne Spießer: Beobachten, erkennen, bestimmen.

FRANZ, A., DEIMEL, I. \& SPILLER, A. (2012). Concerns about animal welfare: A cluster analysis of German pig farmers, in: British Food Journal 114 (10), S. 1445 1462.

FRASER, D. (2008). Understanding animal welfare. In: Acta Veterinaria Scandinavica, 50: $1-7$.

FRASER, D. \& DUNCAN, I. J. H. (1998). Pleasures, pains and animal welfare: toward a natural history of affect. Animal Welfare 7: 383-396.

GENERATION WHAT (2019), www.generation-what.de [abgerufen am 03.04.2020].

GIK (2019). GIK Media, https://gik.media/downloads [abgerufen am 03.04.2018].

GÓMEZ-SUÁREZ, M., QUINONES, M. \& YAGÜE, M. J. (2020). Targeting smart shoppers: a cross-country model. Journal of Business Economics and Management, 21(3), 679-705, https://journals.vgtu.lt/index.php/JBEM/article/view/11851 [abgerufen am 15.09.2020]. 
GOSSARD, M. H. \& YORK, R. (2003). Social structural influences on meat consumption. Human Ecology Review 10: 1-9.

GÖTHLICH, S. E. (2009). Zum Umgang mit fehlenden Werten in großzahligen empirischen Erhebungen. In: Albers, S. et al. (Hrsg.): Methodik der empirischen Forschung, Wiesbaden: 119-136.

GREENPEACE (2020). Schweinefleisch Siegel-Ratgeber https://www.greenpeace.de/sites/www.greenpeace.de/files/publications/e01171siegelratgeber-schweinefleisch-03_17.pdf [abgerufen am 14.10.2020].

GRUBER, M. (2013). Die Zukunft is(s)t vegetarisch. Der Wandel von einer Fleischdominierten Esskultur zu einer vegetarischen Ernährungsweise. Hamburg: Diplomica Verlag GmbH.

GRUEN, L. (2012). The moral status of animals. The Stanford Encyclopedia of Philosophy, Winter 2012 Edition. Stanford University, Stanford.

GRUNERT, K. G. (2006). Future trends and consumer lifestyles with regard to meat consumption. Meat Science 74: 149-160.

HABLESREITER M. \& STUMMERER S. (2020). gut | ESSEN | böse - oder - „Warum Bio nicht Bobo ist und wie Essen die Welt verändern kann“. In: Klotter C., Endres EM. (eds) Gute - Böse Lebensmittelindustrie. Springer, Wiesbaden. https://doi.org/10.1007/978-3-658-26458-1_5 [abgerufen am 01.11.2020].

HAMM, U., HEMMERLING, S., SCHLEENBECKER, R., SPILLER, A., WÄGELI, S., CORDTS, A. \& KARY, V. (2011). Wissensstandsanalyse zum Verbraucher-und Ernährungsverhalten bei ökologischen Lebensmitteln mit Einbezug der Außer-HausVerpflegung.

HAMMANN, P. \& ERICHSON B. (2000). Marktforschung. Gustav Fischer, Stuttgart.

HANDSCHUCH, C. (2014). Traditional Food Crop Production and Marketing in SubSaharan Africa - The Case of Finger Millet in Western Kenya, http://hdl.handle.net/11858/00-1735-0000-0022-5E54-4 [abgerufen am 23.10.2016].

HARWATT, H., SABATÉ, J., ESHEL, G., SORET, S., \& RIPPLE, W. (2017). Substituting beans for beef as a contribution toward US climate change targets. Climatic Change, 143(1), 261-270, https://doi.org/10.1007/s10584-017-1969-1 [abgerufen am 10.07.2020]. 
HEINRICH, S. (2017). Content Marketing : So finden die besten Kunden zu Ihnen: Wie Sie Ihre Zielgruppe anziehen und stabile Geschäftsbeziehungen schaffen. Springer Gabler,http://search.ebscohost.com/login.aspx?direct=true \&db=cat03594a\&AN=H SO.870611054\&lang $=$ de \&site=eds-live [abgerufen am 05.03.2020].

HEDENUS, F., WIRSENIUS, S., \& JOHANSSON, D. J. A. (2014). The importance of reduced meat and dairy consumption for meeting stringent climate change targets. Climatic Change, 124(1), 79-91, https://doi.org/10.1007/s10584-014-1104-5 [abgerufen am 13.03.2020].

HEISE, H., PIRSICH, W. \& THEUVSEN, L. (2016). Kriterienbasierte Bewertung ausgewählter europäischer Tierwohl-Labels. In: Berichte über Landwirtschaft, 94(1).

HEMMERLING, S., SCHÜTZ, K., KRESTEL, N., ZÜHLSDORF, A., \& SPILLER, A. (2016). Trendsegment Foodies: Die neue Leidenschaft für Lebensmittel. Ergebnisse einer Zielgruppenanalyse für den deutschen Lebensmittelmarkt (Textfassung der Studie). Georg-August-Universität, Göttingen.

HÜBNER, A. H. \& KUHN, H. (2012). Retail category management: State-of-the-art review of quantitative research and software applications in assortment and shelf space management. In: Omega, 40(2): 199-209.

HUDDLESTON, P., WHIPPLE, J., NYE, M. R., JUNG LEE, S. (2009). Customer satisfaction in food retailing. Comparing specialty and conventional grocery stores. In: International Journal of Retail \& Distribution Management 37 (1): 63-80.

IKRATH, P. (2015). Die Hipster: Trendsetter und Neo-Spießer. Promedia Verlag.

INDERHEES, P., KIEFER, S., LÜTH, M. \& SPILLER, A. (2004). Determinanten der Kundenzufriedenheit im Fleischerfachhandel. Diskussionsbeitrag 0407, GeorgAugust-Universität Göttingen.

JANSSEN, M., KILIAN, D., \& HAMM, U. (2014). Verbraucherbefragung zur Beurteilung des Regionalfensters. In R. Hermanowski, \& U. Hamm (Eds.), Gemeinsamer Abschlussbericht zu Projekten des Regionalfensters (pp. 78-105). FiBL Deutschland e. V., http://orgprints.org/28149/1/28149-12NA053-12NA05712NA058-fibl-hermanowski-2014-regionalfenster.pdf [abgerufen am 15.10.2019].

JOHANSEN, B. O. (2012). The Buyer Power Of Multiproduct Retailers: Competition With One-Stop Shopping. Working Papers in Economics. https://w2.uib.no/filearchive/wp.03.12.pdf [abgerufen am 04.08.2016]. 
KÄMPER, G. (2003). Emotionen bei Tieren? In: Stephan A, Walter H (Hrsg.) Natur und Theorie der Emotionen. Mentis, Paderborn, S. 34-50.

KANIS, E., GROEN, A. B. F. \& DE GREEF, K. H. (2003). Societal concerns about pork and pork production and their relationships to the production system. In: Journal of Agricultural and Environmental Ethics 16 (2): 137-162.

KAYSER, M., BÖHM, J. \& SPILLER, A. (2012a). Zwischen Markt und Moral. Wie wird die deutsche Land- und Ernährungswirtschaft in der Gesellschaft wahrgenommen? Schriften der Gesellschaft für Wirtschafts- und Sozialwissenschaften des Landbaus e. V. 47: 329-341.

KAYSER, M., SCHLIEKER, K. \& SPILLER, A. (2012b). Die Wahrnehmung des Begriffs „Massentierhaltung“ aus Sicht der Gesellschaft. Berichte über Landwirtschaft 90: 417-428.

KAYSER, M. \& SPILLER, A. (2012). Das Image der verschiedenen Fleischarten aus KonsumentInnensicht. In: Hambrusch J, Hoffmann C, Kantelhardt J, Oedl-Wieser T (Hrsg.) Jahrbuch der österreichischen Gesellschaft für Agrarökonomie Band 21, Heft 1. Österreichische Gesellschaft für Agrarökonomie, Wien, S. 23-33.

KEELING, L., EVANS, A., FORKMANN, B. \& KJÆRNES, U. (2013). Welfare Quality ${ }^{\circledR}$ principles and criteria. In: BLOKHUIS, H., MIELE, M., VEISSIER, I., JONES, B. (Hrsg.): Improving farm animal welfare. The Welfare Quality® approach. Wageningen Academic Publishers, 91-114.

KETELSEN, M., JANSSEN, M., \& HAMM, U. (2020). Consumers' response to environmentally-friendly food packaging-A systematic review. Journal of Cleaner Production, 254, 120123.

KHEIRAVAR, S., \& RICHTER, N. (2016). Neue Technologien im stationären Einzelhandel: Mobile Apps oder stationäre Geräte?. In Digitalisierung im Vertrieb (pp. 609-631). Springer Gabler, Wiesbaden.

KIRSCHKE, D., ODENING, M., HÄGER, A. \& MUßHOFF, O. (2007). Strukturwandel im Agrarsektor. In: Humboldt-Spektrum 1 (2007): 24-31.

KNIERIM, U. (2001). Grundsätzliche ethologische Überlegungen zur Beurteilung der Tiergerechtheit bei Nutztieren. Deutsche Tierärztliche Wochenschrift 109: 261-266. 
KREUTZER, R. T. (2018). Toolbox für Marketing und Management : Kreativkonzepte Analysewerkzeuge - $\quad$ Prognoseinstrumente. Springer Gabler, http://search.ebscohost.com/login.aspx?direct=true $\& d b=$ cat03594a\&AN=HSO.102 7849016\&lang=de\&site=eds-live [abgerufen am 05.03.2020].

KROEBER-RIEL, W. \& ESCH, F.-R. (2004). Strategie und Technik der Werbung. Verhaltenswissenschaftliche Ansätze. Stuttgart: Kohlhammer.

LAGERKVIST, C. J. \& HESS, S. (2011). A meta-analysis of consumer willingness to pay for farm animal welfare. In: European Review of Agricultural Economics, 38(1): $55-78$.

LAI, Y., \& YUE, C. (2020). Consumer Willingness to Pay for Organic and Animal Welfare Product Attributes: Do Experimental Results Align with Market Data?. Journal of Agricultural and Resource Economics.

LANCASTER, K. (1971). Consumer Demand: A New Approach. New York: Columbia University Press. Law and Economics 16 (1), 67-88.

LAZO GALDOS, A. (2011). Effects of country-of-origin and benchmarking on credibility of food quality assurance schemes. Hochschulschrift. Göttingen, Univ., Diss., 2011, http://hdl.handle.net/11858/00-1735-0000-0015-8E54-6 [abgerufen am 16.06.2018].

LEA, E. \& WORSLEY, A. (2001). Influences on meat consumption in Australia. Appetite 36: 127-136.

LEBENSMITTELZEITUNG (2020). Ranking: Top 30 Lebensmittelhandel Deutschland 2018, https://www.lebensmittelzeitung.net/handel/Ranking-Top-30Lebensmittelhandel-Deutschland-2018-134606 [abgerufen am 04.08.2020].

LEE, K. H., NOH, J., \& KHIM, J. S. (2020). The Blue Economy and the United Nations' sustainable development goals: Challenges and opportunities. Environment International, 137, 105528.

LEMKEN, D., A. SPILLER \& SCHULZE-EHLERS, B. (2018). Will consumers substitute meat with legumes? - A clustered binational perspective. GlobalFood Discussion Paper 121, University of Goettingen, http://www.unigoettingen.de/de/213486.html [abgerufen am 10.10.2019].

LOUREIRO, M. L., MCCLUSKEY, J. J. \& MITTELHAMMER, R. C. (2001). Assessing Consumer Preferences for Organic, Eco-labeled, and Regular Apples. Journal of Agricultural and Resource Economics 26(2): 404-416. 
MALHOTRA, N. K. (1984). Reflections on the information overload paradigm in consumer decision making. In: Journal of Consumer Research, 10(4): 436-440.

MANUEL LEITÃO MARQUES, M. (2007). One-Stop Shopping in Portugal. IFC Smart Lessons Brief. World Bank, Washington, DC. (C) World Bank. https://openknowledge.worldbank.org/handle/10986/10629 [abgerufen am 11.05.2017].

MARKEN, G. \& WAGENFELD, N. UNTER MITARBEIT VON DANGEL, V., HETTICH, E., KITZBERGER, M., NESTELE, J. UND RUBIK, F. (2020). Einkaufsgewohnheiten und Einflussfaktoren beim Unverpackt-Einkauf, https://www.bmbf-plastik.de/sites/default/files/202007/Marken\%20\%26\%20Wagenfeld\%20\%282020\%29\%20Einkaufsgewohnheiten\% 20und\%20Einflussfaktoren\%20-\%20Unverpackt-Umfrage.pdf [abgerufen am 14.10.2020].

MASTERTON, M., RENBERG, T., KALVEMARK SPORRONG, S. (2014). Patients' attitudes towards animal testing: "To conduct research on animals is, I suppose, a necessary evil". BioSocieties 9: 24-41.

MAX-RUBNER-INSTITUT (2008). Ergebnisbericht Teil 1, Nationale Verzehrsstudie II. 2008,https://www.bmel.de/SharedDocs/Downloads/Ernaehrung/NVS_Ergebnisberi cht.pdf?_blob=publicationFile [abgerufen am 10.12.2019].

MAY, C. (2016). The application of social marketing to issues in animal welfare, http://search.ebscohost.com/login.aspx?direct=true \&db=edsbas\&AN=edsbas.6E09 8C81\&lang=de\&site=eds-live [abgerufen am 05.03.2020].

MCKENDREE, M. G. S., TONSOR, G. T. \& WOLF, C. A. (2016). Similarities and Differences of Animal Welfare Perceptions between U.S. Cow-Calf Producers and the Public, 2016 Annual Meeting, February 6-9, 2016, San Antonio, Texas, http://search.ebscohost.com/login.aspx?direct=true $\& \mathrm{db}=$ edsrep $\& A N=$ edsrep.p.ags. saea16.230018\&lang=de\&site=eds-live [abgerufen am 16.10.2017].

MENGER, K., FELDMANN, A., DORKEWITZ, K. \& HAMM, U. (2020). Vermarktungskonzepte für Produkte gefährdeter Nutztierrassen, https://orgprints.org/37582/1/37582-15NA168-15NA028-geh-unikassel-feldmannhamm-2020-vermarktung-gefaehrdeter-nutztierrassen.pdf [abgerufen am 19.10.2020].

MEUWISSEN, M. P. M., VAN DER LANS, I. A., HUIRNE, R. B. M. (2007). Consumer preferences for pork supply chain attributes. NJAS - Wageningen Journal of Life Sciences 54: 293-312. 
NAIDOO, R., \& FISHER, B. (2020). Reset sustainable development goals for a pandemic world.

NELSON, P. (1970). Information and Consumer Behavior, Journal of Political Economy (78:20), pp. 311-329.

NIER, H. (2019). So häufig essen Europäer Fleisch. Statista. Statista GmbH, https://de1statista-1 com-1ny53hpia3856.han.sub.uni-goettingen.de/infografik/17195/sohaeufig-essen-europaeer-fleisch [abgerufen am 04.03.2020].

NIJDAM, D., ROOD, T., \& WESTHOEK, H. (2012). The price of protein: Review of land use and carbon footprints from life cycle assessments of animal food products and their substitutes. Food Policy, 37(6), 760-770, https://doi.org/10.1016/j.foodpol.2012.08.002 [abgerufen am 24.08.2016].

NILSSON, E., GÄRLING, T., MARELL, A. \& NORDVALL, A.-C. (2015). Importance ratings of grocery store attributes. In: International Journal of Retail and Distribution Management 43(1): 63-91.

NOCELLA, G., HUBBARD L. \& SCAROA R. (2010). Farm animal welfare, consumer willingness to pay, and trust: results of a cross-national survey. In: Applied Economic Perspective and Policy 32 (2): 275-297.

NORWOOD, F. B. \& LUSK, J. L. (2009). The farm animal welfare debate. Choices 24.

PADEL, S. \& FOSTER, C. (2005). Exploring the gap between attitudes and behaviour. In: British Food Journal, 107(8): 606-625.

PIRSICH, W. (2017). Tierwohl in der Fleischbranche Label - Verbrauchereinstellungen - Vermarktungswege, Dissertation, Universität Göttingen, 2017.

PIRSICH, W., HEISE, H. \& THEUVSEN, L., (2015). Evaluation der Richtlinien deutscher Labelling-Initiativen der Schweinefleischwirtschaft aus Tierwohlperspektive. In: Tagungsband der 15. Fachtagung Fleisch- und Geflügelfleischhygiene für Angehörige der Veterinärverwaltung. Berlin, 109-114

PIRSICH, W., VON HARDENBERG, L. M., \& THEUVSEN, L. (2017). The Pet Food Industry: An innovative Distribution Channel for Marketing Feed Products from Welfare Friendly Production to Consumers?. International Journal on Food System Dynamics, 8(3), 250-261. 
POUTA, E., HEIKKILÄ, J., FORSMAN-HUGG, S., ISONIEMI, M. \& MÄKELÄ, J. (2010). Consumer choice of broiler meat: The effects of country of origin and production methods. In: Food Quality and Preference, 21(5): 539-546.

PRÄTTÄLÄ, R., PAALANEN, L., GRINBERGA, D., HELASOJA, V., KASMEL, A. \& PETKEVICIENE, J. (2007). Gender differences in the consumption of meat, fruit and vegetables are similar in Finland and the Baltic countries. European Journal of Public Health 17: 520-525.

REDLER, J. (2018). Store Brand: Hinführung. In Die Store Brand (pp. 1-21). Springer Gabler, Wiesbaden.

RESPONDI (2019). Echte Insights, https://www.respondi.com/leistungen\#quant [abgerufen am 14.11.2019].

RICHARDSON, N.J., SHEPHERD, R. \& ELLIMAN, N.A. (1993). Current attitudes and future influences on meat consumption in the U.K. Appetite 21: 41-51.

RIEBER, D. (2017). Mobile Marketing : Grundlagen, Strategien, Instrumente. Springer Gabler,http://search.ebscohost.com/login.aspx?direct=true\&db=cat03594a\&AN=H SO.1000115003\&lang=de\&site=eds-live [abgerufen am 31.01.2018].

RÖDIGER, M., \& HAMM, U. (2020). Do consumers care about organic and conventional food prices? An eye tracking study. Organic Agriculture, 10(1), 75-87.

RUTSCHMANN, M. (2018). Kaufprozessorientiertes Marketing: Stop Branding, Start Selling!: wie neueste Erkenntnisse aus der Verhaltensforschung und den Neurowissenschaften Marketing und Vertrieb beflügeln. Springer Gabler, http://widgets.ebscohost.com/prod/customerspecific/s1105463/vpn.php?url=http://s earch.ebscohost.com/login.aspx direct=true $\& d b=$ cat03594a\&AN=HSO 101515885 4\&lang=de \&site=eds-live [abgerufen am 10.07.2019].

SCHIPMANN-SCHWARZE, C. \& HAMM, U. (2020). Exploring drivers and barriers for organic poultry consumption", British Food Journal, https://doi.org/10.1108/BFJ11-2018-0787 [abgerufen am 09.10.2020].

SCHLEICHER, M., CASH, S. B., \& FREEMAN, L. M. (2019). Determinants of pet food purchasing decisions. The Canadian Veterinary Journal, 60(6), 644.

SCHRÖCK, R. (2013). Analyse der Preiselastizitäten der Nachfrage nach Biolebensmitteln. Justus-Liebig-Universität, Gießen, http://orgprints.org/22414/13/22414-08OE148-uni-giessen-herrmann-2013preiselastizitaeten_biolebensmittel.pdf [abgerufen am 30.06.2017]. 
SCHRÖDER, M. J. \& MCEACHERN, M. G. (2004). Consumer value conflicts surrounding ethical food purchase decisions: a focus on animal welfare. International Journal of Consumer Studies, 28(2): 168-177.

SCHULZE, B., LEMKE, D. \& SPILLER, A. (2008). Glücksschwein oder arme Sau? Die Einstellung der Verbraucher zur modernen Nutztierhaltung. In: SPILLER, A., SCHULZE, B. (Hrsg.): Zukunftsperspektiven der Fleischwirtschaft - Verbraucher, Märkte, Geschäftsbeziehungen. Göttingen, Universitätsverlag Göttingen, 465-488.

SCHULZE, B. \& SPILLER, A. (2008). Wer geht noch an die Theke? Ergebnisse einer Verbraucherstudie zu SB-Fleisch. In: Schriften der Gesellschaft für Wirtschafts- und Sozialwissenschaften des Landbaus, 43: 19-28.

$\begin{array}{lll}\text { SCHWEIZER } & \text { TIERSCHUTZVERORDNUNG } & \text { (2008). }\end{array}$ Tierschutzverordnung des Schweizerischen Bundesrates vom 23. April 2008.

SGS GERMANY (2014). Vertrauen und Skepsis: Was leitet die Deutschen beim Lebensmitteleinkauf? SGS Verbraucherstudie 2014. Hamburg.

SINGER (1995). Animal liberation: a new ethics for our treatment of animals. Random House, London.

SPILLER, A. (2006). Zielgruppen im Markt für Bio-Lebensmittel: Ein Forschungsüberblick, https://www.unigoettingen.de/de/104832.htmlde/document/download/aca1194d9bd45c8d6cac6af25 84cff3e.pdf/Zielgruppen\%20Endversion.pdf [abgerufen am 10.08.2018].

SPILLER, A. \& SCHULZE, B. (2008). Trends im Verbraucherverhalten: Ein Forschungsüberblick zum Fleischkonsum. In: SPILLER, A., SCHULZE, B. (Hrsg.): Zukunftsperspektiven der Fleischwirtschaft. Göttingen, Universitätsverlag Göttingen, 230-271.

SPILLER, A., SCHULZE, B. \& CORDTS, A. (2010). Was essen wir in zwanzig Jahren? DLG-Mitteilungen 1: 20-23.

SPILLER, A., KAYSER, M. \& BÖHM, J. (2012). Unternehmerische Landwirtschaft zwischen Marktanforderungen und gesellschaftlichen Erwartungen in Deutschland aus Sicht der Landwirtschaft. Schriften der Gesellschaft für Wirtschafts- und Sozialwissenschaften des Landbaues e.V. 47: 11-22.

SPILLER, A. (2013a). Weg vom üblen Image, in: DLG-Mitteilungen, Nr. 1.2013: 26-27. 
SPILLER A. (2013b). Food Label: Markt- und/oder Politikversagen? Präsentation Dachverband Agrarforschung am 19.11.2013 Berlin, https://www.agrarforschung.de/fileadmin/download/2013_AchimSpiller_DAF.pdf [abgerufen am 10.11.2019].

SPILLER, A. \& NITZKO, S. (2014). Verbraucherverständnis von Transparenz. Ergebnisse einer repräsentativen Konsumentenbefragung. Eine Studie im Auftrag von Die Lebensmittelwirtschaft e.V. Georg-August-Universität, Göttingen.

SPILLER, A., GAULY, M., BALMANN, A., BAUHUS, J., BIRNER, R., BOKELMANN, W., CHRISTEN, O., ENTENMANN, S., GRETHE, H., KNIERIM, U., LATACZ-LOHMANN, U., MARTINEZ, J., NIE-BERG, H., QAIM, M., TAUBE, F., TENHAGEN, B.-A. \& WEINGARTEN, P. (2015). Wege zu einer gesellschaftlich akzeptierten Nutztierhaltung. In: Berichte über Landwirtschaft, 221 (Sonderheft).

SPILLER et al. (2018). Fleischatlas 2018. Ernährungspolitische Leiter, https://www.bund.net/fileadmin/user_upload_bund/publikationen/massentierhaltun g/massentierhaltung_fleischatlas_2018.pdf [abgerufen am 17.10.2019].

SPILLER, A. \& ZÜHLSDORF, A. (2018). Haltungskennzeichnung und Tierschutzlabel in Deutschland: Anforderungen und Entwicklungsperspektiven, Wissenschaftliches Gutachten im Auftrag von Greenpeace Deutschland e. V., Göttingen, https://www.greenpeace.de/sites/www.greenpeace.de/files/publications/haltungsken nzeichnung-tierschutzlabel-gutachten-15.10.2018.pdf [abgerufen am 10.08.2019].

SPILLER, A. (2019). Konferenzdokumentation „Hunger auf nachhaltiges Essen weltweit - Konzepte für eine nachhaltige Ernährungs- und Landwirtschaftspolitik“. Tagung am 4. Dezember 2019 in Berlin, https://germanwatch.org/sites/germanwatch.org/files/Dokumentation\%20Agrarkon gress_04.12.2019.pdf [abgerufen am 20.03.2020].

SPILLER, A., ZÜHLSDORF, A., GAULY, S., \& KÜHL, S. (2019). Wie wichtig ist Verbrauchern das Thema Tierschutz?

SPINKA, M. (2006). How important is natural behaviour in animal farming systems? Applied Animal Behaviour Science 100: 117-128. Springer, Heidelberg.

STAMPA, E., SCHIPMANN-SCHWARZE, C., \& HAMM, U. (2020). Consumer perceptions, preferences, and behavior regarding pasture-raised livestock products: A review. Food Quality and Preference, 82, 103872. 
STAUS, A. (2010). Which household attitudes determine the store type choice for meat? In: Journal of Retailing and Consumer Services 18(3): 224-234.

STEHFEST, E., BOUWMAN, L., VAN VUUREN, D. P., DEN ELZEN, M. G. J., EICKHOUT, B., \& KABAT, P. (2009). Climate benefits of changing diet. Climatic Change, 95(1), 83-102, https://doi.org/10.1007/s10584-008-9534-6 [abgerufen am 10.03.2020].

STEHR, C. \& STRUVE, F. (2017). CSR und Marketing: Nachhaltigkeit und Verantwortung richtig kommunizieren. Springer Gabler, http://search.ebscohost.com/login.aspx?direct=true $\& d b=$ cat03594a\&AN=HSO.882 777815\&lang=de\&site=eds-live [abgerufen am 10.11.2018].

STIFTUNG WARENTEST (2020). Tierwohl-Label. Diese Siegel sollen beim Kauf von Fleisch helfen, https://www.test.de/Tierwohl-Label-Diese-Siegel-sollen-beim-Kaufvon-Fleisch-helfen-5306979-0 [abgerufen am 13.10.2020].

TEISL, M. \& ROE, B. (1998). The Economics of Labeling: An Overview of Issues for Health and Environmental Disclosure. Agricultural and Resource Economics Review. P 140-150.

THE LANCET (2019). Food in the Anthropocene: the EAT-Lancet Commission on healthy diets from sustainable food systems, https://www.thelancet.com/pdfs/journals/lancet/PIIS0140-6736\%2818\%29317884.pdf [abgerufen am 26.02.2020].

THEOBALD, A. (2017). Praxis Online-Marktforschung: Grundlagen Anwendungsbereiche - $\quad$ Durchführung. Springer Gabler, http://search.ebscohost.com/login.aspx?direct=true \&db=cat03594a\&AN=HSO.866 716998\&lang=de\&site=eds-live [abgerufen am 25.10.2018].

THEOBALD, E. \& HAISCH, P. T. (2017). Brand Evolution : moderne Markenführung im digitalen Zeitalter. Springer Gabler, http://search.ebscohost.com/login.aspx?direct=true $\& \mathrm{db}=$ cat03594a\&AN=HSO.871 $119471 \&$ lang $=$ de \&site $=$ eds-live [abgerufen am 25.10.2018].

THEUVSEN, L. \& RECKE, G. (2008). Horizontale Kooperationen in der Schlachtschweinevermarktung: Empirische Ergebnisse aus Nordwestdeutschland In: SPILLER, A., SCHULZE, B. (Hrsg.): Zukunftsperspektiven der Fleischwirtschaft. Göttingen, Universitätsverlag Göttingen, 73-95. 
THIELE, S. (2008). Elastizitäten der Nachfrage privater Haushalte nach Nahrungsmitteln - Schätzung eines AIDS auf Basis der Einkommens- und Verbrauchsstichprobe 2003. Agrarwirtschaft 57: 258-268.

THOGERSEN, J. \& ÖLANDER, F. (2003). Spillover of Environment-Friendly Consumer Behavior. Journal of Environmental Psychology 23(3): 225-236.

THOMAS, A. M., NEWMAN, C. L., FINKELSTEIN, S. R., CHO, Y. N., \& CASCIO, A. (2020). Consumer responses to shopper solutions in service settings. Journal of Services Marketing.

THOMPSON, P. B. (2008). The opposite of human enhancement: nanotechnology and the blind chicken problem. NanoEthics 2: 305-316.

TNS EMNID (2014). Einkaufs- und Ernährungsverhalten in Deutschland. BMEL, Bonn, http://www.bmel.de/SharedDocs/Downloads/Umfragen/TNS-EmnidEinkaufsErnaehrungsVerhaltenInDeutschland.pdf?__blob=publicationFile [abgerufen am 10.06.2016].

TOMASEVIC, I., BAHELKA, I., ČÍTEK, J., ČANDEK-POTOKAR, M., DJEKIĆ, I., GETYA, A. \& SOŁOWIEJ, B. (2020). Attitudes and beliefs of eastern european consumers towards animal welfare. Animals, 10(7), 1220.

TONSOR G. \& OLYNK, N. J. (2010). US meat demand: the influence of animal welfare media coverage. Agricultural Experiment Station and Cooperative Extension Service, Kansas State University, Manhattan.

UMWELTBUNDESAMT (2019). Daten, Umweltzustand und Trends, Chemikalien, https://www.umweltbundesamt.de/daten/chemikalien [abgerufen am 02.11.2019].

UN (2020). THE 17 GOALS, https://sdgs.un.org/goals [abgerufen am 10.10.2020].

UNIVERSITÄT ZÜRICH (2019). UZH - $\quad$ Methodenberatung, https://www.methodenberatung.uzh.ch/de.html [abgerufen am 23.11.2019].

UTOPIA (2019). Fleisch-Labels und -Siegel der Discounter: Sauerei im Kühlregal? 15. Februar 2019 von Benita Wintermantel, https://utopia.de/ratgeber/fleisch-labelsund-siegel-der-discounter-sauerei-im-kuehlregal [abgerufen am 14.10.2020].

VAN KLEEF, E., VAN TRIJP, H., PAEPS, F. \& FERNÁNDEZ-CELEMÍN, L. (2007). Consumer preferences for front-of-pack calories labelling. In: Public Health Nutrition, 11(2): 203-213. 
VAN LOO, E. J., CAPUTO, V., NAYGA, R. M. \& VERBEKE, W. (2014). Consumers' valuation of sustainability labels on meat. In: Food Policy, 49: 137-150.

VANHONACKER, F., VAN POUCKE, E., TUYTTENS, F. \& VERBEKE, W. (2010). Citizens' views on farm animal welfare and related information provision. In: Journal of Agricultural and Environmental Ethics, 23(6), S. 551-569.

VECCHIO, R. \& ANNUNZIATA, A. (2012). Italian consumer awareness of layer hens' welfare standards: a cluster analysis. International Journal of Consumer Studies 36: 647-655.

VERBEKE, W. A. \& VIANE, J. (2000). Ethical challenges for livestock production: meeting consumer concerns about meat safety and animal welfare. Journal of Agricultural and Environmental Ethics 12: 141-151.

VERBRAUCHERZENTRALE (2020). Tierschutz und Tierwohl: Einkaufstipps, https://www.verbraucherzentrale.de/tierwohl [abgerufen am 05.10.2020].

VERHOOG, H., LUND, V. \& ALR $\varnothing$, H. F. (2004). Animal health and welfare in organic agriculture. In: Vaarst, M., Roderick, S., Lund, V., Lockeretz, W. (Hrsg.) Animal health and welfare in organic agriculture. CABI Publishing, Wallingford, S. 73-94.

VERMEIR, I. \& VERBEKE W. (2006). Sustainable food consumption: exploring the consumer "Attitude - Behavioral Intention" gap. Journal of Agricultural and Environmental Ethics 19: 169-194.

VON ALVENSLEBEN, R. (1995). Die Imageprobleme bei Fleisch - Ursachen und Konsequenzen. In: Berichte über Landwirtschaft, 73(1): 65-82.

VON ALVENSLEBEN, R. (2002). Neue Wege in der Tierhaltung Verbraucheransichten und Verbrauchereinsichten. In: KTBL Schrift, 25-32.

VON ROTEN, F. C. (2012). Public perceptions of animal experimentation across Europe, https://journals.sagepub.com/doi/10.1177/0963662511428045 [abgerufen am 02.10.2017].

WBAE (2020). Wissenschaftlicher Beirat für Agrarpolitik, Ernährung und gesundheitlichen Verbraucherschutz beim BMEL. Politik für eine nachhaltigere Ernährung. Gutachten, Berlin, https://www.bmel.de/SharedDocs/Downloads/DE/_Ministerium/Beiraete/agrarpolit ik/wbae-gutachten-nachhaltigeernaehrung.html;jsessionid=55C79A387F3EA4D4E5CA1255871F8280.live841 [abgerufen am 15.11.2020]. 
WCRF (2020). Limit red and processed meat - Eat no more than moderate amounts of red meat and little, if any, processed meat, https://www.wcrf.org/dietandcancer/recommendations/limit-red-processed-meat [abgerufen am 02.08.2020].

WEGMANN, C. (2020). Produktinnovationen. In Lebensmittelmarketing (pp. 17-112). Springer Gabler, Wiesbaden.

WEINRICH, R., KÜHL, S., FRANZ, A. \& SPILLER, A. (2015). Consumer preferences for high welfare meat: self-service counter or service counter? In: International Journal of Food System Dynamics 6 (1), S. 32-49.

WEISSINGER, K. (2020). Online-Kommunikation für Zielgruppen mit einem nachhaltigen Lebensstil: Zielgruppenansprache am Beispiel der LOHAS im Lebensmitteleinzelhandel. Springer-Verlag.

WENZL, C., EVERTS, J., \& RINGEL, F. (2019). Zwischen Corner Shop und Späti - der Bedeutungswandel kleiner inhabergeführter Geschäfte im Quartier. Lokale Ökonomie-Konzepte, Quartierskontexte und Interventionen, 1-18.

WOODWARD, J. (1988). Consumer attitudes towards meat and meat products. British Food Journal 90: 101-104.

WUV (2020). Kantar-Studie: Natur und Nachhaltigkeit stürzen im Werte-Index ab, https://www.wuv.de/marketing/natur_und_nachhaltigkeit_stuerzen_im_werte_inde x_ab [abgerufen am 02.09.2020].

WWF (2019). Nachhaltiger genießen! WWF Einkaufsratgeber Fleisch und Wurst, https://www.wwf.de/fileadmin/user_upload/WWF-

Einkaufsratgeber_Fleisch_und_Wurst.pdf [abgerufen am 05.10.2020].

WWF (2020). Wann, wenn nicht jetzt. Das Maßnahmenprogramm Klimaschutz 2030 der deutschen Zivilgesellschaft, https://www.wwf.de/fileadmin/fm-wwf/PublikationenPDF/Massnahmenprogramm2030.pdf [abgerufen am 15.10.2020].

ZANDER, K., ISERMEYER, F., BÜRGELT, D., CHRISTOPH-SCHULZ, I., SALAMON, P. \& WEIBLE, D. (2013). Erwartungen der Gesellschaft an die Landwirtschaft. Gutachten im Auftrag der Stiftung Westfälische Landschaft. vTI, Braunschweig.

ZENNER, S., WIRTHGEN, B. \& ALTMANN, M. (2004). Analyse des Einkaufsverhaltens beim Direkteinkauf von Lebensmitteln. In: Schriften der Gesellschaft für Wirtschafts- und Sozialwissenschaften des Landbaus, 39: 291-299. 
ZÜHLSDORF, A. \& SPILLER, A. (2012). Trends in der Lebensmittelvermarktung: Begleitforschung zum Internetportal lebensmittelklarheit.de, Göttingen, https://www.vzbv.de/sites/default/files/downloads/LebensmittelvermarktungMarktstudie_Ergebnisse-ZÜHLSDORF-2012.pdf [abgerufen am 10.12.2016].

ZÜHLSDORF, A., SPILLER, A., GAULY, M. \& KÜHL S. (2016). Wie wichtig ist Verbrauchern das Thema Tierschutz? Präferenzen, Verantwortlichkeiten, Handlungskompetenzen und Politikoptionen, https://www.vzbv.de/sites/default/files/downloads/Tierschutz-UmfrageErgebnisbericht-vzbv-2016-01.pdf [abgerufen am 15.11.2019].

ZUKUNFTSINSTITUT (2019). Megatrend Neo-Ökologie, https://www.zukunftsinstitut.de/dossier/megatrend-neo-oekologie [abgerufen am 27.06.2020]. 


\title{
Anhang
}

\section{Anhang A: Fragebogen}

\section{Befragung zur Verbesserung des Produkt- und Beratungsangebots}

von Fleisch- und Wurstwaren aus artgerechterer Haltung im

\author{
deutschen Lebensmitteleinzelhandel
}

\section{Screeningfrage 1:}

Haben Sie in den letzten 12 Monaten für sich oder andere Personen Fleisch- oder Wurstwaren gekauft?

\section{$1 \square \mathrm{Ja}$}

\section{$2 \square$ Nein}

\section{Screeningfrage 2:}

Für viele Menschen sind besondere Produktqualitäten wie zum Beispiel Bio-Qualität, Gentechnikfreiheit, fairer Handel, Umweltfreundlichkeit oder eine artgerechte Tierhaltung in den letzten Jahren immer wichtiger geworden. Haben Sie beim Einkauf von Lebensmitteln schon einmal auf derartige Aspekte geachtet oder können Sie sich vorstellen in Zukunft auf so etwas zu achten?

$1 \square \mathrm{Ja}$

$2 \square$ Nein, das interessiert mich eher weniger 


\section{Befragung zur Verbesserung des Produkt- und Beratungsangebots von Fleisch- und Wurstwaren aus artgerechterer Haltung im deutschen Lebensmitteleinzelhandel} Bearbeitungszeit: ca. 12 Minuten

A Im ersten Teil der Befragung geht es um Ihr persönliches Einkaufs- und Ernährungsverhalten beim Einkauf von Fleisch- und Wurstwaren.

1. Für wen kaufen Sie gewöhnlich Fleisch- oder Wurstwaren ein?
$1 \square$ Nur für mich selber
$2 \square$ Nur für andere Personen
$3 \square$ Für mich und andere Personen

2. Welche der folgenden Einkaufsstätten haben Sie in den letzten 3 Monaten für Ihren Fleisch- und Wursteinkauf genutzt? Sie können mehrere Einkaufsstätten auswählen.

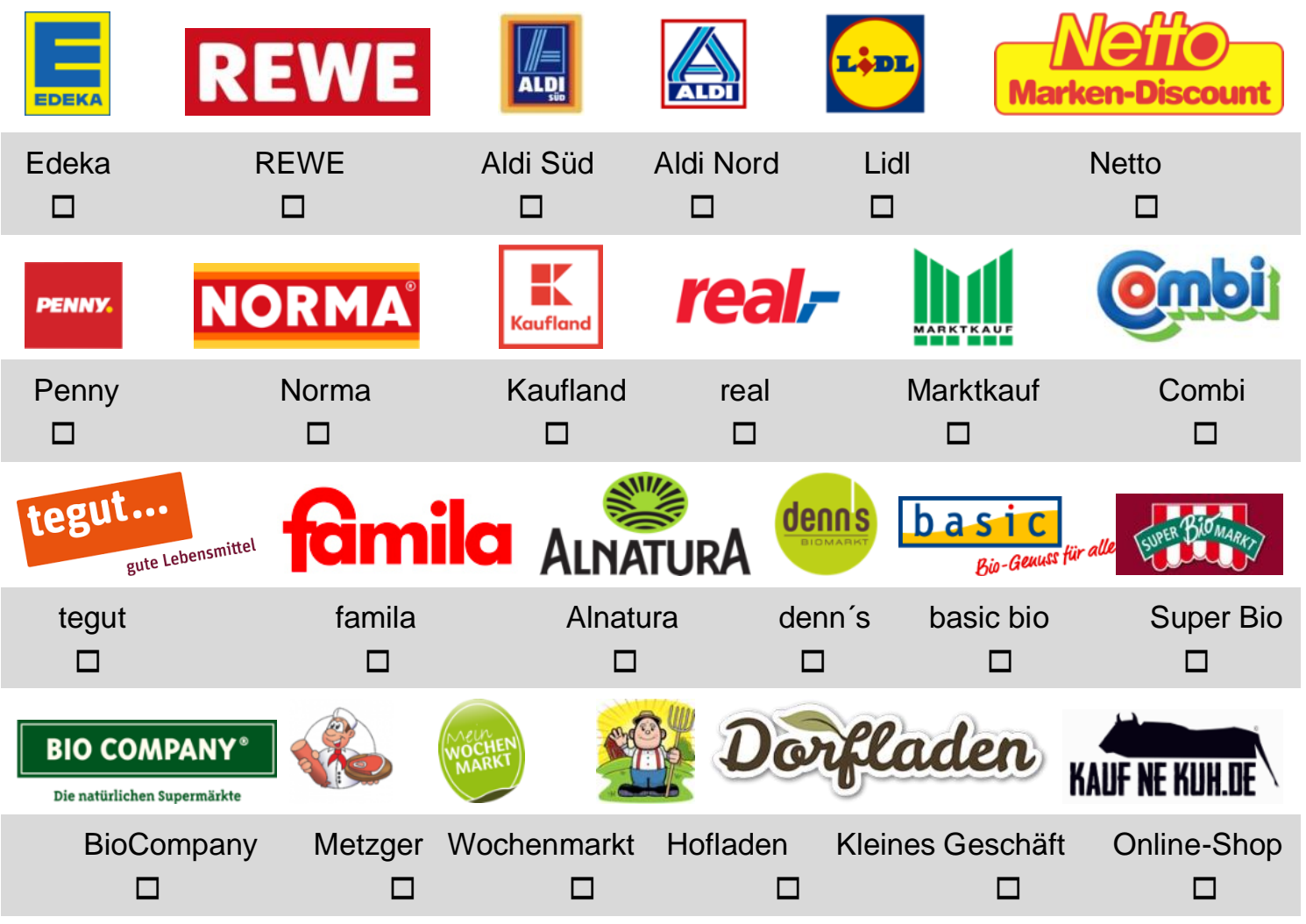

$\square$ Andere Einkaufsstätte (n)

Welche anderen Einkaufsstätten haben Sie in den letzten 3 Monaten für Ihren Fleisch- und Wursteinkauf genutzt? 
3. Sie haben die folgenden Einkaufsstätten ausgewählt, wo Sie Fleisch- und Wurstwaren kaufen. Welche dieser Einkaufsstätten ist lhre Haupteinkaufsstätte für Ihren Fleisch- und Wursteinkauf? Nennen Sie nur eine Haupteinkaufsstätte. (Anm: Es wurden nur Einkaufsstätten gezeigt, die in Frage 2 angegeben wurden)

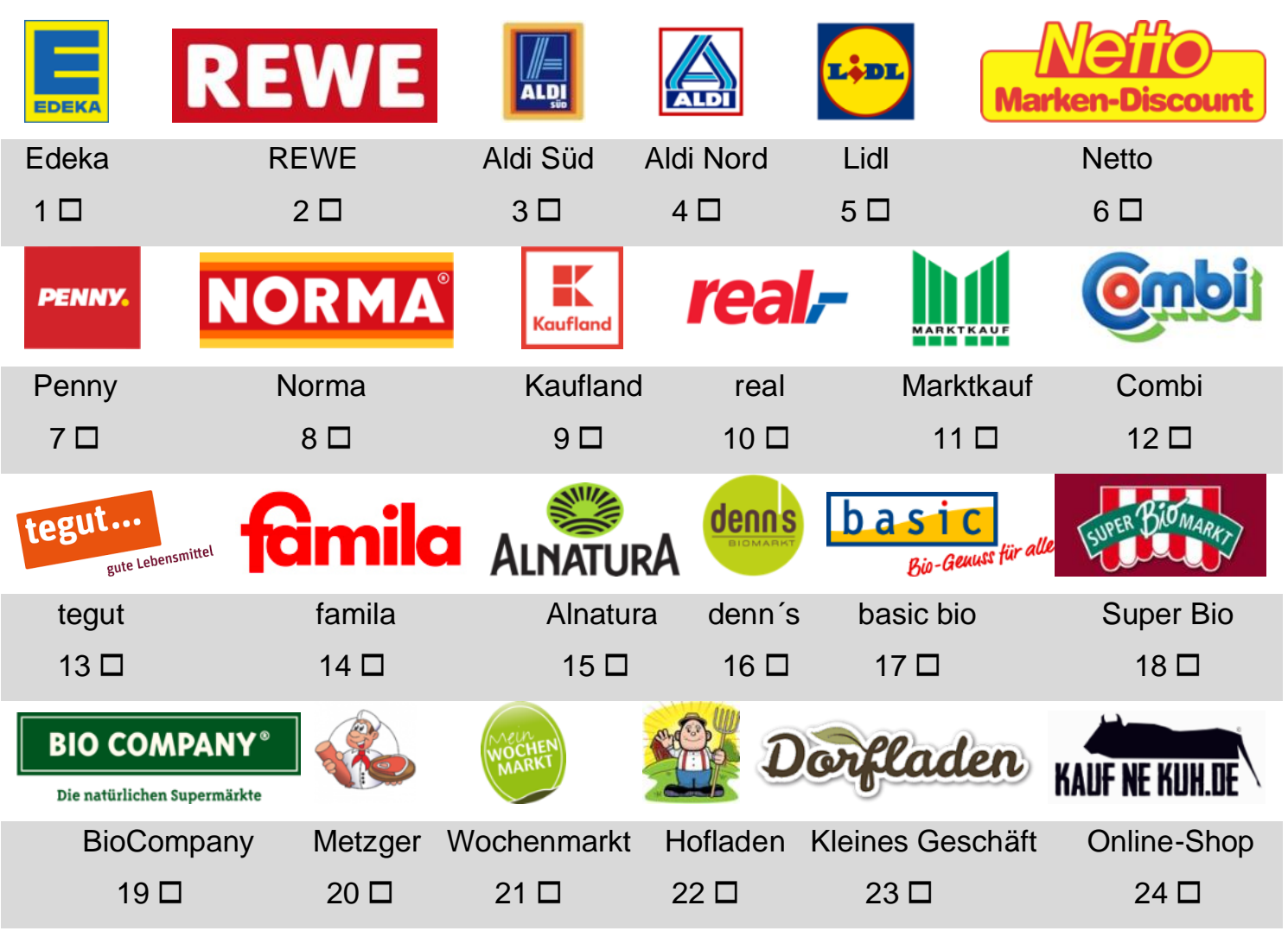

$25 \square$ Andere Einkaufsstätte

Welche andere Einkaufsstätte ist Ihre Haupteinkaufsstätte für Ihren Fleisch- und Wursteinkauf?

4. Kaufen Sie Wurstwaren oder Aufschnitt fürs Brot häufiger beim Verkaufspersonal an der Fleischtheke oder im Selbstbedienungsbereich?

$\begin{array}{ccccc}\text { fast nur an der } & \text { häufiger an der } & \text { ca. gleich viel } & \text { häufiger im SB- } & \text { fast nur im } \\ \text { Fleischtheke } & \text { Fleischtheke } & \text { Bereich } & \text { SB-Bereich } \\ +2 \square & +1 \square & 0 \square & -1 \square & -2 \square\end{array}$

5. Kaufen Sie Fleisch zum Braten oder Grillen häufiger beim Verkaufspersonal an der Fleischtheke oder im Selbstbedienungsbereich?

$\begin{array}{ccccc}\text { fast nur an der } & \text { häufiger an der } & \text { ca. gleich viel } & \text { häufiger im SB- } & \text { fast nur im } \\ \text { Fleischtheke } & \text { Fleischtheke } & \text { Bereich } & \text { SB-Bereich } \\ +2 \square & +1 \square & 0 \square & -1 \square & -2 \square\end{array}$


6. Wie oft werden in Ihrem Haushalt Fleisch- oder Wurstwaren gegessen (auch in Fertiggerichten wie Salami-Pizza etc.)?
$2 \square$ mehrmals täglich
$1 \square$ etwa einmal täglich
$0 \square$ ca. 3-5 mal wöchentlich
$-1 \square$ ca. 1-2 mal wöchentlich
$-2 \square$ weniger als 1 mal wöchentlich

\section{Welche der folgenden Aussagen trifft am ehesten auf Sie zu?}

$5 \square$ Ich esse wie eh und je gerne Fleisch und plane nicht meinen Fleischkonsum zu reduzieren

$4 \square$ Ich esse zwar gerne Fleisch, plane aber in Zukunft etwas weniger Fleisch zu essen

$3 \square$ Ich esse schon weniger Fleisch, möchte aber nicht ganz auf Fleisch verzichten

$2 \square$ Ich esse zwar noch Fleisch, aber ich würde gerne Vegetarier oder Veganer werden

$1 \square$ Ich bin Vegetarier oder Veganer

8. Worauf achten Sie persönlich beim alltäglichen Einkauf von Fleisch- oder Wurstwaren? (Anm.: Vegetarier/Veganer wurden nicht nach Geschmack gefragt)

\begin{tabular}{lccccc} 
Auf... & $\begin{array}{c}\text { achte } \\
\text { ich fast } \\
\text { immer }\end{array}$ & $\begin{array}{c}\text { achte } \\
\text { ich des } \\
\text { Öfteren }\end{array}$ & $\begin{array}{c}\text { achte ich } \\
\text { ab und zu }\end{array}$ & $\begin{array}{c}\text { achte ich } \\
\text { kaum }\end{array}$ & $\begin{array}{c}\text { achte ich } \\
\text { nicht }\end{array}$ \\
$\begin{array}{l}\text { das Aussehen der } \\
\text { Ware }\end{array}$ & $+2 \square$ & $+1 \square$ & $0 \square$ & $-1 \square$ & $-2 \square$ \\
bestimmte Marken & $+2 \square$ & $+1 \square$ & $0 \square$ & $-1 \square$ & $-2 \square$ \\
$\begin{array}{l}\text { den Geschmack (aus } \\
\text { Erfahrung) }\end{array}$ & $+2 \square$ & $+1 \square$ & $0 \square$ & $-1 \square$ & $-2 \square$ \\
\hline Umweltfreundlichkeit & $+2 \square$ & $+1 \square$ & $0 \square$ & $-1 \square$ & $-2 \square$ \\
bestimmte Gütesiegel & $+2 \square$ & $+1 \square$ & $0 \square$ & $-1 \square$ & $-2 \square$ \\
faire Löhne für Landwirte & $+2 \square$ & $+1 \square$ & $0 \square$ & $-1 \square$ & $-2 \square$ \\
Sonderangebot/günstigster & $+2 \square$ & $+1 \square$ & $0 \square$ & $-1 \square$ & $-2 \square$ \\
Preis & $+2 \square$ & $+1 \square$ & $0 \square$ & $-1 \square$ & $-2 \square$ \\
Gesundheitsaspekte & $+2 \square$ & $+1 \square$ & $0 \square$ & $-1 \square$ & $-2 \square$ \\
\hline eine artgerechtere Tierhaltung & $+2 \square$ & $+1 \square$ & $0 \square$ & $-1 \square$ & $-2 \square$ \\
die Frische der Ware & $+2 \square$ & $-1 \square$ \\
eine ansprechende & $+2 \square$ & $+1 \square$ & $0 \square$ & $-1 \square$ & $-2 \square$ \\
Verpackung & $+2 \square$ & $+1 \square$ & $0 \square$ & $-1 \square$ & $-2 \square$ \\
\hline Bio-Qualität & $+2 \square$ & $+1 \square$ & $0 \square$ & $-1 \square$ & $-2 \square$ \\
\hline die regionale Herkunft & & & &
\end{tabular}

$\square$ Andere Aspekte

Auf welche anderen Aspekte achten Sie beim alltäglichen Einkauf von Fleisch- oder Wurstwaren? 
9. Wie häufig kaufen Sie folgende Fleisch- oder Wurstwaren?

\begin{tabular}{|c|c|c|c|c|c|}
\hline & $\begin{array}{c}\text { Mehrmals } \\
\text { pro } \\
\text { Woche }\end{array}$ & $\begin{array}{l}\text { ca. einmal } \\
\text { pro Woche }\end{array}$ & $\begin{array}{l}\text { alle 2-4 } \\
\text { Wochen }\end{array}$ & $\begin{array}{l}\text { alle } 2-6 \\
\text { Monate }\end{array}$ & $\begin{array}{c}\text { fast } \\
\text { nie / } \\
\text { nie }\end{array}$ \\
\hline Frisches Geflügelfleisch wie Hähnchen & $+2 \square$ & $+1 \square$ & $0 \square$ & $-1 \square$ & $-2 \square$ \\
\hline Frisches Rindfleisch wie Steak & $+2 \square$ & $+1 \square$ & $0 \square$ & $-1 \square$ & $-2 \square$ \\
\hline Frisches Schweinefleisch wie Schnitzel & $+2 \square$ & $+1 \square$ & $0 \square$ & $-1 \square$ & $-2 \square$ \\
\hline $\begin{array}{l}\text { Wurstwaren wie Mett, Salami oder } \\
\text { Bratwurst }\end{array}$ & $+2 \square$ & $+1 \square$ & $0 \square$ & $-1 \square$ & $-2 \square$ \\
\hline Aufschnitt als Brotbelag wie Schinken & $+2 \square$ & $+1 \square$ & $0 \square$ & $-1 \square$ & $-2 \square$ \\
\hline $\begin{array}{l}\text { Tiefgekühlte Fleischwaren wie } \\
\text { Hähnchen }\end{array}$ & $+2 \square$ & $+1 \square$ & $0 \square$ & $-1 \square$ & $-2 \square$ \\
\hline Tiefkühl-Gerichte wie Salami-Pizza & $+2 \square$ & $+1 \square$ & $0 \square$ & $-1 \square$ & $-2 \square$ \\
\hline $\begin{array}{l}\text { Dosen-, Tüten-/ Glaswaren wie } \\
\text { Bockwurst }\end{array}$ & $+2 \square$ & $+1 \square$ & $0 \square$ & $-1 \square$ & $-2 \square$ \\
\hline $\begin{array}{l}\text { Fleisch-/ Wurst-Snacks wie Mini- } \\
\text { Salami }\end{array}$ & $+2 \square$ & $+1 \square$ & $0 \square$ & $-1 \square$ & $-2 \square$ \\
\hline
\end{tabular}

$\square$ Andere Fleisch- oder Wurstwaren

Welche anderen Fleisch- oder Wurstwaren kaufen Sie regelmäßig?

B. Im folgenden Abschnitt werden Ihnen Fragen zu Gütesiegeln gestellt, die Ihnen beim Erkennen von Fleisch- oder Wurstwaren aus einer artgerechteren Haltung helfen sollen.

10. Bitte kreuzen Sie alle Gütesiegel an, die Sie schon einmal gesehen haben?
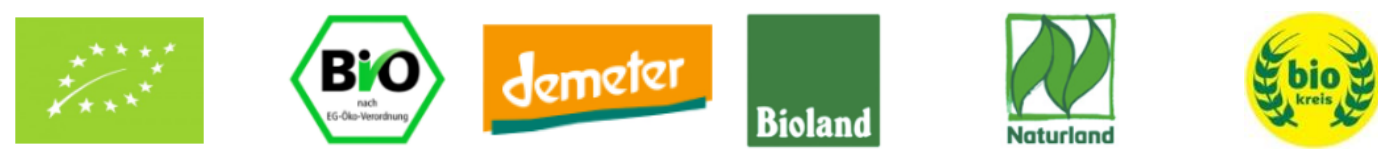

$\square$ EU-Bio $\quad \square$ DE-Bio $\square$ DEMETER $\square$ Bioland $\quad \square$ NATURLAND $\square$ Biokreis
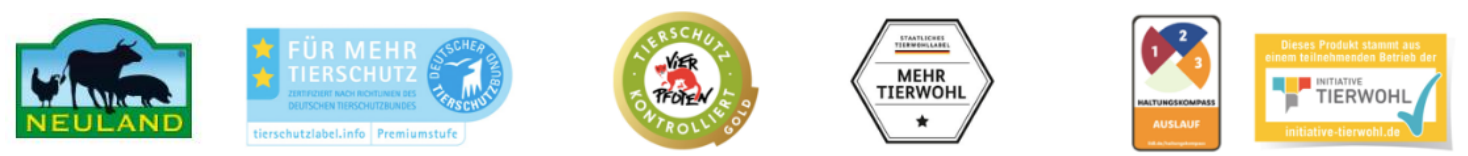

Neuland

Tierschutzbund

$\square$ Vier Pfoten
Landwirtschafts ministerium

Fleisch-

Vielleicht habe ich diese Gütesiegel schon gesehen, sie aber nicht bewusst wahrgenommen $\square$ Ich habe noch keins dieser Gütesiegel gesehen 
11. Welche dieser Gütesiegel haben Sie schon mal beim Fleisch- oder Wurstwarenkauf beachtet bzw. damit gekennzeichnete Produkte gekauft? Sie können mehrere Gütesiegel auswählen. (Anm.: Nur Gütesiegel gezeigt, die in F10 angegeben wurden)
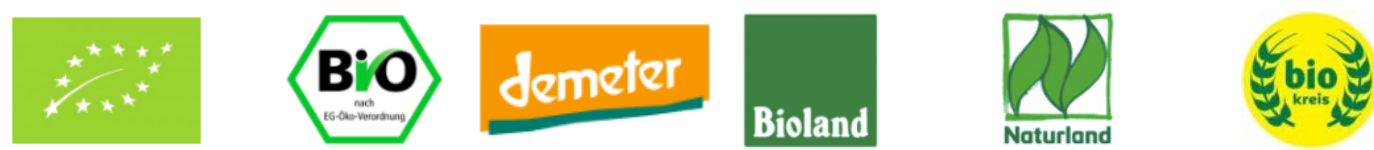

$\square$ EU-Bio $\quad \square$ DE-Bio $\quad \square$ DEMETER $\quad \square$ Bioland $\quad \square$ NATURLAND $\square$ Biokreis

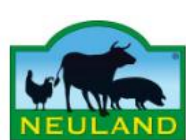

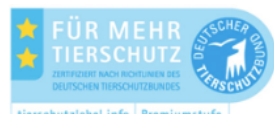

$\square$ Tierschutzbund

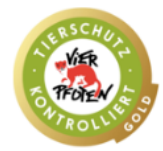

Neuland

$\square$ Vier Pfoten

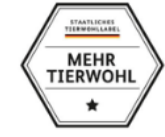

Landwirtschafts ministerium
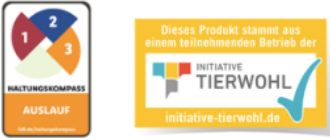

Vielleicht habe ich Produkte mit diesen Gütesiegeln gekauft, aber nicht bewusst darauf geachtet $\square$ Ich habe noch keine Fleisch- oder Wurstwaren mit einem dieser Gütesiegel gekauft

12. Wie häufig kaufen Sie Fleisch- oder Wurstwaren, die mit diesen Gütesiegeln gekennzeichnet sind? (Anm.: Frage nur gezeigt, wenn mind. 1 Gütesiegel in F11)

$2 \square$ Meistens / So gut wie immer

$1 \square$ Wenn möglich, versuche ich diese Produkte zu kaufen

$0 \square$ Gelegentlich / Ab und zu

$-1 \square$ Nur zu besonderen Anlässen

$-2 \square$ Sehr selten

13. Warum haben Sie noch keine Fleisch- oder Wurstwaren mit diesen Gütesiegeln gekauft? Sie können mehrere Gründe angeben. (Anm.: Frage nur gezeigt, wenn kein Gütesiegel in F11)

Dort, wo ich normalerweise einkaufe, sind mir diese Produkte noch nicht aufgefallen

Der Preisunterschied zum normalen Fleisch ist mir zu hoch

Ich bin mir nicht sicher, ob der höhere Preis wirklich den Tieren zu Gute kommt

In der Werbung habe ich noch nie ein Produkt mit diesen Gütesiegeln gesehen

Diese Gütesiegel sind mir nicht wichtig

$\square$ Mir ist nicht bekannt, was diese Gütesiegel bedeuten

Ich vertraue da auf meinen Metzger

Um diese Gütesiegel zu beurteilen, brauche ich mehr Informationen zu den genauen Kriterien

Ich habe bereits nach diesen Produkten gesucht, sie aber nicht gefunden

Andere Gründe

Aus welchen anderen Gründen haben Sie noch keine Fleisch- oder Wurstwaren mit diesen Gütesiegeln gekauft? 
14. Bitte schätzen Sie die folgenden Gütesiegel einmal grob hinsichtlich der Strenge ihrer unterschiedlichen Anforderungen ein. Wenn Ihnen die Unterschiede zwischen den Gütesiegeln nicht bekannt sind, folgen Sie bitte einfach Ihrem Bauchgefühl.

\begin{tabular}{|c|c|c|c|c|c|}
\hline & B'O & demeter & Bioland & No & bio \\
\hline EU-Bio & DE-Bio & DEMETER & Bioland & NATURLAND & biokreis \\
\hline$W$ & AURMEHR Z & sines & & 6 & 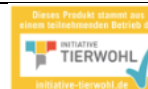 \\
\hline Neuland & Tierschutzbund & Vier Pfoten & $\begin{array}{l}\text { Landwirtschafts- } \\
\text { ministerium }\end{array}$ & Lidl & $\begin{array}{l}\text { Fleisch- } \\
\text { industrie }\end{array}$ \\
\hline
\end{tabular}

Beginnen Sie mit der Einschätzung der Anforderungen an das Wohl der Tiere Ziehen Sie einfach jedes Gütesiegel in den Kasten Ihrer Wahl.

\begin{tabular}{|c|c|c|c|}
\hline Sehr streng +2 & Streng +1 & Weniger streng -1 & Gar nicht streng -2 \\
\hline & & & \\
\hline
\end{tabular}

Schätzen Sie nun die Gütesiegel bezüglich der Umwelt-/Naturschutz-Anforderungen ein. Ziehen Sie wieder einfach jedes Gütesiegel in den Kasten Ihrer Wahl.

\begin{tabular}{|c|c|c|c|}
\hline Sehr streng +2 & Streng +1 & Weniger streng -1 & Gar nicht streng -2 \\
\hline & & & \\
\hline
\end{tabular}

Schätzen Sie abschließend die Anforderungen an die Gesundheit für den Menschen ein. Ziehen Sie wieder einfach jedes Gütesiegel in den Kasten Ihrer Wahl.

\begin{tabular}{|c|c|c|c|}
\hline Sehr streng +2 & Streng +1 & Weniger streng -1 & Gar nicht streng -2 \\
\hline & & & \\
\hline
\end{tabular}

15. Hier sehen Sie noch einmal abschließend alle Gütesiegel, die für eine artgerechtere Nutztierhaltung stehen. Welche der Gütesiegel würden Sie beim Kauf von Fleischund Wurstwaren aus einer artgerechteren Nutztierhaltung berücksichtigen und welche nicht?

\begin{tabular}{|c|c|c|c|c|c|}
\hline$\ldots$ & B'O & demeter & Bioland & Naturand & bioe \\
\hline EU-1 & DE-Bio & DEMETER & Bioland & NATURLAND & biokreis \\
\hline WEVE & * FuR MEHR & sisents & 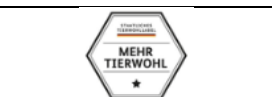 & 6 & 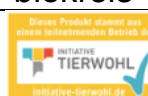 \\
\hline Neuland & Tierschutzbund & Vier Pfoten & $\begin{array}{l}\text { Landwirtschafts- } \\
\text { ministerium }\end{array}$ & Lidl & $\begin{array}{l}\text { Fleisch- } \\
\text { industrie }\end{array}$ \\
\hline
\end{tabular}

Ziehen Sie bitte jedes Gütesiegel in ein Feld Ihrer Wahl.

\begin{tabular}{|c|c|c|c|c|}
\hline Würde ich auf & Würde ich & Bin & Würde ich & Würde ich auf \\
jeden Fall & bestimmt mal & noch & eher nicht & keinen Fall \\
berücksichtigen & berücksichtigen & unentschlossen & berücksichtigen & berücksichtigen \\
+2 & +1 & 0 & -1 & -2 \\
\hline
\end{tabular}


16. Bitte kreuzen Sie nun alle für Sie richtig erscheinenden Aussagen an: „Der übermäßige Verzehr bzw. die hohe Produktion von Fleisch- und Wurstwaren hat vermutlich negative Auswirkungen auf die...

$\square$ Industrie-/ Handelsunternehmen, die mit der Fleischvermarktung Geld verdienen."

$\square$ Qualität unseres (Trink-) Wassers, Luft und Böden.“

Menschen in Dritte-Welt-Ländern."

$\square$ Gesundheit der Menschen in Deutschland."

$\square$ Artenvielfalt in der Natur in Deutschland und in anderen Ländern."

$\square$ Tiere, die für die Produktion von Fleisch- und Wurstwaren gehalten werden."

$\square$ Anzahl der Arbeitsplätze in Deutschland."

$\square$ Höhe des Steueraufkommens in Deutschland."

$\square$ globale Erwärmung (Klimawandel)."

$\square$ Umwelt- und Gesundheitskosten (sehr hohe Kosten)."

$\square$ Landwirte."

Der übermäßige Verzehr bzw. die hohe Produktion von Fleisch- und Wurstwaren hat vermutlich keine negativen Einflüsse. 
17. Stellen Sie sich vor, Sie würden eines der hier gezeigten Produkte kaufen. Welche dieser weiteren Gütesiegel oder Logos könnten sich positiv auf Ihre Kaufentscheidung auswirken? Sie können mehrere Gütesiegel / Logos auswählen.
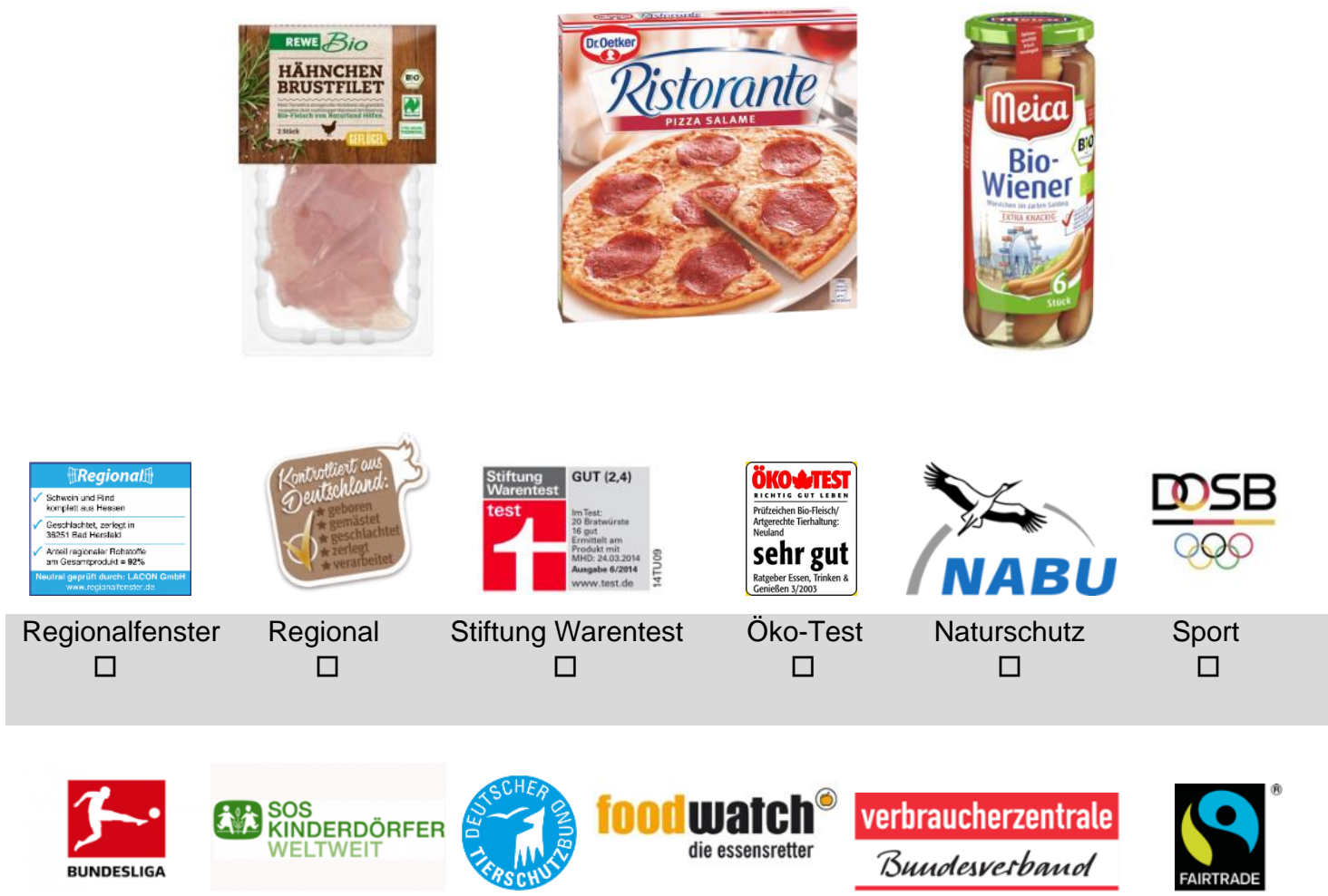

Sportliga Soziales Tierschutz Verbraucherschutz Verbraucherzentrale Fairer Handel 1

$\square$

$\square$

$\square$

$\square$

$\square$
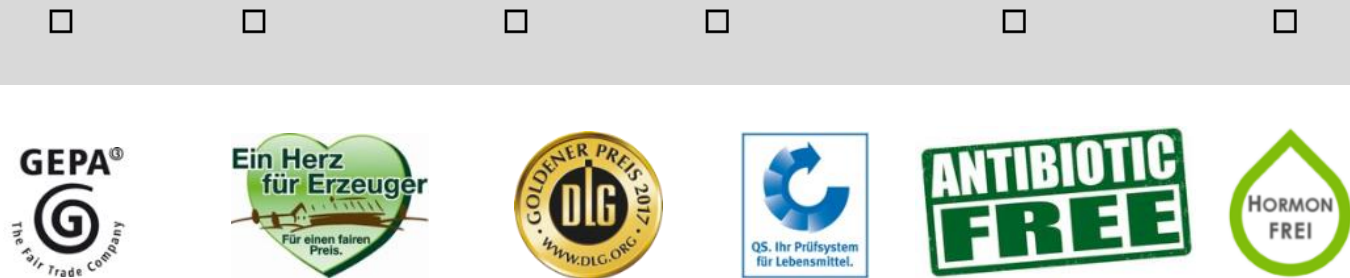

Fairer Handel 2 Fairer Handel 3

Landwirtschaft QS-Siegel

Antibiotikafrei

Hormonfrei

$\square$

$\square$

$\square$

$\square$

$\square$

$\square$
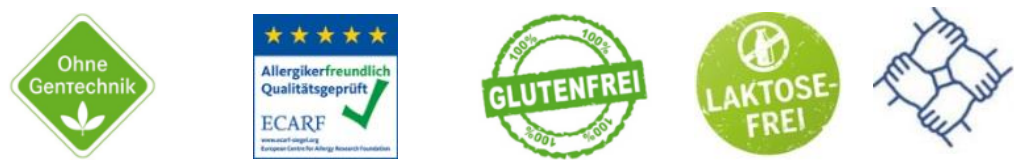

GEmeinsam gegen MRSA

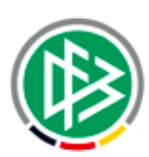

Gentechnikfrei Allergikerfreundlich Glutenfrei

Laktosefrei

Frei von

Sportverband

antibiotikaresistenten

Keimen (MRSA/ESBL)

$\square$

$\square$

$\square$

$\square$

$\square$

Welche anderen Gütesiegel oder Logos könnten sich positiv auf Ihre Kaufentscheidung auswirken? 
18. Angenommen Fleisch- und Wurstwaren aus einer artgerechteren Tierhaltung mit einer vertrauenswürdigen Kennzeichnung wären für Sie gut verfügbar und kaum teurer als herkömmliche Produkte. Könnten Sie sich vorstellen, ab und zu diese Produkte zu kaufen?

$2 \square \mathrm{Ja}$, das kann ich mir sehr gut vorstellen

$1 \square \mathrm{Ja}$, diese Produkte würde ich wahrscheinlich zumindest gelegentlich kaufen

$0 \square$ Das weiß ich nicht

$-1 \square$ Nein, wahrscheinlich würde ich diese Produkte eher nicht kaufen

$-2 \square$ Ich denke, dass ich diese Produkte sicher nicht kaufen werde

19. Wie zufrieden sind Sie mit dem Produkt- \& Beratungsangebot für Fleisch- und Wurstwaren aus einer artgerechteren Tierhaltung in Ihrer Haupteinkaufsstätte.

\begin{tabular}{|c|c|c|c|c|c|c|}
\hline ...bin ich & $\begin{array}{l}\text { rundum } \\
\text { zufrieden }\end{array}$ & $\begin{array}{c}\text { recht } \\
\text { zufrieden }\end{array}$ & $\begin{array}{l}\text { teils } \\
\text { teils }\end{array}$ & $\begin{array}{l}\text { weniger } \\
\text { zufrieden }\end{array}$ & $\begin{array}{l}\text { nicht } \\
\text { zufrieden }\end{array}$ & $\begin{array}{l}\text { Kann ich } \\
\text { nicht } \\
\text { beurteilen }\end{array}$ \\
\hline $\begin{array}{l}\text {...Angebotsvielfalt solcher } \\
\text { Produkte }\end{array}$ & $+2 \square$ & $+1 \square$ & $0 \square$ & $-1 \square$ & $-2 \square$ & $-77 \square$ \\
\hline $\begin{array}{l}\text {...Auffindbarkeit/Erkennbarke } \\
\text { it im Laden }\end{array}$ & $+2 \square$ & $+1 \square$ & $0 \square$ & $-1 \square$ & $-2 \square$ & $-77 \square$ \\
\hline $\begin{array}{l}\text {..Sichtbarkeit solcher } \\
\text { Produkte in } \\
\text { Werbeprospekten, Plakaten, } \\
\text { Anzeigen }\end{array}$ & $+2 \square$ & $+1 \square$ & $0 \square$ & $-1 \square$ & $-2 \square$ & -77 \\
\hline $\begin{array}{l}\text {...persönlichen Beratung zu } \\
\text { Unterschieden einzelner } \\
\text { Haltungsformen/Gütesiegel }\end{array}$ & $+2 \square$ & $+1 \square$ & $0 \square$ & $-1 \square$ & $-2 \square$ & $-77 \square$ \\
\hline $\begin{array}{l}\text {...Auffindbarkeit und } \\
\text { Erkennbarkeit im Online- } \\
\text { Shop (falls } \\
\text { vorhanden/genutzt) }\end{array}$ & $+2 \square$ & $+1 \square$ & $0 \square$ & $-1 \square$ & $-2 \square$ & $-77 \square$ \\
\hline $\begin{array}{l}\text {...Transparenz beim } \\
\text { Engagement für eine } \\
\text { artgerechtere Tierhaltung }\end{array}$ & $+2 \square$ & $+1 \square$ & $0 \square$ & $-1 \square$ & $-2 \square$ & -77 \\
\hline $\begin{array}{l}\text {...Ästhetik der SB- } \\
\text { Verpackungen, Fleischtheken } \\
\text { oder Werbeprospekte }\end{array}$ & $+2 \square$ & $+1 \square$ & $0 \square$ & $-1 \square$ & $-2 \square$ & $-77 \square$ \\
\hline
\end{tabular}

20. Sie stehen in einem Laden vor einem Kühlregal, einer Kühltruhe oder einer Fleischtheke mit verschiedenen Fleisch- und Wurstwaren. Welche Sortierung würden Sie sich persönlich wünschen? Wählen Sie bitte eine der zwei angebotenen Alternativen aus.

$1 \square$ Eigener Bereich (z.B. eigenes Regal) für alle Produkte mit sehr hohen Tierwohl-Standards

$2 \square$ Sortierung nach Produktart (z.B. Salami) - herkömmliche Produkte und mit Gütesiegeln gekennzeichnete Fleischwaren einer Produktart liegen direkt nebeneinander 
21. Stellen Sie sich vor, viele Anbieter von Fleisch- und Wurstwaren verbessern zukünftig Ihr Produkt- und Beratungsangebot zum Thema ,artgerechtere Tierhaltung“. Würden Sie Ihre Haupteinkaufsstätte wechseln, wenn Sie bei Ihrem Anbieter keine Verbesserungen feststellen?

$2 \square \mathrm{Ja}$, dann würde ich sicher wechseln

$1 \square \mathrm{Ja}$, dann würde ich wahrscheinlich wechseln

$0 \square$ Schwer zu sagen, da bin ich unentschlossen

$-1 \square$ Nein, ich würde wahrscheinlich nicht wechseln

$-2 \square$ Nein, ich würde sicher nicht wechseln

C. Viele Verbraucher wünschen sich bessere Informationen zu den Haltungsbedingungen und zur Herkunft der Nutztiere. In diesem Abschnitt können Sie Ihre persönlichen Wünsche äußern.

22. Würden Sie gerne bessere Informationen zu den Haltungsbedingungen der Tiere und zur Herkunft der Fleisch- und Wurstwaren, die Sie kaufen, erhalten?

$1 \square \mathrm{Ja}$, zu diesem Thema sollten bessere Informationen bereitgestellt werden

$2 \square$ Nein, ich habe kein Interesse an Informationen zu diesem Thema

23. Wo würden Sie sich gerne über die Haltungsbedingungen der Nutztiere und die Herkunft der von Ihnen gekauften Fleisch- und Wurstwaren informieren? (Anm.: Frage nur gezeigt, wenn F22 mit Ja beantwortet)

$\begin{array}{ccccc}\text { Trifft voll } & \text { Trifft über- } & \text { teils } & \text { Trifft } & \text { Trifft } \\ \text { und ganz } & \text { wiegend } & \text { teils } & \text { eher } & \text { überhaupt } \\ \text { zu } & \text { zu } & & \text { nicht zu } & \text { nicht zu }\end{array}$

\begin{tabular}{|c|c|c|c|c|c|}
\hline $\begin{array}{l}\text { In den Verkaufsräumen, z.B. } \\
\text { über Broschüren oder Schilder } \\
\text { an Fleischtheke und Kühlregal }\end{array}$ & $+2 \square$ & $+1 \square$ & $0 \square$ & $-1 \square$ & $-2 \square$ \\
\hline $\begin{array}{l}\text { Persönlich beim } \\
\text { Verkaufspersonal, z.B. an der } \\
\text { Fleischtheke }\end{array}$ & $+2 \square$ & $+1 \square$ & $0 \square$ & $-1 \square$ & $-2 \square$ \\
\hline $\begin{array}{l}\text { Auf der Verpackung des } \\
\text { Produkts }\end{array}$ & $+2 \square$ & $+1 \square$ & $0 \square$ & $-1 \square$ & $-2 \square$ \\
\hline $\begin{array}{l}\text { In den kostenlosen } \\
\text { Werbeprospekten }\end{array}$ & $+2 \square$ & $+1 \square$ & $0 \square$ & $-1 \square$ & $-2 \square$ \\
\hline $\begin{array}{l}\text { Auf einem Internetportal mit } \\
\text { Hintergrundinformationen zur } \\
\text { artgerechteren Tierhaltung }\end{array}$ & $+2 \square$ & $+1 \square$ & $0 \square$ & $-1 \square$ & $-2 \square$ \\
\hline $\begin{array}{l}\text { In den Medien, z.B. TV, Radio, } \\
\text { Zeitung. Filme, Bücher }\end{array}$ & $+2 \square$ & $+1 \square$ & $0 \square$ & $-1 \square$ & $-2 \square$ \\
\hline
\end{tabular}


24. Welche der folgenden Institutionen oder Personen sollten weiterführende Informationen zu den Haltungsbedingungen der Tiere im Internet bereitstellen? (Anm.: Frage nur gezeigt, wenn F22 „Ja“ und F23 ,Internetportal“ nicht abgelehnt)
Landwirte / Tierhalter
$\square$ Lebensmittelhersteller oder -verarbeiter
Lebensmitteleinzelhandel
Herausgeber der Gütesiegel
Bundesministerium für Ernährung und Landwirtschaft
$\square$ Europäische Union (EU)
Medien (TV, Radio, Print, Online etc.)
Verbraucherschutzorganisationen
$\square$ Naturschutzorganisationen
$\square$ Tierschutzorganisationen
Verbände der Lebensmittelindustrie
$\square$ Verbände der Landwirte

25. Wäre es für Sie ein Kaufargument, wenn ein Anbieter die Rückverfolgbarkeit von Fleisch- und Wurstwaren bis zurück auf den konkreten Bauernhof, von dem die Tiere stammen, transparent darstellen würde? (Anm.: Frage nur gezeigt, wenn F22 mit Ja beantwortet)

$2 \square$ Ich würde solche Produkte auf jeden Fall bevorzugt kaufen

$1 \square$ Wahrscheinlich würde ich diese Produkte eher kaufen

$0 \square$ Ich bin mir unsicher, ob ich die Herkunft der Produkte beim Kauf berücksichtigen würde

$-1 \square$ Die Angabe der groben Region (z.B. Herkunftsland) reicht mir

$-2 \square$ Herkunft spielt für mich gar keine Rolle

D. Die Entwicklung hin zu einer artgerechteren Nutztierhaltung ist auch aus politischer Perspektive von hoher Relevanz. In diesem Abschnitt werden Ihnen einige ausgewählte Fragen zu diesem Thema gestellt.

26. Es gibt verschiedene Ansätze, die in der Politik diskutiert werden, um das Wohl der Tiere in der Nutztierhaltung und die Kennzeichnung von Fleisch- und Wurstwaren aus einer artgerechteren Haltung zu verbessern. Bitte bringen Sie die folgenden Ansätze in eine für Sie persönlich sinnvolle Reihenfolge (+2 sehr wichtig bis -2 nicht wichtig). Im Hinblick auf die Nutztierhaltung benötigen wir dringend ...

ein deutlich strengeres Tierschutzgesetz mit strengeren Kontrollen und Sanktionen sowie strikten Verboten ethisch nicht vertretbarer Praktiken wie z.B. das Schreddern lebender Küken

eine bundesweit einheitliche und gesetzlich verpflichtende Haltungskennzeichnung für alle Produkte, die tierische Bestandteile enthalten

freiwillige Gütesiegel / Haltungskennzeichnungen, für die sich einzelne Akteure wie Landwirte oder Lebensmitteleinzelhändler frei entscheiden können

eine stärkere staatliche Förderung von tierwohlorientierteren, ökologischeren, sozialeren Betrieben (z.B. durch die bessere Umverteilung von Subventionen)

den Ausbau der freiwilligen „Initiative Tierwohl“ von Fleischindustrie,


27. Welche Form der Haltungskennzeichnung würden Sie persönlich bevorzugen, um beim Fleischkauf die Artgerechtheit der Tierhaltung besser einschätzen zu können? (Anm.: Frage nur gezeigt, wenn F22 mit Ja beantwortet)

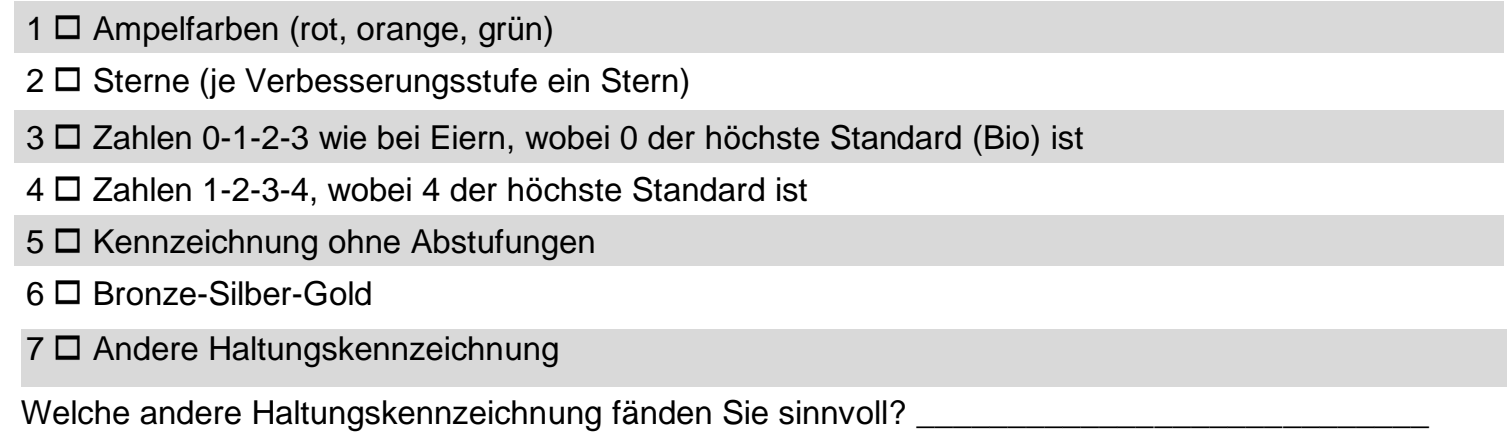

28. Es gibt verschiedene Möglichkeiten, wie sich Menschen für eine verbesserte Tierhaltung einsetzen können. Wie bewerten Sie die aufgeführten Möglichkeiten?

Durch... ...kann ich die Verbesserung der Tierhaltung in Deutschland stark mit evtl. wenig sehr wenig beein- beein- beein- beein- beeinflussen flussen flussen flussen flussen

\begin{tabular}{|c|c|c|c|c|c|}
\hline $\begin{array}{l}\text { mein persönliches Einkaufs- und } \\
\text { Konsumverhalten }\end{array}$ & $+2 \square$ & $+1 \square$ & $0 \square$ & $-1 \square$ & $-2 \square$ \\
\hline die Teilnahme an Petitionen & $+2 \square$ & $+1 \square$ & $0 \square$ & $-1 \square$ & $-2 \square$ \\
\hline $\begin{array}{l}\text { meine Stimmabgabe für eine } \\
\text { Partei bei politischen Wahlen }\end{array}$ & $+2 \square$ & $+1 \square$ & $0 \square$ & $-1 \square$ & $-2 \square$ \\
\hline $\begin{array}{l}\text { die Unterstützung von Tierschutz- } \\
\text { oder Naturschutzorganisationen }\end{array}$ & $+2 \square$ & $+1 \square$ & $0 \square$ & $-1 \square$ & $-2 \square$ \\
\hline $\begin{array}{l}\text { die Teilnahme an } \\
\text { Demonstrationen }\end{array}$ & $+2 \square$ & $+1 \square$ & $0 \square$ & $-1 \square$ & $-2 \square$ \\
\hline $\begin{array}{l}\text { die Unterstützung von } \\
\text { Verbraucherschutzorganisationen }\end{array}$ & $+2 \square$ & $+1 \square$ & $0 \square$ & $-1 \square$ & $-2 \square$ \\
\hline
\end{tabular}

Welche anderen Möglichkeiten, wie sich Menschen für eine verbesserte Tierhaltung in Deutschland einsetzen können, finden Sie sinnvoll?

E. Zum Schluss noch einige Fragen zu Ihrer Person. Alle Angaben in der gesamten Befragung sind anonym.

29. Bitte nennen Sie uns Ihr Geschlecht.
$1 \square$...weiblich
$2 \square . .$. männlich

30. Bitte geben Sie Ihr Alter in Jahren an. __ Jahre 
31. In welchem Bundesland leben Sie?

$1 \square$ Baden-Württemberg
$2 \square$ Bayern
$3 \square$ Berlin
$4 \square$ Brandenburg
$5 \square$ Bremen
$6 \square$ Hamburg
$7 \square$ Hessen
$8 \square$ Mecklenburg-Vorpommern
$9 \square$ Niedersachsen
$10 \square$ Nordrhein-Westfalen
$11 \square$ Rheinland-Pfalz
$12 \square$ Saarland
$13 \square$ Sachsen
$14 \square$ Sachsen-Anhalt
$15 \square$ Schleswig-Holstein
$16 \square$ Thüringen

\section{Wo wohnen Sie (Hauptwohnsitz)?}

$1 \square$ Auf dem Land (Dorf)

$2 \square$ In einer Stadt mit 10.000 Einwohnern oder weniger

$3 \square$ In einer Stadt mit mehr als 10.000 Einwohnern

$4 \square$ In einer Stadt mit mehr als 50.000 Einwohnern

$5 \square$ In einer Stadt mit mehr als 100.000 Einwohnern

$6 \square$ In einer Stadt mit mehr als 200.000 Einwohnern

$7 \square$ In einer Stadt mit mehr als 500.000 Einwohnern

$8 \square$ In einer Stadt mit mehr als 1.000.000 Einwohnern

33. Wenn Sie alle Einkünfte, die Ihrem Haushalt pro Monat für den Lebensunterhalt zur Verfügung stehen, zusammenrechnen: Wie hoch ist das monatliche HaushaltsNettoeinkommen aller Haushaltsmitglieder zusammen nach Abzug von Steuern und Sozialabgaben?

$\begin{array}{llll}1 \square \text { unter } 750 € & 7 \square 2.000 \text { bis unter } 2.250 € & 13 \square 3.500 \text { bis unter } 3.750 € \\ 2 \square 750 \text { bis unter } 1.000 € & 8 \square 2.250 \text { bis unter } 2.500 € & 14 \square 3.750 \text { bis unter } 4.000 € \\ 3 \square 1.000 \text { bis unter } 1.250 € & 9 \square 2.500 \text { bis unter } 2.750 € & 15 \square 4.000 \text { bis unter } 4.250 € \\ 4 \square 1.250 \text { bis unter } 1.500 € & 10 \square 2.750 \text { bis unter } 3.000 € & 16 \square 4.250 \text { bis unter } 4.500 € \\ 5 \square 1.500 \text { bis unter } 1.750 € & 11 \square 3.000 \text { bis unter } 3.250 € & 17 \square 4.500 € \text { und mehr } \\ 6 \square 1.750 \text { bis unter } 2.000 € & 12 \square 3.250 \text { bis unter } 3.500 € & \end{array}$


34. Bitte geben Sie Ihren höchsten Bildungsabschluss an.

$1 \square$ Ohne allgemeinen Bildungsabschluss

$2 \square$ Noch in schulischer Ausbildung (noch ohne Abschluss)

$3 \square$ Volks- oder Hauptschule

$4 \square$ Mittlere Reife / Realschule / Mittelschule

$5 \square$ Allgemeine (Fach-) Hochschulreife / Abitur

$6 \square$ Fachhochschule / Hochschule

$7 \square$ Universität

$8 \square$ Anderer Abschluss

Welchen anderen Bildungsabschluss haben Sie erworben?

35. Wie viele Personen - Sie selbst eingerechnet - leben in Ihrem Haushalt? _Person(-en)

36. Wie viele Kinder unter 14 Jahren leben in Ihrem Haushalt? _ Kind (-er)

37. Wie viele Personen in Ihrem Haushalt sind Vegetarier oder Veganer? _ Person (-en)

38. Wie bzw. mit wem wohnen Sie zusammen (Hauptwohnsitz)? Ich wohne...

$1 \square$ bei meinen Eltern

$2 \square$ mit Partner (-in); Kinder ausgezogen

$3 \square$ mit Partner (-in) und Kindern

$4 \square$ in einer Wohngemeinschaft

$5 \square$ mit Partner (-in); keine Kinder

$6 \square$ mit Kind (-ern); ohne Partner (-in)

$7 \square$ alleine als Single

$8 \square$ alleine; Partner (-in) wohnt woanders

$9 \square$ anders

Wie bzw. mit wem wohnen Sie sonst zusammen (Hauptwohnsitz)?

\section{Haben sie Haustiere? Wenn ja, welche?}

Nein, ich habe kein Haustier

$\square$ Hund

$\square$ Katze

Nagetier (z.B. Maus, Kaninchen, Meerschwein, Hamster, Ratten, Chinchillas, Degus etc.)

$\square$ Fisch (Aquarium oder Teich)

$\square$ Amphibie oder Reptilie (z.B. Frösche, Molche, Schildkröten, Geckos, Schlangen etc.)

Insekt oder Spinne (z.B. Stabheuschrecke, Vogelspinne etc.)

$\square$ Vogel (z.B.Wellensittich, Kanarienvogel etc.)

$\square$ Andere Haustiere

Welche anderen Haustiere haben Sie? 
40. Wenn Sie ein Haustier haben oder in Zukunft vielleicht gerne ein Haustier hätten, würden Sie Tierfutter bevorzugen, wenn die im Tierfutter enthaltenen tierischen Bestandteile aus einer artgerechteren Haltung stammen?

$1 \square \mathrm{Ja}$, das wäre für mich auf jeden Fall ein positives Kaufargument

$2 \square$ Nein, das wäre mir für mein Haustier egal

$3 \square$ Mein Haustier bekommt ohnehin keine Nahrung mit tierischen Bestandteilen

$4 \square$ Ich habe kein Haustier und will auch in Zukunft kein Haustier haben

\section{Welcher Religion gehören Sie an?}

$1 \square$ Christentum römisch-katholisch

$2 \square$ Christentum evangelisch-lutherisch

$3 \square$ Christentum orthodox

$4 \square$ Islam

$5 \square$ Hinduismus

$6 \square$ Buddhismus

$7 \square$ Judentum

$8 \square$ Keine Religionszugehörigkeit

$9 \square$ Andere Religion

Welcher anderen Religion gehören Sie an?

42. Welche Partei haben Sie bei der Bundestagswahl 2017 gewählt?

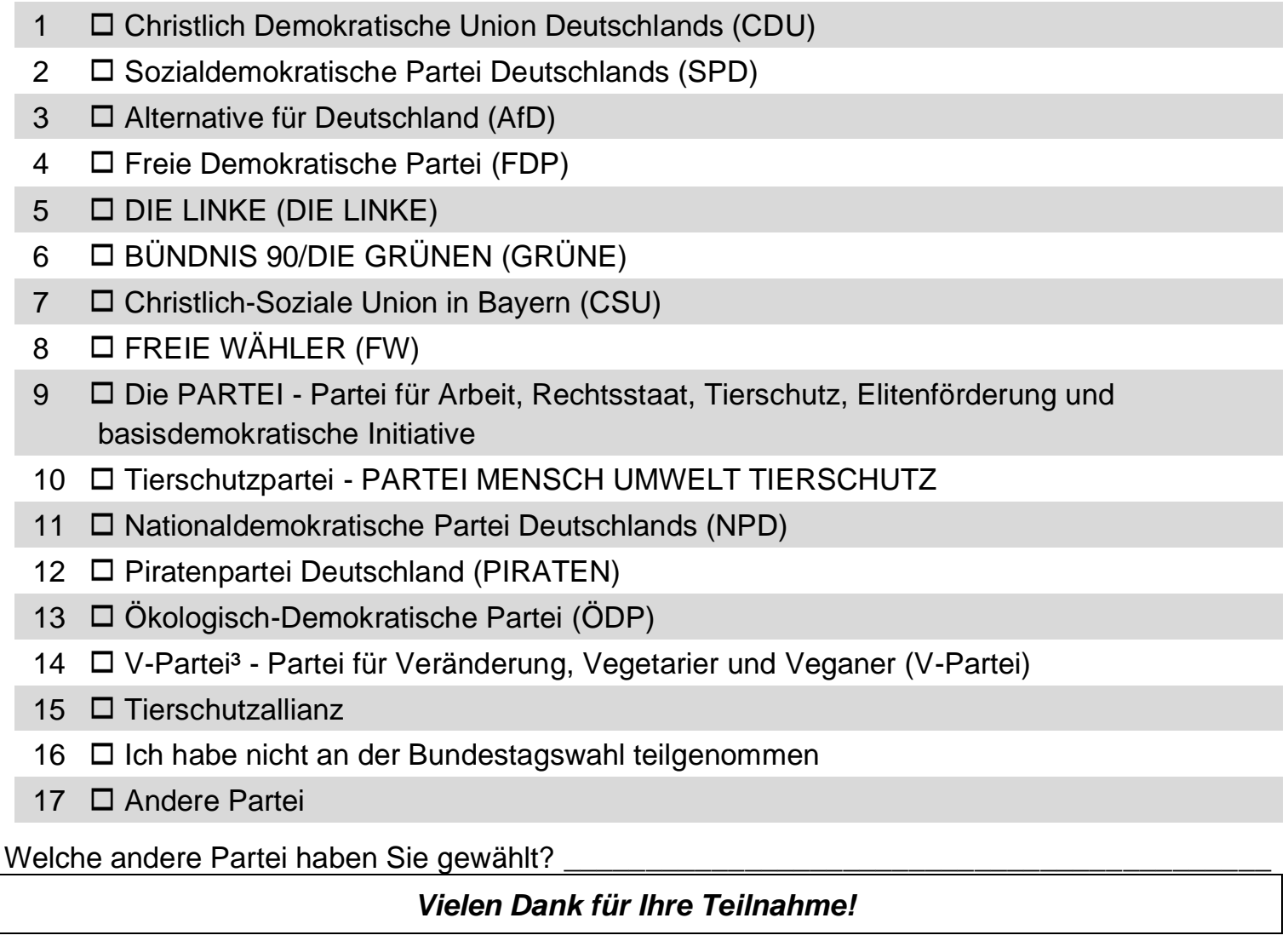




\section{Klassenbildungen und Dichotomisierungen}

- Haupteinkaufsstätten (F3; Haupteinkaufsstätten mit ausreichend N im Logit-Modell als Dummy-Variablen)

- Angebotsform Theke und Selbstbedienung (F4; Top2; Zustimmung 1, Ablehnung 0)

- Konsumhäufigkeit im Haushalt (F6; ca. täglicher Fleischkonsum 2-0=1, relativ selten -1-2=0)

- Reduktionsbereitschaft (F7; normale Fleischesser 4+5 = 1 und Flexitarier 1-3=0)

- Fleischarten und Fleischsorten (F9; Top2; Zustimmung 1, Ablehnung 0)

- Kaufhäufigkeit von Tierwohlprodukten (F12; Top2; Zustimmung 1, Ablehnung 0)

- Strenge von Tierwohl-Labeln (F14; Top2; Zustimmung 1, Ablehnung 0)

- Interesse an Tierwohlprodukten (F18; Top2; Zustimmung 1, Ablehnung 0)

- Kundenzufriedenheit (F19; Top2; Zustimmung 1, Ablehnung 0)

- Wechselbereitschaft (F21; Top2; Zustimmung 1, Ablehnung 0)

- Informationsbedürfnisse (F23; Top2; Zustimmung 1, Ablehnung 0)

- Rückverfolgbarkeit (F25; Top 2 Zustimmung 1, Rest 0)

- Politik-Optionen (F26; Top2; Zustimmung 1, Ablehnung 0)

- Darstellungsvarianten einer gesetzlichen Haltungskennzeichnung (F27; grafische Darstellung oder Zahlenvarianten)

- Politische Partizipation (F28; Top2; bei Zustimmung 1, Ablehnung 0)

- Geschlecht (F29; weiblich 1 und männlich 0)

- Altersklassen (F30; bis 39 jüngere Personen als 0 und ab 40 ältere Personen 3-5=1)

- Ortsgröße (F32; $<10.000=$ auf dem Land und Kleinstädte 1-2=0 und 10.000 und größer 3$8=1)$

- Einkommensklassen (F33; bis unter $3000 €$ niedrigeres Einkommen 1-10=0, ab $3000 €$ höheres Einkommen 11-17=1)

- Bildungsklassen (F34; bis unter Abitur niedrigere Bildung 1-4=0 und Abitur oder Studium höhere Bildung 5-7=1 höher)

- Haustierbesitz (F39; ja/nein), die Religion (F41; ja/nein)

- Parteipräferenz (F42; als konservative Parteien CDU, SPD, AFD sowie FDP und als linksgrüne Parteien Die GRÜNEN und DIE LINKEN, da diese Parteien ausreichend Wähler für die statistische Auswertung vorweisen konnten) 


\section{Anhang B: Soziodemografie}

\begin{tabular}{|l|l|l|l|l|}
\hline \multicolumn{4}{|l|}{ F29:Bitte nennen Sie uns Ihr Geschlecht. } \\
\hline weiblich & 491 & 52,8 & 930 & \\
\hline männlich & 439 & 47,2 & \\
\hline Keine Angabe & 0 & & \\
\hline
\end{tabular}

F30:Bitte geben Sie Ihr Alter in Jahren an.

\begin{tabular}{|l|c|l|l|}
\hline $18-29$ & 175 & 18,8 & 930 \\
\hline $30-39$ & 160 & 17,2 & \\
\hline $40-49$ & 203 & 21,8 & \\
\hline $50-59$ & 237 & 25,5 & \\
$60-69$ & 155 & 16,7 & \\
\hline Keine Angabe & 0 & & \\
\hline
\end{tabular}

\begin{tabular}{|c|c|c|c|c|}
\hline \multicolumn{5}{|c|}{ F31: In welchem Bundesland leben Sie? } \\
\hline Baden-Württemberg & 125 & 13,4 & 930 & \\
\hline Bayern & 158 & 17,0 & & \\
\hline Berlin & 36 & 3,9 & & \\
\hline Brandenburg & 25 & 2,7 & & \\
\hline Bremen & 7 & 0,8 & & \\
\hline Hamburg & 17 & 1,8 & & \\
\hline Hessen & 73 & 7,8 & & \\
\hline Mecklenburg-Vorpommern & 17 & 1,8 & & \\
\hline Niedersachsen & 94 & 10,1 & & \\
\hline Nordrhein-Westfalen & 213 & 22,9 & & \\
\hline Rheinland-Pfalz & 52 & 5,6 & & \\
\hline Saarland & 10 & 1,1 & & \\
\hline Sachsen & 32 & 3,4 & & \\
\hline Sachsen-Anhalt & 24 & 2,6 & & \\
\hline Schleswig-Holstein & 28 & 3,0 & & \\
\hline Thüringen & 19 & 2,0 & & \\
\hline Keine Angabe & 0 & & & \\
\hline
\end{tabular}




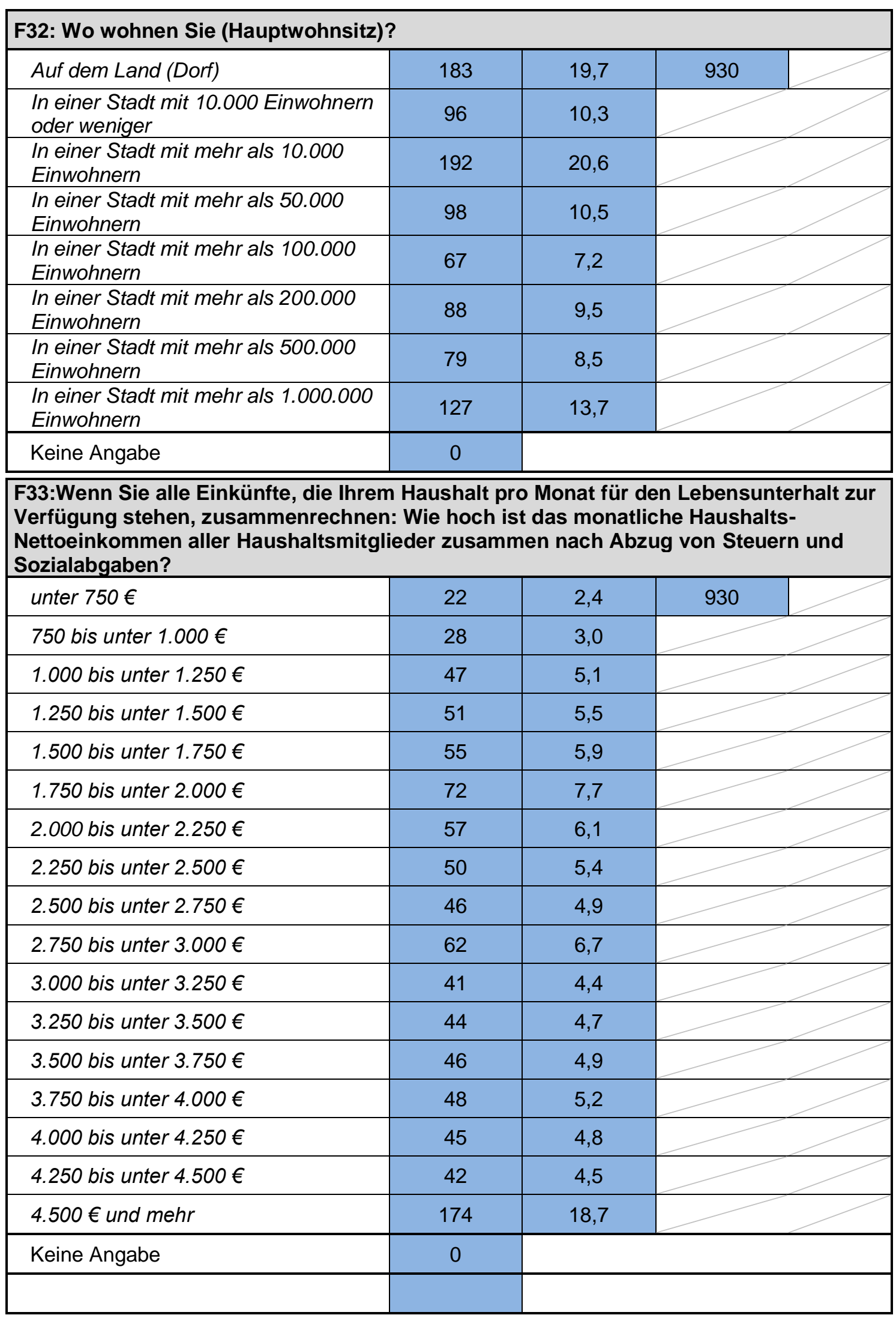




\begin{tabular}{|l|c|c|c|}
\hline \multicolumn{4}{|l|}{ F34:Bitte geben Sie Ihren höchsten Bildungsabschluss an. } \\
\hline Ohne allgemeinen Bildungsabschluss & 7 & 0,8 & 930 \\
\hline Noch in schulischer Ausbildung & 7 & 0,8 & \\
\hline Volks- oder Hauptschule & 236 & 25,4 \\
\hline $\begin{array}{l}\text { Mittlere Reife / Realschule / } \\
\text { Mittelschule }\end{array}$ & 318 & 34,2 & \\
\hline $\begin{array}{l}\text { Allgemeine (Fach-) Hochschulreife / } \\
\text { Abitur }\end{array}$ & 146 & 15,7 & \\
\hline Fachhochschule / Hochschule & 96 & 10,3 & \\
\hline Universität & 120 & 12,9 & \\
\hline Anderer Abschluss & 0 & 0,0 & \\
\hline Keine Angabe & 0 & \\
\hline
\end{tabular}

\begin{tabular}{|l|c|c|c|}
\hline F35:Wie viele Personen - Sie selbst eingerechnet - leben in Ihrem Haushalt? \\
\hline 1 Person & 149 & 16,0 & 930 \\
\hline 2 Personen & 399 & 42,9 & \\
\hline 3 Personen & 192 & 20,6 & \\
\hline 4 Personen & 138 & 14,8 & \\
\hline 5 Personen & 36 & 3,9 & \\
\hline 6 Personen & 10 & 1,1 & \\
\hline mehr als 6 Personen & 6 & 0,2 & \\
\hline Keine Angabe & 0 & & \\
\hline
\end{tabular}

\begin{tabular}{|l|c|c|c|}
\hline \multicolumn{4}{|l|}{ F36: Wie viele Kinder unter 14 Jahren leben in Ihrem Haushalt? } \\
\hline O Kinder & 574 & 61,7 & 930 \\
\hline 1 Kind & 130 & 14,0 & \\
\hline 2 Kinder & 67 & 7,2 & \\
\hline 3 Kinder & 8 & 0,9 & \\
\hline 4 Kinder & 2 & 0,2 & \\
\hline Keine Angabe & 149 & & \\
\hline
\end{tabular}


F37: Wie viele Personen in Ihrem Haushalt sind Vegetarier oder Veganer? (Programmierfehler von Respondi, daher unbrauchbar)

\begin{tabular}{|l|c|c|c|}
\hline 0 Vegetarier/Veganer & 576 & 61,9 & 930 \\
\hline 1 Vegetarier/Veganer & 324 & 34,8 & \\
\hline 2 Vegetarier/Veganer & 23 & 2,5 & \\
\hline 3 Vegetarier/Veganer & 4 & 0,4 & \\
\hline 4 Vegetarier/Veganer & 1 & 0,1 & \\
\hline 5 Vegetarier/Veganer & 2 & 0,2 & \\
\hline Keine Angabe & 0 & & \\
\hline
\end{tabular}

F38: Wie bzw. mit wem wohnen Sie zusammen (Hauptwohnsitz)? Ich wohne...

\begin{tabular}{|l|c|c|c|}
\hline bei meinen Eltern & 54 & 5,8 & 930 \\
\hline $\begin{array}{l}\text { mit Partner (-in); Kind (-er) } \\
\text { ausgezogen }\end{array}$ & 149 & 16,0 \\
\hline mit Partner (-in) und Kind (-ern) & 269 & 28,9 & \\
\hline in einer Wohngemeinschaft & 25 & 2,7 \\
\hline mit Partner (-in); keine Kinder & 229 & 24,6 \\
\hline mit Kind (-ern) ohne Partner (-in) & 37 & 4,0 & \\
\hline alleine als Single & 140 & 15,1 & \\
\hline alleine; Partner (-in) wohnt woanders & 19 & 2,0 & \\
\hline anders & 8 & 0,9 & \\
\hline Keine Angabe & 0 & & \\
\hline Wie bzw. mit wem wohnen Sie zusammen?
\end{tabular}

\begin{tabular}{|l|c|c|c|}
\hline \multicolumn{3}{|l|}{ F39: Haben sie Haustiere? Wenn ja, welche? } \\
\hline Nein, ich habe kein Haustier & 443 & 47,6 & 930 \\
\hline Hund & 213 & 22,9 \\
\hline Katze & 258 & 27,7 & \\
\hline $\begin{array}{l}\text { Nagetier (z.B. Maus, Kaninchen, } \\
\text { Meerschwein, Hamster, Ratten, } \\
\text { Chinchillas, Degus etc.) }\end{array}$ & 60 & 6,5 & \\
\hline Fisch (Aquarium oder Teich) & 71 & 7,6 & \\
\hline $\begin{array}{l}\text { Amphibie oder Reptilie (z.B. Frösche, } \\
\text { Molche, Schildkröten, Geckos, } \\
\text { Schlangen etc.) }\end{array}$ & 23 & 2,5 \\
\hline
\end{tabular}




\begin{tabular}{|l|c|c|}
\hline $\begin{array}{l}\text { Insekt oder Spinne (z.B. } \\
\text { Stabheuschrecke, Vogelspinne etc.) }\end{array}$ & 7 & 0,8 \\
\hline $\begin{array}{l}\text { Vogel (z.B.Wellensittich, Kanarienvogel } \\
\text { etc.) }\end{array}$ & 32 & 3,4 \\
\hline Andere Haustiere & 21 & 2,3 \\
\hline Keine Angabe & 0 & \\
\hline Welche anderen Haustiere haben Sie?
\end{tabular}

\begin{tabular}{|l|c|c|c|}
\hline $\begin{array}{l}\text { F40: Wenn Sie ein Haustier haben oder in Zukunft vielleicht gerne ein Haustier hätten, } \\
\text { würden Sie Tierfutter bevorzugen, wenn die im Tierfutter enthaltenen tierischen } \\
\text { Bestandteile aus einer artgerechteren Haltung stammen? }\end{array}$ \\
\hline $\begin{array}{l}\text { Ja, das wäre für mich auf jeden Fall } \\
\text { ein positives Kaufargument }\end{array}$ & 569 & 61,2 & 930 \\
\hline $\begin{array}{l}\text { Nein, das wäre mir für mein Haustier } \\
\text { egal }\end{array}$ & 109 & 11,7 & \\
\hline $\begin{array}{l}\text { Mein Haustier bekommt ohnehin } \\
\text { keine Nahrung mit tierischen } \\
\text { Bestandteilen }\end{array}$ & 39 & 4,2 & \\
\hline $\begin{array}{l}\text { Ich habe kein Haustier und will auch } \\
\text { in Zukunft kein Haustier haben }\end{array}$ & 213 & 22,9 & \\
\hline Keine Angabe & 0 & \\
\hline
\end{tabular}

\begin{tabular}{|l|c|c|c|}
\hline \multicolumn{4}{|l|}{ F41:Welcher Religion gehören Sie an? } \\
\hline Christentum römisch-katholisch & 253 & 27,2 & 930 \\
\hline Christentum evangelisch-lutherisch & 229 & 24,6 & \\
\hline Christentum orthodox & 14 & 1,5 & \\
\hline Islam & 15 & 1,6 & \\
\hline Hinduismus & 0 & 0,0 & \\
\hline Buddhismus & 10 & 1,1 & \\
\hline Judentum & 0 & 0,0 & \\
\hline Keine Religionszugehörigkeit & 383 & 41,2 & \\
\hline Andere Religion & 26 & 2,8 & \\
\hline Keine Angabe & 0 & & \\
\hline
\end{tabular}




\begin{tabular}{|c|c|c|c|}
\hline F42:Welche Partei haben Sie bei der B & estag & $017 \mathrm{ge}$ & \\
\hline $\begin{array}{l}\text { Christlich Demokratische Union } \\
\text { Deutschlands (CDU) }\end{array}$ & 168 & 18,1 & 930 \\
\hline $\begin{array}{l}\text { Sozialdemokratische Partei } \\
\text { Deutschlands (SPD) }\end{array}$ & 138 & 14,8 & \\
\hline Alternative für Deutschland (AfD) & 93 & 10,0 & \\
\hline Freie Demokratische Partei (FDP) & 68 & 7,3 & \\
\hline DIE LINKE (DIE LINKE) & 95 & 10,2 & \\
\hline $\begin{array}{l}\text { BÜNDNIS 90/DIE GRÜNEN } \\
\text { (GRÜNE) }\end{array}$ & 97 & 10,4 & \\
\hline $\begin{array}{l}\text { Christlich-Soziale Union in Bayern } \\
\text { (CSU) }\end{array}$ & 20 & 2,2 & \\
\hline FREIE WÄHLER (FW) & 9 & 1,0 & \\
\hline $\begin{array}{l}\text { Die PARTEI - Partei für Arbeit, } \\
\text { Rechtsstaat, Tierschutz, } \\
\text { Elitenförderung und } \\
\text { basisdemokratische Initiative }\end{array}$ & 6 & 0,6 & \\
\hline $\begin{array}{l}\text { Tierschutzpartei - PARTEI MENSCH } \\
\text { UMWELT TIERSCHUTZ }\end{array}$ & 20 & 2,2 & \\
\hline $\begin{array}{l}\text { Nationaldemokratische Partei } \\
\text { Deutschlands (NPD) }\end{array}$ & 2 & 0,2 & \\
\hline Piratenpartei Deutschland (PIRATEN) & 5 & 0,5 & \\
\hline $\begin{array}{l}\text { Ökologisch-Demokratische Partei } \\
(O ̈ D P)\end{array}$ & 5 & 0,5 & \\
\hline $\begin{array}{l}\text { V-Partei }{ }^{3} \text { - Partei für Veränderung, } \\
\text { Vegetarier und Veganer (V-Partei) }\end{array}$ & 0 & 0,0 & \\
\hline Tierschutzallianz & 6 & 0,6 & \\
\hline $\begin{array}{l}\text { Ich habe nicht an der } \\
\text { Bundestagswahl teilgenommen }\end{array}$ & 123 & 13,2 & \\
\hline Andere Partei & 75 & 8,1 & \\
\hline Keine Angabe & 0 & & \\
\hline Welche andere Partei haben Sie gewähl & & & \\
\hline
\end{tabular}




\section{Anhang C: Quotierung der Stichprobe}

\begin{tabular}{|c|c|c|}
\hline & Mio. & $\%$ \\
\hline Basis & 54,50 & 100 \\
\hline \multicolumn{3}{|l|}{ Geschlecht } \\
\hline männlich & 27,42 & 50 \\
\hline weiblich & 27,07 & 50 \\
\hline \multicolumn{3}{|l|}{ Alter } \\
\hline 14 bis 19 Jahre & 1,65 & 3 \\
\hline 20 bis 29 Jahre & 9,68 & 18 \\
\hline 30 bis 39 Jahre & 9,83 & 18 \\
\hline 40 bis 49 Jahre & 11,22 & 21 \\
\hline 50 bis 59 Jahre & 12,74 & 23 \\
\hline 60 bis 69 Jahre & 9,36 & 17 \\
\hline \multicolumn{3}{|l|}{ Bundesländer } \\
\hline Schleswig-Holstein & 1,85 & 3 \\
\hline Hamburg & 1,18 & 2 \\
\hline Niedersachsen & 5,30 & 10 \\
\hline Bremen & 0,45 & 1 \\
\hline Nordrhein-Westfalen & 11,76 & 22 \\
\hline Hessen & 4,12 & 8 \\
\hline Rheinland-Pfalz & 2,73 & 5 \\
\hline Baden-Württemberg & 7,18 & 13 \\
\hline Bayern & 8,54 & 16 \\
\hline Saarland & 0,65 & 1 \\
\hline Berlin & 2,35 & 4 \\
\hline Brandenburg & 1,66 & 3 \\
\hline Mecklenburg-Vorpommern & 1,09 & 2 \\
\hline Sachsen & 2,66 & 5 \\
\hline Sachsen-Anhalt & 1,50 & 3 \\
\hline Thüringen & 1,46 & 3 \\
\hline
\end{tabular}




\section{Bildung}

(noch) kein allgemeiner Schulabschluss, noch Schüler

\begin{tabular}{|l|l|}
\hline 0,42 & 1 \\
\hline 3,31 & 6 \\
\hline 13,24 & 24 \\
\hline 18,72 & 34 \\
\hline 8,93 & 16 \\
\hline 9,87 & 18 \\
\hline
\end{tabular}

Hauptschulabschluss; ohne abgeschlossene

Lehre/Berufsausbildung

Hauptschulabschluss mit abgeschlossener

Lehre/Berufsausbildung

\begin{tabular}{|l|l|l|}
\hline Realschulabschluss/Mittlere Reife/Oberschule & 18,72 & 34 \\
\hline Abitur, (Fach-) Hochschulreife ohne Studium & 8,93 & 16 \\
\hline Studium (Universität, Hochschule, Fachhochschule) & 9,87 & 18 \\
\hline
\end{tabular}

Haushaltseinkommen

\begin{tabular}{|l|l|l|}
\hline bis unter 500 Euro & 0,57 & 1 \\
\hline 500 bis unter 750 Euro & 1,07 & 2 \\
\hline 750 bis unter 1.000 Euro & 2,77 & 5 \\
\hline 1.000 bis unter 1.250 Euro & 1,93 & 4 \\
\hline 1.250 bis unter 1.500 Euro & 3,57 & 7 \\
\hline 1.500 bis unter 1.750 Euro & 2,76 & 5 \\
\hline 1.750 bis unter 2.000 Euro & 3,32 & 6 \\
\hline 2.000 bis unter 2.250 Euro & 3,23 & 6 \\
\hline 2.250 bis unter 2.500 Euro & 4,04 & 7 \\
\hline 2.500 bis unter 3.000 Euro & 6,90 & 13 \\
\hline 3.000 bis unter 3.500 Euro & 5,43 & 10 \\
\hline 3.500 bis unter 4.000 Euro & 5,04 & 9 \\
\hline 4.000 bis unter 4.500 Euro & 4,61 & 8 \\
\hline 4.500 bis unter 5.000 Euro & 3,74 & 7 \\
\hline 5.000 Euro und mehr & 5,52 & 10 \\
\hline
\end{tabular}




\section{Anhang D: Eidesstattliche Erklärungen}

Hiermit erkläre ich, Marko Freckmann, geboren am 27.06.1978 in Duderstadt, eidesstattlich, dass:

diese Arbeit weder in gleicher noch in ähnlicher Form bereits anderen Prüfungsbehörden vorgelegen hat.

ich mich an keiner anderen Hochschule um einen Doktorgrad beworben habe.

Göttingen, den 23. Oktober 2020

(Unterschrift)

Hiermit erkläre ich, Marko Freckmann, geboren am 27.06.1978 in Duderstadt, eidesstattlich, dass diese Dissertation selbstständig und ohne unerlaubte Hilfe angefertigt wurde.

Göttingen, den 23. Oktober 2020

(Unterschrift) 


\section{Anhang E: Vermerk Sprachgebrauch}

In der vorliegenden Arbeit sind stets Personen männlichen und weiblichen und diversen Geschlechts gemeint. Wenn in anderen Teilen dieser Arbeit zur Vereinfachung und besseren Lesbarkeit die kürzere männliche Form (z.B. der Verbraucher) verwendet wird, dann sind selbstverständlich ausdrücklich auch weibliche und diverse Personen gemeint. 